Jochen Schellinger

Kim Oliver Tokarski

Ingrid Kissling-Näf Hrsg.

Digitale Transformation

und Unternehmensführung

Trends und Perspektiven für die Praxis 
Digitale Transformation und Unternehmensführung 
Jochen Schellinger - Kim Oliver Tokarski · Ingrid Kissling-Näf

(Hrsg.)

Digitale Transformation und Unternehmensführung

Trends und Perspektiven für die Praxis 
Hrsg.

Jochen Schellinger

Departement Wirtschaft, Berner

Fachhochschule

Bern, Schweiz

Ingrid Kissling-Näf

Departement Wirtschaft, Berner

Fachhochschule

Bern, Schweiz
Kim Oliver Tokarski

Departement Wirtschaft, Berner

Fachhochschule

Bern, Schweiz

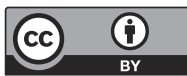

ISBN 978-3-658-26959-3 ISBN 978-3-658-26960-9 (eBook)
https://doi.org/10.1007/978-3-658-26960-9

Die Deutsche Nationalbibliothek verzeichnet diese Publikation in der Deutschen Nationalbibliografie; detaillierte bibliografische Daten sind im Internet Über http://dnb.d-nb.de abrufbar.

\section{Springer Gabler \\ (C) Der/die Herausgeber bzw. der/die Autor(en) 2020. Dieses Buch ist eine Open-Access-Publikation.}

Open Access Dieses Buch wird unter der Creative Commons Namensnennung 4.0 International Lizenz (http://creativecommons.org/licenses/by/4.0/deed.de) veröffentlicht, welche die Nutzung, Vervielfältigung, Bearbeitung, Verbreitung und Wiedergabe in jeglichem Medium und Format erlaubt, sofern Sie den/die ursprünglichen Autor(en) und die Quelle ordnungsgemäß nennen, einen Link zur Creative Commons Lizenz beifügen und angeben, ob Änderungen vorgenommen wurden.

Die in diesem Buch enthaltenen Bilder und sonstiges Drittmaterial unterliegen ebenfalls der genannten Creative Commons Lizenz, sofern sich aus der Abbildungslegende nichts anderes ergibt. Sofern das betreffende Material nicht unter der genannten Creative Commons Lizenz steht und die betreffende Handlung nicht nach gesetzlichen Vorschriften erlaubt ist, ist für die oben aufgeführten Weiterverwendungen des Materials die Einwilligung des jeweiligen Rechteinhabers einzuholen.

Die Wiedergabe von allgemein beschreibenden Bezeichnungen, Marken, Unternehmensnamen etc. in diesem Werk bedeutet nicht, dass diese frei durch jedermann benutzt werden dürfen. Die Berechtigung zur Benutzung unterliegt, auch ohne gesonderten Hinweis hierzu, den Regeln des Markenrechts. Die Rechte des jeweiligen Zeicheninhabers sind zu beachten.

Der Verlag, die Autoren und die Herausgeber gehen davon aus, dass die Angaben und Informationen in diesem Werk zum Zeitpunkt der Veröffentlichung vollständig und korrekt sind. Weder der Verlag, noch die Autoren oder die Herausgeber übernehmen, ausdrücklich oder implizit, Gewähr für den Inhalt des Werkes, etwaige Fehler oder Äußerungen. Der Verlag bleibt im Hinblick auf geografische Zuordnungen und Gebietsbezeichnungen in veröffentlichten Karten und Institutionsadressen neutral.

Springer Gabler ist ein Imprint der eingetragenen Gesellschaft Springer Fachmedien Wiesbaden GmbH und ist ein Teil von Springer Nature.

Die Anschrift der Gesellschaft ist: Abraham-Lincoln-Str. 46, 65189 Wiesbaden, Germany 


\section{Geleitwort}

Der Fortschritt bei den Informations- und Kommunikationstechnologien (IKT) eliminiert ökonomische Handlungseinschränkungen und schafft völlige neue Handlungsmöglichkeiten. Das führt zu Innovationen, die bislang schlicht unvorstellbar waren. Kreativität, Designtalent und Online-Sozialkompetenz rücken ins Zentrum. Neue Ressourcen - beispielsweise intelligent eingesetzte intelligente Maschinen, intelligent genutzte Crowdintelligenz und Wettbewerbe - beschleunigen den Fortschritt. Hohe Flexibilität, Einfühlungsvermögen in andere, die Fähigkeit zu vielfältigen Formen der Zusammenarbeit, Disziplin und Vertrauenswürdigkeit werden zu Schlüsselkompetenzen - insbesondere dort, wo durch agile Ablaufstrukturen die Entwicklungsproduktivität gesteigert wird. Und dort, wo es um wirklich viel geht - Menschenleben, hohe Gewinne oder das Überleben von Unternehmen -, wird aus dem Schlagwort „,disziplinenübergreifende Teamarbeit" gelebte Wirklichkeit.

Gleichzeitig destabilisiert der IKT-Fortschritt grundsätzlich alle Arten von Geschäftsmodellen, frei nach dem Grundsatz: „Irgendwann wird alles kostenlos, nur nicht zur selben Zeit". Denn es ist viel einfacher als früher, Geschäftsmodelle zu kopieren oder durch alternative Geschäftsmodelle zu konkurrenzieren. Schumpeters kreative Zerstörung offenbart in der digitalen Transformation so ihr wahres Wesen, auch wenn dieses oft irritierend anders ist, als wir glaubten, und wir es erst in Ansätzen erkennen. Insbesondere wird Einzigartigkeit für Unternehmen immer schwieriger und ist fast nur mehr durch Street Credibility, durch hoch entwickelte Zusammenarbeitsformen oder durch einzigartige Expertise in der Nutzung und Beherrschung von IKT möglich.

Die durch den IKT-Fortschritt angestoßene digitale Transformation der Wirtschaft wird durch eine Vielzahl sehr unterschiedlicher Phänomene geprägt. Die Märkte und die Gesellschaft verändern sich gleichzeitig, wobei kulturelle und technische Phänomene ineinandergreifen oder zumindest wechselwirken. Das Ergebnis ist ein geradezu berauschender Bedeutungswandel altehrwürdiger ökonomischer und soziologischer Konzepte. Zwei Beispiele veranschaulichen dies. Die Blockchain-Bewegung revoltiert gegen das Establishment ganz ähnlich wie das die 68er taten oder in Zürich die 80er-Bewegung. Ziel der Revolte sind die Institutionen, die durch Distributed-Ledger-Technologien ersetzt werden sollen. Dabei verbinden sich sozialistische und kapitalistische Ideen und 
legen das Fundament für einen möglichen Trend zu Werteökonomien - Ausgang offen. Ein anderes Beispiel ist der Wandel des Stallgeruch-Konzepts, das neu als Street Credibility (Stallgeruch 2.0) gegenüber Kunden auftritt. Wobei schon heute klar ist, dass in Zukunft die Fähigkeit, für Frauen als IKT-Arbeitgeber glaubwürdig zu sein, die entscheidende Street Credibility gegenüber Mitarbeitenden darstellen wird.

Die digitale Transformation führt einerseits zu zahlreichen, oft paarweise gegenläufigen Trends, beispielsweise wenn Cyberwährung gegen den Automatischen Informationsaustausch (AIA) antritt und anderseits $\mathrm{zu}$ einer gewaltigen Ausweitung der wirtschaftlichen und geopolitischen Kampfzone. Wer hier den Fortschritt in der IKT-Nutzung und die damit verbundenen neu entstehenden Geschäftsmodelle und fachlichen und unternehmerischen Geschäftspraktiken aus den Augen verliert, verliert auch schnell die Orientierung und den Sinn für Chancen und Gefahren. Denn gerade weil Digitalisierung ein Enabler für alle ist, ist es nur in Nischen möglich, sie gefahrlos zu ignorieren. Sie schafft neue Konkurrenten, die oft transversal in den Markt eindringen, sie fördert kriminelle Aktivitäten, und sie ordnet das soziale Miteinander in Unternehmen neu. Darauf muss man reagieren, selbst wenn man neue Geschäftsmodelle und Praktiken nicht selber einführen will. Angesichts der Vielzahl neuer Möglichkeiten sollte man aber zuallererst die großartigen Chancen sehen: Digitalisierung ist in erster Linie eine Befreiung von vielen unlösbaren Problemen der Vergangenheit, die durch sie marginalisiert werden.

Resultate der Grundlagenforschung - beispielsweise im Bereich Social Cybersecurity - deuten darauf hin, dass zufällige Prozesse in sozialen Netzwerken immer mehr an Bedeutung gewinnen und sehr anders funktionieren, als wir es bisher angenommen haben. Oft gilt das Gegenteil von dem, was der Hausverstand nahelegt, nicht selten auch das Gegenteil dessen, wovon bisherige Forschung ausging. Denn es ist die Aneignung der IKT, die fast immer den eigentlichen Wandel herbeiführt und oft nicht der technologischen oder ökonomischen oder politischen Logik folgt. Dies schafft scheinbare Paradoxien und führt zu einer scheinbar zufälligen Entwicklung. Bei aller Zufälligkeit wissen wir aber aus der Grundlagenforschung - und vor allem auch aus der angewandten Forschung - zur digitalen Transformation genügend viel Konkretes, dass eine Auseinandersetzung mit diesem Wissen einen großen Wettbewerbsvorteil bringt.

Andere Resultate der Grundlagenforschung, beispielsweise im Bereich der ökonomischen Komplexität, legen nahe, dass systemische Effekte zu großen Chancenungleichheiten für einzelne Länder und Regionen führen. Wachstum gelingt vor allem dort, wo die Fachkompetenzen und innovatives Unternehmertum so zusammenfinden, dass sie die durch die Digitalisierung ermöglichten neuen Handlungsfreiräume nutzen. Was dies für die Bildungspolitik impliziert, ist derzeit ein zentrales Thema für die angewandte Forschung. Klar ist dabei, dass die Bildungspolitik den Wandel antizipieren sollte. Hochschulen müssen dabei nur 10 Jahre voraussehen, dies dafür sehr konkret, und ihre Ausbildungsprogramme entsprechend anpassen. Volksschulen müssen dagegen die nächsten 25 Jahre in Kernzügen antizipieren und insbesondere sicherstellen, dass sie 
die grundlegenden Kompetenzen für die digital transformierte Wirtschaft genderneutral vermitteln.

Der vorliegende Band leistet einen wertvollen Beitrag, die derzeitigen und anstehenden Entwicklungen der Geschäftsmodelle und Praktiken der digitalen Wirtschaft besser $\mathrm{zu}$ verstehen. Er stellt die Beschreibung und Analyse von exemplarischen Innovationsfeldern ins Zentrum. Damit hilft der Band, konkreter zu begreifen, was alles heute möglich ist (beziehungsweise in den nächsten Jahren möglich werden wird), wenn wir das durch den IKT-Fortschritt geschaffene Innovationspotenzial nutzen (oder uns nicht davor wappnen, dass andere dies tun). Dabei machen die einzelnen Beispiele klar, dass in der digital transformierten Wirtschaft altbekannte ökonomische Wirkungsmechanismen weiterhin gelten, dabei aber neue Wirkungsformen und damit teilweise auch neue Gestalt annehmen. Deshalb braucht es neben den traditionellen auch völlig neue Expertisen für eine erfolgreiche Unternehmensführung. Welche dies sind und in welche Richtung der transformative Wandel der Wirtschaft geht, das erfahren Sie in 15 spannenden Fachbeiträgen. Ich wünsche Ihnen viele Erkenntnisse und neue Ideen beim Lesen.

Bern, Schweiz

Reinhard Riedl

im Oktober 2019

Präsident der Schweizer Informatik Gesellschaft (SI) 


\section{Vorwort}

Die Digitalisierung, respektive die mit ihr verbundene digitale Transformation von Wirtschaft und Gesellschaft, sind mittlerweile zu einem Leitthema auch der betriebswirtschaftlichen Forschung geworden. Die Thematik genießt aufgrund ihrer für Kunden und Mitarbeitende alltäglichen Spürbarkeit, die auch über die Produkt- und Arbeitsmarktbeziehungen hinausgeht, wegen der mit ihr verbundenen Chancen und Bedrohungspotenziale derzeit auch weit über den wissenschaftlichen Bereich hinaus eine hohe mediale Aufmerksamkeit. In den vergangenen Jahren haben sich für die verschiedenen funktionalen Handlungsfelder einer Unternehmensführung durch und mit der Digitalisierung eine Fülle neuer Gestaltungsoptionen ergeben. Angesichts der Breite, des Tiefgangs und der dynamischen Expansion digitaler Zugänge zur Unternehmensführung kann in einem weit gefassten Verständnis von einer zunehmenden Digitalisierung der Unternehmensführung oder, verkürzt, auch von einer ,digitalen Unternehmensführung“ als neuer Perspektive für das Management der Zukunft ausgegangen werden. „Digital führen“ heißt in diesem Kontext aber nicht, dass die Führung weitgehend automatisiert und standardisiert durch Systeme und Maschinen erfolgt, sondern dass nach wie vor Menschen als kollaborative und kreative Gestalter agieren und die neuen digitalen Möglichkeiten kundenorientiert nutzen. Der vorliegende Herausgeberband gibt einen exemplarischen Einblick in zentrale Managementbereiche einer solchen digitalen Unternehmensführung.

Die Beiträge befassen sich empirisch mit einzelnen Gestaltungsaspekten der Digitalen Transformation im Umfeld der Schweizer Wirtschaft. Die ausgewählten Themengebiete werden theoretisch-konzeptionell und praktisch-deskriptiv mit dem Ziel der Ableitung von Gestaltungshinweisen für die Unternehmenspraxis analysiert. Der Band zeichnet sich damit durch eine hohe Praxisorientierung aus, was sich auch in der Zusammensetzung der Autorenschaft widerspiegelt. Sämtliche Beiträge wurden durch Autorentandems mit Vertretern aus Wissenschaft und Praxis erstellt. Die Praktiker verfügen über einen ökonomischen Hintergrund und arbeiten überwiegend in Schweizer Unternehmen und Organisationen in verantwortungsvollen Aufgaben und verschiedensten Tätigkeitsgebieten. Die beteiligten Forschenden und Lehrenden des Departements Wirtschaft der Berner Fachhochschule repräsentieren einen stärker wissenschaftsgeprägten Blickwinkel auf die untersuchten Analysefelder. Dieses Setting hat sich auch bereits in einer Reihe früherer 
Publikationen als sehr fruchtbar erwiesen und entspricht in hohem Maße dem anwendungsorientierten Forschungsansatz der Schweizer Fachhochschulen. Aus der Integration der Praxis- und Wissenschaftsperspektiven resultieren eine Reihe von neuen Erkenntnissen zum Forschungsfeld der digitalen Transformation und einer mit ihr verbundenen digitalen Unternehmensführung.

Ganz in Sinne der laufenden digitalen Revolution und der Vorgaben von swissuniversities zur Bereitstellung von öffentlichen Forschungs- und Praxisresultaten haben wir uns entschlossen, den vorliegenden Sammelband als Open-Access-Publikation mit einer Creative-Commons-Lizenz zu erstellen und freuen uns auf eine hiermit verbundene Erweiterung und Vereinfachung des Zugangs zur Nutzung der Forschungsresultate und Diskussionsbeiträge.

Unser Dank gilt allen Autoren für deren Engagement bei der Erstellung des Sammelbandes sowie Prof. Dr. Reinhard Riedl, dem Präsidenten der Schweizer Informatikgesellschaft, für sein Geleitwort. Wieder einmal geht ein besonderes Dankeschön an unsere bewährte Buchprojektkoordinatorin Frau Sabrina Weber, deren wertvolle Unterstützung für das nun abgeschlossene Projekt unabdingbar war. Gleichgerichtet bedanken wir uns auch bei allen Mitwirkenden des Springer Gabler Verlags.

Die Herausgeber wünschen dem Werk über die Schweiz hinausgehend eine hohe Aufmerksamkeit bei Praktikern, bei Studierenden, Lehrenden und Forschenden und allen Lesern viel Freude und Gewinn bei der Lektüre.

Bern, Schweiz

im Oktober 2019
Jochen Schellinger

Kim Oliver Tokarski

Ingrid Kissling-Näf 


\section{Inhaltsverzeichnis}

1 Von der digitalen Transformation zur digitalen

Unternehmensführung

Jochen Schellinger, Kim Oliver Tokarski und Ingrid Kissling-Näf

2 Digitale Transformation und Open Access von

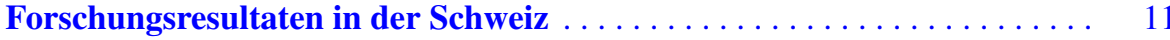

Dirk Verdicchio und Ingrid Kissling-Näf

$3 \quad$ Plattformbasierte Dienstleistungen . . . . . . . . . . . . . . . . . . . . 29

Katinka Weissenfeld, Angelina Dungga und Jan Frecè

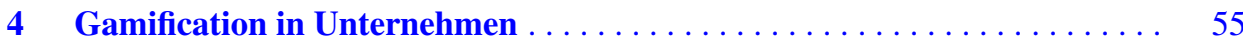

Thomas Ellenberger, Deane Harder und Marie Brechbühler Pešková

5 Cybersicherheit als Führungsaufgabe in Schweizer KMU . . . . . . . . . 83

Dominique Adrian Meier und Daniel Burda

6 Ambidextrie - der organisationale Drahtseilakt . . . . . . . . . . . . 105

Simon Jonathan Schneeberger und Anja Habegger

7 Digitalisierung: Perspektiven für Arbeitsmodelle der Zukunft

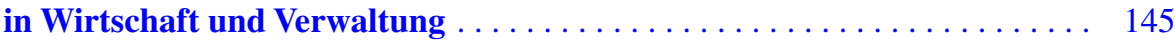

Jochen Schellinger und Gia Le Huynh

8 Digitale Transformation und Human Resource Management . . . . . . . . 183

Jochen Schellinger, Marlies Goedermans,

Lars Patrick Kolb und Yassin Sebai

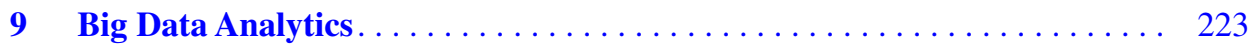

Flurina Fiona Baumann, Nadine Belinda Brunner

und Kim Oliver Tokarski

10 Kryptowährungen. . . . . . . . . . . . . . . . . . . . . . . . . . . 249

Tobias Wenger und Kim Oliver Tokarski 
11 Das Potenzial von Smart Contracts an einem Beispiel aus der Finanzbranche - Smart Rating

Vincent Furrer und Klaus-Georg Deck

12 Erfolgsfaktoren für die Kundengewinnung

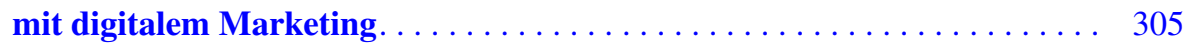

Seraina Ulber und Etienne Rumo

13 Augmented Reality als Entscheidungshilfe beim Möbelkauf . . . . . . . . . 355

David Aemmer, Jonas Bigler, Marco Birkhofer,

Marie Brechbühler Pešková und Deane Harder

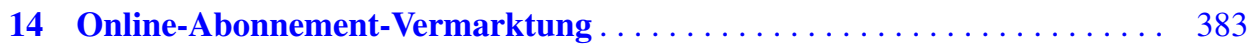

Romain Descloux und Etienne Rumo

15 Social Influencer . . . . . . . . . . . . . . . . . . . . . . . . . . 407

Jonas von Rotz und Kim Oliver Tokarski 


\section{Herausgeber- und Autorenverzeichnis}

\section{Über die Herausgeber}

Prof. Dr. Jochen Schellinger Studiengangsleiter Master of Science in Business Administration an der Berner Fachhochschule. Lehr- und Forschungstätigkeiten in den Bereichen Strategisches Management, Marketing und Personalmanagement. Davor 15 Jahre Praxistätigkeit bei der Deutschen Sparkassenorganisation, im Haniel-Konzern, an der Universität Tübingen sowie in der Daimler AG.

Prof. Dr. Kim Oliver Tokarski Professor für Entrepreneurship und Unternehmensführung und Leiter der Abteilung Weiterbildung am Departement Wirtschaft der Berner Fachhochschule. Er ist Gastprofessor an der Wirtschaftsuniversität Bukarest (ASE Bukarest), Rumänien. Seine Forschungs- und Lehrtätigkeiten liegen in den Bereichen Entrepreneurship, Social Entrepreneurship, Innovation, Digitalisierung, Strategie sowie Leadership. Er ist Autor unterschiedlicher Publikationen in den genannten Themenkontexten.

Prof. Dr. Ingrid Kissling-Näf Direktorin Departement Wirtschaft der Berner Fachhochschule. Forschungserfahrung als Assistenzprofessorin in Ressourcenökonomie an der ETHZ und langjährige Führungserfahrung unter anderem beim Schweizerischen Nationalfonds Abteilung Geistes- und Sozialwissenschaften, bei der Förderagentur für Innovation KTI sowie bei der Akademie der Naturwissenschaften Schweiz. Während zwei Jahren tätig als Beraterin in der Privatwirtschaft bei der Firma Ernst Basler \& Partner AG. Sie ist zudem UNICEF-Delegierte und Stadträtin in Bern. 


\section{Autorenverzeichnis}

David Aemmer Kerzers, Schweiz

Flurina Fiona Baumann Bern, Schweiz

Jonas Bigler Bern, Schweiz

Marco Birkhofer Port, Schweiz

Prof. Dr. Marie Brechbühler Pešková Berner Fachhochschule Wirtschaft, Bern, Schweiz

Nadine Belinda Brunner Muttenz, Schweiz

Prof. Dr. Daniel Burda Berner Fachhochschule Wirtschaft, Bern, Schweiz

Dr. Klaus-Georg Deck Berner Fachhochschule Wirtschaft, Bern, Schweiz

Romain Descloux Bern, Schweiz

Angelina Dungga Berner Fachhochschule Wirtschaft, Bern, Schweiz

Thomas Ellenberger Bern, Schweiz

Dr. Jan Frecè Berner Fachhochschule Wirtschaft, Bern, Schweiz

Vincent Furrer Bern, Schweiz

Marlies Goedermans Bern, Schweiz

Prof. Dr. Anja Habegger Berner Fachhochschule Wirtschaft, Bern, Schweiz

Prof. Dr. Deane Harder Berner Fachhochschule Wirtschaft, Bern, Schweiz

Gia Le Huynh Berner Fachhochschule, Departement Wirtschaft, Bern, Schweiz

Lars Patrick Kolb Ittigen, Schweiz

Dominique Adrian Meier Bülach, Schweiz

Jonas von Rotz Bern, Schweiz

Dr. Etienne Rumo Berner Fachhochschule Wirtschaft, Bern, Schweiz

Simon Jonathan Schneeberger Spiez, Schweiz

Yassin Sebai Bern, Schweiz

Seraina Ulber Chur, Schweiz 
Dirk Verdicchio Digitale Dienste \& Open Science, Universitätsbibliothek Bern, Bern, Schweiz

Katinka Weissenfeld Berner Fachhochschule Wirtschaft, Bern, Schweiz

Tobias Wenger Bern, Schweiz 


\title{
Von der digitalen Transformation zur digitalen Unternehmensführung
}

\author{
Jochen Schellinger, Kim Oliver Tokarski und Ingrid Kissling-Näf
}

\begin{abstract}
Zusammenfassung
Der Metatrend „Digitalisierung“ und die mit ihm einhergehende digitale Transformation gesellschaftlicher und ökonomischer Strukturen und Prozesse stellen eine zentrale Herausforderung für die Unternehmensentwicklung dar. Fundamentale Änderungen der Strukturen und Prozesse der Leistungserstellung, andersartige und neue Leistungsangebote und veränderte Kundenbeziehungen erfordern eine grundlegende Erweiterung der Führungsperspektive in Richtung einer ,digitalen Unternehmensführung“. Für deren funktionale Handlungsfelder ist für die Zukunft von einer weitreichenden und kontinuierlichen Erhöhung des Digitialisierungsgrads auszugehen. Die Beiträge aus den untersuchten Bereichen Führung und Organisation, Human Resource Management, Marketing und Finanzwirtschaft werden zusammengefasst. Sie zeigen das große Gestaltungsspektrum der digitalen Unternehmensführung exemplarisch auf und geben konkrete Hinweise für Problemlösungen in der Unternehmenspraxis.
\end{abstract}

Die Digitalisierung von Wirtschaft und Gesellschaft ist ein Phänomen, dem als derzeit wohl bedeutsamste global wirksam werdende Umweltveränderung für die strategische Entwicklung von Unternehmen eine wachsende Relevanz zukommt. Bei der

J. Schellinger $(\triangle) \cdot$ K. O. Tokarski $\cdot$ I. Kissling-Näf

Departement Wirtschaft, Berner Fachhochschule, Bern, Schweiz

E-Mail: jochen.schellinger@bfh.ch

K. O. Tokarski

E-Mail:kim.tokarski@bfh.ch

I. Kissling-Näf

E-Mail: ingrid.kissling@bfh.ch

J. Schellinger et al. (Hrsg.), Digitale Transformation und Unternehmensführung,

https://doi.org/10.1007/978-3-658-26960-9_1 
Digitalisierung handelt es sich um einen Metatrend, der die Unternehmenswelt und ihre zugrunde liegenden Führungsperspektiven grundlegend verändert und mit einer wachsenden Immaterialisierung von Ressourcen, Prozessen und Produkten einhergeht (Cachelin 2013). Die mit dem Einsatz neuer digitaler Technologien verbundenen gravierenden Veränderungen können unter dem Begriff der ,digitalen Transformation“ subsumiert werden und beeinflussen im ökonomischen Kontext die gesamte Wertschöpfungskette. Ursachen dieser Veränderungen sind die direkten und indirekten Technologieauswirkungen in Bezug auf Rahmenbedingungen und Strukturen des Wirtschaftens sowie auf völlig neuartige Produkt- und Dienstleistungsangebote. Besonders bedeutsame Technologiefelder sind in diesem Zusammenhang der Einsatz von Sensoren, die mobile Kommunikation, der Umgang mit großen Datenmengen (Big Data, Clouds), Maschinenlernen und künstliche Intelligenz, neue Ausprägungen der Mensch-Computer-Interaktion, Simulationstechniken (Virtual und Augmented Reality) sowie der integrierte Einsatz solcher und weiterer Technologiefelder bei der Produktentwicklung (neue digitale Geschäftsmodelle), der Produktion (Industrie 4.0), der Vermarktung und der Kundeninteraktion (Pousttchi 2017). Bei der Wertgenerierung liegt der Fokus dabei auf der Erzielung von Effizienz- und Effektivitätsvorteilen durch die Optimierung von Strukturen und Prozessen der Leistungserstellung, die in der Regel mit einer erheblichen Beschleunigung von Arbeitsabläufen und einer zunehmenden Verdichtung und Komplexität der Arbeitstätigkeiten verbunden sind. In Bezug auf das Leistungsangebot steht eine intelligente Verknüpfung der technologischen Möglichkeiten zur Nutzung ökonomischer Opportunitäten durch neuartige Produkte und Dienstleistungen im Vordergrund. Hinsichtlich der Kundenbeziehungen gilt es, neue kommunikative Möglichkeiten (Online- und Multichannel-Marketing) sowie datenbasierte Optimierungen der Zielgruppenausrichtung und individualisierter Serviceangebote zu nutzen (Pousttchi 2017; Westermann et al. 2014; Bankl 2014, S. 306).

Angesichts der laufenden und weiter noch bevorstehenden gravierenden digitalisierungsbedingten Umwälzungen der Wirtschaftswelt und der Gesellschaft ist neben dem Einbezug dieser internen und externen Umweltveränderungen in der strategischen Planung auch eine grundlegende Erweiterung der Führungsperspektive erforderlich (siehe dazu auch Putzinger 2016; Cole 2017). Die Digitalisierung durchdringt in zunehmendem Maße alle Führungs- und Funktionsbereiche von Unternehmen und Organisationen und verändert die Art und Weise, wie Unternehmen in Zukunft geführt werden. Die mit den technologischen Möglichkeiten verbundene Flexibilisierung und Individualisierung der Wirtschaftsbeziehungen führt $\mathrm{zu}$ zunehmend komplexer werdenden Wertschöpfungs- und Vermarktungsprozessen, die wiederum mit gleichermaßen komplexen technologischen Lösungen bewältigt werden müssen. Gleichzeitig steigt der Bedarf, einfache Lösungen von Kunden- und Betriebsproblemen zu generieren. Agilisierung, Individualisierung, Lateralisierung und Holokratisierung der Führungsbeziehungen und -systeme scheinen hier für die Zukunft wegweisende Entwicklungsrichtungen für in wachsendem Maße selbstorganisierte Netzwerkorganisationen mit fortgeschrittener digitaler Infrastruktur. Im Hinblick auf die funktionalen Handlungsfelder der Unternehmensführung ist für die Zukunft von einer weitreichenden und kontinuierlichen 
Erhöhung des Digitialisierungsgrads der Forschung und Entwicklung, der Beschaffung, der Produktion, des Marketings, von Finanzwirtschaft und Controlling, der Organisation sowie des Human Resource Managements auszugehen. Vor diesem Hintergrund scheint der Terminus einer „digitalen Unternehmensführung“ als Management-Entwicklungsperspektive und Konsequenz der digitalen Transformation, aber auch im Sinne einer unternehmerischen Bewältigungsstrategie für die Digitalisierung von Wirtschaft und Gesellschaft naheliegend.

Einzelne funktionale Handlungsfelder werden im vorliegenden Herausgeberband pointiert und exemplarisch im Sinne von Teilaspekten einer digitalen Unternehmensführung aufgezeigt. Der erste Themenblock befasst sich mit ausgewählten Rahmensetzungen und grundlegenden Aspekten der digitalen Transformation, es folgen Beiträge aus den Bereichen Führung und Organisation, Human Resource Management, Marketing und Finanzwirtschaft.

Für einen ersten Basisaspekt der digitalen Transformation, der wachsenden Verfügbarkeit von Wissen(-schaft), erfolgt im zweiten Beitrag „Digitale Transformation und Open Access von Forschungsresultaten in der Schweiz" eine Bestandsaufnahme der freien Zugänglichmachung von Forschungspublikationen für die Schweiz (Open Access). Grundlagen- und anwendungsorientierte Forschung geben wichtige Impulse für unternehmerische Innovationen und sind ein wichtiges Fundament für den Wohlstand in hochentwickelten Volkswirtschaften. Insofern erscheinen die Entwicklungstendenzen in Verbindung mit der Digitalisierung im Forschungsumfeld der Schweizer Unternehmen als wichtige Signalgeber für zukünftige Entwicklungen, die über die Hochschulwelt hinausgehen. Darüber hinaus werden aber auch der Markt für forschungsbasierte Publikationen und die Geschäftsmodelle für die in diesem Segment tätigen Verlage fundamental verändert. Die digitale Transformation hat einen erheblichen Einfluss auf die Arbeits- und Kommunikationsweisen der Wissenschaft und ermöglicht heute eine weitgehende Transparenz und Offenheit der Forschungsresultate. Die Begriffe „Open Science“ und „Open Access“ stehen für diese Öffnung wissenschaftlicher Prozesse und der Zugänglichmachung von Forschungsergebnissen. Für Open Access - dem freien Zugang zu wissenschaftlichen Publikationen - existieren schweizerische und internationale Programme und Strategien, die von Förderinstitutionen wie dem Schweizerischen Nationalfonds (SNF) und Wissenschaftsorganisationen wie swissuniversities getragen werden. Neben einem Überblick über die zentralen Begriffe und Konzepte geht der Beitrag auf diese Strategien ein. Besondere Beachtung finden die Situation in Europa und der Schweiz sowie der Wandel der Geschäftsmodelle und der Publikationslandschaft.

Mit der digitalen Transformation einher geht eine beschleunigte Entwicklung in Richtung Dienstleistungsgesellschaft als weiterer grundlegender Veränderungsaspekt, der von Beitrag drei „Plattformbasierte Dienstleistungen: Dienstleistungen als Treiber des gesellschaftlichen Wandels" aufgegriffen wird. Peer-to-Peer-Dienstleistungsplattformen (P2P-Plattformen) stellen eine besondere Form von Dienstleistungsplattformen dar, die zum Beispiel Geschäftsmodelle der Sharing Economy in ihrer heutigen Form 
ermöglichen. Als Reaktion auf den aktuell herrschenden Begriffsdschungel rund um Sharing Economy und Dienstleistungsplattformen im Allgemeinen werden zunächst wesentliche Begrifflichkeiten geklärt, bevor Aspekte des gesellschaftlichen Wandels diskutiert werden. Aufgrund iterativer Wechselwirkungen zwischen Sozialstrukturen und sozialen Akteuren werden technische, soziale, ökologische und ökonomische Aspekte des gesellschaftlichen Wandels sowohl als Folge als auch als Vorbedingungen für das Aufkommen von P2P-Plattformen betrachtet. Darauf aufbauend werden Chancen und Herausforderungen diskutiert, die sich aktuell und zukünftig durch P2P-Plattformen für die Gesellschaft ergeben. Dabei ist festzustellen, dass viele Aspekte sowohl Chancen als auch Herausforderungen mit sich bringen, die entsprechend abzuwägen sind.

Der vierte Beitrag „,Gamification in Unternehmen. Beeinflussung der Unternehmenskultur durch spieletypische Elemente" ist gleichzeitig der erste Beitrag im Themenbereich Führung und Organisation und befasst sich mit neuen Möglichkeiten zur zielgerichteten Beeinflussung von Werten und Normen in Unternehmen. Eine „gute“ Unternehmenskultur ist ein wesentlicher Erfolgsfaktor für Unternehmen. Im Rahmen der digitalen Transformation wird zunehmend auch Gamification zur zielgerichteten Kulturbeeinflussung eingesetzt. Dabei werden spieletypische Elemente genutzt, um für Mitarbeitende bezogen auf bestimmte Handlungen Anreize zu schaffen. Im Rahmen einer Fallstudie wurden digital umgesetzte Gamification-Maßnahmen eines Unternehmens bezüglich ihrer Wahrnehmung und Beeinflussung der Arbeitsmotivation analysiert. Die Ergebnisse zeigen, dass Gamification kulturwirksam zur Unternehmensführung genutzt werden kann. Die digitale Umsetzung diente als einheitlicher Impulsgeber und sorgte für Transparenz als Basis für Vertrauen. So konnte die angestrebte „Kultur auf Augenhöhe“ entwickelt werden, geprägt durch Respekt, Toleranz und Mitbestimmung. Der Erfolg der Gamification-Maßnahmen lässt sich vor allem durch die positive Wirkung auf die intrinsische Motivation sowie das gestärkte Zusammengehörigkeitsgefühl der Mitarbeitenden erklären.

In Verbindung mit der digitalen Transformation kristallisiert sich das Thema „Cybersicherheit“" als wichtiger werdendes Führungsfeld auch für kleine und mittlere Unternehmen heraus. Im fünften Beitrag „Cybersicherheit als Führungsaufgabe in Schweizer KMU. Herausforderungen und Chancen im Zuge der Digitalisierung "wird dies aufgegriffen. Diverse Studien zeigen, dass KMU Cybersicherheit als relevantes Handlungsfeld identifizieren, gleichzeitig aber nicht genügend adressieren. Die bestehende Forschung liefert für dieses Verhalten noch keine schlüssigen Erklärungsansätze. Dieses Phänomen wird in der Untersuchung als ,Security Paradox“ definiert und mit Bezug auf die in einem Multiple Case Design untersuchten Schweizer KMU eingehend beschrieben. Es werden dessen Ursachen aufgezeigt und praxisrelevante Empfehlungen für Führungskräfte und Entscheidungsträger im Umgang mit der digitalisierungsbedingten Herausforderung Cybersicherheit gegeben.

Der Beitrag sechs „Ambidextrie - der organisationale Drahtseilakt. Synergie zwischen Exploration und Exploitation als Voraussetzung für die digitale Transformation "widmet sich der Problematik einer organisatorischen Verankerung der Veränderungsfähigkeit 
von Unternehmen, die in Bezug auf die digitale Transformation essenziell ist. Sich disruptiv verändernde Rahmenbedingungen können dazu führen, dass bisher erfolgreiche Geschäftsmodelle innerhalb weniger Jahre obsolet werden. Geschuldet ist dies einer mangelnden Anpassungsfähigkeit, deren Ursache unter anderem in der sogenannten Pfadabhängigkeit wurzeln kann. Die Pfadabhängigkeit bezeichnet eine Situation, in der die Auswirkungen von stark durch die Vergangenheit geprägten Entscheiden dazu führen, dass die Möglichkeit, beziehungsweise Notwendigkeit neuer Geschäftsmodelle nicht erkannt, verpasst oder deren Chancen falsch eingeschätzt werden. Das Ausbrechen aus einer solchen Pfadabhängigkeit ist für den Fortbestand von Unternehmen daher von zentraler Bedeutung. Das Konzept der organisationalen Ambidextrie widmet sich dieser Problemstellung. Ambidextrie beschreibt die Fähigkeit eines Unternehmens, einerseits das Kerngeschäft stetig weiterzuentwickeln, gleichzeitig aber auch neue Wege und Denkweisen zu etablieren und so sicherzustellen, dass Veränderungen im Umsystem rechtzeitig erkannt und die sich dadurch bietenden Chancen für die Zukunft genutzt werden. Eine Fähigkeit, die gerade im Digitalisierungskontext überlebensnotwendig ist.

Die Auswirkungen der Digitalisierung auf die Arbeit und die dahinterstehenden Menschen sind gravierend und müssen vom Human Resource Management aufgegriffen werden. Beitrag sieben „Digitalisierung: Perspektiven für Arbeitsmodelle der Zukunft in Wirtschaft und Verwaltung " greift diesen Transformationsaspekt an der Schnittstelle zwischen Organisation und Human Resource Management mit Fokus auf administrative Arbeitszusammenhänge auf. In Bezug auf die Zusammenarbeit in Unternehmen und Organisationen sind bestehende Arbeitsmodelle für administrative Arbeit anzupassen und neue Formen digitaler Kollaboration einzuführen. Hiermit verbunden sind Änderungen der Arbeitszeitmodelle, der Organisation der Mitarbeitenden, der Art der Zusammenarbeit und Führung sowie der Gestaltung von Büroräumlichkeiten. In einem vergleichenden Case-Study-Ansatz von zwei Schweizer Großunternehmen und einer großen Verwaltungseinheit des Bundes wurden die existierenden Arbeitsmodelle für Büroarbeit analysiert und auf Entwicklungsperspektiven hin untersucht. Als Ergebnis wird unter anderem festgehalten, dass ein derzeit vorhandener Mix aus traditionellen und agilen Arbeitsmodellen sich in Zukunft im digitalen Kontext voraussichtlich unternehmensspezifisch stärker in Richtung einer höheren Agilität weiterentwickeln wird.

Der achte Beitrag „Digitale Transformation und Human Resource Management. Trends und Perspektiven einer zukunftsgerichteten Personalarbeit in Schweizer Großunternehmen “ betrachtet das digitale Führungsfeld Human Resource Management in seiner Gesamtheit, das sowohl als Objekt als auch als ein Treiber der digitalen Veränderungen in Erscheinung treten kann. Strategisch relevante Veränderungen wie Cloud Computing, Big Data und Social Media Management sowie Mobility-Trends wirken sich mittelbar und unmittelbar auch auf die Gestaltungsbereiche betrieblicher Personalarbeit aus. Der Beitrag geht theoretisch und empirisch der Frage nach der Relevanz und Konsequenz der Digitalisierung für das Personalmanagement in Schweizer Großunternehmen nach. Im Mittelpunkt steht die Vorstellung der Ergebnisse einer qualitativen Befragung von sechzehn HR-Managern führender Schweizer Großunternehmen aus verschiedensten 
Wirtschaftsbereichen und eines weltweit marktführenden eHR-Softwareanbieters. Grundlage hierfür war die Entwicklung eines Reifegradmodells, das versucht, die Digitalisierung anhand von acht Dimensionen des digitalen Wandels abgestuft zu spezifizieren. Die Schweizerische Unternehmenslandschaft scheint sich aufgrund der Erkenntnisse der Befragung in Bezug auf die Digitalisierung noch in einer Aufbauphase zu bewegen. Dies gilt auch hinsichtlich des Umsetzungsstands von Digital HRM-Ansätzen in den Unternehmen. Die Personalbereiche können und dürfen sich dem Digitalisierungstrend aber nicht verschließen und sollten künftig eine noch aktivere Rolle bei der Umsetzung von Digitalisierungsstrategien spielen.

Für Aktiengesellschaften und Kapitalmärkte ist der Börsenkurs eine wichtige finanzielle Messgröße. Beitrag neun „Big Data Analytics. Analyse der prädiktiven Fähigkeit von Twitter-Sentiments auf die Entwicklung des Börsenkurses von Technologieunternehmen" verbindet zwei wesentliche Faktoren des Digitalisierungstrends „Big Data“ und "Social Media“ mit der Frage nach der Prognostizierbarkeit von Börsenkursen. Es wird untersucht, inwiefern die Social-Media-Daten die Veränderung von Börsenkursen voraussagbar machen. Die Analyse fokussiert auf die prädiktive Fähigkeit von Twitter-Nachrichten im Zusammenhang mit einem Technologieunternehmen und dessen Börsenkurs anhand von zwei Anwendungsfällen. Konkret wird anhand der Twitter-Nachrichten mithilfe einer Sentimentanalyse die Stimmung der Twitter-Nutzer mit den Veränderungen des Börsenkurses verglichen. Diese Analyse wird anhand der Technologieunternehmen Facebook und Amazon vorgenommen. In einem ersten Schritt wird untersucht, ob eine Beziehung zwischen den Twitter-Sentiments und dem Börsenkurs besteht, in einem zweiten Schritt, ob die Twitter-Sentiments eine Voraussagekraft für die Veränderung des Börsenkurses haben. Die Auswertung zeigt bei beiden Unternehmen eine positive Korrelation der Twitter-Sentiments und des Börsenkurses auf. Weiter konnte mithilfe der Granger-Analyse eine signifikante Voraussagekraft der Twitter-Sentiments für die Börsenkurse beider Unternehmen ermittelt werden. Die Twitter-Sentiments können demnach die Börsenkurse 13 Stunden voraussagen.

Für den Finanzsektor sind Kryptowährungen in den vergangenen Jahren zu einer Herausforderung geworden. Hiermit einher geht die Chance für unternehmerische New Player, neue Marktsegmente in einer bislang eher schwer zugänglichen Branche mittels neuer Geschäftsmodelle zu erschließen. Die Verfasser des zehnten Beitrag „Kryptowährungen. Eine empirisch-qualitative Analyse von Kryptowährungen gegenüber dem traditionellen Währungssystem " sehen die Finanzkrise in Verbindung mit der Digitalisierung als zentrale Haupttreiber dieses „,neuen“ Phänomens der Kryptowährungen. Kryptowährungen sollen sowohl eine Alternative zu dem traditionellen Währungssystem bieten, als auch den Zahlungsverkehr vereinfachen. Es wird ein Vergleich von Kryptowährungen mit dem traditionellen Währungssystem durchgeführt. Dabei liegt der Fokus auf der Kryptowährung Bitcoin und dem Wirtschaftsstandort Schweiz. Die empirischen Untersuchungen wurden mittels qualitativer Erhebungen via Experteninterviews im Bereich des Geldsystems und des Zahlungsverkehrs realisiert. Dabei wurden insbesondere die Aspekte des Vertrauens, der Resilienz sowie der Vereinfachung von Zahlungstransaktionen analysiert. 
Ein weiteres hoch spannendes finanzwirtschaftliches Untersuchungsfeld ist Gegenstand des elften Beitrags „Das Potenzial von Smart Contracts an einem Beispiel aus der Finanzbranche - Smart Rating “. Der Beitrag stellt die Grundsätze von auf Smart Contracts und der Blockchain-Technologie basierenden Rating-Verfahren zur Bonitätsbewertung vor. Es werden die technologischen Grundlagen von Blockchain und Smart Contracts erläutert und diskutiert, inwieweit die Finanzbranche im Bereich des Ratings von dieser Technologie profitieren kann. Zunächst werden die Probleme traditioneller Ratingverfahren und deren Akteure thematisiert und anschließend Lösungsansätze aufgezeigt, um diese zu überwinden. Damit zeigt dieser außerhalb der traditionellen Bankdienstleistungen befindliche Anwendungsfall, dass mit der Blockchain-Technologie neue Potenziale für Geschäftsmodelle entstehen, die etablierte Prozesse grundlegend verändern und damit ein weiteres Mosaik zur Disruption der Finanzbranche darstellen können.

Einer ersten Fragestellung im Marketing wendet sich Beitrag zwölf „Erfolgsfaktoren für die Kundengewinnung mit digitalem Marketing am Beispiel des Online-MarketingTools Google AdWords" zu. Den Absatz zu steigern, ist das übergeordnete Ziel von effizientem Marketing. Die Digitalisierung eröffnet hier über die Umsetzung neuer Online-Marketingaktivitäten neue Chancen. Ein wichtiges Instrument im digitalen Marketing ist Google AdWords, ein Tool, welches es Unternehmen ermöglicht, dass deren Produkte und Dienstleistungen von potenziellen Kunden gefunden werden, wenn auf der Suchmaschine von Google danach gesucht wird. Die Identifikation von Erfolgsfaktoren bei der Arbeit mit Google AdWords ist aufgrund der Schnelllebigkeit des Themenfelds sowie des komplexen AdWords-Auktionssystems eine Herausforderung. Eine Fallstudienanalyse, in deren Rahmen erfahrene Experten in Unternehmen sowie weitere Fachexperten hinsichtlich des Marketings Google AdWords befragt wurden, deckt mögliche Erfolgsfaktoren des Google-AdWords-Einsatzes auf der Maßnahmenebene auf. Eine moderne und nutzerfreundliche Webseite mit relevanten Inhalten sowie eine hohe Relevanz zwischen Keyword, Werbeanzeige und Zielseite haben sich im Rahmen der Analyse als essenzielle Voraussetzungen für erfolgreiche Google-AdWords-Kampagnen herausgestellt.

Beitrag dreizehn „Augmented Reality als Entscheidungshilfe beim Möbelkauf“ untersucht den Einfluss von Augmented Reality (AR) auf den Kaufentscheidungsprozess von Kunden beim Möbelkauf. Ziel der Studie waren konkrete, handlungsleitende und wissenschaftlich fundierte Erkenntnisse für die Praxis im Umgang mit einem wichtigen Treiber der digitalen Transformation. Dafür wurde der Einfluss einer AR-App in der Möbelbranche untersucht. Es konnte gezeigt werden, dass die AR-App ihren Nutzern eine wertvolle Unterstützung im Kaufentscheidungsprozess bietet. Die App funktioniert jedoch nicht als eigenständiger Kanal, sondern entfaltet ihren Nutzen nur im Zusammenspiel mit den bisherigen Kanälen und Hilfsmitteln. Insbesondere der Austausch mit Dritten bietet deutliches Entwicklungspotenzial und Chancen, die Customer Experience weiter zu verbessern. Einen klaren Kundenmehrwert würde eine händlerübergreifende AR-App bieten. Weiterer Forschungsbedarf ergibt sich aus den Rückkopplungseffekten zwischen veränderter Kundeninteraktion und Technologieentwicklung. 
Die Vermarktung von Online-Abonnements steht als vertriebspolitisches Spezialproblem im Mittelpunkt des vierzehnten Beitrags „Online Abonnement-Vermarktung. Erfolgreicher Marketingmix für die Abonnement-Vermarktung von Konsumgütern “. Die Online-Abonnement(Abo)-Vermarktung für Konsumgüter gilt als lukrativer Nischenmarkt im E-Commerce. Sowohl etablierte Firmen als auch Start-ups erarbeiten neue Konzepte hierfür, deren Erfolgsfaktoren bleiben aber bis anhin noch im Dunkeln. Hiervon ausgehend werden im Rahmen des Beitrags diejenigen Schlüsselfaktoren des kuratierten Online-Abo-Marktes untersucht, die den Absatzerfolg fördern. Bisher wurden noch keine entsprechenden branchenspezifischen Analysen durchgeführt, die sich auf den Marketingmix im Online-Abo-Bereich beziehen. Aufgrund der identifizierten Forschungslücke wird der Frage nachgegangen, wie Unternehmen unter Heranziehung des Marketingmix-Ansatzes eine erfolgreiche Online-Abo-Vermarktung für Konsumgüter gestalten können. Im Mittelpunkt steht dabei der kuratierte Online-Abo-Markt. Ausgehend von der theoretischen Verknüpfung von „Subscription Economy“ und der Marketingmix-Thematik wurde eine qualitative Bestandsaufnahme anhand von vier Fallstudien durchgeführt. Die Cross-Case-Untersuchung mit Bezug auf prozentuale Umsatz- und Abonnentenzahlentwicklungen ermöglicht das Herauskristallisieren von Schlüsselfaktoren für eine erfolgreiche Abo-Vermarktung, die auch für andere OnlineAbo-Anbieter relevant sein dürften.

Ein letztes und noch recht neues Handlungsfeld im Online-Marketing, das Social Influencing, wird in Beitrag fünfzehn „Social Influencer. Eine Analyse ausgewählter visueller und auditiver Stile erfolgreicher Social Influencer auf YouTube" behandelt. Visuelle Kommunikation gewinnt im Zusammenhang mit der Digitalisierung immer mehr an Bedeutung. So erfolgt die Kommunikation auf Social-Media-Plattformen wie Facebook, Instagram und YouTube fast nur noch mittels Bild- und Videosprache. Social Influencer nutzen diese Kommunikationsweise, um sich auf den genannten Kanälen eine große Community aufzubauen. Dabei entsteht ein Beziehungsverhältnis zwischen Social Influencer und Empfänger. Der Beitrag untersucht, welche Faktoren dazu führen, dass Social Influencer „Communities“ von mehreren Tausend bis Millionen Menschen aufbauen können, von diesen als Vorbilder und Meinungsmacher angesehen werden, und dass dabei ein Beziehungsverhältnis entsteht. Die Ergebnisse zeigen, dass Social Influencer mittels aktivierender Wirkung des Gesagten die „Interaktion“ fördern. Durch eine selbstoffenbarende und wertschätzende Wirkung wird ein Storytelling realisiert, die Authentizität und Glaubwürdigkeit erhöht und die Entstehung einer Beziehung gefördert. Die Gestik, erhöhte Sprechlautstärke und das schnelle Sprechtempo sorgen dabei beim Empfänger für eine kompetent und glaubwürdig wirkende Erscheinung des Social Influencers, während die Mimik „Freude“ Glücksgefühle beim Adressaten auslöst. Die Ergebnisse liefern somit qualitativ-explorative Erkenntnisse über Stile von Social Influencern und deren Umsetzung auf dem Social-Media-Kanal YouTube. Sie bieten Ansätze zur Erklärung des Beziehungsverhältnisses zwischen YouTuber und Community. 
Die hier im Kontext der digitalen Transformation angeführten Untersuchungen zeigen ansatzweise die große Bandbreite potenzieller Gestaltungsfelder einer digitalen Unternehmensführung auf. Die Analysen stützen sich weitestgehend auf empirische Primärerhebungen und versuchen, auch gestaltungsbezogene Hinweise abzuleiten. Das Feld einer digitalen Unternehmensführung bietet in seiner Gesamtheit, auch angesichts der Entwicklungsdynamik der zugrunde liegenden Digitalisierungsthematik, noch erhebliches Entwicklungspotenzial für zukünftige Forschungsaktivitäten und die konkrete Implementierung in der Unternehmenspraxis.

\section{Literatur}

Bankl, M. (2014). Lebensphasenorientierte Personalarbeit - Ein nachhaltiger Ansatz. In B. Rosenberger (Hrsg.), Modernes Personalmanagement: Strategisch-operativ-systemisch (S. 306-314). Wiesbaden: Gabler Verlag.

Cachelin, J. L. (2013). Digitalisierung als Reifeprozess. Personalmagazin, 11(13), 51-53. https:// www.wissensfabrik.ch/digitalisierung-als-reifeprozess/. Zugegriffen: 25. Apr. 2016.

Cole, T. (2017). Digitale Transformation. Warum die deutsche Wirtschaft gerade die digitale Zukunft verschläft und was jetzt getan werden muss! Impulse für den Mittelstand (2. erw. Aufl.). München: Vahlen.

Pousttchi, K. (2017). Digitale Transformation. In N. Gronau, J. Becker, N. Kliewer, J. M. Leimeister, \& S. Overhage (Hrsg.), Enzyklopädie der Wirtschaftsinformatik. Online-Lexikon. Potsdam. http://www.enzyklopaedie-der-wirtschaftsinformatik.de/. Zugegriffen: 24. Apr. 2019.

Putzinger, R. (2016). Das digitalisierte Unternehmen. Zeit für eine neue Managementphilosophie. Wien: Facultas.

Westerman, G., Bonnet, D., \& McAfee, A. (2014). Leading digital: Turning technology into business transformation. Boston: Harvard Business Press.

Schellinger, Jochen (Prof. Dr./jochen.schellinger@bfh.ch) Studiengangsleiter Master of Science in Business Administration an der Berner Fachhochschule. Lehr- und Forschungstätigkeiten in den Bereichen Strategisches Management, Marketing und Personalmanagement. Davor 15 Jahre Praxistätigkeit bei der Deutschen Sparkassenorganisation, im Haniel-Konzern, an der Universität Tübingen sowie in der Daimler AG.

Tokarski, Kim Oliver (Prof. Dr./kim.tokarski@bfh.ch) Professor für Entrepreneurship und Unternehmensführung und Leiter der Abteilung Weiterbildung am Departement Wirtschaft der Berner Fachhochschule. Er ist Gastprofessor an der Wirtschaftsuniversität Bukarest (ASE Bukarest), Rumänien. Seine Forschungs- und Lehrtätigkeiten liegen in den Bereichen Entrepreneurship, Social Entrepreneurship, Innovation, Digitalisierung, Strategie sowie Leadership. Er ist Autor unterschiedlicher Publikationen in den genannten Themenkontexten. 
Kissling-Näf, Ingrid (Prof. Dr./ingrid.kissling@bfh.ch) Direktorin Departement Wirtschaft der Berner Fachhochschule. Forschungserfahrung als Assistenzprofessorin in Ressourcenökonomie an der ETHZ und langjährige Führungserfahrung unter anderem beim Schweizerischen Nationalfonds Abteilung Geistes- und Sozialwissenschaften, bei der Förderagentur für Innovation KTI sowie bei der Akademie der Naturwissenschaften Schweiz . Während zwei Jahren tätig als Beraterin in der Privatwirtschaft bei der Firma Ernst Basler \& Partner AG. Sie ist zudem UNICEF-Delegierte und Stadträtin in Bern.

Open Access Dieses Kapitel wird unter der Creative Commons Namensnennung 4.0 International Lizenz (http://creativecommons.org/licenses/by/4.0/deed.de) veröffentlicht, welche die Nutzung, Vervielfältigung, Bearbeitung, Verbreitung und Wiedergabe in jeglichem Medium und Format erlaubt, sofern Sie den/die ursprünglichen Autor(en) und die Quelle ordnungsgemäß nennen, einen Link zur Creative Commons Lizenz beifügen und angeben, ob Änderungen vorgenommen wurden.

Die in diesem Kapitel enthaltenen Bilder und sonstiges Drittmaterial unterliegen ebenfalls der genannten Creative Commons Lizenz, sofern sich aus der Abbildungslegende nichts anderes ergibt. Sofern das betreffende Material nicht unter der genannten Creative Commons Lizenz steht und die betreffende Handlung nicht nach gesetzlichen Vorschriften erlaubt ist, ist für die oben aufgeführten Weiterverwendungen des Materials die Einwilligung des jeweiligen Rechteinhabers einzuholen.

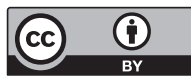




\title{
Digitale Transformation und Open Access von Forschungsresultaten in der Schweiz
}

\author{
Dirk Verdicchio und Ingrid Kissling-Näf
}

\begin{abstract}
Zusammenfassung
Die digitale Transformation hat Einfluss auf die Arbeits- und Kommunikationsweisen der Wissenschaft und ermöglicht eine weitgehende Transparenz und Offenheit der Forschung. Die Begriffe Open Science und Open Access stehen für diese Öffnung wissenschaftlicher Prozesse und die Zugänglichmachung von Forschungsergebnissen. Für Open Access - dem freien Zugang zu wissenschaftlichen Publikationen - existieren schweizerische und internationale Programme und Strategien, die von Institutionen wie dem SNF und swissuniversities getragen werden. Neben einem Überblick über die zentralen Begriffe und Konzepte geht der Beitrag auf diese Strategien ein. Besondere Beachtung finden die Situation in Europa und der Schweiz sowie der Wandel der Geschäftsmodelle und der Publikationslandschaft.
\end{abstract}

\subsection{Was wollen Open Science und Open Access?}

Die digitale Revolution hat die Arbeitsweisen in den Wissenschaften grundsätzlich revolutioniert und das Teilen von Daten und Informationen in großem Ausmaß möglich gemacht. Sie hat damit nicht nur das Selbstverständnis der Wissenschaft verändert, sondern auch die Formen der Kollaboration und der Publikationen weltweit verändert. Eine

D. Verdicchio $(\bowtie)$

Digitale Dienste \& Open Science, Universitätsbibliothek Bern, Bern, Schweiz

E-Mail: dirk.verdicchio@ub.unibe.ch

I. Kissling-Näf

Departement Wirtschaft, Berner Fachhochschule, Bern, Schweiz

E-Mail: ingrid.kissling@bfh.ch

J. Schellinger et al. (Hrsg.), Digitale Transformation und Unternehmensführung,

https://doi.org/10.1007/978-3-658-26960-9_2 
neue Offenheit findet ihren Niederschlag in Begriffen wie Open Science, Open Access und Open Research Data. Open Science steht für den Grundgedanken, den gesamten wissenschaftlichen Prozess möglichst offen und transparent zu gestalten, um wissenschaftliche Forschung möglichst frei zugänglich, nachnutzbar und reproduzierbar zu machen. Das schließt die Beteiligung von Bürgern unter dem Label Citizen Science mit ein. Entsprechend definiert das europäische Projekt FOSTER Open Science wie folgt:

Open Science is the practice of science in such a way that others can collaborate and contribute, where research data, lab notes and other research processes are freely available, under terms that enable reuse, redistribution and reproduction of the research and its underlying data and methods. ${ }^{1}$

Der EU Open Science Monitor gibt einen anschaulichen Überblick über die Charakteristika und Indikatoren von Open Science (Abb. 2.1). Zentral sind hier das Teilen der Forschungsdaten (Open Research Data), die Transparenz des Wissenschaftskommunikationsprozesses (Open Scholarly Communication) sowie der offene Zugang zu wissenschaftlichen Publikationen (Open Access to Publications). Diese Öffnung bedingt strukturelle, kulturelle und praktische Justierungen des wissenschaftlichen Prozesses. Dabei muss betont werden, dass der Begriff der Offenheit weder theoretisch noch praktisch klar umrissen ist und kontextuell interpretiert wird und werden muss (siehe Reichmann 2017; Levin und Leonelli 2017). Illustrieren lässt sich das an den Diskussionen zu Open Research Data und FAIR Data. Die FAIR-Prinzipien, entworfen von FORCE 11, formulieren Findbarkeit, Zugänglichkeit, Interoperabilität und Nachnutzbarkeit von Forschungsdaten als zentrale Momente des Umgangs mit Forschungsdaten. ${ }^{2}$ Das Konzept sieht die Offenheit der Informationen über Forschungsdaten vor, aber nicht zwingend die Offenheit der Forschungsdaten selbst, die sich gerade im Bereich der sensiblen Forschungsdaten nur mit großen Einschränkungen umsetzen lässt.

Open Science verspricht Vorteile im Bereich der Effizienz wissenschaftlicher Forschung, da eine Vielzahl von Ergebnissen für weitere Forschungstätigkeiten genutzt und Duplizierungen von Forschungsergebnissen verhindert werden können. Kollaborationen werden durch Open Science ebenso begünstigt wie die Demokratisierung wissenschaftlichen Wissens, wie es in den Diskursen zu Citizen Science entworfen wird (vgl. Arza und Fressoli 2017). Darüber hinaus verschaffen offene Praktiken Forschenden eine höhere Visibilität und mehr Zitationen (McKiernan et al. 2016). Eine von der Europäischen Kommission in Auftrag gegebene Studie zu den wirtschaftlichen Effekten von FAIR Data kommt $2018 \mathrm{zu}$ dem Schluss, dass ein Verzicht auf das Teilen von Forschungsdaten 10,2 Mrd. EUR jährlich kosten würde und formuliert entsprechende Empfehlungen zur Förderung von Infrastrukturen und Praktiken für Forschungsdaten (European Commission 2018). Bei der Betrachtung der entsprechenden Literatur bleiben - trotz der momentan

\footnotetext{
${ }^{1}$ https://www.fosteropenscience.eu/foster-taxonomy/open-science-definition.

${ }^{2} \mathrm{https} / / / \mathrm{www}$. force11.org/group/fairgroup/fairprinciples.
} 


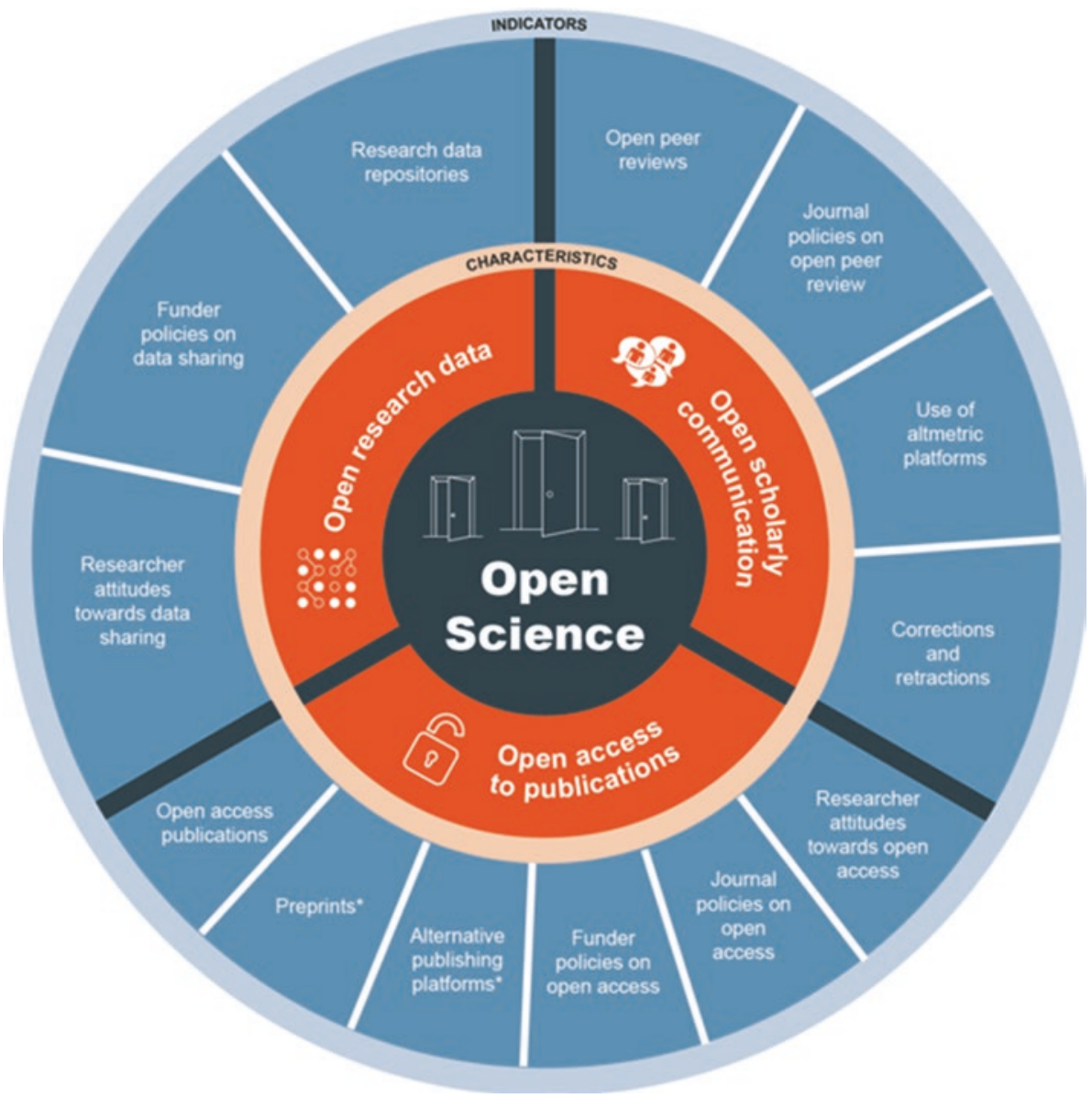

Abb. 2.1 Foster Open Science „Wheel“. (European Commission 2017, S. 6)

noch bestehenden begrifflichen und konzeptuellen Unklarheiten - kaum Zweifel, dass Open Science allen an der Wissenschaft direkt und indirekt Beteiligten zugutekommt. Eine Ausnahme bilden hier vielleicht diejenigen, die von der Regulierung des Zugangs zu wissenschaftlichem Wissen profitieren.

Open Access, der kostenfreie Zugang zu wissenschaftlicher Literatur über das Internet, ist ein wichtiger Baustein von Open Science und blickt auf eine nahezu 30-jährige Geschichte zurück. Die primären Ziele von Open Access sind eng mit den Möglichkeiten der Verbreitung von Informationen über das Internet verknüpft. Die Anfänge von Open Access lassen sich auf die Einrichtung des Servers arXiv durch Alan Ginsparg im Jahr 1991 zurückführen, der die Verbreitung von physikalischen Preprints, die bis dahin per Post versandt wurden, deutlich beschleunigte. Durch arXiv und andere Preprint-Server wurde der Grundstein für den sogenannten grünen Weg gelegt, wie die Zugänglichmachung 
von Texten über Repositorien bezeichnet wird. Ein weiterer technischer Meilenstein war die Gründung der Open Archive Initiative und das im Jahr 2000 entwickelte Open Archive Initiative Protocol for Metadata Harvesting (OAI-PMH), welches das Harvesten (automatisiertes Sammeln) von Daten aus Repositorien erlaubt und die Etablierung von Open-Access-Repositorien förderte (siehe dazu Deppe und Beucke 2017).

In den Jahren 2001 und 2003 fanden in Budapest, Bethesda und Berlin Konferenzen statt, die als Auftakt der Open-Access-Bewegung gesehen werden können. Jede dieser drei Konferenzen mündete in eine Deklaration ${ }^{3}$, die den Open-Access-Gedanken genauer präzisiert. Die Berliner Deklaration von 2003, die in den folgenden Jahren auch vom Schweizerischen Nationalfonds SNF, der Rektorenkonferenzen der Fachhochschulen und der Universitäten, den Akademien der Wissenschaften und den meisten schweizerischen Universitäten unterzeichnet wurde, formuliert das Ziel, wissenschaftliche Informationen weltweit über das Internet frei zugänglich zu machen. Dabei soll allen Nutzenden das Recht zugestanden werden, diese Informationen ,zu kopieren, zu nutzen, zu verbreiten, zu übertragen und öffentlich wiederzugeben sowie Bearbeitungen davon zu erstellen und zu verbreiten, sofern die Urheberschaft korrekt angegeben wird“. Darüber hinaus macht die Berliner Erklärung deutlich, dass Open Access nicht nur auf die Zugänglichmachung von textuellen Publikationen zielt, sondern auch weitere wissenschaftliche Informationen frei zugänglich gemacht werden sollen (siehe Berliner Erklärung ${ }^{4}$ ). Der Umstand, dass die Berliner Erklärung weltweit von über 500 Institutionen unterzeichnet wurde, macht diese Deklaration zu einem zentralen Dokument der Open-Access-Bewegung.

Im Zuge der internationalen Aktivitäten rund um Open Access haben sich folgende Formen und Begriffe etabliert: Grün Open Access wird die Selbstarchivierung, also die Zweitveröffentlichung von wissenschaftlichen Texten in einem Repositorium genannt. Solche Open-Access-Repositorien, wie bspw. BORIS an der Universität Bern, werden in der Regel von Institutionen getragen. Wenn die Erstveröffentlichung bei einem Verlag erfolgte und eine Übertragung der Verbreitungsrechte an den Verlag (copyright transfer agreement) stattgefunden hat, dann bestimmt der Verlag darüber, welche Version des Texts $^{5}$ publiziert werden und mit was für einem Embargo die Zweitveröffentlichung erfolgen darf. Die Erstveröffentlichung von Publikationen in einer Open-Access-Zeitschrift oder als Open-Access-Buch wird Gold Open Access genannt. Die Publikation ist hier mit dem Erscheinen frei zugänglich und ist mit einer Lizenz ausgestattet, die

\footnotetext{
${ }^{3}$ Budapest Open Access Initiative (2002), Bethesda Statement On Open Access Publishing (2003) und die Berlin Declaration on Open Access to Knowledge in the Sciences and Humanities (2003). ${ }^{4}$ https://openaccess.mpg.de/Berlin-Declaration.

${ }^{5}$ In der Regel erlauben die Verlage die Publikation der begutachteten Manuskriptversion (auch post-print oder author accepted manuscript genannt).
} 
die legitime Form der Nachnutzung determiniert. ${ }^{6}$ Einige Open-Access-Zeitschriften verlangen Publikationsgebühren (sogenannte article processing charges oder APCs), andere Zeitschriften finden Wege der Finanzierung, die ohne Zahlungen durch Autoren auskommen (s. u.). Zeitschriften, die keine Publikationsgebühren erheben, werden manchmal auch Diamond-Open-Access-Zeitschriften genannt. Die dritte Form der Umsetzung des Open-Access-Gedankens nennt man Hybrid Open Access. Hier werden Zeitschriftenartikel in klassischen Subskriptionszeitschriften publiziert und gegen Gebühr frei zugänglich gemacht. Hybrid Open Access wird häufig als transitorisches Modell gesehen, das Zeitschriften dabei helfen soll, den geordneten Übergang zu einem Gold-Open-Access-Modell zu bestreiten, indem das Geschäftsmodell nach und nach umgestellt wird. Bisher gibt es jedoch keine Anzeichen dafür, dass dies im großen Stil erfolgen wird. ${ }^{7}$

Neben dem offenen Zugang und der damit verbundenen besseren Verfügbarkeit wissenschaftlichen Wissens, lässt sich durch Open Access die Kommunikation wesentlich beschleunigen und prinzipiell günstiger gestalten. Diesen Zielen stehen die historisch gewachsenen Publikations- und Verbreitungsformen entgegen. Insbesondere das Subskriptionsmodell für Zeitschriften und die damit verbundenen Paywalls unterbinden und beschränken den Zugang zu den Forschungsergebnissen, sie stellen damit ein Hindernis für die wissenschaftliche Forschung und die gesellschaftliche Aneignung wissenschaftlichen Wissens dar und stehen damit dem Grundsatz entgegen, dass wissenschaftliches Wissen für alle zugänglich sein soll (vgl. z. B. Merton 1973).

Die Zugänglichkeit wird zusätzlich durch starke Anstiege der Subskriptionskosten erschwert, die dazu führen, dass Bibliotheken Zeitschriftensubskriptionen abbestellen und Anschaffungen reduzieren müssen. Der Umstand, dass sich auch die Harvard University 2012 gezwungen sah, Zeitschriftenabonnemente zu kündigen, kann als Indiz dafür gesehen werden, dass heute keine Universität ihren Forschenden eine vollständige Versorgung mit wissenschaftlichen Informationen garantieren kann und stellt die ökonomische und wissenschaftliche Nachhaltigkeit der Subskriptionsmodelle insgesamt infrage (Sample 2012).

Das traditionelle Publikationsmodell steht aber auch deswegen in der Kritik, weil der überwiegende Teil wissenschaftlicher Forschung öffentlich finanziert ist, die Ergebnisse der Öffentlichkeit jedoch nicht zur Verfügung stehen. Vielmehr muss der Zugang zu diesem Wissen abermals mit öffentlichen Geldern bezahlt werden. Die Frage nach der Gerechtigkeit eines solchen Modells stellt sich gerade wegen der außerordentlich hohen Gewinne, die einige Verlage mit der Verbreitung öffentlich finanzierter Forschungsergebnisse erzielen, wobei sie Profitraten von bis zu über $40 \%$ erreichen (Siehe z. B. Morrison 2012, S. 11 ff., 2014; Buranyi 2017).

\footnotetext{
${ }^{6}$ Meist handelt es sich dabei um Creative-Commons-Lizenzen (siehe https://creativecommons. org/).

${ }^{7}$ Zu Hybrid Open Access in der Schweiz am Beispiel der ETH Zürich siehe Christen 2017.
} 
Zu den Eigenheiten des wissenschaftlichen Publikationssystems gehört, dass die Verlage weder für die Manuskripte, noch für die redaktionellen Tätigkeiten oder die Qualitätssicherung über Peer Review zahlen, die von Forschenden entweder im Rahmen ihrer Arbeitszeit oder ihrer Freizeit getätigt werden. Dafür treten die Autoren die exklusiven Verbreitungsrechte an ihren Werken an die Verlage ab, die die Verbreitung dieser Werke beliebig monetarisieren und kontrollieren können. So kann es vorkommen, dass die Urheber für die Nachnutzung ihrer eigenen Texte, die sie dem Verlag kostenlos überlassen haben, abermals eine Gebühr bezahlen müssen.

\subsection{Open-Access-Politiken in Europa}

Seit 2012 gibt es in Großbritannien, den Niederlanden und Österreich national koordinierte Pläne zu Open Access ${ }^{8}$, die in der Schweiz als Anlass und Vorbild zum Entwurf einer Nationalen Open-Access-Strategie dienten, die 2017 verabschiedet wurde (s. u.). Für Schweizer Forschende war aber bereits die Open-Access-Politik der Europäischen Union direkt relevant. Das siebte Forschungsrahmenprogramm (FP7) der Europäischen Kommission (2007-2013) empfahl erstmals die Open-Access-Publikation der Ergebnisse der geförderten Forschung. Darüber hinaus wurde ein ,post grant open access fund" eingerichtet, über den sogenannte Article Processing Charges (APC), d. h. Gebühren für Publikationen abgeschlossener FP7-Projekte beantragen konnten. ${ }^{9}$

Im Rahmen von FP7 wurde 2010 das Projekt „Open Access Infrastructure for Research in Europe“ (OpenAire) ins Leben gerufen, das die Zugänglichkeit der Publikationen und Daten sicherstellen soll, die im Rahmen des Programms entstanden sind. $\mathrm{Zu}$ den Infrastrukturen, die hierfür geschaffen wurden, gehört auch das Repositorium Zenodo, das am CERN betrieben wird und für alle Forschenden, unabhängig von der institutionellen Zugehörigkeit, zur Verfügung steht (Manola et al. 2015). Im Jahr 2014 folgte das achte Forschungsrahmenprogramm, das den Titel Horizon 2020 trägt und Open Access mandatiert. Publikationen aus dem Bereich der sogenannten MINT-Fächer müssen spätestens sechs Monate nach Erscheinen Open Access verfügbar gemacht werden und Publikationen aus dem Bereich der Geistes- und Sozialwissenschaften nach spätestens 12 Monaten. Außerdem wird erwartet, dass für jedes Projekt ein Forschungsdatenplan erstellt wird und die Forschungsdaten publiziert werden. Eine wichtige Neuerung von Horizon 2020 ist die Compliance-Überwachung. Wie bei vielen Forschungsförderern und Institutionen, hatte das Open-Access-Mandat bei EU-Projekten bis dahin eher den Charakter einer Empfehlung. Dies änderte sich 2018 als der European

\footnotetext{
${ }^{8}$ Siehe dazu https://ukrio.org/open-access-research-report-of-the-finch-group/, https://www.openaccess.nl/, https://www.oana.at.

${ }^{9} \mathrm{https}: / /$ ec.europa.eu/research/science-society/document_library/pdf_06/open-access-pilot_en.pdf, https://ec.europa.eu/digital-single-market/en/news/results-fp7-post-grant-open-access-pilot.
} 
Research Council (ERC) Universitäten aufforderte, der in der Fördervereinbarung festgehaltenen Verpflichtung zu Open Access nachzukommen.

Im September 2018 hat cOAlition S, eine Allianz von 16 Forschungsförderern, dem ERC und der Europäischen Kommission Open-Access-Richtlinien unter dem Namen Plan S veröffentlicht (cOAlition S 2018; siehe auch Tulney 2018). Die Richtlinien schlagen eine deutliche Verschärfung der Open-Access-Auflagen vor und zielen auf eine Transformation des Publikationswesens. $\mathrm{Zu}$ den zehn Maßnahmen, die Plan S vorsieht, gehören der Verzicht auf die Übertragung der Verbreitungsrechte an die Verlage, die Verpflichtung zur Nutzung einer Lizenz, die der Creative-Commons-Attribution entspricht (CC BY), die Festsetzung einer maximalen Höhe für Publikationsgebühren und Sanktionen bei Nichtbefolgung des Open-Access-Mandats. Mit dem Grundsatz, dass Publikationen in hybriden Open-Access-Zeitschriften nicht akzeptiert werden, soll die Transformation von Subskriptionszeitschriften in Open-Access-Zeitschriften stimuliert werden. Diese Grundsätze sollen für alle geförderten Publikationen gelten, Buchpublikationen eingeschlossen. Plan S hat weltweit Reaktionen provoziert. Der Aufruf zu Rückmeldungen und Kritik des Plans führte zu ca. 600 Eingaben. Während die Gegner einer Verpflichtung zu Open Access den Plan grundsätzlich ablehnen, wurde er von Befürwortern von Open Access zwar prinzipiell begrüßt aber teilweise als zu wenig differenziert und schlecht umsetzbar kritisiert. Dennoch adressiert der Plan S wichtige Punkte, die die Durchsetzung von Open Access behindern und hat daher das Potenzial, die Publikationslandschaft in Richtung Open Access zu verändern.

\subsection{Open-Access-Politik in der Schweiz}

Wie Abb. 2.2 zeigt, führt die Schweiz erstaunlicherweise mit $39 \%$ Open-Access-Publikationen weltweit die Länderliste im Vergleich an. Durchschnittlich sind nur $30 \%$ der Publikationen auf dem goldenen und grünen Weg frei zugänglich, ${ }^{10}$ siehe auch Gutknecht et al. 2016.

Lange Zeit wurde Open Access in der Schweiz eher zurückhaltend verfolgt. Obwohl der Schweizerische Nationalfond (SNF) 2008 eine Verpflichtung zur Selbstarchivierung von Artikeln einführte, wurde die Einhaltung der Vorgabe nicht überwacht. Erst 2013 hat der SNF eine eigentliche Open-Access-Politik postuliert und die Publikationspolitik mit Richtlinien, für die mit öffentlichen Geldern finanzierten Forschungsresultate versehen. Dabei setzte der SNF bewusst auf die Förderung des goldenen Wegs. Article Processing Charges (APCs) für die Publikation von Artikeln in Open Access wurden mit einem Betrag von CHF 3000 unterstützt. Im Jahr 2014 wurde dann die Open-Access-Politik auf Buchpublikationen ausgeweitet. Gefördert werden seitdem nur noch digitale Versionen von Büchern (Produktionskosten). Darüber hinaus müssen Buchpublikationen spätestens 24 Monaten nach deren Erscheinen auf einem Repositorium zugänglich gemacht werden.

\footnotetext{
${ }^{10} \mathrm{https}: / /$ ec.europa.eu/info/research-and-innovation/strategy/goals-research-and-innovation-policy/ open-science/open-science-monitor/trends-open-access-publications_en.
} 


\section{Percentage of Open Access publications (Gold and Green) by country}

Source: Consortium's own analysis of Scopus and Unpaywall databases - Reference date: 20092017

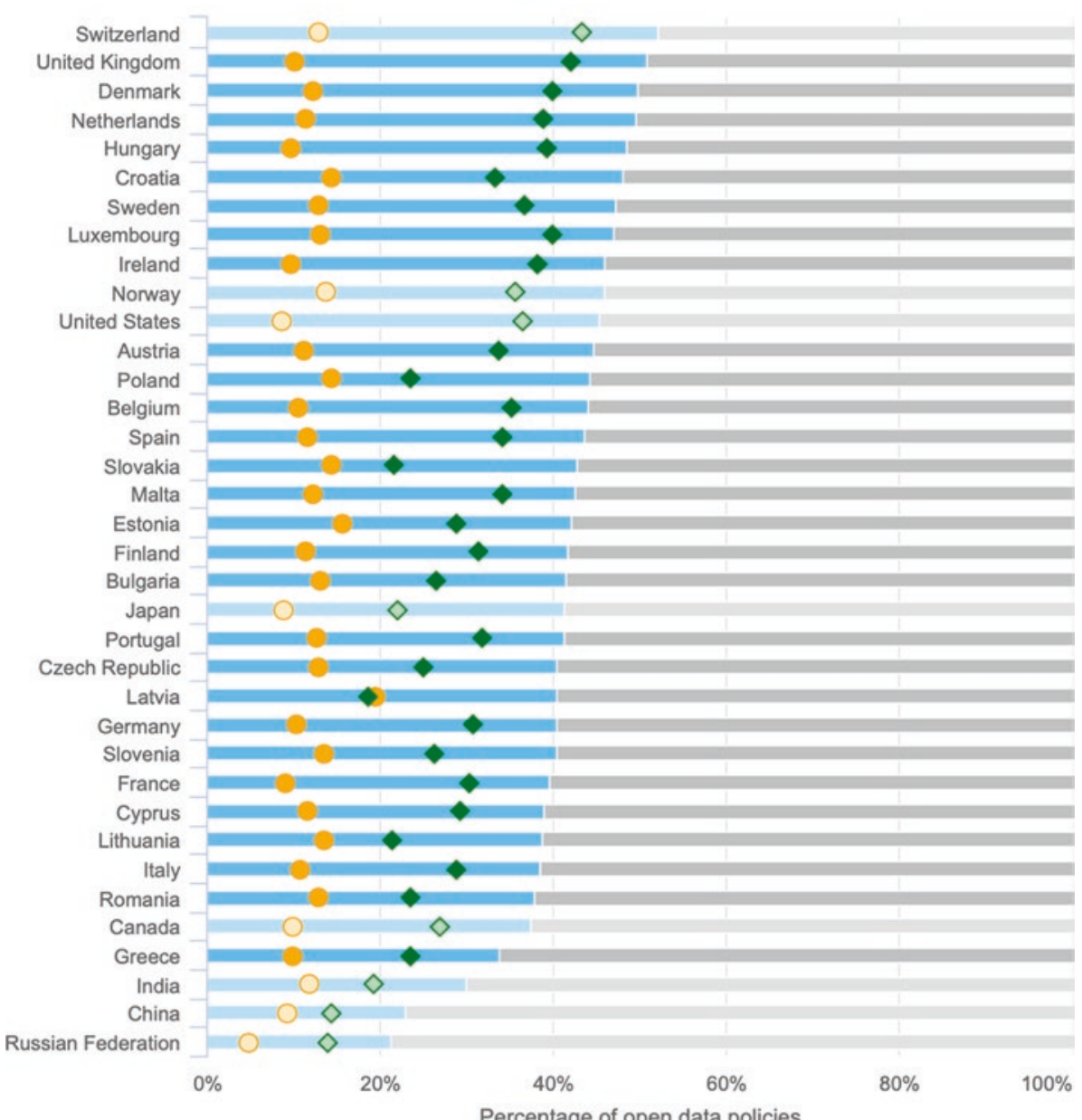

Percentage of open data policies
Green OA
Gold OA
OA
Not OA

Abb. 2.2 EU Open Science Monitor. (European Commission 2019) 


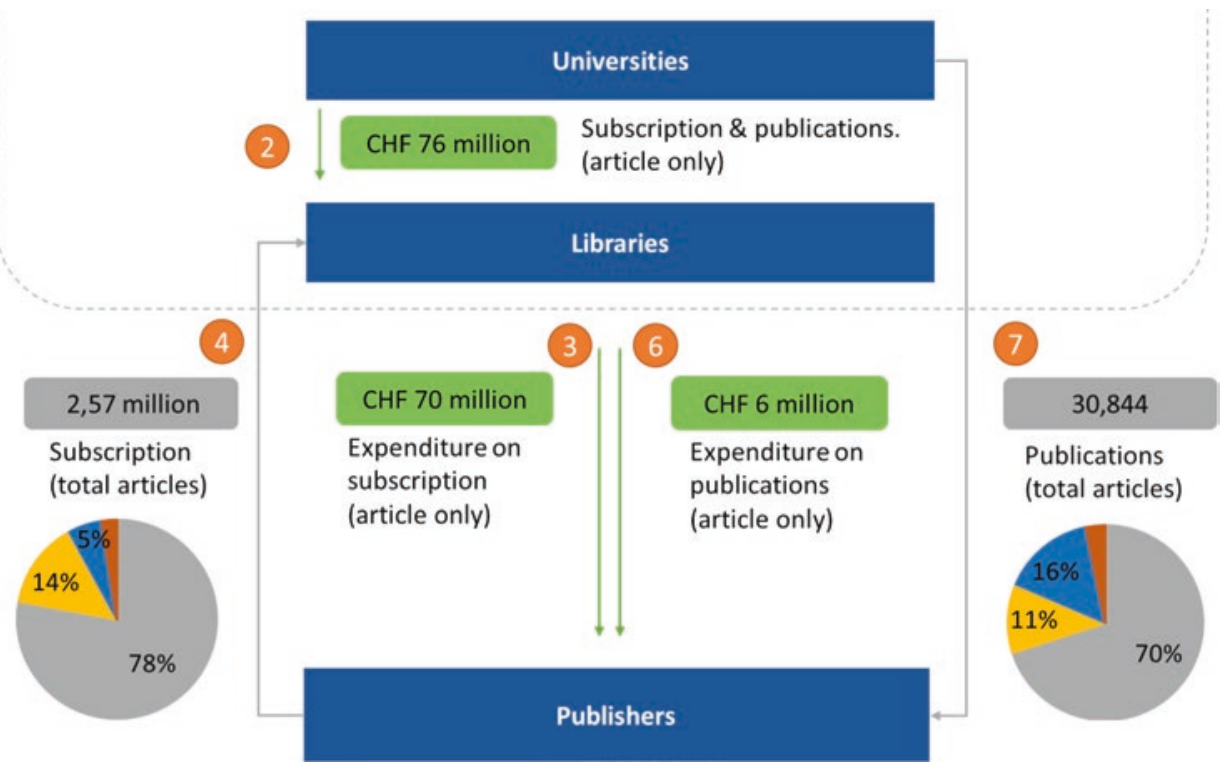

Abb. 2.3 Finanzflüsse im Publikationswesen der Schweiz im Jahr 2015. (CEPA 2017)

Die Richtlinien des SNF erzeugten einen Sturm der Entrüstung, ${ }^{11}$ die Schweizer Verlagshäuser sammelten Unterschriften gegen die Publikationspolitik des SNF ${ }^{12}$ und im Parlament wurde eine Interpellation eingereicht, weil das Schweizer Verlagswesen durch Open Access bedroht sei. ${ }^{13}$ In der Folge fanden im Frühsommer 2014 erste Gespräche zwischen dem SNF und den Verlegern statt, als Kompromiss einigte man sich auf ein Pilotprojekt mit Schweizer und deutschen Verlegern in den Geistes- und Sozialwissenschaften. Das Projekt OAPEN-CH sollte in Anlehnung an Studien, die in den Niederlanden (OAPEN-NL) und Großbritannien (OAPEN-UK) durchgeführt wurden, untersuchen, welche Auswirkungen die Publikation von Büchern in Open Access auf den Verkauf der gedruckten Ausgaben haben. Das Projekt sollte Daten über Kosten und Verkäufe liefern, aber auch einen gemeinsamen Lernprozess von Verlegern, Autoren und Bibliotheken initiieren. Speziell interessierten die Effekte des grünen und goldenen Wegs für die Visibilität und den Absatz von Büchern. In diesem Sinne wurde ein experimentelles Design gewählt, das Open-Access-Bücher mit gedruckten Monografien bezüglich Zitation, Kosten, Absatz etc. verglich. Die Ergebnisse bestätigten die Befunde der Vorgängerstudien in den Niederlanden und Großbritannien. So stellt der Schlussbericht fest, dass

\footnotetext{
${ }^{11}$ Siehe: Weik (2014), Hirschi (2014), Hagner (2014).

${ }^{12}$ „Fonds national suisse de la recherche scientifique FNS-SNSF: L'édition académique en danger! Die akademischen Verlage sind in Gefahr!“.

${ }^{13}$ Savary (2014).
} 
(e)ine digitale, übers Internet frei verfügbare Ausgabe ... die Auffindbarkeit, Sichtbarkeit und Nutzung von Monographien (erhöht). Open Access wirkt sich nicht negativ auf den Verkauf des gedruckten Buches aus. Fazit: Für die akademische Gemeinschaft und die Gesellschaft insgesamt sowie für Verleger und Geldgeber ist es von Vorteil, Open Access für Monographien zu ermöglichen und zu unterstützen (Ferwerda et al. 2018, S. 3).

Ende 2015 beauftragte das Staatssekretariat für Bildung, Forschung und Innovation (SBFI) swissuniversities, gemeinsam mit dem SNF eine nationale Strategie auszuarbeiten. Konkreter Handlungsbedarf wurde darin gesehen, Open Access als übergeordnetes Ziel zu fördern, Kostentransparenz bei öffentlichen Ausgaben zu schaffen und die Aktivitäten der Stakeholder einschließlich der Bibliotheken zu koordinieren (SBFI 2015). Damit war der Grundstein für eine schweizweite Koordination der OpenAccess-Aktivitäten zwischen SNF, Hochschulen, Förderprogrammen und Bibliotheken gelegt. In der Folge wurde eine sogenannte Finanzflussanalyse erarbeitet. Diese wies aus, dass Schweizer Bibliotheken 2015 rund 70 Mio. an Subskriptionenkosten ausgaben, damit Artikel gelesen werden konnten. Die Erhebungen zeigten zudem, dass sich die Kosten der Bibliotheken für Gold Open Access und hybride Publikationen auf rund sechs Millionen Franken beliefen. Zwei Millionen Franken flossen zum damaligen Zeitpunkt in Infrastrukturen für Open Access. Etwa $11 \%$ der Schweizer Artikel wurden Open Access veröffentlicht, $16 \%$ standen auf dem grünen Weg zur Verfügung und $3 \%$ waren hybride Artikel (siehe Abb. 2.3).

Die nationale Strategie Open Access wurde Ende 2017 von der Plenarversammlung von swissuniversities verabschiedet. Sie hält als Zielsetzung fest:

Bis 2024 sollte wissenschaftliches Publizieren in der Schweiz OA sein, alle mit öffentlichen Geldern finanzierten wissenschaftlichen Publikationen müssen im Internet frei zugänglich sein. Die OA-Landschaft wird aus verschiedenen OA-Modellen bestehen (swissuniversities und Schweizerischer Nationalfonds 2016).

Die Strategie betont die Autonomie der Hochschulen und setzt auf eine weitgehende Flexibilität der Umsetzung von Maßnahmen, um das Ziel zu erreichen. Dennoch sollen die Maßnahmen, die in einem Aktionsplan konkretisiert wurden, koordiniert werden: So sollen die Open-Access-Politiken der verschiedenen Akteure abgestimmt werden. Eine Verhandlungsdelegation wurde gegründet, die mit Verlagen Read-and-Publish-Verträge aushandelt. Es sollen, wenn es sinnvoll und möglich ist, Ressourcen gebündelt werden. Und es sollen Alternativen zu den gängigen Publikationsformen geschaffen und unterstützt werden. Darüber hinaus werden Kommunikations- und Sensibilisierungsmaßnahmen ergriffen und ein Monitoring der Umsetzung soll erfolgen. Eine besondere Bedeutung kommt der Reform der Forschungsevaluation zu, die für die Forschenden die Anreize zur Publikation in Open Access schaffen soll. Die Strategie betont zudem die Wichtigkeit eines Zweitveröffentlichungsrechts, das es den Verfassern von wissenschaftlichen Publikationen erlaubt, ihre Publikationen unabhängig von der Einwilligung eines Verlags über den grünen Weg Open Access zugänglich zu machen. 
Der SNF nimmt für die Umsetzung der Open-Access-Politik im Verbund mit den Hochschulen eine Vorreiterrolle ein und strebt an, dass alle von ihm geförderte Forschungsergebnisse bis 2020 frei zugänglich sein sollen. Entsprechend wurden 2018 die Fördermaßnahmen angepasst. So wurde der maximale Förderbetrag für APCs aufgehoben und die Publikationsunterstützung von den Anträgen für Projektbeiträge entkoppelt. Forschende können nun unabhängig von der Laufzeit ihrer Projekte Mittel für Open-Access-Publikationen beantragen. Zudem wurde das Embargo für die Zugänglichmachung von Büchern auf 12 Monate verkürzt und es wurde ein Compliance-Monitoring eingeführt. ${ }^{14}$

\subsection{Geschäftsmodelle und der Wandel der Publikationslandschaft}

Der Publikationsmarkt war in den letzten Jahrzehnten von kommerziellen Verlagen wie Elsevier, Springer und Wiley dominiert. Elsevier z. B. gibt rund 2500 Zeitschriften heraus. Das Oligopol am Publikationsmarkt erschwert zusammen mit den bisher üblichen Non-Disclosure-Agreements, die eine Kommunikation der Lizenzbedingungen und Preise untersagen, die Transparenz der Publikations- und Subskriptionskosten (siehe z. B. Larivière et al. 2015). Diese Konstellation hat zur Folge, dass die Bibliotheken in einer schlechten Verhandlungsposition sind und finanziell zunehmend unter Druck geraten.

Der technologische Wandel und der wachsende Zuspruch, den Open Access und Open Science finden, verändert aber auch die Publikationslandschaft. So erleben kommerzielle Start-ups und nichtkommerzielle Initiativen im Bereich alternativer Metriken, alternativer Publikationsformen und Repositorien eine Blüte. Große Verlage versuchen dagegen das tradierte Finanzierungsmodell mit den ertragreichen Closed Journals so lange wie möglich am Leben zu erhalten und sich zeitgleich über den Aufkauf von Startups und etablierten Firmen ihre oligopolistische Stellung in der neuen Welt zu sichern, indem sie Dienstleistungen und Infrastrukturen anbieten, die eine Kontrolle des gesamten Forschungsprozess erlauben (siehe dazu z. B. Posada und Chen 2018). Open Access und der Wegfall von Subskriptionsgebühren erfordern aber auch die Entwicklung neuer Geschäfts- und Finanzierungsmodelle sowie neuer Publikationsangebote. In der Folge wollen wir vier solcher Veränderungen skizzieren, die unserer Ansicht nach für den Wandel des wissenschaftlichen Publikationssystems stehen. ${ }^{15}$

\footnotetext{
${ }^{14} \mathrm{http}: / / \mathrm{www}$. snf.ch/de/derSnf/forschungspolitische_positionen/open_access/Seiten/default.aspx.

${ }^{15}$ Für eine ausführliche Übersicht über Geschäftsmodelle für Open Access siehe Speicher et al. (2018).
} 
Open-Access-Publikationsgebühren Für diese Form der Finanzierung haben sich die Begriffe Article Processing Charges (APC), Book Processing Charges (BPC) und Book Chapter Processing Charges (BCPC) durchgesetzt. Bezahlt werden die Gebühren entweder von den AutorInnen selbst, von Forschungsförderern oder von den Institutionen, an denen die AutorInnen beschäftigt sind. Da viele Verlage, unabhängig davon ob es sich um eine Open-Access-Publikation handelt oder nicht, Gebühren für die Bearbeitung und Publikation von Texten fordern (für Lektorat, Farbabbildungen, Seitengebühren usw.), passt diese Form der Finanzierung in bereits vorhandene Geschäftsprozesse vieler Verlage. Darüber wie hoch Publikationsgebühren sein müssen oder welche Beträge angemessen sind, gibt es keinen Konsens. So differieren die Kosten zwischen verschiedenen Verlagen oder auch Zeitschriften um mehrere tausend Dollar. Außerdem ist zweifelhaft, ob APC-basiertes Open Access eine Lösung für das Kostenproblem der Wissenschaftskommunikation bieten kann. So kommt eine Studie von Universities UK zu dem Schluss, dass die durchschnittlichen Preise der APCs zwischen 2013 und 2016, die von englischen Universitäten bezahlt wurden, um $16 \%$ gestiegen sind (Universities UK 2017).

Gemeinschaftliche Finanzierung von Publikationen Eine Alternative zur Bezahlung von APCs ist die gemeinschaftliche Finanzierung von Publikationen. Bei diesem mit dem Crowdfunding vergleichbaren Modell werden die Publikationskosten freiwillig von Institutionen übernommen, die sich an der Finanzierung beteiligen. Dieses Modell findet sich häufig bei Non-Profit-Initiativen, wie bspw. Language Science Press oder Open Library of Humanities, die in der fachwissenschaftlichen Community verwurzelt sind.

Neue Universitätsverlage und Academic-led Presses Neben der Tatsache, dass die meisten großen Verlage in der Zwischenzeit Open-Access-Modelle für die Buchpublikationen anbieten, sind seit den letzten Jahren eine Reihe von neuen Universitätsverlagen entstanden (Bargheer und Pabst 2016). Zu nennen sind hier z. B. UCL Press am University College London, Lund University Press und der Universitätsverlag Göttingen. Aber auch Wissenschaftler betätigen sich als Herausgeber von Büchern und Zeitschriften. Zu diesen sogenannten Academic-led Presses gehören bspw. Open Humanities Press oder MayFly Books. Auch wenn sich diese Verlagsangebote im Hinblick auf die Größe und den Umfang der angebotenen Dienstleistungen stark unterscheiden, haben sie gemeinsam, dass sie nicht profitorientiert sind (Adema und Stone 2017). Finanziert werden die Publikationen meist mit Fördergeldern, Crowdfunding oder über Publikationsbeiträge.

Verbreitungsrechte Verfügen die Verlage über die exklusiven Verbreitungsrechte, können sie z. B. für eine weitere Publikation des Texts oder von Varianten des Texts von den Autoren Geld verlangen. Möglich ist das auch bei Texten, die Open Access erscheinen, wenn eine Lizenz gewählt wird, die eine kommerzielle Nutzung ausschließt, der Text aber in einer anderen Verlagspublikation erscheinen soll. Dann kann die Wiederverwendung 
als kommerzielle Nutzung betrachtet werden und die AutorInnen müssen den Verlag, der die Verbreitungsrechte hält, entschädigen. Viele neuere Open-Access-Verlage verzichten zugunsten einer einfachen Publikationsvereinbarung auf eine solche Rechteübertragung. Sollte sich der Verzicht auf die exklusive Übertragung der Verbreitungsrechte an die Verlage durchsetzen, wie das auch der Plan S und die nationale Open-Access-Strategie der Schweiz anstreben, werden der Monetarisierung der Texte durch die Verlage Grenzen gesetzt. Dadurch, dass die Verbreitungsrechte bei den Autoren bleiben, können Verlage für eine nochmalige Publikation der Texte keine Gebühren verlangen.

Der Verzicht auf die Übertragung ausschließlicher Verbreitungsrechte würde jedoch deutlich stärkere Veränderungen bewirken, als der Wegfall gelegentlicher Einnahmen durch die Zweitpublikation von einzelnen Artikeln. Denn ohne die exklusiven Verbreitungsrechte kann mit diesen nicht gehandelt werden. Wenn aber Medienkonzerne andere aufkaufen, Partnerschaften eingehen oder Teile ihres Portfolios verkaufen, geht es gerade um ebendiese Rechte. Blieben die Verbreitungsrechte bei den Autoren, könnte dies deutliche Auswirkungen auf die Dynamik und die fortschreitende Oligopolisierung des wissenschaftlichen Publikationsmarkts haben (siehe dazu Larivière et al. 2015). Ohne den Besitz der Verbreitungsrechte wären Verlage nicht mehr die Besitzer der wissenschaftlichen Literatur, sondern lediglich Dienstleister, die von den Autoren die Erlaubnis zum Publizieren eines Texts erhalten.

\subsection{Abschluss und Ausblick: Open Access in der Schweiz}

Abschließend bleibt festzuhalten, dass in der Schweiz konzertiert und dezidiert an der Erreichung von Open Access gearbeitet wird (siehe Hirschmann und Verdicchio 2017). Im Rahmen der nationalen Open-Access-Strategie laufen Verhandlungen zu Read-and-Publish-Verträgen, die Subskriptionszahlungen mit Gebühren für Open-Access-Veröffentlichungen kombinieren. Ziel der Verhandlungen sind außerdem Kostentransparenz (also der Verzicht auf Geheimhaltungsklauseln) und Kostenneutralität (siehe swissuniversities 2018). Die Strategie des Schweizerischen Nationalfonds ist klar und unterstützt auch explizit Open Access für Buchpublikationen. Einfluss auf die Entwicklung von Open Access in der Schweiz haben nicht zuletzt die wissenschaftlichen Akademien. Die Schweizerische Akademie der Geistes- und Sozialwissenschaften hat 2016 eine Open-Access-Strategie verabschiedet und fördert seitdem die Umstellung der von ihr geförderten Zeitschriften zu Open Access (SAGW 2016). Mit dem SBFI, swissuniversities, dem SNF und der SAGW bekennen sich wichtigsten Wissenschaftsorganisationen und Förderer in der Schweiz zur Forderung und Förderung von Open Access.

Auch an den Universitäten bewegt sich in Bezug auf Open Access einiges. Mit BOP Serials in Bern und HOPE in Zürich stehen bisher zwei Plattformen zur Publikation von Open-Access-Zeitschriften zur Verfügung, die das Hosting von Zeitschriften kostenlos oder kostengünstig anbieten. Weitere Angebote für das Zeitschriftenhosting und der Produktion von Büchern sind im Aufbau. Einige Verlage und Druckereien reagieren auf 
die veränderte Publikationslandschaft, indem sie Verlagsdienstleistungen anbieten, die für universitäre Publikationsdienste attraktiv sein können. Anstatt die Produktion eines Buches vom Manuskript bis zum Verkauf zu organisieren, werden modulare Angebote entwickelt, die einen Ausschnitt des Buchproduktionsprozess abdecken. Inwiefern kleine, alternative Publikationsmöglichkeiten wirklich ,disruptiv“ wirken wie das Adema und Stone (2017, S. 3) erwarten, muss sich allerdings erst noch zeigen. Viel eher scheint es, dass die Medienkonzerne, die die derzeitige Publikationslandschaft dominieren, weiterhin eine wichtige Rolle spielen. Hier bleibt zu hoffen, dass eine Abhängigkeit von wenigen Konzernen wie sie im Publikationswesen besteht, für die anderen Arbeitsbereiche von Open Science vermieden werden kann.

In absehbarer Zeit werden auch für kleinere Hochschulen Infrastrukturen für den grünen Weg zu Open Access zur Verfügung stehen. Dies entweder über Infrastrukturkollaborationen, wie bspw. die Zusammenarbeit zwischen der Berner Fachhochschule mit der Universität Bern, die Hochschulen in Luzern mit der ZHB Luzern oder über die sich im Aufbau befindliche Plattform SONAR, die von kleineren Hochschulen benutzt werden kann. Weitere Entwicklungen in Richtung Open Science werden höchstwahrscheinlich verstärkt zu Dienstleistungskooperationen zwischen verschiedenen Hochschulen, z. B. im Bereich des Forschungsdatenmanagement oder der Datenkuratierung, führen. Wenig verbreitet sind bisher Forschungsinformationssysteme, die für Open Science eine wichtige Rolle spielen können und eine öffentlich sichtbare Verknüpfung von Projekten, Personen, Publikationen und Forschungsdaten leisten. Die Systeme, die bisher in der Schweiz im Einsatz sind, werden außer in Luzern vor allem für interne Zwecke eingesetzt.

$\mathrm{Ob}$ die Schweiz das von der nationalen Strategie formulierte Ziel erreichen wird, muss abgewartet werden. Auch wenn die Open-Access-Rate im internationalen Vergleich gut aussieht, müssen noch erhebliche Anstrengungen unternommen werden, wenn bis 2024 alle öffentlich finanzierten Publikationen offen zugänglich sein sollen. Darüber hinaus kann man gespannt sein, ob und wie es in der Schweiz gelingen wird, den Wandel zu Open Science zu gestalten und dabei lokale und disziplinäre Diversität zu erhalten.

\section{Literatur}

Adema, J., \& Stone C. (2017). Changing publishing ecologies. A landscape study of New University Presses and Academic-Led Publishing. JISC. http://repository.jisc.ac.uk/id/eprint/6666. Zugegriffen: 16. Apr. 2019.

Arza, V., \& Fressoli, M. (2017). Systematizing benefits of open science practices. Information Services \& Use, 37(4), 463-474. https://doi.org/10.3233/isu-170861.

Bargheer, M., \& Pabst, J. (2016). Being small is not a fault': Making sense of the newer generation of German-Language University Presses. Learned Publishing, 29(1), 335-341. https://doi. org/10.1002/leap.1053.

Buranyi, S. (27. Juni 2017). Is the staggeringly profitable business of scientific publishing bad for science? The Guardian. https://www.theguardian.com/science/2017/jun/27/profitable-business-scientific-publishing-bad-for-science. Zugegriffen: 16. Apr. 2019. 
Cambridge Economic Policy Associates Ltd. (CEPA). (2017). Financial flows in Swiss publishing. https://doi.org/10.5281/zenodo.240896.

cAOlition S (2018). Plan S: Accelerating the transition to full and immediate open access to scientific publications. https://www.coalition-s.org/wp-content/uploads/Plan_S.pdf. Zugegriffen: 16. Apr. 2019.

Christen, M. (2017). Aufkommen von hybridem Open Access in der Schweiz am Beispiel der ETH Zürich. o-bib. Das offene Bibliotheksjournal, 4(3), 67-87. https://doi.org/10.5282/obib/2017h3s67-87.

Deppe, A., \& Beucke, D. (2017). Urspünge und Entwicklung von Open Access. In K. Söllner \& B. Mittermaier (Hrsg.), Praxishandbuch Open Access (S. 12-20). Berlin: De Gruyter. https://doi. org/10.5281/zenodo.802639.

European Commission. (2017). Providing researchers with the skills and competencies they need to practise open science. https://doi.org/10.2777/121253.

European Commission. (2018). Cost-benefit analysis for FAIR research data. https://publications. europa.eu/en/publication-detail/-/publication/d375368c-1a0a-11e9-8d04-01aa75ed71a1/language-en. Zugegriffen: 16. Apr. 2019.

European Commission. (2019). Open science monitor. https://ec.europa.eu/info/research-and-innovation/strategy/goals-research-and-innovation-policy/open-science/open-science-monitor/ trends-open-access-publications_en\#open-access-to-publications. Zugegriffen: 16. Apr. 2019.

Ferwerda, E., Snijder, R., Arpagaus, B., Graf, R., Krämer, D., \& Moser, E. (2018). OAPEN-CH Auswirkungen von Open Access auf wissenschaftliche Monographien in der Schweiz. Ein Projekt des Schweizerischen Nationalfonds. https://doi.org/10.5281/zenodo.1219172.

Gutknecht, C., Graf, R., Kissling, I., Krämer, D., Milzow, K., Perini, L., Würth, S., \& Zimmermann, T. (2016). SNSF monitoring report: Open access to publications 2013-2015. Swiss National Science Foundation. http://www.snf.ch/SiteCollectionDocuments/Monitoringbericht_ Open_Access_2015_d.pdf. Zugegriffen: 16. Apr. 2019.

Hagner, M. (23. Mai 2014). Gute Bücher benötigen Zeit und Papier. Neue Zürcher Zeitung. https:// www.nzz.ch/feuilleton/gute-buecher-benoetigen-zeit-und-papier-1.18307870. Zugegriffen: 19. Apr. 2019.

Hirschi, C. (19. Mai 2014). Der Schweizerische Nationalfonds und seine Open-Access-Strategie. Neue Zürcher Zeitung. https://www.nzz.ch/feuilleton/der-schweizerische-nationalfonds-und-seine-open-access-strategie-1.18304812. Zugegriffen: 19. Apr. 2019.

Hirschmann, B., \& Verdicchio, D. (2017). Open Access in der Schweiz. In K. Söllner \& B. Mittermaier (Hrsg.), Praxishandbuch Open Access (S. 215-222). Berlin: De Gruyter. https://doi. org/10.1515/9783110494068-025.

Larivière, V., Haustein, S., \& Mongeon, P. (2015). The oligopoly of academic publishers in the digital era. PLoS ONE, 10(6), e0127502. https://doi.org/10.1371/journal.pone.0127502.

Levin, N., \& Leonelli, S. (2017). How does one ,open" science? Questions of value in biological research. Science, Technology and Human Values, 42(2), 280-305. https://doi. org/10.1177/0162243916672071.

Manola, N., Rettberg, N., \& Manghi, P. (2015). OpenAIREplus project executive report. https:// doi.org/10.5281/zenodo.15464.

McKiernan, E. C. et al. (2016). Point of view: How open science helps researchers succeed. ELife. https://doi.org/10.7554/elife.16800.

Merton, R. K. (1973). The normative structure of science. In R. K. Merton (Hrsg.), The sociology of science: Theoretical and empirical investigations (S. 267-278). Chicago: University of Chicago Press.

Morrison, H. (2012). Freedom for scholarship in the internet age. http://summit.sfu.ca/item/12537. Zugegriffen: 16. Apr. 2019. 
Morrison, H. (2014). Economics of scholarly communication in transition. First Monday. https:// doi.org/10.5210/fm.v18i6.4370.

Posada, A., \& Chen, G. (2018). Inequality in knowledge production: The integration of academic infrastructure by big publishers. In L. Chan \& P. Mouner (Hrsg.), ELPUB 2018. https://doi. org/10.4000/proceedings.elpub.2018.30.

Reichmann, W. (2017). Open Science zwischen sozialen Strukturen und Wissenskulturen. TATuP Zeitschrift für Technikfolgenabschätzung in Theorie und Praxis, 26, 43-48. https://doi. org/10.14512/tatup.26.1-2.43.

Sample, I. (24. April 2012). Harvard University says it can't afford journal publishers' prices. The Guardian. https://www.theguardian.com/science/2012/apr/24/harvard-university-journal-publishers-prices. Zugegriffen: 16. Apr. 2019.

Savary, G. (2014). Interpellation 14.3215: Open Access. Eine Bedrohung für das Verlagswesen? Bundesversammlung - Das Schweizer Parlament 20. März 2014. https://www.parlament.ch/de/ ratsbetrieb/suche-curia-vista/geschaeft?Affairld=20143215. Zugegriffen: 19. Apr. 2019.

Schweizerische Akademie der Geistes- und Sozialwissenschaften (SAGW). (2016). Open-Access-Strategie der Schweizerischen Akademie der Geistes- und Sozialwissenschaften. https:// sagw.ch/fileadmin/user_upload/Open-Access-Strategie_4_.pdf. Zugegriffen: 16. Apr. 2019.

Speicher, L., Armando, 1., Bargheer, M., Eve, M. P., Fund, S., Leão, D., Mosterd, M., Pinter, F., \& Souyioultzoglou, I. (2018). OPERAS open access business models white paper. https://doi. org/10.5281/zenodo.1323708.

Staatssekretariat für Bildung, Forschung und Innovation (SBFI). (2015). Brief an die Präsidentin Swissuniversities vom 04.12.2018, Betr. Nationale Strategie Open Access. https://www.swissuniversities.ch/fileadmin/swissuniversities/Dokumente/Hochschulpolitik/Open_Access/151204_ Brief_SBFI_OA_nationale_Strategie_Swissuniversities_sign.pdf. Zugegriffen: 16. Apr. 2019.

Swissuniversities. (2018). Factsheet zur Verhandlungsstrategie. https://www.swissuniversities.ch/ fileadmin/swissuniversities/Dokumente/Hochschulpolitik/Open_Access/180315_Factsheets_ Verhandlungsstrategie_D.pdf. Zugegriffen: 16. Apr. 2019.

Swissuniversities, \& Schweizerischer Nationalfonds. (2016). Nationale Open-Access-Strategie für die Schweiz. https://www.swissuniversities.ch/fileadmin/swissuniversities/Dokumente/Hochschulpolitik/Open_Access/Open_Access_strategy_final_DE.pdf. Zugegriffen: 16. Apr. 2019.

Tulney, M. (2018). Plan S - Erste Implementierungsrichtlinien für Open-Access-Auflagen. TIB-Blog. https://blogs.tib.eu/wp/tib/2018/11/27/plan-s-erste-implementierungsrichtlinien-fuer-open-access-auflagen. Zugegriffen: 16. Apr. 2019.

Universities UK. (2017) Monitoring the transition to open access. https://www.universitiesuk. ac.uk/policy-and-analysis/reports/Documents/2017/monitoring-transition-open-access-2017. pdf. Zugegriffen: 16. Apr. 2019.

Weik, R. (7. Mai 2014). „Ein Schlag ins Gesicht“. Tagblatt. https://www.tagblatt.ch/ostschweiz/ ein-schlag-ins-gesicht-ld.660820. Zugegriffen: 19. Apr. 2019.

Verdicchio Dirk (Dr. phil./dirk.verdicchio@ub.unibe.ch) Ko-Leiter des Bereichs Digitale Dienste und Open Science an der Universitätsbibliothek Bern. Verantwortlich für Dienstleistungen und Infrastrukturen für Open Science (Open Access, Forschungsdaten, Forschungsinformationen, Publikationsdienste). Dozent für Wissenschaftskommunikation am Studiengang Bibliotheks- und Informationswissenschaft, Universität Zürich. Promotion in Soziologie an der Universität Basel um Thema Wissenschaftskommunikation in Filmen. 
Kissling-Näf, Ingrid (Prof. Dr./ingrid.kissling@bfh.ch) Direktorin Departement Wirtschaft der Berner Fachhochschule.

Forschungserfahrung als Assistenzprofessorin in Ressourcenökonomie an der ETHZ und langjährige Führungserfahrung unter anderem beim Schweizerischen Nationalfonds Abteilung Geistesund Sozialwissenschaften, bei der Förderagentur für Innovation KTI sowie bei der Akademie der Naturwissenschaften Schweiz . Während zwei Jahren tätig als Beraterin in der Privatwirtschaft bei der Firma Ernst Basler \& Partner AG. Sie ist zudem UNICEF-Delegierte und Stadträtin in Bern.

Open Access Dieses Kapitel wird unter der Creative Commons Namensnennung 4.0 International Lizenz (http://creativecommons.org/licenses/by/4.0/deed.de) veröffentlicht, welche die Nutzung, Vervielfältigung, Bearbeitung, Verbreitung und Wiedergabe in jeglichem Medium und Format erlaubt, sofern Sie den/die ursprünglichen Autor(en) und die Quelle ordnungsgemäß nennen, einen Link zur Creative Commons Lizenz beifügen und angeben, ob Änderungen vorgenommen wurden.

Die in diesem Kapitel enthaltenen Bilder und sonstiges Drittmaterial unterliegen ebenfalls der genannten Creative Commons Lizenz, sofern sich aus der Abbildungslegende nichts anderes ergibt. Sofern das betreffende Material nicht unter der genannten Creative Commons Lizenz steht und die betreffende Handlung nicht nach gesetzlichen Vorschriften erlaubt ist, ist für die oben aufgeführten Weiterverwendungen des Materials die Einwilligung des jeweiligen Rechteinhabers einzuholen.

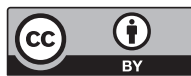




\title{
Plattformbasierte Dienstleistungen
}

\section{Dienstleistungen als Treiber des gesellschaftlichen Wandels}

\author{
Katinka Weissenfeld, Angelina Dungga und Jan Frecè
}

\begin{abstract}
Zusammenfassung
Peer-to-Peer-Dienstleistungsplattformen (P2P-Plattformen) stellen eine besondere Form von Dienstleistungsplattformen dar, die z. B. Geschäftsmodelle der Sharing Economy in seiner heutigen Form ermöglichen. Als Reaktion auf den aktuell herrschenden Begriffsdschungel rund um Sharing Economy und Dienstleistungsplattformen im Allgemeinen werden zunächst Begrifflichkeiten geklärt, bevor Aspekte des gesellschaftlichen Wandels diskutiert werden. Aufgrund iterativer Wechselwirkungen zwischen Sozialstrukturen und sozialen Akteuren werden die technischen, sozialen, ökologischen und ökonomischen Aspekte des gesellschaftlichen Wandels sowohl als Folge als auch als Vorbedingungen für das Aufkommen von P2P-Plattformen betrachtet. Abschließend werden in einem dritten Teil Chancen und Herausforderungen diskutiert, die sich aktuell und zukünftig durch P2P-Plattformen für die Gesellschaft ergeben. Dabei ist festzustellen, dass viele Aspekte sowohl Chancen als auch Herausforderungen mit sich bringen, die entsprechend abzuwägen sind.
\end{abstract}

K. Weissenfeld $(\varangle) \cdot$ A. Dungga $\cdot$ J. Frecè

Berner Fachhochschule Wirtschaft, Bern, Schweiz

E-Mail: katinka.weissenfeld@bfh.ch

A. Dungga

E-Mail: angelina.dungga@bfh.ch

J. Frèce

E-Mail: jan.frece@bfh.ch

J. Schellinger et al. (Hrsg.), Digitale Transformation und Unternehmensführung,

https://doi.org/10.1007/978-3-658-26960-9_3 


\subsection{Einleitung}

Das Phänomen eines gesellschaftlichen Wandels durch die Digitalisierung lässt sich durch das Zitat von Stalder (Stalder 2016) sehr gut wiedergeben: „Erst heute, wo die Faszination für die Technologie abgeflaut ist und ihre Versprechungen hohl klingen, wird die Kultur und Gesellschaft in einem umfassenden Sinne durch Digitalität geprägt“".

Der vorliegende Beitrag befasst sich mit plattformbasierten Dienstleistungen als Treiber für gesellschaftlichen Wandel und zeigt auf, welche Chancen und Herausforderungen für die Gesellschaft damit verbunden sind. Aufgrund der Vielfältigkeit und Komplexität plattformbasierter Dienstleistungen widmet sich der Beitrag digitalen Plattformen, die den direkten Bezug oder Austausch von Waren und Dienstleistungen unter „peers “, also unter gleichgestellten Mitgliedern einer Gruppe ermöglichen. Diese sind Ausdruck einer speziellen Form der Wirtschaft, die gesellschaftliche Veränderungen impliziert und von der ein beträchtliches Wachstum ausgeht. So wird in der EU der Bruttoumsatz solcher Plattformen im Jahr 2015 auf 28 Mrd. EUR geschätzt und es wird eine Verdoppelung des Umsatzes im Vergleich zum Vorjahr in fünf Schlüsselbranchen ${ }^{1}$ festgestellt. Des Weiteren geht die EU von einem zukünftigen Wachstumspotenzial entsprechender Plattformen von 160 bis 572 Mrd. EUR aus (2016). Dies zeigt die große Bedeutung und hohe Relevanz solcher Plattformen für die Gegenwart, aber auch für die nahe Zukunft.

In einem ersten Teil des vorliegenden Beitrags wird zunächst auf die Begriffsklärung von Peer-to-Peer-Dienstleistungsplattformen (P2P-Dienstleistungsplattformen) eingegangen, um so ein einheitliches Verständnis für die nachfolgenden Inhalte zu ermöglichen. Anschließend werden in einem zweiten Teil wesentliche Aspekte beschrieben, die in Interaktion mit P2P-Dienstleistungsplattformen von gesellschaftlicher Relevanz sind. In einem abschließenden dritten Teil stehen Chancen und Herausforderungen im Fokus der Diskussion, denen eine entsprechend hohe gesellschaftliche Brisanz zugesprochen werden und die für eine bestmögliche Entwicklung von ökologischen, gesellschaftlichen und wirtschaftlichen Potenzial von P2P-Dienstleistungsplattformen von Relevanz sind.

\subsection{Peer-to-Peer-Dienstleistungsplattformen}

Dienstleistungen haben über die letzten 60 Jahre hinweg einen zentralen Platz in unserer Gesellschaft eingenommen. Wie bereits in den 1950er-Jahren in der Drei-Sektor-Theorie von Fourastié (1954) beschrieben, gewinnt der tertiäre Sektor, nämlich die Dienstleistung, immer mehr an Bedeutung und dominiert unsere Arbeitswelt vor den primären und sekundären Sektoren Rohstoffgewinnung und Rohstoffverarbeitung. Mittlerweile

\footnotetext{
${ }^{1}$ Als Schlüsselbranchen gelten: Unterkunft (Kurzzeitvermietung), Personenbeförderung, Dienstleistungen für private Haushalte, freiberufliche und technische Dienstleistungen sowie Schwarmfinanzierung (Crowdfunding).
} 
spricht man von einem vierten Sektor (Gottmann 1957) dem Informationssektor, da die Dienstleistungen einen solchen Umfang eingenommen haben, dass gewisse Disziplinen den Bedarf sehen, diese entsprechend aufzuteilen. Der quartäre Sektor ist terminologisch nicht präzise fassbar, deckt aber gemäß Redlich und Wulfsberger (2011) immaterielle Güter wie Informationen, Ideen und Daten ab. Die Kommunikation, Speicherung und Verarbeitung dieser Daten und Informationen ist stark durch die heutigen technologischen Möglichkeiten geprägt (Traiblmaier 2006).

Im Zuge der Digitalisierung können Dienstleistungen, d. h. sowohl personenbezogene Dienstleistungen, wie z. B. Beratungsdienste, Taxi- und Hoteldienste, Weiterbildung und Verwaltungsdienste als auch sachbezogene Dienstleistungen wie Überbrückungsdienste in Form von Transport und Erhaltungsdienste in Form von Wartung und Reparaturen (Bodendorf 1999), immer einfacher einer Vielzahl von Nutzern angeboten werden. Ein weiterer stark zu beobachtender Trend besteht darin, dass Dienstleistungen nicht nur von professionellen Betreibern sondern von Privatpersonen über das Internet angeboten werden. Mittlerweile existieren zahlreiche Plattformen im Internet, über die Privatpersonen mit Internetzugriff kostengünstig und ohne größere Aufwände und Risiken ihre Dienstleistungen unzähligen potenziellen Kunden weltweit anbieten können. Die notwendigen Transaktionen zwischen Leistungserbringer und -bezieher können dabei ohne Einbezug eines Dritten durchgeführt werden.

Wird eine Internetplattform genutzt, um Dienstleistungen $\mathrm{zu}$ präsentieren und einen Leistungserbringer und einen Leistungsbezieher $\mathrm{zu}$ vermitteln, spricht man von einer Dienstleistungsplattform. Hier wird zunächst nicht unterschieden, ob es sich bei Leistungserbringer und -bezieher um Unternehmen, staatliche Einrichtungen oder Privatpersonen handelt. Des Weiteren kann die Rolle des Plattformbetreibers eine reine Vermittlerrolle oder aber auch eine unterstützende Rolle bei der Leistungserbringung sein (z. B. Unterstützung bei der Bezahlung). Beispiele für Dienstleistungsplattformen sind Online-Schalter von Behörden oder Immobilienplattformen wie immoscout24, aber auch Facebook als Kommunikationsplattform und Uber oder Airbnb können unter dem Begriff der Dienstleistungsplattform zusammengefasst werden.

Da die digitale Transformation und die damit einhergehende Vielfalt an Dienstleistungsplattformen neue Möglichkeiten für den geschäftlichen Austausch zwischen Gleichgestellten (Peers), d. h. i. d. R. Privatpersonen, ermöglicht, stützen sich die Ausführungen in Tab. 3.1 auf den vorherrschenden Trend $\mathrm{zu}$ sogenannten P2P-Dienstleistungsplattformen.

Von P2P-Dienstleistungsplattformen spricht man, wenn es sich um dezentrale Plattformen handelt, bei denen Leistungserbringer und Leistungsbezieher zumindest bei der Leistungserbringung direkt und ohne Einbinden eines Dritten agieren. Während traditionelle Geschäfte stark durch Economies of Scale und die Massenproduktion getrieben werden, stehen beim P2P-Business aus Sicht der Beteiligten zunehmend die Economies of Scope im Vordergrund (Quinn 1992). Die Effizienz entsteht hierbei, indem bei der Leistungserbringung zwischen Leistungserbringer und Leistungsbezieher keine Dritten im primären Prozess involviert werden. Bei einem P2P-Business spricht man im 
Tab. 3.1 Begriffsdefinitionen zu Peer-to-Peer. (Eigene Darstellung)

\begin{tabular}{l|l}
\hline Begriff & Eigenschaften und Beispiele \\
\hline Peer-to-Peer (Peers) & $\begin{array}{l}\text { Die Akteure (Peers) weisen eine hohe Heterogenität auf (beide } \\
\text { Akteure sind meistens Privatpersonen) } \\
\text { Peers bieten Dienste an und beziehen auch Dienste } \\
\text { Die Peers agieren autonom und sind selbstorganisiert }\end{array}$ \\
\hline Peer-to-Peer-Business & $\begin{array}{l}\text { Bei der Leistungserbringung zwischen Leistungserbringer und } \\
\text { Leistungsbezieher ist kein Dritter involviert } \\
\text { Leistungserbringer und Leistungsbezieher von Gütern oder } \\
\text { Dienstleistungen agieren unabhängig von anderen Parteien mit- } \\
\text { einander }\end{array}$ \\
\hline Peer-to-Peer-Dienstleistungen & $\begin{array}{l}\text { Dienstleistungen, die direkt zwischen Leistungserbringer } \\
\text { und Leistungsbezieher - ohne Einbezug eines Dritten - aus- } \\
\text { getauscht werden } \\
\text { Beispiele: Beratungsdienste, Fahrdienste, Übernachtungs- } \\
\text { dienste, Weiterbildungsdienste, Finanzdienste, Überbrückungs- } \\
\text { dienste wie Transport und Erhaltungsdienste wie Wartung und } \\
\text { Reparaturen }\end{array}$ \\
\hline Peer-to-Peer-Plattform & $\begin{array}{l}\text { Technologie, die P2P-Dienstleistungen unabhängig von Ort } \\
\text { und Zeit unterstützt, d. h. die Kommunikation zwischen den } \\
\text { Akteuren (Peers) und die Präsentation der Dienstleistung im } \\
\text { Internet ermöglicht } \\
\text { Beispiele: ebay, Airbnb }\end{array}$ \\
\hline
\end{tabular}

Gegensatz zum traditionellen Geschäft daher auch von einem dezentralen Geschäft, bei dem Anbieter und Bezieher von Gütern oder Dienstleistungen unabhängig von anderen Parteien direkt miteinander agieren (aber allenfalls über entsprechende elektronische Plattformen von Dritten entsprechend vermittelt).

Prominente Beispiele hierfür sind eBay und Airbnb, bei denen es sich um Plattformen handelt, die Dienstleistungen (oder Waren) ohne das Einbinden eines Dritten in der Phase der Leistungserbringung ermöglichen.

Diese Art des Geschäfts erinnert stark an das vorindustrielle Zeitalter, in dem Einzelhändler ihre eigens produzierte Ware direkt an Kunden verkauften (etwa durch die Eliminierung teurerer persönlicher Makler). Die digitale Transformation ermöglicht nun, dieses eigentlich veraltete Businessmodell in einigen Bereichen auf eine neue Weise wiederzubeleben. Wichtige und neue Akteure für das Business-Modell von P2P-Dienstleistungen sind die Plattformbetreiber, die nicht direkt in den Leistungserbringungsprozess involviert sind, jedoch essenzielle Grundlage für P2P-Dienstleistungen schaffen. Mit den heute existierenden P2P-Plattformen wurde eine effiziente und moderne Lösung geschaffen, die P2P-Dienstleistungen unabhängig von Ort und Zeit ermöglicht (Chtacherbia et al. 2002). 
Bei den heutigen P2P-Plattformen unterscheidet man die folgenden Ausprägungen (Botsman und Rogers 2010):

- die Akteure Leistungserbringer und Leistungsbezieher handeln ökonomisch getrieben und nutzen die P2P-Plattform, um kommerzielle Geschäfte abzuwickeln, wie dies beispielsweise bei eBay der Fall ist;

- Plattformen, bei denen Leistungserbringer und Leistungsbezieher nicht nur rein ökonomisch getrieben handeln, sondern auch weltanschauliche Aspekte wie etwa das Teilen und damit das Schonen von Ressourcen ebenfalls im Vordergrund stehen. Diese Form von P2P-Plattformen wird häufig auch als Sharing-Economy-Plattform bezeichnet (Botsman und Rogers 2010). Beispiele hierzu sind u. a. Airbnb und BlaBlaCar. Zur Sharing-Economy zählen neben dem Aspekt des Teilens auch das Tauschen und Leihen von Dienstleistungen und Produkten (Keller 2013);

- unterschiedliche Personen werden über eine P2P-Plattform vernetzt, um gemeinsam an Projekten zu arbeiten (z. B. OpenInnov);

- es wird Wissen über eine Plattform zur Verfügung gestellt, das von jedem abgerufen werden kann und damit geteilt wird (z. B. Wikipedia);

- Kommunikationsplattformen, wie beispielsweise Facebook, Retroshare oder Tox.

Im Folgenden wird auf alle genannten Ausprägungen Bezug genommen. Im Fokus stehen jedoch Plattformen, über die kommerzielle Geschäfte abgewickelt werden und bei denen eine neue Art der Geschäftsbeziehung durch Teilen entsteht.

\subsection{Aspekte gesellschaftlichen Wandels in Interaktion mit Peer-to-Peer-Dienstleistungsplattformen}

Das Aufkommen von P2P-Dienstleistungsplattformen beeinflusst eine Gesellschaft in verschiedener Hinsicht und führt dabei einen Wandel in Verhalten und Wahrnehmung vieler Menschen herbei. Dieser Wandel geht in einer modernen Gesellschaft so weit, dass von einer Veränderung der Struktur eines sozialen Systems die Rede sein kann, also von einem gesellschaftlichen Wandel. ${ }^{2}$

Nach einer sozialen Innovation durch P2P-Dienstleistungen, tritt der gesellschaftliche Wandel schrittweise durch ein Wechselspiel von bestehender Struktur des sozialen Systems und neu auftretendem Verhalten ein. P2P-Dienstleistungsplattformen sind also nicht nur Ausdruck gesellschaftlichen Wandels, sondern gleichzeitig dessen Vorbedingung.

\footnotetext{
${ }^{2}$ Aus den zahlreichen Definitionen des Begriffs „Gesellschaftlicher Wandel“ wird hier derjenige von Giddens (Hamari et al. 2016) und Stones (Weber 1922) verwendet, da dieser die Wechselwirkung zwischen Agenten und Struktur besonders herausarbeitet.
} 
Da Mitglieder sozialer Gemeinschaften häufig ähnliche Lebenswelten pflegen und sich diese gegenseitig bestätigen und somit auch ein „Wir“-Gefühl schaffen (Hillmann 1994), findet Fortschritt innerhalb einer Gesellschaft i. d. R. gruppenweise statt. Die nachfolgenden Ausführungen adressieren folglich nie die Gesamtheit einer Gesellschaft, sondern immer nur gesellschaftliche Teilgruppen und ihre Perspektive(n) auf einmal.

Wie gesellschaftlicher Wandel sich im Einzelnen entwickeln wird, ist im Voraus kaum abzuschätzen, aber es ist mit einiger Sicherheit vorauszusagen, welche Aspekte in der Ausgestaltung des gesellschaftlichen Wandels von Relevanz sein werden. Aus diesem Grund beschreiben die nachfolgenden Abschnitte eine Vielzahl von Aspekten, die im Zusammenhang mit P2P-Dienstleistungsplattformen einen gesellschaftlichen Wandel vorantreiben können. Zunächst werden technologisch ausgerichtete Themen wie Nutzerzahlen und Vertrauen in die P2P-Dienstleistungsplattformen thematisiert. AnschlieBend werden die sozialen Voraussetzungen und die beispielhaften Mechanismen des gesellschaftlichen Wandels beschrieben bevor auf die potenziellen ökologischen Folgen und die ökonomischen Aspekte, d. h. die Auswirkungen der neuen Dienstleistungen auf das Marktangebot, eingegangen wird.

\subsubsection{Technologische Aspekte}

Die Möglichkeit für plattformbasierte Dienstleistungen ist primär durch den technologischen Wandel der letzten Dekaden gegeben. Zum einen ist neben den technischen Voraussetzungen die nötige internationale Akzeptanz und Verbreitung der Technologien vorhanden. Zum anderen gewinnt mit dem Aspekt der technologischen Entwicklung die Sicherstellung von Qualität und Vertrauen eine neue Dimension. Im Folgenden werden die steigenden Nutzerzahlen von digitalen Plattformen im Detail beleuchtet und der Aspekt von Qualität und Vertrauen bezüglich digitaler Plattformen diskutiert.

\subsubsection{Steigende Nutzerzahlen von digitalen Plattformen}

Die International Telecommunication Union (ITU) publiziert jährlich Zahlen zur IKT-Nutzung weltweit. Die neuesten Daten zeigen, dass im Jahre $2017 \mathrm{mehr}$ als zwei Drittel der Weltbevölkerung in Gebieten leben, die über eine hohe mobile Breitbandabdeckung verfügen. Zudem werden IKT-Leistungen immer preiswerter. Ca. $80 \%$ der Bevölkerung in Industriestaaten nutzen das Internet, wobei der Anteil der Haushalte mit Internetanschluss sogar bei über $80 \%$ liegt. Wird die Anzahl Registrierungen an das mobile Netz betrachtet, so sind ungefähr $90 \%$ der Bevölkerung aus Industriestaaten an mobile Netze angeschlossen (ITU 2017).

Zahlen der OECD bestätigen zudem, dass in den OECD-Ländern eine Verlagerung der Wirtschaft in das Internet stattfindet (OECD 2014). Ca. $65 \%$ der 25- bis 44-jährigen Internetnutzer haben bereits Waren oder Dienstleistungen über das Internet bestellt, wobei die Top-3-Länder (Großbritannien, Deutschland, Dänemark) einen Anteil von ca. $90 \%$ ausmachen. Auch bei den über 65-Jährigen führten im Jahr 2013 ca. $40 \%$ 
eine Bestellung über das Internet durch. Wird der Anteil der E-Commerce-Teilnehmer an der gesamten Bevölkerung gemessen, so kann zwischen 2007 und 2013 ein Wachstum von ca. 18 Prozentpunkten festgestellt werden. Werden die Unternehmen nach Unternehmensgröße heruntergebrochen, wird ersichtlich, dass v. a. große Unternehmen (>250 Mitarbeiter) Online-Transaktionen anbieten. Gleichzeitig hält der Trend zur höheren Nutzung von Smartphones an und überholte 2013 die Nutzung von herkömmlichen stationären Telefonen. Dieser Trend scheint sich stark auf Dienstleistungsplattformen auszuwirken. So stieg die Nutzung von Facebook via Smartphone von $28 \%$ aller Facebook-Nutzer auf $75 \%$ im Jahre 2013. Dieser Trend wird auch durch die Nutzungszahlen von Wikipedia bestätigt. Der Zugriff via Smartphone auf die Webseite lag im Juni 2010 bei fast Null und stieg auf über 4 Mrd. Ende des Jahres 2013. Der Anteil an Zugriffen über Smartphones steigt im Vergleich zu anderen Kanälen heute stetig (OECD 2014).

Die weite Verbreitung des Internets und die Verlagerung wirtschaftlicher Kommunikationen und Transaktionen in das Internet legen nahe, dass auch P2P-Dienstleistungsplattformen von dieser Entwicklung profitieren. Ebenfalls ist zu berücksichtigen, dass Dienstleistungsplattformen zu jeder Zeit und von jedem Ort erreichbar seien können, da viele Nutzer die Möglichkeiten über das Smartphone verstärkt nutzen.

\subsubsection{Qualität und Vertrauen auf digitalen Plattformen}

Vertrauen ist ein multidimensionaler und subjektiver Faktor, der sowohl für Leistungsempfänger als auch für Leistungserbringer eine zentrale Rolle spielt, wenn Dienstleistungen (online) bezogen werden. Im Zuge der Digitalisierung werden Dienstleistungen immer häufiger über das Internet angeboten, sodass Leistungsempfänger und Leistungserbringer sich nicht persönlich kennenlernen und teilweise ausschließlich virtuelle Geschäfte abwickeln. In der Regel existieren Verträge und mögliche Reputationen über die Leistungsempfänger und Leistungserbringer, die das Vertrauen in den jeweiligen Geschäftspartner und damit in die Qualität der Dienstleistung der Leistungserbringer respektive in die Zahlungsmoral der Leistungsempfänger stärken sollen.

Werden Dienstleistungen über P2P-Plattformen angeboten, können die Geschäftspartner gegenseitiges Vertrauen nur virtuell aufbauen. Hierzu existieren bereits viele verschiedene Hilfsmittel, so etwa das Bewerten von Leistungsempfänger und Leistungserbringer und deren Leistungen oder aber allenfalls Gütesiegel, die über eine Zertifizierung durch Externe vergeben werden. Die Bewertungen durch Leistungsempfänger und Leistungserbringer finden für jede Plattform separat statt und sind nur auf der jeweiligen Plattform an die virtuelle Identität des einzelnen Leistungsempfängers und Leistungserbringers geknüpft. Über die Qualität der Dienstleistung oder der Ware ist ebenfalls nur das bekannt, was der Leistungserbringer in seiner subjektiven Wahrnehmung darüber berichtet.

So werden Angebote der sogenannten Sharing Economy, die auf dem kollaborativen Lebensstil basiert, beispielsweise nur von rund $20 \%$ der Bevölkerung genutzt, obwohl das Potenzial viel höher ist. Hauptbedenken der Nutzer ist die Unsicherheit und das damit nicht vorhandene Vertrauen in den jeweiligen Leistungserbringer bzw. 
Leistungsempfänger (BITKOM 2013). Des Weiteren wird der Bedarf nach Regulierungen und Standards für den neuen Markt der Sharing Economy immer größer, d. h. dass auch Standards für Reputationen und damit für die Vertrauenswürdigkeit der Akteure ein wichtiger Erfolgsfaktor für ein gesichertes Wachstum der Sharing Economy bzw. P2P-Dienstleistungsplattformen ist. $\mathrm{Zu}$ bedenken ist hierbei jedoch die enorme Vielfalt an P2P-Dienstleistungsplattformen, für die eine einheitliche Regulierung und Standardisierung über eine Vielzahl von Rechtsräumen hinweg kaum möglich ist. Eine Regulierung, die Gültigkeit für bestimmte Sektoren hat und für Online- und physische Dienstleistungen gleich ist, scheint eine angemessene Vision für die Zukunft zu sein (level playing field) (BMWi Bundesministerium für Wirtschaft und Energie 2016).

\subsubsection{Soziale Aspekte}

Grob definiert besteht gesellschaftlicher Wandel aus einer Iteration zweier Elemente:

1. Soziale Akteure reagieren auf die kulturelle, politische oder soziale und natürliche Umwelt betreffend Wandel mit neuen Weltanschauungen, Rollenvorstellungen etc.

2. Bestehende soziale Institutionen und konservative soziale Akteure reagieren auf neuartige Ideen und Handlungen.

Die wiederholte Wechselwirkung dieser beiden Elemente führt dabei zu einem gesellschaftlichen Prozess und im Idealfall schlussendlich zu einem gesellschaftlich stabilen Kompromiss. Wie stark in diesem spezifischen Fall

- die Interessen der Nutzer (billigere und/oder bessere Dienstleistung),

- die Interessen des jeweiligen Gewerbes (Marktanteil halten, bisheriges Geschäftsmodell ausschöpfen) oder

- die Interessen des Staates (klare legale Regelung, verlässliche Steuereinnahmen in genügender Höhe, soziale Ruhe) gewichtet werden, ist eine Frage der Machtdisparität verschiedener Gruppen einer Gesellschaft und ihrer Fähigkeit und Bereitschaft, Kompromisse einzugehen.

Die nachfolgenden Kapitel beleuchten für das Beispiel von P2P-Dienstleistungsplattformen relevante Elemente gesellschaftlichen Wandels: Das erste Kapitel schildert die gesellschaftlichen Vorbedingungen, welche dem Aufkommen von Communities im 20. und 21. Jahrhundert überhaupt erst den Boden bereitet haben, während sich die beiden folgendem Kapitel dem Einfluss von und der Teilhabe an technologischem Fortschritt zuwenden. Das letzte Kapitel beschreibt schließlich institutionellen Wandel am Beispiel von P2P-Dienstleistungsplattformen und zeigt beispielhaft davon betroffene Institutionen auf. 


\subsubsection{Stärkung des Community-Gedankens durch Stärkung des Einzelnen}

Die Gemeinschaft ist die ursprünglichste Zusammenlebensform des Menschen und damit das historische Grundelement jeder Gesellschaft. Der Begriff der Gemeinschaft, obwohl zuerst geprägt von Tönnies (1887), wird heute in diesem Zusammenhang eher im Sinn von Weber verwendet und bezeichnet somit eine soziale Beziehung, ,wenn und soweit die Einstellung des sozialen Handelns ... auf subjektiv gefühlter (affektueller oder traditionaler) Zusammengehörigkeit der Beteiligten beruht“ (Weber 1922). Im Gegensatz dazu stellt der Begriff der Gesellschaft eine soziale Beziehung dar, ,wenn und soweit die Einstellung des sozialen Handelns auf rational (wert- oder zweckrational) motiviertem Interessenausgleich oder Interessenverbindung beruht“ (Weber 1922). Beide Phänomene kommen allerdings nur in seltensten Fällen in ihrem „ganz reinen Typus“ (Weber 1922) vor, sodass Gemeinschaften z. B. zweckrationale Motivationen aufweisen dürfen, ohne dadurch als eine Vergesellschaftung zu gelten.

Das heute zu beobachtende Erstarken der Gemeinschaft in Form von Online Communities (Kardorff 2006) ist maßgebend durch folgende soziale Phänomene ermöglicht und/ oder vorangetrieben:

- Ein Großteil der Bevölkerung ist 24/7 mit dem Internet verbunden (Eurostat 2015). Dies ermöglicht eine breite und alltägliche Nutzung der Innovation.

- Eigentum als traditionelles Statussymbol verliert beständig an Bedeutung (Belk 2014). Erst durch den beginnenden sozialen Bedeutungsverlust von Eigentum zugunsten von funktionalem Besitz ${ }^{3}$ und vor allem Zugang, kann Teilen sozial akzeptabel werden.

- Individualisierte Lebensführung wird höher gewertet als sozial akzeptierte Lebensentwürfe und Lebensläufe (Beck 1986; Dahrendorf 1961; Thome 1985). Traditionelle Anbieter haben dadurch nicht per se den Vorzug vor neuartigen Angeboten.

- Von Dienstleistungsplattformen akzeptierte Zahlungsmittel sind großflächig im Einsatz. Eine Teilnahme an diesen Plattformen ist dadurch praktisch ohne Zusatzaufwand (Registration auf der Plattform) möglich.

- Ressourcenverbrauch wird nicht mehr im selben Maß mit Status verbunden (Weller 2015). Recycling und effizientere Nutzung von Ressourcen wird - zunächst noch als fortschrittliches Verhalten gewertet - zunehmend zum Mainstream, was den Erfolg von P2P-Dienstleistungsplattformen befeuert. Würde Verbrauch immer noch als Ausdruck von Erfolg und Reichtum gesehen, wäre diesen Plattformen ein entsprechendes soziales Stigma sicher.

Die Tatsache, dass eine verstärkte Individualisierung erst ein Erstarken von Communities ermöglicht, erscheint auf den ersten Blick paradox, sollte doch das „Wir“ bei Communities

\footnotetext{
${ }^{3}$ Unter funktionalem Besitz wird hier der Wille verstanden, über einen Gegenstand zu verfügen, um ihn zu gebrauchen.
} 
im Fokus stehen und nicht das „Ich“. Dabei ist zu bedenken, dass die Existenz einer Vielzahl unterschiedlicher, zum Teil selbstverwalteter Communities, die nach Belieben entstehen und vergehen können, einen fundamentalen Bruch mit den sozialen Konventionen in der Vorstellung der 1950er- und 1960er-Jahre des 20. Jahrhunderts und den allermeisten vorhergehenden Sozialkonventionen darstellt. Dieser Bruch wurde dadurch ermöglicht, dass das Individuum in der Betrachtung der Gesellschaft an Wichtigkeit gewann und vermehrt als einzelne Persönlichkeit und nicht als bloße Manifestation der Gemeinschaft oder später Gesellschaft gesehen wurde.

Erst als die individuelle Persönlichkeit unabhängig genug sein durfte, um subjektiv ein effektuiertes oder traditionelles Zusammengehörigkeitsgefühl zu entwickeln - zuvor wurden solche Tendenzen als gefährliche soziale Anomalien gesehen, die es im Auge zu behalten galt -, wurde die Bildung von Communities und eventuell darin gelebter Subkulturen sozial denkbar und machbar. In Kombination mit neuen Technologien, welche die Bildung einer Online Community unabhängig von einem geografischen Ort ermöglichen, aber auch einer voranschreitenden Technisierung früher technikfernerer Gruppen, rückten globale Communities aus dem Bereich des Denkbaren ins Machbare.

\subsubsection{Technischer Fortschritt und gesellschaftlicher Wandel}

Neues Wissen oder technische Errungenschaften ziehen seit Jahrhunderten einen Wandel der davon betroffenen Gemeinschaften und Gesellschaften nach sich. Berufszweige entstehen und verschwinden, soziale Wertzuschreibungen verschieben sich, selbst der Stellenwert von religiösen Weltanschauungen kann von Fortschritten in Technik und Wissen beeinflusst werden.

Kurzfristige, tief greifende technische Innovationen, wie sie P2P-Dienstleistungsplattformen zweifellos darstellen, durchbrechen nicht nur Rollenerwartungen, sondern können völlig neue Ansprüche an Lernende stellen, die von einigen Mitgliedern der Gruppe potenziell nicht mehr oder nur unter zusätzlichem Aufwand zu leisten sind. Vor allem grundlegend innovative Entwicklungen, welche nicht nur eine leichte Anpassung des Bekannten, sondern tief greifend neue, vielleicht auch kontraintuitive Perspektiven und Denkweisen voraussetzen, verlangen Adaptionsfähigkeit. Im Versagensfall wird das Nichtadaptieren entsprechender Denkweisen mit Statusverlust oder gar sozialem Ausschluss sanktioniert.

Eine qualitative Änderung des gesellschaftlichen Wissenskorpus bedeutet auf der einen Seite immer den Statusverlust der bis dahin Wissenden und stellt für die Stabilität der gesellschaftlichen Struktur eine Gefährdung dar. Auf der anderen Seite bietet das neue Wissen denjenigen, die das neue Wissen aufzunehmen und zu nutzen wissen, neue Chancen sozial oder ökonomisch aufzusteigen (Mead 1934). Sozial machtvollen Institutionen oder Individuen kann folglich nur an gesellschaftlicher Veränderung liegen, wenn sie sich zutrauen, die Veränderung zu ihrem Vorteil zu nutzen. Je nach Macht dieser Institutionen oder Personen kann es in der Folge zu Einschränkungen der Nutzung oder Verbreitung neuen Wissens und/oder einer Subvention herkömmlichen Wissens kommen. 
Gilt diese Einschränkung nicht vollständig, sickert die Innovation über die Early Adopter einer Gesellschaft langsam durch. Wenn auch der Gewinn an sozialem Status häufig nicht sofort einsetzt, da die ,guten alten Dinge“ noch eine Weile mit einem Nostalgievorteil im sozialen Rennen verbleiben, setzen die funktionalen Vorteile der Nutzung einer neuen Technologie unmittelbar ein. Menschen, welche das neue Wissen adaptiert und die Anpassung gemeistert haben, erhalten durch die Anwendung des neuen Wissens Vorteile. Diese werden von anderen erkannt (Mead 1934). Je bekannter und beliebter ein Anwender neuer Technologie ist und je zugänglicher und übertragbarer die durch die neue Technologie hervorgebrachten Vorteile sind, desto mehr Menschen schreiben der neuen Technologie positive Eigenschaften zu und empfinden sie als erstrebenswert (Hill 2010). Dies geschieht auch gegen den geltenden sozialen Konsens, was diesen wiederum schrittweise schwächt. Ist gesellschaftlicher Wandel einmal so weit vorgedrungen, ist es nicht mehr Macht, die Wissen beschränkt, sondern neues Wissen, das bestehende Machtverhältnisse ins Wanken zu bringen vermag. Es ist also sehr wahrscheinlich, dass tief gehenden Innovationen Widerstand von jenem Teil der Gesellschaft erhalten, der durch einen Wandel der Verhältnisse an Macht und Status verliert.

$\mathrm{Ob}$ eine Gesellschaft den potenziellen Ausschluss einzelner Gruppen als Preis für die Inklusion oder den Aufstieg anderer Gruppen in Kauf nimmt, hängt ab von relativen Faktoren, wie z. B. der Größe, Reputation und dem Machtpotenzial der betroffenen Gruppen, aber auch absoluten Aspekten, wie z. B. dem Selbstverständnis der urteilenden Bevölkerungsgruppen, legalen Standards, zur Verfügung stehenden Ressourcen, sozialen Traditionen usw. (Warschauer 2004; The Tomás Rivera Policy Institute 2015). Jede Gesellschaft steht in all ihrer Einzigartigkeit der gleichen Herausforderung des gesellschaftlichen Wandels gegenüber: Der Modernisierung von Institutionen, ohne dass aus Gründen des Machterhalts Teile von der Modernisierung ausgeschlossen werden und ohne dass Teile der Gesellschaft aufgrund ihrer traditionellen Haltung sanktioniert werden. Breite soziale Teilhabe ist für offene Gesellschaften zentral, um ein Wir-Gefühl zu schaffen und zu erhalten, das den Zusammenhalt offener Gesellschaften langfristig überhaupt erst ermöglicht (Popper 1975).

Einerseits ermöglichen P2P-Dienstleistungsplattformen eine Privatisierung der Dienstleistungserbringung, was insbesondere für eine Dienstleistungsgesellschaft eine Art teilweise Vergemeinschaftung der Gesellschaft bedeutet. Damit ist zwar nicht eine De-facto-Rückkehr zur gemeinschaftlichen Lebensform gemeint, sondern das Phänomen, dass der Handelspartner trotz des zumindest anfänglich klar funktionalen Beziehungscharakters sein Gegenüber nicht primär als Rollenvertreter einer Institution ansieht, dem an der Bewahrung der bestehenden Verhältnisse liegt, sondern als Privatperson wie sich selbst, daher der Begriff „Peer“ (Deaux 1996).

\subsubsection{Institutionen im gesellschaftlichen Wandel}

Die Identifikation und Trennung von privaten und juristischen Personen ist in den sozialen Institutionen Europas seit Jahrhunderten institutionell verankert, um der jeweiligen 
Rolle und den damit verbundenen sozialen, aber auch rechtlichen Erwartungen gerecht werden zu können. Die Aufteilung in private und gewerbliche Handlungen und deren Regelung hat historischen Hintergrund, ist somit keine soziale Konstante und daher durch gesellschaftlichen Wandel jederzeit umformbar. So existiert bei P2P-Dienstleistungsplattformen solch eine klare Unterteilung zwischen privatem und gewerblichem Handeln nicht mehr. Beispielsweise ist das Vermieten der eigenen (Ferien-)Wohnung an Freunde und Bekannte, das Verleihen des eigenen Autos oder das Teilen von Fahrtkosten durch Fahrgemeinschaften keineswegs ein neues Phänomen, da diese Handlungen von der Gesellschaft nicht als gewerblich betrachtet wurden. Die Auflösung gewohnter Rollen kommt im Falle der P2P-Dienstleistungsplattformen erst durch den neuen Maßstab der Nutzung solcher Dienstleistungen zustande. Ein weiterer Aspekt hierbei ist die Ausweitung der Geschäftspartner von Familie, Freunden und vielleicht Freunden zweiten Grades auf jede beliebige Person, die den aktuellen Anforderungen der jeweiligen Plattform genügt.

Eine Auflösung bestehender Verhältnisse geht selten ohne Widerstände vonstatten, denn sie birgt Risiken. Traditionell gut im Markt positionierte Marktteilnehmer oder gar Marktführer spüren den Druck der neuen durch den gesellschaftlichen Wandel hervorgebrachten Konkurrenz und Geschäftsmodelle (beispielsweise im Taxigeschäft im Gegensatz zu Uber). Wenn das neue Geschäftsmodell Bedürfnisse systemisch besser $\mathrm{zu}$ befriedigen weiß und damit eine bessere Dienstleistung zu erbringen vermag, bleibt dieser Druck naturgemäß bestehen. Die traditionellen Branchenführer müssen sich entscheiden, ob sie sich dem Wandel unterwerfen oder ob sie finanzielles, politisches und soziales Wissenskapital dafür einsetzen, den laufenden gesellschaftlichen Wandel zu stoppen oder zu verzögern und so die Führungsrolle wenigstens vorübergehend zu behalten.

Die Chancen neuer Institutionen für eine Gemeinschaft oder Gesellschaft bestehen aus den daraus neu entstehenden Rollen, welche die bestehenden Rollendefinitionen zwar infrage stellen und dadurch wie beschrieben ein Risiko für Etablierte darstellen, sich aber im Lösen einiger aktueller Probleme besser bewähren und dadurch ihren Nutzern Vorteile bringen. So widersprechen Geschäftsmodelle wie sie von ebay, Uber oder Airbnb betrieben werden zwar mit dem institutionalisierten Verständnis der Trennung von Gewerbe und Privatpersonen, befriedigen aber Bedürfnisse der Konsumenten besser als die etablierten Dienstleister und stellen deren Anspruch auf die Befriedigung ebendieser Bedürfnisse dadurch infrage. Dadurch entstehen Chancen auf bessere Lösungen bekannter Probleme, unter der Voraussetzung, die institutionellen Anpassungen können in der Folge gemeistert werden.

Durch P2P-Dienstleistungsplattformen kann das institutionalisierte, soziale Rollenverständnis neu diskutiert werden, da zumindest für einen Teil der Gesellschaft offensichtlich wird, dass ein Beibehalten des Bekannten neue, attraktive Lösungen verunmöglichen würde. Die gesellschaftliche Herausforderung besteht folgerichtig darin, soziale Institutionen an die neuen Verhältnisse anzupassen, wobei die Stabilität der neu ausgerichteten Institutionen stark von einem breiten sozialen Konsens abhängt, der dem 
individuellen Anspruch auf verbesserte Problemlösung wie auch dem gesellschaftlichen Anspruch auf stabile Verhältnisse gerecht wird (Seyfert 2011).

P2P-Dienstleistungsplattformen stellen naturgemäß vor allem Institutionen im Bereich der Erwerbsarbeit infrage, allerdings nicht nur. Die nachfolgenden Beispiele sollen einen Eindruck davon vermitteln, welche Fragen institutioneller Wandel nach sich ziehen kann, auch wenn er wie in diesem Fall nur ein sehr beschränktes Gebiet betrifft.

Die Unterscheidung zwischen Angestellten und Selbstständigen ist nach herkömmlichen Maßstäben unschwer zu leisten, abgesehen vielleicht von der Kategorie der Scheinselbstständigen, die sich allerdings auch nur als Folge der weitläufigen Institutionalisierung von „Outsourcing“ etabliert hat. Doch in welche Kategorie ein Taxifahrer fällt, der zwar seinen Lohn von einer Vermittlungsplattform überwiesen bekommt, die für sich Provision einstreicht, allerdings nicht von dieser für den Job als Taxifahrer angestellt wurde, muss u. a. auch juristisch erst geklärt werden. Ob die Schaffung einer solchen neuen Kategorie Auswirkungen auf die bereits bestehenden Erwerbskategorien hat, ist dabei nicht vorwegzunehmen und zeigt den dynamischen Charakter gesellschaftlichen Wandels. Ähnliche Fragen stellen sich zu Themen wie diversen Versicherungen, Vorsorge, Reichweite der eigenen Haftpflicht, Versteuerung der Einkünfte/Umsätze, Kündigungsschutz, Verzollung von Handelsware, Nutzung von Business-to-BusinessAngeboten usw.

\subsection{3 Ökologische Aspekte}

Klimawandel und die Ausschöpfung der natürlichen Ressourcen unseres Planeten setzen Nationen vor große ökologische Herausforderungen. Der ökologische Handlungsdruck wächst und wirkt sich gleichermaßen auf Staat, Wirtschaft und Bevölkerung aus. Die Schonung der natürlichen Ressourcen steht auf der weltweiten politischen Agenda und wurde in 2015 von der Generalversammlung der UNO verabschiedete Resolution A/RES/70/1 - „Transforming our world: the 2030 Agenda for Sustainable Development" adressiert (UN DESA United Nations Department of Economic und Social Affairs 2016). In der Schweiz beschäftigt sich die Impulsgruppe Dialog Grüne Wirtschaft mit der Thematik und hält fest, dass die Menschheit daran ist, die Belastbarkeit des Planeten $\mathrm{zu}$ überschreiten und damit die Umweltbedingungen $\mathrm{zu}$ verlassen, welche für ein langfristiges Überleben der Menschheit günstig sind. Als Treiber für die Übernutzung des Planeten nennt sie das starke Anwachsen der Weltbevölkerung, die Steigerung der Wirtschaftskraft und damit des Konsumniveaus als wesentliche Einflussfaktoren auf diese Entwicklung. Das Konsumniveau in der Schweiz bildet den Großteil der Umweltbelastung in der Schweiz. Würden alle Länder pro Kopf so viel konsumieren wie die Schweiz, würde die Belastbarkeitsgrenze des Planeten massiv überschritten. Die umweltrelevantesten Konsumbereiche sind die Ernährung, das Wohnen und die Mobilität (Impulsgruppe Dialog Grüne Wirtschaft 2016). 
Die ökologischen Herausforderungen, vor denen die Menschheit steht, wurden in der Schweiz u. a. durch den Aktionsplan Grüne Wirtschaft 2013 aufgenommen. In seinem Zwischenbericht an den Bundesrat hat das Bundesamt für Umwelt (BAFU) im April 2016 Maßnahmen für eine ressourcenschonende, zukunftsfähige Schweiz formuliert. Die Maßnahmen zielen darauf $a b$, die Leistungsfähigkeit der Wirtschaft und damit die Wohlfahrt insgesamt zu stärken, indem es eine Wirtschafts- und Konsumweise fördert, welche die Knappheit begrenzter Ressourcen und die Regenerationsfähigkeit erneuerbarer Ressourcen berücksichtigt und die Ressourceneffizienz steigert. Für die Legislaturperiode 2016-2019 sieht der Bericht u. a. Sensibilisierungsmaßnahmen vor, welche ressourcenschonende Kauf- und Nutzungsentscheide fördert (Umwelt 2016). Die Impulsgruppe Dialog Grüne Wirtschaft sieht in der Sharing Economy ein verstecktes Potenzial zur Ressourcenschonung. Sie will neue Formen des Konsums fördern, indem sie Konsumenten dafür sensibilisiert und die Kreislaufwirtschaft in die Praxis umsetzt. Sie treibt damit die Idee des Nutzens statt Besitzens voran, wie sie im Rahmen der Sharing Economy bereits praktiziert wird.

P2P-Plattformen eröffnen Raum für neue Geschäftsmodelle, die das Teilen einer Ressource in den Vordergrund stellen. Geschäftsmodelle, welche ungenutzte Waren oder Kapazitäten, wie z. B. die Vermietung von ansonsten leerstehenden Räumen oder das Vermieten einer ansonsten ungenutzten Mitfahrgelegenheit, fördern die Ressourceneffizienz und können zur Veränderung in Richtung einer Kreislaufwirtschaft beitragen.

\subsection{4 Ökonomische Aspekte}

Die Entfaltung neuer Verhaltensweisen in Bezug auf Konsum und neue Beschäftigungsmöglichkeiten werden durch P2P-Plattformen geschaffen. Diese Entwicklung bietet große Chancen für eine größere Inklusion und wirtschaftliches Wachstum, stellt aber die herkömmliche Wirtschaft und auch soziale Institutionen vor neuen Herausforderungen.

\subsubsection{Veränderung im Konsumverhalten}

Der klassische Konsument war lange Zeit primär ein Verbraucher. In den westlichen, kapitalistisch geprägten Gesellschaften bemaß sich daher der gesellschaftliche Status stark an den finanziellen Möglichkeiten der Mitglieder, die sich wiederum für alle wahrnehmbar am klarsten im Ressourcenverbrauch manifestierten.

Der stärker werdende Community-Gedanke ermöglichte in den letzten Jahrzehnten allerdings alternative Statuszuschreibungen. Dies hat zur Folge, dass der Konsum der jüngeren Generationen bereits in großen Teilen anders gesehen wird, als dass dies bei der Elterngeneration noch der Fall war (Bucic et al. 2012).

Wie sich diese neuen Formen von Marktwirtschaft von den traditionellen - auf die Gewinner/Verlierer-Dichotomie fixierten - Formen unterscheiden, ist dabei abschließend nicht beurteilbar. Grund dafür ist die zunehmende Abwesenheit für große Gruppen 
gültiger Sozialskripts, die das Aufkeimen kleinerer Communities überhaupt ermöglicht hat. Diese ist aber auch dafür verantwortlich, dass sich Communities kaum mehr an einem einzigen Vorbild orientieren, sondern, gestärkt durch den Siegeszug des Individualismus eine Community nach ihren eigenen Bedürfnissen ausrichten. Dass diese Bedürfnisse sich - im Vergleich zur Elterngeneration - weniger am materiellen Erfolg (und Verbrauch) des Einzelnen orientieren, sondern das gesellschaftlich effiziente Nutzen von Ressourcen in den Mittelpunkt stellen (Ghosh 2006), mag vielerlei Motivationen haben. Zwei in diesem Zusammenhang zentrale Strömungen sind die Umweltbewegung und die Debatte um Corporate Social Responsibility (CSR). Beide bestanden zwar schon seit dem ausgehenden 19. Jahrhundert, gewannen aber erst anfangs der 1970er-Jahre an gesellschaftlicher Bedeutung (Marens 2010; Marens 2013; McCormick 1991). Obwohl ab 1987 theoretisch durch die Nachhaltigkeitsdebatte vereint, verbleibt der Umweltgedanke dominierend, was auch an der Ausrichtung der neuen Geschäftsmodelle abzulesen ist. Wenn Ressourcen geschont werden sollten, so handelt es sich zumeist um stoffliche Ressourcen und seltener um soziale Ressourcen. Das Spielfeld ist groß und damit sind die Möglichkeiten zur erfolgreichen Realisierung alternativer, kollaborativer Geschäftsmodelle sicherlich noch längst nicht ausgeschöpft.

Eine weitere Entwicklung zeichnet die Auflösung der funktionalen Differenzierung der gesellschaftlichen Sphären „Konsum“ und „Produktion“ auf. Wurde damals der Konsument als passiver Käufer und Verbraucher gesehen, nimmt er heute eine immer aktivere Rolle bei der Leistungserbringung ein. Dem sogenannten arbeitenden Konsumenten wird seit Ende der 1990er-Jahre vermehrt eine Mitverantwortung bei der Leistungserbringung übertragen, wie z. B. im E-Commerce oder E-Banking (Kleemann et al. 2009). P2P-Plattformen stützen sich teilweise stark auf den arbeitenden Konsumenten ab: Wikipedia beispielsweise, indem die Nutzenden die inhaltlichen Beiträge verfassen oder Dienstleistungsplattformen, wie z. B. Airbnb, indem Konsumenten dazu aufgerufen werden, die Leistungsbezieher $\mathrm{zu}$ bewerten. Eine Besonderheit von P2P-Plattformen in dieser Hinsicht liegt auch darin, dass der Konsument auf derselben Plattform gleichzeitig auch Produzent sein kann. So kann dieselbe Person auf derselben Plattform einmal eine Fahrgelegenheit anbieten und ein anderes Mal eine in Anspruch nehmen.

\subsubsection{Veränderungen der Anbieterlandschaft}

P2P-Plattformen erleichtern den Marktzugang für neue Leistungsanbieter. Die Eintrittshürde selbst als Leistungsanbieter auf dem Markt aufzutreten, ist dank der Existenz von geeigneten Plattformen sehr klein. Will man freien Wohnraum für Kurzaufenthalte anbieten, seine Fahrdienste für kurze Personentransporte oder seine handwerklichen Fähigkeiten für kleine Reparaturarbeiten in der Nachbarschaft zur Verfügung stellen, so genügen ein paar Klicks auf der entsprechenden Plattform, um sich als Leistungserbringer zu registrieren. Einmal registriert, wird die angebotene Leistung auf dem Markt sofort ersichtlich und für Leistungsbezieher rasch auffindbar. 
Der Anreiz, ungenutzte Kapazitäten zu monetarisieren steigt und steht für große Kreise der Gesellschaft offen. Entsprechende Leistungen auf dem Markt anzubieten, ist nunmehr nicht einem bestimmten Berufskreis vorbehalten, sondern steht auch Privatpersonen offen. Die Folge davon ist das Aufkommen einer großen Anzahl von selbstständig Erwerbstätigen auf dem Markt. Mithilfe von P2P-Plattformen können neue Einkommensquellen erschlossen werden und neue Beschäftigungschancen mit flexiblen Arbeitszeitregelungen entstehen (Europäische Kommission 2016). Der Kreis der Leistungserbringer wird damit wesentlich erweitert und auch die Vielfältigkeit der Angebote.

Wie auch der Eintritt zum Markt, gestaltet sich die Änderung oder auch der Rückzug eines Angebots vom Markt dank P2P-Plattformen sehr einfach. Dank dieser Einfachheit können Leistungserbringer ihr Angebot flexibel an ihre eigenen Kapazitäten oder an die Nachfrage anpassen. Sind die Kapazitäten ausgeschöpft, kann der Leistungserbringer leicht sein Angebot reduzieren oder zurückziehen. Sieht der Leistungsanbieter seine Teilnahme auf der P2P-Plattform als eine Möglichkeit, sich einen Nebenverdienst zu verschaffen (opportunity providers), kann er im Falle einer Verschlechterung der Bedingungen auf der Plattform mit einem Austritt reagieren. Zunehmend sind auf P2P-Plattformen jedoch Leistungsanbieter unterwegs, für die die Einnahmen aus der Plattform die primäre Einkommensquelle darstellen (necessity providers). Ihre Teilnahme an P2P-Plattformen ist weniger von den Bedingungen auf der Plattform selbst als von der Gesamtwirtschaftslage abhängig. Eröffnen sich dem Leistungserbringer Beschäftigungsmöglichkeiten außerhalb der Plattformwirtschaft, wird er diese nutzen (EPRS European Parliamentary Research Service 2016).

Der einfache Marktzutritt und -austritt wirkt sich auf den bestehenden Markt aus und kann die Angebotslandschaft verändern, indem die bestehenden Leistungen durch mehr oder neuartige Leistungen erweitert wird, bestehende Leistungen wegfallen oder das bestehende Leistungsangebot durch neue Leistungsangebote substituiert wird. Diese Veränderungen durch P2P-Plattformen beeinflusst auch die Nachfrageseite. Die Erweiterung des Angebots ist für die Leistungsempfänger von Vorteil, da die Angebote oft ihre Bedürfnisse besser abdecken und preisgünstiger sind. Für Leistungserbringer stellen sich dadurch jedoch Fragen rund um die Sozialleistungen. Für bereits im Markt etablierte Akteure stellen diese neuen Leistungserbringer eine neue Herausforderung dar, welche die bestehenden Verhältnisse teilweise grundlegend verändern.

\subsubsection{Kommerzialisierung bisheriger Praktiken des Teilens}

Teilen ist ein Phänomen, das es in unserem gesellschaftlichen Umgang immer schon gegeben hat. Ein prominentes Beispiel ist das Teilen von Landmaschinen. Bauern investieren gemeinsam in Landmaschinen, die sie nur einige Male im Jahr nutzen, und teilen sich die Maschinen dann. Dies wird schon lange so praktiziert und erfolgt auf persönlicher Kommunikationsebene, da die Bauern i. d. R. benachbart sind und teilweise auch schon über Generationen von der Option des Teilens Gebrauch machen. Auch die 
schon lange existierenden Wohngemeinschaften sind ein Beispiel dafür, dass das Phänomen des Teilens kein neuer Gedanke ist.

Neu ist allerdings, dass durch die Möglichkeiten, die die digitale Transformation bietet, sich solche einfachen Praktiken des Teilens mittlerweile in deutlich größeren Dimensionen und vor allem über größere Distanzen realisieren lassen. Hieraus entstand in den letzten zwei Jahrzehnten ein neues Geschäftsmodell in Form von Online-Plattformen, was den ursprünglichen Tauschgedanken erweitert und eine Kommerzialisierung des Tauschs von Dienstleistungen und Gütern herbeiführte. Standen beim ursprünglichen Gedanken des Teilens neben einer kosteneffizienten Lösung der Aspekt der Nachhaltigkeit und der soziale Aspekt häufig im Vordergrund, ist heutzutage ganz klar zu beobachten, dass der Trend stark geworden ist, aus kommerzieller Motivation heraus zu teilen (Hamari et al. 2016).

Auch Airbnb, eines der prominentesten Beispiele für eine erfolgreiche P2P-Plattform zur Wohnungsvermittlung, entstand, indem zwei Mitgründer von Airbnb in finanzieller Not waren und ein freistehendes Zimmer in ihrer Wohnung an Reisende vermittelten. Da schnell klar war, dass die Nachfrage und Zahlungsbereitschaft bei einem solchen Konzept sehr groß ist, war das neue Geschäftsmodell- über eine P2P-Plattform freistehende Zimmer und Wohnungen zu vermitteln - schnell geboren (Hofmann 2016). Die Alliance for a New Economy (LAANE) zeigt auf, dass der Großteil des Umsatzes der Plattform heute durch kommerzielle Anbieter generiert wird (Samaan 2015).

In Deutschland sind bereits über 100 Online-Plattformen verfügbar, auf denen P2PSharing-Dienstleistungen angeboten werden. Dabei liegt der Fokus mittlerweile auf Mobilität und Gebrauchsgegenständen (vgl. Abb. 3.1). Einen großen Teil machen dabei Angebote zu Co-Using und Verkauf aus.

Abb. 3.2 stellt die Neuregistrierungen von P2P-Plattformen dar, die in Deutschland genutzt werden können. Hier ist klar zu erkennen, dass ein besonderer Hype für neue P2P-Plattformen in den Jahren 2010-2014 existierte und danach die Neuanmeldungen drastisch zurückgingen. Erste Plattformen in den 1990er-Jahren waren ebay und Homeexchange.
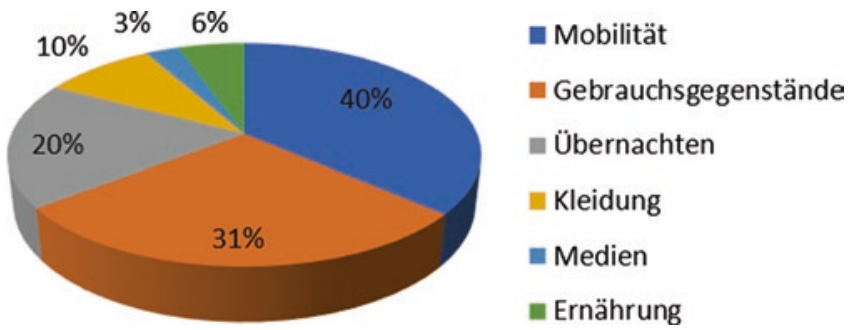

Abb. 3.1 Anzahl von P2P-Plattformen in Deutschland nach Konsumbereichen. (Eigene Darstellung; nach: Harnisch 2017) 


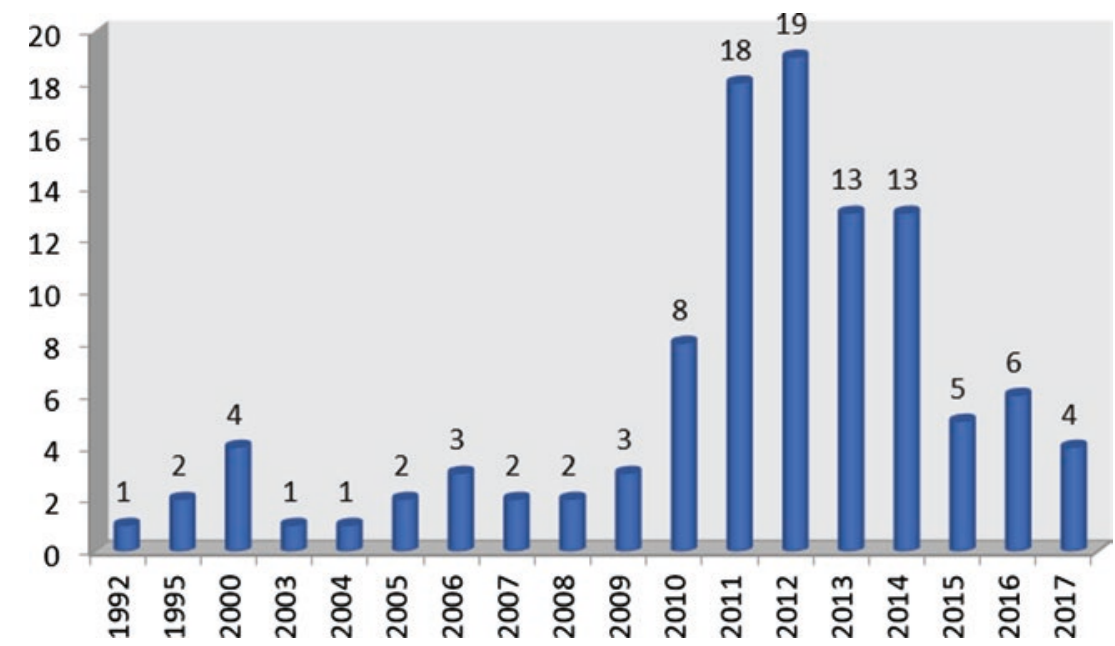

Abb. 3.2 Anzahl von Neugründungen von P2P-Plattformen in Deutschland. (Eigene Darstellung; nach: Harnisch 2017)

\subsection{Chancen und Herausforderungen für die Gesellschaft durch P2P-Dienstleistungsplattformen}

P2P-Dienstleistungsplattformen bergen für eine Gesellschaft neben zahlreichen Chancen auch einige Herausforderungen. Im Folgenden werden einige ausgewählte Chancen und Herausforderungen betrachtet, die bei P2P-Dienstleistungen von zentraler Bedeutung sind.

\subsubsection{Chancen durch P2P-Plattformen}

\section{Zeit- und ortsunabhängige Dienstleistungsangebote}

Durch die Digitalisierung besteht die Chance, möglichst viele Personen unabhängig von Ort und Zeit zu erreichen und somit bei P2P-Dienstleistungen aus der Perspektive von Leistungsbezügern ein reichhaltiges Angebot von Dienstleistungen zu erhalten. Aus der Sicht von Leistungserbringern kann über P2P-Plattformen eine einfache und schnelle Kommunikation zu potenziellen Kunden aufgebaut werden.

\section{Erweiterung der Angebotslandschaft}

Durch die direkte Vermittlung zwischen Peers können Leistungen, die üblicherweise über Vermittlungsfirmen oder andere Institutionen (traditionelle Intermediäre) angeboten wurden, innovativer gestaltet werden. Prominentes Beispiel hierfür sind P2P-Kredite. 
Erfolgte die Vergabe von Krediten traditionell über Kreditinstitute, können Kreditgeber und Kreditnehmer mittels P2P-Plattformen, wie z. B. zopa.com auf Finanzinstitute als Akteur verzichten. Dadurch eröffnen sich neue Möglichkeiten der Finanzierung, wie z. B. crowd-lending. Auch im Verkehrsbereich setzen sich neue innovative Modelle zunehmend durch. Waren für die Personenbeförderung im Fernverkehr üblicherweise Busgesellschaften oder öffentliche Verkehrsmittel die herkömmliche Anlaufstelle, können heute alternativ dazu auch P2P-Plattformen, welche Mitfahrgelegenheiten vermitteln, genutzt werden.

\section{Volkswirtschaftliches Wachstum}

Das Ausmaß des volkswirtschaftlichen Potenzials, das durch P2P-Plattformen geschaffen wird, ist schwierig einzuschätzen. Jedoch wird ihr ein enormes Potenzial für volkswirtschaftliches Wachstum zugeschrieben. Gemäß Studien der Europäischen Kommission konnte in 2013 dank P2P-Plattformen weltweit ein Zusatzeinkommen von 5,5 Mrd. US\$ generiert werden. Der Umsatz aus P2P-Plattformen für 2013 wird weltweit auf 20 Mrd. US\$ geschätzt (EPRS European Parliamentary Research Service 2016).

Die im vorangehenden Kapitel beschriebenen Veränderungen legen ein weiteres Wachstum von auf P2P-Plattformen basierende Geschäftsmodelle nahe. Eine Einschätzung des European Parliamentary Research Services (EPRS) ergibt, dass fast die Hälfte der für den Privatkonsum hergestellten Waren und Dienstleistungen potenziell über P2P-Plattformen angeboten werden könnten (EPRS European Parliamentary Research Service 2016). P2P-Plattformen würden so den Konsum bestimmter Waren und Dienstleistungen ankurbeln, auch von solchen, die auf den herkömmlichen Märkten fehlen.

\section{Professionalisierung bisheriger sozialer Praktiken}

Eine Professionalisierung sozialer Praktiken bringt zumeist eine Standardisierung gewissen Grades mit sich, um die Dienstleistung replizierbar und damit planbar zu halten. Dies wiederum erleichtert es anderen, die gleiche oder zumindest eine ähnliche Dienstleistung anzubieten, was die Erreichbarkeit der Dienstleistung stark erhöht.

Zum Beispiel: Durch den Großteil der Menschheitsgeschichte war Altenpflege eine soziale Praxis, die nahezu ausschließlich innerhalb der Kerngruppe angefragt und geleistet wurde. Hatte jemand aus irgendwelchen Gründen keinen Anschluss an solch eine Kerngruppe, war Altenpflege kaum erreichbar. Erst durch die moderne Nachfrage nach Altenpflege in der Nachkriegszeit des 20. Jahrhunderts als Folge demografischer und medizinischer Entwicklungen wurde diese Dienstleistung professionalisiert und steht seitdem einem immer breiterem Publikum offen, sodass Altenpflege heutzutage nicht mehr gezwungenermaßen vom Sozialnetz abhängt und somit auch alleinstehende ältere Menschen Aussicht auf einen umsorgten Lebensabend haben. 


\subsubsection{Herausforderungen durch P2P-Plattformen}

\section{Vertrauensverlust durch Anonymität in einer virtuellen Welt}

In herkömmlichen geschäftlichen Beziehungen sind die Akteure den persönlichen und physischen Kontakt gewöhnt und können sie so auf zwischenmenschlicher Ebene eine Vertrauensbasis schaffen. Durch die digitale Transformation und den zunehmenden Trend, geschäftliche Beziehungen auf Online-Plattformen abzuwickeln, entfällt der persönlichen Kontakt und damit die Vertrauensgrundlage. Sowohl für Leistungserbringer als auch für Leistungsempfänger müssen neue Dienste geschaffen werden, die ein solches Vertrauen auch auf Online-Plattformen herstellen. Vernetzungen über soziale Netzwerke, Bewertungssysteme und das Einbinden von vertrauenswürdigen Stellen sind Beispiele, dem Phänomen der Anonymität entgegenzuwirken. So bietet beispielsweise Airbnb die Möglichkeit an, dass Fotos des offerierten Objekts von zertifizierten Stellen gemacht werden, um so das Vertrauen in die Echtheit der Fotos zu stärken.

Neben der Gefahr von Vertrauenseinbußen in den jeweiligen Geschäftspartner durch die im digitalen Business vorhandene Anonymität besteht die Unsicherheit für Nutzende von P2P-Dienstleistungsplattformen bezüglich der Verarbeitung ihrer Daten auf solchen Plattformen. Digitale Plattformen speichern die Daten ihrer Nutzer in der Regel und verwenden sie u. a. für zielgerichtete Werbung (targeted advertising). Für die Nutzenden von Dienstleistungsplattformen und damit auch von P2P-Plattformen ist es nach wie vor kaum nachvollziehbar, was mit ihren Daten geschieht. Auch dieses Phänomen birgt einen Vertrauensverlust für Nutzende von P2P-Plattformen in das digitale Geschäftsmodell gegenüber traditionellen Geschäftsmodellen mit sich.

\section{Schwächung der gesellschaftlichen Strukturen}

Eine Professionalisierung sozialer Praxen kann zu einer leichteren Erreichbarkeit der Dienstleistung führen, da sie nicht mehr vom Grad des Sozialanschlusses abhängt. Dasselbe Phänomen kann allerdings auch schnell dazu führen, dass eine Praxis, z. B. die Altenpflege, nicht mehr als Teil der institutionalisierten Rolle der Familie gesehen, sondern als normale Dienstleistung empfunden wird. Hat jemand die finanzielle Mittel nicht, sich eine solche Dienstleistung zu erwerben, kann er nicht mehr auf den automatischen Rückhalt seiner Kerngruppe zählen, selbst wenn diese vorhanden wäre. Die Professionalisierung der Altenpflege hat diese aus dem Selbstverständnis der Kerngruppe entfernt, was zu einer Schwächung der sozialen Strukturen innerhalb von Kerngruppen führt. Pointiert formuliert sind die Mitglieder einer Gruppe weniger aufeinander angewiesen, wenn Dienstleistungen von außen bezogen werden können und sehen sich daher auch weniger in der gegenseitigen Verantwortung.

\section{Neue Formen des Arbeitens}

Verschiedene Formen des Arbeitens waren lange Zeit stabil institutionalisiert. Die neue Unsicherheit, ob Arbeitsformen der privaten oder der gewerblichen Sphäre zuzuordnen sind, birgt Herausforderungen für alle Beteiligten. Ohne eine klare Unterscheidung, 
fehlt dem Erbringer der Dienstleistung die Gewissheit, welche Steuern und Abgaben zu entrichten sind und welche Freiheiten bzw. Pflichten ihm zustehen. Abgesehen von der lähmenden Wirkung von Unsicherheiten auf die Wirtschaft, tendieren unregulierte Situationen dazu, das Recht des Stärkeren zu begünstigen. Im hochregulierten Verhältnis von Arbeitnehmer und Arbeitgeber ist ein Rückzug darauf allerdings unakzeptabel, weshalb staatliche Institutionen bemüht sind, diese zeitnah klar unterscheiden zu können. Orientierungspunkte für die Differenzierung gibt die Europäische Kommission in den überarbeiteten Leitlinien für die Richtlinie über unlautere Geschäftspraktiken. Dabei empfiehlt sie die Prüfung der Häufigkeit der Dienstleistungen, die Gewinnorientierung und die Umsatzhöhe. Eine bestimmte Kombination kann darauf hinweisen, dass es sich bei einem Anbieter um einen Gewerbetreibenden handelt. Klare Regelungen und Kategorien fehlen allerdings derzeit.

\section{Konkurrenz zu herkömmlichen Branchen}

Bieten die Leistungsanbieter auf P2P-Plattformen Leistungen an, die anderweitig auch angeboten werden, so stellen sie eine direkte Konkurrenz zu diesen Anbietern dar. Dies ist z. B. der Fall in der Übernachtungsbranche oder in der Taxibranche: P2P-Plattformen, welche Fahrgelegenheiten anbieten, substituieren die Angebote in der Taxibranche; P2P-Plattformen, welche Übernachtungsmöglichkeiten für Kurzaufenthalte anbieten, substituieren die Angebote auf dem Hotelbranche. Anbieter, welche ihre Leistungen nicht mittels P2P-Plattformen anbieten, werfen der Regierung ungleiche Spieße vor. So seien die Leistungsanbieter auf P2P-Plattformen nicht an dieselben Auflagen gebunden wie sie und erfahren deshalb einen Vorteil.

P2P-Plattformen können sich auch auf die Branche auswirken, indem sie Angebote, die anderweitig nachgefragt werden, für sich beanspruchen. So hat Samaan (2015) festgestellt, dass die auf Airbnb angebotenen Liegenschaften zu einer Abnahme der für die städtische Bevölkerung zur Verfügung stehenden Liegenschaften geführt hat.

Dass sich P2P-Plattformen auf die herstellende Industrie der auf der Plattform angebotenen Güter auswirken, ist naheliegend. Fördern P2P-Plattformen doch die Wiederverwendung von einmal gekauften Gütern, ist die daraus resultierende Folge eine Abnahme der Nachfrage nach diesen Gütern. Studien belegen eine Reduktion der Anzahl Fahrzeugbesitzer in Zusammenhang mit Car-Sharing-Angeboten (Cervero et al. 2007; Martin et al. 2010).

\section{Rebound-Effekte}

Im Rahmen plattformbasierter Dienstleistungen wird sehr häufig ein positiver Einfluss hinsichtlich Ressourcenschonung vorangestellt. Insbesondere bei Dienstleistungsmodellen wie Car-Sharing wird dies sehr gerne als ein wesentliches Argument für die Stärkung solcher Konzepte angeführt. Werden Autos geteilt, so impliziert dies zunächst, dass weniger Autos genutzt werden, was eine positive Auswirkung auf die Umwelt haben sollte. Bedenkt man jedoch, dass eine Ausbreitung des Car-Sharing-Konzepts möglichweise dazu führt, dass immer mehr Personen die Dienstleistungen von 
Car-Sharing-Anbietern beziehen und damit aber auch weniger öffentliche Verkehrsmittel nutzen, stellt sich die Frage, ob die positiven Einflüsse bezüglich Ressourcenverbrauch wirklich vorhanden sind. Dieser Effekt wird als Rebound-Effekt bezeichnet. Es handelt sich dabei um einen Effekt, bei dem das vermeintliche Einsparpotenzial von Effizienzsteigerung nur bedingt existiert, da es durch andere Faktoren nahezu aufgehoben wird (Thomas 2012).

\subsection{Ausblick}

Dieser Beitrag zeigt auf, dass der gesellschaftliche Boden für P2P-Plattformen, der von vorangegangen Tendenzen zur Individualisierung und Wertfreiheit geschaffen wurde, gesellschaftliche Entwicklungen in diese Richtung weiter vorantreibt und damit einen breiten Einfluss auf die Wirtschaft aufweist. Während P2P-Plattformen das Gefühl einer Gemeinschaftszugehörigkeit zu stärken vermögen, kann auch festgestellt werden, dass Gruppen, welche die Zugangshürden, z. B. finanzieller, technischer oder sprachlicher Art, nicht meistern, von dieser Gemeinschaft eher ausgeschlossen werden. Die zunehmende Nutzung von P2P-Plattformen eröffnet die Möglichkeit, die Potenziale der P2P-Dienstleistungsplattformen auszuschöpfen, wobei die mit der Nutzung dieser Plattformen verbundenen Unsicherheiten sich aktuell noch als große Hürde herausstellen. Die neuen Beschäftigungschancen, die sich dank P2P-Plattformen ergeben, stehen Rechtsunsicherheiten in Bezug auf die soziale Sicherheit gegenüber. Die Idee der Förderung der schonenderen Nutzung von Ressourcen kann durch Rebound-Effekte kontraproduktive Wirkungen zeitigen.

Staatsregierungen sehen in einer sogenannten P2P-Economy ein großes Potenzial für wirtschaftliches Wachstum und eine effizientere Nutzung von natürlichen Ressourcen. Sowohl in der Schweiz wie auch auf EU-Ebene sollen Maßnahmen ergriffen werden, die es erlauben dieses Potenzial voll auszuschöpfen.

\section{Literatur}

BAFU Bundesamt für Umwelt. (2016). Bericht an den Bundesrat. Grüne Wirtschaft. Massnahmen des Bundes für eine ressourcenschonende, zukunftsfähige Schweiz. https://www.uvek.admin.ch/ uvek/de/home/umwelt/gruene-wirtschaft.html. Zugegriffen: 5. Apr. 2019.

Beck, U. (1986). Risikogesellschaft: Auf dem Weg in eine andere Moderne. Frankfurt a. M.: Suhrkamp.

Belk, R. (2014). You are what you can access: Sharing and collaborative consumption online. Journal of Business Research, 67(8), 1595-1600.

BITKOM. (2013). Das Internet schafft eine Kultur des Teilens. https://www.bitkom.org/Presse/ Presseinformation/Das-Internet-schafft-eine-Kultur-des-Teilens.html. Zugegriffen: 16. Aug. 2016. 
BMWi Bundesministerium für Wirtschaft und Energie. (2016). Grünbuch Digitale Plattformen. http://www.bmwi.de/BMWi/Redaktion/PDF/G/gruenbuch-digitale-plattformen,property $=$ pdf,bereich $=$ bmwi2012, sprache $=$ de,rwb=true.pdf. Zugegriffen: 18. Dez. 2016.

Bodendorf, F. (1999). Wirtschaftsinformatik im Dienstleistungsbereich. Berlin: Springer.

Botsman, R., \& Rogers, R. (2010). What's mine is yours - How collaborative consumption is changing the way we live. New York: HarperBusiness.

Bucic, T., Harris, J., \& Arli, D. (2012). Ethical consumers among the millennials: A cross-national study. Journal of Business Ethics, 110(1), 113-131.

Cervero, R., Golub, A., \& Nee, B. (2007). City carshare: Longer-term travel demand and car ownership impacts. Transportation Research Record: Journal of the Transportation Research Board, 1992(1), 70-80.

Chtacherbia, E., Mittermeier, L., Oberhausen, R., \& Wieland, T. (2002). Peer to Peer in Theorie und Praxis. JavaSpectrum, 04(2002), 23-28.

Dahrendorf, R. (1961). Gesellschaft und Freiheit - Zur soziologischen Analyse der Gegenwart. München: Piper.

Deaux, K. (1996). Social identification. In E. T. Higgins \& A. W. Kruglanksi (Hrsg.), Social psychology: Handbook of basic principles (S. 777-798). New York: Guilford.

EPRS European Parliamentary Research Service. (2016). The cost of Non-Europe in the sharing economy. Economic, social and legal challenges and opportunities. http://www.europarl.europa. eu/RegData/etudes/STUD/2016/558777/EPRS_STU(2016)558777_EN.pdf. Zugegriffen: 10. Jan. 2017.

Europäische Kommission. (2016). Europäische Agenda für die kollaborative Wirtschaft. https:// ec.europa.eu/transparency/regdoc/rep/1/2016/DE/1-2016-356-DE-F1-1.PDF. Zugegriffen: 2. Juni 2016.

Eurostat. (2015). Households with internet access at home. http://ec.europa.eu/eurostat/web/ products-datasets/-/tin00088. Zugegriffen: 20. Juli 2016.

Fourastié, J. (1954). Die große Hoffnung des 20. Jahrhunderts. Köln-Deutz: Bund-Verlag.

Gottmann, J. (1957). Megalopolis or the urbanization of the Northeastern seaboard. Economic Geography, 33(3), 189-200.

Hamari, J., Sjöklint, M., \& Ukkonen, A. (2016). The sharing economy: Why people participate in collaborative consumption. Journal of the Association for Information Science and Technology, 7(9), 2047-2059.

Harnisch, R. (2017). Landkarte des online-gestützten Peer-to-Peer Sharing in Deutschland 2017. http://www.peer-sharing.de/veroeffentlichungen/landkarte-peer-to-peer-sharing.html. Zugegriffen: 5. Apr. 2019.

Hill, B. (2010). The sociology of innovation. Cambridge. MIT. http://mako.cc/academic/generals/ bmh-generals-sociology_innovation.pdf. Zugegriffen: 4. Aug. 2016.

Hillmann, K. (1994). Wörterbuch der Soziologie. Stuttgart: Kroener.

Hofmann, A. (2016). Der Airbnb-Gründer über die Geschichte hinter seinem Milliarden-Startup. http://www.gruenderszene.de/allgemein/nathan-blecharczyk-airbnb-interview/2. Zugegriffen: 25. Apr. 2016.

Impulsgruppe Dialog Grüne Wirtschaft. (2016). Go for impact. https://www.gruenewirtschaft. admin.ch/grwi/de/home/go-for-impact/Go-for-Impact.html. Zugegriffen: 10. Jan. 2017.

ITU. (2017). ICT Facts and figures 2017. https://www.itu.int/en/ITU-D/Statistics/Documents/facts/ ICTFactsFigures2017.pdf. Zugegriffen: 10. Jan. 2017.

Kardorff, E. (2006). Virtuelle Netzwerke - Eine Neue Form Der Vergesellschaftung? In B. Hollstein \& F. Straus (Hrsg.), Qualitative Netzwerkanalyse (S. 63-97). Wiesbaden: VS Verlag.

Keller, A. (2013). Teilen heisst das neue Haben. Absatzwirschaft, 56(6), 32-37. 
Kleemann, F., Voß, G., \& Rieder, K. (2009). Crowdsourcing und der Arbeitende Konsument. Sozialwissenschaftlicher Fachinformationsdienst. Industrie- und Betriebssoziologie, 2009(1), 9-23.

Marens, R. (2010). Destroying the village to save it: Corporate social responsibility, labour relations, and the rise and fall of American hegemony. Organization, 17(6), 743-766.

Marens, R. (2013). What comes around: The early 20th century American roots of legitimating corporate social responsibility. Organization, 20(3), 454-476.

Martin, S., Shaheen, A., \& Lidicker, J. (2010). Impact of carsharing on household vehicle holdings. Transportation Research Record: Journal of the Transportation Research Board, 2143(1), 150-158.

McCormick, J. (1991). Reclaiming paradise: The global environmental movement. Bloomington: Indiana University Press.

Mead, G. (1934). Mind, self, and society. Chicago: University of Chicago Press.

OECD. (2014). Measuring the digital economy: A new perspective. http://www.oecd-ilibrary.org/ science-and-technology/measuring-the-digital-economy_9789264221796-en. Zugegriffen: 10. Jan. 2017.

Popper, K. (1975). Die offene Gesellschaft und ihre Feinde. München: Francke.

Quinn, J. B. (1992). Intelligent enterprise: A knowledge and service based paradigm. United States of America: Free Press.

Redlich, T., \& Wulfsberg, J. (2011). Wertschöpfung in der Bottom-up-Ökonomie. Heidelberg: Springer.

Samaan, R. (2015). IAANE policy brief: Short-term rentals and Los Angeles' lost housing. http:// www.laane.org/wp-content/uploads/2015/08/Short-Term_RentalsLAs-Lost_Housing.pdf. Zugegriffen: 2. Jan. 2017.

Seyfert, R. (2011). Das Leben der Institutionen: Zu einer allgemeinen Theorie der Institutionalisierung. Weilerswist: Velbrück.

Stalder, F. (2016). Kultur der Digitalität. Frankfurt a. M.: Surkamp.

The Tomás Rivera Policy Institute. (2015). Access \& use of technology - Disparities in access and use of information technology. http://immigrantservices.uscmediacurator.com/disparities-in-access-and-use-of-information-technology-it/. Zugegriffen 29. Dez. 2016.

Thomas, S. (2012). Energieeffizienz spart wirklich Energie - Erkenntnisse zum Thema „ReboundEffekte“. Energiewirtschaftliche Tagesfragen, 62(8), 8-11.

Thome, H. (1985). Wandel zu postmaterialistischen Werten?: Theoretische und empirische Einwände gegen Ingleharts Theorie-Versuch. Soziale Welt, 36(1), 27-59.

Tönnies, F. (1887). Gemeinschaft und Gesellschaft: Abhandlung des Communismus und des Socialismus als empirische Culturformen. Leipzig: Fues's.

Traiblmaier, H. (2006). Datenqualität und individualisierte Kommunikation. Wiesbaden: Deutscher Universitätsverlag.

UN DESA United Nations Department of Economic and Social Affairs. (2016). Sustainable development goals. https://sustainabledevelopment.un.org/sdgs\#. Zugegriffen: 10. Jan. 2017.

Warschauer, M. (2004). Technology and social inclusion: Rethinking the digital divide. Cambridge: Mass. \& MIT.

Weber, M. (1922). Wirtschaft und Gesellschaft. Tübingen: Mohr.

Weller, I. (2015). Freizeit und Lebensqualität in der Postkonsumgesellschaft. In R. Freericks \& D. Brinkmann (Hrsg.), Handbuch Freizeitsoziologie (S. 255-275). Wiesbaden: Springer. 
Weissenfeld, Katinka (Dipl. Wirtsch. Ing./katinka.weissenfeld@bfh.ch) Dozentin am Institut Public Sector Transformation des Departements Wirtschaft der Berner Fachhochschule für Projektmanagement und Wirtschaftsinformatik. Mehrjährige Projektmanagementaufgaben im IT-Umfeld. Forschungsschwerpunkte E-Accessibility, Privacy, Web Technologien.

Dungga, Angelina (lic.phil.hist./angelina.dungga@bfh.ch) Research Associate am Institut für Public Sector Transformation des Departements Wirtschaft der Berner Fachhochschule. Forschungsinteresse: Public Sector Innovation, E-Inclusion. Dozentin für empirische Sozialforschung und digitale Transformation in Gesellschaft und Politik. Davor Leiterin Dienst Nomenklaturen im Bundesamt für Statistik der Schweiz.

Frecè, Jan Thomas (Dr. des./jan.frece@bfh.ch) Lehr- und Forschungstätigkeit in den Themengebieten dezentrale Identitäten und Daten, digitale Transformation, nachhaltige Unternehmensführung und Unternehmenswerte in den Instituten Public Service Transformation und Sustainable Business der Berner Fachhochschule, davor Projekte zur betrieblichen Nachhaltigkeit am Institut für Unternehmensführung der Fachhochschule Nordwestschweiz, davor Project Manager, Service Solution Architect und Root Cause Analyst bei IBM Schweiz.

Open Access Dieses Kapitel wird unter der Creative Commons Namensnennung 4.0 International Lizenz (http://creativecommons.org/licenses/by/4.0/deed.de) veröffentlicht, welche die Nutzung, Vervielfältigung, Bearbeitung, Verbreitung und Wiedergabe in jeglichem Medium und Format erlaubt, sofern Sie den/die ursprünglichen Autor(en) und die Quelle ordnungsgemäß nennen, einen Link zur Creative Commons Lizenz beifügen und angeben, ob Änderungen vorgenommen wurden.

Die in diesem Kapitel enthaltenen Bilder und sonstiges Drittmaterial unterliegen ebenfalls der genannten Creative Commons Lizenz, sofern sich aus der Abbildungslegende nichts anderes ergibt. Sofern das betreffende Material nicht unter der genannten Creative Commons Lizenz steht und die betreffende Handlung nicht nach gesetzlichen Vorschriften erlaubt ist, ist für die oben aufgeführten Weiterverwendungen des Materials die Einwilligung des jeweiligen Rechteinhabers einzuholen.

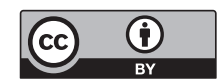




\title{
Gamification in Unternehmen
}

\section{Beeinflussung der Unternehmenskultur durch spieletypische Elemente}

\author{
Thomas Ellenberger, Deane Harder und Marie Brechbühler Pešková
}

\begin{abstract}
Zusammenfassung
Eine ,gute“ Unternehmenskultur ist ein wichtiger Erfolgsfaktor für Unternehmen. Im Rahmen der digitalen Transformation wird zunehmend Gamification zur Kulturbeeinflussung eingesetzt. Dabei werden spieletypische Elemente genutzt, um für Mitarbeitende für bestimmte Handlungen Anreize zu schaffen. Im Rahmen einer Fallstudie wurden digital umgesetzte Gamification-Maßnahmen eines Unternehmens bezüglich ihrer Wahrnehmung und Beeinflussung der Arbeitsmotivation analysiert. Die Ergebnisse zeigen, dass Gamification kulturwirksam zur Unternehmensführung genutzt werden kann. Die digitale Umsetzung diente als einheitlicher Impulsgeber und sorgte für Transparenz als Basis für Vertrauen. So konnte die angestrebte „Kultur auf Augenhöhe“ entwickelt werden, geprägt durch Respekt, Toleranz und Mitbestimmung. Der Erfolg der Gamification-Maßnahmen lässt sich vor allem durch die positive Wirkung auf die intrinsische Motivation sowie das gestärkte Zusammengehörigkeitsgefühl der Mitarbeitenden erklären.
\end{abstract}

T. Ellenberger $(\bowtie)$

Bern, Schweiz

E-Mail: thomas.ellenberger@gmx.ch

D. Harder

Berner Fachhochschule Wirtschaft, Bern, Schweiz

E-Mail: deane.harder@bfh.ch

M. Brechbühler Pešková

Institut Unternehmensentwicklung, Berner Fachhochschule Wirtschaft, Bern, Schweiz

E-Mail: marie.brechbuehler@bfh.ch

J. Schellinger et al. (Hrsg.), Digitale Transformation und Unternehmensführung,

https://doi.org/10.1007/978-3-658-26960-9_4 


\subsection{Einleitung}

„In every job that must be done, there is an element of fun.

You find the fun, and - SNAP - the job's a game!“"

Mary Poppins

Im Verständnis vieler Experten ist der Mensch ein spielendes Wesen, ein Homo ludens. Rund 2,2 Mrd. Gamer verbringen weltweit mehr als $3 \mathrm{Mrd}$. h pro Woche mit Videospielen (McDonald 2017; McGonigal 2012). Das ergibt etwa 156 Mrd. h pro Jahr. Videospiele sind längst keine Randerscheinung mehr, sie wurden während den letzten Jahren immer mehr zu einem Massenphänomen. Dabei werden die allermeisten Menschen nicht fürs Spielen bezahlt, sondern investieren ihre Zeit auf freiwilliger Basis. Mit dem enormen Erfolg von Videospielen kam in den vergangenen Jahren der Trend auf, spieletypische Elemente auch in spielefremde Kontexte zu verwenden. Dieses Vorgehen wird als Gamification bezeichnet. Tätigkeiten, Arbeitsabläufe oder ganze Prozesse können mithilfe von spielerischen Attributen und Prinzipien für die Anwender interessanter gestaltet werden.

Demografischer Treiber dieser Entwicklung sind vor allem die Generationen X, Y und Z. Also jene Menschen, die seit kurz vor der Jahrtausendwende geboren wurden und nun immer mehr in die Arbeitswelt drängen. Viele von ihnen sind sich durch ihre Freizeitaktivitäten eine ständige Stimulation der Sinne gewohnt und mögen es, mit Gamification-Elementen angesprochen zu werden (Ryan et al. 2013). Sie verlangen nach Spielerlebnissen in einer Art in der Freizeit, aber auch zunehmend bei der Arbeit, die ältere Generationen oft wenig verstehen (McGonigal 2012). Sie wollen eine erfüllende, für sie sinnvolle Tätigkeit ausüben, in der sie ihr Können einbringen und die Möglichkeit zur Selbstverwirklichung haben (Wikipedia 2018). Gamification verspricht genau diese Anforderungen erfüllen zu können. Mit entsprechend gestalteten Arbeitssituationen soll die Arbeitsmotivation der Mitarbeitenden gezielt und positiv angesprochen werden.

\subsubsection{Zielsetzungen der Studie}

Ziel dieser Studie war das Analysieren und Dokumentieren von digital umgesetzten Gamification-Maßnahmen zur Kulturbeeinflussung in Unternehmen. Dafür wurden die Maßnahmen der Firma Acme Apps $A G^{1}$ mit dem Octalysis-Modell zur Gamification untersucht und aufbereitet. Zudem sollte herausgefunden werden, wie die Gamification-Maßnahmen von Mitarbeitenden wahrgenommen werden und welche Wirkung diese auf die Arbeitsmotivation von Einzelnen und die Unternehmenskultur insgesamt haben.

\footnotetext{
${ }^{1}$ Name geändert.
} 


\subsubsection{Relevanz des Themas}

Bereits im Jahr 2013 zählte das Wirtschaftsmagazin Forbes Gamification zu einem der wichtigsten disruptiven Trends. Damals, auf dem Höhepunkt des sogenannten Gartner Hype Cycles, wurde jedoch auch vorhergesagt, dass bereits im Jahr 2014 etwa $80 \%$ der bestehenden Gamification-Maßnahmen aufgrund von fehlerhaftem Design fehlschlagen würden (Burke 2013). Im Jahr 2015 erschien der Begriff Gamification zum letzten Mal auf dem Gartner Hype Cycle. Gemäß diesem war die Zeit der übertriebenen Erwartungen an die Gamification vorbei und der Trend befand sich in der Phase des „Tals der Enttäuschung“. Es wurde vorausgesagt, dass der Trend in 2 bis 5 Jahren zum Mainstream gehören und das „Plateau der steigenden Produktivität“ erreichen würde (Levy 2015). Ein Jahr später, im August 2016, prognostizierte Forbes, Gamification würde bereits ab dem Jahr 2017 zu einer der zentralen Business-Strategien zählen (Newman 2016). In den Jahren des Hypes litt die Reputation von Gamification als unternehmerische Maßnahme. Wie durch Burke (2013) vorhergesagt, waren viele der bis dahin implementierten Maßnahmen unausgereift und deren Resultate konnten den hohen Erwartungen nicht gerecht werden.

Gemäß Gamification-Experten Yu-kai Chou (2016) lag dies an der Überbetonung bestimmter Gamification-Elemente. Solche fehldesignten Maßnahmen zeichnen sich dadurch aus, dass sie vor allem auf Punkte, Abzeichen und Ranglisten fokussieren (englisch Points, Badges and Leaderboards oder kurz PBL). Zur besseren und einfacheren Gestaltung von Gamification-Maßnahmen in der Praxis entwickelte Chou das sogenannte Octalysis-Modell (Chou 2018). Es eignet sich aber auch, um bereits bestehende Maßnahmen zu analysieren und bildet die methodische Grundlage der vorliegenden Studie.

\subsection{Unternehmenskultur, Gamification und Motivation}

Zum Verständnis der Studie werden nachfolgend einige der zentralen Begriffe theoretisch aufgearbeitet und definiert. Zuerst werden die Theorien zur Unternehmenskultur und zur Motivation vorgestellt. Ziel des Einbezugs dieser Theorien ist in erster Linie die Erarbeitung einer einheitlichen Terminologie. Daran anschließend wird Gamification erläutert und ein Überblick darüber gegeben, was das Thema alles beinhaltet und wie dieses in der Praxis angewendet werden kann. Ergänzt wird die Theorie durch die Vorstellung des Octalysis-Modells, das zur Analyse der Maßnahmen zur Kulturbeeinflussung verwendet wurde. 


\subsubsection{Definition Unternehmenskultur}

Einer der ersten und bis heute mit am weitesten verbreiteten Definition zur Unternehmenskultur stammt von Edgar H. Schein (1984). Er definiert Kultur als: „ein Muster gemeinsamer Grundprämissen, das die Gruppe bei der Bewältigung ihrer Probleme mit externen Anpassungen und interner Integration erlernt hat, das sich bewährt hat und das somit als bindend gilt; und das daher an neue Mitglieder als rational und emotional korrekter Ansatz für den Umgang mit diesen Problemen weitergegeben wird. " Herget und Strobl (2018) betonen dagegen eher normative Aspekte: „Kultur stellt die zentralen Spiel- und Kommunikationsregeln auf, die das faktische Leben innerhalb der Organisation entscheidend prägen. Sie legt auf eine informelle Art und Weise fest ,was man hier macht und was nicht', was als ,gut' belohnt wird und was als ,schlecht" sanktioniert wird.“ Eine ähnliche Definition bietet Sackmann (2002): „Unternehmenskultur sind die gemeinsamen grundlegenden Überzeugungen in einer Gruppe, die diese charakterisieren. Diese Überzeugungen beeinflussen Wahrnehmung, Denken, Handeln und Fühlen der Gruppenmitglieder. Sie können sich auch in den Artefakten manifestieren. Die Überzeugungen werden nicht mehr bewusst gehalten, sie sind aus der Erfahrung der Gruppe entstanden und haben sich durch die Erfahrung der Gruppe weiterentwickelt, d. h. sie sind gelernt und werden an neue Gruppenmitglieder weitergegeben“ (Sackmann 2002). Zentral bei diesen Definitionen sind also die impliziten Spielregeln und dass Kultur emergent und evolutionär ist, sie also von sich aus erwächst und nicht geplant oder verordnet werden kann.

\subsubsection{Beeinflussung der Unternehmenskultur}

Studien haben gezeigt, dass eine starke Unternehmenskultur mit dem Erfolg eines Unternehmens korreliert (Kotter und Heskett 1992). So wurde herausgefunden, dass mit einer „guten“ Unternehmenskultur sowohl der Umsatz, der Nettogewinn, der Aktienkurs sowie der Bestand an Mitarbeitenden signifikant stärker wuchsen (Jost 2003). Die Beeinflussung der Unternehmenskultur in eine positive Richtung ist also stark im Interesse des Unternehmens.

Eine Unternehmenskultur kann nicht direkt verändert werden. Um eine Unternehmenskultur verändern zu können, ist es zunächst wichtig, dass die Führungskräfte einen Veränderungsbedarf erkennen und gewillt sind, eine Veränderung herbeizuführen. In einem zweiten Schritt sollten die Mitarbeitenden für den Prozess gewonnen und ihnen die Notwendigkeit zur Veränderung plausibel und glaubwürdig vermittelt werden. Für die Erarbeitung der tatsächlichen Maßnahmen sollten möglichst viele Betroffene miteinbezogen und die Maßnahmen gemeinsam erarbeitet werden. Mitarbeitende sollten dabei jederzeit das Gefühl haben, vom Management unterstützt zu werden. Ansonsten kann der ganze Prozess nach einer anfänglichen Euphorie schnell im Sand verlaufen. Anschließend ist eine ständige Überprüfung und Weiterentwicklung der Kultur und der Maßnah- 
men sinnvoll (Zelesniack und Grolman 2018). Auch wenn der prinzipielle Prozess relativ einfach ist, sollte stets bedacht werden, dass Kulturveränderungen nicht so zielgerichtet bewirkt werden kann wie die Umsetzung eines Projektplans. Vielmehr bedarf es einen kontinuierlichen Abgleich zwischen gewünschter und tatsächlicher Wirkung von Maßnahmen und entsprechenden Anpassungen im weiteren Vorgehen.

\subsection{Motivation}

Motivation ist ein oft verwendeter Ausdruck, wenn es darum geht, etwas Bestimmtes anzupacken oder sein zu lassen. Motivation ist der ,Zustand einer Person, der sie dazu veranlasst, eine bestimmte Handlungsalternative auszuwählen, um ein bestimmtes Ergebnis zu erreichen und der dafür sorgt, dass diese Person ihr Verhalten hinsichtlich Richtung und Intensität beibehält" (Maier und Kirchgeorg 2018). Etwas anschaulicher ist Motivation die Triebkraft, die uns dazu bringt, bei mehreren Optionen die für uns ansprechendste auszuwählen und uns dazu bringt, eine Aktivität weiter zu verfolgen. Unsere Motivation ist dabei immer abhängig von einem aktuellen Bedürfnis. Zudem bestimmt sie, mit welcher Intensität und welcher Ausdauer wir uns in welche Richtung bewegen (De Luca 2017). Die gleiche Aufgabe kann dabei verschiedene Menschen auf unterschiedliche Art motivieren. Nicht jeder Mensch ist für die gleichen Aufgaben motiviert und selbst wenn, sind die Menschen nicht zwingend aus den gleichen Gründen motiviert (Mühlenhof 2018).

In der Psychologie wird zwischen extrinsischer und intrinsischer Motivation unterschieden. Von extrinsischer Motivation spricht man, wenn die Belohnung für eine Aufgabe von außerhalb kommt. Sind Menschen extrinsisch motiviert, führen sie eine Aufgabe nicht der Aufgabe willen aus, sondern wegen der Belohnung, die sie dafür bekommen. Ein einfaches Beispiel einer extrinsischen Belohnung, die Leute motivieren kann, ist der monetäre Lohn, den sie für eine Arbeit erhalten (De Luca 2017). Von intrinsischer Motivation spricht man, wenn eine Aufgabe wegen eines inneren Anreizes ausführt wird, der in der Tätigkeit selbst steckt. Wenn Menschen also eine Aufgabe gerne ausführen, weil sie Spaß macht, für sinnvoll erachtet wird, eine Herausforderung darstellt oder einfach interessiert (Mühlenhof 2018). Bei der Arbeit kommen typischerweise beide Formen der Motivation vor, allerdings gibt es zunehmend Bemühungen, den Anteil an intrinsischer Motivation bei der Arbeit zu erhöhen.

\subsection{Gamification}

Spiele können Menschen für eine lange Zeit fesseln. Sie können das kreative Potenzial fördern und bedeutsame Beziehungen zwischen Menschen aufbauen (Chou 2016). Die meisten Menschen betrachten Spiele jedoch hauptsächlich als Freizeitaktivität. Etwas, das wir freiwillig machen und an dem wir Spaß haben. Gamification beschäftigt sich 
damit, die Konzepte, die Spiele so vergnüglich und spannend machen, in einen spielefremden Kontext zu implementieren. Das folgende Kapitel beschäftigt sich mit der detaillierten Definition von Gamification und damit, wie Gamification eingesetzt werden kann. Auf die einzelnen Treiber hinter den Spielen und damit auch hinter Gamification wird anschließend in Zusammenhang mit dem Octalysis-Framework eingegangen.

\subsubsection{Definition Spiele}

Im Gegensatz zum Deutschen unterscheidet man im Englischen sprachlich zwischen „Game“ und „Play“. Dabei bezeichnet „Game“ vor allem das regelbasierte Spiel; „Play“ wird dagegen eher für Spiele genutzt wird, die mit wenigen oder gar keinen Regeln auskommen (Engelhard 2014). Im Rahmen dieser Arbeit wird ausschließlich auf den „Game“-Aspekt von Spielen eingegangen. McGonigal (2012) definiert hierfür vier Charakteristiken, die in sämtlichen Spielen vorhanden sind: Ein Ziel, Regeln, ein Feedbacksystem und Freiwilligkeit. Eine ergänzende Definition liefert Adams (2014), worin Spiele definiert werden als ,eine Form der Aktivität, die in einem Kontext einer vorgetäuschten Realität stattfinden und in der Teilnehmende versuchen, wenigsten ein willkürliches, nichttriviales Ziel zu erreichen, indem sie sich gemäß bestimmter Regeln verhalten." Zusammenfassend lässt sich also festhalten, dass Spiele nicht inhaltlich definiert werden, sondern über den Prozess und den dabei geltenden Rahmenbedingungen.

\subsubsection{Definition Gamification}

Der Begriff Gamification wurde in den letzten Jahren von diversen Autoren mit unterschiedlichen Definitionen verwendet. Der Begriff selbst setzt sich aus den Wörtern „Game“, Englisch für Spiel, und dem Suffix ,,-ification“, der Prozess etwas zu werden (Wiktionary 2018), zusammen. Eine mögliche Übersetzung wäre also in etwa: „Der Prozess, wie etwas zum Spiel wird“. Ungefähr 2008 ist der Begriff erstmals in den digitalen Medien aufgetaucht. Erst 2010, nachdem diverse Firmen begannen den Begriff zu verwenden, wurde ihm jedoch wirklich Beachtung geschenkt (Deterding et al. 2011). Eine der ersten Definitionen stammt von Deterding et al. (2011): „Gamification ist der Einsatz von Spiele-Elementen in einem Nicht-Spiele-Kontext.“ Dies ist bis heute eine der am weitesten verbreiteten Definitionen und wird beispielsweise im Wiktionary in ähnlicher Form verwendet: „Der Einsatz von Spielmechaniken in Nicht-Spiel-Anwendungen“ (Wiktionary 2017). Eine etwas weiterführende Definition schlug Kapp (2012) vor: „Gamification verwendet Spiele-basierte Mechanismen, Ästhetik und Spiellogik um Menschen anzusprechen, Handlungen zu motivieren, Lernen zu fördern und Probleme zu lösen“. Bei dieser Definition wurde gezielt der Teil „Nicht-Spiele-Kontext“ ausgelassen. Dies, weil frühere Autoren nie eine konkrete Definition dazu liefern konnten, was denn nun genau ein Nicht-Spiele-Kontext alles umfasst. Neu an dieser Definition ist 
dafür der Begriff der Ästhetik in diesem Zusammenhang, was erstmals impliziert, dass Gamification ansprechende Grafiken einsetzt und positive wahrgenommene Nutzererlebnisse vermittelt. Ebenfalls hinzugekommen ist „Menschen anzusprechen, Handlungen zu motivieren“, was zwei Schlüsselziele der Gamification in die Definition mit einschließt, nämlich, wie es gelingt, dass sich Leute beteiligen und engagieren und dass sie motiviert werden, eine bestimmte Aktion auszuführen (Engelhard 2014). Yu-kay Chou (2016) definiert den Begriff wie folgt: „Aus meiner Sicht ist Gamification die Kunst Spaß zu vermitteln und Elemente zu nutzen, die typischerweise in Spielen vorkommen, und diese auf produktive Handlungen in der Realität anzuwenden. Diesen Prozess meine ich, wenn ich von ,Human-Focused Design“ spreche.“ Etwas ausführlicher bedeutet diese Definition, Mechaniken aus Spielen mit Motivationspsychologie sowie Verhaltensökonomie zu verbinden und diesen auf einer technologischen Plattform ein ansprechendes User Interface zu geben. Für Chou (2016) ist eine Gamification-Maßnahme zudem im besten Fall für den Nutzer so gut wie unsichtbar. Er vergleicht dies mit einer Türklinke. Sie ist da und alle benutzen sie unbewusst mehrmals täglich, ihr wird jedoch nur selten spezifisch Beachtung geschenkt.

Chou gibt zu bedenken, dass Gamification eine Form der Manipulation ist. Diesen Einwand gilt es jedoch zu relativieren. Erstens sind Menschen ständigen Beeinflussungen ausgesetzt und nahezu jede Handlung wird bewusst oder unbewusst durch Interventionen geleitet. Zweitens sollten Gamification-Maßnahmen stets freiwillig und transparent sein. Benutzende sollten also die Gamification klar erkennen und sich dadurch nicht negativ beeinflusst oder ausgetrickst fühlen (Chou 2016).

\subsubsection{Human Centered Design}

In Gamification-Theorien wird häufig das Konzept des Human Centered Designs erwähnt. Es geht dabei darum, eine Maßnahme zu entwickeln, bei der Mensch als Nutzer im Mittelpunkt steht (Norman 2013). Human Centered Design stellt die Bedürfnisse von Nutzern stets über die Bedürfnisse der Unternehmung oder des Urhebers der Gamification-Maßnahme. Es geht darum, ob und wieso Nutzer eine bestimmte Funktion brauchen. Bei jeder Design-Entscheidung sollte man sich deshalb fragen: Wie profitieren Nutzer von diesem System (Nicholson 2012)? Ein wichtiger Bestandteil davon ist es, Gamification-Maßnahmen für Nutzer transparent zu machen. Diese sollen sehen, was genau mit der Maßnahme bezweckt wird und nicht nur, wie viele Punkte erreicht wurden, ohne zu wissen, was der Sinn dieser Maßnahme ist. Die meisten Maßnahmen, die lediglich ein Punktesystem oder eine Rangliste präsentieren, und so die extrinsische Motivation von Benutzenden ansprechen, folgen deshalb nicht den Prinzipien des Human Centered Designs (Nicholson 2012). 


\subsubsection{Gamification in Unternehmen}

Bei der Umsetzung von Gamification in internen Prozessen von Unternehmen gibt es einige wichtige Punkte zu beachten. Hier geht es beispielsweise nicht darum, jemanden zu einem Impulskauf zu überzeugen, den er kurz später bereits wieder bereut (,Buyer's Remorse“). Vielmehr steht die Langzeitmotivation der Mitarbeitenden im Fokus. Von zentraler Wichtigkeit ist, zuerst das Bedürfnis der Mitarbeitenden zu identifizieren. Man sollte die generelle Akzeptanz der Benutzer gegenüber einer solchen Maßnahme sowie deren Präferenzen kennen (Dale 2014). Dabei gilt es, die verschiedenen Spielertypen zu beachten und sicherzustellen, dass sich möglichst alle in der Maßnahme wiederfinden (Bartle 1996). Bereits hier sollte auch an die verschiedenen zeitlichen Phasen einer Gamification-Maßnahme gedacht werden (Stieglitz 2015). Außerdem sollte auch die bestehende Unternehmenskultur berücksichtigt werden (Dale 2014). Ebenfalls notwendig ist die Analyse der vorhandenen IT-Infrastruktur, da die meisten Gamification-Maßnahmen IT-gestützt sind. Da viele Gamification-Experten eine ansprechende Benutzeroberfläche empfehlen, sollte unbedingt im Voraus abgeklärt werden, ob die geplanten Maßnahmen überhaupt entsprechend umgesetzt und unterstützt werden können (Stieglitz 2015). Des Weiteren wird empfohlen, die Gamification-Maßnahme an einen geeigneten Prozess zu koppeln und sicherzustellen, dass die Mechaniken und die Ansätze, die mit der Maßnahme verfolgt werden, unmissverständlich klar sind. Ist die Maßnahme dann einmal implementiert, sollte sie wie jeder Prozess regelmäßig überprüft und, wenn nötig, angepasst werden. Eine Beurteilung der Maßnahme im Laufe der Zeit ist deshalb anzuraten, da die Maßnahmen teilweise nur kurzfristige Erfolge verzeichnen und nach einer gewissen Zeit durch die Benutzer als bedeutungslos oder gar störend wahrgenommen werden können (Stieglitz 2015).

\subsubsection{Warum Gamification?}

Warum sollte ein Unternehmen Gamification einsetzten? Warum nicht eine andere, konventionelle, bewährte Methode verwenden? Die Idee eine Arbeit durch Spielelemente interessanter $\mathrm{zu}$ machen, ist gar nicht so neu. Bereits 1984 stellte Charles Coonradt (2012) in seinem Werk „The Game of Work“ die Frage, warum Menschen für Hobbys oder im Sport bezahlen um noch mehr oder noch härter arbeiten zu dürfen, während sie für das Ausführen ihrer Arbeit einen Lohn als Motivator verlangen. IT-gestützte Spiele waren damals aber noch eine Randerscheinung und die mit ihnen einhergehenden Konzepte den Menschen weniger vertraut. Heutzutage sind Videogames zum Massenmedium geworden. Im Jahr 2012 war das Durchschnittsalter der Gamer in den USA 35 Jahre. Jeder vierte davon war über 50 Jahre alt. Außerdem spielten dort $97 \%$ aller Jugendlichen irgendeine Art von Games (McGonigal 2012). Durch die Spiele werden die Benutzer gefesselt und angeregt. Viele können den Feierabend kaum erwarten, wenn sie sich endlich wieder ihren Spielen widmen können. 
Volkswagen generierte 33 Mio. Aufrufe auf ihrer Website durch die Gamification-Maßnahme Perfect Car und erhielt dadurch 119.000 neue Ideen für Autos. Im Spiel Foldit, in dem Spieler neue Proteine designen können, halfen 240.000 registrierte Benutzer eine fehlende Verbindung in der Aids-Forschung zu finden. Ein Problem, an dem Forscher 15 Jahre lang gearbeitet hatten, konnte von Gamern in nur 10 Tagen gelöst werden. Eine Lernplattform für Studenten, die fürs GMAT lernten, konnte mit Gamification-Maßnahmen die Zeit, die die Studierenden darauf verbrachten und versuchten ihr Testresultat zu verbessern, um 370 \% erhöhen. Des Weiteren gibt die US Armee, deren Zielgruppe vor allem junge Männer sind, heute mehr Geld für Rekrutier-Spiele aus, als für jede andere Werbeplattform (Chou 2016). Dies sind nur ein paar Beispiele, wie Gamification in allen möglichen Situationen zu enormen Erfolgen führen kann. Somit dürfte deutlich geworden sein: Gamification ist längst keine belächelte Randerscheinung mehr, sondern ein Business mit enormem Potenzial, das im Zuge der digitalen Transformation mehr und mehr genutzt werden wird.

\subsection{Das Octalysis-Framework}

Das Octalysis-Modell ist ein Modell, das dazu dient, gamifizierte Maßnahmen zu planen, zu gestalten, zu analysieren oder zu verbessern. Das Modell wurde vom Gamification-Experten Yu-kai Chou empirisch anhand seiner eigenen Erfahrungen mit Spielen entwickelt und später mit wissenschaftlichen Theorien ergänzt und begründet. Aufgrund des fehlenden psychologischen Hintergrundes nennt Chou das Modell auch einen „Framework" und nicht psychologische Studie oder Theorie (Chou 2016). Dennoch ist es aus Anwendungssicht nützlich und wird auch in akademischen Kreisen als solide Grundlage für kulturelle Interventionen diskutiert.

Das Modell baut auf den sogenannten Core Drives auf, die verschiedenen Motivatoren hinter einer Aktion repräsentieren (Abb. 4.1). Die Core Drives stellen dabei theoretische Treiber dar, die Nutzer dahingehend motivieren, eine gewisse Aktion auszuführen. Chou (2016) weist dabei darauf hin, dass diese Core Drives nicht nur in Spielen, sondern in sämtlichen menschlichen Handlungen eine Rolle spielen. Fehlen die Core Drives komplett, ist keine Motivation vorhanden und es kommt infolgedessen keine Aktion zustande. Die Spieleindustrie ist lediglich die erste Industrie, die die Core Drives soweit gemeistert hat, dass sie Nutzer gezielt über viele Stunden motivieren können (Chou 2016).

Hauptziel des Octalysis-Modells ist es, Motivatoren zu identifizieren oder aufzuzeigen, wie zusätzliche Motivation für eine Aktion angeregt werden kann. Dabei sollte stets darauf geachtet werden, wie sich der Nutzer fühlt und weniger, wie man Gamification-Elemente in eine Aktion integrieren kann. Zudem sollte man es vermeiden, sich beim Planen von Maßnahmen von Spielen beeinflussen zu lassen, sondern sich stattdessen darauf konzentrieren, wie und welche der acht Core Drives in eine Maßnahme implementiert werden können. Dazu gibt es zu jedem Core Drive mehrere Gamification-Techniken, die speziell 


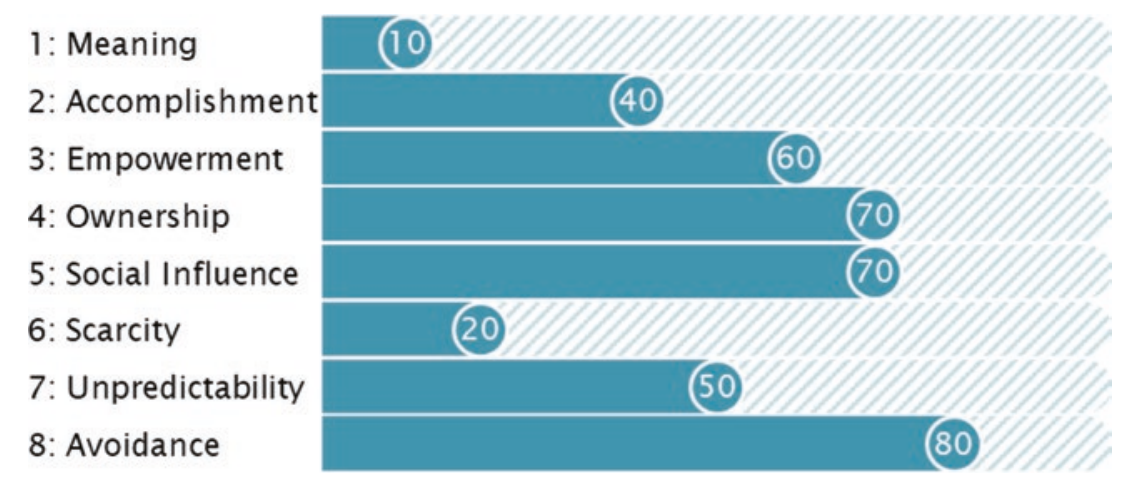

Abb. 4.1 Visualisierung des Octalysis-Modells zur Gestaltung und Analyse von gamifizierten Maßnahmen. (Illustratives Beispiel; eigene Darstellung)

dafür gedacht sind, diesen Core Drive in einem Arbeitsumfeld effizient umsetzen zu können (Chou 2016; aus Platzgründen wird in dieser Arbeit nicht auf die einzelnen Gamification-Techniken eingegangen, diese finden sich im Internet und in der Originalliteratur).

Durch das Gewichten sämtlicher Core Drives auf einer Skala von 0 bis 100 bezüglich einer Maßnahme kann diese bewertet werden; innerhalb des Modells wird dies als Octalysis-Score bezeichnet. Da theoretisch jede Aktion die Core Drives in einem geringen Maß beinhaltet, ist ein Score von weniger als 50 kein Indiz, dass hier spezielle Gamification-Techniken angewandt werden. Der Score ist außerdem als absolute Zahl wenig aussagekräftig, da die Gewichtung der Maßnahmen subjektiv ist. Anstatt damit eine Maßnahme als ,gut“ oder ,schlecht“ zu beurteilen, sollte er eher dafür benutzt werden, zu identifizieren, wo die relativen Stärken liegen und wo noch Potenzial für Verbesserungen vorhanden ist (Chou 2016).

\subsubsection{Octalysis Design Levels}

Das Octalysis-Modell ist in der bisherigen Ausarbeitung auf drei Levels ausgelegt: Core Drives, Spielephasen und Spielertypen (Tab. 4.1). In den meisten Situationen ist jedoch bereits das erste Level ausreichend (Core Drives), um eine Maßnahme bezüglich Gamification zu analysieren oder zu entwickeln (Chou 2012). Für die Studie wurde deshalb nur der erste Level zur Analyse der kulturverändernden Maßnahmen verwendet.

Der erste Level des Octalysis-Frameworks beinhaltet das namensgebende Oktagon mit den acht Core Drives. Aus Gründen der besseren Lesbarkeit wurde die ursprünglich achteckige Visualisierung als Balkendiagramm linearisiert. Es zeigt auf, welche Faktoren die Motivatoren bei Spielen sind (Tab. 4.2). Für die Auswertung einer bestehenden Maßnahme wird analysiert, welcher Core Drive darin in welchem Ausmaß vorhanden 
Tab. 4.1 Die drei konzeptionellen Level im Octalysis-Modell. (angelehnt an Chou (2016))

\begin{tabular}{l|l|l}
\hline Level & Konzept & Beschreibung \\
\hline 1. & Core Drives & $\begin{array}{l}\text { 4 Faktoren, die als Motivatoren bei Spielen wirken: Epic Meaning \& } \\
\text { Calling, Development \& Accomplishment, Empowerment of Creativity } \\
\text { \& Feedback, Ownership \& Possession, Social Influence \& Relatedness, } \\
\text { Scarcity \& Impatience, Unpredictability \& Curiosity, Loss \& Avoidance }\end{array}$ \\
\hline 2. & Spielephasen & $\begin{array}{l}\text { Phasen, die Spieler über den Gesamtverlauf eines Spiels durchleben: } \\
\text { Discovery, Onboarding, Scaffolding, Endgame }\end{array}$ \\
\hline 3. & Spielertypen & Achievers, Explorers, Socializers, Killers \\
\hline
\end{tabular}

ist. Anschließend wird jedem Core Drive eine entsprechende Zahl zwischen 0 und 100 zugeordnet. Aus den Einschätzungen ergibt sich ein Profil der untersuchten Maßnahme.

\subsubsection{White Hat and Black Hat Gamification}

Das Octalysis-Framework wird in White-Hat-Gamification- und Black-Hat-Gamification-Maßnahmen unterteilt (Tab. 4.3). In der Visualisierung des Modells sind dabei die oberen drei Core Drives White-Hat- und die untersten drei Black-Hat-Maßnahmen (Abb. 4.2). Die Core Drives vier und fünf können je nach Anwendung sowohl White-Hat- wie auch Black-Hat-Einflüsse haben, sind jedoch für keines davon typisch. Black-Hat-Elemente sind nicht typischerweise schlecht und White Hat gut. Für den Erfolg einer Maßnahme braucht es oft Einflüsse von beiden Typen. Die Black Hat Core Drives beinhalten jedoch diejenigen Maßnahmen, die, über einen längeren Zeitraum angewendet, eher zu Frust oder Abhängigkeit führen können. Deshalb sollte man beim Entwickeln einer Gamification-Maßnahme auch zuerst die White Hat Core Drives betonen (Chou 2016).

White Hat Core Drives sind die Elemente, die für eine Langzeitmotivation sorgen und zufriedenstellend wirken. Sie geben uns das Gefühl, die Kontrolle über unser eigenes Leben und unsere Aktionen zu haben. Hat eine Maßnahme keine White-Hat-Elemente, ist die Chance groß, dass sie langfristig nicht erfolgreich ist. Die große Schwäche der White Hat Core Drives ist, dass sie kein Gefühl der Dringlichkeit im Benutzer auslösen. Deshalb scheitern Maßnahmen, die sich nur darauf konzentrieren, meist bereits in den Discovery- und Onboarding-Phasen. Beim Arbeiten in einem Unternehmen, in welchem diese beiden Phasen nach der Anstellung jedoch sehr rasch überwunden sind und es um eine Langzeitverbindung geht, sollte man sich vorwiegend auf White-Hat-Maßnahmen fokussieren. Dies führt dazu, dass sich Mitarbeitende gut fühlen, mit dem Unternehmen wachsen können und auch länger dabeibleiben (Chou 2016).

Dies deckt sich mit der Self-Determination-Theorie von Deci (2008) bzw. deren Interpretation durch Pink (2011). Diese besagt, dass Menschen insbesondere durch drei intrinsische Treiber motiviert werden: Erstens Autonomie, das Bedürfnis selbst 
Tab. 4.2 Die acht Faktoren, die als Motivatoren bei Spielen wirken. (angelehnt an Chou (2016))

\begin{tabular}{|c|c|c|}
\hline $\mathrm{Nr}$. & Core Drive & Erklärung \\
\hline 1. & Epic Meaning \& Calling & $\begin{array}{l}\text { Dieser Core Drive wirkt, wenn jemand das } \\
\text { Gefühl hat, Teil von etwas Größerem, Wich- } \\
\text { tigerem als sich selbst zu sein, oder zu etwas } \\
\text { Bestimmten berufen zu sein }\end{array}$ \\
\hline 2. & Development \& Accomplishment & $\begin{array}{l}\text { Dieser Core Drive spricht die innere Moti- } \\
\text { vation an, Fortschritte zu erzielen und die } \\
\text { eigenen Fähigkeiten zu verbessern um im } \\
\text { Endeffekt eine Herausforderung überwinden } \\
\text { zu können }\end{array}$ \\
\hline 3. & Empowerment of Creativity \& Feedback & $\begin{array}{l}\text { Hier geht es darum, den Benutzer immer } \\
\text { wieder einen kreativen Prozess durchlaufen } \\
\text { zu lassen, ihn zu ermutigen, Neues auszu- } \\
\text { probieren und ihm eine unmittelbare Rück- } \\
\text { meldung dazu zu geben. Dieser Core Drive } \\
\text { beinhaltet was die meisten Menschen unter } \\
\text { „spielen“ verstehen }\end{array}$ \\
\hline 4. & Ownership \& Possession & $\begin{array}{l}\text { Hier geht es darum den Benutzern das Gefühl } \\
\text { zu geben, etwas zu besitzen oder zu kontrol- } \\
\text { lieren. Entscheidungen werden hier vor allem } \\
\text { analytisch getroffen und Besitztum ist der } \\
\text { primäre Motivationsfaktor }\end{array}$ \\
\hline 5. & Social Influence \& Relatedness & $\begin{array}{l}\text { Core Drive } 5 \text { beinhaltet sämtliche sozialen } \\
\text { Elemente einer Maßnahme, wie Mentoring, } \\
\text { soziale Akzeptanz, soziales Feedback, Gesell- } \\
\text { schaft, Rivalität, der Wettbewerb mit anderen } \\
\text { Menschen oder auch Neid. Grundsätzlich } \\
\text { zählen dazu sämtliche Aktivitäten, bei denen } \\
\text { es darum geht, wie sich andere Menschen } \\
\text { uns gegenüber verhalten und was sie über uns } \\
\text { denken oder sagen }\end{array}$ \\
\hline 6. & Scarcity \& Impatience & $\begin{array}{l}\text { Dieser Core Drive kommt zum Zug, wenn wir } \\
\text { etwas haben wollen, einfach nur, weil es sel- } \\
\text { ten, nicht sofort erreichbar oder das Erreichen } \\
\text { mit großen Schwierigkeiten verbunden ist. } \\
\text { Menschen haben eine natürliche Tendenz } \\
\text { dazu, zu wollen, was sie nicht haben können. } \\
\text { Exklusivität zieht Menschen an und bringt sie } \\
\text { dazu, mehr für etwas zu tun oder zu bezahlen }\end{array}$ \\
\hline 7. & Unpredictability \& Curiosity & $\begin{array}{l}\text { Dieser Core Drive beinhaltet alles was mit } \\
\text { Zufällen oder Chancen sowie mit Situationen, } \\
\text { bei denen man nicht genau weiß, was als } \\
\text { Nächstes passiert zu tun hat }\end{array}$ \\
\hline
\end{tabular}


Tab. 4.2 (Fortsetzung)

\begin{tabular}{l|l|l}
\hline Nr. & Core Drive & Erklärung \\
\hline 8. & Loss \& & Menschen tendieren dazu, etwas, das man \\
& Avoidance & $\begin{array}{l}\text { bereits erreicht hat, nicht mehr verlieren zu } \\
\text { wollen oder verhindern zu wollen, dass etwas } \\
\text { Schlechtes eintritt }\end{array}$ \\
\hline
\end{tabular}

Tab. 4.3 Einteilung in White-Hat- und Black-Hat-Gamification. (angelehnt an Chou (2016))

\begin{tabular}{l|l|l|l}
\hline White Hat & \multicolumn{3}{l}{ Black Hat } \\
\hline Core Drive 1 & Epic Meaning \& Calling & Core Drive 6 & Scarcity \& Impatience \\
\hline Core Drive 2 & $\begin{array}{l}\text { Development \& Accomplish- } \\
\text { ment }\end{array}$ & Core Drive 7 & Unpredictability \& Curiosity \\
\hline Core Drive 3 & $\begin{array}{l}\text { Empowerment of Creativity \& } \\
\text { Feedback }\end{array}$ & Core Drive 8 & Loss \& Avoidance \\
\hline Right Brain & \multicolumn{2}{l}{ Left Brain } & \\
\hline Core Drive 3 & $\begin{array}{l}\text { Empowerment of Creativity \& } \\
\text { Feedback }\end{array}$ & Core Drive 2 & $\begin{array}{l}\text { Development \& Accomplish- } \\
\text { ment }\end{array}$ \\
\hline Core Drive 5 & Social Influence \& Relatedness & Core Drive 4 & Ownership \& Possession \\
\hline Core Drive & Unpredictability \& Curiosity & Core Drive 6 & Scarcity \& Impatience \\
\hline
\end{tabular}

White Hat

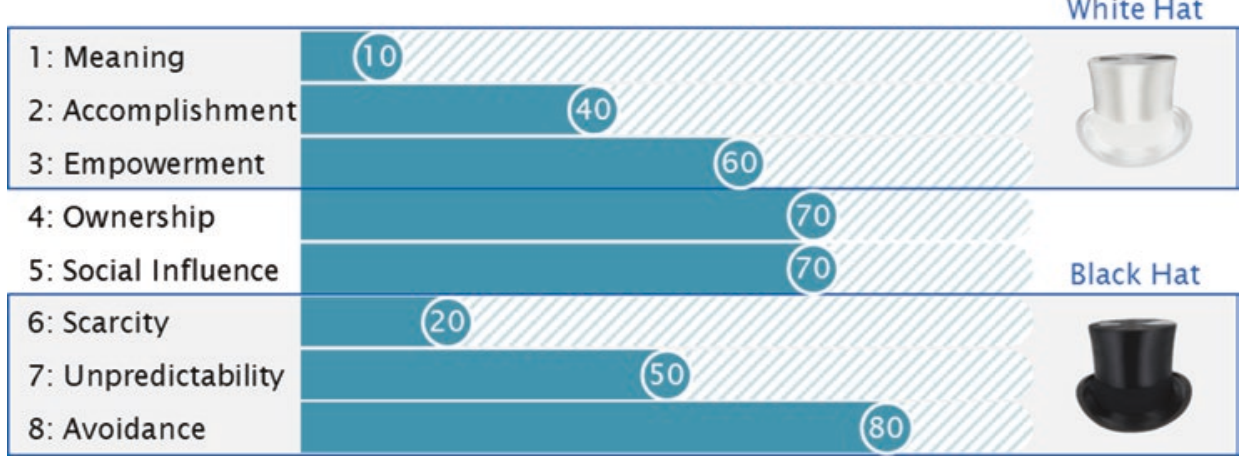

Abb. 4.2 Einteilung der Core Drives in White-Hat- und Black-Hat-Motivatoren. (Eigene Darstellung)

verantwortlich zu sein und selbst entscheiden zu können was sie machen. Zweitens die Meisterschaft einer Disziplin, also seine Fähigkeiten verbessern zu können und stetig dazu zu lernen. Drittens, einen Sinn zu sehen, in dem was sie tun. Diese Motivatoren finden sich alle in den White Hat Core Drives wieder. 


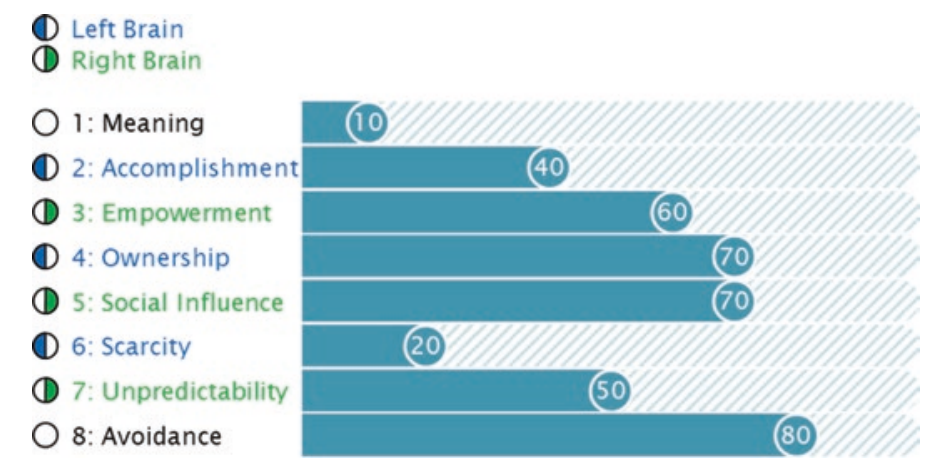

Abb. 4.3 Einteilung der Core Drives in Right-Brain- und Left-Brain-Motivatoren. (Eigene Darstellung)

Black Hat Drives führen in der Regel zu größeren kurzfristigen Erfolgen, indem sie den Menschen ein deutliches Gefühl der Dringlichkeit vermitteln. Damit können sie gerade zu Beginn einer Maßnahme sehr erfolgreich eingesetzt werden. Wie bereits erwähnt heißt Black Hat nicht, dass diese Elemente automatisch schlecht sind. Viele Menschen setzten sich freiwillig Black-Hat-Beeinflussungen aus, um sich selbst für etwas zu motivieren. Diese Core Drives haben das Potenzial, rasch viele Menschen zu mobilisieren. Im Endgame fallen diese dann jedoch oft ab und können, wenn man ihnen zu lange und in zu großen Dosen ausgesetzt ist, gar einen gegenteiligen Effekt haben und zu Frust oder auch zu Suchtverhalten führen. Dies kommt oft mit dem Gefühl, die eigenen Aktionen nicht mehr selbst unter Kontrolle zu haben und hinterlässt deshalb oft einen fahlen Beigeschmack. Da Black-Hat-Elemente oft rasche und zahlengetriebene Resultate liefern, werden in der Industrie vornehmlich diese angewandt. Insbesondere im Verkauf, wo es darum geht, dass jemand einen Kaufvorgang erfolgreich abschließen soll, funktionieren sie gut. Die Langzeitmotivation spielt in diesem Szenario meist keine Rolle. Hat eine Maßnahme gar keine Black-Hat-Elemente, besteht zudem das Risiko, dass sie gar nicht entdeckt wird und deshalb nie die Chance bekommt ein durchschlagender Erfolg zu werden (Chou 2016).

Neben der Einteilung nach Black Hat und White Hat wird das Octalysis-Framework in Right Brain und Left Brain Gamification unterteilt (Abb. 4.3). Die Core Drives 1 und 8 gehören dabei weder zum einen noch zum anderen. Die Right-Brain- und Left-Brain-Terminologie des Octalysis-Frameworks hat nichts mit dem physikalischen Gehirn und dessen zwei Hälften zu tun. Die Begriffe wurden symbolisch gewählt, um aufzuzeigen, welche Core Drives eher vom logischen Denken beeinflusst werden und welche eher vom emotionalen. Dabei fokussieren sich die Right Brain Core Drives insbesondere auf intrinsische Motivation während bei den Left Brain Core Drives die extrinsische Motivation im Vordergrund steht. Left Brain Core Drives sind dabei typischerweise einfacher zu entwickeln, weshalb sie in der Arbeitswelt auch viel häufiger genutzt werden. Es ist 
viel einfacher, einer Aktivität einen Preis zuzuordnen, als die Aktivität selbst spannend zu gestalten (Chou 2016). Für die Gestaltung von Gamification-Maßnahmen sollten jedoch beide Gruppen berücksichtigt werden.

Die Charakteristika von Right Brain Core Drives sind Kreativität, soziale Aspekte und Neugier. Dies korrespondiert mit intrinsischen Motivatoren, die einen Menschen antreiben. Intrinsische Motivation kommt davon, wenn einem eine Aufgabe positive Gefühle auslöst und man diese für sich selbst macht und nicht, weil jemand einem eine Belohnung dafür gibt. Right Brain Core Drives sind von Natur aus erfahrungsorientiert und eher auf den Prozess fokussiert als auf das Resultat (Chou 2016). Die drei Right Brain Core Drives finden sich auch in den Forschungsarbeiten von Jane McGonigal (2012) wieder, in der die wichtigsten intrinsischen Belohnungen, um glücklich zu sein, folgendermaßen beschrieben werden: eine befriedigende Arbeit, das Gefühl, im Laufe der Zeit besser zu werden, soziale Kontakte pflegen sowie das Gefühl, Teil von etwas Größerem zu sein.

Left Brain Core Drives haben vor allem mit Logik, mit Eigentum und mit analytischem Denken zu tun. Sie korrespondieren mit extrinsischen Motivationsfaktoren. Extrinsische Motivation kommt von einem Ziel, einem Zweck oder einer Belohnung, die uns jemand anderes gibt, und fokussieren auf Resultate. Wir erledigen eine Aufgabe, die nicht interessant ist, unter Umständen trotzdem, wenn uns jemand eine Belohnung dafür gibt. Dies kann dabei helfen Leute für eine monotone Arbeit zu begeistern oder um das initiale Interesse von jemandem zu gewinnen. Extrinsische Belohnungen können aber auch gefährlich sein. Hat eine Arbeit nur extrinsische Anreize, machen wir sie oft so rasch wie möglich oder so, dass sie gerade noch gut genug ist um die extrinsische Belohnung zu erhalten (Chou 2016). Dies deckt sich auch mit psychologischen Studien, die im Bereich der Arbeitsentlohnung gemacht wurden. Viele Vorgesetzte in Unternehmen denken, dass Mitarbeitende besser arbeiten, wenn man sie besser bezahlt. Dies funktioniert zwar bei monotonen oder mechanischen Arbeiten. Bei konzeptionellen Arbeiten wirkt dies jedoch nur kurzfristig. Jemand mag eine Arbeit einer anderen vorziehen, weil er für diese besser bezahlt wird. Langfristig macht man ohne intrinsische Motivation seine Arbeit hingegen nur gut genug, um seinen Job nicht $\mathrm{zu}$ verlieren (Pink 2011). Schlimmer noch: egal ob Geld, Schulnoten, Beförderungen, Aufmerksamkeit oder andere materielle Belohnungen, Forscher sind sich einig, dass extrinsischen Belohnungen nachzueifern, ein sicherer Weg ist, die eigene Freude zu sabotieren. Zudem kann das Hinzufügen von extrinsischen Belohnungen zu einer Tätigkeit, die wir freiwillig verrichten sogar gegenteilige Auswirkungen haben. Oft ist Leuten eine Tätigkeit verleidet, nachdem sie dafür eine Bezahlung erhalten haben, da sie nun das Gefühl bekamen, ihre Zeit sei mehr wert oder sie haben den Spaß verloren, da die Tätigkeit nun nicht mehr freiwillig war, sondern für die Bezahlung erledigt werden musste (McGonigal 2012). 


\subsection{Case Study und Methodik}

Im folgenden Kapitel wird die Case Study eingeführt und die Methodik zur Erhebung der empirischen Daten für diese Untersuchung erörtert. Es wird dabei zuerst die Methode für die Abbildung der bestehenden Maßnahmen der zu untersuchenden Firma festgelegt. Anschließend wird die Methode für die Interviews zur Beantwortung der Zielsetzungen erklärt.

\subsubsection{Charakterisierung des Unternehmens der Case Study}

Die Firma Acme Apps AG wurde 2010 gegründet. Sie spezialisierte sich darauf, benutzerfreundliche digitale Produkte zu entwickeln, die das tägliche Leben der Benutzer vereinfachen. Dabei decken sie die gesamte Wertschöpfungskette ab, von der ersten Idee über User Research, Konzept und Design bis hin zur technischen Entwicklung und dem Marketing. Das Konzept war ein Erfolgsmodell und die Acme Apps $A G$ wuchs rasch. Heute beschäftigt die Firma rund 30 Personen an ihrem Sitz in einer Schweizer Großstadt. Noch immer entwickeln sie Apps und weitere digitale Produkte, haben ihr Portfolio jedoch auch auf Workshops, das Gestalten von Benutzeroberflächen und interaktiven Erlebnissen sowie systematischer App-Vermarktung erweitert.

\subsubsection{Maßnahmen zur Kulturveränderung}

Unternehmenskultur wird bei Acme Apps AG großgeschrieben. „Kultur ist König“ steht dann auch als erste Überschrift auf der Website der Firma. Für den Unternehmensgründer ist die Kultur in seinem Unternehmen das wertvollste Gut. Da die Arbeit ein bedeutender Teil des Lebens ist, soll diese den Mitarbeitenden ein gewisses Maß an Zufriedenheit bereiten und einen Gewinn darstellen; nicht nur finanziell, sondern auch menschlich und geistig. So soll die Unternehmenskultur die Mitarbeitenden motivieren, unterstützen und glücklicher machen. Die Firma Acme Apps AG strebt dabei eine Kultur auf Augenhöhe an, die durch Respekt, Toleranz und Mitbestimmung getragen wird. Deshalb sollen auch alle ihre Bedürfnisse mitteilen können und ernst genommen werden. Um dies sicherzustellen, hat Acme Apps AG die traditionelle Geschäftsleitung abgeschafft und die meisten Entscheidungen werden nun in verschiedenen Boards getroffen, an welchen alle Mitarbeitenden teilnehmen können. Eines davon ist das Kultur-Board, in dem auch die drei Maßnahmen zur Kulturbeeinflussung entwickelt wurden: „Time to Play“, „Abzeichen \& Ehrenmedaillen“ sowie „Ämtlikarten“, die nachfolgend kurz skizziert werden. 


\subsubsection{Time to Play}

Im Büro von Acme Apps $A G$ hat es diverse Spielemöglichkeiten wie Ping Pong, Darts oder Tischfußballspiel. Diese wurden aufgestellt, da kurze Spiele während der Arbeitszeit als wichtige Ablenkung und Entspannung angesehen werden. Die Mitarbeitenden sollten die Möglichkeit haben, kurz aufzustehen und den Kopf durchlüften zu können. Diese kurze Ablenkung und der geförderte soziale Kontakt sollen die Arbeitsqualität verbessern. Da immer jemand das Spielen initiieren und Mitspielende mobilisieren musste, wurden die Möglichkeiten jedoch selten genutzt. Um dies zu ändern, wurde im Kultur-Board beschlossen, einen digitalen Zufallsgenerator zu erstellen. Dieser geht zweimal in der Woche während der Arbeitszeit los und fordert sämtliche Mitarbeitenden auf, für zehn Minuten gemeinsam zu spielen. Dank dieser Maßnahme wird nun regelmäßig gespielt, der Kopf gelüftet, Bewegung initiiert und der soziale Kontakt in einem anderen Rahmen gepflegt.

\subsubsection{Abzeichen und Ehrenmedaillen}

Acme Apps $A G$ hat es sich zum Ziel gesetzt, faire Löhne zu zahlen und transparente Lohnkriterien dafür anzuwenden. Außerdem sollen Lohnverhandlungen gänzlich abgeschafft werden. Dies wurde durch die Entwicklung eines eigenen Lohnsystems umgesetzt. So basiert der Lohn für jeden Mitarbeitenden nun auf genau festgelegten Kriterien, wie die absolvierte Ausbildung, Facherfahrung im entsprechenden Arbeitsgebiet, Firmentreue und Verantwortung. Bewusst hat man sich dabei gegen eine individuelle Bewertung der Leistung als Lohnfaktor entschieden. Die Hauptgründe hierfür waren, dass die erbrachte Leistung oft nur subjektiv einschätzbar ist und die Zusammenarbeit im Team gefördert werden soll. Um sowohl die Firmentreue noch weiter zu fördern, aber auch besondere Verdienste doch auf eine Weise zu würdigen, wurde ein Achievementsystem ins Leben gerufen. Dafür wurden spezielle Medaillen entwickelt und mithilfe eines 3D-Druckers gedruckt. Medaillen gibt es sowohl für Firmentreue wie auch für außerordentliches Engagement in der Projektarbeit. Die Medaillen kommen dabei in unterschiedlichen Stufen. So erhält man nach drei Jahren Firmentreue eine Bronzemedaille, nach fünf Jahren die Silbermedaille und nach sieben Jahren die Goldmedaille. Für außerordentliches Engagement bekommt man zuerst die Auszeichnung „Hirsch“, wird danach zum „Meister“, dann zum „Held“ und schlussendlich zum „Guru“ ernannt. Erhält man eine Medaille, darf man zusätzlich auch noch einen Wunsch äußern, der nach Möglichkeit erfüllt wird, oder man erhält eine kleine Bargeldprämie. Die Auszeichnungen werden den Mitarbeitenden jeweils an den Teammeetings feierlich übergeben. Das (stolze) Tragen oder Ausstellen der Medaille ist anschließend jedem selbst überlassen.

\subsubsection{3 Ämtlikarten}

Damit im Büro von Acme Apps $A G$ alles funktioniert, gibt es viele kleinere, wöchentlich anstehende Aufgaben („Ämtli“). So müssen beispielsweise die Pflanzen gegossen, das Altpapier entsorgt, die Kaffeemaschine gepflegt, aktuelle Tweets vom Firmen-Account aus getätigt oder das nächste Teammeeting geleitet werden. Ursprünglich wurden 
diese Arbeiten freiwillig von Mittarbeitenden übernommen, doch wurde es mit der Zeit immer aufwendiger, sämtliche Aufgaben zu verteilen. Deshalb wurden aufgrund eines Inputs vom Kultur-Board Karten erstellt, mit denen die Aufgaben zufällig verteilt werden konnten. Nach den ersten erfolgreichen Ziehungen wurde dann ein komplettes eigenes Kartenset entwickelt, mit allen vorhandenen Aufgaben und genügend Nieten für die restlichen Mitarbeitenden. Die Karten werden als eine einfachere Maßnahme als ein konventioneller „Ämtliplan“ angesehen, zudem wird die Maßnahme mit einer Portion Nervenkitzel angereichert. Die Ziehung ist jedes Mal ein kleines, positiv aufgeladenes Ereignis und macht allen Beteiligten Spaß. Die Aufgaben selbst werden seither nicht mehr so negativ, sondern als Teil des Spiels angesehen und von allen zuverlässig ausgeführt. Die Erledigung der Arbeiten wird jeweils vor der Ziehung in der Folgewoche kontrolliert.

\subsubsection{Interviewerhebung zur Wahrnehmung der Maßnahmen}

Um die Fragestellungen der Studie beantworten zu können, wurden strukturierte Interviews mit Mitarbeitenden von Acme Apps AG durchgeführt. Es handelte sich um qualitative Interviews, da mit diesen die kollektiven Strukturen herausgearbeitet werden sollten. Da lediglich wenige Antworten benötigt wurden, diese jedoch umso ausführlicher ausfallen sollten, wurden die Interviews schriftlich geführt. Dadurch wurde den Interviewpartnern mehr Zeit zur durchdachten Beantwortung der Fragen gegeben. Die zu erwartende niedrigere Rücklaufquote wurde dabei bewusst in Kauf genommen, da von den rund dreißig Mitarbeitenden lediglich drei bis fünf Antworten benötigt wurden. Damit die Interviewfragen ohne Vorkenntnisse der Theorie beantwortet werden konnten, wurden diese vorgängig operationalisiert. Die Interviews wurden anonymisiert und in einer zusammenfassenden Inhaltsanalyse aufgearbeitet.

\subsection{Ergebnisse}

Im folgenden Kapitel geht es darum, die Theorie mit der Praxis zu verknüpfen. Dazu wurden die drei Maßnahmen „Time to Play“, „Abzeichen \& Ehrenmedaillen“ sowie „Ämtlikarten“ ausgewertet. In einem ersten Schritt wurden diese mithilfe des Octalysis-Modells analysiert. Hierfür wurde ausgewertet, welche Core Drives sich in welchen Maßnahmen wiederfinden und wie diese konzipiert wurden (Tab. 4.4). In einem zweiten Schritt wurden anschließend die in den Interviews erhobenen Daten mit dem Framework verknüpft, um zu eruieren, ob die Mitarbeitenden die Core Drives als solche wahrnehmen. Anschließend wurde untersucht, ob die Maßnahmen tatsächlich zur Unternehmenskultur beitragen und ob sie die Motivation der Mitarbeitenden anregen.

Untersucht man die Maßnahme „Time to Play“, so fällt zunächst der Core Drive 7: 
Tab. 4.4 Ergebnisse der Interviews und Analyse der kulturverändernden Maßnahmen. (angelehnt an Chou (2016))

\begin{tabular}{l|l|l|l|l}
\hline & Core Drive & Time to Play & Abzeichen \& Ehrenmedaillen & Ämtlikarten \\
\hline 1. & Epic Meaning \& Calling & - & $\checkmark$ & $\checkmark$ \\
\hline 2. & $\begin{array}{l}\text { Development \& Accomplish- } \\
\text { ment }\end{array}$ & - & $\checkmark$ & $\checkmark$ \\
\hline 3. & $\begin{array}{l}\text { Empowerment of Creativity \& } \\
\text { Feedback }\end{array}$ & - & $\checkmark$ & $\checkmark$ \\
\hline 4. & Ownership \& Possession & - & - & $\checkmark$ \\
\hline 5. & $\begin{array}{l}\text { Social Influence \& Related- } \\
\text { ness }\end{array}$ & $\checkmark$ & $\checkmark$ & $\checkmark$ \\
\hline 6. & Scarcity \& Impatience & - & $\checkmark$ & $\checkmark$ \\
\hline 7. & Unpredictability \& Curiosity & $\checkmark$ & $\checkmark$ & $\checkmark$ \\
\hline 8. & $\begin{array}{l}\text { Loss \& } \\
\text { Avoidance }\end{array}$ & - & - & - \\
\hline
\end{tabular}

Unpredictability \& Curiosity auf. Insbesondere der Teil der Unvorhersehbarkeit ist im Design dieser Maßnahme stark verankert. Gemäß Aussagen des CEO wurde die Maßnahme gezielt so erarbeitet, dass eine „höhere Macht“ zu zufälligen Zeiten alle Mitarbeitenden zusammenbringt.

Das Miteinander und damit Core Drive 5: Social Influence \& Relatedness ist der zweite auffällige Aspekt dieser Maßnahme. Ziel davon ist es, nebst der Ablenkung und der Entspannung, insbesondere den sozialen Austausch zu fördern. Dies passiert, indem die Maßnahme alle Anwesenden zum gemeinsamen Spielen auffordert und nicht nur einzelne. Dies kann sogar Kunden oder Auftraggeber beinhalten, die sich zur Zeit des Alarms zufälligerweise im Gebäude aufhalten.

Da der Alarm lediglich zweimal in der Woche losgeht, könnte man denken, dass auch Core Drive 6: Scarcity \& Impatience in einem gewissen Masse vorhanden ist. Dies ist jedoch nicht der Fall, da die Mitarbeitenden von Acme Apps AG theoretisch jederzeit eine kurze Pause fürs Spielen einlegen können. Es finden sich in dieser Maßnahme hauptsächlich zwei Core Drives wieder. Beide korrespondieren mit intrinsischen Motivatoren (Right Brain). Dies deutet darauf hin, dass die Nutzer sich gerne freiwillig der Maßnahme aussetzen werden, da die Maßnahme selbst belohnend wirkt. Core Drive 7: Unpredictability \& Curiosity wird außerdem den Black-Hat-Gamification-Maßnahmen zugeordnet. Dies deutet darauf hin, dass durch die Maßnahme ein gewisser Sinn für Dringlichkeit entsteht. Man muss nämlich zu genau diesem Zeitpunkt aufstehen und spielen, sonst verpasst man die Chance. Dies kann zwar durchaus eine Langzeitmotivation generieren, durch die Black-Hat-Einflüsse aber über längere Zeit auch als lästig angesehen werden und die erhoffte Wirkung verlieren.

Durch die Auswertung der Interviews wird klar, dass die Ungewissheit, die Teil des Designs dieser Maßnahme ist, durch die Mitarbeitenden nicht mehr als solche angesehen 
wird. Zwar ist die Zeit, zu welcher der Alarm losgeht, noch immer zufällig, doch die Sicherheit, dass er zweimal in der Woche kommt, relativiert dies. Core Drive 7: Unpredictability \& Curiosity wird also nicht als treibendes Element dieser Maßnahme wahrgenommen. Ob dies nie der Fall war oder dieser Core Drive sich über die Zeit langsam abgebaut und an Bedeutung verloren hat, ließ sich nicht mehr ermitteln.

Anders sieht dies bei Core Drive 5: Social Influence \& Relatedness aus. Dieser wird als treibende Kraft hinter der Maßnahme angesehen und durch die Mitarbeitenden sehr geschätzt. Der zwanglose Austausch während einer anderen Aktivität als der täglichen Arbeit lässt es zu, Arbeitskollegen von einer anderen Seite kennenzulernen.

Die Maßnahme „Abzeichen \& Ehrenmedaillen“ ist eine typische Form des Core Drive 2: Development \& Accomplishment. Man erhält eine Auszeichnung, wenn man lange genug in der Firma gearbeitet oder wenn man etwas Außerordentliches geleistet hat. Dies in Form von physikalischen Medaillen zu tun, verleiht der Maßnahme noch ein bisschen Core Drive 5: Social Influence \& Relatedness. Dies einerseits durch die Art, wie sie verliehen werden, während des Teammeetings und mit Fanfaren, aber anderseits auch dadurch, dass man die Medaille später aufstellen, aufhängen oder tragen kann. Damit kann man andere stets daran erinnern, dass man die Auszeichnung erhalten hat. Weil die Auszeichnung vor dem gesamten Team vorgenommen wird, und nicht während eines Mitarbeitergesprächs, bekommen die Auszeichnung alle mit, was zur Verbundenheit beiträgt. Die Tatsache, dass nicht einfach jeder eine Medaille hat, sondern man dafür entweder schon lange dabei sein oder etwas wirklich Herausragendes geleistet haben muss, gibt der Maßnahme zudem ein bisschen von Core Drive 6: Scarcity \& Impatience. In sehr geringem Maße sind auch noch weitere Core Drives vertreten. Die Treuemedaillen haben ein bisschen etwas von einem Meilenstein, weshalb man sie Core Drive 3: Empowerment of Creativity \& Feedback zuordnen könnte. Die Projektabzeichen kommen meist unverhofft, was etwas von Core Drive 7: Unpredictability \& Curiosity hat. Da man sich aber kaum ständig fragt, wann man nun endlich eine Medaille erhält, ist das wohl nur sehr schwach vorhanden. Außerdem könnten die Auszeichnungen zu einer Art Elite führen. Einer Gruppe von Personen, die alle schon lange bei der Firma arbeiten oder die alle schon einmal für besondere Verdienste ausgezeichnet wurde. Dies könnte man Core Drive 1: Epic Meaning \& Calling zuordnen. Da die Maßnahme von fast jedem Core Drive zumindest ein wenig hat, kann man auf eine gut ausbalancierte Maßnahme schließen, die sowohl die intrinsische wie auch die extrinsische Motivation anspricht. Durch die starke Dominanz von Core Drive 2: Development \& Accomplishment steht die extrinsische Belohnung jedoch im Vordergrund. Trotz des Vorhandenseins von Core Drive 6: Scarcity \& Impatience sowie Core Drive 7: Unpredictability \& Curiosity ist die Maßnahme vor allem eine White-Hat-Maßnahme und eignet sich deswegen besonders gut, um eine Langzeitmotivation aufrechtzuerhalten.

In den Interviews wurde die Analyse teilweise bestätigt. Es wurde deutlich, dass bei dieser Maßnahme der Core Drive 2: Development \& Accomplishment durch die Mitarbeitenden als einer der Haupttreiber erkannt wird. Sie fühlen sich gut und motiviert dadurch, eine Auszeichnung zu erhalten. Dass nicht nur Firmentreue, sondern auch spezielle 
Anstrengungen belohnt werden, verstärkt dieses Gefühl noch. Das Gefühl, etwas erreicht $\mathrm{zu}$ haben und dafür wertgeschätzt $\mathrm{zu}$ werden, ist deutlich vorhanden. Auch Core Drive 5: Social Influence \& Relatedness ist deutlich vorhanden. Durch das Spektakel, das beim Verleihen veranstaltet wird, entsteht das Gefühl, dass die anderen die eigene Anstrengung honorieren. Außerdem freuen sich die Kollegen mit einem über den Erhalt, was der Sache einen deutlichen sozialen Bezug gibt. Dadurch, dass man eine Ehrenmedaille direkt nach dem sehr erfolgreichen Abschluss einer Arbeit erhält und nicht erst am Ende des Jahres beim Mitarbeitergespräch, wird der Aspekt des unmittelbaren Feedbacks von Core Drive 3: Empowerment of Creativity \& Feedback betont. Dieser Core Drive wird durch die Mitarbeitenden vermutlich stärker wahrgenommen, als dies bei der Planung angedacht war. Core Drive 6: Scarcity \& Impatience wird dagegen überhaupt nicht als solcher wahrgenommen. Zu Core Drive 1: Epic Meaning \& Calling sowie Core Drive 7: Unpredictability \& Curiosity fehlen in Bezug auf diese Maßnahme gezielte Aussagen in den Interviews. Auch hier muss deshalb davon ausgegangen werden, dass diese von den Mitarbeitenden kaum als solche wahrgenommen werden.

Die Verteilung der „Ämtlikarten“ ist in erster Linie ein Glücksspiel. Damit ist der vorrangige Core Drive dieser Maßnahme Core Drive 7: Unpredictability \& Curiosity. Dieser zielt darauf ab, dass man nicht genau weiß, ob und welche Aufgabe man erhält. Durch die Ziehung und die Kontrolle im Plenum ist auch Core Drive 5: Social Influence \& Relatedness vertreten. Aus Sicht der Gamification geht es hier vor allem darum, damit angeben zu können, dass man keine Aufgabe gezogen hat. Aber auch um das Teilen von Emotionen, sei dies Schadenfreude oder Mitgefühl. Etwas weniger stark, aber dennoch vorhanden, ist Core Drive 3: Empowerment of Creativity \& Feedback. Hier ist der Feedbackteil vertreten, indem man einerseits unmittelbar nach der Ziehung der Karten ein Feedback erhält, ob man ein Gewinner oder Verlierer ist, und andererseits am Ende der Woche das Feedback, ob die Aufgabe erfüllt wurde oder nicht. Dies geht ebenfalls ein bisschen in Richtung Core Drive 2: Development \& Accomplishment, wenn man am Ende der Woche verkünden kann, die Aufgabe erfüllt zu haben. Des Weiteren kann in der Maßnahme „Ämtlikarten“ etwas von Core Drive 1: Epic Meaning \& Calling gefunden werden. Dies da die Erledigung der Arbeit das Gefühl wecken kann, dass man die Aufgabe zum Wohle aller oder zum Wohle der Firma erledigt. In ganz kleinen Teilen vertreten ist außerdem Core Drive 4: Ownership \& Possession, da man die gezogene Aufgabe als seine eigene ansehen kann und deshalb versucht, diese bestmöglich zu erledigen, sowie auch Core Drive 6: Scarcity \& Impatience, da man nicht jederzeit erneut das Glück herausfordern und neue Karten ziehen kann. Die Maßnahme „Ämtlikarten“ ist klar right-brain-lastig und spricht damit die intrinsische Motivation an. Dies ist interessant, da es eigentlich um die Verteilung unliebsamer Arbeiten geht. Mit dem starken Fokus auf Core Drive 7: Unpredictability \& Curiosity ist es zudem eher eine Black-Hat-Gamification-Maßnahme, was darauf schließen ließe, dass die Motivation lediglich kurzfristig ist und nach einer gewissen Zeit nachlässt. Die Maßnahme hat kumuliert jedoch genügend kleine White-Hat-Elemente, dass auch eine gewisse Langzeitmotivation vorhanden sein dürfte. 

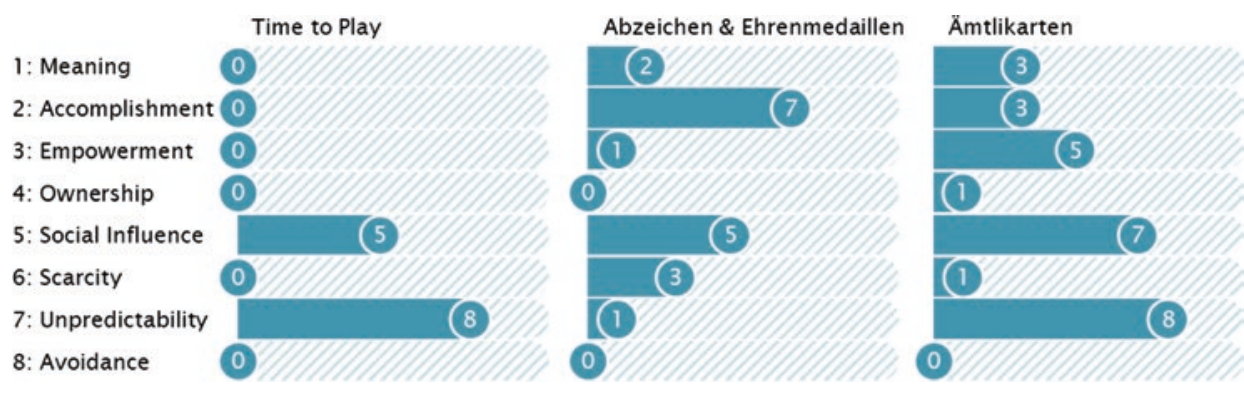

Abb. 4.4 Auswertung der Maßnahmen nach dem Octalysis-Modell (Eigene Darstellung)

Durch die Mitarbeitenden wird der Core Drive 7: Unpredictability \& Curiosity in dieser Maßnahme sofort erkannt. Dem zufälligen Verteilen der Karten wird Gamblingcharakter nachgesagt und es ist auch bei der Wahrnehmung das vorherrschende Gefühl. Auch Core Drive 5: Social Influence \& Relatedness wird klar erkannt und durch die Maßnahme gestärkt. Die Ziehung wird als gesellschaftliches Ritual angesehen und verbindet dadurch, dass man gegenseitig mitfühlt oder gemeinschaftliche Schadenfreude zum Ausdruck bringt. Auch in der Gleichstellung sämtlicher Mitarbeitenden des Unternehmens wird ein starker Aspekt von Core Drive 5: Social Influence \& Relatedness gesehen. Core Drive 1: Epic Meaning \& Calling wird als solcher wahrgenommen, tendenziell noch stärker als in der Planung der Maßnahme erwartet. Das Gefühl etwas Unliebsames zum Wohle aller zu erledigen, ist definitiv vorhanden. Auch Core Drive 2: Development \& Accomplishment sowie Core Drive 3: Empowerment of Creativity \& Feedback werden mehr oder weniger so wahrgenommen, wie dies für diese Maßnahme vorgesehen war. Durch das Feedback in der darauffolgenden Woche ist man eher gewillt, die Aufgabe zu erledigen und man hat auch das Gefühl, etwas erreicht zu haben. Core Drive 4: Ownership \& Possession und Core Drive 6: Scarcity \& Impatience werden hingegen gar nicht als solche wahrgenommen. Keine Aussage lässt darauf schließen, dass man die Aufgabe zuverlässiger erledigt, da man diese als seine eigene ansieht. Auch hat offensichtlich niemand das Verlangen, das Glück erneut herauszufordern, da alles, was man bekommen kann, als eher negativ angesehen wird.

Nachfolgend wurden die mit den Mitarbeitenden der Firma Acme Apps AG geführten Interviews weiter ausgewertet. Das Ziel der Interviews war es zu untersuchen, wie gut die drei vorgestellten Maßnahmen aufgenommen werden, ob diese zur Unternehmenskultur beitragen und ob dadurch die Arbeitsmotivation gesteigert werden kann. In einem ersten Schritt werden dafür die empirisch erhobenen Daten vorgestellt, die anschließend in einem zweiten Schritt interpretiert wurden (Abb. 4.4). Insgesamt wurden Interviews mit vier Mitarbeitenden geführt. Da die Option der Anonymisierung von einzelnen wahrgenommen wurde, werden die Aussagen in der Arbeit keinen spezifischen Urhebern zugewiesen. 
In Bezug auf die Unternehmenskultur sind sich die Interviewpartner einig, dass diese bei Acme Apps AG sehr offen sei. Auch das demokratische, selbstbestimmende, beziehungsweise das Mitspracherecht aller, wird als besonders großer Pluspunkt angesehen. Die Bezeichnung „familiär“ wird außerdem mehrmals als Charakterisierung genannt. Dies entspricht der durch die Führung angestrebten offenen Kultur auf Augenhöhe, die durch ein Mitspracherecht aller getragen wird. Der Einfluss der Unternehmenskultur auf die Arbeitsmotivation wurde von allen Interviewpartnern als sehr stark beurteilt. Besonders motivierend wirke sich dabei das Mitspracherecht aus, wie auch das Gefühl, Teil eines Teams, eines größeren Ganzen zu sein. Aber auch die Arbeitsgestaltung, beziehungsweise die Work-Life-Balance sowie die Arbeit selbst wurden genannt. Insofern kann gefolgert werden, dass die Mitarbeitenden durch die vorherrschende Kultur im Unternehmen motiviert werden, die zu erledigende Arbeit selbst aber auch motiviert.

Die Maßnahme „Time to Play“ kommt bei allen Befragten gut bis sehr gut an. Viele sehen den Wert der Pause darin, dass man anschließend wieder motivierter und konzentrierter arbeiten könne. Wann genau der Alarm losgeht, wird dabei kaum als Ungewissheit bewertet. Zwar wird es als toll angesehen, dass nicht alles streng geregelt und an fixe Zeiten gebunden ist, doch scheint dies mittlerweile beinahe zum Tagesablauf zu gehören und wird nicht mehr als etwas Spezielles wahrgenommen. Als sehr positiv wird die soziale Komponente wahrgenommen. Nicht nur bringe der Alarm auch Leute zum Spielen, die sonst eher weniger mitmachen, sondern lasse beim Spielen auch persönliche Kontakte und Gespräche zu, die sich nicht um die tägliche Arbeit drehen. Man lerne sich so auf eine andere Art kennen als während der ,,produktiven“ Arbeit.

Die Maßnahme „Abzeichen \& Ehrenmedaillen“ wird grundsätzlich als etwas sehr Positives angesehen. Die Mitarbeitenden finden es schön, wenn sie für gute Arbeit ein besonderes Lob erhalten. Dass Firmentreue belohnt wird, wird dabei als gerecht betrachtet. Insbesondere in einer Zeit, in der es nicht mehr selbstverständlich ist, dass man allzu lange beim gleichen Arbeitgeber bleibt. Der Erhalt einer Ehrenmedaille wirke sich durchaus motivierend aus und bestärke einem in der Arbeit, die man tue. Auch motiviere, dass ein geleisteter besonderer Aufwand anerkannt und honoriert werde.

Wenn jemand anderes eine Medaille erhält, wird dies dabei nicht als negativ betrachtet, sondern man freut sich mit diesen zusammen. Dass die Medaillen fair verteilt werden, scheint aber dennoch ein wichtiger Aspekt zu sein. Da die Medaille jedoch aus unterschiedlichsten Gründen vergeben werden kann, erscheint dies bisher nicht als Problem. Mehrmals wird erwähnt, dass diese Maßnahme die Motivation insgesamt stärke. Besonders schön sei dabei, dass dies nicht nur pro forma gemacht werde, sondern wirklich ein Spektakel sei. Es wird aber auch erwähnt, dass die Maßnahme die tägliche Motivation zur Erledigung der Tagesarbeit kaum beeinflusse.

Die Ziehung der „Ämtlikarten“ wird als wöchentliches Highlight angesehen, das eine gute Stimmung verbreite. Besonders gut wird dabei die Tatsache aufgenommen, dass sich alle an der Ziehung beteiligen. Nicht nur verleihe dies dem Ritual eine soziale Komponente, sondern es wird darin trotz der Zufallskomponente auch die Fairness gesehen. 
Jedes „Ämtli“ könne jeden treffen. Dass dies dabei womöglich zweimal hintereinander dieselbe Person sein kann, wird nicht als unfair betrachtet, sondern als Pech. Die Maßnahme wird somit auch als spannend angesehen und es wird ihr gar Glücksspielcharakter nachgesagt. Die Mehrheit der Befragten ist sich einig, dass die Maßnahme die Kultur im Unternehmen positiv beeinflusse. Sie fördere die Gleichstellung aller Mitarbeitenden, was ein Kulturziel der Acme Apps $A G$ darstellt. Zudem profitiere auch das Gemeinschaftsgefühl davon. Dennoch wird auch einmal erwähnt, dass die Maßnahme keinen Einfluss auf die Motivation für die restlichen Arbeiten habe.

Die vorherrschende Kultur bei Acme Apps $A G$ wird von den Interviewpartnern als einer der zentralen Erfolgsfaktoren der Firma angesehen. Dabei wird es als erfrischend betrachtet, dass das Unternehmen hier eine etwas andere Herangehensweise hat als andere Unternehmen. Die analysierten Maßnahmen helfen dabei, die Kultur und speziell den Teamzusammenhalt zu fördern. Es wird aber auch angemerkt, dass die Maßnahmen allein keine gute Kultur ausmachen, sondern lediglich eine existierende fördere. Denn auch andere Unternehmen haben Maßnahmen für ähnliche Szenarien, haben diese lediglich anders aufgebaut. Es ist also nicht nur die Vergabe kleiner Aufgaben oder das Honorieren von Unternehmenstreue, die motiviert, sondern vor allem, wie dies in einem vorhandenen kulturellen Kontext umgesetzt wird.

\subsection{Diskussion und Schlussfolgerungen}

Die vorliegende Studie soll Aufschluss darüber geben, ob und welche Gamification-Elemente in den Maßnahmen von Acme Apps AG integriert wurden. Anhand der Auswertung der Maßnahmen kann das Vorhandensein von Gamification klar bestätigt werden. Alle drei Maßnahmen beinhalten Elemente aus mehreren Core Drives gemäß dem Octalysis-Modell. Relativiert wird dieses Ergebnis durch die Prämisse des Modells, dass ohne Anwesenheit mindestens eines Core Drives überhaupt keine Aktion stattfindet und dass auch nicht spezifische Maßnahmen, die nicht nach Gamification-Kriterien gestaltet wurden, einen geringen Octalysis-Score aufweisen. Es kann deshalb geschlussfolgert werden, dass zumindest die Maßnahme „Time to Play“ nicht als spezifische Gamification-Maßnahme aufgefasst werden sollte. Abschließend sollten die Maßnahmen als Teile von etwas größerem Ganzen angesehen werden. So trägt jede Maßnahme zum Ziel bei, die Unternehmenskultur bei Acme Apps AG zu beeinflussen, ohne dass diese notwendigerweise als gamifiziert betrachtet werden.

Die zweite Zielsetzung war es, zu eruieren, ob die Maßnahmen von Acme Apps AG die Unternehmenskultur tatsächlich beeinflussen. Diese Fragestellung kann anhand der ausgewerteten Interviews ebenfalls positiv beantwortet werden. Von allen Interviewpartnern wird mehrmals erwähnt, dass die Maßnahmen insbesondere das Zusammengehörigkeitsgefühl unter den Mitarbeitenden fördern. Sie bauen einen Teamspirit auf und geben den Mitarbeitenden das Gefühl ein Teil von Acme Apps AG zu sein. Damit tragen die Maßnahmen klar zur angestrebten offenen Kultur auf Augenhöhe bei. 
Die dritte Zielsetzung war es, zu erörtern, ob die Unternehmenskultur von Acme Apps $A G$ die Motivation der Mitarbeitenden dahingehend anregt, sich für ihre Arbeit einzusetzen.

Auch diese Fragestellung kann aufgrund der Antworten der Interviews mit „Ja“ beantwortet werden. Die Interviewpartner haben übereinstimmende Aussagen gemacht, dass die Unternehmenskultur bei Acme Apps AG ein wichtiger Motivator für sie ist. Spezifisch wurde hier die gefühlte Mitverantwortung für das Team, beziehungsweise das Unternehmen erwähnt, das sich aufgrund der Kultur ergibt. Dies bewirkt, dass die Mitarbeitenden einen tieferen Sinn in ihrer Tätigkeit sehen und dadurch auch längerfristig motiviert bleiben.

Die Studie zeigt, dass Gamification ein angemessenes Werkzeug für Kulturveränderung sein kann. Durch die Anwendung verschiedener Core Drives können vielfältige Maßnahmen gezielt gestaltet und auf ihre Wirkungen getestet werden. Gleichzeitig wird deutlich, dass nicht jede Maßnahme unbedingt gamifiziert werden muss, sondern es eine Mischung aus Maßnahmen mit unterschiedlich stark ausgeprägten Gamification-Elementen geben kann. Der Einsatz von Gamification wurde von den Betroffenen und Beteiligten als insgesamt positiv wahrgenommen. Für Führungskräfte eröffnet sich hier ein Feld an Möglichkeiten, dass die digitale Transformation erst ermöglicht hat, sowohl von der technischen Seite als auch, was die kulturelle Akzeptanz und Offenheit von Mitarbeitenden anbelangt. Insgesamt ist Gamification noch ein junges und wenig ausgelotetes Gebiet, bei dem es sich lohnt, selbst einfache, kleine Experimente durchzuführen.

\section{Literatur}

Adams, E. W. (2014). Fundamentals of game design (3. Aufl.). London: Pearson Education Inc.

Bartle, R. (1996). Hearts, clubs, diamonds, spades: Players who suit MUDs. Colchester: MUSE Ltd. http://mud.co.uk/richard/hcds.htm. Zugegriffen: 8. Apr. 2018.

Burke, B. (2013). The gamification of business. Forbes, 21.01.2013. https://www.forbes.com/sites/ gartnergroup/2013/01/21/the-gamification-of-business. Zugegriffen: 12. Apr. 2018.

Chou, Y.-K. (2012). http://yukaichou.com/gamification-examples/octalysis-complete-gamification-framework/\#.WvX8pIiFO70. Zugegriffen: 8. Apr. 2018.

Chou, Y.-K. (2016). Actionable gamification. Freemont: Octalysis Media.

Chou, Y.-K. (2018). http://www.yukaichou.com. Zugegriffen: 8. Apr. 2018.

Coonradt, C. (2012). The game of work (revised and updated Aufl.). Layton: Gibbs Smith.

Dale, S. (2014). Gamification: Making work fun, or making fun work? Business Information Review 31(2), 82-90. http://journals.sagepub.com/toc/bira/31/2/82. Zugegriffen: 10. Apr. 2018.

De Luca, A. (2017). What is salary? Bachelor Thesis. Bern: Berner Fachhochschule.

Deterding, S., et al. (2011). Gamification: Toward a definition. Vancouver: o. V. http://hci.usask.ca/ uploads/219-02-Deterding,-Khaled,-Nacke,-Dixon.pdf. Zugegriffen: 10. Apr. 2018.

Engelhard, M. (2014). Gamification in Theorie und Praxis. Masterarbeit. Würzburg: Julius -Maximilians-Universität, Lehrstuhl für Wirtschaftsinformatik und Systementwicklung.

Herger, J., \& Strobl, H. (2018). Unternehmenskultur in der Praxis. Wiesbaden: Springer. 
Jost, H. R. (2003). Unternehmenskultur. Zürich: Orell Füssli.

Kapp, K. M. (2012). The gamification of learning and instructions. San Francisco: Pfeiffer.

Kotter, J. P., \& Heskett, J. L. (1992). Corporate culture and performance. New York: Free Press.

Levy, H. P. (2015). 5 key trends in Gartner's 2015 digital marketing hype cycle. Gartner, 14.10.2015. https://www.gartner.com/smarterwithgartner/five-key-trends-in-gartners-2015-digital-marketing-hype-cycle/. Zugegriffen: 10. Apr. 2018.

Maier, G. W., \& Kirchgeorg, M. (2018). Motivation. Gabler Wirtschaftslexikon. https://wirtschaftslexikon.gabler.de/definition/motivation-38456. Zugegriffen: 20. Apr. 2018.

McDonald, E. (2017). The global games market will reach $\$ 108.9$ Billion in 2017 with mobile taking 42\%. Newzoo, 20.04.2017. https://newzoo.com/insights/articles/the-global-games-market-will-reach-108-9-billion-in-2017-with-mobile-taking-42/. Zugegriffen: 12. Apr. 2018.

McGonigal, J. (2012). Reality is broken. London: Vintage.

Mühlenhof, M. C. (2018). Chefsache intrinsische motivation. Wiesbaden: Springer.

Newman, D. (2016). Top 10 trends for digital transformation in 2017. Forbes, 30.08.2016. https://www.forbes.com/sites/danielnewman/2016/08/30/top-10-trends-for-digital-transformation-in-2017/. Zugegriffen: 12. Apr. 2018.

Nicholson, S. (2012). A user-centered theoretical framework for meaningful gamification, Madison. http://scottnicholson.com/pubs/meaningfulframework.pdf. Zugegriffen: 14. Apr. 2018.

Norman, D. A. (2013). The design of everyday things (Revised and expanded Aufl.). New York: Basic Books.

Pink, D. H. (2011). Drive: The surprising truth about what motivates us. New York: Riverhead Books. (Reprint).

Ryan, M. et al. (2013). Why gamification is serious business. Outlook, 2013(1): Accenture. https:// www.accenture.com/us-en/insight-outlook-why-gamification-is-serious-business. Zugegriffen: 14. Apr. 2018.

Sackmann, S. (2002). Unternehmenskultur. München: Hermann Luchterhand.

Schein, E. H. (1984). Coming to a new awareness of organizational culture. Sloan Management Review, 25(2), 3-16. http://www.sietmanagement.fr/wp-content/uploads/2016/04/culture_ schein.pdf. Zugegriffen: 14. Apr. 2018.

Stieglitz, S. (2015). Gamification - Vorgehen und Anwendung. Praxis der Wirtschaftsinformatik, 52(6), 816-825.

Wikipedia. (2018). Generation Y. https://de.wikipedia.org/wiki/Generation_Y. Zugegriffen: 14. Apr. 2018.

Wiktionary. (2017). Gamification. https://en.wiktionary.org/wiki/gamification. Zugegriffen: 14. Apr. 2018.

Wiktionary. (2018). -ification. https://en.wiktionary.org/wiki/-ification. Zugegriffen: 14. Apr. 2018.

Zelesniack, E., \& Grolman, F. (2018). Unternehmenskultur. https://organisationsberatung.net/ unternehmenskultur-kulturwandel-in-unternehmen-organisationen/. Zugegriffen: 20. Apr. 2018.

Ellenberger, Thomas (B.Sc. BA/thomas.ellenberger@gmx.ch) Abgeschlossenes, berufsbegleitendes Bachelorstudium in Betriebsökonomie mit Vertiefung in Management in Practice: Strategy. Während dem Studium angestellt als Implementation Manager bei der Swisscom (Schweiz) AG sowie als Sachbearbeiter Fachstelle Cicero beim Berufsbildungsverband der schweizerischen Versicherungswirtschaft (VBV) 
Harder, Deane (Prof. Dr./deane.harder@bfh.ch) Tätigkeit in Lehre und Forschung an der Berner Fachhochschule, Departement Wirtschaft. Schwerpunkte sind Innovation, Verhaltensökonomie und Systemik im Bereich Nachhaltigkeit. Vorher 10 Jahre Praxiserfahrung in der Unternehmensberatung und -entwicklung. Studium und Promotion in Biologie an den Universitäten Freiburg i. Br. (Deutschland) und Otago University (Neuseeland).

Brechbühler Pešková, Marie (Prof. Dr./marie.brechbuehler@bfh.ch) ist in der Lehre und Forschung an der Berner Fachhochschule, Departement Wirtschaft tätig. Sie unterrichtet insbesondere Module in den Bereichen „Nachhaltige Wirtschaft, Strategie und Innovation und International Business. Sie leitet der Forschungsschwerpunkt „Nachhaltigkeit“ und widmet sich in Ihre angewandte und praxisorientierte Forschungstätigkeit der Themen „Transformation zur nachhaltigen Gesellschaft und Wirtschaft“, „Nachhaltige Kreisläufe: Sustainable Consumption, Circular Economy, nachhaltige Geschäftsmodelle, Recycling“ sowie „Corporate Sustainability / CSR/ social Entrepreneurship“.

Open Access Dieses Kapitel wird unter der Creative Commons Namensnennung 4.0 International Lizenz (http://creativecommons.org/licenses/by/4.0/deed.de) veröffentlicht, welche die Nutzung, Vervielfältigung, Bearbeitung, Verbreitung und Wiedergabe in jeglichem Medium und Format erlaubt, sofern Sie den/die ursprünglichen Autor(en) und die Quelle ordnungsgemäß nennen, einen Link zur Creative Commons Lizenz beifügen und angeben, ob Änderungen vorgenommen wurden.

Die in diesem Kapitel enthaltenen Bilder und sonstiges Drittmaterial unterliegen ebenfalls der genannten Creative Commons Lizenz, sofern sich aus der Abbildungslegende nichts anderes ergibt. Sofern das betreffende Material nicht unter der genannten Creative Commons Lizenz steht und die betreffende Handlung nicht nach gesetzlichen Vorschriften erlaubt ist, ist für die oben aufgeführten Weiterverwendungen des Materials die Einwilligung des jeweiligen Rechteinhabers einzuholen.

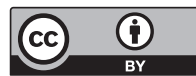




\title{
Cybersicherheit als Führungsaufgabe in Schweizer KMU
}

\section{Herausforderungen und Chancen im Zuge der Digitalisierung}

\author{
Dominique Adrian Meier und Daniel Burda
}

Cybersicherheit gewinnt im Kontext der Digitalisierung auch in KMU zunehmend an Wichtigkeit und verdient die Aufmerksamkeit von Führungskräften und Entscheidungsträgern

\begin{abstract}
Zusammenfassung
Durch die digitale Transformation gewinnt Cybersicherheit für kleine und mittlere Unternehmen (KMU) zunehmend an Bedeutung und rückt damit auch auf die Tagesordnung der Unternehmensleitung. Diverse Studien zeigen, dass KMU Cybersicherheit als relevantes Handlungsfeld identifizieren, gleichzeitig aber nicht genügend adressieren. Bestehende Forschung liefert für dieses Verhalten keine Erklärung. Dieser Beitrag widmet sich der Untersuchung dieses Phänomens, welches im Beitrag als „Security Paradox“ definiert wird. Dabei werden dessen Ursachen aufgezeigt und praxisrelevante Empfehlungen für Führungskräfte und Entscheidungsträger im Umgang mit der Herausforderung „Cybersicherheit im Zuge der Digitalisierung“ abgegeben.
\end{abstract}

D. A. Meier $(\bowtie)$

Bülach, Schweiz

E-Mail: me@dominiquemeier.ch

D. Burda

Berner Fachhochschule Wirtschaft, Bern, Schweiz

E-Mail: daniel.burda@bfh.ch 


\subsection{Einleitung}

Die digitale Transformation stellt kleine und mittlere Unternehmen (KMU) sowie deren IT vor neue große Herausforderungen. Ein zentrales Thema in diesem Zusammenhang stellt die Cybersicherheit ${ }^{1}$ dar, die auch in KMU zunehmend an Bedeutung gewinnt und auf die Tagesordnung der Unternehmensleitung rückt. Gemäß EY (2018) rechnen $41 \%$ der im Rahmen des Unternehmensbarometers 2018 befragten Schweizer Unternehmen mit Schwierigkeiten bei der Sicherung ihrer IT-Infrastruktur. Ferner zeigen Studien wie die der KPMG (2017, S. 38) sowie andererseits Berichterstattungen zu Cybersicherheitsvorfällen bei Schweizer KMU (Inside-IT 2018), dass Cybersicherheit ein aktuelles Kernthema für Schweizer Unternehmen darstellt. Rund $88 \%$ der im Rahmen der Studie befragten Schweizer Unternehmen wurden im vergangenen Jahr Ziel von Cyberangriffen. Auch eine Studie der Zürich Versicherungs-Gesellschaft (2016, S. 1) identifiziert Cyberkriminalität als relevantes Schlüsselrisiko für Schweizer KMU. Untermauert werden diese Aussagen durch Entwicklungen am Sicherheitsdienstleistermarkt in der Schweiz. So hat 2017 ein Zusammenschluss von verschiedenen IT- und Security-Dienstleistern stattgefunden, die sich drauf spezialisieren, Security-Dienstleistungen für Schweizer KMU zu erbringen (Inside-Channels 2017).

Andererseits zeigen Studien, wie die der Zürich Versicherungs-Gesellschaft (2016) auf, dass trotz des Bewusstseins für Cyberkriminalität in KMU lediglich 2,5\% der befragten Unternehmen über einen ausreichenden Schutz verfügen. Aufgrund der erheblichen Differenz zwischen Risikobewusstsein und Ergreifen von konkreten Maßnahmen leiten die Autoren der Studie ab, dass die Mehrheit der KMU mit der Thematik Informationssicherheit überfordert ist. Dass es Schweizer KMU im Umgang mit dem Thema Informationssicherheit an Wissen fehlt, bestätigen auch Hirschi und Portmann (2017, S. 8-10). Sie zeigen auf, dass lediglich $46 \%$ der befragten KMU Standards bei der Umsetzung von Informationssicherheit berücksichtigen. In derselben Studie gaben $74 \%$ der befragten KMU an, kein Informationssicherheitsmanagementsystem (ISMS) einzusetzen, wodurch Informationssicherheit nicht systematisch adressiert wird. Dass Cybersicherheit durch KMU zwar als relevantes Handlungsfeld identifiziert, gleichzeitig aber nicht genügend adressiert wird, zeigen auch die Studien von Renaud (2016) und

\footnotetext{
${ }^{1}$ In Anlehnung an den allgemeinen Sprachgebrauch und wissenschaftliche Veröffentlichungen (vgl. Eckert 2017; Klipper 2015) werden die Begriffe „IT-Sicherheit“" und „Informationssicherheit“ in der vorliegenden Arbeit unter dem Begriff „Cybersicherheit“ zusammengefasst, wobei Cybersicherheit eine inhaltliche Erweiterung des Begriffs Informationssicherheit und dieser wiederum eine inhaltliche Erweiterung des Begriffs IT-Sicherheit darstellt. Diese eingeführte Terminologie wird bestärkt durch Erkenntnisse aus bestehenden Studien wie beispielsweise der von gfs-zürich (2017) oder der von Hirschi und Portmann (2017). Aus den Studien geht hervor, dass KMU in der Regel keine Unterscheidung zwischen den Begriffen vornehmen. So wird in der vorliegenden Analyse ausschließlich der übergeordnete Begriff „Cybersicherheit“ verwendet, der Informationssicherheit und IT-Sicherheit mit einschließt.
} 
gfs-zürich (2017). Dieses Phänomen wurde ansatzweise von Straub (1990) bereits zu Beginn der 1990er-Jahre beschrieben:

Over the last several decades, managers have become aware that information and information systems are critical organizational resources. It is reasonable to expect therefore, that they would consider the security of information to be a crucial activity. Curiously, this is not the case (Straub 1990, S. 255).

Auch eine Studie des Bundesamts für Sicherheit in der Informationstechnik (BSI 2011, S. 99) hinsichtlich des IT-Sicherheitsniveaus von KMU in Deutschland kommt zu einem ähnlichen Schluss - das Bewusstsein für das Thema ist sehr ausgeprägt, der Umsetzungsgrad von Maßnahmen sowie die Etablierung eines systematischen IT-Sicherheitsmanagement lassen jedoch zu wünschen übrig.

Bestehende Forschung liefert aktuell keine Erklärung bezüglich dieser Intentionsverhaltenslücke. Die vorliegende Untersuchung setzt an dieser Forschungslücke an und untersucht mithilfe eines qualitativen Forschungsansatzes die Ursachen für dieses Phänomen bei Schweizer KMU im Bereich Cybersicherheit. Vor diesem Hintergrund lassen sich folgende Forschungsfragen als Ausgangspunkt der Analyse formulieren:

- Forschungsfrage 1: Kann eine Intentionsverhaltenslücke (IVL) hinsichtlich der Cybersicherheit bei Schweizer KMU nachgewiesen werden?

- Forschungsfrage 2: Welche Ursachen sind ausschlaggebend für das Vorhandensein der IVL hinsichtlich Cybersicherheit in Schweizer KMU?

Durch die Beantwortung obiger Forschungsfragen soll die vorliegende Analyse eine Hilfestellung für Führungskräfte von Schweizer KMU bei der Adressierung von Cybersicherheit bieten und einen bedeutenden Meilenstein in dem noch jungen Themengebiet Cybersicherheit bei Schweizer KMU darstellen.

\subsection{Forschungsmodell}

Für die vorliegende Analyse wurde ein Forschungsmodell (siehe Abb. 5.1) auf Basis einer umfassenden Literaturrecherche erarbeitet, das gemäß vorhergehender Untersuchungen Determinanten umfasst, die entscheidend dafür sind, ob eine Organisation adäquate Cybersicherheitsmaßnahmen ergreift. Im Rahmen der vorliegenden Analyse werden diese Determinanten basierend auf empirischen Daten aus den durchgeführten Fallstudien analysiert. So sollen das Vorhandensein und die Ursache der Intentionsverhaltenslücke, hinsichtlich Cybersicherheit bei Schweizer KMU, nachgewiesen werden.

Das für die vorliegende Analyse erarbeitete Forschungsmodell orientiert sich dabei primär an den Forschungsmodellen von Browne et al. (2015), Liang und Xue (2009) sowie Renaud (2016). Zur Erarbeitung des Forschungsmodell wurden zudem die folgenden Theorien und Modelle analysiert und als Determinanten im Forschungsmodell verankert: 


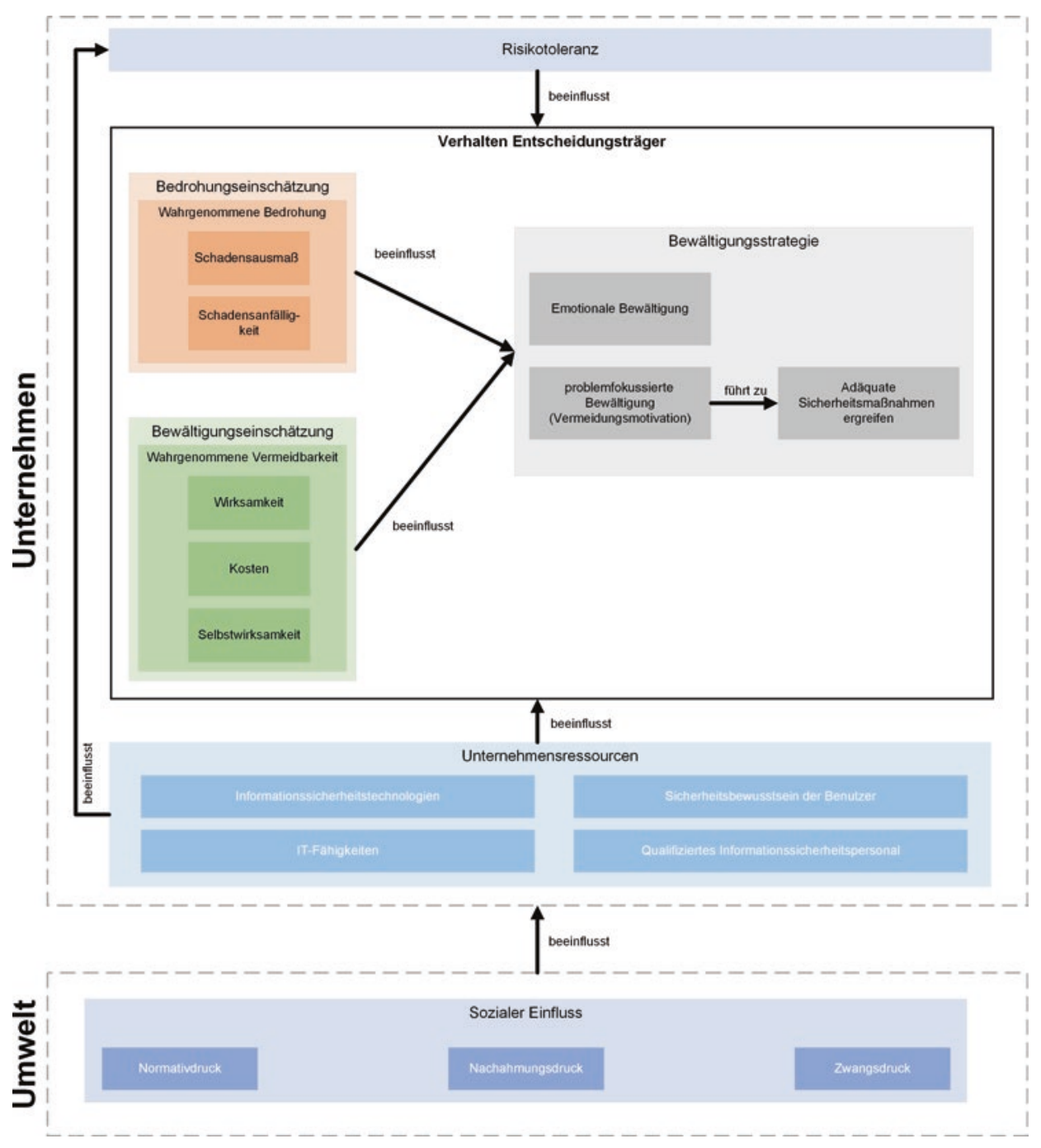

Abb. 5.1 Forschungsmodell. (Eigene Darstellung)

A. Cybersicherheit Ressourcen

Damit ein KMU in der Lage ist, Cybersicherheit adäquat zu adressieren, benötigt es bestimmte Unternehmensressourcen (Cavusoglu et al. 2015, S. 385). Cavusoglu et al. identifizierten basierend auf dem Unternehmensressourcenmodell von Grant (2010, S. 127) vier unterschiedliche aber zusammenhängende Dimensionen von Informationssicherheitskontrollressourcen, die für das Umsetzen von Informationssicherheitskontrollen essenziell sind: 
a) Informationssicherheitstechnologien (präventive und detektivische technische Lösungen zur Behebung von Schwachstellen innerhalb der IT-Infrastruktur),

b) qualifiziertes Informationssicherheitspersonal (Ausmaß an professionellen Mitarbeitenden zur Definition, Ausführung und Pflegen des ISMS),

c) Sicherheitsbewusstsein der Benutzer (Ausmaß des Sensibilisierungsgrads hinsichtlich Cybersicherheit der Mitarbeitenden),

d) IT-Fähigkeiten (Ausmaß an professionellen IT-Mitarbeitenden und vorhandenes IT Wissen).

B. Risikotoleranz

Browne et al. (2015, S. 35) argumentieren, dass die Risikotoleranz eines KMU einen Einfluss auf den Umgang mit wahrgenommenen Cybersicherheit-Bedrohungen hat. Sie gehen davon aus, dass die Risikotoleranz die Vermeidungsmotivation mindert und gleichzeitig eine emotionale Bewältigung von Bedrohungen fördert. Zudem folgern sie aus der von ihnen untersuchten Fachliteratur, dass die Risikotoleranz eines Unternehmens in starker Abhängigkeit zu dessen Herkunft, Größe und Reifegrad steht. Dies ist ihrer Ansicht nach ein Indikator dafür, dass sich die Risikotoleranz von KMU zu KMU stark unterscheidet und deshalb als Determinante für das Umsetzen von adäquaten Sicherheitsmaßnahmen infrage kommt. Im Forschungsmodell zur vorliegenden Analyse wird die Risikotoleranz deshalb ebenfalls als potenzielle Determinante eingeführt.

C. Verhaltensmodelle (Verhalten von Führungskräften)

Die für die Erarbeitung des Forschungsmodells analysierten sozialpsychologischen Verhaltensmodelle beziehen sich jeweils auf das Verhalten von Individuen (vgl. Ajzen 1985; Rogers 1975; Kelman 2006; Liang und Xue 2009). Wie Browne et al. (2015, S. 34) in ihrer Forschungsarbeit aufzeigen, besteht im Kontext von KMU jedoch eine gewisse Legitimität, Organisationsverhalten zu anthropomorphisieren. Grund dafür ist die bei KMU bestehende Abhängigkeit zu dominanten Einzelpersonen. In der vorliegenden Analyse werden deshalb die nachfolgend beschriebenen Verhaltensmodelle auf das Organisationsverhalten in Bezug auf die Vermeidung von Cybersicherheitsbedrohungen und das Umsetzen von Cybersicherheitsmaßnahmen übertragen.

a) Protection Motivation Theory

Um Cybersicherheitsbedrohungen $\mathrm{zu}$ vermeiden, müssen Unternehmen eine Reihe von Beurteilungs- und Bewältigungsschritten hinsichtlich einer spezifischen Bedrohungslage vornehmen (Browne et al. 2015, S. 34). Die von Rogers (1975) entwickelte Theorie der Schutzmotivation (engl. Protection Motivation Theory) beschreibt, wie von einem Individuum erlebte Bedrohungen dazu führen, dass es sein Verhalten ändern will. Gemäß der Theorie führt der Erhalt von überzeugenden Informationen über die unerwünschten Folgen eines bestimmten Ereignisses zu zwei Beurteilungsprozessen, von denen die Verhaltensintention des Individuums abhängt: 
1. Bedrohungseinschätzung - Dabei wird beurteilt, welchen Schweregrad eine Bedrohung aufweist und wie die eigene Verwundbarkeit wahrgenommen wird.

2. Bewältigungseinschätzung - Dabei wird beurteilt, ob ein Individuum selbst in der Lage ist, Maßnahmen gegen eine Bedrohung zu ergreifen (Selbstwirksamkeitserwartung ${ }^{2}$ ), ob das Umsetzen von Maßnahmen tatsächlich gegen die Bedrohung schützt (Handlungsergebniserwartung) und wie viel die Umsetzung der Maßnahmen kostet.

Wird eine Bedrohung von einem Individuum als hoch eingeschätzt und die Bewältigungseinschätzung fällt positiv aus, kommt es beim Individuum zu einer Verhaltensintention, die der Bedrohung entgegenwirkt (Rogers, 1975). Dass die Wahrnehmung einer Cybersicherheitsbedrohung ausschlaggebend dafür ist, wie stark Individuen motiviert sind, diese zu verhindern, zeigte eine Studie von Workman, Bommer und Straub (2008, S. 2813-2814). Sie stellten fest, dass Sicherheitsmaßnahmen mit größerer Konsequenz umgesetzt werden, wenn eine Bedrohung als schwerer empfunden wird, als wenn eine Bedrohung als harmlos angesehen wird. Eine weitere Erkenntnis der Studie war, dass bei Individuen ein gegensätzliches Verhalten eintritt, wenn der Grad an Bedrohungsmeldungen ein chronisches Ausmaß annimmt und diese in der Folge keine Maßnahmen umsetzen. Dies ist vermutlich auf eine reduzierte Selbstwirksamkeitserwartung und reduzierte Handlungsergebniserwartung, bedingt durch eine Überhäufung mit Bedrohungsmeldungen, zurückzuführen.

b. Model of Social Influence

Der soziale Einfluss stellt eine bedeutende Variable in jedem Verhaltensmodell dar (vgl. Burnkrant und Cousineau 1975; Deutsch und Gerard 1955). So wurde die Bedeutung von sozialem Einfluss auf das Verhalten von Individuen im Kontext von IT- und Informationssystemen bereits breit erforscht und bewiesen (vgl. Pavlou und Fygenson 2006; Taylor und Todd 1995; Venkatesh und Davis 2000; Venkatesh et al. 2003). Wie in den Forschungsmodellen von Browne et al. (2015) und Liang und Xue (2009) wird der soziale Einfluss auch im Forschungsmodell der vorliegenden Analyse als Determinante eingeführt. Basierend auf Erkenntnissen bestehender Forschung (vgl. Cavusoglu et al. 2015; Kelman 2006; Liang und Xue 2009) werden im vorliegenden Forschungsmodell die folgenden Arten von sozialem Einfluss unterschieden:

\footnotetext{
${ }^{2}$ Es wird davon ausgegangen, dass die Ausführung eines Verhaltens umso wahrscheinlicher ist, je größer die subjektive Überzeugung ist, das Verhalten zu bestimmen, das heißt je mehr ein Individuum der Ansicht ist, über genügend Fähigkeiten, Fertigkeiten oder Ressourcen zu verfügen, um das Verhalten zu realisieren (Ajzen 1985). Diese subjektive Überzeugung wird auch als Selbstwirksamkeitserwartung (engl. Perceived Self Efficacy) bezeichnet. Hohmann und Schwarzer (2009, S. 67) weisen in ihrem Werk darauf hin, dass Selbstwirksamkeitserwartung von den Fähigkeiten eines Individuums unterschieden werden muss, da geringe Fähigkeiten durchaus mit hoher Selbstwirksamkeitserwartung einhergehen können oder umgekehrt.
} 


\section{Normativdruck (Normativer Einfluss)}

Das soziale Umfeld eines Individuums generiert gemäß Kelman (2006) normativen Einfluss auf den drei Ebenen Compliance (das Bedürfnis eines Individuums nach Akzeptanz und Belohnung oder Angst vor Bestrafung), Internalisierung (das Entwickeln einer Übereinstimmung zwischen den Werten und Zielen eines Individuums und denen einer Gruppe) und Identifikation (die selbstbestimmte Beziehung eines Individuums $\mathrm{zu}$ einem anderen Individuum oder einer Gruppe. Als Konsequenz aus dieser Beziehung verhält sich ein Individuum entsprechend den Werten und Zielen seines sozialen Umfelds).

2. Nachahmungsdruck

Unter Nachahmungsdruck verstehen Cavusoglu et al. (2015, S. 387-388) den Druck, den Organisationen spüren, wenn erfolgreiche Praktiken bei Konkurrenten beobachtet werden. Diese Beobachtung führt zu einem Nachahmungsdruck, die als erfolgreich beobachteten Maßnahmen in der eigenen Organisation umzusetzen.

3. Zwangsdruck

Nach Cavusoglu et al. (2015, S. 388) entspricht Zwangsdruck dem Einfluss, den Regulierungsbehörden direkt auf bestimmte Organisationen und Branchen ausüben. Durch diesen Einfluss entsteht ein Zwangsdruck für Organisationen, gesellschaftliche und politische Erwartungen zu erfüllen.

Basierend auf den empirischen Daten aus ihrer Forschung konnten Cavusoglu et al. (2015, S. 396) beweisen, dass normativer und zwanghafter Druck einen starken direkten Einfluss auf das Sicherheitsbedürfnis und die Informationssicherheitskontrollressourcen einer Organisation haben. Ein starker Einfluss durch Nachahmungsdruck konnte jedoch nicht nachgewiesen werden. Cavusoglu et al. schlussfolgern daraus, dass Organisationen nicht dazu neigen, zu glauben, dass erfolgreiche Sicherheitsmaßnahmen ihrer Konkurrenten auch ihre Sicherheitsprobleme lösen können.

c. Technology Threat Avoidance Theory

Liang und Xue (2009) entwickelten durch die Zusammenführung von verschiedenen Theorien aus den Forschungsgebieten Psychologie, Gesundheitspsychologie, Informationssysteme, Management, Marketing und Finanzen eine Technologie-Bedrohungs-Vermeidungstheorie (engl. Technology Threat Avoidance Theory $=$ TTAT). Die von Liang und Xue beschriebene Theorie erklärt das Verhalten eines einzelnen IT-Anwenders bei der Vermeidung von Bedrohungen durch Informationstechnologien. Die Theorie zeigt auf, dass das Bedrohungsvermeidungsverhalten eines Benutzers in Abhängigkeit von den zwei kognitiven Prozessen Bedrohungseinschätzung (engl. Threat-Appraisal) und Bewältigungseinschätzung (engl. Coping-Appraisal) steht. Dabei beziehen sie sich auf die Protection Motivation Theory. Im Kontext der Bewältigungsstrategie (engl. Coping) unterscheiden Liang \& Xue (2009, S. 77) zwischen problemorientierter Bewältigung (engl. Problem-Focused Coping) und emotionaler Bewältigung (engl. Emotion-Focused Coping). Abhängig von der Bedrohungs- und Bewältigungseinschätzung eines 
Benutzers tendiert dieser zu einer der obigen Bewältigungsstrategien. Die problemorientierte Bewältigung führt gemäß Liang und Xue zur Umsetzung von Sicherheitsmaßnahmen und emotionale Bewältigung führt beispielsweise zu Verneinung im Sinne von Ablehnung der Situation oder Wunschdenken, d. h. beispielsweise, nicht interessant für einen Angreifer zu sein.

\subsection{Methode und Sample}

Die für die vorliegende Analyse gewählte Forschungsmethode basiert auf einem holistischen Multiple-Case-Design nach Yin (2014, S. 50) und durchläuft die drei Phasen „Define and Design“, „Prepare, Collect and Analyze“ und „Analyze and Conclude“. In der Phase Define and Design wurden die theoretischen Grundlagen zur vorliegenden Analyse erarbeitet, die Forschungsfragen präzisiert, mögliche Wirtschaftspartner (Fälle) kontaktiert und selektiert. Im Rahmen der vorliegenden Analyse wurden insgesamt drei Fallstudien durchgeführt. Jede Fallstudie bezieht sich dabei auf den realen Kontext eines Schweizer KMU. Um potenzielle Fallstudienpartner zu finden, wurden Schweizer KMU mit mindestens 10 Mitarbeitenden sowie einem Tätigkeitsfeld im tertiären Sektor im Internet, auf sozialen Netzwerken und im privaten Umfeld gesucht. Insgesamt konnten so drei Fallstudienpartner für die vorliegende Forschungsarbeit gewonnen werden. Da davon ausgegangen wird, dass es sich bei der zu untersuchenden Intentionsverhaltenslücke um ein grundsätzliches Phänomen bei Schweizer KMU handelt, wurden als Auswahlkriterien lediglich Unternehmensgröße und Wirtschaftssektor definiert und eine Kombination von gezieltem und Convenience-Stichprobenziehungsverfahren angewandt. Die Fallstudienanzahl $(n=3)$ wurde dabei aufgrund der im Rahmen der vorliegenden Analyse zur Verfügung stehenden Ressourcen festgelegt.

In der Phase Prepare, Collect and Analyze werden die einzelnen Fälle betrachtet. Dabei wird jeder Fall isoliert untersucht und aufbereitet. Die Erhebung der empirischen Daten für die vorliegende Analyse basierte auf Interviews und Dokumenten, die im Kontext der drei Fallstudien erhoben wurden. Alle Interviews wurden nach Zustimmung durch die Teilnehmenden aufgenommen und im Anschluss transkribiert. Die Transkription und Codierung (Kategorienzuweisung) der Interviews erfolgte mit der Software MAXQDA ${ }^{3}$. Eisenhardt (1989, S. 540) folgend wurden in einem ersten Analyseschritt fallinterne Muster identifiziert, bevor generalisierte fallübergreifende Muster im Rahmen der abschlieBenden Phase Analyse and Conclude abgeleitet wurden. Die Musterbildung orientierte sich an der qualitativen Inhaltsanalyse nach Gläser und Laudel (2010, S. 46). Dazu wurde ein Kategoriensystem einerseits deduktiv aus der analysierten Literatur (Theorie) sowie andererseits induktiv aus den Erkenntnissen der empirischen Daten gebildet. Die definierten Kategorien bilden bewusst sämtliche Determinanten aus dem Forschungsmodell (siehe Abschn. 5.2) ab. Das finale Kategoriensystem wird in der Tab. 5.1 dargestellt.

\footnotetext{
${ }^{3}$ Software zur qualitativen Datenanalyse - https://www.maxqda.de/.
} 
Tab. 5.1 Kategoriensystem. (Eigene Darstellung)

\begin{tabular}{|c|c|c|}
\hline ID & Kategorie & Beschreibung \\
\hline A & Intentionsverhaltenslücke & $\begin{array}{l}\text { A1: Wichtigkeit der IT-Landschaft } \\
\text { A2: Umgesetzte Sicherheitsmaßnahmen } \\
\text { (Technologie, Prozess, Mensch) } \\
\text { A3: Angestrebtes Cybersicherheitsniveau }\end{array}$ \\
\hline B & Unternehmensressourcen & $\begin{array}{l}\text { B1: Informationssicherheitstechnologien } \\
\text { B2: Qualifiziertes Informationssicherheits- } \\
\text { personal } \\
\text { B3: Sicherheitsbewusstsein der Benutzer } \\
\text { B4: IT-Fähigkeiten }\end{array}$ \\
\hline $\mathrm{C}$ & $\begin{array}{l}\text { Bewältigungsmanagement (Bewältigungs- } \\
\text { einschätzung) }\end{array}$ & $\begin{array}{l}\text { C1: Selbstwirksamkeit } \\
\text { C2: Wirksamkeit } \\
\text { C3: Kosten }\end{array}$ \\
\hline $\mathrm{D}$ & $\begin{array}{l}\text { Bedrohungsmanagement (Bedrohungsein- } \\
\text { schätzung) }\end{array}$ & $\begin{array}{l}\text { D1: Schadensausmaß } \\
\text { D2: Schadensanfälligkeit }\end{array}$ \\
\hline $\mathrm{E}$ & Sozialer Einfluss & $\begin{array}{l}\text { E1: Normativdruck } \\
\text { E2: Nachahmungsdruck } \\
\text { E3: Zwangsdruck }\end{array}$ \\
\hline $\mathrm{F}$ & IT-Risikomanagement & Keine Subkategorie \\
\hline $\mathrm{G}$ & Selbsteinschätzung & Keine Subkategorie \\
\hline $\mathrm{H}$ & Motivation & Keine Subkategorie \\
\hline I & Subjektive Wahrnehmung & Keine Subkategorie \\
\hline $\mathrm{J}$ & Hilfsmittel & Keine Subkategorie \\
\hline K & Erfolgsfaktoren & Keine Subkategorie \\
\hline
\end{tabular}

Der gewählte Forschungsprozess nach Yin (2014) erlaubt dabei, die Datenerhebungsmethoden während des Forschungsprozesses basierend auf ermittelten Erkenntnissen anzupassen. So wurde sichergestellt, dass eine bedeutende Entdeckung innerhalb einer Fallstudie bereits während des laufenden Forschungsprozesses in die noch bevorstehende Forschung einfließen konnte (Yin 2014, S. 59 ff.).

\subsubsection{Sample}

Die Fallstudienpartner der vorliegenden Forschungsarbeit werden in der Tab. 5.2 kurz vorgestellt. Auf Wunsch der Fallstudienpartner werden diese anonymisiert und nicht namentlich vorgestellt. 
Tab. 5.2 Fallstudienpartner. (Eigene Darstellung)

\begin{tabular}{l|l}
\hline Fallstudienpartner 1 & $\begin{array}{l}\text { Beim Fallstudienpartner 1 handelt es sich um ein Schweizer KMU mit } \\
\text { Sitz im Zürcher Oberland. Das Kerngeschäft des Fallstudienpartners 1 } \\
\text { ist die Erbringung von Hauswartungs- und Reinigungsdienstleistungen. } \\
\text { Aktuell beschäftigt das Unternehmen etwas mehr als 200 Mitarbeitende. } \\
\text { Rund 50 Mitarbeitende arbeiten regelmäßig mit IT-Mitteln der Unter- } \\
\text { nehmung. Als Interviewpartner stellte sich der Geschäftsführer zur Ver- } \\
\text { fügung. Er entscheidet in seiner Rolle als Geschäftsführer über sämtliche } \\
\text { IT-Fragen innerhalb der Unternehmung }\end{array}$ \\
\hline Fallstudienpartner 2 & $\begin{array}{l}\text { Der Fallstudienpartner 2 ist ein Schweizer KMU mit Hauptsitz in der } \\
\text { Zentralschweiz. Das Unternehmen besitzt zwei Zweigstellen, die sich } \\
\text { ebenfalls in der Zentralschweiz befinden. Das Kerngeschäft des Fall- } \\
\text { studienpartners 2 sind Unternehmens-, Steuer- und Rechtsberatungen } \\
\text { sowie weitere Treuhand- und Revisionsdienstleistungen. Das Unter- } \\
\text { nehmen beschäftigt zurzeit 50 Mitarbeitende. Als Interviewpartner stellte } \\
\text { sich der ICT-Verantwortliche des Fallstudienpartners zur Verfügung }\end{array}$ \\
\hline Fallstudienpartner 3 & $\begin{array}{l}\text { Beim Fallstudienpartner 3 handelt es sich um ein im Bereich Touris- } \\
\text { musmarketing tätiges Schweizer KMU mit Hauptsitz in Zürich sowie 33 } \\
\text { Außenstandorten weltweit. Aktuell beschäftigt das Unternehmen rund } \\
\text { 245 Mitarbeitende. Die gesamte IT-Landschaft wird dabei vom Hauptsitz } \\
\text { in Zürich bewirtschaftet. Als Interviewpartner stellten sich der IT-Leiter } \\
\text { sowie ein Senior-System-Engineer zur Verfügung }\end{array}$ \\
\hline
\end{tabular}

\subsection{Ergebnisse}

In diesem Abschnitt werden die aus den empirischen Daten gewonnenen Erkenntnisse anonymisiert aufgezeigt und die definierten Forschungsfragen beantwortet. Die vorliegende Veröffentlichung fokussiert sich auf die Erkenntnisse aus der Cross-CaseAnalyse und verzichtet bewusst auf die Ausführung der Erkenntnisse der einzelnen Within-Case-Analysen (siehe Abschn. 5.2).

\subsubsection{Intentionsverhaltenslücke}

Die Analyse der empirischen Daten zeigt, dass bei allen Entscheidungsträgern der Fallstudienpartner die Verhaltensintention ,ein adäquates Cybersicherheitsniveau zu erreichen" besteht. Dies äußerte sich in Interviewaussagen der Entscheidungsträger sowie in firmeninternen Dokumenten, welche zusammengefasst festhalten, dass die Informationstechnologie für die Kontinuität der Unternehmen eine entscheidende Rolle spielt und deshalb der Schutz der Daten und IT-System besonders wichtig ist. Die Wichtigkeit der IT-Landschaft wird dabei von den Entscheidungsträgern mit dessen Verfügbarkeits- oder Vertraulichkeitsanforderungen argumentiert. Die bestehende 
Verhaltensintention lässt vermuten, dass somit durch die Unternehmen oder deren Entscheidungsträger als für sie relevant wahrgenommene Cybersicherheitsbedrohungen durch adäquate Maßnahmen adressiert werden (Ausführung des Zielverhaltens vgl. Abschn. 5.2). Die Analyse der empirischen Daten zeigt jedoch, dass häufig das Gegenteil der Fall ist. Als relevant wahrgenommene Bedrohungen werden nicht oder lediglich teilweise durch adäquate Maßnahmen adressiert. So werden beispielsweise Phishing-Angriffe $^{4}$ von allen Entscheidungsträgern als relevante Bedrohung wahrgenommen, möglich Abwehrmaßnahmen, bspw. zur Stärkung der Mitarbeitersensibilisierung, werden jedoch nicht umgesetzt. Ein Interviewpartner des Fallstudienpartner 1 unterstreicht sein Verhalten sinnbildlich mit folgender Aussage:

Man sagt ja bekanntlich der Deckel kommt erst dann auf den Brunnen, wenn das Kind hineingefallen ist. Wenn etwas passieren würde, wäre das Thema natürlich von einer Sekunde auf die andere extrem wichtig für mich.

Das dabei gezeigte Verhalten entspricht der Form einer emotionalen Bewältigungsstrategie. Diese Form von Bewältigung führt zu Verleugnung ,diese Bedrohung wird bei mir nicht eintreten“ oder Wunschdenken ,niemand will meiner IT-Landschaft Schaden zufügen“ (Liang und Xue 2009, S. 86). Das bereits in mehreren Studien (vgl. BSI 2011; Hirschi und Portmann 2017; Renaud 2016; Zürich Versicherungs-Gesellschaft 2016; gfs-zürich 2017) sowie von Straub (1990, S. 255) aufgezeigte Phänomen der Intentionsverhaltenslücke hinsichtlich Cybersicherheit konnte auf Basis der erhobenen empirischen Daten der vorliegenden Analyse somit auch bei Entscheidungsträgern in Schweizer KMU beobachtet werden. Basierend auf den erhobenen empirischen Daten sowie der bestehenden Literatur kann zudem davon ausgegangen werden, dass es sich dabei um ein großflächig vorhandenes Phänomen bei KMU handelt, das unabhängig von der Größe, Branche und Herkunft der KMU vorkommt.

Das Phänomen der Intentionsverhaltenslücke hinsichtlich Cybersicherheit hat in der bestehenden Forschung keine einheitliche Bezeichnung und wird in der vorliegenden Forschungsarbeit als Security-Paradox ${ }^{5}$ bezeichnet. Nachfolgend wird der Versuch einer Definition des Begriffs Security-Paradox vorgenommen.

Als Security-Paradox wird bei einer Organisation eine beobachtbare Intentionsverhaltenslücke hinsichtlich der Bestrebung ${ }^{6}$ eines gewünschten Cybersicherheitsniveaus

\footnotetext{
${ }^{4}$ Die betrügerische Praxis, E-Mails zu versenden, die angeblich von seriösen Absendern stammen, um Einzelpersonen dazu zu bringen, persönliche Daten wie Passwörter und Kreditkartennummern preiszugeben.

${ }^{5}$ Der Begriff IT-Sicherheits-Paradox wurde erstmalig von Buxmann (2017) in einem Blogeintrag erwähnt.

${ }^{6}$ Die Bestrebung ergibt sich dabei aus der Wichtigkeit der IT-Landschaft der Unternehmung.
} 
(Verhaltensintention) und der fehlenden Umsetzung von hierfür notwendigen Maßnahmen (Ausführung des Zielverhaltens) bezeichnet.

Das Security-Paradox darf dabei nicht mit den Begriffen Privacy-Paradox oder Datenschutzparadox verwechselt werden, die sich auf das Phänomen beziehen, dass Privatsphäre und Datenschutz für die Menschen im digitalen Zeitalter ein Hauptanliegen sind, während auf der anderen Seite Einzelpersonen persönliche Informationen für relativ kleine oder gar keine Belohnungen preisgeben (Kokolakis 2017, S. 122). Buxmann (2017), der den Begriff IT-Sicherheits-Paradox erstmalig 2017 in einem Blogbeitrag erwähnt, führt aus, dass unrealistischer Optimismus eine der Hauptursachen für das Vorhandensein des Security-Paradox sein könnte. Dies gründet auf seiner Feststellung, dass Entscheidungsträger dazu tendieren, ihr eigenes Cybersicherheitsniveau im Vergleich mit jenem zu konkurrierenden Unternehmen als deutlich besser einschätzen. Gemäß Buxmann wird dieses Verhalten in der Psychologie als unrealistischer Optimismus beschrieben, bei welchem Menschen dazu tendieren, ihr eigenes Risiko im Vergleich mit anderen verzerrt wahrzunehmen und zu unterschätzen. Dasselbe Verhalten von Entscheidungsträgern konnte auch in den im Rahmen der vorliegenden Analyse durchgeführten Fallstudien beobachtet werden. So schätzen zwei der drei Entscheidungsträger ihr eigenes Cybersicherheitsniveau als deutlich besser ein, als jenes von konkurrierenden Unternehmen. Im folgenden Kapitel werden die potenziellen Ursachen für das Vorhandensein des Security-Paradoxes diskutiert.

\subsubsection{Ursachenanalyse}

Die dieser Analyse zugrunde liegende Forschungsarbeit fokussierte sich auf die Ursachenanalyse für das Vorhandensein des Security-Paradoxes. Die folgenden Analyseergebnisse wurden von den Autoren bewusst für die Führungskräfte und Entscheidungsträger aufbereitet. So werden Determinanten, für welche kein oder lediglich ein geringer Einfluss auf das Vorhandensein des Security-Paradoxes festgestellt werden konnte, nicht berücksichtigt. Gleiches gilt für Determinanten, die von Führungskräften und Entscheidungsträgern nicht beeinflusst werden können. Das Lesen der Analyseergebnisse soll Führungskräfte und Entscheidungsträger somit befähigen, bessere Entscheidungen betreffend Cybersicherheit im Rahmen der zu bewältigenden Digitalisierung treffen zu können.

\subsubsection{Unternehmensressourcen}

Cavusoglu et al. (2015, S. 396) stellten fest, dass die IT-Fähigkeiten einer Organisation einen maßgeblichen Einfluss auf ihr Cybersicherheitsniveau besitzen. Dieser Sachverhalt konnte auch in den empirischen Daten der vorliegenden Forschungsarbeit nachgewiesen werden und wird in der Abb. 5.2 illustriert. 


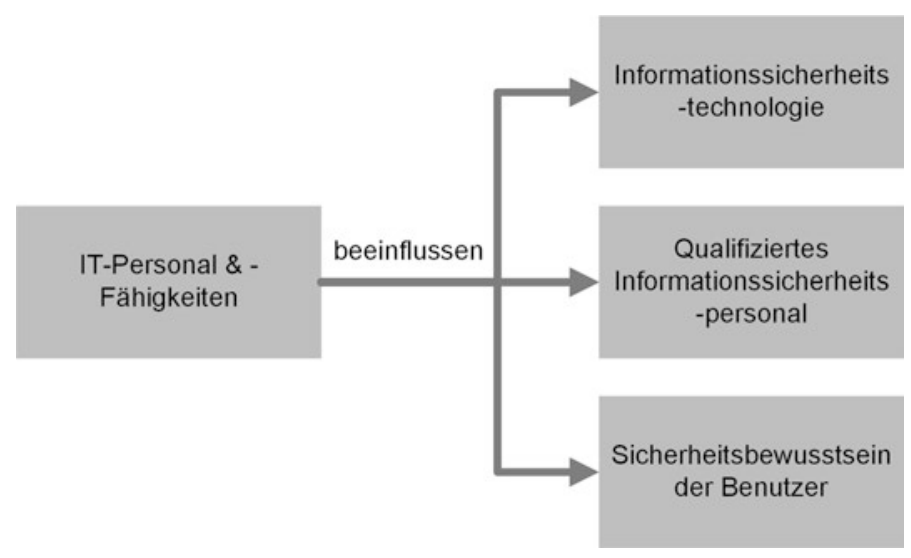

Abb. 5.2 Einfluss IT-Fähigkeiten. (Eigene Darstellung)

So haben die Fallstudienpartner, die über eine interne IT-Abteilung verfügen, ein merklich höheres Cybersicherheitsniveau als jene ohne interne IT-Abteilung. Zudem wurde festgestellt, dass dieselben Fallstudienpartner ihre eigene Selbstwirksamkeit deutlich höher einschätzen. Die Literaturrecherche zeigt, dass die Ausführung eines Verhaltens umso wahrscheinlicher ist, je höher die Selbstwirksamkeitserwartung ausfällt (siehe Abschn. 5.2). Im heutigen digitalen Wandel ist es deshalb umso wichtiger, dass Führungskräfte und Entscheidungsträger sicherstellen, dass eine Unternehmung über ausreichend IT-Fähigkeiten verfügt. Gleichwohl reicht das ledigliche Vorhandensein von IT-Fähigkeiten jedoch für die meisten Schweizer KMU nicht aus, um ein für sie angemessenes Cybersicherheitsniveau zu erreichen. Ferner ist es wichtig, dass Führungskräfte und Entscheidungsträger Cybersicherheit als Führungsaufgabe wahrnehmen und eine Cybersicherheitskultur etablieren. Dazu müssen notwendige Verantwortlichkeiten definiert, das Sicherheitsbewusstsein der Mitarbeiter gestärkt, Topmanagement-Unterstützung für das Thema gezeigt und notwendiges Fachwissen beschafft werden. Kurzfristig ist nicht davon auszugehen, dass in Zukunft jedes Schweizer KMU einen Cybersicherheitsverantwortlichen benennt und diesen mit Unmengen an Unternehmensressourcen ausrüstet - dies soll auch keinesfalls die Quintessenz der vorliegenden Analyse sein - jedoch müssen sich Führungskräfte der Verantwortung, ein für ihre Unternehmung angemessenes Cybersicherheitsniveau zu erreichen, stellen. Dies kann beispielsweise bereits mit dem Durchführen institutionalisierter Sensibilisierungsmaßnahmen für Mitarbeitende, dem Umsetzen von technologischen Grundschutzmaßnahmen und der Definition grundlegender Prozesskontrollen, wie bspw. dem Vieraugenprinzip erreicht werden. 


\subsubsection{Bedrohungs- und Bewältigungsmanagement}

Die Erkenntnisse aus den empirischen Daten der vorliegenden Analyse zeigen, dass bei keinem der untersuchten Fallstudienpartner ein systematischer Ansatz zum Bedrohungs- oder Bewältigungsmanagement vorhanden ist. Die kognitiven Prozesse der Bedrohungs- und Bewältigungseinschätzung sind jedoch ausschlaggebend dafür, ob eine Verhaltensintention, welche einer Bedrohung entgegenwirkt, entsteht oder nicht und wie mit dieser Verhaltensintention umgegangen wird (siehe Abschn. 5.2). Die dabei fehlende Systematik zur Behandlung des Themas führt dazu, dass die Adressierung stark von den persönlichen Erfahrungen, Fähigkeiten und dem Wissen der jeweiligen Entscheidungsträger abhängt. Dies birgt hohes Potenzial für das Auftreten einer emotionalen Bewältigungsstrategie und damit einhergehenden Fehleinschätzungen (vgl. Buxmann 2017) und unvollständigen Ansätzen bei der Behandlung des Themas Cybersicherheit. Beispielsweise wenn der Entscheidungsträger nicht über genügend Wissen über die Materie verfügt oder nicht genügend stark sensibilisiert ist. So wurde bei der Analyse der empirischen Daten festgestellt, dass die Entscheidungsträger aller Fallstudienpartnern eine klare Präferenz für technologische Maßnahmen gegenüber organisatorischen oder prozessualen Maßnahmen zur Stärkung ihrer Cybersicherheit besitzen, die von den Entscheidungsträgern erkannten Bedrohungen jedoch häufiger organisatorische oder prozessuale Maßnahmen nahelegen würden (bspw. menschliches Fehlverhalten). Um dieser Herausforderung erfolgreich zu begegnen, sollten sich Entscheidungsträger und Führungskräfte deshalb bei der Analyse von Cybersicherheitsbedrohungen und bei der Festlegung von Cybersicherheitsmaßnahmen an etablierten Standards (z. B. ISO 2700x ${ }^{7}$, NIST Cybersecurity Framework ${ }^{8}$, IT-Grundschutz des BSI ${ }^{9}$ oder den IKT-Minimalstandard des Bundesamt für wirtschaftliche Landesversorgung ${ }^{10}$ ) orientieren und sofern sinnvoll, externe Fachspezialisten beiziehen.

Die Literaturrecherche und die empirischen Daten zeigen, dass ein ganzheitlicher Ansatz zur adäquaten Adressierung von Cybersicherheit notwendig ist. Dies bedeutet, dass gerade sich im digitalen Wandel befindliche Schweizer KMU Maßnahmen auf organisatorischer, prozessualer sowie technologischer Ebene ergreifen müssen, um ein angemessenes Cybersicherheitsniveaus zu erreichen. Dabei ist die Aufklärung und Schulung der Mitarbeitenden in Bezug auf Cybersicherheit ein zentraler Aspekt. Eine angemessene Aufklärung fördert das allgemeine Bewusstsein und das Verständnis bei den Mitarbeitenden und ermöglicht die Etablierung einer Cybersicherheitskultur. In dieser Kultur teilen die Mitarbeitenden gemeinsame Werte, Normen und Überzeugungen bezüglich Cybersicherheits-Praktiken (Woodhouse 2008, S. 247; Tu und Yuan 2014, S. 1878-1879). Dieses gemeinsame Verständnis erzeugt einen sich bei den Mitarbeitenden auf Cybersicherheit positiv auswirkenden sozialen

\footnotetext{
${ }^{7}$ https://www.iso.org/news/ref2266.html.

${ }^{8}$ https://www.nist.gov/cyberframework.

${ }^{9}$ https://www.bsi.bund.de/DE/Themen/ITGrundschutz/itgrundschutz_node.html.

${ }^{10} \mathrm{https} / / /$ www.bwl.admin.ch/bwl/de/home/themen/ikt/ikt_minimalstandard.html.
} 
Einfluss in Form von Normativdruck (siehe Abschn. 5.2). Um das für ein KMU passende Cybersicherheitsniveau identifizieren zu können, sollte zudem ein unternehmensweites Risikomanagement etabliert werden. Dies hilft bei der Auswahl passender Sicherheitsmaßnahmen sowie bei der realistischen Einschätzung von Bedrohungs- und Bewältigungsmöglichkeiten (Tu und Yuan 2014, S. 1878-1879).

\subsubsection{Verhalten Entscheidungsträger}

Die Analyse der empirischen Daten zeigt, dass bei allen drei Fallstudienpartnern eine klare Definition hinsichtlich der Verantwortlichkeiten und Aufgaben zur Cybersicherheit nicht oder nur teilweise vorhanden sind. Auch fehlt bei sämtlichen Fallstudienpartnern ein systematisches Bewältigungs-, Bedrohungs- und/oder Risikomanagement (siehe Abschn. 5.4.2.2). Diese beiden Tatsachen führen dazu, dass das Cybersicherheitsniveau einer Organisation stark dem Einfluss des jeweiligen IT-Verantwortlichen unterliegt. So zeigte sich bei den Fallstudienpartnern 2 und 3, dass der Vorgänger des jeweiligen IT-Verantwortlichen dem Cybersicherheitsniveau der jeweiligen IT-Landschaft lediglich geringe Beachtung schenkte. Durch den Wechsel zu den heutigen IT-Verantwortlichen konnte das Cybersicherheitsniveau der IT-Landschaften merklich gesteigert werden. Der IT-Leiter des Fallstudienpartners 3 hält diesbezüglich fest:

Wenn IT- und Informationssicherheit nicht systematisch in einer Unternehmung ausgeübt wird, entscheiden die Erfahrungen, die Interessen und die Fokusse des IT-Verantwortlichen, ob Security adäquat adressiert wird oder nicht.

Fehlendes Wissen oder Erfahrung beim IT-Verantwortlichen führen so zu einer nicht realistischen Bedrohungs- und Bewältigungseinschätzung. Diese Fehleinschätzung ${ }^{11}$ führt wiederum zur Nichtergreifung von adäquaten Sicherheitsmaßnahmen. Führungskräfte und Entscheidungsträger von Schweizer KMU müssen sich diesem Entscheidungsbias bewusst werden. Um diesem entgegen zu wirken, sollten sich Führungskräfte und Entscheidungsträger stärker auf Sicherheitsstandards zur Adressierung von Cybersicherheit berufen. Bestehende Studien (vgl. Hirschi und Portmann 2017; gfs-zürich 2017) zeigen jedoch, dass lediglich zirka $30 \%$ der Schweizer KMU Sicherheitsstandards zur Adressierung von Cybersicherheit verwenden. Von den untersuchten Fallstudienpartnern verwendet keines der Unternehmen Sicherheitsstandards. Die Verwendung von Sicherheitsstandards hätte für KMU jedoch den Vorteil, eine durch den Standard geführte, ganzheitliche Betrachtung von Cybersicherheit sicherzustellen. Dabei gilt es jedoch anzumerken, dass eine Vielzahl verfügbarer Sicherheitsstandards (z. B. ISO 2700x, NIST Cybersecurity Framework, IT-Grundschutz des BSI usw.) sich für KMU zwar grundsätzlich eignen, diese aber in der Praxis Mangels an Erfahrung mit solchen Werken für KMU schwer umsetzbar sind. Gemäß Kardel (2011, S. 44-48) müssen Sicherheitsstandards

\footnotetext{
${ }^{11}$ Siehe Abschn. 5.4.1 - Fehleinschätzung betreffend „Cybersicherheitsniveau im Vergleich mit konkurrierenden Unternehmen“.
} 
deshalb an den KMU-Kontext angepasst werden. Eine Möglichkeit für KMU, diese Kontextanpassung zu umgehen, ist die direkte Verwendung von KMU-spezifischen Hilfsmitteln, wie beispielsweise:

- Merkblatt IT-Sicherheit für KMU von der Melde- und Analysestelle Informationssicherung und dem Swiss Government Computer Emergency Response Team (MELANI \& GovCert 2018)

- 10-Punkte-Regel zur Stärkung der Cybersicherheit des Schweizer KMU Portal (KMU Portal, 2018)

- Mehr Informationssicherheit für Klein- und Mittelbetriebe (KMU) (KMU Portal, 2016)

- Informationssicherheits- und Datenschutzstandard für KMU der ENISA (Manso et al. 2015)

\subsection{Diskussion}

Die vorliegende Forschungsarbeit identifizierte erstmalig ${ }^{12}$ mittels empirischer Forschung Ursachen, die zum Vorhandensein des Security-Paradoxes führen. Es ist allerdings zu beachten, dass die kleine Anzahl beobachteter Fälle in dieser Forschungsarbeit eine wesentliche Einschränkung bei der Interpretation der Ergebnisse bedeutet und damit Vorsicht geboten ist, die vorliegenden Ergebnisse für sämtliche Schweizer KMU oder grundsätzlich für KMU zu verallgemeinern. Stattdessen gilt es durch den Leser zu prüfen, ob die Ergebnisse im relevanten KMU-Kontext wiedererkannt werden und wie die beschriebenen Empfehlungen situationsgerecht adaptiert werden können.

Bezugnehmend auf die Forschungsfragen dieser Arbeit lassen sich folgende Beobachtungen zusammenfassend festhalten. 1) Eine Intentionsverhaltenslücke (Security-Paradox) hinsichtlich Cybersicherheit konnte bei allen drei Fallstudienpartnern beobachtet werden (siehe Abschn. 5.4.1). Bestehende Studien und die empirischen Ergebnisse lassen somit die Vermutung zu, dass es sich beim Security-Paradox um ein verbreitetes Phänomen handelt, das auch bei Schweizer KMU zu beobachten ist. Die vorliegende Analyse liefert dazu jedoch keinen stichhaltigen Nachweis. 2) Basierend auf dem entwickelten Forschungsmodell und den erhobenen empirischen Daten wurde für folgende, von Führungskräften und Entscheidungsträgern beinflussbaren, Determinanten ein starker Einfluss auf das Vorhandensein des Security-Paradox beobachtet ${ }^{13}$ :

\footnotetext{
${ }^{12} \mathrm{Gemäß} \mathrm{dem} \mathrm{durch} \mathrm{die} \mathrm{Autoren} \mathrm{dieser} \mathrm{Veröffentlichung} \mathrm{erhobenen} \mathrm{aktuellen} \mathrm{Forschungsstand.}$

${ }^{13}$ Nebst den drei genannten Determinanten wurde für die Determinante „Sozialer Einfluss“ ebenfalls ein starker Einfluss auf das Vorhandensein des Security-Paradoxes beobachtet. Da Zwangsdruck beispielsweise durch Regulatorien einen starken Einfluss auf das Verhalten von Entscheidungsträgern besitzt. Dies wurde in der vorliegenden Veröffentlich jedoch nicht detaillierter behandelt, da Entscheidungsträger nicht verantworten ob ein Zwangsdruck besteht oder nicht.
} 
- Unternehmensressourcen (siehe Abschn. 5.4.2.1)

- Bewältigungs- und Bedrohungsmanagement (siehe Abschn. 5.4.2.2)

- Verhalten Entscheidungsträger (siehe Abschn. 5.4.2.3)

Im Rahmen der Ursachenanalyse zeigte sich jedoch, dass die Zusammenhänge und Wechselwirkungen der Determinanten vielschichtig sind und so nicht eine einzelne Determinante für das Vorhandensein des Security-Paradoxes identifiziert werden konnte. Kritisch merken die Autoren der vorliegenden Analyse an, dass aufgrund der eingeschränkten Datenbasis möglicherweise relevante Ursachen nicht identifiziert wurden. Zukünftige Forschungsarbeiten könnten an diesen Punkt anknüpfen und die in dieser Forschungsarbeit identifizierten Ursachen validieren und allfällig erweitern.

Zusammenfassend stellen die Autoren der vorliegenden Forschungsarbeit fest: Cybersicherheit ist generell sowie spezifisch für KMU ein Thema, das im Kontext der Digitalisierung an Wichtigkeit gewinnt und somit auch in Zukunft die Aufmerksamkeit von Führungskräften und Entscheidungsträger verdient.

\subsection{Zusammenfassung und Empfehlungen für die Praxis}

In dem Versuch das Vorhandensein des Security-Paradoxes zu vermeiden, ist es für Führungskräfte und Entscheidungsträger von Schweizer KMU unerlässlich, zu verstehen, welche Faktoren einen erheblichen Einfluss auf dieses Phänomen besitzen. Dazu wurden in der vorliegenden Forschungsarbeit, die für Führungskräfte und Entscheidungsträger relevanten Determinanten aufbereitet (siehe Abschn. 5.4.2) und nachfolgende Empfehlungen zusammengestellt:

\section{Cybersicherheit als Führungsaufgabe wahrnehmen}

Um die Herausforderung der Cybersicherheit, im Zuge der Digitalisierung zu meistern, müssen Entscheidungsträger und Führungskräfte von Schweizer KMU sich ihrer Verantwortung hinsichtlich Cybersicherheit bewusst werden und sie wahrnehmen. Unterstützung durch Entscheidungsträger (Management Support) wird in der bestehenden Forschung als einer der wichtigsten Erfolgsfaktoren zur erfolgreichen Adressierung von Cybersicherheit genannt (vgl. Chew et al. 2008; Dirks et al. 2016; Schwyter und Wisler 2013; Tu und Yuan 2014; Woodhouse 2008; Manso et al. 2015). Diese Tatsache wird in den empirischen Daten der vorliegenden Forschungsarbeit klar bestätigt. Es konnte eine starke Abhängigkeit zwischen der Möglichkeit einer Organisation, Sicherheitsmaßnahmen zu ergreifen und der Unterstützung für die Maßnahmenumsetzung durch die Geschäftsleitung (in der Regel der Entscheidungsträger) erkannt werden. So entscheidet bei allen drei Fallstudienpartnern die Geschäftsleitung über die Umsetzung von Maßnahmen zur Stärkung der Cybersicherheit. Es ist aus diesem Grund wichtig, dass Entscheidungsträger einer Organisation ein Bewusstsein für Cybersicherheit besitzen. Fehlt dieses Bewusstsein bei den Entscheidungsträgern, bleiben Investitionen in die Stärkung der Cybersicherheit mit 
hoher Wahrscheinlichkeit aus. Die Sensibilisierung der Entscheidungsträger von Schweizer KMU wird aktuell einerseits durch den Staat und andererseits durch die Privatwirtschaft (Gewerbeverbände) bewusst gefördert. Um die Unterstützung von Schweizer KMU im Bereich Cybersicherheit zu stärken, wurde 2015 von der damaligen Bundesrätin Eveline Widmer-Schlumpf die Expertengruppe Zukunft der Datenbearbeitung und Datensicherheit eingesetzt. ${ }^{14}$ In einem Interview mit der Neuen Zürcher Zeitung gibt Brigitta M. Gadient als Präsidentin der Expertengruppe an (Mäder 2017):

Wir wollen bei den kleinen und mittleren Unternehmen eine Sensibilisierung für die IT-Sicherheit erreichen.

Im September 2018 wurde von der Expertengruppe gemeinsam mit den wichtigsten Verbänden und Gruppierungen aus dem IT-Bereich sowie dem Bundesamt für wirtschaftliche Landesversorgung in einer gemeinsamen Initiative ein Schnelltest ${ }^{15}$ zur Cyberresilienz von KMU lanciert. Der Schnelltest liefert Erkenntnisse, in welchen Bereichen eines KMU hinsichtlich Cybersicherheit Lücken und allfälliger Handlungsbedarf bestehen (ICTswitzerland 2018).

\section{Etablierte Standards verwenden}

Bestehende Studien (vgl. Hirschi und Portmann 2017; gfs-zürich 2017) zeigen, dass lediglich ein geringer Teil (zirka $30 \%$ ) der Schweizer KMU Sicherheitsstandards zur Adressierung von Cybersicherheit verwenden. In den empirischen Daten der vorliegenden Forschungsarbeit zeigte sich ein noch deutlicheres Bild - keiner der Fallstudienpartner verwendet Sicherheitsstandards zur Adressierung von Cybersicherheit. Die Verwendung von Sicherheitsstandards hätte für KMU jedoch den Vorteil, eine durch den Standard geführte, ganzheitliche und auf etablierten Praktiken basierende Betrachtung von Cybersicherheit sicherzustellen. So können die relevanten organisatorischen, technologischen und prozessualen Sicherheitsmaßnahmen zur Erreichung eines adäquaten Sicherheitsniveaus bestmöglich definiert werden, was für einige Entscheidungsträger und Führungskräfte aufgrund fehlender Erfahrungswerte zunehmend eine Herausforderung darstellt (Expertengruppe zur Zukunft der Datenbearbeitung und Datensicherheit 2018, S. 64). Das systematische Vorgehen anhand eines Sicherheitsstandards zur Einführung sowie zur Unterhaltung eines Cybersicherheit-Managementsystems ist ein relevanter Erfolgsfaktor für die erfolgreiche Adressierung von Cybersicherheit (Woodhouse 2008, S. 247). Es empfiehlt sich somit für Entscheidungsträger und Führungskräfte von Schweizer KMU, sich mit bestehenden Sicherheitsstandards (z. B. ISO 2700x, NIST Cybersecurity Framework, IT-Grundschutz des BSI usw.) auseinanderzusetzen und diese bei der Bearbeitung des Themas zu berück-

\footnotetext{
${ }^{14}$ Motion 13.3841 - https://www.parlament.ch/de/ratsbetrieb/suche-curia-vista/geschaeft?Affai$\mathrm{rId}=20133841$.

${ }^{15}$ https://www.cybersecurity-check.ch/.
} 
sichtigen. Nach Ansicht der Autoren stellt der Schnelltest zur Cyberresilienz von KMU einen guten Ausgangspunkt zur erstmaligen systematischen Adressierung des Themas dar.

\section{Cybersicherheitskultur etablieren}

Um Cybersicherheit nachhaltig in einem Unternehmen zu verankern und das Verhalten der Mitarbeitenden langfristig positiv zu verändern, bedarf es der Definition notwendiger Verantwortlichkeiten und einer flächendeckenden Sensibilisierung aller Mitarbeitenden. Eine angemessene Aufklärung fördert das allgemeine Bewusstsein und das Verständnis bei den Mitarbeitenden und ermöglicht die Etablierung einer Cybersicherheitskultur. Dabei teilen die Mitarbeitenden gemeinsame Werte, Normen und Überzeugungen bezüglich Cybersicherheitspraktiken. Durch dieses gemeinsame Verständnis entsteht eine sich positiv auswirkende Form von Normativdruck (siehe Abschn. 5.2). Diese verankert einen sicheren Umgang mit IT-Mitteln im Unternehmen und fördert so eine nachhaltige Stärkung des Cybersicherheitsniveaus. Entscheidungsträger und Führungskräfte müssen dazu ihre Verantwortung hinsichtlich Cybersicherheit wahrnehmen, mit gutem Beispiel vorangehen und die entsprechenden Werte, Normen und Überzeugungen vorleben, um diese als Unternehmenskulturwerte zu verankern.

Abschließend halten die Autoren der vorliegenden Forschungsarbeit fest: Die Digitalisierung kann für ein KMU nur dann nachhaltig sein, wenn sie auf Vertrauen in eine organisatorische, prozessuale und technologische sichere IT beruht und Cybersicherheit dabei als Führungsaufgabe wahrgenommen wird.

\section{Literatur}

Ajzen, I. (1985). From intentions to actions: A theory of planned behavior. In: J. Kuhl \& J. Beckman (Hrsg.), Action-control: From cognition to behavior (S. 11-39). Heidelberg: Springer.

Bundesamt für Sicherheit in der Informationstechnik (BSI). (2011). Studie zur IT-Sicherheit in kleinen und mittleren Unternehmen. https://www.bsi.bund.de/SharedDocs/Downloads/DE/BSI/ Publikationen/Studien/KMU/Studie_IT-Sicherheit_KMU.pdf. Zugegriffen: 9. Apr. 2018.

Burnkrant, R. E., \& Cousineau, A. (1975). Informational and normative social influence in buyer behavior. Journal of Consumer Research, 2(3), 206-215. https://doi.org/10.1086/208633.

Buxmann, P. (2017). Das IT-Sicherheits-Paradox: Warum Unternehmen zu wenig in IT-Sicherheit investieren. http://www.peterbuxmann.de/2017/08/03/das-it-sicherheits-paradox-warum-unternehmen-zu-wenig-in-it-sicherheit-investieren/. Zugegriffen: 19. März 2018.

Browne, S., Lang, M., \& Golden, D. W. (2015). Linking threat avoidance and security adoption: A theoretical model for SMEs. BLED 2015 Proceedings. https://aisel.aisnet.org/bled2015/35. Zugegriffen: 11. März 2018.

Cavusoglu, H., Cavusoglu, H., Son, J.-Y., \& Benbasat, I. (2015). Institutional pressures in security management: Direct and indirect influences on organizational investment in information security control resources. Information \& Management, 52(4), 385-400. https://doi.org/10.1016/j. im.2014.12.004. 
Chew, E., Swanson, M., Stine, K. M., Bartol, N., Brown, A., \& Robinson, W. (2008). Performance measurement guide for information security. Gaithersburg: National Institute of Standards and Technology. https://doi.org/10.6028/NIST.SP.800-55r1.

Deutsch, M., \& Gerard, H. B. (1955). A study of normative and informational social influences upon individual judgement. Journal of Abnormal Psychology, 51(3), 629-636. https://doi. org/10.1037/h0046408.

Dirks, N., Schemmer, S., \& Schumann, R. (2016). Etablierung effektiver Informationssicherheit. In: S. Helmke \& M. Uebel (Hrsg.), Managementorientiertes IT-Controlling und IT-Governance (S. 239-251). Wiesbaden: Springer. https://doi.org/10.1007/978-3-658-07990-1_15.

Eckert, C. (2017). Cybersicherheit beyond 2020! Informatik-Spektrum, 40(2), 141-146. https://doi. org/10.1007/s00287-017-1025-6.

Eisenhardt, M. K. (1989). Building theories from case study research. The Academy of Management Review, 14(4), 532-550. https://doi.org/10.2307/258557.

Expertengruppe zur Zukunft der Datenbearbeitung und Datensicherheit. (2018). Bericht der Expertengruppe zur Zukunft der Datenbearbeitung und Datensicherheit. https://www.newsd. admin.ch/newsd/message/attachments/53591.pdf. Zugegriffen: 10. Jan. 2019.

EY. (2018). Schweizer Unternehmen so optimistisch wie lange nicht mehr. http://www.ey.com/ $\mathrm{ch} / \mathrm{de} /$ newsroom/news-releases/medienmitteilung-ey-schweizer-unternehmen-so-optimistisch-wie-lange-nicht-mehr. Zugegriffen: 11. März 2018.

gfs-zürich. (2017). Cyberrisiken in Schweizer KMU. Zürich: gfs-zürich. https://ictswitzerland.ch/ media/dateien/Studien/Schlussbericht_Cyberrisk_KMU_2017.pdf. Zugegriffen: 25. März 2018.

Gläser, J., \& Laudel, G. (2010). Experteninterviews und qualitative Inhaltsanalyse (4. Aufl.). Wiesbaden: VS Verlag.

Grant, R. M. (2010). Contemporary strategy analysis: Text only (7. Aufl.). Hoboken: Wiley.

Hirschi, O., \& Portmann, A. (2017). Nationale Studie zur Informationssicherheit in Schweizer KMU. Zustand der Informationssicherheit in Schweizer KMU (Veröffentlichte Studie). Luzern: Hochschule Luzern.

Hohmann, C., \& Schwarzer, R. (2009). Selbstwirksamkeitserwartung. In J. Bengel \& M. Jerusalem (Hrsg.), Handbuch der Gesundheitspsychologie und Medizinischen Psychologie (S. 61-67). Göttingen: Hogrefe.

ICTswitzerland. (2018). Medienmappe. Cybersecurity-Schnelltest für KMU. https://ictswitzerland. $\mathrm{ch} /$ media/dateien/Cyber_Security/Minimalstandards/Medienmappe_Cybersecurity-Schnelltest_ KMU_2018_09_03_de.pdf. Zugegriffen: 18. Jan. 2019.

Inside-Channels. (2017). Initiative soll Schweizer KMU-Security verbessern. http://www.inside-channels.ch/articles/49147. Zugegriffen: 26. Okt. 2017.

Inside-it. (2018). Hacker stehlen Kundendaten von Waadtländer IT-Unternehmen. https://www. inside-it.ch/articles/50941. Zugegriffen: 30. Apr. 2018.

Kardel, D. (2011). IT-Sicherheitsmanagement in KMU. HMD Praxis der Wirtschaftsinformatik, 48(5), 44-51. https://doi.org/10.1007/BF03340623.

Kelman, H. C. (2006). Interests, relationships, identities: Three central issues for individuals and groups in negotiating their social environment. Annual Review of Psychology, 57(1), 1-26. https://doi.org/10.1146/annurev.psych.57.102904.190156.

Klipper, S. (2015). Cybersicherheit. Wiesbaden: Springer. https://doi.org/10.1007/978-3-658$11577-7$.

KMU Portal für kleine und mittlere Unternehmen (KMU Portal). (2016). Mehr Informationssicherheit für Klein- und Mittelbetriebe (KMU). https://www.kmu.admin.ch/dam/kmu/de/ dokumente/savoir-pratique/Informatique-et-IT/InfoSurance_10_Points_Programme_FR.pdf. Zugegriffen: 16. Mai 2018. 
KMU Portal für kleine und mittlere Unternehmen (KMU Portal). (2018). Zehn Regeln für die Informationssicherheit im KMU. https://www.kmu.admin.ch/kmu/de/home/aktuell/monatsthema/2018/zehn-regeln-fuer-die-informationssicherheit-im-kmu.html. Zugegriffen: 17. Mai 2018 .

Kokolakis, S. (2017). Privacy attitudes and privacy behaviour: A review of current research on the privacy paradox phenomenon. Computers \& Security, 64, 122-134. https://doi.org/10.1016/j. cose.2015.07.002.

KPMG. (2017). Clarity on Cybersicherheit. https://assets.kpmg.com/content/dam/kpmg/ch/pdf/clarity-on-Cybersicherheit-2017-en.pdf. Zugegriffen: 4. Juni 2018.

Mäder, L. (11. Dez. 2017). KMU sollen eine Grundhygiene für IT-Sicherheit beachten. Neue Zürcher Zeitung. https://www.nzz.ch/schweiz/kmu-sollen-eine-grundhygiene-fuer-it-sicherheit-beachten-ld.1337191. Zugegriffen: 17. Mai 2018.

Manso, C. G., Rekleitis, E., Papazafeiropoulos, F., \& Maritsas, V. (2015). Information security and privacy standards for SMEs. https://www.enisa.europa.eu/publications/standardisation-for-smes. Zugegriffen: 11. Mai 2018.

Melde- und Analysestelle Informationssicherung (MELANI), \& Swiss Government Computer Emergency Response Team (GovCERT). (2016) Merkblatt IT-Sicherheit für KMU. https:// www.melani.admin.ch/dam/melani/de/dokumente/2015/01/merkblatt_it-sicherheitfuerKMU. pdf.download.pdf/merkblatt_it-sicherheitfuerKMU.pdf. Zugegriffen: 10. Apr. 2018.

Liang, H., \& Xue, Y. (2009). Avoidance of information technology threats: A theoretical perspective. MIS Quarterly, 33(1), 71-90. https://doi.org/10.2307/20650279.

Pavlou, P. A., \& Fygenson, M. (2006). Understanding and predicting electronic commerce adoption: An extension of the theory of planned behavior. MIS Quarterly, 30(1), 115-143. https:// doi.org/10.2307/25148720.

Renaud, K. (2016). How smaller businesses struggle with security advice. Computer Fraud \& Security, 2016(8), 10-18. https://doi.org/10.1016/S1361-3723(16)30062-8.

Rogers, R. W. (1975). A protection motivation theory of fear appeals and attitude change. Journal of Psychology, 91(1), 93-114. https://doi.org/10.1080/00223980.1975.9915803.

Schwyter, F., \& Wisler, A. (2013). Informationssicherheit für KMU. Sicherheitskonzepte \& praktische Umsetzung (2. Aufl.). Rheinfelden: BPX-Edition.

Straub, D. W. (1990). Effective IS security: An empirical study. Information Systems Research, 1(3), 255-276. https://doi.org/10.1287/isre.1.3.255.

Taylor, S., \& Todd, P. A. (1995). Understanding information technology usage - A test of competing models. Information Systems Research, 6(2), 144-176. https://doi.org/10.1287/isre.6.2.144.

Tu, Z., \& Yuan, Y. (2014). Critical success factors analysis on effective information security management: A literature review. In: Association for Information Systems (Hrsg.), 20th Americas Conference on Information Systems (AMCIS 2014): Smart sustainability: The information systems opportunity (S. 1874-1886). Red Hook: Curran.

Woodhouse, S. (2008). Critical success factors for an information security management system. In: Fifth international conference on information technology \& applications 2008: ICITA2008, D. Tien, \& M. Kavakli (Hrsg.), (S. 244-249). Bathurst: Macquarie Scientific Publishing.

Workman, M., Bommer, W. H., \& Straub, D. (2008). Security lapses and the omission of information security measures: A threat control model and empirical test. Computers in Human Behavior, 24(6), 2799-2816. https://doi.org/10.1016/j.chb.2008.04.005.

Venkatesh, V., \& Davis, F. D. (2000). A theoretical extension of the technology acceptance model: Four longitudinal field studies. Management Science, 46(2), 186-204. https://doi.org/10.1287/ mnsc.46.2.186.11926. 
Venkatesh, V., Morris, M. G., Davis, G. B., \& Davis, F. D. (2003). User acceptance of information technology: Toward a unified view. MIS Quarterly, 27(3), 425-478. https://doi. org/10.2307/30036540.

Yin, R. K. (2014). Case study research. Design and methods (5. Aufl.). Los Angeles: Sage.

Zürich Versicherungs-Gesellschaft. (2016). Schweizer KMU sind nicht vor Cybercrime geschützt. Abgerufen von https://www.zurich.ch/de/ueber-uns/medien/medienmitteilungen/2016/20161123-medienmitteilung. Zugegriffen 4. Nov. 2017.

Meier, Dominique Adrian (M.Sc. WI/me@dominiquemeier.ch) Konsekutives Masterstudium in Wirtschaftsinformatik an der Zürcher Fachhochschule für Angewandte Wissenschaften. Langjährige Tätigkeit als Berater im Bereich IT- und Informationssicherheit. Aktuell als Partner \& Head of Operations in der Geschäftsleitung bei einem auf Informationssicherheit spezialisierten Schweizer Beratungsunternehmen (https://www.redguard.ch).

Burda, Daniel (Prof. Dr./daniel.burda@h-da.de) ist Professor für Wirtschaftsinformatik am Fachbereich Informatik der Hochschule Darmstadt. Zuvor war er an der Berner Fachhochschule als Professor für Wirtschaftsinformatik tätig. Vor seiner Berufung zum Professor war Herr Burda mehrere Jahre als SAP und Business Process Consultant u.a. bei SAP in Deutschland und der Schweiz tätig.

Open Access Dieses Kapitel wird unter der Creative Commons Namensnennung 4.0 International Lizenz (http://creativecommons.org/licenses/by/4.0/deed.de) veröffentlicht, welche die Nutzung, Vervielfältigung, Bearbeitung, Verbreitung und Wiedergabe in jeglichem Medium und Format erlaubt, sofern Sie den/die ursprünglichen Autor(en) und die Quelle ordnungsgemäß nennen, einen Link zur Creative Commons Lizenz beifügen und angeben, ob Änderungen vorgenommen wurden.

Die in diesem Kapitel enthaltenen Bilder und sonstiges Drittmaterial unterliegen ebenfalls der genannten Creative Commons Lizenz, sofern sich aus der Abbildungslegende nichts anderes ergibt. Sofern das betreffende Material nicht unter der genannten Creative Commons Lizenz steht und die betreffende Handlung nicht nach gesetzlichen Vorschriften erlaubt ist, ist für die oben aufgeführten Weiterverwendungen des Materials die Einwilligung des jeweiligen Rechteinhabers einzuholen.

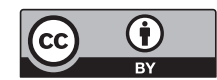




\title{
Ambidextrie - der organisationale Drahtseilakt
}

\section{Synergie zwischen Exploration und Exploitation als Voraussetzung für die digitale Transformation}

\author{
Simon Jonathan Schneeberger und Anja Habegger
}

\begin{abstract}
Zusammenfassung
Sich disruptiv verändernde Rahmenbedingungen können dazu führen, dass bisher erfolgreiche Geschäftsmodelle innert weniger Jahren obsolet werden. Geschuldet ist dies einer mangelnden Anpassungsfähigkeit, deren Ursache unter anderem in der sogenannten Pfadabhängigkeit wurzeln kann. Die Pfadabhängigkeit bezeichnet eine Situation, in der die Auswirkungen von stark durch die Vergangenheit geprägten Entscheiden dazu führen, dass die Möglichkeit bzw. Notwendigkeit neuer Geschäftsmodelle nicht erkannt, verpasst oder deren Chancen falsch eingeschätzt werden. Das Ausbrechen aus der Pfadabhängigkeit ist für den Fortbestand von Unternehmen daher von zentraler Bedeutung. Das Konzept der organisationalen Ambidextrie widmet sich dieser Problemstellung. Ambidextrie beschreibt die Fähigkeit eines Unternehmens, einerseits das Kerngeschäft stetig weiterzuentwickeln, gleichzeitig aber auch neue Wege und Denkweisen zu etablieren und so sicherzustellen, dass Veränderungen im Umsystem rechtzeitig erkannt und die sich dadurch bietenden Chancen für die Zukunft genutzt werden. Eine Fähigkeit, die gerade im Zeitalter der Digitalisierung an Wichtigkeit gewinnt.
\end{abstract}

S. J. Schneeberger $(\square)$

Spiez, Schweiz

E-Mail: simonschneeberger@bluewin.ch

A. Habegger

Berner Fachhochschule Wirtschaft, Bern, Schweiz

E-Mail: anja.habegger@bfh.ch 


\subsection{Wachsende Bedeutung organisationaler Ambidextrie}

Organisationslehre war und ist bekannt als ein Denken in Strukturen, Abläufen und Funktionsbeschreibungen. Gesucht ist die perfekte Organisation, schlank und effizient, ohne Doppelspurigkeiten, klar definiert. Gefunden wurde sie bislang nicht. Und im Schatten der Theorien zur Effizienzsteigerung schlug sachte ein Begriff Wurzeln: Ambidextrie. Ein Begriff, der alles andere als strukturierend, klar definiert und greifbar ist. Und doch, oder gerade deshalb, birgt er das Potenzial, die bisherige Management-Denkweise stark zu verändern. Denn auf einmal geht es nicht mehr nur um Prozessverbesserungen, Effizienz, Stabilität und Total Quality Management. Nun geht es zusätzlich um Flexibilität, Exploration, die Suche nach neuen Geschäftsmodellen, um das Ausprobieren, Scheitern und Gewinnen. Und das Ganze innerhalb einer einzelnen Organisation. Innerhalb einer ambidextren, „beidhändigen“ Organisation. Ohne dass die organisationale Ambidextrie als Begriff eine große Reichweite hat, lassen sich ihre Ideen in vielen Unternehmen wiederfinden. Überall dort, wo in auf Effizienz getrimmten Organisationen ,plötzlich“ Exploration erlaubt ist, das Denken und Gehen abseits der bekannten Pfade. So erhielt die Ambidextrie nicht nur eine theoretische, sondern auch eine praktische Relevanz. Schließlich fordern wirtschaftliche und gesellschaftliche Trends die etablierten Geschäftsmodelle von Unternehmen heraus. Disruptive Geschäftsmodelle lauern auf den richtigen Moment, um Umbrüche auf den Märkten zu verursachen. Vor diesem Hintergrund muss ein Unternehmen ambidexter sein. Es muss einerseits sein bestehendes Geschäftsmodell bestmöglich unterhalten, auf der anderen Seite müssen die Verantwortlichen beginnen, neue Geschäftsmodelle zu suchen, auszuprobieren und im Idealfall zu skalieren. Diesem Drahtseilakt, der Kombination dieser zwei hochrelevanten Aufgaben, widmet sich das Konzept der Ambidextrie.

In diesem Beitrag wird nach der Auseinandersetzung mit der zugrunde liegenden Theorie und der empirischen Untersuchung eines Unternehmens erstmals ein Entwurf eines Reifegradmodelles vorgestellt. Dieses soll den erfolgreichen Transfer der Theorie in die Praxis unterstützen und dazu dienen, geeignete Maßnahmen auf dem Weg zur organisationalen Ambidextrie anzustoßen.

\subsubsection{Pfadabhängigkeit einer Organisation}

Die Beschreibung der Ambidextrie beginnt mit der Annäherung an das Phänomen der Pfadabhängigkeit, die überhaupt erst Konzepte, wie jenes der organisationalen Ambidextrie, notwendig werden lässt. Kurz erklärt, bedeutet Pfadabhängigkeit, als Unternehmung bewusst oder unbewusst an Ineffizienzen festhalten zu müssen, obwohl es bessere Lösungen gäbe. Das Modell der Pfadabhängigkeit beschäftigt sich mit dem Phänomen, warum an ineffizienten Handlungsweisen festgehalten wird, weshalb alte Bindungen beibehalten werden, obwohl es keinen objektiven Grund dafür gibt (Schreyögg 2014, S. 1). 
Als Paradebeispiel dient, seit den ersten Untersuchungen zu diesem Thema, das Tastaturlayout QWERTZ. Ein ineffizientes Layout, für das es schon seit längerer Zeit Alternativen gäbe, die sich aber nicht durchsetzen können (David 1985, S. 332). Übertragen in die Betriebswirtschaft, stellt dieses Phänomen drei zentrale Denkweisen zur Disposition: Es wird infrage gestellt, dass erstens, der Marktdruck dafür sorge, dass die effizientesten Methoden überleben, zweitens, dass auf getroffene Entscheide grundsätzlich zurückgekommen werden könne und, drittens, dass diese auch revidiert würden, sobald sich bessere Lösungen bieten (Sydow et al. 2005, S. 5). Beides scheint nicht immer der Fall zu sein. Die Erklärung dafür findet sich in der Tatsache ,,bygones are rarely bygones“ (Teece et al. 2008, S. 522), dass also die Summe aller früheren Entscheide von zentraler Bedeutung für gegenwärtige Handlungsalternativen ist und diese stark einschränken kann. Dieser Umstand hat durchaus auch Vorteile: Entscheidungen können effizienter getroffen werden, die Unsicherheit und der Implementierungsaufwand von neuen Lösungen sind kleiner. So steigen die Gewinne zusätzlich, was zur Beurteilung führt, dass die Entscheide richtig gewesen sind. Dies befeuert eine Spirale von sich selbst verstärkenden Entscheidungsprozessen, da auch beim nächsten Investitionsentscheid wieder auf dieses bewährte Muster zurückgegriffen wird (Sydow et al. 2005, S. 6).

Problematik der Increasing Returns Diese Spirale von positiven Rückkoppelungsschleifen wird auch ,increasing returns“ genannt. Sie äußern sich unter anderem als Koordinationseffekte (Schreyögg 2014, S. 7), Netzwerkeffekte (Arthur 1996, S. 103), „Sunk Costs“ (Dievernich 2012, S. 62) oder Lerneffekte (Schreyögg 2014, S. 8) aber auch in Form von Erwartungserwartungen, Emotionen, Macht, Legitimität, Funktionalität und Konformität (Roedebeck und Holtmann 2008, S. 79). Grundlegend für diese „,increasing returns“ ist, dass bereits getroffene Entscheidungen an vorher getroffene ansetzen. Dies schränkt die zur Verfügung stehenden Optionen im Voraus ein (Dievernich 2012, S. 62). Es ist nun nicht mehr möglich rational über eigentlich alle zur Verfügung stehenden Alternativen zu entscheiden, weil die Auswahl von gewissen Optionen bereits durch frühere Entscheide eingeschränkt wurde. In kleinen Schritten führt diese Entscheidungsdynamik über den Zeitverlauf zu einem „Lock-In“, zu einer gravierenden Einschränkung der Handlungsalternativen. Die Pfadabhängigkeit tritt also nicht durch große, falsche Entscheidungen ein. Es sind vielmehr kleinere Entscheidungen oder Ereignisse, die retrospektiv betrachtet zur Pfadabhängigkeit geführt haben (Schreyögg 2014, S. 5). Diese äußert sich dann darin, dass die technische Ausstattung Produkte oder Dienstleistungen nur noch auf eine gewisse Art produzieren lässt oder dass die Kapazitäten aufeinander abgestimmt sind und somit auch eine weitere Skalierung schwierig wird. Gleichzeitig beeinflusst die Pfadabhängigkeit das Managementverhalten und die Unternehmenskultur, was sich wiederum auf die Produkte, Marketingmaßnahmen und schließlich die Unternehmensperformance auswirkt.

An dieser Stelle muss erwähnt werden, dass sich selbstverstärkende Effekte keineswegs nur negativ sind. Gerade in einem dynamischen Umfeld müssen Entscheide schnell und mit größtmöglicher Sicherheit gefällt werden, die oft auf der Erfahrung aus 
bisherigen Entscheiden basiert. Das Festhalten an Markt-Ineffizienzen ist zudem im Zusammenhang mit der Sicherung von Arbeitsplätzen (Dievernich 2012, S. 65), Wohlstandsdenken oder aus ökologischen Gründen vielfach nötig und gewollt.

Rolle der lernenden Organisation Im vorangehenden Absatz wurden verschiedene sich selbstverstärkende Effekte beschrieben. Einige davon haben mit „lernen“ zu tun. Eine Organisation lernt durch ihre Mitglieder. Insofern ist entscheidend, ob und wie diese lernen. In der Literatur werden drei verschiedene Typen des produktiven Lernens bei Organisationen unterschieden (Argyris und Schön 1999, S. 35):

- Typ 1: „Organisationale Untersuchungen, instrumentales Lernen, das zu einer Leistungsverbesserung bei den Aufgaben der Organisation führt“.

- Typ 2: „Untersuchungen, mit denen eine Organisation Werte und Kriterien erkundet und umstrukturiert, durch die sie definiert, was sie unter verbesserter Leistung versteht“. Dieser Lerntyp wird auch „Doppelschleifen-Lernen“ genannt.

- Typ 3: „Untersuchungen, mit denen eine Organisation ihre Fähigkeiten zum Erlernen der Typen (1) und (2) steigert.“

Das Speichern von gelerntem Wissen und dessen kontinuierliche, inkrementelle Verbesserung sind mit ein Grund, weshalb die Unternehmung in die Pfadabhängigkeit gerät. Dieses instrumentale Lernen, auch als „Einschleifen-Lernen“ bezeichnet (Argyris und Schön 1999, S. 35), ist unabdingbar, um eine Organisation immer effektiver und effizienter zu gestalten, die Prozesse zu stabilisieren und Skalen- und Netzwerkeffekte zu nutzen. Einschleifen-Lernen fördert Effizienz und Stabilität, indem die Organisation mittels positiven Rückkoppelungsschleifen lernt, was gut und was schlecht funktioniert (Tushman und O'Reilly 1996, S. 18). Die Kehrseite der Medaille: Stabilität bedeutet auch Inflexibilität (Ackermann 2001, S. 86). Einer auf Effizienz getrimmten Organisation fehlt die Möglichkeit, flexibel auf größere und unter Umständen disruptive Veränderungen zu reagieren. Es ist also destruktiv für eine Organisation, den Fokus alleine auf die Exploitation zu richten (March 1991, S. 85). Nach Simon (2007) ist dies der Moment, an dem die Organisation nicht mehr lernt und gemäß Sydow (2005) in den sogenannten „Lock-In“ der Pfadabhängigkeit gerät. Das Lernen nach Typ 1, das instrumentale Lernen, führt also in die Pfadabhängigkeit.

\subsubsection{Vermeiden und Brechen der Pfadabhängigkeit}

Auch wenn aus gesellschaftlicher Sicht Pfadabhängigkeit teilweise notwendig ist, so kann sie für den Fortbestand eines einzelnen Unternehmens gefährlich werden. Früher oder später werden diskontinuierliche Veränderungen das erfolgreiche Geschäftsmodell und die daran angepasste Organisationsarchitektur infrage stellen (Tushman und O’Reilly 1996, S. 12). Es ist daher wichtig, dass die Organisation versucht, ihre Situation 
selbst zu erkennen, einzuschätzen und entsprechende Maßnahmen dagegen zu ergreifen. Im strategischen Management helfen dabei Instrumente der Früherkennung, wie das Konzept der „Schwachen Signale“. Dabei wird davon ausgegangen, dass disruptive Veränderungen sich mit schwachen Signalen ankünden. Die Signale entwickeln sich dann zu immer stärkeren Hinweisen, bis die disruptive Veränderung eintritt (Ansoff 1975, S. 23). Grundvoraussetzung dafür, dass man diese schwachen Signale deuten kann, ist, dass sich die Organisation ihrer eigenen Situation aber auch der Umweltsituation bewusst ist. Die Suche nach schwachen Signalen hat einen explorativen Charakter. Die damit betrauten Personen müssen ein Gefühl dafür haben, wo und wie sich schwache Signale ausbreiten können. Dies kann in Zeitungen, Zeitschriften, in den Sozialen Medien, an Messen, in Fachbüchern oder auch nur durch Influencer sein (Welsch 2010, S. 45).

Die Suche nach schwachen Signalen ist gleichzusetzen mit dem Lerntyp 2. Das „Doppelschleifen-Lernen“ betrifft Untersuchungen von Organisationsmitgliedern, die sich damit beschäftigen, einerseits zu explorieren, neues Wissen zu generieren und bisher bestehende Werte und Kriterien zu hinterfragen. Dies beinhaltet das Aufbrechen bisher gültiger Normen und Werte und eröffnet die Möglichkeit, auf disruptive Veränderungen in der Umwelt zu reagieren (Argyris und Schön 1999, S. 36 f.). Das Doppelschleifen-Lernen muss konkret etabliert werden. Das bedeutet: Exploration zulassen (He und Wong 2004, S. 481), Geld und Personal aus der Exploitation entfernen, neu organisieren und auf die Suche nach neuen Geschäftsfeldern schicken. Exploration zuzulassen heißt aber auch, die daraus entstandenen Erkenntnisse in die bestehende Organisation zu integrieren. Die bestehende Organisation muss dazu mit all ihren Akteuren bereit sein, Neues auszuprobieren, Neues zu wagen und im Erfolgsfall Neues umzusetzen. Bereit sein bedeutet, dass vor allem die Denkhaltung von Total Quality Management, Business Process Engineering, Hierarchien und quantitativen Kennzahlen befreit werden muss (Benner und Tushman 2003, S. 239).

Die Fokussierung auf die Exploration als Lösung, um aus der Pfadabhängigkeit zu gelangen, festigt die Bedeutung der organisationalen Ambidextrie. Aus einer auf Effizienz getrimmten Organisation heraus müssen nun Ressourcen zur Verfügung gestellt werden, um in die Exploration investieren zu können. Damit wird ein Spannungsfeld zwischen Exploration und Exploitation aufgebaut, dessen Handling von entscheidender Bedeutung für das Überleben der Organisation ist.

Exploration und Exploitation March (1991), der einen vielzitierten Beitrag zu der Thematik geliefert hat, spricht in seiner Untersuchung davon, dass eine passende Balance zwischen Exploration und Exploitation gefunden werden muss. Da das ganze Konzept der organisationalen Ambidextrie auf diesen beiden Aktivitätsmustern aufbaut, ist es wichtig, dass diese zuerst definiert werden. March (1991) schreibt: „Exploration includes things captured by terms such as search, variation, risk taking, experimentation, play, flexibility, discovery, innovation“. Diese Umschreibung wurde jeweils weiter ergänzt, beispielsweise mit „organic structures, loosely coupled systems, path breaking, improvisation, 
autonomy and chaos, and emerging markets and technologies“ (He und Wong 2004, S. 481). Damit ein Unternehmen langfristig anpassungs- und wettbewerbsfähig bleibt, müssen durch Exploration entsprechende neue Chancen gefunden werden (Reichert 2017, S. 24). Ohne finanzielle Ressourcen kann allerdings die Exploration nicht funktionieren. Dieser Umstand ist es auch, der die wechselseitige Abhängigkeit von Exploration und Exploitation verdeutlicht. Die Exploitation stellt sicher, dass überhaupt Ressourcen zur Verfügung gestellt werden können. Wird zu sehr auf die Exploration fokussiert, besteht die Gefahr, dass diese unzureichend kapitalisiert werden kann (Reichert 2017, S. 24). Daher ist es wichtig, dass die Exploitation, die als ,such things as refinement, choice, production, efficiency, selection, implementation, execution“ (March 1991, S. 71) definiert wird, weiterhin ihre Wichtigkeit behält. Auch die Definition von Exploitation wurde durch verschiedene Autoren ergänzt: „Exploitation is associated with mechanistic structures, tightly coupled systems, path dependence, routinization, control and bureaucracy, stable markets and technologies“ (He und Wong 2004, S. 481). Mitarbeitende, die exploitativ arbeiten, haben das Ziel, immer besser und besser zu werden und vorgegebene Kennzahlen zu erreichen, während explorativ tätige Personen versuchen müssen, die Herausforderungen und Geschäftsmodelle der Zukunft zu erkennen, bevor es andere tun (Euchner und Tushman 2015, S. 16). Bezüglich Exploitation ist die Einsicht relevant, dass darin auch das „refinement“ eingeschlossen ist. Es handelt sich hierbei um die kontinuierliche Weiterentwicklung des bisherigen Geschäftsmodells. Es ist also keineswegs so, dass die Exploitation nur darin besteht, einzelne Prozesse effizienter zu machen. Exploitative Aktivitäten umfassen alle Handlungen und Tätigkeiten, die bestehende Fähigkeiten und Kompetenzen verbessern, um bereits vorhandene Business Cases besser abzuschöpfen. „Incremental technological innovations and innovations designed to meet the needs of existing customers are exploitative and build upon existing organizational knowledge“ (Benner und Tushman 2003, S. 243). Wird der Begriff Exploitation auf die kontinuierlichen Verbesserungen ausgedehnt, schärft sich dadurch die Exploration. Sie benötigt neues Wissen und neue Fähigkeiten, mit denen gänzlich neue Ressourcen und Märkte identifiziert und bearbeitet werden sollen (Levinthal und March 1993, S. 105). Wie bereits ausgeführt, müssen und können die beiden Aktivitätsmuster simultan ausgeführt werden. Die Problematik besteht jedoch darin, dass dabei eine unterschiedliche Führungs- und Unternehmenskultur und eine andere Organisationsstruktur vonnöten sind. So verlangen explorative Aktivitäten eine Führungskultur, die Kompetenzen und Verantwortung delegiert, Freiheiten lässt und einen kooperativen Führungsstil fördert. Die Unternehmenskultur wird unter anderem als offen und transparent definiert, während die Organisationsstruktur interdisziplinär, dezentralisiert und informal sein soll. Demgegenüber werden die exploitativen Tätigkeiten durch eine eher hierarchische, transaktionale Führungskultur, eine aufgabenorientierte Unternehmenskultur und eine funktionale, zentralisierte Organisationsstruktur unterstützt (Fojcik 2015, S. 22). Das gleichzeitige Vorhandensein von zwei verschiedenen Unternehmensmodellen führt zu den beschriebenen Spannungsmomenten, die durch ambidextre Fähigkeiten entspannt werden können. 
Widerspruch, Balance oder Synergie zwischen Exploration und Exploitation Es ist nicht unbestritten, dass eine Balance zwischen Exploration und Exploitation tatsächlich einen positiven Einfluss auf die Unternehmensperformance hat (Fojcik 2015, S. 79 ff.). In der Forschung zur Ambidextrie haben sich dazu folgende Meinungen herauskristallisiert:

- Exploration und Exploitation können durch eine einzelne Unternehmung nicht ausgeführt werden, da sie sich komplett widersprechen. Dies sei auf Abstimmungs- und Opportunitätskosten bei der Koordination der beiden Aktivitätsmuster zurückzuführen (Fojcik 2015, S. 58). Die simultane Umsetzung von Exploitation und Exploration führe zu einem klassischen ,stuck-in-the-middle“ (Raisch und Birkinshaw 2008, S. 392; Tempelaar 2010, S. 6).

- Exploration und Exploitation können durch eine einzelne Unternehmung nicht gleichzeitig ausgeführt werden. Die einzige Möglichkeit bestünde in der Abwechslung zwischen Exploration und Exploitation im Zeitablauf. So könne die Wirkung der beiden Aktivitätsmuster zu unterschiedlichen Zeitpunkten maximiert werden (Gupta et al. 2006, S. 697). Dass dieses Vorgehen nicht sinnvoll ist, erschließt sich bereits aus der Tatsache, dass eine Unternehmung nicht „einfach und plötzlich“ von Exploitation auf Exploration und umgekehrt umstellen kann. Zudem stellt sich die Frage, wann die Umstellung zu erfolgen hätte, wie lange die jeweiligen Perioden dauern sollen und was passieren würde, wenn in der Phase der maximalen Exploration die Ressourcen ausgingen, bevor neue Geschäftsmodelle gefunden werden.

Die beiden ersten Sichtweisen widersprechen der Prämisse von Ambidextrie, welche mit den Arbeiten von March (1991); Tushman und O'Reilly (1996) und vielen anderen begründet wurde. Die Prämisse besagt, dass Unternehmen Exploration und Exploitation gleichzeitig ausführen können und auch sollen (Raisch und Birkinshaw 2008, S. 392). Daran schließt die folgende Argumentation an:

- Exploration und Exploitation können durch eine einzelne Unternehmung ausgeführt werden. So äußerten sich unter anderen He \& Wong (2004) dahin gehend, dass die Balance für Unternehmen überlebenswichtig sei und dadurch Nachteile in der Spezialisierung und der Rendite in der Gegenwart in Kauf zu nehmen seien. Dies ist der Preis dafür, dass die Unternehmung längerfristig erfolgreich sein kann. Diese synthetische Sichtweise einer Balance wurde auch empirisch belegt (He und Wong 2004, S. 492).

- Exploration und Exploitation können durch eine einzelne Unternehmung ausgeführt werden. Anstatt dass sich die beiden Dimensionen gegenseitig ausschließen, schaffen sie Synergien, verhalten sich komplementär zueinander und beeinflussen einander somit positiv. Grund dafür ist der wechselseitige Einfluss, den beide Aktivitätsmuster aufeinander haben. So kann ein hoher Grad an Exploitation die Effektivität der Exploration steigern (Cao et al. 2009, S. 784). Die komplementäre Wirkung der beiden Aktivitätsmuster kann dann dazu führen, dass der gemeinsame Wirkungsgrad höher ist, als er in einer reinen Balance erzielt werden könnte (Fojcik 2015, S. 62). 
Die Erhaltung der Balance oder die Kombination der beiden Aktivitätsmuster ist eine große Herausforderung. Es handelt sich um einen Drahtseilakt, da die Gefahr besteht, dass die Organisation das eine oder andere Aktivitätsmuster bevorzugt und sich damit in eine nicht nachhaltige Position bringt. Sofern es möglich ist, hat die Organisation klar ihre Präferenz: Die Exploitation. Dies wurde bereits im Rahmen der Pfadabhängigkeit diskutiert. Die Organisation sucht den Weg des geringsten Widerstands und daher ,,von sich aus" eher die Exploitation als die Exploration (Levinthal und March 1993, S. 107).

\subsubsection{Definition von Ambidextrie im vorliegenden Beitrag}

Nachdem verschiedene Argumentationen beleuchtet wurden, stellt sich die Frage, welche der alternativen Definitionen für den vorliegenden Beitrag verwendet werden soll. O'Reilly und Tushman (2004) sprechen von einer ambidextren Organisation, wenn Unternehmen die explorativen Geschäftsbereiche organisatorisch von den exploitativen Geschäftsbereichen trennen. Andere führen den Begriff weniger konkret aus und betrachten die Fähigkeit einer Organisation ,to be aligned and efficient in their management of today's business demands while simultaneously adaptive to changes in the environment" (Raisch und Birkinshaw 2008, S. 375).

Als zielführend erweist sich eine Eingrenzung der organisationalen Ambidextrie auf die „Fähigkeit mittels derer Unternehmen exploitative und explorative Aktivitäten gleichzeitig umsetzen können“ (Fojcik 2015, S. 22). Wichtig dabei erscheint, dass die folgenden Grundaussagen geklärt sind:

- Die Anerkennung der wechselseitigen Abhängigkeit von explorativen und exploitativen Aktivitäten.

- Der Umgang mit dem Widerspruch aber auch der Synergie von explorativen und exploitativen Aktivitätsmustern.

- Die Fähigkeit, mit diesen Aktivitätsmustern simultan, gleichzeitig, umzugehen.

Diese Konkretisierung bietet sich an, weil sie in der Praxis feststellbar ist. Bei der Beobachtung von Tätigkeiten einer Unternehmung lassen sich diese in exploitative und explorative oder bereits ambidextre Aktivitäten einteilen und machen so die Ambidextrie eines Unternehmens explizit sichtbar, sofern beide Aktivitätsmuster auch vorhanden sind. Diese Definition stellt auch den Zusammenhang mit den Ausführungen von March (1991) her, der als primären Erfolgsfaktor für das längerfristige Überleben einer Unternehmung eine geeignete Balance zwischen Exploitation und Exploration sieht (March 1991, S. 71). Gleichzeitig lässt die Definition auch eine Weiterentwicklung im Sinne der synergetischen Sichtweise zu und lässt sich auch passend zum Untersuchungsgegenstand mit Beispielen konkretisieren und beschreiben. 


\subsection{Untersuchung zur organisationalen Ambidextrie}

Forschungslücke Die Problematik am Konzept der organisationalen Ambidextrie ist der noch ungenügende empirische Beweis, dass eine ambidextre Organisation einen positiven Einfluss auf die Unternehmensperformance hat. Hingegen zeigt die Auseinandersetzung mit der Literatur und deren Vergleich mit der Praxis, dass ein Großteil der in der Theorie entwickelten Merkmale einer ambidextren Organisation durchaus angewandt werden (können). Seltsam erweist sich allerdings, dass in der Literatur sehr viel über die Konzeption und über die Ambidextrie selbst geschrieben wird, nicht aber über deren Verankerung oder Auftreten in Unternehmen. Es fehlt ein Instrument, mit welchem anhand von konkreten Merkmalen gemessen oder zumindest beschrieben werden kann, ob überhaupt oder in welchem Grad die aktuelle Struktur und Kultur einer Unternehmung ambidexter sind.

Um dieser Forschungslücke zu begegnen, wurde in der vorliegenden Untersuchung zunächst nach sinnvollen Stellhebeln zur Implementierung der Ambidextrie im Unternehmen gesucht. Diese Gestaltungselemente orientieren sich an bewährten Methoden der Strategieumsetzung und werden in den folgenden Abschnitten beschrieben. Anhand des Untersuchungsrahmens aus Gestaltungselementen wurde die Literatur aufgearbeitet und die sich daraus ergebenden Erkenntnisse anschließend den Aussagen aus Praxis-Interviews und -Beobachtungen gegenübergestellt. Dieses Vorgehen erlaubt einen umfassenden Einblick in die Theorie der Ambidextrie und zeigt gleichzeitig deren Ausprägung in der Praxis. Die kombinierten Aussagen zu den fünf Gestaltungselementen wurden zusammengefasst und anschließend in den Entwurf eines Reifegradmodelles implementiert, das als Instrument zur Analyse von Praxisbeispielen dienen kann.

Untersuchte Unternehmung Das untersuchte Unternehmen ist in einigen Bereichen seiner Tätigkeit Markführerin in seinem Heimmarkt mit zaghaftem Branchenwachstum ( $2 \%$ p. a.), erwirtschaftet einen Umsatz von knapp 3 Mrd. EUR mit einer Eigenkapitalrendite von über $9 \%$ und zählt über 5000 Mitarbeitende.

Beim untersuchten Unternehmen handelt es sich um ein geeignetes Forschungsobjekt, da sich der bislang stabile Markt in den nächsten Jahren durch Markteintritte globaler Playern wie Google/Amazon verändern wird, das Unternehmen diese Gefahr wahrgenommen hat und nun mit großer Kraft versucht, sich auf die Zukunft vorzubereiten. Aus dieser Ausgangslage lassen sich interessante Aussagen zur Ambidextrie gewinnen.

Das Unternehmen bot praktisch uneingeschränkten Zugang zu Informationen und ließ Interviews mit Personen aller Hierarchiestufen von der Geschäftsleitung bis hin zu den Fachexperten zu. Ziel war es, den Begriff Ambidextrie und die dazu allfällig vorhandenen Maßnahmen und Strukturen innerhalb der Organisation aus verschiedenen Blickwinkeln beurteilen zu können. Ergänzend zum Literaturreview wurden insgesamt 13 leitfadengestützte Interviews geführt. Dabei wurden 11 Personen aus der Unternehmung ausgewählt und zusätzlich eine externe Expertin und ein externer Experte befragt. 
Gleichzeitig wurden während eines halben Jahres Eindrücke zur Arbeitsweise, Unternehmenskultur, Kommunikation und anderen Merkmalen der Unternehmung beobachtet.

\subsection{Systematik des Reifegradmodelles}

Für die Entwicklung des Konzeptes für ein Reifegradmodell wird auf das Top-DownVerfahren von De Bruin et al. (2005) zurückgegriffen. Der Kern des Top-Down-Verfahrens liegt in der Gestaltung des Modellbereiches. In dieser Untersuchung startet die Entwicklung bei den allgemeinen Theorien der Strategieimplementierung mit Hilfe von Gestaltungselementen und konkretisiert sich in definierbaren Implementierungsmechanismen, die neu aus der Empirie gewonnen werden. Die Interpretation der Resultate aus der Empirie führt dann zu einer letzten Verfeinerung und Unterteilung in feststellbare Abstufungen.

Gestaltungselemente der Ambidextrie Auf der Suche nach einer optimalen Kombination von Exploration und Exploitation wird immer wieder festgestellt, dass im Zeitverlauf exploitative Aktivitäten die explorativen Aktivitäten verdrängen. Grund dafür ist, dass Exploitation sicherere, besser messbare und raschere Erfolge generiert als die Exploration, deren Erfolg mehr als unsicher ist (Levinthal und March 1993, S. 106). Damit diese Verdrängung nicht stattfinden kann, muss die Unternehmensarchitektur Gestaltungselemente beinhalten, welche die Exploration fördern und nachhaltig sichern. Die Fähigkeit, gleichzeitig exploitative und explorative Aktivitäten umzusetzen, ist dabei nicht nur eine Frage der Organisationsstruktur, wie sie auf einem Organigramm dargestellt werden kann. Die Fähigkeit beginnt im Denken des einzelnen Individuums und endet in dessen Handlung für die Organisation. Die Organisationsstruktur ist hierbei nur Mittel zum Zweck. Das ambidextre Denken und Handeln wird von unzähligen Faktoren beeinflusst. Diese lassen sich, unter vielen anderen, in Gestaltungselemente wie Strategien, Anreizsysteme, Führungsstile, Prozesse, Differenzierungs- und Integrationsmechanismen, Unternehmenskultur und Werte einteilen (Fojcik 2015, S. 31). Bei dieser Fülle an Gestaltungselementen tut eine Reduktion auf sinnvolle Untersuchungseinheiten Not. Dabei kann auf das strategische Management zurückgegriffen werden. Zur Unterstützung der Strategieumsetzung wurde durch McKinsey das 7S+P-Schema entworfen (Lombriser und Abplanalp 2010, S. 368). Nach dessen Elementen wurde im Literaturreview zur Ambidextrie gezielter gesucht und gefunden wurden Aussagen zu vier der sieben Elemente, während zwei Elemente unter „Ressourcenzuteilung“ zusammengefasst werden können. Die Resultate darauf führten $\mathrm{zu}$ einem auf die Ambidextrie angepassten Untersuchungsrahmen mit Gestaltungselementen und den dazugehörigen Integrationsmechanismen. 
Übersicht und Definition der Gestaltungselemente Die Unternehmung wird anhand folgender, aus der Literatur gewonnener Gestaltungselemente und den damit verbundenen Untersuchungsfragen analysiert:

- Management: Ist sich das Management bewusst, dass für den nachhaltigen Erfolg der Unternehmung eine ambidextre Organisationsarchitektur gewählt werden muss?

- Strategie: Besteht für die Unternehmung eine klare, aussagekräftige und umgesetzte Strategie, welche die Grundlagen der organisationalen Ambidextrie berücksichtigt?

- Organisationsstruktur: Ist die Organisationsstruktur so gestaltet, dass die Grundlagen der organisationalen Ambidextrie damit abgedeckt sind?

- Ressourcenzuteilung: Sind die Ressourcen in einem Verhältnis zugeteilt, welches erlaubt, die organisationale Ambidextrie umzusetzen?

- Unternehmenskultur: Trägt die Unternehmenskultur zur optimalen Umsetzung der organisationalen Ambidextrie bei?

\subsubsection{Integrationsmechanismen}

Die Prozesse und Systeme, welche im 7S+P-Schema (Lombriser und Abplanalp 2010, S. 368) relativ weit gefasst sind, wurden in der Literatur zur Ambidextrie teilweise erwähnt. Es handelt sich insgesamt um sogenannte Integrationsmechanismen. Das sind einerseits Kommunikation, Anreizsysteme und Kontrollmechanismen sowie Prozesse und Abläufe allgemein (Fojcik 2015, S. 31), die sich anhand von festgestellten Denkweisen, konkreten Maßnahmen, beobachtbaren Artefakten und Prozessen beschreiben lassen. Diese können nachgelagert den Hauptelementen zugeordnet werden, wobei schließlich auf drei sinnvolle Integrationsmechanismen pro Gestaltungselement fokussiert wurde. Es wurden dabei diejenigen Aussagen berücksichtigt, über die ein genereller Konsens bestand oder die mit empirischen Belegen fundiert sind.

\subsubsection{Reifegradstufen}

Für jeden der Integrationsmechanismen wurden die fünf Stufen mit Kriterien und Beispielen (wo vorhanden) beschrieben. Die Einstufung und Zuordnung von Kriterien erfolgte in einem kreativen Mindmapping-Prozess an mehreren Tagen unter Berücksichtigung der oben erwähnten Aussagen aus der Literatur, den Interviews und Beobachtungen. Die Tatsache, dass in der Praxis bei der Unternehmung je nach Geschäftsbereich die ambidextre Denk- und Handlungsweise unterschiedlich ausgeprägt und feststellbar war, diente der Definition der Reifegradstufen in entscheidender Weise. Ein Großteil der Reifegradstufen ließ sich so anhand der Interviews und der Beobachtungen beschreiben. 


\subsection{Gestaltungselement Management und Führung}

Die zu beantwortende Fragestellung zu diesem Gestaltungselement lautet: Denkt und handelt das Management nach den Grundsätzen des Ambidextrie-Konzeptes?

\subsubsection{Integrationsmechanismus „Umgang mit der Pfadabhängigkeit"}

Aussagen aus der Theorie Die wichtigste Funktion des Managements ist, dass es Entscheide dort trifft, wo die Organisation ansonsten den Weg des geringsten Widerstandes sucht (Birkinshaw und Gupta 2013, S. 293) und so in eine Pfadabhängigkeit gerät. Aus der Theorie der Pfadabhängigkeit abgeleitet, kann festgestellt werden, dass die sich selbstverstärkenden Effekte einer Organisation aktiv verhindert oder aufgebrochen werden müssen. Das einheitliche Auftreten aber auch das Treiben von Maßnahmen in die vereinbarte Richtung, senden klare Signale an die Stakeholder aus, dass von Ambidextrie nicht nur gesprochen, sondern diese auch konsequent durchgesetzt wird (Lubatkin et al. 2006, S. 647). Dabei darf das Ziel nicht aus den Augen verloren werden: Eine Organisation, die sehr nahe am Kunden und am Markt denkt, da sonst Chancen vergeben werden, um neue Geschäftsfelder zu finden (Tushman und O’Reilly 1996, S. 28).

Exploration braucht Zeit und Geld und wird unter Umständen keine Profite abwerfen. Trotzdem darf das Management nicht in Versuchung geraten, explorative Einheiten aufgrund von kurzfristigen Renditezielen zu streichen oder gar nicht erst aufzubauen. Es benötigt das Verständnis, dass die Exploration eine Investition in die langfristige Zukunft des Unternehmens darstellt (Benner und Tushman 2003 S. 248). Gleichzeitig muss das Management fähig sein, eingeschlagene Wege auch wieder abzubrechen. Das bedeutet, das „Sunk-Cost-Syndrom“ zu überwinden, wenn festgestellt wird, dass ein Explorationsprojekt nicht in die richtige Richtung verläuft (Tokarski et al. 2016, S. 98). Das Management muss also jederzeit bereit sein, die eigenen Maßnahmen zu hinterfragen und gegebenenfalls grundlegend zu verändern (Tushman und O’Reilly 1996, S. 24).

Aussagen aus der Praxis Die Wichtigkeit des Topmanagements im Zusammenhang mit der Ambidextrie wird von allen Interviewten herausgestrichen. „Sie müssen zunächst wissen, dass es viele Veränderungen gibt, sie müssen das Bewusstsein haben“ (Zitat Interviewpartner). Es ist auch ihre Aufgaben, dieses Bewusstsein in die ganze Organisation zu tragen. Das Topmanagement, die Geschäftsleitung und der Verwaltungsrat müssen die Grundlagen schaffen, damit Ambidextrie entstehen kann. Die Organisation selbst funktioniert ohne Druck einfach weiter, bis es nicht mehr geht. Deshalb muss das Topmanagement genau dort dem Druck entgegenhalten können. Es müssen visionäre Gedanken in die Unternehmung kommen. Sie müssen den Wandel sowie die Innovationen voraussehen und die Gefahren kennen. 


\section{Ausprägung der Reifegradstufen}

Stufe 5: Instrumente zum Brechen der Pfadabhängigkeit und Kreation neuer Pfade werden eingesetzt.

Stufe 4: Selbstverstärkende Effekte werden festgestellt, jedoch nicht aktiv bekämpft.

Stufe 3: Feststellung, dass die Organisation träge, undynamisch oder entscheidungsschwach ist. Jedoch ohne Ursachensuche.

Stufe 2: Ansicht, dass, solange kleinere Effizienzverbesserungen geschehen, alles in Ordnung ist.

Stufe 1: Die Pfadabhängigkeit oder ähnliche Theorien kennt das Management nicht.

\subsubsection{Integrationsmechanismus „Bewusstsein für Ambidextrie“}

Aussagen aus der Theorie Hinsichtlich der strukturellen Ambidextrie spielt das Management eine entscheidende Rolle bei der Koordination zwischen Exploitation und Exploration. Die Verantwortlichen müssen zwei verschiedene Welten kombinieren und diese mit einem anderen Führungsstil steuern. Sie müssen ein tiefergehendes Verständnis entwickeln, damit sie die Allokation von Ressourcen zwischen Exploration und Exploitation expliziter, geplanter, vornehmen können (He und Wong 2004, S. 492). Sie müssen einerseits denken wie in einem Start-up, anderseits haben sie einen auf Effizienz getrimmten, großen Bereich zu führen. Das bedeutet, dass das Management fähig sein muss, die damit einhergehenden scheinbaren Paradoxien zu akzeptieren (Lewis 2000, S. 769). Es muss auf der einen Seite Entscheide bewusst zentral fällen, während es auf der anderen Seite die kreativen Spezialisten machen lassen und unter Umständen dadurch auch Kontrolle abgeben muss (Benner und Tushman 2003, S. 248).

Aussagen aus der Praxis Auch wenn der Begriff Ambidextrie den meisten Interviewpartnern unbekannt war, so besteht doch ein großes, intuitives Verständnis dafür. Die Interviewpartner sprechen von zwei gegensätzlichen Welten, von einem Spagat oder einem Spannungsfeld. Sie sprechen von kontinuierlicher Veränderung aber auch von Austesten und Entdecken. Beide Welten schließen sich nicht aus. „Ich glaube es ist extrem wichtig, dass man das Positive aus beiden Welten nimmt und man die Stärke aus dem Kerngeschäft nimmt, um auch explorativ tätig zu sein“ (Zitat Interviewpartner). Die Erkenntnis, dass es überhaupt zwei Welten gibt und dass diese trotz ihrer Widersprüchlichkeiten kombiniert werden müssen, ist bereits ein erster Schritt hin zur Ambidextrie. Ein zweiter besteht in der Tatsache, dass das Topmanagement beide Welten akzeptiert und mit ihnen arbeitet. „Ich finde das Topmanagement in einer ambidextren Organisation muss Wertschätzung für beide Welten haben“ (Zitat Interviewpartner). Die interviewten Geschäftsleitungsmitglieder sehen die Notwendigkeit, dass sie die Lücke zwischen traditionellen Werten und der Innovation zusammenführen müssen, so, dass sich die Tradition weiterentwickelt und die Innovation nicht zu weit weg vom Kerngeschäft entsteht. 
Die Leute sollen nicht denken „es sind Spinner, die etwas auf dem Mars basteln“ (Zitat Interviewpartner).

Die Interviewten teilen die Ansicht, dass das Topmanagement grundsätzlich eine visionäre Aufgabe hat und sich selbst um die künftigen Herausforderungen kümmern muss. Steht die langfristige Perspektive im Vordergrund, so kommt das Management nicht umhin, ambidextre Strukturen $\mathrm{zu}$ initiieren. Im Fall des untersuchten Unternehmens wurde dies mit der Einführung von sogenannten Raumstationen, als Bild für das Kerngeschäft, und Raumsonden, als Bild für explorative Projekte, gemacht. Dabei wird die Raumstation als weiter $\mathrm{zu}$ entwickelndes, zu renovierendes Kerngeschäft betitelt, während die Raumsonden auslaufen, um neue Geschäftsfelder zu erforschen. „Wir unterstützen dies mit Geschäftsleitungsmitgliedern, die in Steering-Commitees sind, die die Raumsonden begleiten“ (Zitat Interviewpartner).

\section{Ausprägung der Reifegradstufen}

Stufe 5: Der Kombination von Exploitation und Exploration wird hohe Wichtigkeit zugeschrieben und Maßnahmen dazu werden umgesetzt.

Stufe 4: Ambidextrie wird anerkannt, mittelfristig sollen entsprechende Maßnahmen ergriffen werden.

Stufe 3: Ambidextrie wird anerkannt. Diskussionen dazu laufen.

Stufe 2: Ambidextrie wird nicht anerkannt. Kontinuierliche Verbesserungen des Geschäftsmodelles reichen aus.

Stufe 1: Ambidextrie wird nicht anerkannt. Das Geschäftsmodell bleibt grundsätzlich, wie es ist.

\subsubsection{Integrationsmechanismus „Veränderungsbereitschaft"}

Aussagen aus der Theorie Die untersuchte Literatur vermittelt ein eindeutiges Bild: Ambidextrie muss von „oben her“ kommen. Das Topmanagement muss fähig sein, eine Revolution in der Unternehmenskultur loszutreten. Nicht nur das Managementteam selbst muss ambidexter sein, die Mitarbeitenden und die ganze Unternehmenskultur müssen dies auch sehen und verstehen (Tushman und O'Reilly 1996, S. 20). Die Rolle des Managements in ambidextren Unternehmen unterscheidet sich von der traditionellen hierarchischen Rolle. Das Management kann Ambidextrie besser in die Unternehmung implementieren und vorleben, wenn es sich stark interhierarchisch engagiert und sich auf die an der Front arbeitenden Organisationsmitglieder einlässt und deren Denk- und Handlungsweise versteht. Insofern ist Ambidextrie nicht nur ein reiner Top-Down-Ansatz, sondern es finden sich darin auch Bottom-Up-Ansätze (Raisch et al. 2009, S. 687). Das Management soll die Mitarbeitenden nicht zwingen, die neue Strategie und Denkweise umzusetzen, sondern sie immer wieder davon überzeugen, dass der Weg der Ambidextrie in eine nachhaltige Zukunft führt. Insofern ist die Rolle eher definiert als „Dirigent“, 
denn als „General“ (Tushman und O’Reilly 1996, S. 27). Damit dieses Verständnis auch bei den Mitarbeitenden entsteht, müssen soziale Interaktionen wie Zuhören und Wissensaustausch stattfinden. Offenes Feedback führt dazu, dass die Mitarbeitenden Vertrauen aufbauen, was gerade für die Exploration, insbesondere im Zusammenhang mit der Fehlerkultur, von größter Wichtigkeit ist (Lubatkin et al. 2006, S. 651).

Zum richtigen Zeitpunkt muss das Management schlussendlich entscheiden können, dass es auch auf das aus der Exploration entstandene Geschäftsfeld setzt, dort das Risiko fährt und bedeutende Investitionen für die Skalierung des Geschäftsmodelles tätigt (Tushman und O’Reilly 1996, S. 10).

Aussagen aus der Praxis Der Weg hin zu einer ambidextren Organisation benötigt eine hohe Veränderungsbereitschaft des gesamten Systems. „Wenn das Topmanagement nicht dahinter steht, kann man es vergessen“ (Zitat Interviewpartner). Dabei ist von Bedeutung, dass die Geschäftsleitung ein gemeinsames Commitment zur Strategie abgibt und die getroffenen Maßnahmen die Strategieumsetzung unterstützen. „Wenn du fünf, sechs Leute hast, von sieben oder acht Personen, die enorm am Heute und Jetzt hängen, dann wird das mit größter Wahrscheinlichkeit nicht eine wahnsinnig innovative Geschichte“" (Zitat Interviewpartner).

Ein großes Thema innerhalb des untersuchten Unternehmens war die Umgestaltung der Büroräumlichkeiten am Hauptsitz. Dort ging der CEO voran und verzichtete als erster auf sein Büro. Die meisten Geschäftsleitungsmitglieder sind diesem Vorbild gefolgt. Auch andere Änderungen müssen einerseits vorgelebt, anderseits auch erklärt werden. Die Mitarbeitenden müssen verstehen, wohin die Reise geht. „Denn wir müssen unsere Leute mitnehmen auf diese Reise" (Zitat Interviewpartner). Dieses Mitnehmen hat viel mit Überzeugen und nicht mit Befehlen zu tun. Die Offenheit und Neugierde muss auf die Mitarbeitenden übertragen und es muss aufgezeigt werden, „,dass der Mitarbeitende diese Veränderung auch machen kann“ (Zitat Interviewpartner). Es ist jedoch auch Aufgabe des Topmanagements zu schauen, dass der Organisation nicht zu viel zugemutet wird und Veränderungen, die nicht $\mathrm{zu}$ den Werten und zur Kultur passen, nicht zugelassen werden.

\section{Ausprägung der Reifegradstufen}

Stufe 5: Die Veränderung wird vorgelebt und deren Notwendigkeit immer wieder erwähnt und von der Organisation verstanden.

Stufe 4: Projektteams werden mit der Veränderung beauftragt, „es läuft etwas“. Leichte Verzettelungen der Maßnahmen.

Stufe 3: Veränderung wird propagiert, umgesetzt wenig.

Stufe 2: „Ist so, weil ist so“. Veränderung sollte passieren, tut es aber nicht (passive Haltung).

Stufe 1: Was bisher gut war, ist auch für die Zukunft gut genug. 


\subsection{Gestaltungselement Strategie}

Die zu beantwortende Fragestellung zu diesem Gestaltungselement lautet: Deckt die Unternehmensstrategie die Grundsätze der Ambidextrie ab?

\subsubsection{Integrationsmechanismus „,Strategie enthält Grundsätze der Ambidextrie"}

Aussagen aus der Theorie Traditionell wurde eine hybride Strategie, also die Kombination von Exploration und Exploitation als negativ für die Unternehmensperformance eingestuft. Grund dafür war die Einschätzung, dass „stuck in the middle“ einen mangelnden Fokus darstelle, welcher für den langfristigen Erfolg einer Unternehmung von herausragender Bedeutung wäre (Porter 1985, S. 16). Es gäbe kein Ambidextrie-Konzept, wäre die wissenschaftliche Argumentation auf dieser Linie geblieben. Burgelman (1991) trat den Gegenbeweis an. Er argumentierte, dass Unternehmen durchaus zwei verschiedene Strategien fahren könnten. Einerseits eine, die, Porter (1985) folgend, auf den Stärken der Organisation aufbaute. Andererseits sollte eine davon unabhängige Strategie etabliert werden können, die sich mehr auf die Erforschung des weiteren Umfelds bezieht. Exploitation und Exploration waren als sich ergänzende Strategien geboren (Burgelman 1991, S. 256). Weitergeführt wurde die Forschung im Rahmen von „dynamic capabilities“. Diese werden beschrieben ,as the firm's ability to integrate, build and reconfigure internal and external competences to address rapidly changing environments“ (Teece et al. 2008, S. 516). Diese Strategie erlaubt es den Unternehmen, schneller als die Mitbewerber auf diskontinuierliche Veränderungen zu reagieren, was einen Wettbewerbsvorteil darstellt.

Ausgehend vom resource-based-view, das heißt aufbauend auf den vorhandenen internen und externen Ressourcen, stellen die dynamic capabilities die Fähigkeit dar, diese vorhandenen Ressourcen immer wieder sehr schnell neu zu konfigurieren. Diese „dynamic capabilities“ sind unter dem Strich die neu gewonnenen Fähigkeiten der Unternehmung, sobald Ambidextrie erfolgreich implementiert wurde. Schließlich erfordert die Integration von Exploration und Exploitation genau jene Fähigkeiten, die notwendig sind, um diskontinuierliche Veränderung von außen in der Organisation so zu verarbeiten, dass daraus ein Wettbewerbsvorteil entsteht. Ambidextrie ist in diesem Zusammenhang also die Begründung, weshalb Wettbewerbsvorteile im Rahmen des „Dynamic capabilities“-Ansatzes erlangt werden können. Mit dieser Grundlage erlebte die Ambidextrie ihren Einzug auch in das strategische Management und wurde so um eine Perspektive reicher (Fojcik 2015, S. 42). Trotz allem lässt sich jedoch nicht feststellen, ob aus Sicht des strategischen Managements die Ambidextrie effektiv bereits ,angekommen“" ist. 
Aussagen aus der Praxis Es muss ein theoretischer Input gewesen sein, der in der Unternehmung dazu führte, aus der Exploitation heraus die Exploration konsequent einzuführen und damit den Weg hin zur organisationalen Ambidextrie frei zu machen. Die Begriffe Raumstation und Raumsonden wurden als Allegorien eingeführt und werden seither in der Kommunikation immer wieder und konsequent verwendet. Dabei wurden die Raumsonden zu Beginn fast belächelt. Etwas später konnte man dem Ausprobieren bereits etwas abgewinnen, ohne solchen Versuchen jedoch eine große Erfolgswahrscheinlichkeit zuzugestehen. „Und jetzt habe ich den Eindruck, man hat das Raumsonden-Gedankengut in das tägliche Geschäft übernommen“" (Zitat Interviewpartner). Diese klare Strategie mit der sinnbildlichen Darstellung durch zwei Raumfahrzeuge wurde daraufhin immer wieder kommuniziert, erklärt und vor allem an Beispielen erläutert. Eine konkrete Maßnahme bestand darin, dass in den IT-Projektorganisationen neue Projektmanagementmethoden eingeführt wurden. Diese basieren auf der Planung und Umsetzung kleiner Projektteile, die innerhalb von drei Monaten durch kleine interdisziplinäre Teams erarbeitet werden. Alle drei Monate werden die Projekte wieder priorisiert. Diese neue Methode hat dazu beigetragen, dass sich von der IT her die agile Denkweise immer mehr in das Kerngeschäft verbreitet hat.

Interessant ist die Entwicklung hinsichtlich der Distanz (inhaltlich, strukturell und kulturell) der Raumsonden zur Raumstation. Diese wurde über die Jahre und als Reaktion auf gewonnene Erkenntnisse immer kleiner. Zunächst wurden Raumsonden unabhängig vom Kerngeschäft losgeschickt, später wurden sie einzelnen Kategorien zugeordnet und heute in definierten Ökosystemen eingesetzt. Es wäre zu erwarten gewesen, dass sich, je näher die Exploration am Kerngeschäft platziert wird, die Kreativität, Freiheit und somit das wirklich Explorierende abhandenkommen würde. Dem war jedoch nicht so. Vielmehr hat sich die Denkweise des Kerngeschäfts in Richtung Exploration oder zumindest in einen offeneren Mindset gewandelt. Mit der Betonung der stetigen Weiterentwicklung der Raumstation und gezielten Investitionen wird auch die exploitative Seite stark berücksichtigt. Das Double-Loop-Lernen erfolgt gleichzeitig in speziellen Workshops (Design Thinking, Agilität).

Damit die ganze Thematik sich jedoch nicht verselbstständigt, wurde ein neuer Geschäftsleitungsbereich gegründet: „Operations“. Dieser Bereich hat die Aufgabe, die Kräfte zu bündeln und zu koordinieren. Die Gründung von „Operations“ ist eine direkte Folge der Erkenntnis, dass die Raumsonden nicht zu weit weg vom Kerngeschäft Ideen entwickeln sollten, sondern in Ökosystemen anzusiedeln sind, die auch einen Bezug zum Kerngeschäft haben.

\section{Ausprägung der Reifegradstufen}

Stufe 5: Die Kombination von Exploration/ Exploitation, Single- und Double-Loop-Lernen sind in der Strategie explizit erwähnt.

Stufe 4: Strategie enthält implizit Komponenten der Exploration und Exploitation und des organisationalen Lernens. 
Stufe 3: Strategie enthält Aussagen über Innovation und Effizienzsteigerung.

Stufe 2: Strategie enthält einseitige Aussagen entweder zu Effizienzverbesserung oder zu großen Forschungsanstrengungen.

Stufe 1: Strategie beinhaltet keine Entwicklungsperspektive.

\subsubsection{Integrationsmechanismus „Formulierung der ambidextren Strategie"}

Aussagen aus der Theorie Die Pfadabhängigkeit, führt im Zeitverlauf zu einer sich selbst verstärkenden Unternehmensstrategie. Deshalb muss regelmäßig eine sorgfältige und konsequente Strategieüberprüfung, von der Geschäftsleitung oder vom Verwaltungsrat initiiert, durchgeführt werden. Die Unternehmensstrategie muss so formuliert sein, dass sie die Kombination von Exploration und Exploitation bereits beinhaltet, indem sie einerseits den nachhaltigen Erfolg, aber auch den Durchbruch in neue Geschäftsmodelle umfasst (Andriopoulos und Lewis 2009, S. 708).

Die Implementierung von Ambidextrie erfolgt zeitgleich mit dem Brechen der Pfadabhängigkeit. In der Literatur zu Ambidextrie sind nicht viele Methoden erwähnt, wie das Konzept konkret, Schritt für Schritt eingeführt werden soll. Die grundsätzliche Vorgehensweise wird sich an den bekannten Theorien und Konzepten zur Strategieumsetzung orientieren. Instrumente dazu können aus der betriebswirtschaftlichen Standardliteratur entnommen werden. Darin wird die Anwendung von Instrumenten wie Zielsetzungssysteme, Balanced Scorecard, Aktionsprogramme, Ressourcenzuteilung und Budgetierung sowie Maßnahmen in der Kommunikation (Lombriser und Abplanalp 2010, S. 352 ff.) vorgeschlagen. Teil der Strategieumsetzung ist auch die Organisationsgestaltung. Details dazu können ebenfalls aus der grundlegenden Literatur, wie Mintzberg (1979), entnommen werden. Die Umsetzung der Organisationsstruktur erfolgt anhand von Gestaltungsprinzipien, welche die aus der Strategie entwickelten wichtigsten Ansprüche an die Organisation festhalten (Thom und Wenger 2010, S. 102).

Aussagen aus der Praxis Die gesamte Strategieumsetzung hat mit Überzeugen zu tun. Dabei spielt die Unternehmenskommunikation eine entscheidende Rolle. Diverse Schulungs- und Informationsanlässe, Video-Logs des CEO, Intranet, viel persönlicher Austausch und immer wieder praktische Beispiele werden kommuniziert. Die Kommunikation muss offen und transparent erfolgen. Die Konsequenzen, ob positiv, ob negativ, müssen klar formuliert werden. Die Mitteilung der Botschaft über die Ambidextrie hat kontinuierlich zu erfolgen. „Man darf auf keinen Fall das Gefühl haben, man könne es einfach kommunizieren und dann sei es klar ..., nein, man sagt es hunderttausend Mal, man sagt es immer anders, man zeigt es mit anderen Beispielen“ (Zitat Interviewpartner). 


\section{Ausprägung der Reifegradstufen}

Stufe 5: Die Strategieformulierung ist klar, unmissverständlich und einfach. Beantwortet „wie“ und „warum“. Sie wird regelmäßig kommuniziert. Erfolge, Fehlschläge und Beispiele werden regelmäßig geteilt.

Stufe 4: Die Strategieformulierung ist klar, unmissverständlich, beantwortet jedoch das „warum“ nur ansatzweise. Sie wurde einmal ausgiebig kommuniziert, danach unregelmäßig.

Stufe 3: Die Strategieformulierung beantwortet, wie man vorwärtsgehen möchte, ist aber wenig konkret und erklärt nicht weshalb.

Stufe 2: Die Strategieformulierung richtet sich an die Führungskräfte und ist deshalb schwer verständlich und schwer nachvollziehbar.

Stufe 1: Es besteht keine oder nur eine vage Strategieformulierung. Niemand weiß eigentlich, wohin es gehen soll.

\subsubsection{Integrationsmechanismus „Berücksichtigung der Mitarbeitenden"}

Aussagen aus der Theorie Die Berücksichtigung der Mitarbeitenden beschränkt sich, mit wenigen Ausnahmen, in der Literatur auf die Führungskräfte, die entweder mitziehen oder aber aus dem Unternehmen ausscheiden (O'Reilly und Tushman 2004, S. 79). Ambidextrie betrifft jedoch den Großteil der Mitarbeitenden, da die Organisation durch ihre Mitglieder lernt (Argyris und Schön 1999, S. 26 ff.) und das Ausbrechen aus der Pfadabhängigkeit nur unter Einbezug der ganzen Organisation funktioniert und einen sozialen Prozess darstellt (Schreyögg et al. 2003, S. 285), der die Mitarbeitenden explizit berücksichtigt.

Aussagen aus der Praxis Es ist Teil der Strategie, dass keine „Unternehmung 2.0“ auf der grünen Wiese aufgebaut wird. Den Veränderungen soll auf der Basis der bestehenden Organisation begegnet werden. Ziel ist es, die Mitarbeitenden auf diese Veränderungen vorzubereiten und „fit“ zu halten. Die Raumstation soll kontinuierlich weiterentwickelt werden, indem Prozesse verbessert und damit Leute für andere Aufgaben freigespielt werden können. Es wurden keine Angestellten entlassen, obwohl stetig Prozesse automatisiert wurden. Man hat mit diesen Stellen die Exploration aufgebaut. Daneben wurden auch viele Maßnahmen ins Leben gerufen, die sich auf die Weiterentwicklung von Mitarbeitenden konzentrieren. Beispielsweise wurde ein Büro im Silicon Valley gegründet, wo sich ständig Mitarbeitende der Unternehmung befinden und neue Dinge, vor allem in Bezug auf die Digitalisierung, erforschen. Eine wichtige Rolle spielen auch die zugekauften Tochtergesellschaften, wo ebenfalls Stellen für Mitarbeitende des Unternehmens geschaffen wurden, die dort das Geschäftsmodell untersuchen und neue Prozesse erlernen sollen. 
Trotz aller Kommunikationsbemühungen und Maßnahmen wurde das Konzept nicht überall mit gleicher Euphorie aufgenommen und die Einführung war teilweise mit Ängsten verbunden. Den Blick weg vom aktuellen Tagesgeschäft, hin zu den Herausforderungen der Zukunft zu richten, ist nicht für alle Mitarbeitenden gleich einfach. Ob es gelingt, alle Mitarbeitenden ,mitzunehmen“ ist fraglich. Weiterbildungen und Schulungen werden gefördert, allerdings meist auf vollkommen freiwilliger Basis. Die Notwendigkeit, dass auch langjährige Mitarbeitende wieder Schulungen besuchen sollten, wurde noch nicht mit Nachdruck kommuniziert. An die Eigenverantwortung zu appellieren, greift hingegen zu kurz und beinhaltet die Gefahr, dass einige Mitarbeitende nicht mithalten können. So wird von einem Teil der Belegschaft über die Begriffe „Agilität““ und „Raumsonden“ mit einem gewissen Zynismus gesprochen. Unabhängig des Aktivitätsmusters, in welchem die Mitarbeitenden arbeiten, sollen alle auf eine neue Ebene der Unternehmenskultur mitgenommen werden. Der Abbau von Hierarchie, die Verkürzung der Kommunikationswege und der unkomplizierte Umgang miteinander hören sich zwar in Bereichen der Exploration theoriekonform an, widerspricht aber den in der Literatur formulierten Merkmalen eines exploitativen Bereiches, der per Definition starre, klare und damit hierarchische Strukturen enthält.

\section{Ausprägung der Reifegradstufen}

Stufe 5: Die Strategie ist darauf ausgerichtet, wenn möglich alle Mitarbeitenden in die ambidextre Welt mitzunehmen und entsprechend zu fördern.

Stufe 4: Die Strategie ist darauf ausgerichtet, nicht zu große Schritte zu unternehmen. Auf Maßnahmen zur Förderung der Mitarbeitenden wird aber weitgehend verzichtet.

Stufe 3: Die Strategie beinhaltet einen großen Schritt in die Zukunft, den nicht alle Mitarbeitenden mitmachen können.

Stufe 2: Die Strategie basiert auf der Verbesserung des Shareholder-Values.

Stufe 1: Die Strategie beinhaltet die Wegrationalisierung von Arbeitsplätzen durch Effizienzsteigerungen.

\subsection{Gestaltungselement Organisationsstruktur}

Die zu beantwortende Fragestellung zu diesem Gestaltungselement lautet: Ist die Organisationsstruktur so gestaltet, dass die Grundlagen der organisationalen Ambidextrie damit abgedeckt sind?

\subsubsection{Integrationsmechanismus „Einordnung der Struktur“}

Aussagen aus der Theorie Die Bedeutung der Organisationsstruktur wird in der Literatur zur Ambidextrie stark hervorgehoben (u. a. Levinthal und March 1993, S. 107; 
Adler et al. 1999; Benner und Tushman 2003, S. 247; Birkinshaw und Gibson 2004, S. 50). Wird von der verwendeten Definition von Ambidextrie ausgegangen, interessiert deren organisationale Umsetzungsmöglichkeit innerhalb des Unternehmens. Die Lösungskonzepte lassen sich wie folgt unterscheiden:

- Strukturelle Ambidextrie: Die theoretische Basis für die strukturelle Ambidextrie legte Duncan (1976), der stipulierte, dass Managementanforderungen mit unterschiedlichem Zeithorizont voneinander zu trennen seien (Duncan 1976, S. 180). Diese Argumentation wurde dahingehend weiterentwickelt, dass ein Unternehmen Exploration und Exploitation nur simultan umsetzen kann, wenn diese Aktivitäten strukturell innerhalb der Organisation voneinander getrennt werden. Dies hat einen empirisch bewiesenen positiven Einfluss auf die Unternehmensperformance (Jansen 2005, S. 130).

- Sequenzielle Ambidextrie: Im Rahmen der sequenziellen Ambidextrie wird die Tatsache bejaht, dass ein Unternehmen Exploitation und Exploration zu verschiedenen Zeitpunkten ausführen kann, jedoch nicht gleichzeitig. Die Abwechslung im Zeitablauf soll dazu führen, dass beide Aktivitätsmuster mit dem vollen Wirkungsgrad umgesetzt werden können. Bislang konnte der empirische Beweis jedoch noch nicht erbracht werden, dass die Performance dadurch gesteigert wird (He und Wong 2004).

- Kontextuelle Ambidextrie: Als Alternative zur strukturellen Ambidextrie besteht das Konzept der kontextuellen Ambidextrie. Der Fokus liegt hierbei auf den einzelnen Mitarbeitenden der Unternehmung und weniger auf den Organisationsstrukturen. Die Voraussetzungen für die kontextuelle Ambidextrie werden mit dem Aufbau eines „High-Performance Organizational Context" geschaffen. Birkinshaw und Gibson (2004) streichen heraus, dass ein hoher ,,social support“ und ein starkes ,performance management“ zu einem ,high performance context“ führen. Dies sei die Grundbedingung für die kontextuelle ambidextre Organisation. Das ,performance management" soll dabei alle Mitarbeitenden zu Höchstleistungen stimulieren und sie verantwortlich für ihren Arbeitsbereich machen. Die zweite Dimension ,social support" bezeichnet eine Kombination von Unterstützung und Vertrauen, die den Mitarbeitenden die notwendige Sicherheit und Freiheit bietet, die sie benötigen, um die hohe Leistung zu erbringen.

- Hybride strukturelle/integrative Ansätze: Anstatt dass sich die Individuen selbst zwischen Exploration und Exploitation entscheiden, werden die Funktionen auf Abteilungsebene entsprechend verteilt. Mit Jobrotation können die Mitarbeitenden beide Aktivitätsmuster ausüben (Birkinshaw et al. 2016, S. 37).

- Rein separative Ansätze: Das Unternehmen richtet sich ausschließlich auf Exploration oder Exploitation. Eine rein exploitativ ausgerichtete Unternehmung wird sich die notwendige Exploration also von Dritten einkaufen. Nach Fojcik (2015) wäre dies die Reinform der separativen Lösungsansätze. In diesem Zusammenhang wird argumentiert, dass es unvorteilhaft ist, innerhalb der Organisation beide 
Aktivitätsmuster auszuführen. Insofern sei eine Fokussierung auf eines der beiden Aktivitätsmuster vorzuziehen. Weiter kann neues Wissen über den Einbezug von Allianzen oder den Zukauf von explorativ tätigen Unternehmen entstehen. Für die Unternehmensperformance sei dies positiv (Stettner und Lavie 2014, S. 1923).

Aussagen aus der Praxis Grundsätzlich muss sich eine Organisation überlegen, ob sie Exploration selbst betreiben will oder nicht. Die Frage, die sich stellt ist: „Warum soll überhaupt das Unternehmen das machen. Warum macht es nicht ein Startup?“ (Zitat Interviewpartner). Dies mag womöglich für die Pharmabranche oder auch die Digitalbranche zutreffen, wo die Exploration teilweise eingekauft wird. Es besteht durchaus auch in der Branche des untersuchten Unternehmens die Möglichkeit, einzelne Produkte und Geschäftsmodelle einzukaufen. So übernahm das Unternehmen einen führenden Nischenmarktanbieter, allerdings nicht wegen dessen Portefeuille und Produkten, sondern weil der Anbieter große Erfahrung mit digitalen Vertriebs- und Geschäftsmodellen hat. Das Gleiche gilt für eine $50 \%$-Beteiligung an einem Anbieter in einem rein digitalen Markt, der als Zuliefermarkt des Unternehmens verstanden werden kann. Dieser Kauf eröffnete interessante Chancen in den bearbeiteten Ökosystemen. „Man hat ja gesagt, es macht Sinn, dass wir uns in gewissen Ökosystemen spezialisieren und wir dort eine starke Stellung einnehmen und den Kunden eben auf seiner ganzen Journey begleiten können“ (Zitat Interviewpartner).

\section{Ausprägung der Reifegradstufen}

Stufe 5: Die strukturelle Ambidextrie wurde konsequent umgesetzt. Integrative Ambidextrie tritt in sinnvollen Bereichen auf.

Stufe 4: Organisationsstruktur mit Elementen der strukturellen Ambidextrie.

Stufe 3: Konsequent durchgesetzte kontextuelle Ambidextrie.

Stufe 2: Es wird eine Form der sequenziellen Ambidextrie angewandt.

Stufe 1: Stark hierarchische Struktur ohne Elemente, die Exploration ermöglichen; oder keine Strukturen, die Exploitation ermöglichen.

\subsubsection{Integrationsmechanismus „Integration der Exploration“}

Aussagen aus der Theorie Die Implementierung von stärker formalen Strukturen wird gegenüber informellen Strukturen bevorzugt (Jansen et al. 2009, S. 808). Dabei sollen die explorativen Einheiten klein und dezentral, mit offenen Kulturen und kreativen Prozessen ausgestaltet sein, während die exploitativen Einheiten größer, zentralisierter, mit stark strukturierten Prozessen und hierarchisch geprägten Unternehmenskultur gestaltet sind (Benner und Tushman 2003, S. 247). Die Verbindung der beiden Aktivitätsmuster erfolgt durch das Topmanagement, welches die Rolle des Koordinators zwischen den beiden Welten spielt. Diese zwei Welten müssen so koordiniert werden, dass 
sie sich nicht gegenseitig behindern (Jansen 2005, S. 139). Nachteile dieser strukturellen Ambidextrie finden sich in eben dieser Trennung vom Kerngeschäft. Die Innovationsabteilungen kreieren Lösungen, die Gefahr laufen, vom Kerngeschäft nicht akzeptiert zu werden. Um dieser Problematik beizukommen, wurden Unterformen der Ambidextrie angewandt. So sollen einzelne Mitarbeitende aus der Linie genommen werden, um über einen begrenzten Zeitraum in interdisziplinären Teams zu arbeiten. Andere etablieren kleine Teams direkt als Stabstelle bei den Linien (Birkinshaw und Gibson 2004, S. 49).

Aussagen aus der Praxis $\mathrm{Zu}$ Beginn waren die Raumsonden als explorative Vehikel gedanklich und thematisch ziemlich weit weg vom Kerngeschäft. Aus diesen Fehlern wurde gelernt, diverse Raumsonden wurden gestoppt. Mit der Definition von Ökosystemen konnten die Suchaktivitäten gezielter gestaltet werden. Die Raumsonden blieben eigenständige Start-ups, welche in einem für die Unternehmung interessanten Ökosystem unterwegs sind. Die Unternehmung beteiligt sich mit Geld und Personal an diesen Start-ups. Organisatorisch sind die unternehmensseitigen Funktionen im Zusammenhang mit den Raumsonden dem Geschäftsleitungsbereich Operations angegliedert. Strukturell sind die Raumsonden also von der Raumstation unabhängig und laufen autonom. Dies wird grundsätzlich positiv bewertet: „Im Separierten ist die Chance größer, eine neue Kultur zu entwickeln, andere Arten von Mitarbeitenden zu finden, also alles anders zu machen“ (Zitat Interviewpartner). Der Bereich „Operations“"wird als Bindeglied und Koordinationsstelle aller explorativer Tätigkeiten, welche die Unternehmung ausführt, verstanden. Allerdings auch als Koordinationsstelle aller kontinuierlichen Weiterentwicklungsbemühungen im Rahmen der Exploitation. Es ist eine neu gegründete Organisationseinheit, ,in der eigentlich die wesentlichen Weiterentwicklungsthemen, wie Daten, Ökosysteme, die Innovationen, das Projektportfolio und das M\&A vereint werden“" (Zitat Interviewpartner). Dies mit dem Ziel, die Kräfte zu bündeln und in eine strategisch relevante Richtung zu führen. Die Interviewpartner stützen die Sichtweise, dass dies für ein Unternehmen einer gewissen Größe die vorzuziehende Lösung sei. „Ich finde wir haben einen guten Weg gewählt mit der neuen Organisationseinheit, die man etwas loskoppelt von den Kernkompetenzen“" (Zitat Interviewpartner). Wird diese Struktur mit der Theorie verglichen, so kann eine strukturelle Ambidextrie festgestellt werden.

\section{Ausprägung der Reifegradstufen}

Stufe 5: Die Exploration findet einerseits völlig losgelöst, anderseits in definierten Ökosystemen und in angegliederten Tochterfirmen statt.

Stufe 4: Die Exploration findet in nahegelegenen, definierten Ökosystemen statt.

Stufe 3: Die Exploration erfolgt völlig losgelöst vom Kerngeschäft.

Stufe 2: Die Exploration beschränkt sich auf den Kauf von oder bei Dritten.

Stufe 1: Es gibt keine Exploration. 


\subsubsection{Integrationsmechanismus „Unterstützung des Wissenstransfers"}

Aussagen aus der Theorie Ambidextrie basiert ebenfalls auf dem Wissenstransfer von Mitarbeitenden untereinander. Ein reger Wissenstransfer hilft, einerseits die bestehenden Prozesse mithilfe anderer Mitarbeitender immer besser zu verstehen, andererseits können durch die Kombination mehrerer Sichtweisen neue Probleme aufgedeckt und neue Lösungen gefunden werden (Caniëls et al. 2017, S. 1099). Erst der Transfer von Wissen ermöglicht die Anschlussfähigkeit der explorativen Fähigkeiten an das exploitative Kerngeschäft. Dieser Umstand muss in der Organisationsstruktur berücksichtigt werden. Trotz aller Freiheiten müssen sich die explorativen Aktivitäten in einem Umfeld abspielen, welches Erkenntnisse zulässt, die im Kerngeschäft verstanden und verarbeitet werden können. Auf der anderen Seite bedeutet dies aber auch, dass das Kerngeschäft selbst auf einen höheren Wissensstand gebracht werden muss, damit Neuerungen anschlussfähig werden. Diese absorptiven Fähigkeiten müssen mit den verfügbaren Ressourcen ebenfalls aufgebaut werden (Jansen 2005, S. 139). Dafür sieht die Literatur zwei Methoden vor: Erstens die bewusste Bildung eines organisationalen Slacks, also eines Puffers an personellen Ressourcen. Diese unterstützen die Organisation dabei, dass sich auch in exploitativen Bereichen Mitarbeitende um die Weiterentwicklung des Kerngeschäfts kümmern können. Das dient der Anschlussfähigkeit an die Exploration und ermöglicht später auch, neue Wege zu denken (Adler et al. 1999, S. 62). Eine weitere Möglichkeit für die Verbesserung des Wissenstransfers und damit der Anschlussfähigkeit ist die Einführung von Metaroutinen. Ein Unternehmensprozess muss einerseits stabil und standardisiert laufen, anderseits muss er flexibel genug sein, damit er rasch angepasst werden kann. Der Prozess selbst kann diese Funktionen jedoch nicht erfüllen. Infolgedessen können Metaroutinen eingeführt werden. Dabei geht es um etablierte Overhead-Prozesse, durch die definiert ist, wie die Hauptprozesse bei Bedarf angepasst werden können. Prozesseigner dieser Metaroutinen ist ein Pilotteam. Dieses kümmert sich darum, die neuen Prozesse in Zusammenarbeit mit den betroffenen Mitarbeitenden zu designen und mittels Schulungen umzusetzen. Das Team besteht aus den Teamleadern verschiedener Produktionsgruppen (Adler et al. 1999, S. 50). Es handelt sich somit um ein interdisziplinäres Team, welches außerhalb der angestammten Linien Prozesse entwickelt, welche den Übergang von neuen Erkenntnissen aus der Exploration in das Kerngeschäft orchestriert. Allerdings dürften nur größere Unternehmen die Kapazität haben, Slackressourcen oder Metaroutinen aufzubauen (Cao et al. 2009, S. 785). Zu viel Slack kann zudem dazu führen, dass die exploitativen Bereiche plötzlich selbst explorativ tätig werden und dadurch unternehmensweit die explorativen Fähigkeiten unkoordiniert werden (Reichert 2017, S. 31).

Aussagen aus der Praxis Der neu gegründete Geschäftsleitungsbereich „Operation“ ist eine Plattform, auf welcher die Ideen und Anforderungen der verschiedenen Unternehmensbereiche zusammengefasst und angeglichen werden können. Damit soll ein 
Multiplikatoreffekt erzielt werden. Dieser Nutzen steht außer Frage. „Das gibt Ruhe im Tagesgeschäft" (Zitat Interviewpartner) und es gebe wohl im Moment keinen anderen Weg, als diese Parallelstruktur. Die Nachteile liegen, wie dies auch bereits aus der Theorie hervorgeht, in der problematischen Integration in das Kerngeschäft. Die strukturelle Ambidextrie hemmt die Umsetzung von Projekten, da es zwei Welten sind, die sich unter Umständen schlecht verstehen. Insofern scheint diese Organisationsform, wenn auch in diesem Umfeld sinnvoll, nicht auszureichen. Denn gerade die Vertreter aus der IT und der Business Analyse betrachten die Organisationsstruktur als sekundär. Wichtiger seien interdisziplinäre Teams, in welchen Leute beider Aktivitätsmuster anzutreffen sind. Exploratorinnen und Exploitatoren. Im Vordergrund dürfe daher nicht die Organisationsstruktur stehen, sondern die Lösung des Integrationsproblems. Es bestehen zwar Aussagen, welche die Innovation (hier gleichgestellt mit Exploration) auch auf Teambasis implementieren würden. „Es gibt einfach Leute mit verschiedenen Rollen. Zunehmend ist es interdisziplinär. Es ist nicht jeder Typ der Explorator. Er muss dies auch nicht sein. Es ist aber wichtig, dass die zusammenarbeiten können. Es braucht beide“ (Zitat Interviewpartner). Dass jeder Einzelne, wie dies in der Theorie zur kontextuellen Ambidextrie beschrieben ist, ambidexter sein muss, wird nicht bestätigt. Der Wissenstransfer wird in manchen Bereichen noch klassisch mit Sitzungen, Schulungen und informalem Wissenstransfer sichergestellt. Angebote für Jobrotation und Jobenrichment bestehen, werden aber selten genutzt. Die interdisziplinäre bzw. interhierarchische wissensbasierte Zusammenarbeit hat sich noch nicht überall, wo es sinnvoll wäre, durchgesetzt.

\section{Ausprägung der Reifegradstufen}

Stufe 5: In sinnvollen Bereichen werden interdisziplinäre und interhierarchische Teams und Projektgruppen gebildet. In exploitativen Bereichen werden Slackressourcen bewusst aufgebaut.

Stufe 4: Jobrotation, Jobenrichment und andere interdisziplinäre oder weiterbildende Maßnahmen werden regelmäßig genützt.

Stufe 3: Jobrotation, Jobenrichment und andere interdisziplinäre oder weiterbildende Maßnahmen wären möglich.

Stufe 2: Ein Wechsel der Perspektiven ist nur mit einem internen Stellenwechsel möglich und wird nicht gerne gesehen.

Stufe 1: Es gibt keine Flexibilität. Die Strukturen sind starr oder es herrscht vollkommene Flexibilität, wie z. B. Hollocracy.

\subsection{Gestaltungselement Ressourcenzuteilung}

Die zu beantwortende Fragestellung zu diesem Gestaltungselement lautet: Sind die Ressourcen in einem Verhältnis zugeteilt, welches erlaubt, die organisationale Ambidextrie umzusetzen? 


\subsubsection{Integrationsmechanismus „Zuteilung der Ressourcen“}

Aussagen aus der Theorie Die Ressourcenzuteilung ist eine der zentralen Fragen im Zusammenhang mit Ambidextrie und wird bereits in den ersten Grundlagen zur Thematik beschrieben (Levinthal und March 1993; March 1991). Dabei geht es um die Problematik, dass aus exploitativen Strukturen Ressourcen herausgebrochen und an explorative Strukturen neu vergeben werden müssen. Dabei stellt sich die Frage nach dem Umfang oder dem Verhältnis der Zuteilung sowie der langfristigen Sicherung der Kombination. In der Literatur lassen sich keine Hinweise finden, wie groß die Menge oder die prozentuale Verteilung der Ressourcen zwischen Exploration und Exploitation sein muss. Die genaue Balance zwischen Exploration und Exploitation ist schwer festzustellen (Levinthal und March 1993, S. 105). Sie ist sehr stark abhängig von der Marktdynamik oder auch der Dynamik des technologischen Wandels (He und Wong 2004, S. 493). Die Ressourcenzuteilung geschieht top-down. Das Managementteam entscheidet über das Verhältnis der zugeteilten Ressourcen und beauftragt die nachgelagerten Stufen mit der Umsetzung. Die Mitarbeitenden selbst entscheiden dann noch, mit wie viel Effort und Disziplin sie die jeweilige Aufgabe erfüllen. Das bedeutet, dass jede betroffene Stufe einen Einfluss auf das Verhältnis zwischen Exploration und Exploitation hat. Während in der Führung über Personaleinsatz und Geld entschieden wird, entscheiden Mitarbeitende via Fähigkeiten, Motivation und Wissen, wie effektiv der Einsatz im jeweiligen Aktivitätsmuster aussieht (Andriopoulos und Lewis 2009, S. 708).

Aussagen aus der Praxis Die Verteilung der Ressourcen ist innerhalb des Unternehmens immer mit einem gewissen Zerren und Ringen, mit Macht und Notwendigkeit verbunden. Im Zusammenhang mit Ambidextrie kommt eine weitere Komponente dazu: Die Exploration kann in der kurzen Frist schlecht gemessen werden. Sie verfügt selten über einen klaren Business Case. Raumsonden können verglühen, der Ertrag ist ungewiss. Im Verteilkampf um die Ressourcen hat die Exploration immer das Nachsehen, wenn sie nicht geschützt wird. In der untersuchten Unternehmung sind sich die Interviewten insofern einig, dass genügend Mittel für die Exploration und auch die Weiterentwicklung im Rahmen der Exploitation vorhanden sind. „Erstens glaube ich, dass wir gut aufgestellt sind. Wir haben gute Ressourcen für die Innovationsaktivität, wenn man mit anderen Unternehmen vergleicht. Vor allem mit Leuten, die das nur Vollzeit machen“ (Zitat Interviewpartner). Zum goldenen Verhältnis zwischen den beiden Aktivitätsmustern konnten keine konkreten Angaben gemacht werden. „Jetzt zu sagen, $75 \%$ auf Effizienz und $25 \%$ auf Veränderungsfähigkeit zu setzen, das wäre Humbug“. Die meisten Interviewten sind sich einig, dass die Verteilung von der Situation abhängt und nicht generell beantwortet werden kann. Mitentscheidend ist die Situation, in der die Unternehmung steckt. In einer Krisensituation müssen über eine kürzere Zeit viel mehr Mittel in die Exploration gesteckt werden als bei erfolgreichem Geschäftsverlauf. 


\section{Ausprägung der Reifegradstufen}

Stufe 5: Die Ressourcenzuteilung an die explorativen Bereiche erfolgt dynamisch, je nach Erfolgsaussichten und Bedarf. Die exploitativen Bereiche erhalten ein zusätzliches Explorationsbudget.

Stufe 4: Die Investitionen in die Exploration sind in absoluten Zahlen und in einem Mehrjahresbudget festgelegt. Die Exploitation wird normal budgetiert.

Stufe 3: Die Exploration wird in den Globalbudgets miteingerechnet und es erfolgt ein Controlling über deren Einsatz.

Stufe 2: Die Exploration wird in den Globalbudgets miteingerechnet. Es erfolgt kein Controlling über diesen Betrag.

Stufe 1: Alle Ressourcen werden entweder in die Exploitation oder in die Exploration investiert.

\subsubsection{Integrationsmechanismus „Verteilkämpfe“}

Aussagen aus der Theorie In der Literatur wurden verschiedene Unternehmen untersucht. Dort wurde festgestellt, dass jeweils eine bittere Auseinandersetzung im Management geführt wurde, ob in neue Technologien investiert werden solle, welche unter Umständen das bestehende Geschäft kannibalisieren würden (Tushman und O'Reilly 1996, S. 9). Diese Verteilkämpfe bremsen die ambidextre Organisation aus. Auf der Ebene der Führungskräfte kann der Kampf um die Ressourcen dazu führen, dass die verfügbaren Mittel mit hoher Priorität in die eigenen Aufgaben investiert werden, während dem Austausch mit anderen, insbesondere explorativen Bereichen, praktisch keine Beachtung geschenkt wird. Der Wissensaustausch ist jedoch eine wichtige Komponente im Konzept der Ambidextrie und wird mit diesem Verhalten abgewürgt (De Clercq et al. 2013, S. 21). Dabei gilt es als erwiesen, dass die funktionsübergreifende Zusammenarbeit die Unternehmensperformance steigert. Insofern müssen Voraussetzungen geschaffen werden, damit Wissens- und Erfahrungsaustausch aber auch der Austausch von Werten funktionieren (Xueming Luo et al. 2006, S. 77). Die Verteilkämpfe nehmen $\mathrm{ab}$, wenn sich die Führungskräfte in der grundsätzlichen Richtung einig sind. Ist dies nicht der Fall, so bleibt vielfach nichts anderes mehr übrig, als die unkonstruktiven Verhinderer aus der Führungsfunktion zu entlassen (O'Reilly und Tushman 2007, S. 35).

Aussagen aus der Praxis Die Verteilkämpfe, welche in der Literatur beschrieben werden, fanden auch im untersuchten Unternehmen statt. Allerdings wurde dies von den jeweiligen Vertretern der Aktivitätsmuster unterschiedlich wahrgenommen. Die Seite der Exploration hat keine Verteilkämpfe festgestellt: „Direkt habe ich es nie gehört“ (Zitat Interviewpartner). Die Entscheidungsträger betrachten die Sache als normal: „Grundsätzlich ist es gut. Das zeigt, dass sich die Firma weiterentwickelt“ (Zitat Interviewpartner). 
Die bereits ambidextren Abteilungen sind sich uneinig: „Ich habe nie etwas gehört" (Zitat Interviewpartner), während andere sagen: „Es besteht ein harter Verteilkampf zwischen operativem Geschäft und dem Projektgeschäft" (Zitat Interviewpartner). Auf der exploitativen Seite wurden Projekte gestoppt, Business Cases abgelehnt, Stellen nicht bewilligt. Letztlich dürfte die Unternehmung davon profitiert haben, dass ganz grundsätzlich genügend Mittel für alle da waren, weil das Unternehmen erfolgreich unterwegs war und nicht in einer Krisensituation agieren musste. Dies führt zu einem differenzierten Bild.

\section{Ausprägung der Reifegradstufen}

Stufe 5: Es bestehen keine Verteilkämpfe zwischen Exploitation und Exploration. Es ist allen Beteiligten klar, dass nicht mit gleichen Ellen gemessen werden kann und beides notwendig ist.

Stufe 4: Es bestehen Verteilkämpfe. Sie sind Bestandteil einer diskussionsoffenen Kultur.

Stufe 3: Es bestehen Verteilkämpfe und Futterneid ist zu spüren, wenn Themen Ressourcen erhalten, die kurz- und mittelfristig nicht rentabel sein könnten.

Stufe 2: Es bestehen Verteilkämpfe, die Exploitation gewinnt in der Regel aufgrund überlegener Business Cases.

Stufe 1: Es gibt keine Verteilkämpfe, da es nur das Eine gibt.

\subsubsection{Integrationsmechanismus „Personal“}

Aussagen aus der Theorie Der Integrationsmechanismus „Personal“ besteht aus Teilen des Konzepts der kontextuellen Ambidextrie. Die Rolle von Mitarbeitenden wird im Zusammenhang mit der gleichzeitigen Ausführung von Exploration und Exploitation aufgrund ihrer Fähigkeit, Ideen zu generieren und zu teilen, hervorgehoben (Tempelaar 2010, S. 94). Von ihnen wird erwartet, dass sie selbstständig die Initiative ergreifen, die Kooperation mit anderen suchen und selbst entscheiden, wann sie im Rahmen ihres Tagesgeschäfts tätig sind und wann sie explorativ arbeiten. Sie denken dabei mit Vorliebe über den Rand ihres Tätigkeitsbereiches hinaus und fühlen sich wohl dabei, jeweils zwei verschiedene Hüte zu tragen. Innerhalb der Organisation nehmen diese Mitarbeitenden die Aufgabe wahr, verschiedene Bereiche und Wissensquellen zu verbinden (Birkinshaw und Gibson 2004, S. 51). Das bedeutet aber auch, dass sie auf der einen Seite wissen, was in anderen Bereichen des Unternehmens von Wichtigkeit ist und können so bei der kontinuierlichen Weiterentwicklung in ihrem eigenen Bereich tätig werden (De Clercq et al. 2013, S. 20). Es handelt sich hierbei um das exploitative Aktivitätsmuster. Auf der anderen Seite müssen sie fähig sein, radikal neues Wissen zu generieren. Dieses finden sie außerhalb der Organisation. Damit sie aber überhaupt verstehen, welches neue 
Wissen für sie relevant ist, müssen sie selbst ihr Wissensniveau erhöhen. Dies geschieht in der Regel mit einem gut strukturierten internen Wissensaustausch und entsprechenden Wissensdatenbanken (Lane und Lubatkin 1998, S. 464). Weitere mögliche Maßnahmen sind Jobenrichment- oder Jobrotation-Angebote (Hill und Birkinshaw 2012, S. 1922).

Aussagen aus der Praxis Als wichtig wird erachtet, dass in Zusammenhang mit der Ambidextrie nicht nur über Geld gesprochen wird. Denn wichtiger sind die Personen, die auf Exploration oder Exploitation angesetzt werden. Kompetente Arbeitnehmende, welche die eine oder andere Rolle ausfüllen können, finden sich nicht leicht. Insofern ist ihr richtiger Einsatz von Bedeutung. ,Man müsste wahrscheinlich schon die größten Talente auf jene Veränderungsinitiativen ansetzen, wo man sich am meisten daraus erhofft" (Zitat Interviewpartner). Die Mitarbeitenden wechseln intern allerdings nicht so leicht aus der Exploitation in die Exploration. Der Anteil an Jobrotation ist gering. Der Aufbau der Exploration hat bei der Unternehmung erst in den letzten Jahren angezogen. Geräuschlos ist dies nicht vonstattengegangen. Gerade im Kerngeschäft wunderte man sich über eine Sparpolitik, Kämpfe um jedes Stellenprozent, während Millionen von Franken in teure Experimente und teure Experten investiert wurden - und dies mit einem überschaubaren Nutzen. „Rein von der Historie her ist die neue Welt erst in den letzten Jahren entstanden. So hat man dort im Verhältnis viele Leute eingestellt“ (Zitat Interviewpartner). Für die Exploration werden zudem vielfach Leute (Hochschulabsolventen) eingestellt, die keinen Branchen-Background haben, was nur bis zu einem gewissen Grad als sinnvoll erachtet wird.

\section{Ausprägung der Reifegradstufen}

Stufe 5: Es besteht ein reger Austausch von Talenten zwischen allen Bereichen. Dies fördert den Wissenstransfer.

Stufe 4: Die Weiterentwicklung von Talenten in explorativen Bereichen wird breit unterstützt. Ist die Projektarbeit beendet, können sie in ihre Stammlinie zurückkehren. Entsprechende Ausgleichsbudgets für die Linie sind vorhanden.

Stufe 3: Es besteht ein vehementer Widerstand, wenn Talente für z. B. Projektarbeiten abgegeben werden müssen. Das Tagesgeschäft hat klaren Vorrang.

Stufe 2: Die größten Talente werden in der Exploitation eingesetzt.

Stufe 1: Es gibt kein Seilziehen um Talente, da es nur das Eine gibt.

\subsection{Gestaltungselement Unternehmenskultur}

Die zu beantwortende Fragestellung zu diesem Gestaltungselement lautet: Trägt die Unternehmenskultur zur optimalen Umsetzung der organisationalen Ambidextrie bei? 


\subsubsection{Integrationsmechanismus „Einstellung zu Unternehmen und Arbeit"}

Aussagen aus der Theorie Die Unternehmenskultur ist nicht nur für Mitarbeitende entscheidend. Sie unterstützt oder verhindert auch die Umsetzungsfähigkeit des Managements. Der Wechsel zu einer ambidextren Unternehmung muss in den Normen und Werten abgebildet sein. Starre Verhaltensregeln, Prozesse und enge Überwachung müssen durch Werte ersetzt werden, die von allen Mitarbeitenden geteilt werden können und die im Zusammenhang mit der Weiterentwicklung des Unternehmens stehen. Da diese Werte geteilt werden, etabliert sich eine Art soziale Kontrolle und die Mitarbeitenden engagieren sich vermehrt für die Weiterentwicklung der ambidextren Unternehmung, während sie gleichzeitig ihre Kollegen anhalten, es ihnen gleichzutun (Tushman und O’Reilly 1996, S. 22).

Im Zusammenhang mit Ambidextrie ist es wichtig, dass die Unternehmenskultur kreative Entscheidungen des Managements möglich macht. Dazu muss eine Kultur geschaffen werden, die es erlaubt, Fehler zu machen. Ansonsten wird der „Kampf“ des Managements gegen die Pfadabhängigkeit verhindert (Birkinshaw und Gupta 2013, S. 293). Eine Fehlerkultur führt dazu, dass Mitarbeitende aus ihren Fehlern lernen können und diese nutzen, um die Unternehmung kontinuierlich weiter zu entwickeln. Eine Fehlerkultur bietet aber auch Sicherheit für jene, die Unsicheres wagen (Caniëls et al. 2017, S. 1101). Dies führt zu einer gewissen Autonomie der Mitarbeitenden, die sie bestenfalls auch dazu verwenden, neue Wege zu erforschen, also explorativ tätig zu sein. Wer mit langfristiger Perspektive denkt und handelt, kann auf diese Art vermehrt Vertrauen bei den Mitarbeitenden und weiteren Stakeholdern schaffen (Adler et al. 1999, S. 62). Führungskräfte, die Mitarbeitende dazu ermutigen, autonom zu sein, Risiken einzugehen und auch Fehler zu machen, genießen ein erhöhtes Vertrauen, weil im Falle eines Fehlers Unterstützung anstatt einer Sanktion geboten wird. Mehr Vertrauen führt zu einer höheren explorativen Fähigkeit der Mitarbeitenden und somit der Organisation (Caniëls et al. 2017, S. 1103).

Aussagen aus der Praxis „Die Unternehmung ist erfolgreich und das macht fett und schläfrig" (Zitat Interviewpartner). Nicht auf den einzelnen Mitarbeitenden bezogen, sondern auf die gesamte Organisation mit ihren Prozessen, ihrer informalen Struktur und ihrem Selbstverständnis, dass das Geschäftsmodell auch künftig funktionieren wird. Dies sind einige der Schattenseiten des unternehmerischen Erfolges. Die Unternehmung war gut im Konzeptionieren, im lange und viel Nachdenken, Dokumentieren und saubere Papiere entwickeln. „Dies war jedoch eher hinderlich für die Umsetzungsgeschwindigkeit“ (Zitat Interviewpartner), obwohl Veränderung und Anpassung ständige Bestandteile der Unternehmenskultur waren, wie auch das langfristige, nachhaltige Denken. Die Interviewten führten dies zurück auf die Tatsache, dass die Unternehmung aufgrund der Eigentumsverhältnisse keinen Shareholder hat, der die Unternehmung zwingt, in Quartalsabschlüssen zu denken. 
Die Kultur innerhalb der Unternehmung hat sich dann seit einem Führungswechsel markant geändert. ,Wir müssen auf der einen Seite weit genug vorausschauen und auf der anderen Seite sind wir auch unseren Mitarbeitenden gegenüber verpflichtet, dass wir sie mitnehmen. Das war ein bewusster Entscheid. Wir wollen die Unternehmung, wie sie ist, weiterentwickeln. Wir haben das fast zwei Jahrhunderte fertiggebracht, also werden wir das auch hier fertigbringen. Weil unsere Mitarbeitenden absolut fähig sind, sich zu adaptieren“ (Zitat Interviewpartner). Es wurde eine offene, lockere Kommunikationskultur etabliert, die von den Mitarbeitenden sehr geschätzt wird. Sie waren bereit, sich auch auf ungewisse Dinge einzulassen. Markant gesteigert wurde, vor allem in der IT und der Business-Analyse, die Arbeit in interdisziplinären Teams, was als sehr großer Vorteil angesehen wird. Weitere Veränderungen kann man mit Design-Thinking-Workshops anstoßen, die bislang allerdings nur ausgewählte Führungskräfte besuchen konnten. Weiter wurden Kurse zur Agilisierung angeboten, die für ein breiteres Publikum geöffnet waren. In der IT wurden danach einzelne Teams, die das Potenzial dazu hatten, vorangetrieben und auf eine agile Arbeitsweise umgestellt. Andere Teams haben dies dann realisiert und zogen schon bald nach. Es wird auch festgehalten, dass den Mitarbeitenden eine große Veränderungsbereitschaft zugetraut werden kann, ohne dass diese zuerst explizit gefördert werden müssten.

Eine ambidextre Unternehmenskultur beinhaltet gemäß den Interviewpartnern Neugier, Offenheit, das Grundverständnis, dass sich alles verändert, aber auch die Denkhaltung, dass nicht alles prognostizierbar ist. Man muss bereit sein, in mehr Initiativen zu investieren, als schließlich erfolgreich sein werden. Dazu muss eine Trialand Error-Mentalität zur Gewohnheit werden, manche Dinge muss man auch wieder beerdigen. Es ist dabei durchaus möglich, dass das Unternehmen in zwei verschiedenen Unternehmenskulturen unterwegs ist. Werden diese voneinander getrennt, wie in der strukturellen Ambidextrie, so können parallel verschiedene Unternehmenskulturen bestehen. Dies wäre grundsätzlich nichts Neues, da es verschiedene Kulturen, im Sinne von Subkulturen, schon immer gegeben hat. Als wichtiger eingeschätzt wird die Tatsache, dass eine ambidextre Unternehmenskultur nicht mehr von Hierarchien lebt. Die Zusammenarbeit muss interhierarchisch und interdisziplinär funktionieren. Dies bedingt aber in einer Unternehmung mit stark gewachsenen Strukturen und Hierarchien einen großen Veränderungswillen und die Bereitschaft, sich bewegen zu wollen.

\section{Ausprägung der Reifegradstufen}

Stufe 5: Der Einsatz für die Unternehmung ist auch außerhalb des Tagesgeschäfts sehr hoch. Ziel ist es, täglich einen Unterschied zu machen. Offenheit und Neugierde zeigen sich.

Stufe 4: Trotz anspruchsvollem Tagesgeschäft, wird versucht, im Rahmen der Möglichkeiten sich selbst und die Unternehmung weiterzubringen. „KeinProblem"-Mentalität. 
Stufe 3: Arbeit wird gerne, aber in den definierten Strukturen gemacht. Bloß keine Überraschungen. Das Tagesgeschäft ist anspruchsvoll genug.

Stufe 2: Der Chef hat gesagt, man soll Ideen einbringen, aber eigentlich ist es egal. Entschieden wird oben.

Stufe 1: Die Arbeit ist nur Mittel zum Zweck. Es gilt, einfach den Job zu machen und den Lohn abzuholen.

\subsubsection{Integrationsmechanismus „Anreize für ambidextres Denken und Handeln"}

Aussagen aus der Theorie Wie lassen sich die Führungskräfte oder auch die Mitarbeitenden dazu motivieren, ambidexter zu denken und zu handeln? Unter Umständen reicht es nicht, Vision und Strategie zu kommunizieren und die Notwendigkeit aufzuzeigen. Um die (Denk-)Routine aufzubrechen, werden zusätzliche Anreize benötigt. Anreizsysteme sind eine klassische Antwort auf die nachhaltige Sicherung der Exploration. Grundsätzlich ist der Anreiz für die Exploration so gesetzt, dass diejenigen, welche den Effort erbringen und explorativ tätig sind, die erreichten Erfolge auch behalten oder davon profitieren sollen. Dies soll für das Risiko entschädigen, welches für die Exploration eingegangen werden musste. In der Gesamtwirtschaft werden die Resultate aus der Exploration mit dem Patentrecht geschützt. Zudem wird durch Gesetze und die Verwendung von „fremdem“ Geld der Explorator, oder genauer der oder die Entrepreneurin, vor eigenen Verlusten geschützt. Entsprechende Systeme könnten auch innerhalb der Unternehmung angewendet werden (Levinthal und March 1993, S. 107). Die Ausgestaltung von Anreizsystemen muss grundsätzlich auf die Ambidextrie ausgerichtet sein. Wenn Führungskräfte und Mitarbeitende Ziele vereinbaren, die sich lediglich auf Messgrößen im Tagesgeschäft abstützen, werden sie ihr Verhalten nicht in Richtung Exploration ändern. Es müssen also andere Anreize geschaffen werden, damit die Mitarbeitenden sich an der Strategieumsetzung beteiligen. Diese können so aussehen, dass in Zielvereinbarungen nicht nur das Was und Wieviel, sondern auch das Wie, also das Verhalten, gemessen wird (Tushman und O'Reilly 1996, S. 21). Ein Ziel könnte lauten „Einreichen von zwei Innovationen pro Monat“ und würde entsprechend gewichtet und honoriert (Adler et al. 1999, S. 61). Andere Anreizsysteme koppeln die Gewährung eines Bonus an die Gesamtunternehmungsleistung und die Jobrotation von Führungskräften (Jansen 2005, S. 32) oder deren Erfolg im explorativen und exploitativen Bereich (Tushman et al. 2006, S. 20). Es bestehen verschiedene, auf die Arbeit bezogene Anreize, welche die Etablierung einer ambidextren Organisation unterstützen. Eine Art davon ist die ,job rotation“, die bewirkt, dass die Mitarbeitenden ganz allgemein für Prozessneuerungen offener werden, abgesehen davon, dass dadurch die Routine der Arbeit unterbrochen wird (Adler et al. 1999, S. 60).

Aussagen aus der Praxis Die Interviewpartner sind sich einig, dass keine finanziellen Anreize nötig waren, um die festgestellte Veränderung zu fördern. Vielmehr wurden 
weiche Faktoren angesprochen. Da ist einerseits die Feststellung, dass Ideen und Vorhaben von Vorgesetzten oder anderen Mitarbeitenden nicht mehr als störend betrachtet werden, sondern als Versuch, die Unternehmung weiterzubringen. Andererseits konnte mit dem Aufzeigen von Beispielen, seien diese negativ oder positiv, die Denkhaltung stetig verändert werden. Vermutlich ist die Mehrzahl der Mitarbeitenden daran interessiert, ihre Arbeitsstelle interessant zu gestalten. Mit dem Aufzeigen von Erfolgen, auch kleinen, können sie motiviert werden, selber etwas auszuprobieren und anzustoßen. Die Unternehmung kann so immer wieder kleine Erfolge von Teams kommunizieren, was als Anreiz für all jene dient, die auch Freude an Erfolgen haben. Wenn die Organisation, die Führungskräfte und die Arbeitskollegen zulassen, dass kleinere Ideen verwirklicht werden können, dann ist dies der größte individuelle Anreiz. Die Organisation muss also Zeit und Raum geben können, in der Mitarbeitende und Teams Ideen weiterverfolgen können. Weitere Maßnahmen sind klassischer Natur: Jobrotation sowie Jobenrichment. Generell müssen die Anreize mit der Unternehmenskultur übereinstimmen. So darf nicht kommuniziert werden, dass man eine offene Fehlerkultur haben möchte, Fehler dann aber sanktioniert werden.

\section{Ausprägung der Reifegradstufen}

Stufe 5: Es braucht keine finanziellen Anreize, da die Entlohnung bereits sehr gut ist. Wichtiger ist das Anbieten von neuen Perspektiven, interhierarchische Zusammenarbeit, Wertschätzung und Freizeit.

Stufe 4: Weiterbildung, interne Schulungen und flexible Arbeitsgestaltung, flache Hierarchien und ein Chef als Coach unterstützen die ambidextre Handlungsweise. Ideen und Vorschlägen, offenen Diskussionen wird mit Wertschätzung begegnet.

Stufe 3: Anreize bestehen durch flexible Arbeitszeitgestaltung, Weiterbildungsmöglichkeiten und das interne Ideenmanagement mit interessanten Sachpreisen.

Stufe 2: Finanzielle Anreize helfen, insbesondere wenn die Entlohnung unterdurchschnittlich ist.

Stufe 1: Anreize sind nicht vonnöten, da die Mitarbeitenden nicht in die Weiterentwicklung der Unternehmung involviert werden sollen.

\subsubsection{Integrationsmechanismus „Sichtbare Artefakte"}

Aussagen aus der Theorie Eine Eigenschaft der ambidextren Unternehmenskultur liegt darin, dass diese gleichzeitig eng und lose sein sollte. Dies bedeutet, dass einerseits eine gewisse Offenheit, Autonomie und Risikokultur herrschen soll, um kreative Prozesse zu fördern. Auf der anderen Seite muss die Unternehmenskultur auch so ausgerichtet sein, dass in entscheidenden Momenten, vor allem unter Zeitdruck, die Kommunikationswege und Hierarchien eingehalten werden (Tushman und O'Reilly 1996, S. 26). Wie dies in der Unternehmung allerdings sichtbar sein könnte, darüber schweigt sich die Theorie aus. 
Aussagen aus der Praxis Angesprochen darauf, wie sich diese Eigenschaften innerhalb des Unternehmens feststellen lassen, wiesen die Interviewpartner auf verschiedene Artefakte hin. Als wichtigstes Beispiel erwähnten sie die neuen Arbeitszonen, die freie Platzwahl und das mobile Arbeiten. Während bei einige Mitarbeitenden Ängste in diesem Zusammenhang bestanden, freuten sich andere sehr auf die neuen Arbeitswelten. Diese Umstellung führte auch dazu, dass der CEO und die Mehrzahl der Geschäftsleitungsmitglieder keine eigenen Büros mehr haben und sich irgendwo in den neu gestalteten Arbeitsbereichen niederlassen. Dies wurde als Beispiel für ein „Vorbild sein“ betrachtet. Andererseits wurde begonnen, ohne dass dies, wie in anderen Betrieben üblich, von oben verordnet wurde, einander zu duzen. Es war überraschend zu sehen, wie schnell die Organisation bereit war, solche Neuerungen aufzunehmen. Beim Konstruieren von Artefakten, welche die Unternehmensstrategie unterstützen sollen, muss jedoch stark darauf geachtet werden, dass dies nicht zu Zynismus führt. Ein neuer Anstrich reicht nicht, um einen Wandel zu beschwören. Erst wenn das Managementteam und die Verantwortlichen sich auch im Sinne dieser Artefakte verhalten, lassen sich Verhaltensänderungen anstoßen.

\section{Ausprägung der Reifegradstufen}

Stufe 5: Helle, offene Landschaften mit Rückzugsmöglichkeiten für konzentriertes Arbeiten; freie Platzwahl; viele Gelegenheiten, um ad-hoc in kleinen Gruppen zu arbeiten; Früchtekörbe, Austausch mit externen Personen, Du-Kultur, Möglichkeit, mobil zu arbeiten, Kunst, offene Pausenzonen (auch zum Arbeiten geeignet).

Stufe 4: Eine Kombination zwischen 3 und 5.

Stufe 3: Einzelbüros und zu lärmige, enge, standardisierte Großraumbüros, mehrere kleine Pausenzonen; wenige Gelegenheiten, um ad hoc Gruppen zu bilden; dunkle, immer besetzte oder reservierte Sitzungszimmer; dunkle Gänge, uniforme Kleidung, stationäre PC-Infrastruktur, Katzen- und Fußballbilder, persönliche Objekte.

Stufe 2: Eine Kombination zwischen 1 und 3.

Stufe 1: Gesichtslose, austauschbare, pseudo-moderne Bürolandschaft ohne eigenen Arbeitsplatz, dafür mit eigenem Rollkorpus; grau-schwarze Einheitsmöbel; mehrere abgetrennte Bereiche mit Badge-Zugangskontrolle; geschlossene Türen; kein Grüßen auf dem Gang; Pausenecken ohne Sitzgelegenheiten.

\section{$6.9 \quad$ Fazit}

Die Merkmale, welche ambidextre Organisationen auszeichnen, wurden mit der Analyse von rund 100 wissenschaftlichen Beiträgen, Büchern und Zeitschriftenartikeln ausgewählt und beschrieben. Wichtig ist zunächst die Tatsache, dass Entscheidungsträger über die 
Problematik der Pfadabhängigkeit, deren Ursache und Lösung (organisationales Lernen) Bescheid wissen. Ist sich das Managementteam der Problematik bewusst, so spielt es eine herausragende Rolle bei der Formulierung und Umsetzung einer Strategie mit dem Ziel, die Organisation ambidexter zu gestalten. Dabei muss das Managementteam eine angepasste Organisationsstruktur zusammen mit einer entsprechenden Kultur schaffen. Dies bedingt, dass das Managementteam die Maßnahmen auch vorlebt und der Organisation als Beispiel vorangeht. Die Strategie muss klar und deutlich formuliert sein und es muss den verschiedenen Stakeholdern, insbesondere der Belegschaft, verständlich gemacht werden, dass ein Verharren in einer bislang möglicherweise komfortablen Lage nicht ausreicht, um langfristig am Markt bestehen zu können. Nachdem eine Strategie formuliert wurde, müssen die Organisationsstrukturen entsprechend angepasst werden. Dabei empfiehlt sich, mindestens zu Beginn, die Trennung zwischen Exploration und Exploitation. Die Exploration muss im herrschenden Verteilkampf in gesonderten Strukturen geschützt werden. Mit der Etablierung einer Unternehmenskultur, die versteht, weshalb es Exploration braucht und die solche Prinzipien auch lebt, kann Ambidextrie im Unternehmen erfolgreich sein.

In der Praxis werden die meisten Aussagen aus der Literatur bestätigt. Bestätigt wird zum einen die Notwendigkeit von Exploration und Exploitation. Sichtbar sind das starke Involvement des Managementteams, die Strategieformulierung, die angepassten Organisationsstrukturen und die Bemühungen, eine ambidextre, offene, neugierige Unternehmenskultur zu schaffen. Sichtbar in der Praxis sind noch viele weitere Merkmale, die in der Theorie noch nicht diskutiert wurden. Beispielsweise die Veränderung oder Schaffung von Artefakten, welche die Etablierung einer ambidextren Denkhaltung unterstützen können. Oder das effektive Ausmaß der explorativen Tätigkeiten.

Die Kombination aus Merkmalen aus der Theorie und der Praxis ergaben den Entwurf eines einfachen Ambidextrie-Reifegradmodells. Dieses deckt die fünf Gestaltungselemente der Organisation ab und definiert für je drei Integrationsmechanismen die fünf Reifestufen. Das Reifegradmodell wurde daraufhin anhand der untersuchten Unternehmung ausgetestet und ein erstes Resultat erzielt. Damit wurde auch eine Forschungslücke geschlossen, die daraus bestand, dass die Ambidextrie bislang nie ausreichend konkret beschrieben wurde, sodass daraus Handlungsanweisungen abgeleitet werden konnten.

Kritik am Konzept der Ambidextrie Die Tatsache, dass Ambidextrie, je nach Definition, als Konzept für praktisch alle unternehmerischen Herausforderungen hinzugezogen werden kann, ist vermutlich eine Stärke aber gleichzeitig auch die größte Schwäche (Birkinshaw und Gupta 2013, S. 291). So stößt man auf der Suche nach geeigneten Anschauungsobjekten für Ambidextrie auf Beispiele wie die ABB vor ihrem Crash in den Nullerjahren. In der frühen Ambidextrie-Literatur werden die dezentrale Struktur, die Profitcenter und die Fehlerkultur nicht nur hervorgehoben, sondern ausdrücklich als gute Beispiele erwähnt (Tushman und O’ Reilly 1996, S. 25). Was mit ABB daraufhin 
passierte, ist allgemein bekannt. Auch Nokia wird im Jahre 2004 als Vorzeigebeispiel dargestellt (Birkinshaw und Gibson 2004, S. 47). Die Finnen waren dabei, viele neue technologische Errungenschaften auf dem Gebiet der mobilen Technologie auszuprobieren, während sie ihre Handysparte kontinuierlich weiterentwickelten. Zu dieser Zeit entwickelte Apple jedoch bereits die erste iPhone-Version mit Touchscreen, Internet und Apps (Murtazin 2010). Die weitere Geschichte von Nokia ist bekannt. Wenn also Nokia als ambidextres Unternehmen galt, wie konnten sie in der langfristigen Perspektive dermaßen versagen? Diese Beispiele zeigen auf, dass die Eigenschaften und die Implementierung von Ambidextrie innerhalb eines Unternehmens noch nicht genügend erforscht sind und der Beitrag zum Unternehmenserfolg noch $\mathrm{zu}$ wenig fundiert beschrieben werden kann.

Ist Ambidextrie noch state-of-the-art? Werden Aussagen berücksichtigt, die für die exploitativen Bereiche eine hierarchische, stark strukturierte Organisation vorsehen, so stellt sich die Frage, weshalb aktuell über ganze Unternehmen hinweg flache Hierarchien und interdisziplinäre Teams eingeführt werden. Gleichzeitig werden Jobenrichment und Jobrotation über das ganze Unternehmen hinweg angeboten. Gemäß der Literatur, insbesondere zur strukturellen Ambidextrie, müssten diese Maßnahmen auf die explorativen Bereiche beschränkt werden. Ist dies allenfalls ein Schritt zu weit in das explorative Aktivitätsmuster und laufen die Unternehmen Gefahr, gänzlich ineffizient zu werden? Oder ist auf der anderen Seite in diesem Bereich das Konzept der Ambidextrie überholt? Vermutlich nicht. Vielmehr entwickeln sich die Eigenschaften des exploitativen Aktivitätsmuster laufend weiter. Möglicherweise ist sogar die ambidextre Denkweise ein Treiber dieser Entwicklung. Damit die exploitativen Bereiche überhaupt anschlussfähig bleiben, bewegen sich diese auf die explorativen Bereiche $\mathrm{zu}-$ und nicht etwa umgekehrt. Die Spannungen zwischen diesen beiden Bereichen können so massiv verkleinert werden. Innovation wird in den exploitativen Bereichen dadurch besser angenommen und dabei verändert sich die ganze Denkweise.

Weiterer Forschungsbedarf Die empirischen Belege für den Nachweis des positiven Einflusses der Ambidextrie auf den nachhaltigen Erfolg eines Unternehmens sind weiterhin dünn. Vermutlich hat dies auch damit zu tun, dass bislang ,die“ ambidextre Unternehmung noch nicht bestimmt werden konnte und daher eine ambidextre Strategie noch nicht auf deren langfristigen Erfolg hätte getestet werden können. Der in diesem Artikel vorgestellte Entwurf für ein Ambidextrie-Reifegradmodell könnte ein erster Schritt sein, um ambidextre Unternehmen zu erkennen, zu kennzeichnen und auf ihren langfristigen Erfolg zu untersuchen. Dies bedingt allerdings, dass das Reifegradmodell weiterentwickelt würde, sowohl in seiner Breite (weitere Gestaltungselemente) als auch in seiner Tiefe (weitere Integrationsmechanismen je Gestaltungselement definieren). Dabei besteht allerdings die Gefahr, dass das Kind mit dem Bade ausgeschüttet wird. Schließlich bestehen schon Anzeichen dafür, dass die Ambidextrie-Theorie überinterpretiert wird (Birkinshaw und Gupta 2013, S. 290) und weitere, empirisch schlecht fun- 
dierte Aussagen zum Untergang des Konzeptes führen könnten. Auf der anderen Seite darf im wissenschaftlichen Kontext nicht vergessen gehen, dass es sich bei der Ambidextrie um ein Phänomen handelt, das aus der Praxis heraus entstanden ist. Weshalb also nicht zuerst dort beginnen und das Feld wieder von hinten aufrollen? Das Vorpreschen mit einem Reifegradmodell würde auf jeden Fall das agile Vorgehen in der Weiterentwicklung des Konzeptes anheizen. Vielleicht bedürfte auch die Organisationsforschung einer gewissen Ambidextrie. Also ausprobieren, testen, um dann die Erkenntnisse in den strukturierten Forschungsprozess zu implementieren.

\section{Literatur}

Ackermann, R. (2001). Pfadabhängigkeit, Institutionen und Regelreform. Tübingen: Mohr Siebeck.

Adler, P. S., Goldoftas, B., \& Levine, D. I. (1999). Flexibility versus efficiency? A case study of model changeovers in the Toyota production system. International Abstracts in Operations Research, 10, 43-68. https://doi.org/10.1057/iaor.2002.289.

Andriopoulos, C., \& Lewis, M. W. (2009). Exploitation-exploration tensions and organizational ambidexterity: Managing paradoxes of innovation. Organization Science, 20, 696-717.

Ansoff, H. I. (1975). Managing strategic surprise by response to weak signals. California Management Review, 18, 21-33. https://doi.org/10.2307/41164635.

Argyris, C., \& Schön, D. (1999). Die Lernende Organisation: Grundlagen, Methode, Praxis. Stuttgart: Klett-Cotta.

Arthur, W. B. (1996). Increasing returns and the new world of business. Harvard Business Review, 74, 100-109.

Benner, M. J., \& Tushman, M. L. (2003). Exploitation, exploration, and process management: The productivity dilemma revisited. Academy of Management Review, 28, 238. https://doi. org/10.2307/30040711.

Birkinshaw, J., Gibson, C. (2004). Building ambidexterity into an organization. MIT Sloan Management Review, 45, 47-55.

Birkinshaw, J., \& Gupta, K. (2013). Clarifying the distinctive contribution of ambidexterity to the field of organization studies. Academy of Management Perspectives, 27, 287-298. https://doi. org/10.5465/amp.2012.0167.

Birkinshaw, J., Zimmermann, A., \& Raisch, S. (2016). How Do Firms Adapt to DiscontinuousChange? Bridging the Dynamic Capabilities and Ambidexterity Perspectives. California Management Review 58, 36-58. https://doi.org/10.1525/cmr.2016.58.4.36.

Burgelman, R. A. (1991). Intraorganizational ecology of strategy making and organizational adaptation: Theory and field research. Organization Science, 2, 239-262.

Caniëls, M., Neghina, C., \& Schaetsaert, N. (2017). Ambidexterity of employees: The role of empowerment and knowledge sharing. Journal of Knowledge Management. https://doi.org/10.1108/ JKM-10-2016-0440

Cao, Q., Gedajlovic, E., \& Zhang, H. (2009). Unpacking organizational ambidexterity: Dimensions, contingencies ans synergistic effects. Organization Science, 20, 781-796.

David, P. A. (1985). Clio an the economics of QWERTY. American Economic Review, 75, 332-337.

De Bruin, T., Freeze, R., Kulkarni, U., Rosemann, M. (2005). Understanding the main phases of developing a maturity assessment model. In: ACIS 2005 Proceedings. Presented at the 16th Australasian Conference on Information Systems, Association for Information Systems, Sydney. 
De Clercq, D., Thongpapanl, N., \& Dimov, D. (2013). Contextual ambidexterity in SMEs: The roles of internal and external rivalry. Small Business Economics, 42, 191-205. https://doi. org/10.1007/s11187-013-9471-2.

Dievernich, F. (2012). Pfadabhängigkeitstheoretische Beiträge zur Zukunftsgestaltung. In V. Tiberius (Hrsg.), Zukunftsgenese (S. 57-72). Wiesbaden: Springer Fachmedien.

Duncan, R. B. (1976). The ambidextrous organization: Designing dual structures for innovation. In R. H. Kilmann, L.R. Pondy, \& D. Slevin (Hrsg.), The management of organizational design (S. 167-188). New York: Elsevier.

Euchner, J., \& Tushman, M. (2015). The challenges of ambidextrous leadership. Research-Technology Management, 58, 16-20.

Fojcik, T. M. (2015). Ambidextrie und Unternehmenserfolg bei einem diskontinuierlichen Wandel: Eine empirische Analyse unter besonderer Berücksichtigung der Anpassung und Veränderung von Organisationsarchitekturen im Zeitablauf, Strategisches Kompetenz-Management. Wiesbaden: Springer-Gabler.

He, Z.-L., \& Wong, P.-K. (2004). Exploration vs. exploitation: An empirical test of the ambidexterity hypothesis. Organization Science, 15, 481-494. https://doi.org/10.1287/orsc.1040.0078.

Hill, S., \& Birkinshaw, J. (2012). Ambidexterity and Survival in Corporate Venture Units. Journal of Management, 40, 1899-1931. (Issue published: 2014).

Jansen, J. P. (2005). Ambidextrous organizations: A multiple-level study of absorptive capacity, exploratory and exploitative innovation and performance, ERIM Ph.D. series research in management. Erasmus Research Inst. of Management (ERIM), Rotterdam.

Jansen, J. P., Tempelaar, M. P., van den Bosch, F. A. J., \& Volberda, H. W. (2009). Structural differentiation and ambidexterity: The mediating role of integration mechanisms. Organization Science , 20, 797-811. https://doi.org/10.1287/orsc.1080.0415.

Lane, P. J., \& Lubatkin, M. (1998). Relative absorptive capacity and interorganizational learning. Strategic Management Journal, 19, 461-477. https://doi.org/10.1002/10970266.

Levinthal, D. A., \& March, J. G. (1993). The myopia of learning. Strategic Management Journal, 14, 95-112. https://doi.org/10.1002/smj.4250141009.

Lewis, M. W. (2000). Exploring paradox: Toward a more comprehensive guide. Academy of Management Review, 25, 760. https://doi.org/10.2307/259204.

Lombriser, R., \& Abplanalp, P. A. (2010). Strategisches Management. Zürich: Versus.

Lubatkin, M. H., Simsek, Z., Ling, Y., \& Veiga, J. F. (2006). Ambidexterity and performance in small-to medium-sized firms: The pivotal role of top management team behavioral integration. Journal of Management, 32, 646-672. https://doi.org/10.1177/0149206306290712.

Luo, X., Slotegraaf, R. J., \& Pan, X. (2006). Cross-functional “coopetition”: The simultaneous role of cooperation and competition within firms. Journal of Marketing, 70, 67-80.

March, J. G. (1991). Exploration and exploitation in organizational learning. Organization Science, 2, 71-87

Mintzberg, H. (1979). The Structuring of organizations. London: Pearson.

Murtazin, E. (2010). Apple's Phone: From 1980s' sketches to iPhone. Mob.-Rev.

O'Reilly, C. A., \& Tushman, M. L. (2004). The ambidextrous organization. Harvard Business Review, 82(4), 74-81.

O'Reilly, C. A., \& Tushman, M. L. (2007). Ambidexterity as a dynamic capability: Resolving the innovator's dilemma. Research in Organizational Behavior, 28, 185-206.

Porter, M. E. (1985). Competitive advantage. New York: Free Press.

Raisch, S., \& Birkinshaw, J. (2008). Organizational ambidexterity: Antecedents, outcomes, and moderators. Journal of Management, 34, 375-409. https://doi.org/10.1177/0149206308316058.

Raisch, S., Birkinshaw, J., Probst, G., \& Tushman, M. L. (2009). Organizational ambidexterity: Balancing exploitation and exploration for sustained performance. Organization Science, 20, 685-695. 
Reichert, I. (2017). Der Status-Effekt: Bestseller und Exploration im Literaturmarkt. Wiesbaden: Springer VS.

Roedebeck, M., \& Holtmann, P. (2008). Raus aus der Pfadabhängigkeit - Rein in die Pfadgestaltung. Organisations Entwicklung, 2008(4), 76-84.

Schreyögg, G. (2014). Pfadabhängigkeit und Pfadbruch in Unternehmen. Schmalenbachs Zeitschrift für betriebswirtschaftliche Forschung, 66, 17.

Schreyögg, G., Sydow, J., \& Koch, J. (2003). Organisationale Pfade - Von der Pfadabhängigkeit zur Pfadkreation. Managementforschung, 13, 257-294.

Simon, F. (2007). Die Kunst nicht zu lernen. Heidelberg: Carl-Auer (Erstveröffentlichung 1997).

Stettner, U., \& Lavie, D. (2014). Ambidexterity under scrutiny: Exploration and exploitation via internal organization, alliances, and acquisitions: Exploration and Exploitation. Strategic Management Journal, 35, 1903-1929. https://doi.org/10.1002/smj.2195.

Sydow, J., Schreyögg, G., \& Koch, J. (2005). Organizational paths: Path dependency and beyond. In: Path dependence and creation processes in the emergence of markets, technologies and institutions. Presented at the 21st EGOS Colloquium, Berlin, S. 42.

Teece, D. J., Pisano, G., \& Shuen, A. (2008). Dynamic capabilities and strategic management, In: Technological know-How, organizational capabilities, and strategic management. WORLD SCIENTIFIC, S. 27-51. https://doi.org/10.1142/9789812834478_0002

Thom, N., \& Wenger, A.P. (2010). Die optimale Organisationsform. Wiesbaden: Gabler. https:// doi.org/10.1007/978-3-8349-8830-0.

Tokarski, K. O., Schellinger, J., \& Berchtold, P. (Hrsg.). (2016). Unternehmensentwicklung: Strategien und Instrumente aus Forschung und Praxis. Wiesbaden: Springer Gabler.

Tushman, M. L., \& O’Reilly, C. A. (1996). Ambidextrous organizations: Managing evolutionary and revolutionary change. Strategic management journal, 38, 8-29. https://doi. org/10.2307/41165852.

Tushman, M. L., Smith, W. K., Wood, R. C., Westerman, G., \& O’Reilly, C. A. (2006). Organizational designs and innovation streams. IIndustrial and Corporate Change, 19, 1331-1366. https://doi.org/10.1093/icc/dtq040.

Welsch, C. (2010). Organisationale Trägheit und ihre Wirkung auf die strategische Früherkennung von Unternehmenskrisen. Wiesbaden: Gabler. https://doi.org/10.1007/978-3-8349-8485-2.

Schneeberger, Simon (M.Sc. BA/simonschneeberger@bluewin.ch) Absolvent Masterstudiengang der Berner Fachhochschule BFH-W, Mitglied des Kaders einer Schweizer Versicherung, Eidg. Dipl. Versicherungswirtschafter HF.Habegger, Anja (Prof. Dr./anja.habegger@bfh.ch) Leiterin Studiengang Bachelor Betriebsökonomie (BScBA) und Leiterin Abteilung Lehre sowie stellvertretende Departementsleiterin der Berner Fachhochschule Wirtschaft (BFH-W). Forschungs- und Lehrtätigkeiten in den Themenbereichen Personalmanagement und Organisation. Studium und Promotion in Betriebswirtschaftslehre an der Universität Bern. 
Open Access Dieses Kapitel wird unter der Creative Commons Namensnennung 4.0 International Lizenz (http://creativecommons.org/licenses/by/4.0/deed.de) veröffentlicht, welche die Nutzung, Vervielfältigung, Bearbeitung, Verbreitung und Wiedergabe in jeglichem Medium und Format erlaubt, sofern Sie den/die ursprünglichen Autor(en) und die Quelle ordnungsgemäß nennen, einen Link zur Creative Commons Lizenz beifügen und angeben, ob Änderungen vorgenommen wurden.

Die in diesem Kapitel enthaltenen Bilder und sonstiges Drittmaterial unterliegen ebenfalls der genannten Creative Commons Lizenz, sofern sich aus der Abbildungslegende nichts anderes ergibt. Sofern das betreffende Material nicht unter der genannten Creative Commons Lizenz steht und die betreffende Handlung nicht nach gesetzlichen Vorschriften erlaubt ist, ist für die oben aufgeführten Weiterverwendungen des Materials die Einwilligung des jeweiligen Rechteinhabers einzuholen.

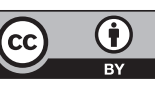




\title{
Digitalisierung: Perspektiven für Arbeitsmodelle der Zukunft in Wirtschaft und Verwaltung
}

\author{
Jochen Schellinger und Gia Le Huynh
}

\begin{abstract}
Zusammenfassung
Die Digitalisierung ist derzeit einer der wichtigsten Wandlungstreiber in Wirtschaft und Gesellschaft. Die aus den neuen technischen Möglichkeiten resultierenden Veränderungen wirken sich auch auf das Verhalten von Kunden und Mitarbeitenden aus. In Bezug auf die Zusammenarbeit in Unternehmen und Organisationen sind bestehende Arbeitsmodelle für administrative Arbeit anzupassen und neue Formen digitaler Kollaboration einzuführen. Hiermit verbunden sind Änderungen der Arbeitszeitmodelle, der Organisation der Mitarbeitenden, der Art der Zusammenarbeit und Führung sowie der Gestaltung von Büroräumlichkeiten. In einem vergleichenden Case-Study-Ansatz von zwei Schweizer Großunternehmen und einer großen Verwaltungseinheit des Bundes werden die existierenden Arbeitsmodelle für Büroarbeit analysiert und auf Entwicklungsperspektiven hin untersucht. Ein derzeit vorhandener Mix aus traditionellen und agilen Arbeitsmodellen wird sich in Zukunft im digitalen Kontext voraussichtlich unternehmensspezifisch stärker in Richtung einer höheren Agilität weiterentwickeln.
\end{abstract}

J. Schellinger $(\bowtie) \cdot$ G. Le Huynh

Berner Fachhochschule, Departement Wirtschaft, Bern, Schweiz

E-Mail: jochen.schellinger@bfh.ch

G. Le Huynh

E-Mail: gia-le.huynh@gmx.net 


\subsection{Einleitung}

Die Arbeitswelt befindet sich im Wandel. Globalisierung, die Alterung der Gesellschaft, die Pluralisierung von Lebensmodellen, aber auch die Digitalisierung treiben diesen Wandel weiter voran. Insbesondere Letztere verändert die Struktur der Arbeitswelt grundlegend (Neufeind 2016, S. 58). Damit Unternehmen die mit der Digitalisierung verbundenen Herausforderungen bewältigen können, braucht es stimmigere und agilere Arbeitsmodelle und Strukturen (Schweizer Verband der Telekommunikation 2015, S. 20 f.). In der Literatur finden sich bislang nur wenig konkrete Aussagen über die Auswirkungen der Digitalisierung für die Implementierung praxistauglicher Arbeitsmodelle für administrative Arbeit. Ein deskriptives Herangehen in Bezug auf aktuelle Umsetzungsmodelle in sich entwickelnden größeren Organisationen soll dabei helfen, denkbare Zukunftsszenarien digitaler Arbeitswelten zu entwickeln. Im Mittelpunkt des Interesses steht die Frage nach dem Aussehen zukünftig erfolgversprechender Arbeitsmodelle für Büroarbeit. Hiermit verbunden sind Fragestellungen hinsichtlich der gegenwärtigen Ausprägung erfolgswirksamer Arbeitsmodelle, der Digitalisierungschancen und -risiken, der konkreten Veränderungsbedarfe, relevanter Rahmensetzungen sowie der Implementierung neuer, beziehungsweise veränderter Arbeitsmodelle.

\subsection{Gegenstand und allgemeine Auswirkungen der Digitalisierung auf Großunternehmen}

Eine Analyse der Auswirkungen von Digitalisierung auf Arbeitsmodelle setzt zunächst eine Klärung von Gegenstand, Antriebskräften und möglichen Auswirkungen der Digitalisierung voraus. Ferner stellt sich die Frage, ob und wie der Digitalisierungsgrad von Unternehmen gemessen werden kann und welche Potenziale die Digitalisierung für die hier im Vordergrund stehenden Großunternehmen und -organisationen mit sich bringt.

\subsubsection{Begriff und Eigenschaften der Digitalisierung}

Der Begriff „Digitalisierung“ wird in der Literatur sehr vielfältig verwendet und interpretiert. Er wird häufig synonym zu den Begriffen „digitale Transformation“ und „digitales Zeitalter" verwendet. Der Begriff steht insbesondere für die digitale Vernetzung aller Bereiche in Wirtschaft und Gesellschaft sowie für die durch die Digitalisierung ausgelösten Veränderungsprozesse (Schallmo und Rusnjak 2017). Grundlage der Digitalisierung sind digitale Technologien für die Datengewinnung, -erfassung, -speicherung und die technische Datenverarbeitung (Blaeser-Benfer und Pollety 2017, S. 14). In Verbindung mit menschlicher Arbeit werden in der Unternehmenspraxis vielfach mit dem Begriff digitale Arbeitsprozesse und -inhalte, eine Beschleunigung der Arbeit und mobil-flexible und papierlose Arbeitsformen verbunden und mit digitalen Phänomenen 
wie Social Media, Big Data, künstlicher Intelligenz oder Industrie 4.0 in Verbindung gebracht (Genner et al. 2017, S. 4 f.; Ittermann und Niehaus 2015, S. 34 f.). Insbesondere der letzte Begriff steht auch als Synonym für eine vierte industrielle Revolution hin zur Wissens- und Informationsgesellschaft, einer neuen Ära der digitalen Automatisierung durch die Mustererkennung in riesigen Datenmengen, cloudbasierte Verfahren, Internet of Things, Smart-Industry-Konzepte, Robotik, Maschinenlernen, Sensorik u. a. Digitalisierung kann aber auch im eher evolutionären Sinne lediglich als Fortführung einer zunehmenden Computerisierung verstanden werden (Raveling 2017b; Schwab 2016, S. $17 \mathrm{f}$.).

\subsubsection{Treiber und Auswirkungen der Digitalisierung}

Disler (2018) identifiziert insgesamt sechs primäre Treiber der Digitalisierung, die nachfolgend eingehender betrachtet werden:

- Globalisierung.

- New Economy.

- Internet of Things.

- Big Data.

- Kunden.

- Transaktionskosten.

Die „Globalisierung“ steht für die wachsende weltweite Verflechtung von Wirtschaft, Politik, Kultur sowie Umwelt und den weltweiten Austausch von Produkten, Ressourcen, Technologien und Kapital. Die Globalisierung fördert die Agilität in allen Bereichen der Volkswirtschaft. Der Begriff „New Economy“ versinnbildlicht den Fokuswechsel weg von der Warenproduktion hin zu webbasierten Dienstleistungen. Das „Internet of Things (IoT)“" verbindet die virtuelle mit der physischen Welt und ermöglicht ein Netzwerk aus Geräten, die miteinander weltweit kommunizieren und interagieren können (Machine-to-Machine-Funktion). Mit „Big Data“ verbindet man die Wertgenerierung aus der Verarbeitung und Analyse riesiger Datenmengen, z. B. um zielgruppenorientierte Kundenbedarfe zu identifizieren oder Absatzmengen vorherzusagen. Zudem hat sich das Konsum- und Kaufverhalten in den letzten Jahren massiv verändert: Die „Kunden“ treiben die Digitalisierung voran, indem sie neue Anforderungen an Transparenz, Kauferlebnisse und die Geschwindigkeit von Lösungen und Services stellen. „Transaktionskosten“ sind Kosten, die durch die Nutzung des Marktes im Zusammenhang mit der Transaktion von Verfügungsrechten entstehen. Vereinfachungen von Abläufen und Konzentration auf Kundenbedürfnisse dienen u. a. der Reduktion der Transaktionskosten (Disler 2018). Vor allem diese sechs Digitalisierungstreiber haben dazu geführt, dass der Digitalisierungsprozess in der Unternehmenswelt, aber auch in administrativen serviceorientierten Organisationen, mit hoher Dynamik und wachsendem Wirkungskreis vorangeht. 
Die Digitalisierung und die hinter ihr stehenden Treiber haben große Auswirkungen auf die Unternehmen. Veränderten Kundenerwartungen kann durch datenbasierte, transparenzschaffende Analyseverfahren besser erkannt und begegnet werden. Dies geht gleichzeitig mit einer Machtverschiebung in Richtung Verbraucher einher (Schwab 2016, S. 82 f.). Erweiterungen von Produkten um digitale Fähigkeiten führen ferner zu Kundennutzensteigerungen und ermöglichen neue Geschäftsmodelle (Schwab 2016, S. 85 f.). Angesichts der Schnelligkeit, Innovation und Disruption, die die neuen Technologien mit sich bringen, braucht es neue Formen der kooperativen Zusammenarbeit mit Partnern. Es ist von Bedeutung, Kundenerfahrungen, datenbasierte Dienstleistungen sowie Leistungsbeurteilungen gegenseitig auszutauschen (Schwab 2016, S. 87 f.). Durch neue Technologien, geänderte Produktionsverfahren und veränderte Kundenerwartungen müssen Unternehmen ihre Geschäftsmodelle überdenken. In Verbindung mit relevanter werdenden Plattformstrategien erfolgen zunehmend Schwerpunktverlagerungen weg vom Produktverkauf hin zum Erbringen von Dienstleistungen (Schwab 2016, S. 89).

Die Digitalisierung hat auch fundamentale Auswirkungen auf die Arbeitswelt und somit auf die Arbeitnehmer und wirkt über die Unternehmenswelt hinaus zudem in das Privatleben der Beschäftigten hinein. Digitale Technologien und Kommunikationskanäle sind für die meisten Menschen mittlerweile auch in der Freizeit über Smartphones, Tablets oder Social Networking zum festen Lebensbestandteil geworden (Glaser 2016). Wie wir einkaufen, wie wir Musik hören und wie wir unser Leben gestalten, das alles wird durch die Digitalisierung beeinflusst (Süddeutsche Zeitung, o. J.). Angesichts der neuen Kommunikationsmöglichkeiten und der jederzeitigen Erreichbarkeit der Beschäftigten vermischen sich Arbeitswelt und Freizeit in zunehmendem Maße und die Grenzziehung zwischen diesen Sphären wird aufgeweicht.

\subsubsection{Ansätze zur Messung der Digitalisierung}

Zur Bewertung des Digitalisierungsgrads von Unternehmen und Organisationen können Reifegradmodelle herangezogen werden. Es handelt sich dabei um Referenzmodelle, die sich mithilfe von definierten Entwicklungsstufen (Reifegrade) mit dem aktuellen Entwicklungszustand, Änderungen in Organisationen sowie mit den eingesetzten Technologien befassen (Leyh et al. 2016, S. 3). Die Reifegradmessung hilft, ein gemeinsames Verständnis für die Digitalisierung herzustellen. Sie hilft bei der Standortbestimmung und Aufdeckung von Wahrnehmungsdifferenzen und ermöglicht Vergleiche mit anderen Unternehmen (Berghaus et al. 2017, S. 7).

Es gibt mittlerweile eine Reihe von Reifegradmodellen zur Messung der digitalen Reife von Unternehmen, die sich in Art und Umfang der herangezogenen Transformationsfaktoren, respektive Digitalisierungsmerkmale unterscheiden (Berlin 2016, S. 6). Die beiden wohl bekanntesten wissenschaftlichen Verfahren sind der „Four-Types-of-Digital-Maturity“-Ansatz des Centers for Digital Business am Massachusetts Institute of Technology (MIT) und das „Digital Transformation Assessment“ von Forrester Research 
(Digital Transformer Institute 2017; Ruoss 2015). Das „Digital Maturity Model“ der Universität St. Gallen (Berlin 2016, S. 6) scheint im Besonderen für die spezifischen Rahmenbedingungen in der Schweiz geeignet zu sein. Das Institut für Wirtschaftsinformatik der Universität St. Gallen und die Strategieberatung Crosswalk untersuchen seit 2014 die digitale Reife von Unternehmen in der Schweiz, Deutschland und Österreich. Zwischenzeitlich nehmen 452 Unternehmen an der Studie teil (Institut für Wirtschaftsinformatik 2017; Berghaus et al. 2017, S. 6, 11). Das „Digital Maturity Model“ besteht aus den neun Dimensionen Customer Experience, Produktinnovation, Strategie, Organisation, Prozessdigitalisierung, Zusammenarbeit, Informationstechnologie, Kultur \& Expertise sowie Transformationsmanagement, die mittels 64 Indikatoren in Online-Fragebögen erhoben werden (Berghaus et al. 2017, S. 8). Der Reifegrad eines Unternehmens wird in drei Schritten mittels Indikatoreneinstufung auf einer fünfstufigen Likert-Skala, einer Schwierigkeitsgradbewertung pro Indikator und einer Clusterbildung ermittelt (Berghaus et al. 2017, S. 8 f.).

\subsubsection{Potenziale der Digitalisierung für Großunternehmen}

Großunternehmen haben gegenüber Klein- und mittleren Unternehmen (KMU) nicht nur mehr Beschäftigte, sondern verfügen auch über mehr Ressourcen und Handlungsmöglichkeiten. Sie weisen zudem in der Regel einen höheren digitalen Reifegrad als kleinere Unternehmen auf und eignen sich somit besser für die Analyse der digitalisierungsbasierten Veränderung von Arbeitsmodellen.

Es gibt in der Schweiz rund 1570 Großunternehmen, welche 1,4 Mio. Personen beschäftigen, was einem Anteil von rund $30 \%$ der Beschäftigten entspricht. Als Großunternehmen gilt in der Schweiz ein Unternehmen, welches mindestens 250 Personen beschäftigt (Schweizerische Eidgenossenschaft 2018a; Moneyhouse 2017). Losgelöst von der Unternehmensgröße wenden viele Unternehmen bereits digitale Technologien an, um die Effizienz sowie den Mehrwert ihrer Produkte und Dienstleistungen zu verbessern. Schweizer Unternehmen sehen in Verbindung mit der Digitalisierung insbesondere folgende Potenziale zur Verbesserung ihrer Unternehmensperformance (Deflorin et al. 2015, S. 58 ff.):

- Effizienzsteigerung und Automatisierung von Produktion und Prozessen.

- Überwachung von Prozessen.

- Verbesserung von Dienstleistungsmehrwert und Dienstleistungsangebot.

- Verbesserung von Produktmehrwert sowie Einführungszeit von Produkten.

- Einbettung von digitalen Schnittstellen in die Produkte für die Erhöhung von Marktund Kundenwissen.

- Gemeinsame digitale Plattformen für unterschiedliche Funktionsbereiche, wie beispielsweise Forschung und Entwicklung, Produktion sowie Marketing.

- Erhöhung der Integration von Funktionen und Prozessen.

- IT-Schnittstellen zu externen Partnern in der Wertschöpfungskette, auch international. 
Aus den Potenzialen können kompetitive Chancen, wie Kostensenkungen, vermehrte Kundenbindung sowie individualisierte neue Produkte und Dienstleistungen entstehen (Deflorin et al. 2015, S. 61; Deloitte 2016, S. 6). Hierfür müssen Unternehmen ihre Geschäftsstrategie und -ziele anpassen, digitale Initiativen starten und adäquate Strukturen und Arbeitsmodelle sowie eine Innovationskultur einführen (Deflorin et al. 2015, S. 61; Deloitte 2016, S. 17). Eine explizit auf Großunternehmen mit mehr als 500 Mitarbeitenden beschränkte Studie in Deutschland kommt zu ähnlichen Ergebnissen. Das Potenzial der Digitalisierung wird von diesen Großunternehmen aber als noch größer eingeschätzt als von KMU (Rotter 2017).

Die jüngsten Ergebnisse des Digital Maturity Report können Hinweise darauf geben, welche Herangehensweisen für Großunternehmen in der Schweiz bei der Digitalisierung relevant sein können, auch wenn die Untersuchung nicht nur auf diese Unternehmenskategorie ausgerichtet ist (Berghaus et al. 2017, S. 17). Es konnten fünf typische Vorgehensweisen zur Digitalisierungsumsetzung identifiziert werden (Berghaus et al. 2017, S. 20 f.):

- Top-down: Die Digitalisierung wird von der Führungsebene getrieben. Das Hauptaugenmerk wird dabei auf die Erstellung und Umsetzung einer digitalen Transformationsstrategie gesetzt.

- Bottom-up: Die Mitarbeitenden ergreifen Initiativen und konzentrieren sich auf die Konsolidierung von bestehenden Initiativen.

- IT-Fokus: Die Anforderungen an die IT sind Treiber der Digitalisierung. Im Mittelpunkt stehen die Erneuerung und Bereitstellung geeigneter IT-Infrastruktur und -Systeme.

- Kanal-Fokus: Das Unternehmen wird von den digitalen Kundenerwartungen getrieben. Hierbei wird auf die Erneuerung und Verbesserung der digitalen Kanäle fokussiert.

- Innovations-Fokus: Digitalisierung wird durch Experimentierfreude oder Gefährdung des aktuellen Geschäftsmodells vorangetrieben. Das Ausprobieren neuer Technologien und die Erarbeitung möglicher neuer Geschäftsmodelle sind dabei zentral.

Von den in der Studie befragten 662 Unternehmen wählt das Gros von $23 \%$ einen Top-Down-Ansatz (Berghaus et al. 2017, S. 20 f.). Ferner sind Unternehmen mit niedrigem Digitalisierungsreifegrad häufig IT-fokussiert oder wenden den Bottom-Up-Ansatz an. Unternehmen mit hohem Reifegrad präferieren eine Top-downoder innovationsfokussierte Herangehensweise vor (Berghaus et al. 2017, S. 20 f.). Das Gros der Unternehmen weist auf der fünfstufigen Reifegradskala einen Wert von 3 auf, der durchschnittliche Reifegrad der 452 einbezogenen Unternehmen liegt bei 2,8. Großunternehmen mit mehr als 10.000 Angestellten verfügen über die höchsten Digitalisierungsreifegrade. Großunternehmen mit einer Beschäftigtenzahl von 251 bis 500 Mitarbeitenden liegen aber nahezu gleichauf. Insgesamt liegen die Reifegrade der Großunternehmen leicht oder spürbar über dem durchschnittlichen Reifegradswert 
(Berghaus et al. 2017, S. 18). Branchenbezogen liegen die IT, Telekommunikation \& Beratung, Versicherungen sowie die Banken mit einem durchschnittlichen Reifegrad ab 3 im vorderen Bereich der Digitalisierungsimplementierer (Berghaus et al. 2017, S. 19).

\subsection{Arbeitsmodelle für administrative Arbeit in der Schweiz}

In den folgenden Abschnitten werden Arbeitsmodelle im Schweizer Kontext inhaltlich anhand von fünf Gestaltungsdimensionen charakterisiert und die wichtigsten gesetzlichen Rahmenbedingungen für die Ausgestaltung von Arbeitsmodellen für administrative Arbeit aufgezeigt.

\subsubsection{Begriff und Gegenstand von Arbeitsmodellen}

Auch der Begriff „Arbeitsmodell“ ist in der Literatur vielfältig belegt. Oft wird er mit Arbeitszeitmodellen gleichgesetzt, was jedoch der heutigen veränderten Arbeitswelt mit einer Verschmelzung von Arbeitszeit-, Arbeitsform-, Arbeitsführungs-, Arbeitsstrukturund Arbeitsraumdimensionen nicht mehr gerecht wird. Arbeitsmodelle im Verständnis des vorliegenden Beitrags umfassen alle diese dimensionalen Ausprägungen von (Zusammen-)Arbeit. In der nachfolgenden Kennzeichnung dieser Dimensionen wird der Fokus auf in diesem Beitrag im Vordergrund stehende administrative Tätigkeiten gelegt.

\subsubsection{Arbeitszeitdimension von Arbeitsmodellen}

Die Arbeitszeitdimension kann anhand der Hauptausprägungen von Arbeitszeitmodellen charakterisiert werden. Im Digitalisierungskontext kommt vor allem auf Flexibilität und Eigenverantwortung setzenden Zeitmodellen eine größere Bedeutung zu. Bei der stark verbreiteten Vertrauensarbeitszeit verzichten Arbeitgeber auf Zeitkontrollen. Die Mitarbeitenden handeln eigenverantwortlich und orientieren sich am Resultat ihrer Arbeit. Bei der Umsetzung sind Restriktionen der Arbeitsgesetzgebung zu berücksichtigen, was dazu führt, dass Vertrauensarbeitszeitmodelle vor allem bei Führungskräften zum Einsatz kommen (Lienhart 2015). In flexiblen Arbeitszeitmodellen können die Beschäftigten in einem bestimmten Rahmen selbst auf ihre Arbeitszeit Einfluss nehmen. Das bekannteste, in der Praxis bewährte und beliebte Modell ist das Gleitzeitmodell. Es lässt sich gut mit den arbeitsrechtlichen Vorschriften vereinbaren. Ein Gleitzeitreglement regelt, dass sowohl durch Minussaldo als auch Plussaldo Abweichungen von der durchschnittlichen Arbeitszeit entstehen können und wie diese ausgeglichen werden. Gleitzeitregelungen sorgen in erster Linie dafür, dass für Arbeitnehmer und für Arbeitgeber mehr Flexibilität entsteht. Amorphe Arbeitszeitmodelle sind dadurch gekennzeichnet, dass für Mitarbeitende lediglich festgehalten wird, wie viel in einem bestimmten Zeitraum gearbeitet 
werden muss, aber nicht, wann und wie lange gearbeitet wird (Wochen-, Monats-, Jahres- und Lebensarbeitszeitmodelle). Zu viel geleistete Arbeitszeit wird auf einem Arbeitszeitkonto festgehalten und kann für Sabbaticals, Familienpausen oder eine vorzeitige Pensionierung angespart werden. Die Mehrarbeit der Mitarbeitenden stellt eine geldwerte Leistung dar und muss in der Bilanz des Unternehmens als Schuld ausgewiesen werden. Viele Unternehmen wählen diese Modellvariante wegen dieser Offenlegungspflicht nicht. Jahresarbeitszeit kommt vor allem bei Unternehmen mit starken Schwankungen im Arbeitsanfall zum Tragen. Lebensarbeitszeitmodelle sind Weiterführungen des Jahresarbeitszeitmodells mit weitergehenderen Kompensationsoptionen. Sie kommen in der Schweiz vor allem in der öffentlichen Verwaltung zur Anwendung (Arbeitgeberverband Basel 2014).

\subsubsection{Arbeitsformdimension von Arbeitsmodellen}

Unter Arbeitsformen werden hier das Jobsharing, die Teilzeitarbeit sowie die Arbeit auf Abruf subsumiert. Den angeführten Arbeitsformen kommt hinsichtlich der im Digitalisierungskontext besonders wichtigen Flexibilisierung und Mobilisierung der Arbeit eine größere Relevanz zu.

Jobsharing ist dadurch gekennzeichnet, dass eine Arbeitsstelle auf mehrere Arbeitnehmer aufgeteilt wird und die zugehörigen Aufgabenstellungen gemeinsam, aber zeitversetzt als Gruppe bewältigt werden. Die Jobsharer arbeiten teilzeit und können die Arbeitszeit im definierten Rahmen selber einteilen. Hauptmerkmal der Teilzeitarbeit ist eine gegenüber der betriebsüblichen Arbeitszeit reduzierte Arbeitszeit. Oft arbeiten Teilzeitbeschäftigte für mehrere Arbeitgeber unter der Voraussetzung, dass nicht gegen die Sorgfalts- und Treuepflicht verstoßen wird. Bei der Arbeit auf Abruf veranlasst der Arbeitgeber den Einsatz der Beschäftigten bedarfsabhängig. Hierbei sind Abrufarbeiten mit und ohne Befolgungspflicht zu unterscheiden. Beim Abruf mit Befolgungspflicht sind Mitarbeitende verpflichtet dem Abruf zu folgen, und die Beschäftigten müssen auch außerhalb der betrieblichen Arbeitszeit für einen Arbeitseinsatz zur Verfügung stehen (entschädigungspflichtiger Bereitschaftsdienst). Eine Abrufarbeit mit Befolgungspflicht ist in der Regel auch eine Teilzeitarbeit. Bei der Abrufarbeit ohne Befolgungspflicht können Beschäftigte frei entscheiden, ob sie dem Abruf Folge leisten oder nicht. Prinzipiell entsteht dabei durch jeden neuen Arbeitseinsatz ein neuer Vertrag (Bürgi und Nägeli Rechtsanwälte o. J.).

\subsubsection{Arbeitsführungsdimension von Arbeitsmodellen}

Je nach Arbeitssituation und Personenkreis kommen in den Unternehmen heutzutage verschiedene Personalführungsansätze zur Anwendung. In der Literatur gibt es eine Fülle von Führungsmodellen, die sich in der praktischen Anwendung im Laufe der vergangenen 
Jahrzehnte zwar hinsichtlich Schwerpunktsetzung und Variabilität verändert haben, in ihrer Grundform aber eine hohe Kontinuität vorweisen. Eine bekannte klassische Grundkategorisierung von Führungsansätzen ist das „Kontinuummodell“ nach Tannenbaum und Schmidt (Grannemann 2015). Es ordnet die in Anlehnung an Lewin abgegrenzten Führungsstile entlang eines Kontinuums, das durch zunehmende Entscheidungsspielräume der Gruppe und abnehmende Entscheidungsspielräume der Vorgesetzten gekennzeichnet ist. Die Bandbreite der Personalführungsperspektive reicht hier von autoritären, über konsultative, partizipative bis hin zu kooperativ-demokratischen Entscheidungsfindungsmodellen (Grannemann 2015). Im Zuge der Digitalisierung gewinnen Gruppenansätze noch mehr an Bedeutung, sodass im Rahmen eines situativen Führungsansatzes vermehrt kooperative, oder, noch weitergehend, auf Selbstorganisation setzende, vertrauensbasierte laterale Führungsmodelle an Bedeutung gewinnen.

\subsubsection{Arbeitsstrukturdimension von Arbeitsmodellen}

Arbeitsmodelle sind immer auch untrennbar mit der Frage nach strukturellen Festlegungen zur Zusammenarbeit verbunden. Die Grundkategorisierung von in der Unternehmenspraxis vorhandenen Organisationsformen nach Sattelberger et al. (2015) gibt einen Überblick über vier Basisformen zur Organisation der Arbeit, die sich durch ihr Organisationsdesign und die Rollen der Mitarbeitenden unterscheiden. Das Organisationsdesign kann gemäß dem Typologieschema gesteuert oder selbstorganisierend ausgerichtet werden und die Rollen der Mitarbeitenden werden entweder als umsetzungsorientiert oder als gestaltungsorientiert beschrieben (Sattelberger et al. 2015, S. 270 ff.). Je nach Kombination dieser zweidimensionalen Ausprägungsformen resultieren vier Organisationsformen, die in Verbindung mit der Digitalisierung der Arbeitswelt mehr oder weniger gut geeignet sein können.

Gesteuerte und umsetzungsorientierte Weisungs- und Kontrollorganisationen haben eine lange Tradition und sind vor allem dann geeignet, wenn man durch Routineprozesse Effizienz generieren möchte. In der Top-Down-Struktur stehen Mitarbeitende im Vordergrund, die sich durch effiziente Ausführungen vorgegebener Prozesse auszeichnen. Kreativität und Innovation werden durch Hierarchie und Standardisierung allerdings nicht gefördert (Sattelberger et al. 2015, S. 271 f.). Selbstorganisierende und umsetzungsorientierte überlastete Organisationen sind dadurch gekennzeichnet, dass veränderte Marktbedürfnislagen dazu geführt haben, dass anstelle von nur ausführenden Mitarbeitenden von diesen zunehmend mehr Eigenverantwortung, innovatives Verhalten und Selbstorganisation der Arbeit erwartet wird. Dies funktioniert jedoch nur, wenn die Mitarbeitenden hierfür befähigt werden, was in der Praxis oft nicht der Fall ist und dann zu Überbelastung der Beschäftigten führt (Sattelberger et al. 2015, S. 272). Ähnlich problematisch können sich gesteuerte und gestaltungsorientierte Schattenorganisationen entwickeln, die durch Mitarbeitende gekennzeichnet sind, die sich aktiv als Mitentscheider am Unternehmenserfolg beteiligen möchten, jedoch von den vorhandenen 
autoritären, starren Strukturen ausgebremst werden. Mitarbeitende verfolgen in diesem Umfeld zunehmend ihren eigenen Weg und entwickeln oftmals eine hohe Eigendynamik, die nur bedingt mit den strategischen Rahmensetzungen kompatibel sein muss. Oft verlassen gute und an sich motivierte Mitarbeitende solche Organisationen (Sattelberger et al. 2015, S. 274-275). Selbstorganisierende und gestaltungsorientierte agile Netzwerke verfügen über ein flexibles vertrauensbasiertes Organisationsdesign und eigenverantwortliche Mitarbeitende mit unternehmerischen Gestalterqualitäten. Mitarbeitende werden möglichst umfassend bei wichtigen Entscheidungen einbezogen. Sie arbeiten eigenständig, denken unternehmerisch und erschließen und überführen komplexe Sachverhalte in Konzepte (Sattelberger et al. 2015, S. 276 f.). Diese auf Innovation ausgerichtete Organisationsform scheint in Verbindung mit digitalisierungsbedingten Anpassungen von Arbeitsmodellen von besonderer Bedeutung zu sein. Agilität und Digitalisierung sind Begriffe, die in hohem Maße miteinander kompatibel sind.

\subsubsection{Arbeitsraumdimension von Arbeitsmodellen}

Neben der Zeitgestaltung, Arbeitsarten, Führung und der Organisation trägt auch der Arbeitsort im Sinne einer physischen Festlegung dessen, wo und wie Mitarbeitende zusammenarbeiten, zur Produktivität und Zufriedenheit der Angestellten bei. Traditionelle Büroraumlösungen sind dadurch gekennzeichnet, dass sie für Mitarbeitende konzipiert sind, die ihre Arbeiten fix an einem Standort und zu festen Zeiten erledigen. Die Räume sind in der Regel hierarchisch strukturiert. Es gibt Einzel- und Teambüros und jeder Mitarbeitende hat seinen eigenen Arbeitsplatz. Etwa zehn Prozent der Schweizer Unternehmen nutzen noch diese klassische Form der Bürogestaltung. Flexible Büros sind durch flache hierarchische Strukturierungen und einen geringen Anteil von Einzelbüros gekennzeichnet. Homeoffice ist zwar möglich, wird aber nicht wirklich gefördert. Etwa fünf Prozent der Schweizer Unternehmen verwenden heute solche flexibleren Raumlösungen. Mobile Büros, die bei etwa $16 \%$ der Schweizer Unternehmen zum Einsatz kommen, sind auf mobil-flexibles Arbeiten mit geeigneter Infrastruktur ausgerichtet. Hierarchien spielen, wie bei den flexiblen Raumlösungen, eher keine Rolle bzw. sind flach und es werden Open-Space-Lösungen als moderne Form des Großraumbüros implementiert. Zwei Drittel der Schweizer Unternehmen befinden sich entweder im Wechsel in Richtung flexible oder mobile Lösungen oder sind nicht eindeutig einer Kategorie zuordenbar (Witzig the office company 2014).

\subsubsection{Arbeitsrechtliche Rahmenbedingungen}

Bei der Ausgestaltung der Arbeitsmodelle entlang der angeführten Gestaltungsdimensionen im Hinblick auf Aspekte der digitalen Transformation sind die arbeitsrechtlichen Regelungen als Rahmensetzung zu berücksichtigen. Diese sind für die Schweiz 
im Obligationenrecht (OR) und im Arbeitsgesetz (ArG) festgelegt (Schweizerische Eidgenossenschaft o. J.a.). Hinsichtlich Flexibilisierung und Mobilisierung in zukünftigen digitalen Arbeitswelten sind insbesondere die gesetzlichen Regelungen für Überstunden, Nacht- und Sonntagsarbeit, zur Arbeitszeiterfassung und zur Mehrbeschäftigung relevant.

Sofern Arbeitsstunden, die vertraglich vereinbarte Arbeitszeit übersteigen, spricht der Gesetzgeber von Überstunden, die nur in Ausnahmefällen zu leisten sind. Beschäftigte müssen körperlich und psychisch Überstunden leisten können, die Anforderungen der Überzeitarbeit müssen zumutbar sind und die maximale Wochenarbeitszeit für Büroarbeit von $45 \mathrm{~h}$ muss eingehalten werden (Ausnahmeregelungen für bestimmte Berufsgattungen). Die geleisteten Überstunden können monetär abgegolten werden (Schweizerische Eidgenossenschaft o. J.c.). Für Nacht- und Sonntags- sowie Feiertagsarbeit gelten strengere Regeln. Diese ist zunächst grundsätzlich verboten. Nur bei bewilligten Ausnahmen und bei speziellen Unternehmen sowie Berufsgattungen gibt es eine Sondererlaubnis. Im Falle einer solchen Erlaubnis sind die Unternehmen in besonderem Maße für die Sicherheit und Gesundheit der Mitarbeitenden verantwortlich (Schweizerische Eidgenossenschaft 2015b). Die Arbeitszeiterfassung ist im Arbeitsgesetz (ArG) und den zugehörigen Verordnungen (ArGV) als zwingende Norm des öffentlichen Rechts geregelt (insbesondere Art. 46 ArG und Art. 73 ArGV 1, Art. 73a ArGV 1 und Art. 73b ArGV 1). Die Arbeitszeit beschreibt die tatsächliche zeitliche Beanspruchung des Mitarbeitenden (Arbeitgeberverband Basel 2014). Die Arbeitgeber sind gesetzlich verpflichtet, die Arbeitszeiten der Arbeitnehmer zu erfassen (Schweizerische Eidgenossenschaft 2016). Die Arbeitgeber müssen die geleisteten Arbeitszeiten der Beschäftigten, inklusive Ausgleichs- und Überzeitarbeit, nachweisen können. Ebenfalls müssen die Ruhe- oder Ersatzruhetage sowie die gesetzlich verpflichtenden Pausen dokumentiert werden. Bei Nichteinhaltung der Regelung drohen Strafmaßnahmen, von Verwarnungen zu Bußgeldern bis hin zur Schließung des Unternehmens. Ausgenommen von der Dokumentationspflicht sind lediglich Angestellte des Topmanagements, die an wichtigen Unternehmensentscheidungen beteiligt sind und ihre Arbeitszeit selbstständig organisieren können. Darüber hinaus gibt es seit Januar 2016 zwei zusätzliche Ausnahmeoptionen, um dem zunehmenden Flexibilisierungs- und Mobilitätsbedarf der Wirtschaft entsprechen zu können. Mitarbeitende, die über ein Jahreseinkommen von mehr als CHF 120.000 verfügen, können von der Arbeitszeiterfassung befreit werden (Art. 73a ArGV 1). Dies gilt allerdings nicht für Sonntags- oder Nachtarbeit. Außerdem ist seither neu auch eine partielle oder vereinfachte Zeiterfassung möglich, bei welcher nur die Gesamtdauer der täglichen Arbeitszeit einzutragen ist (Art. 73b ArGV 1) (Schweizerische Eidgenossenschaft 2016). In Verbindung mit Mehrfachbeschäftigung sind Arbeitgeber verpflichtet, die gesamten Arbeits- und Ruhezeiten ihrer Angestellten sicherzustellen, auch wenn diese mehreren Erwerbstätigkeiten nachgehen, was gesetzlich erlaubt ist. Die Nebenerwerbstätigkeit darf allerdings nicht mit dem Geschäft des Hauptarbeitgebers konkurrieren, dem Ansehen des Unternehmens schaden und keine negativen Auswirkungen auf die Haupttätigkeit haben (Treuepflicht der Beschäftigten) (Schweizerische Eidgenossenschaft 2015a). 


\subsection{Implikationen der Digitalisierung für Arbeitsmodelle}

Nachfolgend wird die Fragestellung vertieft, wie die Digitalisierung bestehende Arbeitsmodelle für Büroarbeit beeinflusst und Impulse für neue Arbeitsmodelle geben kann. In dem Zusammenhang werden außerdem mögliche Chancen und Problemstellungen von mit der Digitalisierung einhergehenden Arbeitsmodellen hinterfragt.

In der Literatur wird auch in Verbindung mit der Digitalisierung grundlegend eine Tendenz der Unternehmen zu mehr Flexibilität, Mobilität sowie Autonomie der Beschäftigten ausgemacht. In der Praxis hat sich dies in der Schweiz unter anderem in der Gründung der Work Smart-Initiative durch die Swisscom, die Post, die SBB, die Mobiliar sowie die Firma Witzig im Jahr 2015 niedergeschlagen. Das Ziel dieser Initiative ist es, flexible und ortsunabhängige Arbeitsmodelle in der Schweiz zu fördern. Seit der Gründung haben sich über 150 Schweizer Unternehmen dieser Initiative angeschlossen (Work Smart Initiative 2018). Übergreifende Darstellungen zur digitalisierungsbasierten Veränderung von Arbeitsmodellen sind nur bedingt vorhanden, sodass eine Charakterisierung der Änderungsbewegungen unter Bezugnahme auf die fünf herangezogenen Ausprägungsdimensionen erfolgt. Generell kann aber festgehalten werden, dass heute in großen Unternehmen flexible und agile Arbeitsmodelle bereits eingesetzt und zukünftig vermehrt relevant werden dürften, da eine fortlaufende, fließende Ausrichtung der Arbeitswelten an den Bedürfnissen der Kunden und Mitarbeitenden immer wichtiger wird (Zinser und Boch 2007, S. 57). Alle in den fünf Dimensionen angeführten Arbeitsmodellkomponenten bzw. -varianten sind mehr oder minder von der Digitalisierung betroffen und entwickeln sich im Digitalisierungskontext in Richtung flexiblerer, mobilerer und autonomerer dimensionaler Ausprägungsformen für die bestehenden Arbeitsmodelle oder bringen in Kombination auch neue Arbeitsmodelltypen hervor.

Hinsichtlich der Dimension Arbeitszeit kann festgehalten werden, dass bereits heute für administrative Tätigkeiten Vertrauensarbeitszeit, flexible Arbeitszeitmodelle und amorphe Arbeitszeit in den Unternehmen zunehmend zur Anwendung kommen und von den Mitarbeitenden in der Regel geschätzt werden. Diese Tendenz wird sich in Zukunft vermutlich weiter verstärken (Arbeitgeberverband Basel 2014). Im Hinblick auf die Arbeitsmodelldimension Arbeitsform kann angenommen werden, dass die Arbeitsarten Teilzeitarbeit, Jobsharing und Arbeit auf Abruf weiterhin und vermehrt genutzt werden. Auch diese begünstigen bereits die Flexibilität sowie die Vereinbarkeit von Beruf und Freizeit. Kombinationsmöglichkeiten mit neueren Entwicklungsformen wie Freelancer-Modelle, Human-Cloud-Ansätze sowie mobil-flexibles Arbeiten sind vorstellbar. In Bezug auf die Arbeits-, respektive Personalführung sind Veränderungen bereits vielfach realisiert. So werden Meetings und Gespräche beispielsweise vermehrt ortsungebunden virtuell durchgeführt und Führungskräfte haben bei ihren Führungsentscheidungen oft relevante Informationen in Echtzeit zur Verfügung (Mühlenbeck 2017). In Verbindung mit der Digitalisierung scheinen sich ferner die Erwartungen der Mitarbeitenden an die Führungskräfte stark zu verändern, sodass Unternehmen ihre 
Führungskultur entsprechend hinterfragen und, in Anlehnung an die Erkenntnisse der Studie von Genner et al. (Genner, S. et al. 2017, S. 17), vermehrt in Richtung Selbstführung der Mitarbeitenden, Führung auf räumliche Distanz, identifikations- und zielorientierte Führung, Führung über digitale Kanäle und flache Teamhierarchien anpassen müssen. In Bezug auf die Arbeitsstrukturen zeichnet sich eine zunehmende Entwicklung hin zu agilen Netzwerkstrukturen ab, die durch Selbstorganisation, Vertrauensbasierung, Unternehmertum und ein hohes Maß an Autonomie der Mitarbeitenden gekennzeichnet sind (Sattelberger et al. 2015, S. 272, 276 ff.). Die Ausgestaltung der Büroarbeitsräume wird voraussichtlich im Zuge der Digitalisierung in immer höherem Maß auf mobil-flexibles Arbeiten in Open-Space-Arbeitswelten hin ausgerichtet sein, die der Tendenz Rechnung tragen, dass die in Büros vor Ort verbrachte Zeit der Angestellten sich weiter reduzieren wird. So sind etwa bereits heute selbst im Umfeld traditioneller Arbeitsformen nur maximal $70 \%$ der Büroarbeitsplätze während der Arbeitszeit gleichzeitig besetzt (Zinser und Boch 2007, S. 58). In Verbindung mit den digitalisierungsbedingten und -unterstützten Änderungstendenzen der Arbeitsmodelldimensionen sind auch weitere Änderungen der arbeitsrechtlichen Rahmensetzungen in der Schweiz für die Zukunft zu erwarten. Dem entsprechend hat der Bund 2017 einen Bericht verabschiedet, der die Rahmenbedingungen der Wirtschaft in Hinblick auf die Digitalisierung analysiert. Vor dem Hintergrund der Zielsetzung der Arbeitsplatz- und Wohlstandssicherung wird festgehalten, dass die heutigen Gesetzesgrundlagen zwar weitgehend bereits Spielraum für den Wandel gewährleisten, aber nicht mehr nötige Regulierungen sollen punktuell angepasst und die Rahmenbedingungen für die digitale Arbeitswelt weiter verbessert werden. Hierzu erteilt der Bundesrat Prüfaufträge (Schweizerische Eidgenossenschaft 2018a).

\subsubsection{Entwicklung neuer digitalisierungsbasierter Arbeitsmodelle}

Grundlegende Entwicklungstendenzen für die einzelnen Arbeitsmodelldimensionen wurden unter Bezugnahme auf die bestehende Literatur bereits aufgezeigt. In einem weiteren Konkretisierungsschritt werden exemplarisch mögliche neuere Ausprägungsformen identifiziert, die für sich, oder auch in Kombination mit anderen dimensionalen Ausprägungen, neuartige agile Formen der Zusammenarbeit generieren und in hohem Maße mit den Anforderungen und Möglichkeiten der Digitalisierung kompatibel sind.

Mobil-flexibles Arbeiten steht für eine Kombination verschiedener Arbeitsmerkmale wie etwa Homeoffice, gleitender Arbeitszeit oder Vertrauensarbeitszeit mit räumlich ungebundenem Arbeiten innerhalb oder außerhalb des Unternehmens (Genner et al. 2017, S. 12). Insgesamt $75 \%$ der Schweizer Bevölkerung arbeiten mit steigender Tendenz mittlerweile bereits im Dienstleistungssektor, in dem vorwiegend Wissens- und Kopfarbeit erbracht wird. Von dieser Mehrheit der Beschäftigten arbeiten schon heute fast $30 \%$ mindestens einen halben Tag pro Woche von zu Hause aus (Zobrist und Grampp 2016, S. 10). Es ist davon auszugehen, dass dieser Wert im Zuge der Digitalisierung noch 
deutlich ansteigen wird. Digitalisierung steht dabei auch und insbesondere für den Einsatz mobiler Geräte wie Smartphones, Tablets oder Laptops in Kombination mit Cloudlösungen und VPN-Verbindungen. Hiermit verbunden ist eine wachsende Autonomie der Beschäftigten bei der Art und Weise der Arbeitsdurchführung (Genner et al. 2017, S. 12). Beschäftigte, die in mobil-flexiblen Arbeitsmodellen arbeiten, geben an, dass sie produktiver arbeiten und zufriedener sind, was sich aus Sicht der Unternehmen auch in einer höheren Arbeitsleistung niederschlägt (SBB AG und Swisscom (Schweiz) AG 2013, S. 4).

Freelancer sind freie Mitarbeitende, die ein Honorar für geleistete Dienste erhalten und somit nicht in einem traditionellen Arbeitsverhältnis arbeiten. Zumeist arbeiten Freelancer für verschiedene Auftraggeber. Freelancer sind vor allem in wissensintensiven Berufen tätig, wie etwa als Berater, Informatiker, Grafiker oder Übersetzer (Zobrist und Grampp 2016, S. 7 f.). Gemäß einer repräsentativen Befragung von Deloitte Schweiz und Research Now ist heute bereits jeder vierte Schweizer haupt- oder nebenberuflich als Freelancer tätig (Zobrist und Grampp 2016, S. 8). Der Einsatz von Freelancern ist für Unternehmen mit einer hohen professionellen Flexibilität verbunden, dem entgegen stehen höhere Transaktionskosten und Informationsrisiken. Zobrist und Grampp unterscheiden fünf Arten von Freelancern (Zobrist und Grampp 2016, S. 8 f.):

- Independent Contractor: „Klassische“ Freelancer, die hauptberuflich temporäre und projektbasierte Arbeiten erledigen (ca. $37 \%$ ).

- Moonlighter: Freelancer in Nebentätigkeit, die hauptberuflich einer „traditionellen“ Arbeit nachgehen (ca. $30 \%$ ).

- Diversified Worker: Beschäftigte, die ihr Einkommen mit unterschiedlichen Auftraggebern verdienen (z. B. Teilzeitarbeitnehmerin in einem Unternehmen, die nebenbei für Uber fährt und Online-Englischunterricht gibt) (ca. $23 \%$ ).

- Business Owner: Unternehmer mit bis zu fünf Mitarbeitenden, die sich selbst als Freelancer bezeichnen (ca. $8 \%$ ).

- Temporary Worker: Freelancer, die für einen einzigen Arbeitgeber oder Kunden temporär arbeiten (ca. $2 \%$ ).

Es kann davon ausgegangen werden, dass der Anteil der Freelancer im Sinne einer mit der Digitalisierung einhergehenden weiteren Flexibilisierung der Arbeit in den kommenden Jahren weiter ansteigen wird.

Eng verbunden mit dem Freelancer-Ansatz sind Human Cloud-Arbeitslösungen. Human Cloud-Plattformen wie upwork.com, freelancer.com oder peopleperhour.com zeichnen sich dadurch aus, dass auf ihnen von Arbeitsanbietern und -nachfragern online rund um die Uhr und weltweit Angebote zur Aufgabenwahrnehmung oder für Projektarbeiten platziert werden. Berufliche Tätigkeiten werden in konkrete Aufgaben und separate Projekte aufgeteilt und über die virtuelle Plattform an verschiedene ortsunabhängige selbstständige Arbeitskräfte verteilt, respektive von diesen als befristete Dienstleistungen eingekauft. Im Falle des Erbringens der Dienstleistungen von selbstständig Erwerbstätigen 
sind die einkaufenden Leistungsbezieher nicht verpflichtet, Mindestlöhne und Sozialversicherungsbeiträge zu zahlen (Schwab 2016, S. 73 f.). Für die anbietenden Arbeitskräfte bedeutet diese Form der Arbeitsbeschaffung eine weitgehend autonome Festlegung des Work-Life-Mixes mit größtmöglicher Flexibilität und Mobilität (Schwab 2016, S. 75).

Coaching- und Mentoringansätze der Führung sind sich abzeichnende Antworten auf die geänderten Führungsanforderungen in den flachen Hierarchien zunehmend selbstorganisierter Systeme. In einem solchen Kontext ist die wichtigste Aufgabe von Führungskräften die Schaffung von Verbindlichkeit in flexiblen Netzwerkstrukturen, das Inspirieren und Motivieren ihrer Teams und die kompetenzorientierte Optimierung der Aufgabenzuordnungen zu Personen sowie die systematische Förderung der Kompetenzentwicklung der Mitarbeitenden (Sattelberger et al. 2015, S. 133 f.). Beispiele für eine entsprechende Führungsperspektive sind die kontextabhängigen Ansätze einer systemischen oder symbolischen Führung. Die systemische Führung kommt vor allem bei laufenden Veränderungsprozessen und bei komplexen und unsicherheitsbehafteten Situationen zur Anwendung. Vor diesem Hintergrund führt der Vorgesetzte vor allem indirekt und legitimiert seine Autorität aus der Aufgabe und nicht aus der hierarchischen Position heraus (WEKA 2016). Vorgesetzte agieren hier als Navigatoren in selbstorganisierten Systemen und unterstützen die Mitarbeitenden beim Umgang mit Unsicherheit und Mehrdeutigkeiten. Bei der symbolischen Führung führt der Vorgesetzte über Zeichen, die für bestimmte Verhaltensweisen, Überzeugungen, Selbstbilder und Erwartungen stehen (WEKA 2016). Führen heißt hier vor allem reflektierte Inszenierung gewünschter Verhaltensweisen und Einstellungen durch die Führungskraft über symbolisches Handeln, wodurch auch die kulturellen Rahmensetzungen zum Ausdruck kommen.

In struktureller Hinsicht sehen Sattelberger et al. in Verbindung mit der Digitalisierung für die Zukunft vor allem drei alternative neuartige Organisationstypen, die im Organisationsentwicklungsprozess miteinander in Konkurrenz stehen (Sattelberger et al. 2015, S. 48 ff.). In „flachen Macht-Pyramiden“ kommt die Macht unverändert von oben, und es wird top-down gesteuert, allerdings mit nur wenigen Hierarchieebenen und unterstützt durch dosierte Kulturveränderungen in Verbindung mit den neuen Arbeitsformen. Bei der „Machtverteilung“ liegt ein holokratisches Strukturverständnis zugrunde, das Partizipation und Transparenz in demokratischen und agilen Netzwerkstrukturen favorisiert. Es gibt mehr Teilhabe und Souveränität der Mitarbeitenden, die sich weitgehend selbst führen, sowie Open Innovation. Anstelle der klassischen Strukturen und der hierarchischen Position der Führung werden in holokratischen Organisationsformen „Rollen“ gelebt, die alle Mitarbeitenden innehaben (Meyer 2016). „Machtkonzentration“ steht als letzter hybrider Organisationstypus für eine zweifache Machtkonzentration. Machtbasis ist die Innovation, und die Machtspitze wird durch die strategische und finanzielle Führung repräsentiert. Die operative Handlungskompetenz ist hier über das Empowerment der Mitarbeitenden dezentralisiert, die Steuerung erfolgt jedoch nach feudalistischen Prinzipien. Loyale und transformationsfähige Mitarbeiter, welche als Kernbelegschaft das Wissen des Unternehmens sichern, werden spirituell vereinnahmt. 
Berufliche Karrieren werden entweder über Projekte oder als Expertenkarrieren innerhalb einer offenen Wissenscommunity entwickelt (Sattelberger et al. 2015, S. 48 ff.).

Neuere Ansätze der Büroraumgestaltung tragen diesen strukturellen und führungsbezogenen Veränderungen im physischen Arbeitsumfeld Rechnung. Mobil-flexible Büronetzwerke integrieren die Büro- und Heimarbeitswelten und mobil-flexibles Arbeiten, das Arbeitsinhalte in Projekten strukturiert, wird immer mehr zu einer Normalität, wie sie in vielen Großunternehmen heute schon vorgefunden werden kann (Witzig the office company 2014). In Verbindung mit der aufstrebenden Sharing Economy erfolgt eine Erweiterung der Raumperspektive über das Homeoffice hinaus auf temporär genutzte und angemietete Büroräume. Coworking Spaces sind derzeit im Trend und werden über Plattformanbieter wie LiquidSpace oder ShareDesk vermarktet. Insbesondere Freelancer, Jungunternehmer und Mitarbeitende von Unternehmen nutzen die stundenweise Anmietbarkeit von Büroplätzen oder Meetingräumen in zumeist zentralen City-Lagen immer mehr (Zobrist und Grampp 2016, S. 11). Im Jahr 2017 gab es in der Schweiz bereits an über 180 Standorten auf mehr als 70.000 Quadratmetern Arbeitsfläche rund 6700 Coworking-Arbeitsplätze. Der Median hinsichtlich der Zahl der Arbeitsplätze liegt bei 20, die verfügbare Arbeitsfläche schwankt dabei zwischen 30 und 2500 Quadratmeter. Insbesondere Unternehmen mit mehr als 1000 Mitarbeitenden möchten in den kommenden Jahren unter Flexibilitäts- und Kostengesichtspunkten für ihre Mitarbeitenden solche Coworking Spaces dauerhaft zur Verfügung stellen (Auf der Maur 2017, S. 7 ff.). Ein visionäres Zukunftsbild digitaler Arbeitswelten entwickeln Zinser und Boch (2007, S. 34 f.): In vernetzten Städten sind Büro-, Projekt- und Ideenräume und Think Tanks als intelligente Knotenpunkte präsent, die urbanes Wohnen und Arbeiten integrierbar machen.

\subsubsection{Chancen und Risiken der Digitalisierung für Arbeitsmodelle}

Mit der Digitalisierung und den angeführten komplementären und unterstützenden Entwicklungstendenzen bei der Ausgestaltung von Arbeitsmodellen sind für Unternehmen Chancen und Risiken verbunden.

Auf der Chancenseite neuer flexibler und mobilitätsorientierter Arbeitsmodelle werden aus Sicht der Angestellten vor allem eine höhere persönliche Flexibilität, eine bessere Vereinbarkeit von Familie und Beruf sowie die Reduktion der Arbeitswegzeiten angeführt. Unternehmen profitieren von einer effizienteren Nutzung der Büroflächen, sparen Kosten, können sich als attraktiver Arbeitgeber am Arbeitsmarkt positionieren und haben produktivere und zufriedenere Mitarbeiter. Vermietet das Unternehmen zudem Arbeitsplätze an Externe, können zusätzliche Einnahmen generiert sowie neue Netzwerke erschlossen werden, auch im Hinblick auf die steigende Anzahl an Freelancern (Zobrist und Grampp 2016, S. 16). Generell ermöglicht die Digitalisierung eine erleichterte Flexibilisierung des Arbeitsmarkts (Reynard und Derder 2017, S. 4). Mit den neuen 
Arbeitsmodellen im digitalen Zeitalter gehen auch eine höhere Innovationsfähigkeit und potenzielle Schübe für die Entwicklung neuer Produkte und Dienstleistungen einher (Schweizer Verband der Telekommunikation 2015, S. 20).

Der digitale Fortschritt ermöglicht heute ein Arbeiten, wann und wo immer man dies möchte. Dies hat jedoch aus Sicht der Arbeitnehmenden auch seine Schattenseiten, denn gleichzeitig haben immer mehr Menschen Schwierigkeiten, Arbeit und Privatleben sowie berufliche und familiäre Verpflichtungen in Einklang zu bringen. Dies resultiert in einer zunehmenden psychischen Arbeitsbelastung in Form von Stress, zu der auch das Mehr an Verantwortung und Autonomie beitragen kann. Gemäß der repräsentativen Schweizer Job-Stress-Index-Studie von 2016 ist jeder vierte Erwerbstätige gestresst im Job. Die Ausfälle von Mitarbeitenden aufgrund von Stress kosten die Schweizer Arbeitgeber jährlich ca. 5,7 Mrd. Franken. In welchem Umfang hierbei spezifische Stressfaktoren des digitalen Wandels der Arbeitswelt beteiligt sind, ist allerdings noch unklar und schwer einschätzbar (Genner et al. 2017, S. 39 f.). Die neuen Arbeitsmodellformen können bei den betroffenen Beschäftigten auch Ängste auslösen. Nicht jeder bringt von vorneherein das ideale Soll-Kompetenz- und Persönlichkeitsprofil eines selbstorganisierten, autonom und hochflexibel arbeitenden Experten mit, der sich souverän in agilen Netzwerkstrukturen bewegen kann. Außerdem geht mit der Digitalisierung der Arbeit auch bei vielen die Sorge einher, dass derzeitige menschliche Bürotätigkeiten immer mehr von intelligenten digitalen Systemen ersetzt werden. So sind gemäß der Studie „The Future of Employment" von Frey und Osborne aus dem Jahr 2013 fast 50 \% der derzeitigen Arbeitsplätze dem Risiko einer Eliminierung durch digitale Rationalisierung ausgesetzt (Frey und Osborne 2013). In Verbindung mit der Gestaltung der Rahmenbedingungen neuer Arbeitsformen und -strukturen (z. B. physische und virtuelle Präsenzzeiten und -orte, Arbeits- und Ruhezonenraumgestaltung etc.) besteht bei entsprechenden Defiziten die Gefahr, dass sich bei den Mitarbeitenden ein Identifikations- und Bindungsverlust gegenüber dem Arbeitgeber einstellt und der soziale Austausch mit Kollegen leidet oder gar verloren geht. Die erhöhte Flexibilität und räumliche Ungebundenheit mit globaler Datenverfügbarkeit geht ferner mit nicht unbeträchtlichen Risiken bezüglich der Sicherheit von sensiblen Informationen und Daten einher (Zobrist und Grampp 2016, S. 17). Im Hinblick auf die arbeitsrechtlichen Rahmensetzungen besteht bei einem Ausnutzen rechtlicher Grauzonen (z. B. Mindestlohnthematik) bei der Nutzung von Arbeitsvermittlungsplattformen auf Dauer die Gefahr, dass mit restriktiven Interventionen des Gesetzgebers gerechnet werden muss und gegebenenfalls die Reputation als Arbeitgeber leiden kann.

\subsection{Zwischenfazit der konzeptionellen Analyse}

Digitalisierung steht in Verbindung mit der Arbeitswelt heute insbesondere für digitale Arbeitsprozesse und -inhalte, die Beschleunigung von Arbeit und mobil-flexible Arbeitsformen. Neue Ausprägungsformen von Arbeitsmodellen im Sinne von mehrdimensionalen 
Systemen der Zusammenarbeit können anhand der fünf Dimensionen Arbeitszeit, Arbeitsform, Arbeitsführung, Arbeitsstruktur und Arbeitsraum charakterisiert werden. In Verbindung mit der Digitalisierung weisen diese jeweils für sich oder in Kombination Entwicklungstendenzen in Richtung Flexibilisierung, Individualisierung, Selbstorganisation, Autonomie der Mitarbeitenden, partnerschaftliche systemisch-symbolische Führung, Holokratie, Agilität und Mobilität auf. Bei der Einführung der zukunftsgerichteten Arbeitsmodelle sind die geltenden gesetzlichen Regelungen als Restriktion zu berücksichtigen. Die digitalisierungsbasierten oder -unterstützten Arbeitsmodelle sind für die Unternehmen mit einer Vielzahl an Effizienz-, Effektivitäts- und Innovationspotenzialen verbunden, weisen aber auch eine Reihe von Risiken auf, die mit einer defizitären Einführung und Umsetzung, beziehungsweise fehlender oder falsch gesetzter Rahmenbedingungen verbunden sind. Die konzeptionelle Grundsystematik des Zusammenhangs von Digitalisierung, Arbeitsmodellen und Unternehmenserfolg ist in Abb. 7.1 dargestellt.

In Bezug auf die Einführung und Anwendung modifizierter oder neuer Arbeitsmodelle, die die neuen Möglichkeiten der Digitalisierung nutzen, beziehungsweise auf diesen basieren, gibt es in empirischer Hinsicht noch eine geringe wissenschaftliche Aufbereitung der Thematik. Die nachfolgende deskriptive Analyse des Einsatzes moderner Arbeitsmodelle in Schweizer Großunternehmen setzt an dieser identifizierten Forschungslücke mit der Intention an, Hinweise für Best Practices und Optimierungspotenziale von Arbeitsmodellen in der Praxis abzuleiten.

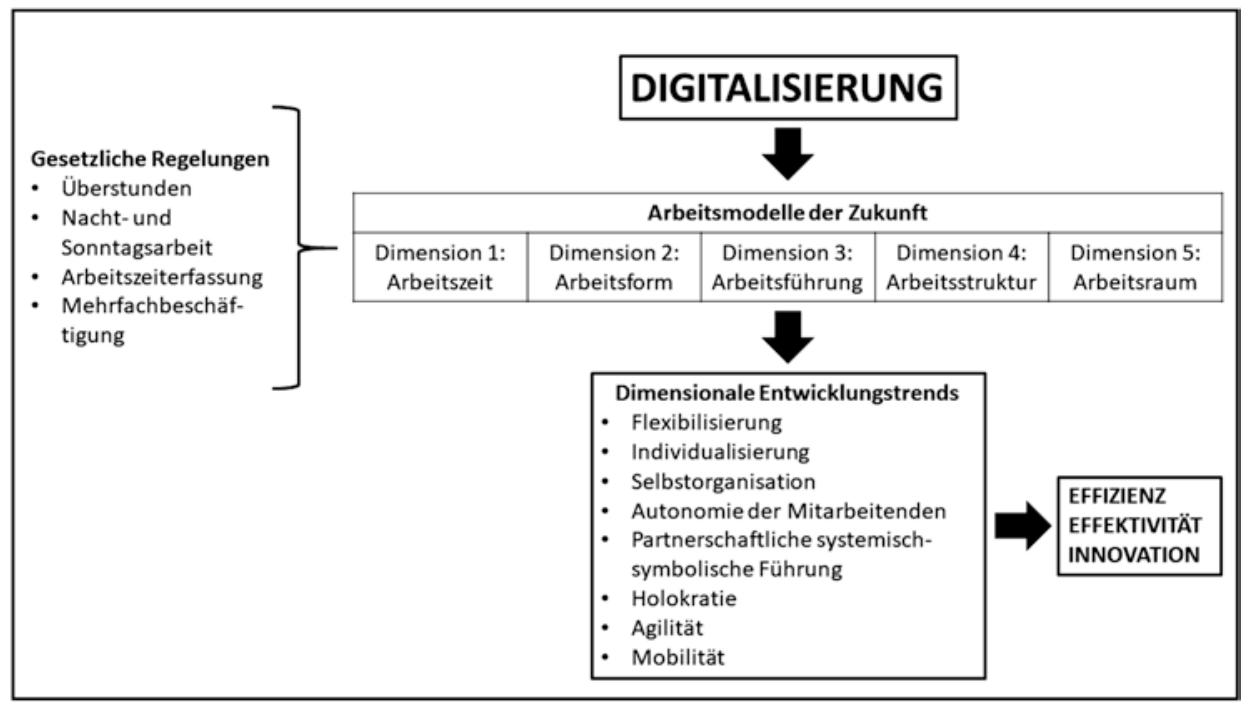

Abb. 7.1 Digitalisierung, Arbeitsmodelle und Unternehmenserfolg. (Eigene Darstellung) 


\subsection{Empirische Untersuchung}

In einem ersten Schritt wird im folgenden Abschnitt zunächst das methodische Vorgehen der empirischen Analyse gekennzeichnet. Es folgen danach eine Darstellung der wichtigsten Ergebnisse der Erhebung und eine abschließende Diskussion.

\subsubsection{Forschungsmethodik}

Bei der Festlegung des Forschungsansatzes wurde das Vorgehen an der Prozessdarstellung von Glogner-Pilz (2012) gemäß Abb. 7.2 ausgerichtet.

Vor dem Hintergrund der interessierenden offenen Fragestellungen wurde kein quantitatives, sondern ein interpretatives qualitatives Vorgehen für die Erhebung gewählt, bei dem das subjektbezogene Verstehen von Zusammenhängen, Erfahrungen und Verhaltensweisen in Bezug auf ausgewählte Gesprächspersonen im Vordergrund steht (Röbken und Wetzel 2016, S. 12 ff.). In Verbindung mit der Fokussierung auf die Realsituation in Großunternehmen wurde ein vergleichender Fallstudienansatz auf der Grundlage leitfadengestützter Experteninterviews als geeignet angesehen.

Insgesamt wurden zehn Interviews in drei Schweizer Großunternehmen durchgeführt, bei denen vermutet werden konnte, dass bereits Erfahrungen bei der Umsetzung von digitalen Veränderungsprozessen und der Anwendung von hiermit verbundenen neuen Arbeitsmodellen bestehen. Selektionskriterien für die Samplebildung, respektive Fallauswahl waren die Klassifizierung als Großunternehmen mit mehr als 250 Mitarbeitenden und Hauptstandort in der Schweiz, als besonders innovativ bekannte Großunternehmen oder als Unternehmen, die die Charta der Work Smart-Initiative (Work Smart Initiative 2018) unterzeichnet haben. Außerdem wurde themenbezogen ein Schwerpunkt auf besonders von der Digitalisierung betroffene kundenorientierte Unternehmen und Organisationen in Dienstleistungsbranchen und der öffentlichen Verwaltung gelegt. Vor diesem Hintergrund konnten 25 potenziell geeignete Unternehmen identifiziert und im März/April 2018 angefragt werden. Fünf der angefragten Unternehmen/Organisationen waren grundsätzlich für eine Mitwirkung an der Studie offen, von den fünf wurden im Hinblick auf die Kriterienerfüllung drei ausgewählt, die alle auch die Charta der Work Smart-Initiative unterzeichnet haben. Konkret handelt es sich bei den ausgewählten Organisationen um die Schweizerische Bundesbahnen (SBB) mit fünf Interviewpartnern, das Bundesamt für Informatik (BIT) mit drei Interviewpartnern und ein namhaftes Versicherungsunternehmen mit zwei Interviewpartnern, das sich anonym an der Studie beteiligt hat. Die Interviews wurden anhand eines Leitfadens im Frühling/Sommer 2018 vor Ort am jeweiligen Arbeitsplatz der Interviewpartner durchgeführt. Der Interviewleitfaden wurde auf Wunsch den Befragten vorgängig zugestellt. Die Interviews wurden zudem alle in Mundart geführt, damit keine sprachlichen Hürden entstanden. Die Befragten gehören verschiedenen Funktionen und Hierarchieebenen an und zeichnen sich alle durch ein Arbeitsumfeld aus, das durch neue Formen der Zusammenarbeit und 
Abb. 7.2 Prozessablauf der empirischen Untersuchung. (Eigene Darstellung in Anlehnung an Glogner-Pilz 2012)

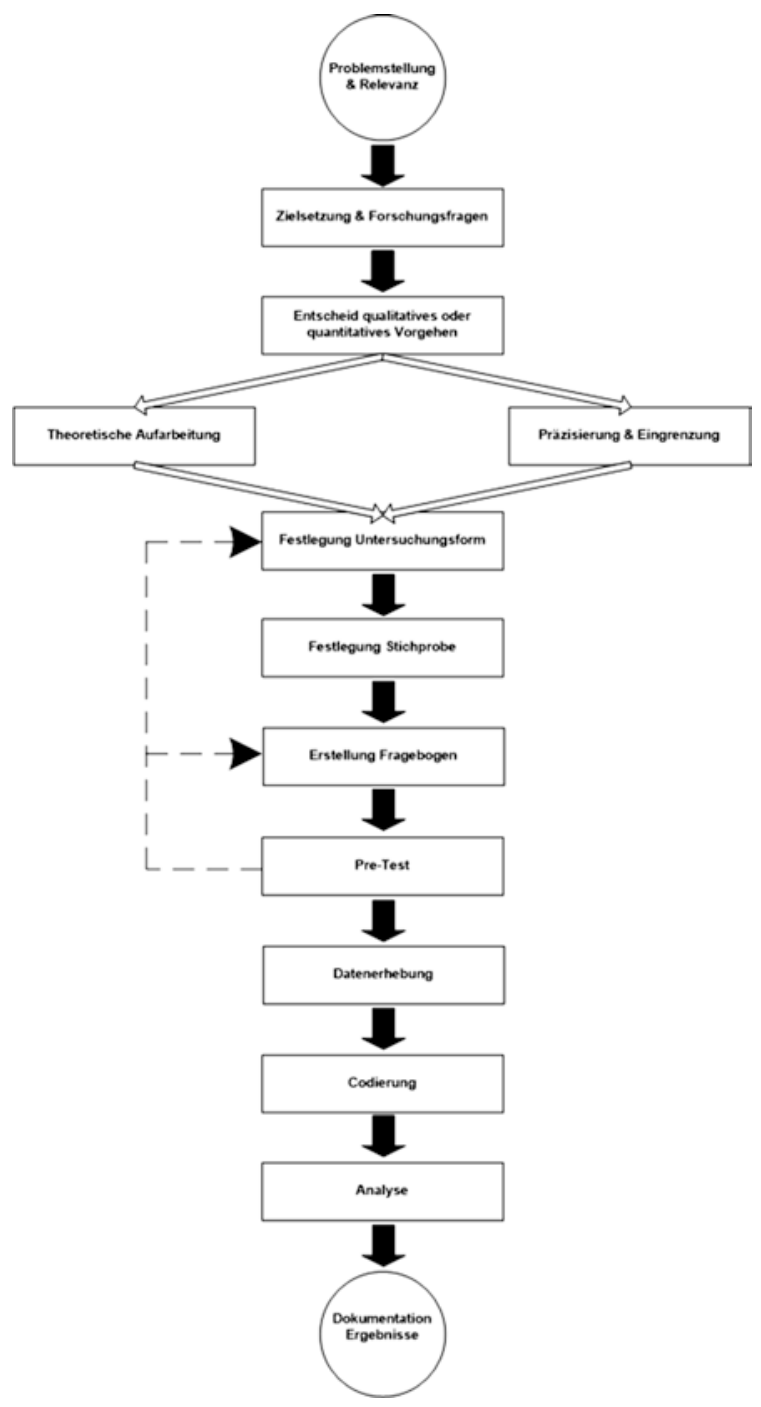

Entwicklungen im Umfeld der Digitalisierung geprägt ist (Human Resource Management, IT-Abteilungen, Organisationsentwicklung, Projektmanagement). Die Breite der Profile soll eine Abdeckung verschiedener Aspekte der Thematik Digitalisierung und Arbeitsmodelle gewährleisten.

Für die Interviews wurde ein leitfadengestützter Fragebogen mit sieben Hauptforschungsfragen und achtundzwanzig Subfragen erarbeitet. Die Fragen wurden bewusst sehr offen formuliert, um die Thematik nicht zu sehr konzeptionsgeleitet vorzustrukturieren und Raum für ungelenkte subjektive Praxisperspektiven zu schaffen. Der 
Interviewleitfaden wurde im Rahmen eines Pre-Tests mit mehreren neutralen Personen geprüft und angepasst.

Die durchgeführten und aufgezeichneten Interviews wurden anschließend transkribiert. Die Transkripte wurden danach in Anlehnung an die Themenstruktur des Fragebogens codiert und mittels der Analyse-Software ATLAS.TI ausgewertet.

\subsubsection{Ergebnisse}

Die Ergebnisse werden themenfeldbezogen zunächst anhand fallstudienübergreifender Konsensaussagen der Interviewten vorgestellt und dann fallbezogen weiter konkretisiert.

\subsubsection{Bedeutung und Auswirkungen der Digitalisierung für Unternehmen und Mitarbeitende}

Die Breite der begrifflichen Festlegung dessen, was „Digitalisierung“ inhaltlich bedeutet, hat sich auch bei den Experteninterviews gezeigt. Alle Teilnehmenden der Befragung sehen die Aktualität und Relevanz des Themas und verbinden dieses vor allem mit der Effizienzsteigerung elektronischer Prozesse und neuer Geschäftsmodelle. Vereinzelt wird der Begriff auch mit „Automatisierung“ gleichgesetzt. Die hohe Bedeutung der Digitalisierung für auf alle Bereiche der Gesellschaft und für die menschliche Arbeit wird von allen Befragten wahrgenommen (BIT (P6, P7, P9) 2018; SBB (P1, P2, P3, P5, P10) 2018; Versicherungsunternehmen (P4, P8) 2018). Die Befragten der SBB betrachten darüber hinaus die Digitalisierung zudem als Synonym für die papierlose Vernetzung der Daten und Einheiten mit Hard- und Software und sehen mit ihr auch eine bestimmte Arbeitskultur und Denkhaltung für digitale Veränderungen und den Umgang mit wachsender Volatilität, Unsicherheit, Komplexität und Mehrdeutigkeiten verbunden (SBB (P1, P2, P3, P5, P10) 2018). Beim BIT wurde ferner auf einen definitorischen Ansatz der Universität St. Gallen Bezug genommen: „Unter digitaler Transformation verstehen wir die Kombination von Veränderungen in Strategie, Geschäftsmodellen, Organisationsprozessen und -kulturen in Unternehmen durch Einsatz von digitalen Technologien mit dem Ziel, die Wettbewerbsfähigkeit zu steigern“"(BIT (P6) 2018, Z. 12 f.). Ergänzend hierzu wird im BIT im Rahmen der Digitalisierung ein vermehrter Einsatz von Maschinen mit intelligenten Programmen und mit künstlicher Intelligenz verknüpft (BIT (P6, P7, P9) 2018). Im Versicherungsunternehmen wird in einem engeren Verständnis Digitalisierung mit der Zurverfügungstellung digitaler Daten gleichgesetzt und in einem weiteren Sinne eine digitale Prozessgestaltung und -programmierung sowie ein hohes Maß von Automatisierung als wesentlich betrachtet (Versicherungsunternehmen (P4, P8) 2018).

Die Auswirkungen der Digitalisierung auf die Unternehmen und deren Mitarbeitende sind untrennbar miteinander verbunden. Alle Befragten sehen eine generelle Beschleunigung der Aktivitäten und gestiegene Erwartungshaltungen der Kunden, die durch das Internet eine wachsende Markttransparenz in Bezug auf die Qualität und Preise der Produkte und Dienstleistungen für sich nutzen. Dies führt insgesamt zu 
einem höheren Konkurrenzdruck (BIT (P6, P7, P9) 2018; SBB (P1, P2, P3, P5, P10) 2018; Versicherungsunternehmen (P4, P8) 2018). Bei der SBB wird die Digitalisierung ferner als vergleichbar mit anderen Veränderungen wahrgenommen: „Es wird Sachen geben, welche es zukünftig nicht mehr geben wird, aber es wird auch Neues entstehen, wovon wir aktuell noch gar nicht wissen, dass wir es einmal brauchen werden“ (SBB (P1) 2018, Z. 286 f.). Insgesamt sei durch die Digitalisierung heute schon die Zusammenarbeit anders geworden und die Kommunikation über digitale Kanäle habe zugenommen. Mit dem „Working Anywhere“ hat die SBB ein Arbeitsmodell eingeführt, das es den Mitarbeitenden ermöglicht, ortsunabhängig und flexibel zu arbeiten, sodass auch die Züge entlastet werden. Wichtig bei der Digitalisierung sei, sich zu fragen, wohin man wolle, sich auf die wesentlichen Fragestellungen zu konzentrieren und dann die erforderlichen Ressourcen einzusetzen (SBB (P1, P2, P3, P5, P10) 2018). Das Versicherungsunternehmen arbeitet mit einem gleichgerichteten Modellansatz „Work Anywhere, Anytime and Smart“. Darüber hinaus wurde die Entwicklungsabteilung „Unit Development" initialisierend komplett digitalisiert. Aus Sicht der Befragten des Versicherungsunternehmens ist die Digitalisierung die nächste ,industrielle Revolution“. Sie habe die gleichen Auswirkungen wie die Erfindung der Dampfmaschine. Dank der Digitalisierung würden nicht nur neue Jobprofile entstehen, sondern auch ergänzende neue Kommunikationskanäle zwischen Mitarbeitenden und Kunden: „Kunden nutzen andere Zugänge; über WhatsApp werden Kundenberater heute kontaktiert, was noch vor ein paar Jahren undenkbar gewesen wäre“ (Versicherungsunternehmen (P4) 2018, Z. 14 f.). Ferner würden durch die Digitalisierung auch neue Geschäftsmodelle ermöglicht, die die Konkurrenzfähigkeit auch in Zukunft gewährleisten sollen (Versicherungsunternehmen (P4, P8) 2018). Aus Sicht der Befragten des BIT verändert die Digitalisierung das Zusammenarbeiten und das Führen von Mitarbeitenden nachhaltig: Die Freiheitsgrade werden größer, es entstehen neue Arbeitsformen und übergreifende Tätigkeiten und die Orientierung an individuellen Bedürfnissen wird begünstigt. Außerdem verbessere sich der Umgang mit Unsicherheit und Ungewissheit. Hier stehe man bei den Digitalisierungsauswirkungen allerdings erst am Anfang einer weitgehenden Veränderung (BIT (P6, P7, P9) 2018).

\subsubsection{Aktuelle Arbeitsmodelle - Ausprägungsformen und Erfolgswirksamkeit}

Die Frage danach, welche aktuellen Arbeitsmodelle derzeit bei den untersuchten Großunternehmen/-organisationen zum Einsatz kommen, wurde zunächst von den Teilnehmenden mit Bezug auf die Arbeitszeitgestaltung hin beantwortetet. Sie verwiesen durchgängig auf vorhandene Jahresarbeitszeit-, Vertrauensarbeitszeit- und Gleitzeitmodelle. Obwohl die untersuchten Organisationen somit alle im Grundsatz eine flexible Arbeitsgestaltung ermöglichen, gibt es in verschiedenen Bereichen und für bestimmte Arbeitstätigkeiten nach wie vor noch klassische Arbeitsmodelle (BIT (P6, P7, P9) 2018; SBB (P1, P2, P3, P5, P10) 2018; Versicherungsunternehmen (P4, P8) 2018). So sind beispielsweise bei der SBB Nichtbürotätigkeiten im Betrieb vielfach noch traditionell 
hierarchisch organisiert. Andere Bereiche wie IT oder HR arbeiten stattdessen mit agileren Arbeitsformen. In der HR-Abteilung der SBB wurde beispielsweise vor kurzem „Holacracy“ als Strukturmodell eingeführt. Charakteristisch für solche agilen Arbeitsmodelle bei der SBB seien Teamorientierung, Selbstorganisation, eine erhöhte Autonomie und eine geringere Anzahl an Führungsstufen, respektive flache Hierarchien. Die agilen Arbeitsformen seien leistungs-, output-, und zusammenarbeitsorientiert. Im Weiteren habe man weniger Präsenzzeiten und Homeoffice sei in Absprache mit dem/der Vorgesetzten erlaubt. Auch flexible Pensionierungen sind in der SBB möglich. So kann man etwa ein Zeitguthaben ansparen und in einem gewissen Umfang selber entscheiden, wann man in den Ruhestand eintritt (SBB (P1, P2, P3, P5, P10) 2018). Beim Versicherungsunternehmen gibt es eine klassische „Run-the-Business“-Arbeitsperspektive und agilere, projektbasierte „Change-the-Business“-Arbeitsweisen. Das Versicherungsunternehmen arbeitet in Projekten vor allem mit der agilen Methode „Scrum“ und der Anwendung der ,SAFe“-Logik (Scaled Agile Framework), anhand derer Umsetzungsvorhaben vorgängig gesammelt, priorisiert, unternehmensweit abgestimmt und den Teams zugeteilt werden. Ziel hierbei ist es, die Vorhaben sinnvoll zu bündeln und Konflikte zwischen den verschiedenen Schnittstellen zu senken. Es kommen dabei stabile interdisziplinäre und autonome Teams zum Einsatz (Versicherungsunternehmen (P4, P8) 2018). Das BIT verwendet verschiedene Arbeitsmodelle, die zu den jeweiligen Lebenssituationen und -abschnitten der Mitarbeitenden passen. Für Einsteiger bietet das BIT einerseits die Lehre, und andererseits auch ein praxisintegriertes Bachelorstudium oder ein Berufseinsteiger-Programm für Hochschulpraktikanten an. Für Mitarbeitende, welche eine Familie gründen wollen oder diese bereits haben, besteht die Möglichkeit, in Teilzeit oder Jobsharing zu arbeiten. Für ältere Personen können „Bogenkarrieren“ derart umgesetzt werden, dass Führungspersonen Verantwortung abgeben, und stattdessen eine Beratungsfunktion übernehmen. Ferner sind auch frühzeitige Pensionierungen oder Teilpensionierungen möglich. Darüber hinaus haben Mitarbeitende des BIT die Möglichkeit, Überzeiten für ein Sabbatical-Konto anzusammeln oder sich diese auszahlen zu lassen. Im BIT besteht ferner die Option, „Remote Work“ im Sinne eines orts- und zeitunabhängigen Arbeitens zu praktizieren. Mitarbeitende im BIT haben nicht automatisch ein Anrecht auf Homeoffice, sondern dies wird fallweise betrachtet. Beim BIT ist die Jahresarbeitszeit das dominante Modell für alle Mitarbeitenden. Bei Führungskräften kommt die Vertrauensarbeitszeit zum Einsatz. Die entsprechenden Festlegungen können aber personen- und teamabhängig variieren. Da das BIT aufgabenbedingt über arbeitszeitbezogene Sonderbewilligungen des Bundes verfügt, stellt flexibles Arbeiten beispielsweise auch an Wochenenden kaum ein Problem dar (BIT (P6, P7, P9) 2018).

Hinsichtlich der Fragestellung, ob und in welcher Form sich die bisherigen Arbeitsmodelle bewährt haben, wurde seitens der Befragten der SBB darauf verwiesen, dass die Erfolgswirksamkeit der eingesetzten Modelle eine Sache der Gewohnheit, Einfachheit und der sozialen Normierung sei. Arbeitsmodelle müssten zur aktuellen Entwicklung und der gegenwärtigen Unternehmenssituation passen. Deshalb gäbe es bei der SBB Arbeitsmodelle, die früher schon funktioniert haben und auch heute noch funktionieren 
würden. Die kontinuierliche Fortführung und Weiterentwicklung von Arbeitsmodellen bringe Stabilität und Sicherheit (SBB (P1, P2, P3, P5, P10) 2018). In eine ähnliche Richtung geht die Einschätzung der BIT-Teilnehmenden. Die praktizierten BIT-Arbeitsmodelle hätten bislang die Bedürfnisse der einzelnen Mitarbeitenden gut abdecken können und seien an der Individualisierung und Flexibilisierung der Arbeit ausgerichtet. Dies fördere auch die Zufriedenheit der Mitarbeitenden (BIT (P6, P7, P9) 2018). Beim Versicherungsunternehmen gibt es Arbeitsmodelle, die sich mehr bewährt und solche, die sich weniger bewährt haben. Besonders bewährt hätten sich diejenigen Arbeitsmodelle, die effizienzorientiert seien. In einem explorativen oder Projektumfeld hätten sich insbesondere die flexiblen Arbeitsmodelle bewährt (Versicherungsunternehmen ( $\mathrm{P} 4$, P8) 2018).

\subsubsection{Chancen und Problemfelder für bestehende Arbeitsmodelle}

Alle Befragten waren sich einig darüber, dass mit der Digitalisierung und der damit einhergehenden Möglichkeiten der Arbeitsmodellgestaltung viele Vorteile und Chancen verbunden seien. Eine bessere Vereinbarung von Beruf und Privatleben wurde bei allen Fallstudienteilnehmenden als positiver Effekt genannt. Auch die gestiegene Flexibilität sowie orts- und zeitunabhängiges Arbeiten sehen alle als Vorteil für das Unternehmen/ die Organisation und die Mitarbeitenden. Außerdem werden für die Mitarbeitenden mehr Möglichkeiten zur Übernahme von Verantwortung, zur Veränderung und zur Erweiterung des eigenen Horizonts durch interdisziplinäres Arbeiten und Wissensaustausch angeführt. Die Befragten sehen vor allem die flexiblen Arbeitsmodelle als wichtigen Faktor, um sich am Arbeitsmarkt als attraktiver Arbeitgeber positionieren zu können. Als nachteilig in Verbindung mit den praktizierten modernen Arbeitsmodellen erachten die Befragungsteilnehmenden, dass die Mitarbeitenden durch erhöhte Anforderungen und mehr Verantwortung auch überfordert oder psychisch überlastet werden können. Zudem werde der persönliche Kontakt durch die digitalen technologischen Möglichkeiten geringer (BIT (P6, P7, P9) 2018; SBB (P1, P2, P3, P5, P10) 2018; Versicherungsunternehmen (P4, P8) 2018). Bei der SBB werden agile Arbeitsmodelle auch mit Einsparungen an Büroflächen in Verbindung gebracht. Viele Mitarbeitende würden von zu Hause aus oder an anderen Orten außerhalb des Büros arbeiten. Für die Arbeitnehmer reduziere sich mit der flexiblen Arbeitsgestaltung vielfach der Stress, da sie auch private Termine wahrnehmen könnten und nicht fix im Büro bleiben müssten bis alle Pendenzen erledigt seien. Im Kontext der Digitalisierung wurden bei der SBB eine Reihe von Projekten zur Arbeitsmodell(weiter)entwicklung gestartet. Ein exemplarisches Projekt ist „MEET“ das zum Ziel hat, Mitarbeitende aller Hierarchieebenen miteinander digital zu verbinden. Arbeitsrelevante Informationen sollen durch gemeinsame digitale Ablagen (eSpace) einfacher für alle zugänglich gemacht werden. Die Mitarbeitenden sollen dadurch ihr Wissen verbreitern und weniger auf direkte Information durch Vorgesetzte angewiesen sein, die dadurch wiederum entlastet werden und sich stärker um ihre Mitarbeitenden und die Personalführung kümmern können (SBB (P1, P2, P3, P5, P10) 2018). Die Befragten des Versicherungsunternehmens sehen durch die angewandten modernen Arbeitsmodelle 
eine Beschleunigung und Effizienzsteigerung bei den Prozessen. Außerdem würden komplexe Aufgaben in interdisziplinären Teams effektiver bearbeitet und seien stärker an den Kundenbedürfnissen ausgerichtet. In diesem Zusammenhang sei auch die Vielfalt der gelebten Arbeitsmodelle im Vergleich zu früher größer geworden (Versicherungsunternehmen (P4, P8) 2018). Im BIT werden die Erweiterungen der Arbeitsmodelle als Reaktion auf die gestiegenen Anforderungen der Mitarbeitenden an die Arbeitgeber wahrgenommen. Hieraus seien neue Rollen geschaffen worden und das Führungsverhalten habe sich stärker in Richtung selbstorganisierter Teams entwickelt. Mitarbeitende würden dadurch autonomer und flexibler arbeiten, was wiederum deren Motivation steigere. Ein stärkeres Involvement der Kunden in Arbeits- und Entwicklungsprozesse trage zur Verbesserung der Kundenzufriedenheit bei (BIT (P6, P7, P9) 2018).

Von den Befragten der SBB wird neben einer möglichen psychischen Überlastung von Mitarbeitenden durch die veränderten Arbeitsweisen wahrgenommen, dass es auch Mitarbeitende gibt, die die avisierte Flexibilität und Autonomie nicht wollen und lieber in stabilen hierarchischen Strukturen arbeiten. Gründe hierfür könnten Angst, Unsicherheit oder der Wunsch nach klaren Aufträgen oder Arbeitsleitplanken sein. Auch die Angst vor Arbeitsverlust oder einer unerwünschten Änderung der Arbeitstätigkeit spielten hierbei eine Rolle. So würden etwa zunehmend die bisherigen Arbeitstätigkeiten von Maschinen/Systemen ausgeführt und vom Menschen nur noch kontrolliert. Probleme in Verbindung mit der Digitalisierung und veränderten Arbeitswelten würden sich vor allem dann einstellen, wenn einzelne Menschen noch nicht in der Lage seien, mit der Digitalisierung und deren Auswirkungen auf ihre Arbeit umzugehen, denn „es hat noch keine Transformation gegeben, bei welcher alle mitgenommen wurden“ (SBB P2 2018, Z. 67 f.). Auch für die Vorgesetzten ändere sich die Führung: Sie müssten Macht an die Mitarbeitenden abgeben und sich aufgrund der erhöhten Flexibilität und Mobilität verstärkt mit den Mitarbeitenden abstimmen. Die Abstimmungen erfolgten dabei vermehrt nicht mehr persönlich, sondern digital. Man sitze nicht mehr im gleichen Raum und könne daher auch Mimik, Gestik, Stimmung und Atmosphäre schwerer wahrnehmen (SBB (P1, P2, P3, P5, P10) 2018). Für die Studienteilnehmenden des Versicherungsunternehmens sind mit den neuen Arbeitsmodellen im Digitalisierungskontext auch Unklarheiten bezüglich der Rollenverteilung verbunden. Ferner gingen durch die wachsende Agilität zunehmend Strukturen und Stabilität im Arbeitsalltag verloren. Mit den heutigen Arbeitsmodellen stoße man an operative Grenzen, da immer mehr Mitarbeitende agil und in Projekten arbeiten möchten, was etwa eine Zielplanung und -vereinbarung erschwere (Versicherungsunternehmen (P4, P8) 2018). Die Befragten des BIT sehen einen weiteren Nachteil der neuen Arbeitsmodellvarianten darin, dass feste Arbeitszeiten und der bis anhin fixe Arbeitsort nun zu einer Abmachungssache geworden seien. Man könne nicht mehr erwarten, die Kollegen im Büro automatisch anzutreffen, sondern müsse Termine im Vorfeld planen. Ferner sei das hohe Maß der eigenen Variabilität und Flexibilität bei der Zusammenarbeit mit Partnerunternehmen und -organisationen oftmals schwierig für die Zusammenarbeit, da nicht alle auf einem vergleichbaren Agilitätsniveau unterwegs seien (BIT (P6, P7, P9) 2018). 


\subsubsection{Treiber für die Veränderung der Arbeitsmodelle}

Die wichtigsten Auslöser und Triebkräfte für die Weiter- und Neuentwicklung von Arbeitsmodellen sind bei allen untersuchten Organisationen mehrheitlich entweder nur auf Leitungsebene (top-down) oder in einem Leitungs-Basis-Mix (top-down - bottom-up) positioniert. Ausgangspunkt für Anpassungen sind (digitalisierungsbedingte) Veränderungen des Umfeldes, wie die Kundenanforderungen, die Bedürfnisse der Mitarbeitenden und die technologischen und kulturellen Entwicklungen sowie die Globalisierung und die Entwicklung neuer Medien. Externe Beratungsunternehmen spielen dabei bei den befragten Organisationen bislang keine Rolle, denn es sei schwierig von außen unternehmensspezifisch geeignete Arbeitsmodelle zu bestimmen (BIT (P6, P7, P9) 2018; SBB (P1, P2, P3, P5, P10) 2018; Versicherungsunternehmen (P4, P8) 2018). Bei der SBB wurden in Bezug auf den Anstoß und das Voranbringen von Arbeitsmodellanpassungen sowohl Top-down-, als auch Bottom-up- oder integrierte Vorgehensweisen ausgemacht. Im besten Falle kämen Impulse für Anpassungen direkt und selbstorganisiert aus den Teams heraus, was jedoch ein starkes Miteinander und eine hohe intrinsische Motivation der Teammitglieder voraussetze. Oftmals brauche es aber einen „Fahnenträger“, der die Digitalisierung und die Anpassung der Arbeitsmodelle von innen nach außen vorantreibe. Angesichts der Unternehmensgröße seien im Thema aber auch Top-Down-Prozesse erforderlich, um zu vermeiden, dass nicht jeder Bereich abgekoppelt für sich etwas erarbeite (SBB (P1, P2, P3, P5, P10) 2018). Beim Versicherungsunternehmen kommt der Anstoß für Veränderungen aus dem HR-Bereich und vom CEO. Besonders wichtig dabei sei, dass man den Mitarbeitenden erkläre, warum die Anpassungen vorgenommen werden müssten und was deren Nutzen sei (Versicherungsunternehmen (P4, P8) 2018). Auch beim BIT wird die Digitalisierung und Entwicklung der Arbeitsmodelle primär von der Geschäftsleitung in Verbindung mit den veränderten Geschäftsanforderungen der Kunden vorangetrieben. Derzeit steht dabei der Prozessverbesserungsansatz „DevOps“ im Mittelpunkt, der das Zusammenwachsen von Entwicklung, Engineering und Betrieb zum Gegenstand hat. Mit DevOps einher geht ein Arbeitsmodell, das den End-to-End-Aspekt fokussiert. Ein DevOps-Team bearbeitet dabei ein Projekt oder einen Kundenauftrag von Anfang bis Ende. Das Team setzt sich aus verschiedenen Mitarbeitenden verschiedener Bereiche zusammen, die über die benötigten Skills verfügen. Ein wichtiger externer Treiber für die Weiterentwicklung der Arbeitsmodelle sind beim BIT auch die massiven Engpässe am Arbeitsmarkt für hochqualifizierte Arbeitskräfte und Talente. Dies erfordere zwingend eine Positionierung als attraktiver zukunftsorientierter Arbeitgeber. In diesem Zusammenhang wurde eine strategische Initiative durch die Geschäftsleitung auf den Weg gebracht, die eine Analyse der Rahmenbedingungen zum Gegenstand hat, um zukünftige Arbeitsmodelle und attraktive Arbeitsbedingungen zu fundieren (BIT (P6, P7, P9) 2018).

\subsubsection{Vorgehen bei der Einführung und Anpassung von Arbeitsmodellen}

Keines der untersuchten Unternehmen arbeitet mit spezifischen und stark standardisierten Verfahren und Prozessen für die Wahl und Einführung neuer Arbeitsmodelle, 
sondern greift auf geläufige interne Projektmethoden zurück. Ein „One-size-fits-all“-Arbeitsmodell gibt es ebenfalls nicht. Wichtig für die erfolgreiche Implementierung von Arbeitsmodellen sei es, dass diese zum Unternehmen, zu den Mitarbeitenden, zur Aufgabenstellung sowie zur Kultur passten, die Bedürfnisse abdeckten und für die Organisation einen Mehrwert generierten. Nur so könne das Arbeitsmodell erfolgreich werden. Die Grundstruktur des Vorgehens bei der Einführung oder Anpassung von Arbeitsmodellen ist jedoch bei allen Organisationen vergleichbar. In der Regel werden zunächst neue Bedürfnisse und Anforderungen abgeklärt. Anschließend werden die betroffenen Personen so weit wie möglich zu Beteiligten gemacht, und es wird gemeinsam schrittweise und mit Pilotumsetzungen an einer Lösung gearbeitet (BIT (P6, P7, P9) 2018; SBB (P1, P2, P3, P5, P10) 2018; Versicherungsunternehmen (P4, P8) 2018). Bei der SBB werden üblicherweise grundlegende Veränderungen in Form von Projekten umgesetzt. Bei der Auswahl und Einführung neuer Arbeitsmodelle werden unterschiedliche betroffene Personen und Bereiche eingebunden (z. B. HR, Immobilien, Infrastruktur etc.). Beispielhaft wurde das interne Projekt „BYTE“ für die Arbeitsplatzgestaltung des neuen SBB-Gebäudes in Bern Wankdorf angeführt. Für die Betrachtung des Arbeitsumfeldes wurden Workshops mit den beteiligten Personen durchgeführt, um deren Bedürfnisse abzuholen und gemeinsam Gestaltungsmöglichkeiten zu erarbeiten. Der hohe Partizipationsgrad benötigte zwar Zeit und Ressourcen, hat sich aber gelohnt. Eine weitere Entscheidungsgrundlage waren Beobachtungen im Arbeitsumfeld und Befragungen der Mitarbeitenden mittels Fragebogen. Bei den Reaktionen in den ersten Umsetzungstagen zeigte sich wie gut die neuen Arbeitsstrukturen und Raumkonzepte ankamen, und wo noch Verbesserungen erforderlich waren (SBB (P1, P2, P3, P5, P10) 2018). Beim Versicherungsunternehmen kommt bei Aufgabenstellungen wie der Einführung oder Anpassung von Arbeitsmodellen ein Reifemodell zum Einsatz, das eine Phasenstrukturierung zugrunde legt und eine kontinuierliche kommunikative Begleitung des Veränderungsprozesses vorsieht. Vorgängig muss dabei Sinn und Zweck der Anpassung eingehend geklärt werden (Versicherungsunternehmen (P4, P8) 2018). Das BIT hebt bei Arbeitsmodellanpassungen und -einführungen auf experimentelle Pilotierungen ab. Ist die Pilotierung erfolgreich, wird das Modell breiter im Unternehmen kommuniziert und top-down ausgerollt. Der Einführungsprozess ist in einen gremiengestützten Entscheidungsrahmen eingebettet, in dem Vertreter aus den HR- und Finanzbereichen die Wirtschaftlichkeit veränderter Arbeitsmodelle mittels KostenNutzen-Betrachtungen analysieren und die grundsätzliche Realisierbarkeit des Veränderungsprojektes prüfen (BIT (P6, P7, P9) 2018).

Ein wichtiger Erfolgsfaktor der Arbeitsmodelleinführung und -weiterentwicklung, der sich bei den Interviews durchgängig herauskristallisiert hat, ist das Einbeziehen unterschiedlicher Stakeholder im Einführungsprozess. Eine weitere wichtige Erfolgskomponente scheint die rechtzeitige Qualifizierung der Mitarbeitenden im Hinblick auf die neuen Arbeitsanforderungen hinsichtlich Agilität und dem Mehr an Verantwortung zu sein. Wichtig bei der Einführung von neuen Arbeitsmodellen ist ferner, dass diese auch nach der Einführung begleitet werden, um ein Rückfallen in alte Verhaltensmuster 
$\mathrm{zu}$ vermeiden. Es braucht ferner eine nachvollziehbare Storyline und eine logische Erklärung für die Veränderung für alle Betroffenen (insbesondere Kundennutzen, Nutzen für die Mitarbeitenden, bessere Zusammenarbeit und Effizienz) (BIT (P6, P7, P9) 2018; SBB (P1, P2, P3, P5, P10) 2018; Versicherungsunternehmen (P4, P8) 2018).

Als größte Hürde für Neueinführungen und Anpassungen von Arbeitsmodellen wurde von allen Interviewten der Faktor Mensch angeführt. Personen, die die neue Arbeitswelt nicht wollen und sich in ihr nicht zurechtfinden, stellen eine zentrale Herausforderung dar. Wenn Arbeitsmodelle angepasst werden, gehe es zwar einerseits um Organisationsentwicklung, vor allem aber auch um die Persönlichkeitsentwicklung der Betroffenen: „Der kleine Haken, den diese Arbeitsformen haben, ist, dass wenn man sie konsequent umsetzen möchte, sie etwas mit Persönlichkeitsentwicklung zu tun haben, nicht mit Personalentwicklung. Und diese Persönlichkeitsentwicklung ist meistens schmerzhafter. Weil ich mich nicht mit meinen Fähigkeiten und Kompetenzen auseinandersetzen muss, sondern ich muss mich mit mir und meiner Person auseinandersetzen." (SBB (P2) 2018, Z. 86 ff.). Es wird immer Personen geben, die mit der neuen Arbeitsweltgestaltung nicht einverstanden sind. Es gibt Personen, die blockieren oder gar sabotieren. Der nachvollziehbare Grund hierfür sind überwiegend jahrelang etablierte Gewohnheiten im früheren Arbeitsumfeld. Die Widerstände müssen akzeptiert und konstruktiv angegangen werden und die Mitarbeitenden sind auf ihrem Weg mit Überzeugungsarbeit und Qualifizierungsmaßnahmen zu begleiten (SBB (P1, P2, P3, P5, P10) 2018; Versicherungsunternehmen (P4, P8) 2018).

\subsubsection{Rahmenbedingungen der Arbeitsmodelle}

Die gesetzlichen Rahmenbedingungen spielen bei allen untersuchten Organisationen in Verbindung mit den angewandten und zukünftigen Arbeitsmodellen eine große Rolle. Auf deren Einhaltung wird konsequent geachtet. Es sind sich alle Befragten einig darüber, dass die heutigen gesetzlichen Rahmenbedingungen bis $\mathrm{zu}$ einem gewissen Grad Flexibilität zulassen, aber in einigen Bereichen an ihre Grenzen stoßen und auf die erhöhten Flexibilitätsanforderungen der Zukunft hin angepasst werden müssen. Anpassungen seien im Hinblick auf die weitere Flexibilisierung von Arbeitszeiten und Leistungserbringungen vor allem beim Schutz der Mitarbeitenden, hinsichtlich Leistungsmessungen und hinsichtlich der Entlohnung nötig (BIT (P6, P7, P9) 2018; SBB (P1, P2, P3, P5, P10) 2018; Versicherungsunternehmen (P4, P8) 2018). Aus Sicht der Befragten der SBB sind die gesetzlichen und internen Regelungen noch wichtiger als früher, weil durch die Digitalisierung frühere Rahmensetzungen wegfallen und neu entwickelnde Grauzonen klare Leitplankensetzungen erforderlich machen. Heutige Rahmenbedingungen funktionieren größtenteils noch, sind aber durch die Ausrichtung auf traditionellere Arbeitsmodelle teilweise intransparent und unfair und weisen Anpassungsbedarfe auf. Dazu gehöre zum Beispiel die Handhabung von Nachtund Sonntagsarbeit, da aufgrund der Autonomie und Flexibilität die Mitarbeitenden manchmal von sich aus abends und an Wochenenden arbeiten und dafür andere Tage frei nehmen. Daraus resultiere etwa die Fragestellung, ob solche Arbeiten dann auch 
zulagenberechtigt sind. Für das Human Resource Management ist es zudem wichtig, dass der Arbeitnehmerschutz (z. B. Ruhezeiten) auch für die neuen flexiblen Arbeitsmodelle gewährleistet bleibt, da eine führungsseitige Kontrolle nur mehr bedingt oder gar nicht mehr gegeben ist. Rahmenbedingungen sollten in dem Zusammenhang eine möglichst klare Trennung von Arbeitszeit und Freizeit ermöglichen (SBB (P1, P2, P3, P5, P10) 2018). Ein weiteres defizitäres Regelungsfeld im Hinblick auf aktuelle und zukünftige neue Arbeitsmodelle ist die Flexibilisierung von Lebensabschnitten während der Erwerbszeit. Wenn man in der Phase der Familiengründung ist, sollte man beispielsweise mehr Zeit für die Familie haben können. Weitere Regelungslücken sind die fehlende Förderung von Jobsharing im Topmanagement oder die Teilzeitarbeit von Mann und Frau. Seitens einigen Befragten wurde auch auf Regelungsdefizite beim Datenschutz im Kontext der Zielsetzung einer jederzeitigen Verfügbarkeit von Unternehmensdaten für einen großen Personenkreis hingewiesen (SBB (P1, P2, P3, P5, P10) 2018; Versicherungsunternehmen (P4, P8) 2018). Seitens der Befragten des BIT werden die Rahmensetzungen für die Auftragsvergaben an Unternehmen oder an externe Mitarbeitende (z. B. Freelancer) als Bereich mit weiterem Regelungs-, beziehungsweise Öffnungsbedarf betrachtet. Heute dauerten Vergabeverfahren zu lange und seien zu kompliziert. Zudem sei die Vertrauensarbeitszeit noch nicht für alle möglich, weil diese an das Lohnklassensystem gekoppelt sei. Generell müssten sich die gesetzlichen und internen Rahmenbedingungen den neuen Gegebenheiten schneller anpassen können. Denkbar wäre in diesem Kontext die Definition zeitlich befristeter Experimentierfelder, damit der Gesetzgeber für einen bestimmten Zweck einige bisherige Rahmenbedingungen außer Kraft setzen könnte und die hiermit verbundenen Effekte beurteilen und für dauerhafte Festlegungen berücksichtigen könnte (BIT (P6, P7, P9) 2018).

\subsubsection{Zukunftsperspektiven der Arbeitsmodelle im Lichte der Digitalisierung}

Nahezu alle Befragten hoben für die Zukunft eine weitere Forcierung von organisations- und unternehmensübergreifenden Zusammenarbeitsformen auf der Grundlage skillbasierter Teams und Netzwerke hervor. Von den neuen agilen Arbeitsmodellen, wie beispielsweise Holacracy oder projektbasiertes Arbeiten nach Scrum oder Kaizen werden in der Zukunft in Verbindung mit einer transparenzfördernden Digitalisierung weitere Impulse für noch mehr orts- und zeitunabhängiges sowie effizienteres Arbeiten erwartet. Die Mitarbeitenden können sich zukünftig noch mehr bei der Bearbeitung von Aufgaben und Veränderungsprozessen einbringen, mitgestalten und mitbestimmen, was zur Erhöhung der Arbeitsmotivation beitragen sollte. Ferner wird für die Zukunft ein Mehr an Optionen für den Einsatz von Freelancern erwartet (BIT (P6, P7, P9) 2018; SBB (P1, P2, P3, P5, P10) 2018; Versicherungsunternehmen (P4, P8) 2018).

Zukünftig werden die Mitarbeitenden vermutlich aufgrund weiter reduzierter Präsenzzeiten noch mehr auf Distanz geführt, und der unmittelbare persönliche Kontakt mit Teamkollegen wird geringer. Gleichzeitig steigt der Bedarf für eine engere und intensivere Zusammenarbeit, da die Aufgabenstellungen zunehmend komplexer werden und 
schneller erledigt werden müssen. Die Unternehmen werden in Zukunft vermutlich vermehrt dazu übergehen, Büroräume flexibel anzumieten, um Fixkosten zu senken. Trotzdem werden Büroarbeitsplätze am Standort voraussichtlich auch in zukünftigen Arbeitsmodellen weiterhin eine Rolle dabei spielen, wenn es um den Aufbau von informellen Beziehungen und eine Identifikation und Verbindung zum Unternehmen, respektive der Organisation geht. Bei den Befragten der SBB wurde vermutet, dass zukünftig Großunternehmen sich vermehrt in vernetzte kleinere und flexiblere Einheiten aufteilen werden. Holacracy wird von den bei der SBB interviewten Personen auch für die Zukunft vor allem in den Konzernleitungsbereichen sowie der IT und dem Human Resource Management ein hohes Effizienzverbesserungspotenzial zugeschrieben. Hiermit verbunden dürfte die Arbeit durch regelmäßige Rollenwechsel abwechslungsreicher und überflüssige Meetings und formalisierte Personalbeurteilungsformen und Personalführungsprozesse deutlich weniger werden. Allerdings ist Holacracy nicht für alle Bereiche geeignet. Die Experten der SBB rechnen für die Zukunft auch mit einem generellen Wachstum bei Mehrfachanstellungen, sodass sich das traditionelle eingleisige Arbeitgeber-Arbeitnehmerverhältnis spürbar verändern wird. Die Bedeutung von News und Feeds dürfte für die Zukunft ferner anstelle des bisherigen Mailverkehrs deutlich zunehmen (SBB (P1, P2, P3, P5, P10) 2018).

Seitens der Befragten des Versicherungsunternehmens wird ebenfalls das zu erwartende Wachstum im Freelancer-Bereich und bei den Ich-AGs betont, auch wenn es sehr wahrscheinlich nach wie vor traditionelle Beschäftigungsverhältnisse mit einer gewissen Jobsicherheit geben wird. In dem Zusammenhang kommt dann Lebensarbeitsmodellen eine wachsende Bedeutung zu (Versicherungsunternehmen (P4, P8) 2018).

Die Experten des BIT erwarten für die Zukunft unter anderem auch kostenbedingt eine wachsende und intensivere Zusammenarbeit mit Freelancern. Im öffentlich-rechtlichen Umfeld benötigt es aber hierfür klare Rahmensetzungen. Multispace-Büroräume mit Rückzugs-, Projekt- und Begegnungszonen werden vermutlich weiter ausgedehnt. Zyklische Wechsel von Teams und Projekten dürften in wachsendem Maße für das Gros der Mitarbeitenden zur Selbstverständlichkeit werden. Trotz der Entwicklungstendenz hin zu agilen Strukturen wird aber auch für die Zukunft Bedarf an coachenden, moderierenden und koordinierenden Führungspersonen gesehen (BIT (P6, P7, P9) 2018).

Potenzielle Problemfelder für die Zukunft sehen die Interviewten hinsichtlich einer reduzierten bilateralen und persönlichen Kommunikation in agileren und digitalen (Führungs-)Strukturen und damit verbundenen Unsicherheiten und Störungen im sozialen Arbeitsumfeld mancher Beschäftigter. Das Mehr an Verantwortung in instabilen Umfeldern könne bei Mitarbeitenden auch zu Überforderung oder Selbstausbeutung führen. Insofern kann das Weniger an Struktur auch zu einem Mehr an Regelungsbedarfen führen (BIT (P6, P7, P9) 2018; SBB (P1, P2, P3, P5, P10) 2018; Versicherungsunternehmen (P4, P8) 2018).

\subsubsection{Zusammenfassung der Ergebnisse}

Die befragten Großunternehmen/-organisationen setzen heute eine Bandbreite von Arbeitsmodellen mit traditionellen und agilen Elementen ein. In den Unternehmen 
kommen Jahres- und Vertrauensarbeitszeit- sowie amorphe Arbeitszeitmodelle zum Einsatz. Im Vordergrund stehen bei den Mitarbeitenden die Gleitzeit-/Jahresarbeitszeitmodelle. Vertrauensarbeitszeitmodelle sind primär für Führungskräfte mit einem definierten Gehaltsniveau relevant. In den untersuchten Organisationen wird Teilzeitarbeit, Jobsharing und mobil-flexibles Arbeiten praktiziert. Vielfach arbeiten Mitarbeitende in Projekten mit agilen Projektstrukturen. Teilweise wird mit effizienz- und flexibilitätsfördernden Methoden wie Scrum, Kaizen oder DevOps gearbeitet. Freelancing und Human-Cloud-Ansätze werden primär als Optionen für die Zukunft gesehen und sind heute noch wenig relevant. Homeoffice und Remote Working ist in der Regel in Absprache mit den Vorgesetzten möglich. Karrierebezogen kommen Einsteigerprogramme für junge Mitarbeitende und für ältere Mitarbeitende Optionen wie Bogenkarrieren und Teil- oder Frühpensionierungen zum Einsatz. Im Bereich der Personalführung kommen situativ verschiedenste Ansätze von traditionell-hierarchisch bis kooperativ zum Tragen. Der Umfang der direkten Führung wird tendenziell in Verbindung mit einem Mehr an selbstorganisierenden Teams heute schon geringer. Coachingansätze der Führung, zielorientierte holokratische Führungsperspektiven, systemische Führung und Führung auf Distanz werden, zumindest in einzelnen Bereichen, bereits gelebt. Symbolische Führung wurde in den Interviews nicht thematisiert. Alle Großunternehmen/-organisationen verfügen über Großraumbüros mit Rückzugsmöglichkeiten, Sitzungs- sowie Projekträumen. Multi-Space-Welten sind bereits Standard. Klassische Bürolösungen mit Einzelbüros werden nicht, respektive kaum mehr genutzt. Auch bei den strukturellen Lösungsansätzen für Zusammenarbeit wird situativ eine Bandbreite von traditionell-hierarchisch (bei effizienzorientierten, standardisierten Arbeiten) bis agil (bei Projektarbeiten) abgedeckt. Struktur- und Führungsmodelle hängen dabei eng miteinander zusammen. In projektorientierten Arbeitsumfeldern kommen holokratische agile Netzwerkstrukturen mit autonomen Teams zum Einsatz, die auch durch orts- und zeitunabhängiges Arbeiten gekennzeichnet sind. Auf die Einhaltung der gesetzlichen Rahmenbedingungen wird in allen Organisationen konsequent geachtet. Die technischen Voraussetzungen und die Infrastruktur für agiles Arbeiten sind in allen Organisationen vorhanden. Wenn möglich, werden Sonderbewilligungen für Arbeitszeitflexibilisierungen eingeholt. Die realisierten Arbeitsmodelle scheinen insgesamt in ihrem gegenwärtigen Mix aus stabilitäts- und flexibilitätsbezogenen Komponenten gut zu funktionieren und werden von den Mitarbeitenden großteils als passend wahrgenommen.

Die derzeit umgesetzten Arbeitsmodelle haben situationsabhängige Vor- und Nachteile. Chancen für die Zukunft werden vor allem in Verbindung mit agilen holokratischen Arbeitsmodellen gesehen, die flexibler und kundenorientierter sind und sich voraussichtlich weiter ausdehnen werden. Die neueren agilen Ansätze der Zusammenarbeit sind nicht nur für die Wettbewerbsfähigkeit des Unternehmens relevant, sondern für neue jüngere Mitarbeitende auch eine wichtige Komponente der Arbeitgeberattraktivität. Ein intensiverer Einsatz von digitalen Technologien ist auch mit einer flexibleren Ausgestaltbarkeit der Arbeitsmodelle verbunden, schafft Freiräume für Führungsarbeit, reduziert die Raumkosten, erhöht die Vereinbarkeit von Freizeit und Beruf, verbessert 
die Datenverfügbarkeit, die Motivation und senkt die Wegzeiten der Mitarbeitenden. Mit der Einführung mobilerer und flexiblerer agiler Strukturen einher geht allerdings auch das Risiko einer Überforderung von Mitarbeitenden und einer Vermischung von Arbeitsund Freizeitsphären, die sich auch negativ auf Commitment, Motivation und Gesundheit der Mitarbeitenden auswirken kann. In dem Zusammenhang ist auch mit auf Ängsten und Gewohnheit basierendem Widerstand von Mitarbeitenden bei der Einführung agiler Arbeitsmodelle zu rechnen.

Für die Anpassung bestehender und Einführung neuer flexiblerer Arbeitsmodelle im Kontext der Veränderung der digitalen Rahmenbedingungen gibt es derzeit keine Standardrezepte. Dies erfolgt sehr kontextabhängig, eher stufen- und bereichsweise, oftmals in Verbindung mit geänderten Anforderungen der Kunden und Mitarbeitenden. Die Einführung neuer Arbeitsmodelle erfolgt dabei tendenziell eher top-down, wird aber in der Regel mit Bottom-up-Kopplungen kombiniert. Externe Beratungen spielen bei der Einführung in den befragten Organisationen eher keine Rolle. Bei der üblicherweise als Projekt erfolgenden stufenweisen Einführung neuer Formen der Zusammenarbeit sind die betroffenen Mitarbeitenden mittels Bedürfniserhebung, Überzeugungsarbeit und Mitwirkungsoptionen einzubinden. Die neuen Arbeitsmodelle müssen zur Arbeitstätigkeit und Kultur passen, was in Pilotumsetzungen getestet werden kann. Die Führungskräfte sollten die eingeforderten neuen Arbeitsweisen als Vorbilder selbst auch vorleben. Darüber hinaus ist für den Erfolg das Erkennen eines klaren kunden-, mitarbeitenden- und effizienzbezogenen Mehrwerts von Bedeutung. Es sollte insofern für alle Betroffenen deutlich werden, dass es bei der Einführung eines neuen Arbeitsmodells nicht um das bloße Aufgreifen eines Managementtrends geht, sondern um eine Verbesserung der Wettbewerbsfähigkeit. Pointiert können somit unter Bezugnahme auf die erfolgten Interviews folgende Handlungsempfehlungen für die Einführung und Änderung neuer Arbeitsmodelle abgeleitet werden:

- Nachvollziehbare Begründungen für den Wandel (Sinnstiftung).

- Kontextabhängige, iterative Projektimplementierung (situativer Projektansatz).

- Stimmige Veränderung des Mindsets (Struktur-Kultur-Fit).

- Einbindung und Mitgestaltung der Mitarbeitenden (Faktor Mensch).

Die Digitalisierung ermöglicht und beschleunigt die Einführung von agilen Projekt- und Netzwerkstrukturen und systemischer Führung. Sie fördert orts- und zeitunabhängiges, organisationsübergreifendes und interdisziplinäres Zusammenarbeiten etwa über Cloudund mobile Kommunikationslösungen wie Feeds und News. Sie ermöglicht auch neue Formen der Zusammenarbeit im Arbeitssegment der selbstständig mitarbeitenden Freelancer und bei Mehrfachbeschäftigten. Neben der Flexibilisierung der Arbeit geht mit der Digitalisierung auch eine potenzielle Flexibilisierung der Strukturen einher, die für Großunternehmen zum Beispiel in die Aufteilung in kleinere Netzwerkeinheiten münden könnte. So kann den gestiegenen Anforderungen der Kunden und Mitarbeitenden 
besser entsprochen werden. Digitalisierung begünstigt ferner den Einsatz moderner, auf Flexibilität abhebender Arbeitsmethoden wie Scrum oder Design Thinking.

Die neuen Arbeitsmodelle sind mit zusätzlichen Freiräumen verbunden, für die ein stabiler Handlungsrahmen von Bedeutung ist. Die heutigen gesetzlichen Rahmenbedingungen in der Schweiz werden teilweise nur bedingt den Anforderungen von agileren und flexibleren Arbeitsmodellen der Zukunft gerecht. Als relevante Regelungsfelder mit Entwicklungspotenzial erscheinen etwa die zunehmende Verschmelzung von beruflichen und privaten Lebenssphären, die derzeit restriktiven Vorgaben hinsichtlich Wochenend- und Sonntagsarbeit, die Rahmenbedingungen für eine Zusammenarbeit mit Freelancern sowie für Mehrbeschäftigungsverhältnisse, Jobsharing oder lebensphasenorientierte Rahmensetzungen.

\subsubsection{Schlussbetrachtung und Ausblick}

Die empirische Untersuchung anhand der drei interviewbasierten Fallstudien bestätigt im Wesentlichen das Bild hinsichtlich der Relevanz der Digitalisierung für neue Arbeitsmodelle und deren Umsetzungsformen sowie Entwicklungstendenzen in Richtung Agilität, Flexibilisierung und Mobilisierung der Arbeit. Diese Tendenzen entsprechen großteils auch den veränderten heutigen Bedürfnislagen der Mitarbeitenden und stehen in einem engen Zusammenhang mit einer effizienteren und fokussierteren Arbeitsdurchführung. Mit den neuen Arbeitsformen einher geht auch eine Flexibilität in Bezug auf sich im Phasenverlauf des Erwerbslebens verändernde Bedürfnislagen der Mitarbeitenden. Die untersuchten Organisationen haben in einigen Anwendungsbereichen und -dimensionen bereits einen hohen Entwicklungsstand erreicht und entwickeln ihre Arbeitsmodelle kontinuierlich weiter. „Neue Arbeitsmodelle“ sind insofern heute bereits Realität. Die inhaltlichen Aussagen der befragten drei Fallstudienpartner weisen eine große Schnittmenge für nahezu alle angeschnittenen Themenfelder auf. Angesichts der hohen Kontingenzabhängigkeit der Thematik, der Fallzahl und der begrenzten Anzahl der Interviewpartner ist dennoch eine Generalisierung der Erkenntnisse nur bedingt möglich. Insofern ist die Untersuchung als ein methodisch fundierter deskriptiver Pinselstrich von vielen zu verstehen, die skizzenhaft versuchen, ein mehrdimensionales Bild von der Arbeitswelt von morgen zu zeichnen.

Auch wenn heute noch niemand genau vorhersagen kann, wie Arbeitsmodelle in Verbindung mit der voranschreitenden Digitalisierung in zehn oder fünfzehn Jahre voraussichtlich aussehen werden, so scheinen die angeführten Entwicklungstendenzen und die hiermit verbundenen Schlagworte „Kundenzentrierung“, „Agilität“, „Selbstorganisation“, „Flexibilisierung“, „Dynamisierung“, „Mobilität“, „Teamorientierung“, „Kollaboration“ und „Vernetzung“ der Arbeit doch zumindest einen möglichen Entwicklungskorridor aufzuzeigen. Heute noch bestehende Grenzziehungen zwischen Arbeits- und Privatwelt sowie Unternehmensinnen- und -außenwelten scheinen zunehmend fluid $\mathrm{zu}$ werden, 
es wird aber voraussichtlich dennoch kontextabhängig auch auf absehbare Zeit noch Arbeitsmodelle mit effizienzorientierten und innovationsfördernden Dimensionsausprägungen in standortbasierten Arbeitswelten geben.

\section{Literatur}

Arbeitgeberverband Basel. (2014). Personalradar. http://www.personalradar.ch/arbeitsrecht-kategorien/arbeitszeitmodelle-und-ihre-rechtlichen-folgen/10052. Zugegriffen: 28. März 2018.

Auf der Maur, M. (2017). Büroflächenmarkt Schweiz: Coworking Spaces liegen im Trend. Schweizer Immobilienbrief, 20, 7-9.

Berghaus, S., Back, P., \& Kaltenrieder, B. (2017). Digital Maturity \& Transformation Report 2017. St. Gallen: Institut für Wirtschaftsinformatik, Universität St. Gallen, Crosswalk AG.

Berlin, B. B. (2016). Mittelstand im Wandel - Wie ein Unternehmen seinen digitalen Reifegrad ermitteln kann. Berlin: HBP University Press.

Blaeser-Benfer, D., \& Pollety, W. (2017). Digitalisierung, Betriebliche Handlungsfelder der Unternehmensentwicklung. Frankfurt a. M.: Frankfurter Societäts-Medien GmbH.

Bürgi und Nägeli Rechtsanwälte. (o. J.). Arbeitsrecht - Job sharing. https://www.arbeits-recht.ch/ job-sharing. Zugegriffen: 3. Apr. 2018.

Deflorin, P., Hauser, C., \& Scherrer-Rathje, M. (2015). Schweizer Unternehmen sehen Digitalisierung als Chance. o. $\mathrm{O}$.

Deloitte. (2016). Deloitte. https://www2.deloitte.com/content/dam/Deloitte/ch/Documents/consumer-business/ch-de-cip-swiss-transformation.pdf. Zugegriffen: 27. März 2018.

Digital Transformer Institute. (2017). Digitaler Reifegrad Check. http://www.digitaltransformerinstitute.ch/services/reifegrad-check/\#1452765442453-b921a1fb-6ec8. Zugegriffen: 14. Febr. 2018.

Disler, A. (2018). adigiconsult GmbH. https://www.adigiconsult.ch/6-treiber-der-digitalisierung/. Zugegriffen: 12. Apr. 2018.

Frey, C., \& Osborne, M. A. (2013). The future of employment. How suspectible are jobs to computerisation? Oxford Martin School (OMS), Working Paper. Oxford: University of Oxford.

Genner, S., Probst, L., Huber, R., Werkmann-Karcher, B., Grundrum E., Majkovic, A.-L. (2017). IAP Studie 2017. Der Mensch in der Arbeitswelt 4.0. Zürich: IAP Institut für Angewandte Psychologie der ZHAW Zürcher Hochschule für Angewandte Wissenschaften.

Glaser, N. (2016). Markenkonstrukt. https://www.markenrebell.de/2016/07/19/so-wirkt-sich-die-digitalisierung-aus/\#.Wvhfh2cUmM8. Zugegriffen: 2. Apr. 2018.

Glogner-Pilz, P. (2012). Publikumsforschung - Grundlagen und Methoden. Wiesbaden: Springer Gabler.

Grannemann, U. (2015). Leadion. https://www.leadion.de/2015/10/18/kooperativer-bis-autoritaerer-fuehrungsstil-das-kontinuummodell-von-tannenbaum-und-schmidt/. Zugegriffen: 6. Apr. 2018.

Institut für Wirtschaftsinformatik. (2017). Digital Maturity \& Transformation Studie. https://aback. iwi.unisg.ch/kompetenz/digital-maturity-transformation/. Zugegriffen: 12. Febr. 2018.

Ittermann, P., \& Niehaus, J. (2015). Digitalisierung industrieller Arbeit. Baden-Baden: Nomos.

Leyh, C., Schäffer, T., \& Forstenhäusler, S. (2016). SIMMI 4.0 - Vorschlag eines Reifegradmodells zur Klassifikation der unternehmensweiten Anwendungssystemlandschaft mit Fokus Industrie 4.0. Dresden: o. V.

Lienhart, A. (2015). WEKA. https://www.weka.ch/themen/personal/arbeitszeit-und-absenzen/ arbeitszeitmodelle/article/vertrauensarbeitszeit-in-der-schweiz-weshalb-das-vertrauen-genaue-planung-und-kontrolle-braucht/. Zugegriffen: 28. März 2018. 
Meyer, M. (2016). Bilanz. https://www.bilanz.ch/management/das-ende-der-hierarchien-holacracyschafft-den-chef-ab-761343. Zugegriffen: 07. Apr. 2018.

Moneyhouse. (2017). 99,8 Prozent der Unternehmen in der Schweiz sind KMU. http://ratgeber. moneyhouse.ch/article/998-prozent-der-unternehmen-in-der-schweiz-sind-kmu/. Zugegriffen: 25. März 2018.

Mühlenbeck, F. (2017). Die Wirkungsweise der digital Transformation - Ein Modell. http://transformieren.com/digital-transformation-wirkungsweise-modell/. Zugegriffen: 25. März 2018.

Neufeind, M. (2016). Generation Y, Wie eine kreative Generation heute Grenzen verschiebt. Berlin: De Gruyter.

Raveling, J. (2017b). Wirtschaftsförderung Bremen GmbH. https://www.wfb-bremen.de/de/page/ stories/digitalisierung-industrie40/geschichte-der-digitalisierung-teil-zwei. Zugegriffen: 11. Febr. 2018.

Reynard, \& Derder. (2017). Kurzfassung: Auswirkungen der Digitalisierung auf Beschäftigung und Arbeitsbedingungen - Chancen und Risiken. o. O.: o. V.

Röbken, P. D., \& Wetzel, K. (2016). Universität Oldenburg. https://www.uni-oldenburg.de/filead$\mathrm{min} /$ user_upload/c31/Studiengaenge/BABusinessAdmin/Download/Leseproben/bba_leseprobe_ quli_quanti_forschungsmethoden.pdf. Zugegriffen: 12. Apr. 2018.

Rotter, D. (2017). Beraternews.net. http://www.beraternews.net/wirtschaft/unternehmen-nutzenchancen-der-digitalisierung-kaum-35712279/. Zugegriffen: 27. März 2018.

Ruoss, S. (2015). Digitale transformation. https://www.firegroup.ch/de/blog/digitale-transformation-teil-8. Zugegriffen: 14. Febr. 2018.

Sattelberger, T., Welpe, P., \& Boes, P. (2015). Das demokratische Unternehmen. Freiburg: Haufe-Lexware.

Schallmo, D., \& Rusnjak, A. (2017). Roadmap zur Digitalen Transformation von Geschäftsmodellen. In D. Schallmo, A. Rusnjak, J. Anzengruber, T. Werani, \& M. Jünger (Hrsg.), Digitale Transformation von Geschäftsmodellen - Grundlagen, Instrumente und Best Practices. Wiesbaden: Springer Gabler.

Schwab, K. (2016). Die Vierte Industrielle Revolution. München: Pantheon.

Schweizer Verband der Telekommunikation. (2015). asult-Bulletin. Bern: KMU und Digitalisierung.

Schweizerische Bundesbahnen (SBB AG), \& Swisscom (Schweiz) AG. (2013). Workanywhere. Bern: PrintMedia Services.

Schweizerische Eidgenossenschaft. (2015a). Mehrfachbeschäftigung. https://www.kmu.admin. $\mathrm{ch} / \mathrm{kmu} / \mathrm{de} / \mathrm{home} /$ praktisches-wissen/personal/arbeitsrecht/mehrfachbeschaeftigung.html. Zugegriffen: 3. Apr. 2018.

Schweizerische Eidgenossenschaft. (2015b). Nacht- und Sonntagsarbeit. https://www.kmu.admin. $\mathrm{ch} / \mathrm{kmu} / \mathrm{de} / \mathrm{home} /$ praktisches-wissen/personal/arbeitsrecht/nacht-und-sonntagsarbeit.html. Zugegriffen: 3. Apr. 2018.

Schweizerische Eidgenossenschaft. (2016). Arbeitszeiterfassung: Pflicht für Arbeitgebende. https://www.kmu.admin.ch/kmu/de/home/praktisches-wissen/personal/arbeitsrecht/arbeitszeiterfassung-pflicht-arbeitgeber.html. Zugegriffen: 3. Apr. 2018.

Schweizerische Eidgenossenschaft. (2018a). Digitale Wirtschaft. https://www.seco.admin.ch/ seco/de/home/wirtschaftslage—wirtschaftspolitik/wirschaftspolitik/digitalisierung.html. Zugegriffen: 8. Apr. 2018.

Schweizerische Eidgenossenschaft. (o. J.a.). Arbeitsrecht: die gesetzlichen Grundlagen. https:// www.kmu.admin.ch/kmu/de/home/praktisches-wissen/personal/arbeitsrecht.html. Zugegriffen: 3. Apr. 2018.

Schweizerische Eidgenossenschaft. (o. J.c.). Überstunden. https://www.kmu.admin.ch/kmu/de/ home/praktisches-wissen/personal/arbeitsrecht/ueberstunden.html. Zugegriffen: 3. Apr. 2018. 
Süddeutsche Zeitung. (o. J.). Digitales Morgen. http://www.sueddeutsche.de/thema/Digitales_Morgen. Zugegriffen: 2. Apr. 2018.

Wissen. Weiterbildung. Lösungen (WEKA). (2016). Führungsstil: Übersicht und Anwendungstipps. https://www.weka.ch/themen/fuehrung-kompetenzen/mitarbeiterfuehrung/fuehrungsinstrumente/article/fuehrungsstil-uebersicht-und-anwendungstipps/. Zugegriffen: 6. Apr. 2018.

Witzig the office company. (2014). FlexWork - die Vorteile des flexiblen Arbeitens. https://www. witzig.ch/de/post/20140406/flexwork-die-vorteile-des-flexiblen-arbeitens. Zugegriffen: 7. Apr. 2018.

Work Smart Initiative. (2018). Work smart initiative. http://work-smart-initiative.ch/de/\%C3\%BCberuns/charta-unterzeichnen/. Zugegriffen: 27. Febr. 2018.

Zinser, S., \& Boch, D. (Hrsg.). (2007). Flexible Arbeitswelten. Zürich: vdf Hochschulverlag AG an der ETH Zürich.

Zobrist, L., \& Grampp, D. (2016). Der Arbeitsplatz der Zukunft, Wie digitale Technologie und Sharing Economy die Schweizer Arbeitswelt verändern. o. O.: Deloitte AG.

\section{Interviews}

Bundesamt für Informatik und Telekommunikation BIT (P6). (2018). Digitalisierung und Arbeitsmodelle, Interview vom 06.04.2018.

Bundesamt für Informatik und Telekommunikation BIT (P6, P7, P9). (2018). Digitalisierung und Arbeitsmodelle, Interviews vom April bis Mai 2018.

Schweizerische Bundesbahnen (SBB AG) (P1). (2018). Digitalisierung und Arbeitsmodelle, Interview vom 23.03.2018.

Schweizerische Bundesbahnen (SBB AG) (P1, P2, P3, P5, P10). 2018. Digitalisierung und Arbeitsmodelle, Interviews von März bis April 2018.

Schweizerische Bundesbahnen (SBB AG) (P2). (2018). Digitalisierung und Arbeitsmodelle, Interview vom 26.03.2018.

Versicherungsunternehmen (P4). (2018). Digitalisierung und Arbeitsmodelle, Interview vom 01.05.2018.

Versicherungsunternehmen (P4, P8). (2018). Digitalisierung und Arbeitsmodelle, Interviews im Mai 2018.

Schellinger, Jochen (Prof. Dr./jochen.schellinger@bfh.ch) Studiengangsleiter Master of Science in Business Administration an der Berner Fachhochschule. Lehr- und Forschungstätigkeiten in den Bereichen Strategisches Management, Marketing und Personalmanagement. Davor 15 Jahre Praxistätigkeit bei der Deutschen Sparkassenorganisation, im Haniel-Konzern, an der Universität Tübingen sowie in der Daimler AG.Huynh, Gia Le (M.Sc. BA/gia-le.huynh@gmx.net) Masterstudium in Betriebsökonomie mit Vertiefung in Corporate/Business Development an der Berner Fachhochschule. Langjährige Tätigkeit in Anlagensteuerung, LifeCycle- und Produktmanagement, Leistungscontrolling sowie Strategie- und Organisationsprojekten. Aktuell bei der Schweizerischen Bundesbahnen AG als Senior strategische Anlagenmanagerin im Kundenanlagen- und FacilityManagement. 
Open Access Dieses Kapitel wird unter der Creative Commons Namensnennung 4.0 International Lizenz (http://creativecommons.org/licenses/by/4.0/deed.de) veröffentlicht, welche die Nutzung, Vervielfältigung, Bearbeitung, Verbreitung und Wiedergabe in jeglichem Medium und Format erlaubt, sofern Sie den/die ursprünglichen Autor(en) und die Quelle ordnungsgemäß nennen, einen Link zur Creative Commons Lizenz beifügen und angeben, ob Änderungen vorgenommen wurden.

Die in diesem Kapitel enthaltenen Bilder und sonstiges Drittmaterial unterliegen ebenfalls der genannten Creative Commons Lizenz, sofern sich aus der Abbildungslegende nichts anderes ergibt. Sofern das betreffende Material nicht unter der genannten Creative Commons Lizenz steht und die betreffende Handlung nicht nach gesetzlichen Vorschriften erlaubt ist, ist für die oben aufgeführten Weiterverwendungen des Materials die Einwilligung des jeweiligen Rechteinhabers einzuholen.

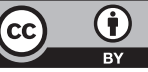




\title{
Digitale Transformation und Human Resource Management
}

\section{Trends und Perspektiven einer zukunftsgerichteten Personalarbeit in Schweizer Großunternehmen}

\author{
Jochen Schellinger, Marlies Goedermans, Lars Patrick Kolb \\ und Yassin Sebai
}

\begin{abstract}
Zusammenfassung
Die voranschreitende digitale Transformation elementarer Unternehmensprozesse schließt auch die betrieblichen Querschnittsfunktionen mit ein. Dies gilt in besonderem Maße für das Human Resource Management von Unternehmen, das sowohl als Objekt als auch als ein Treiber dieser digitalen Veränderungen in Erscheinung treten kann. Strategisch relevante Veränderungen wie Cloud Computing, Big Data und Social Media Management sowie Mobility-Trends wirken sich mittelbar und unmittelbar auch auf die Gestaltungsbereiche betrieblicher Personalarbeit aus. Der vorliegende Beitrag setzt sich theoretisch und empirisch mit der Frage nach der Relevanz und Konsequenz der Digitalisierung für das Personalmanagement in Schweizer Großunternehmen auseinander. Im Mittelpunkt steht die Vorstellung der Ergebnisse einer qualitativen Befragung von sechzehn HR-Managern führender Schweizer Großunternehmen aus verschiedensten Wirtschaftsbereichen und eines weltweit marktführenden eHR-Softwareanbieters. Grundlage hierfür war die Entwicklung eines Reifegradmodells, das versucht, die Digitalisierung anhand von acht Dimensionen des digitalen Wandels
\end{abstract}

J. Schellinger $(\square) \cdot$ M. Goedermans $\cdot$ Y. Sebai

Bern, Schweiz

E-Mail: jochen.schellinger@bfh.ch

M. Goedermans

E-Mail: marlies.goedermans@sap.com

Y. Sebai

E-Mail: yaessu@msn.com

L. P. Kolb

Ittigen, Schweiz

E-Mail: lars.kolb@gmx.ch 
abgestuft zu spezifizieren. Die Schweizerische Unternehmenslandschaft scheint sich aufgrund der Erkenntnisse der Befragung in Bezug auf die Digitalisierung noch in einer Aufbauphase zu bewegen. Dies gilt auch hinsichtlich des Umsetzungsstands von Digital HRM-Ansätzen in den Unternehmen. Die Personalbereiche können und dürfen sich dem Digitalisierungstrend aber nicht verschließen und sollten künftig eine noch aktivere Rolle bei der Umsetzung von Digitalisierungsstrategien spielen.

\subsection{Einleitung}

Mit „Digitalisierung“ wird ein globaler Entwicklungstrend bezeichnet, der zunehmend alle Lebens- und Wirtschaftsbereiche durchdringt. Im ökonomischen Kontext steht Digitalisierung für die Vereinfachung und Effizienzsteigerung von Prozessen durch neue Möglichkeiten digitaler Technologien. Auch der betriebliche Personalbereich ist hiervon nicht ausgenommen. In welchem Ausmaß und in welcher Art und Weise die Digitalisierung Personalprozesse verändert, ist für das Human Resource Management (HRM) in Schweizer Unternehmen empirisch allerdings noch kaum untersucht. Diese Grundfragestellung kann anhand von fünf leitenden Forschungsfragen weiter konkretisiert werden:

- Welche unter dem Begriff der Digitalisierung subsumierbaren Trends sind für das HRM weshalb von Bedeutung?

- Was sind in personaler Hinsicht zentrale Chancen und Problemfelder der Digitalisierung?

- Welche Konsequenzen und Gestaltungsempfehlungen lassen sich aus den wichtigsten Trends für die einzelnen Politikfelder des HRM ableiten?

- Wie sind einzelne Digitalisierungstrends aktuell im HRM Schweizer Unternehmen eingegangen?

- Wie bzw. in welche Richtung entwickelt sich das HRM Schweizer Unternehmen im Kontext der Digitalisierung in den nächsten Jahren weiter?

Als systematisierender Ausgangspunkt für die Identifikation von Digitalisierungspotenzialen im HRM dienen die acht Personalprozesse nach Nicolai (2009) sowie die vier Querschnittsfunktionen des Personalmanagements nach Thom (2001). Dabei wird von einer wechselseitigen Beeinflussung von HR-Hauptprozessen und -Querschnittsfunktionen ausgegangen. Bei der theoretisch-konzeptionellen Aufbereitung wurden 120 einschlägige Quellen aus dem deutschen und angelsächsischen Sprachraum zur Fundierung der qualitativen Befragungen von 17 (e)HR-Fachexperten Schweizer Unternehmen analysiert und die Erkenntnisse anhand eines Reifegradmodells zur Digitalisierung in der Schweiz zusammengefasst, das im empirischen Teil der Untersuchung den Rahmen für eine praxeologische Prüfung und Interpretation absteckt. 


\subsection{Personalbezogene Aspekte der digitalen Transformation}

Startpunkt für die konzeptionelle Aufbereitung zur Beantwortung der Forschungsfragen ist eine Präzisierung relevanter Begrifflichkeiten und Rahmensetzungen aus der Human Resource Management-Literatur. Es folgen eine Konkretisierung des Digitalisierungskontexts besonders relevanter Ansätze und Einzelaspekte für das HRM sowie eine Marktbetrachtung für digitale HR-Produkte und -Services. Im Anschluss an eine Reflexion potenzieller Problemfelder wird das für die empirische Analyse strukturgebende Reifegradmodell abgeleitet.

\subsubsection{Begriffliche und konzeptionelle Grundlagen}

Wie nahezu für alle Managementbegrifflichkeiten gibt es auch für das HRM nicht eine Definition, sondern viele. Human Resource Management wird oft synonym mit den Begriffen Personalwirtschaft, Personalwesen, Personalmanagement oder Strategisches Personalmanagement verwendet (Karaus 2003, S. 8 f.). Es geht bei allen Begriffen aber stets um den betrieblichen Produktionsfaktor Mensch und dessen Leistungen sowie dessen richtungsweisende Führung (Kolb 2010, S. 5). Seit den 1990er-Jahren wird HRM als zunehmend bedeutsame Unternehmensfunktion gesehen, und die Mitarbeitenden werden als wertgenerierendes zentrales Kapital des Unternehmens betrachtet. Das HRM hat als betriebliche Funktion die Aufgabe, die richtigen Menschen zur richtigen Zeit in der richtigen Anzahl mit der adäquaten Qualifikation am richtigen Ort der Leistungserstellung in der Organisation einzusetzen (Haubrock 2004, S. 17 f.). Seit der Jahrtausendwende haben neue unternehmensinterne und -externe Herausforderungen das HRM geprägt: der wachsende Wettbewerbsdruck erfordert ein kontinuierliches Kosten- und Veränderungsmanagement; die Globalisierung forciert unternehmerisches Agieren und führt zu globalen Organisationen mit multinationalen Kulturen; die technologische Entwicklung erfordert neue Qualifikationen der Mitarbeitenden sowie deren Anpassungsfähigkeit; der demografische Wandel beeinflusst sehr stark die internen und externen Arbeitsmärkte und den Erwartungen der Mitarbeitenden in Bezug auf Arbeitszeitmodelle, Mitbestimmung am Arbeitsplatz oder Formen neuer Arbeitsorganisationen muss Rechnung getragen werden (Wertewandel). Hieraus resultieren neue Belegschafts- und Leistungsstrukturen (Selke 2012, S. 207 f.).

Eine Vielzahl der Unternehmen misst heute dem HRM einen hohen Stellenwert bei. Je höher die strategische Priorisierung des Funktionsfelds, desto weiter oben findet sich die Personalabteilung und das HR-Management in der Unternehmenshierarchie (Nicolai 2009, S. 20). Die hierarchische Aufhängung korrespondiert dabei oftmals mit der Unternehmensgröße (Heymann 2009, S. 26). Kleine und mittelständische Unternehmen (KMU) weisen der Personalabteilung in der Regel nicht eine höchste Priorität zu. Der Personalbereich ist bei mittleren Unternehmen im Normalfall erst auf der 
zweiten Hierarchieebene positioniert. Häufig wird die Personalabteilung dann einer Verwaltungsabteilung unterstellt oder ist in andere Abteilungen integriert. In Kleinunternehmen existiert aufgrund des limitierten Aufgabenumfangs in der Regel keine originäre Personalabteilung. Die Aufgaben werden meist von einer administrativen Verwaltungsstelle oder dem Eigentümer selbst ,nebenbei“ erledigt (Nicolai 2009, S. 20).

In Verbindung mit der Personalfunktion werden in Unternehmen in der Regel ökonomische Ziele (optimale Personalressourcenallokation und Steigerung der Arbeitsleistung) und soziale Ziele im Sinne einer Bedürfnisbefriedigung der Mitarbeitenden verfolgt. Das resultierende Spannungsverhältnis muss bei der Ausgestaltung der Personalstrategien und -prozesse vom HRM berücksichtigt werden (Scherm und Süss 2011, S. 6). Die zunehmende Komplexität und Verrechtlichung der Arbeitsbeziehungen macht diese Aufgabe nicht leichter.

Die Aufgabenstellungen des HRM können in Anlehnung an die Prozessperspektive von Nicolai (2009) in insgesamt acht Teilprozesse untergliedert werden: Personalbedarfsplanung, Personalbeschaffung, Personalauswahl, Personaleinführung und -einarbeitung, Personaleinsatz und -erhaltung, Personalbeurteilung sowie Personalentwicklung und -freisetzung. Bei der elementaren Personalbedarfsplanung geht es um die zukunftsgerichtete Bestimmung der notwendigen personellen Ressourcen zur Erreichung der Unternehmensziele. Ziel ist die kontinuierliche Deckung des quantitativen und qualitativen Bedarfs an Arbeitsleistung unter Beachtung der zeitlichen und örtlichen Notwendigkeit (Nicolai 2009, S. 31 ff.). Die Personalbeschaffung verfolgt das Ziel, die richtige Anzahl an Mitarbeitenden mit der passenden Qualifikation zum richtigen Zeitpunkt und für die richtige Zeitspanne am passenden Arbeitsort zu positionieren (a. a. O., S. 48 ff.). Sie kann intern oder extern erfolgen, wobei in Verbindung mit der Digitalisierung für die externe Personalbeschaffung das E-Recruiting in den letzten Jahren massiv an Bedeutung gewonnen hat. Bei der Personalauswahl wird das Ziel verfolgt, diejenigen Bewerber zu ermitteln, die mit ihren Qualifikationen und ihrer Persönlichkeit am geeignetsten zur Besetzung vakanter Stellen erscheinen. Fehlerhafte Auswahlentscheidungen sind in der Regel mit beträchtlichen Kosten verbunden (Scherm und Süss 2013, S. 54). Zur Personalauswahl stehen diverse Selektionsverfahren zur Verfügung, die üblicherweise kombiniert werden. Nach Arbeitseintritt dienen Einführungs- und Entwicklungsmaßnahmen einer systematischen Integration ausgewählter Personen in die Organisation (Nicolai 2009, S. 125). Personaleinsatz und Personalerhaltung stehen für sämtliche Aspekte des HRM, bei denen es darum geht, Mitarbeitende optimal zu beschäftigen, ihre Qualifikationen sowie ihr Potenzial für die Organisation zu erhalten, zu fördern und den Unternehmensbedürfnissen anzugleichen. Die Mitarbeitenden sollen zu Leistungssteigerungen motiviert und perspektivisch an das Unternehmen gebunden werden (Nicolai 2009, S. 133). Von besonderer Bedeutung sind hierbei adäquate Anreizsysteme zur materiellen und immateriellen Incentivierung. Die Personalbeurteilung dient als institutionalisiertes System und Verfahren, welches die Leistungsergebnisse, das Arbeits-, Führungs- und Sozialverhalten sowie das Potenzial des Mitarbeiters kontrolliert (Nicolai 2009, S. 236 ff.). Aktuell zeichnet sich in der 
Praxis ein Trend zur Durchführung von Mehrfachbeurteilungen ab (z. B. 360-Feedbacks). Die Personalentwicklung stellt gemäß Nicolai (2009, S. 266) eine ,immaterielle Investition ins Humankapital“ dar. Im Zentrum stehen die Vermittlung und der Erwerb von neuen Qualifikationen der Mitarbeitenden zur Nachwuchssicherung und zur Instandhaltung und Intensivierung des betrieblichen Wissens (Wachter 2015). In Verbindung mit der Digitalisierung kann vor allem auch auf die zunehmende Bedeutung von agilen E-Learning-Ansätzen verwiesen werden (z. B. Blended Learning/Distance Learning) (Kolb 2008, S. 473 f.). Unter Personalfreisetzung versteht man sämtliche Maßnahmen zur Dezimierung einer personellen Überdeckung in qualitativer und quantitativer sowie zeitlicher und örtlicher Perspektive (Nicolai 2009, S. 326 f.). Häufigste Beweggründe zur Personalfreisetzung sind in der Praxis Sanierungen, Reorganisationen und Standortschließungen (Stock-Homburg 2008, S. 216 f.). Die sorgfältige Durchführung des Freistellungsprozesses hat einen beträchtlichen Einfluss auf das Unternehmensimage und führt zu entsprechenden Wettbewerbsvorteilen oder -nachteilen bei der künftigen Personalbeschaffung auf dem Arbeitsmarkt.

Die angeführten Personalprozesse stehen in enger Wechselwirkung mit den personalen Querschnittsfunktionen zur Erschließung von im HR-Bereich verankerten Erfolgspotenzialen (Thom 2001). Dem Personalcontrolling kommt die Aufgabe zu, Planungs-, Steuerungs- und Kontrollfunktionen zu unterstützen und wahrzunehmen sowie die personale Informationsversorgung sicherzustellen (Thom 2001, S. 125 f.). Ein systematisches Personalcontrolling kommt zumeist im Einsatz von Personalkennzahlensystemen zum Ausdruck. Die Digitalisierung dürfte in dem Zusammenhang zu völlig neuen integrierten Analyse- und Steuerungsmöglichkeiten beitragen. Das Personalmarketing setzt sich vorrangig mit dem Employer Branding und der hiermit einhergehenden Positionierung als attraktiver Arbeitgeber auf dem Arbeitsmarkt auseinander (Thom 2001, S. 126 f.). Die Digitalisierung hat auch hier eine zunehmende Fülle neuer zielgruppenorientierter Vermarktungsoptionen hervorgebracht (z. B. Social Media-HR-Marketing) (Beck 2008, S. 49). Im Rahmen der Personalinformation ist es Aufgabe des HRM, eine verständnisfördernde innerbetriebliche Kommunikation (Cantin 1999) zu realisieren. Eine jüngere Untersuchung bei den 500 umsatzstärksten Unternehmen Deutschlands (Spachmann und Huck-Sandhu 2013, 2015) zeigt in Verbindung mit der Digitalisierung die Tendenz auf, dass sich die Personalinformation zunehmend weg von einer einseitigen Sprachrohrfunktion der Geschäftsleitung hin zu verselbstständigten dialogorientierten Austauschplattformen verschiebt, die neue Kollaborationsmöglichkeiten eröffnen und zu einer offenen Kommunikationskultur beitragen (Huck-Sandhu 2015, S. 7 f.). Die Organisation des Personalmanagements schließlich befasst sich mit der Regelung des Zusammenspiels der involvierten Aufgabenträger innerhalb des Personalbereichs und zwischen HRM und Linienführungkräften (Arbeitsteilung und Koordination) (Thom 2001, S. 127 f.).

Das strategische Personalmanagement ist als potenzialorientierte Metafunktion über den Personalprozessen und den HR-Querschnittsfunktionen positioniert und befasst sich mit grundlegenden Gestaltungsoptionen zur personalseitigen Implementierung der 


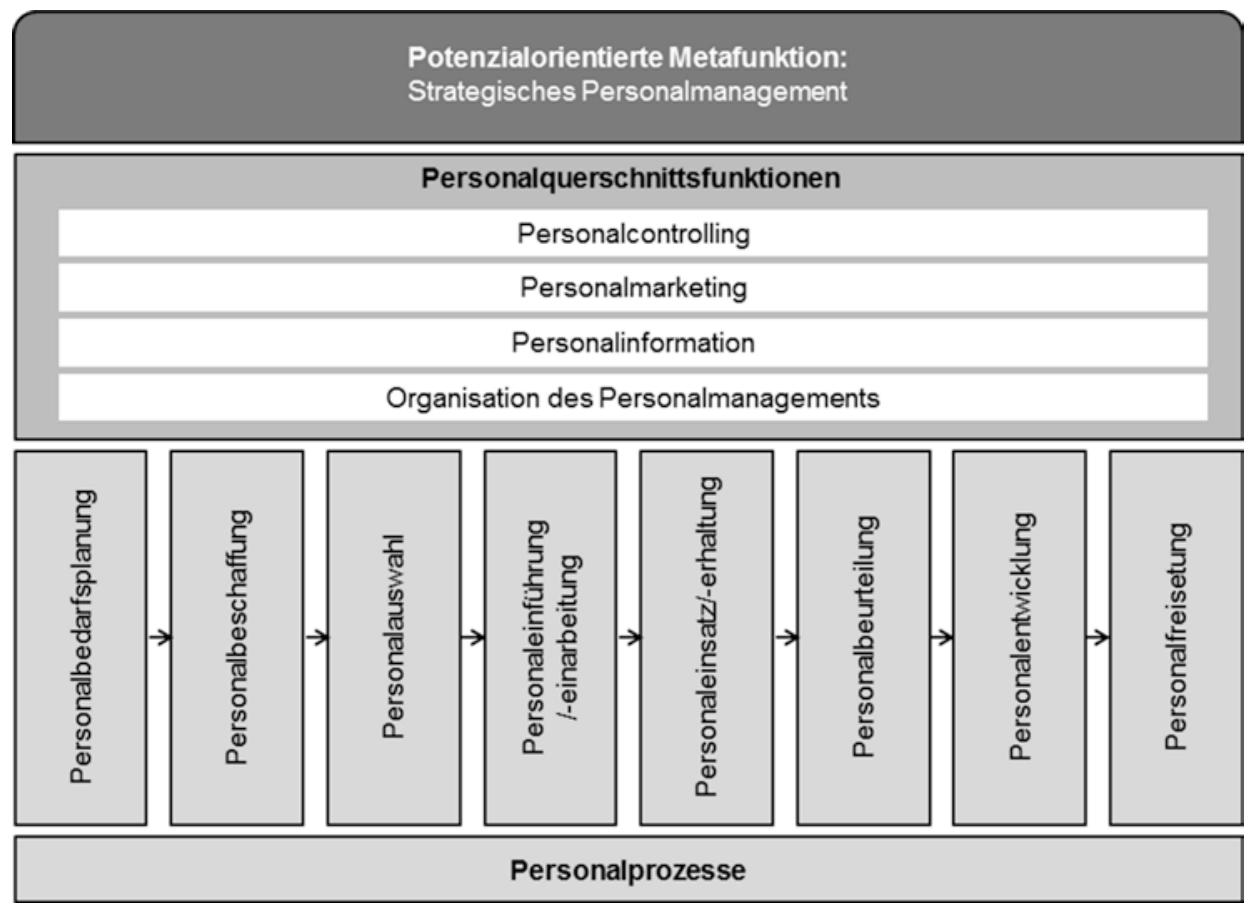

Abb. 8.1 Handlungs- und Gestaltungsbereiche des HRM. (Eigene Darstellung in Anlehnung an Nicolai 2009, S. 5 f.; Thom 2001, S. 118)

Unternehmensstrategie. Die drei Handlungs- und Gestaltungsbereiche des HRM sind gesamthaft in Abb. 8.1 dargestellt und dienen als erster Bezugsrahmen für die Analyse der HR-bezogenen Digitalisierungsaspekte.

\subsubsection{Human Resource Management im Digitalisierungskontext}

Die Digitalisierung kann als Metatrend verstanden werden, der eine Veränderung der Arbeitswelt, der Unternehmens- und Wissenskultur sowie der damit korrespondierenden Führungsverständnisse nach sich zieht (Cachelin 2013a). Westermann et al. (2014) sehen die digitale Transformation als technikbasierte Performance- oder Reichweitenerhöhung von Unternehmen, die mit einer Transformation von Betriebsprozessen, Kundenerlebnissen und der Geschäftsmodelle verbunden ist. Digitalisierung steht ferner für eine zunehmende Immaterialisierung von Produkten, Prozessen und Ressourcen von Unternehmen (Cachelin 2013a) und geht mit einer Mediatisierung, einer rapiden Beschleunigung der Arbeitsabläufe sowie einer wachsenden Verdichtung und Komplexitätssteigerung der Tätigkeiten einher (Bankl 2014, S. 306). 
In Bezug auf das HRM gibt es eine Fülle von Verbindungslinien zum Digitalisierungskontext. Eine eindeutige Festlegung dessen, was Digitalisierung für das HRM bedeutet, gibt es derzeit noch nicht. Hackl und Gerpott (2014) sehen als Folge der Digitalisierung eine Verschmelzung von physikalischer und virtueller Arbeitswelt, die sich auf das HRM nachhaltig auswirken wird. Eine zunehmende Virtualisierung der Arbeit und Interaktionsbedarf mit intelligenten Maschinen, erhöhte Mitarbeitermobilität und neue personale Anforderungsprofile führen zu Neuerungen vor allem hinsichtlich der Auswahl und Entwicklung von Arbeitskräften mit den künftig benötigten technologischen Kompetenzen sowie in Bezug auf die Neugestaltung von Arbeits- und Geschäftsmodellen. Die Digitalisierung geht einher mit einer resultatsorientierten Auf- und Ablauforganisation der Personalprozesse (Gora 2016) bei der administrative Routinearbeiten automatisiert werden. Die Vernetzung der HRM-Instrumente erlaubt das Einbringen fundierterer und neuer HR-Informationen in den strategischen Entwicklungsprozess. So geht aus dem Ergebnisbericht der HR-Trendstudie 2015 von Kienbaum (2015) hervor, dass rund $70 \%$ aller Studienteilnehmenden in Data Analytics ein hochgradiges Nutzungspotenzial sehen. In diesem Zusammenhang wird der Einsatz von künstlicher Intelligenz im HRM spürbar zunehmen und Tools wie Social Media, Mobile Applications und Cloud-Lösungen werden künftig zum HRM-Standard gehören. In Bezug auf die zu entwickelnden Führungskonzepte werden Fähigkeiten zum Change Management sowie zur Vernetzung und wertschätzenden Kommunikation noch wichtiger. Insgesamt gewinnt das HRM durch die Digitalisierung wohl an Bedeutung (Holtbrügge 2015, S. 5).

Es gibt zwar eine ständig wachsende Zahl an theoretisch-konzeptionellen Beiträgen, literarischen Werken und empirischen Studien, die das Ziel verfolgen, in den Umgang mit der digitalen Transformation auf der Metaebene einzuführen und Entwicklungspotenziale und geschaffene Herausforderungen für eine Volkswirtschaft, Branche oder Unternehmung aufzuzeigen. Die wenigsten Arbeiten beschäftigen sich jedoch explizit mit der Digitalisierung im Kontext HRM. Gemäß einer Studie von Ernst \& Young (2016) bei 700 mittelständischen Schweizer Firmen mit 30 bis 2000 Mitarbeitenden kann aus der Unternehmenspraxis heraus festgehalten werden, dass mehr als die Hälfte der untersuchten Fälle den digitalen Technologien nur eine marginale Bedeutung zuweisen. Jedoch muss angemerkt werden, dass in der Schweiz offenbar ein „digitaler Graben“ zwischen Großkonzernen und KMU besteht. Während Großunternehmen sich seit geraumer Zeit mit den Chancen und Herausforderungen der Digitalisierung beschäftigen, hinken kleinere und mittlere Betriebe hinterher. Eine in jüngerer Zeit durchgeführte Studie der Hochschule für Wirtschaft Zürich (HWZ) mit 463 befragten Personen mit und ohne Kaderfunktion in Schweizer Unternehmen stellte entsprechend fest, dass über $50 \%$ der Unternehmen als ,digitale Dinosaurier“ kategorisiert werden können. Insgesamt scheint in der Schweizer Wirtschaft nach wie vor digitales Know-how sowohl auf Führungs- als auch auf Mitarbeiterebene zu fehlen (HWZ 2015). Der Digital Transformation Report von Berghaus et al. (2015) hat versucht, per Online-Umfrage mit 196 Teilnehmenden, von denen $83 \%$ aus der Schweiz stammen, die digitalen Fähigkeiten 
der befragten Unternehmen zu ermitteln. Durch die Gewinnung der Daten konnte ein Digital Maturity Model entwickelt werden, das neun relevante Fähigkeiten für die digitale Transformation zusammenfasst. Die digitale Reife einer Unternehmung konnte anhand von fünf Reifegraden aufgezeigt werden. Berghaus et al. (2015, S. 7) betonen in dem Zusammenhang, dass es für eine erfolgversprechende digitale Transformation entscheidend sei, dass die Geschäftsleitung eine unterstützende Rolle einnehme, das mittlere Management zum Handeln aufgefordert werde, die Transformation eine entsprechende Strategie benötige, der Spirit einer digitalen Unternehmung spürbar sein sowie aus einzelnen Projekten ein Programm gemacht werden müsse.

Forschungsseitig ist die Thematik Digitalisierung und HRM in der Schweiz im Vergleich zu Deutschland, Österreich und vor allem den USA noch relativ wenig erschlossen. Cachelin veröffentlicht seit 2012 in regelmäßigen Abständen wissenschaftliche Beiträge zur Bedeutung des technologischen Wandels und der resultierenden Relevanz neuer technologischer Tools für die Personalabteilungen. Er sieht die Digitalisierung als Auslöser eines umfassenden Change-Prozesses (Cachelin 2014a). Die Metauntersuchung „HRM-Trendstudie 2013“ (Cachelin 2014b) analysiert zehn Studien, die sich mit der Zukunft der Personalarbeit befassen. Sie fokussiert auf die Darstellung der benötigten wertschaffenden HR-Kompetenzen in einer digitalen Welt: die Schaffung eines Digitalisierungsverständnisses, die Nutzung von digitalen HR-Instrumenten zur verstärkten Wirkung des HRM, das Erlangen der Datenkompetenz zur Erarbeitung einer Datenstrategie und eines effektiven Controllings sowie die Positionierung des HR-Bereichs innerhalb des Unternehmens durch das richtige Angebot und konsequentes Marketing. Die Folgestudie zu den Risiken eines digitalen Arbeitsumfelds mit 232 Teilnehmenden aus Deutschland, Österreich und der Schweiz zeigt auf, dass eine Annäherung von HR- und IT-Abteilungen für ein integriertes Risiko- und Innovationsmanagement erforderlich scheint (Cachelin 2015). Die HRM-Trendstudie von Kienbaum (2015) basiert auf einer Befragung von 187 Personalverantwortlichen führender Unternehmen im deutschsprachigen Raum (Anteil Deutschland 86\%, Schweiz $2 \%$ ). Sie zeigt erste Trends in der Nutzung von Digitalisierungs-Tools im HRM auf und räumt der digitalen Transformation ein hohes Erfolgspotenzial ein. Die Befragten sehen derzeit noch einen geringen Nutzungsgrad von Data Analytics, künstlicher Intelligenz, Social Media, Mobile Applications und Cloud-Lösungen im HRM, gehen aber von einem großen Potenzial für das HRM der Zukunft aus. Auf die wichtige Bedeutung eines adäquaten Umgangs mit dem Thema Datenschutz im Kontext der Digitalisierung verweist die Deutschlandstudie von Olsok et al. (2016). Die umfassende Befragung von 532 Führungskräften im deutschsprachigen Raum (Anteil Schweiz $32 \%$ ) durch Eilers et al. (2016) kommt zum Schluss, dass die digitale Transformation in Unternehmen nur dann gelingen kann, wenn dabei verstärkt auch kulturelle und soziale Aspekte einbezogen werden. Die jüngst erfolgte breitere Befragung von 480 HRM-Fachexperten (75\% aus Schweizer Unternehmen) identifiziert vor allem bei der Digitalisierung und im faktenbasierten HRM zukünftige Investitionsbedarfe (Winkler 2016). 


\subsubsection{Digitalisierungsansätze mit besonderer HRM-Relevanz}

Insgesamt scheinen vor allem vier technologische Entwicklungslinien der Digitalisierung für das HRM von besonderer Relevanz zu sein: Social Media, Data Mining, Cloud Computing und Mobility.

\section{Social Media}

In der digitalisierten Welt hat sich das Internet vom Informations- zum Mitmachmedium verändert, in dem das, was man von sich aktiv auf den Social-Media-Plattformen preisgibt, entscheidend dafür ist, wie man von anderen Personen und Unternehmen wahrgenommen wird (Schwarb und Vecchia 2016, S. 50). Folgende Eckzahlen mit Stand Anfang 2016 belegen eindrücklich die heutige Allgegenwart der sozialen Medien (Schwarb und Vecchia 2016, S. 52):

- 3,42 Mrd. Menschen sind aktiv im Internet (=46\% der Weltbevölkerung).

- $31 \%$ der Internetuser nutzen soziale Medien wie Facebook, Twitter etc.

- 1,5 Mrd. Menschen haben einen Facebook-Account.

- 3,5 Mio. Facebook-User gibt es in Österreich.

- 28 Mio. Facebook-User gibt es in Deutschland.

- 3,7 Mio. Facebook-User gibt es in der Schweiz.

- 3,1 Mio. Menschen sind mit mobilen Geräten in der Schweiz auf sozialen Medien aktiv.

Gerade junge Menschen diskutieren in den sozialen Medien wie Facebook, Xing oder LinkedIn über ihre Arbeitgeber, geben sich gegenseitig Tipps zur Bewerbung oder rekrutieren sogar Freunde als zukünftige Mitarbeiter (Cole 2015, S. 172). Das heutige Web 2.0 ermöglicht es außerdem, interessante Stellenangebote mit Freunden zu teilen oder Interessierten aus dem Bekanntenkreis direkt Links zu senden. So bieten zum Beispiel „Buddybroker“ die Möglichkeit, Freunden konkrete Jobangebote zu empfehlen (PwC 2013, S. 8). Deshalb nimmt die Bedeutung der Mitarbeitenden als Markenbotschafter eines Unternehmens deutlich zu. Die Digitalisierung ist außerdem mit einem zunehmenden Maß an Transparenz am Arbeitsmarkt verbunden. Ein Beleg hierfür sind etwa Arbeitgeberbewertungsplattformen wie Kununu (Jäger und Petry 2012, S. 21). Unternehmen müssen sich heute also im Rahmen eines „Employer Branding“ auch im Internet und in den sozialen Medien präsentieren und inszenieren (Cole 2015, S. 174). Die Auswahl relevanter Plattformen für in der Regel sehr zeitintensive Social Media-Aktivitäten hängt meist von der anzusprechenden Zielgruppe ab. Plattformen mit direkt steuerbaren, d. h. selbst erstellten Inhalten sind dabei von Plattformen mit nicht direkt steuerbaren, d. h. von Internetnutzern erstellten Inhalten abzugrenzen. Letztere werden von den Internetusern als vertrauenswürdiger eingestuft werden (Schwarb und Vecchia 2016, S. 57). Ein Überblick über die bekanntesten steuerbaren und nicht steuerbaren Social-Media-Plattformen und zugehörige HRM-Handlungsfelder gibt Tab. 8.1. 
Tab. 8.1 Steuerbare und nichtsteuerbare Social Media-Plattformen und HRM-Handlungsfelder. (Eigene Darstellung in Anlehnung an Schwarb und Vechia 2016, S. 71)

\begin{tabular}{l|l|l|l|l|l}
\hline \multicolumn{2}{|c|}{} & \multicolumn{2}{l}{ Relevante HRM-Handlungsfelder } \\
\hline Plattformen & $\begin{array}{l}\text { Direkte } \\
\text { Steuerbarkeit }\end{array}$ & $\begin{array}{l}\text { Employer } \\
\text { Branding }\end{array}$ & Stellenan-zeigen & $\begin{array}{l}\text { Bewerber- } \\
\text { recherche }\end{array}$ & $\begin{array}{l}\text { Active } \\
\text { Sourcing }\end{array}$ \\
\hline 1) Kununu & Nein & Ja & Ja & Nein & Nein \\
\hline 2) Glassdoor & Nein & Ja & Ja & Nein & Nein \\
\hline $\begin{array}{l}\text { 3) Staufenbiel } \\
\text { (Stellenbör- } \\
\text { senforum) }\end{array}$ & Nein & Nein & Ja & Nein & Nein \\
\hline 4) Xing & Ja & Ja & Ja & Ja & Ja \\
\hline 5) LinkedIn & Ja & Ja & Ja & Ja & Ja \\
\hline 6) Google+ & Ja & Ja & Ja & Ja & Ja \\
\hline 7) Facebook & Ja & Ja & Ja & Nein & Indirekt \\
\hline 8) Instagram & Ja & Ja & Ja & Nein & Nein \\
\hline 9) Snapchat & Ja & Bedingt & Bedingt & Nein & Nein \\
\hline 10) Twitter & Ja & Bedingt & Ja & Nein & Nein \\
\hline 11) WhatsApp & Ja & Bedingt & Bedingt & Nein & Nein \\
\hline
\end{tabular}

Bewertungsplattformen: 1) - 3)/Business-Plattformen: 4) - 5)/Private Netzwerke: 6) - 11)

Die Nutzung von Social Media im HRM steckt jedoch immer noch in den Kinderschuhen: Auch wenn in einer jüngeren Studie immerhin zwei Drittel der befragten 100 Unternehmen im Social Media-Bereich aktiv sind, so war bei lediglich knapp $20 \%$ Social Media ein etablierter Bestandteil des HRM (PwC 2013). Ein Social Media-Einsatz erfolgt überwiegend in der erfolgstransparenten Frühphase des Mitarbeitendenzyklus bei der Rekrutierung (91\%) sowie im Employer Branding (89\%). Ferner scheinen der Kontakthaltung zu ehemaligen Mitarbeitenden (29\%) und der Mitarbeitendenbindung (21\%) eine gewisse Bedeutung zuzukommen (PwC 2013, S. 15). Die vier von den Unternehmen am häufigsten genutzten Plattformen stehen mit Facebook (59\%), Xing (52\%), Linkedin (52\%) und YouTube (44\%) auch für die bekanntesten und etabliertesten Betreiber (PwC 2013, S. 12).

\section{Data Mining (Big Data/Business Intelligence)}

Unser Alltag wird mit der Zeit immer digitaler, was mit einer zunehmenden Datenproduktion einhergeht. Wir hinterlassen im Internet mit jedem Klick eine Spur, sei es beim Buchen der Ferien, beim Lesen eines Zeitungsartikels, beim Posten auf Facebook oder beim Bestellen eines Kochbuches. Gleiches gilt auch für die Arbeit: Jedes geführte Telefonat, jedes verschickte Mail, jedes abgespeicherte Dokument und jede besuchte Internetseite ist Teil der beruflichen Datenspur. Der Begriff „Big Data“, bzw. „Data Mining“ steht synonym für diese digitalisierungsbedingten riesigen Datensätze und deren Verwendung (Cachelin 2013c). 
Mit Big Data verbunden ist die Umwandlung des „Rohstoffs“ Informationen in verwertbares Wissen. Die bei der Big-Data-Verwertung eingesetzten Technologien und Methoden dienen dazu, Korrelationen und Muster zu erkennen, wo Menschen nur Datenchaos sehen (Cole 2015, S. 35). Es geht also um die Herstellung von „Transparenz“, die jedoch nicht immer willkommen ist. Gemäß der HR-Trendstudie 2013 denken $78 \%$ der Befragten, dass Big Data zu einem stärkeren Überwachen der Mitarbeitenden führt. Die Angst davor, dass der Datenschutz des eigenen Unternehmens nicht eingehalten wird, ist hoch. Lediglich $38 \%$ der Befragten wissen Bescheid, was ihr Unternehmen mit HR-Daten anstellt. Es besteht also ein großer Erklärungsbedarf hinsichtlich eines eventuellen HR Data Mining (Cachelin 2013b, S. 29).

Im HRM können Big Data-Analysen (Business Intelligence) dazu beitragen, Verbesserungspotenzialen auf die Spur zu kommen, sei es zum Beispiel bei der Identifikation von unterstützungsbedürftigen Mitarbeitenden oder bei der Effizienzsteigerung von Personalentscheidungen und -prozessen. Entsprechende HRM-Datenanalysen heben in der Regel auf die Beantwortung folgender Fragestellungen ab (Wagner AG 2016):

- Was ist passiert? (Beschreibung)

- Warum ist es passiert? (Analyse)

- Was wird passieren? (Voraussage)

- Was sollte geschehen? (Beeinflussung).

Große Anbieter wie SAP, Oracle und Microsoft bieten zur Beantwortung dieser Fragen dabei für Großunternehmen aber auch für KMU relativ kostengünstige Analytics-Lösungen an. Das Analytics-Tool von Microsoft etwa ist so konzipiert, dass auch Anwender ohne großes IT-Know-how einfach und schnell auf Personaldaten zugreifen, selbst Berichte erstellen und mit anderen Mitarbeitenden teilen können. Personalverantwortliche können Daten schnell und ohne Mühe visualisieren. Mit dem Analytics Tool sind Fluktuationen, demografische Entwicklungen, Abwesenheiten, Arbeitseintritte oder Lohnsummen analysierbar, visuell darstellbar und können für strategische Personalentscheidungen differenziert genutzt werden (Pentaho 2012, S. 1). Außerdem sind die Auswertungen auch über eine Mobile App auf dem Smartphone, Tablet oder Notebook jederzeit abrufbar.

\section{Cloud Computing}

„Cloud Computing“ steht für die Ausführung von Softwareprogrammen, die nicht auf lokalen Rechnern installiert sind, sondern von externen Rechnern, bzw. über das Internet aufgerufen werden. Im digitalen Zeitalter stellt die Verbindungsgeschwindigkeit über das Internet in der Regel kein Problem mehr dar, sodass immer mehr Dienste in diese „Software-Wolke“ verlagert werden. Anwendungen und der Speicherplatz für die Daten werden von einem externen Dienstleister mit Server-Farmen über das Internet bezogen, sodass man im Prinzip jederzeit und überall auf die Daten zugreifen kann (Focus 2010). Eine Mitarbeiterin kann so beispielswiese auf ihrem Tablet Dokumente erstellen und 
diese später auf dem Laptop abrufen und weiterbearbeiten. Es existieren unterschiedliche Arten von Cloud Computing: Infrastructure as a Service (IaaS - Miete von IT-Infrastruktur plus Wartung), Platform as a Service (PaaS - Bereitstellung von Entwicklungsumgebungen als Frameworks für selbst erstellte Programme) und Software as a Service (SaaS - Nutzung browserbasierter Standardsoftware via Internetbrowser) (Tetzel 2016; Botfrei Blog 2012).

Eine PwC-Studie (PwC 2014), an der sich knapp 270 in den USA ansässige Unternehmen beteiligten, veranschaulicht die Auslagerung von HR-Applikationen in die Cloud. Die Nutzung von Cloud-Lösungen unterschied sich demnach in Abhängigkeit von der Unternehmensgröße. So lagerten $70 \%$ der befragten Unternehmen mit weniger als 5000 Mitarbeitenden ihre HR-Applikationen in eine Cloud aus, aber nur $10 \%$ der Unternehmen mit mehr als 10.000 Mitarbeitenden. Mit zunehmender Kleinheit von Unternehmen konnte eine Tendenz zur HR-Cloud-Auslagerung ausgemacht werden. Die wichtigsten Gründe waren die Innovationsförderung, die Erhöhung der Flexibilität und eine Kostenreduzierung. Man konnte außerdem feststellen, dass Unternehmen welche HR-Anwendungen aus der Cloud einsetzen, mehr mobile Technologien einsetzen, deren Gebrauch ebenfalls deutlich zugenommen hat (2014 ca. $68 \%$ gegenüber $30 \%$ in 2013).

\section{Mobility}

Im Zeitalter der digitalen Vernetzung kommunizieren viele Mitarbeitende zunehmend auch von Zuhause aus mit Arbeitskollegen, Kunden, Lieferanten usw. Es ist für immer mehr Arbeitnehmende egal, wo sie arbeiten, da sie überall gleichermaßen gut erreichbar sind. Vor diesem Hintergrund verwenden Büroplaner und Architekten schon heute in wachsendem Maße die Formel $70 \mathrm{zu}$ 30: $70 \%$ der Mitarbeitenden befinden sich normalerweise an ihrem Schreibtisch im Unternehmen und $30 \%$ sind woanders. Dies geht einher mit der immer häufigeren Möglichkeit von Homeoffice-Tagen (Cole 2015, S. 178 f.). Eine Reihe von Unternehmen sieht diese Entwicklung aber durchaus auch kritisch. Sie befürchten einen Kontrollverslust. Die Digitalisierung fördert aber zwar eine Reduzierung der Arbeitskontrolle, erlaubt jedoch eine Beibehaltung der primär erfolgsrelevanten und unverzichtbaren Zielkontrolle (Jäger und Petry 2012, S. 127). Flexibilität wird von Kritikern ferner mit sinkender Produktivität in Verbindung gebracht. Letzteres ist aber heute wohl weniger eine Frage der Örtlichkeit als eine Frage der Unternehmenskultur. In dem Zusammenhang kann Homeoffice sogar dazu führen, dass Mitarbeitende effizienter arbeiten, denn sie sind motivierter und müssen Aufgaben verstärkt wertorientiert priorisieren.

\subsubsection{Demografie und Neupositionierung des HRM}

Zwei zentrale Aspekte für Digitalisierungsprozesse im HRM sind der demografische Wandel in der Schweizer Bevölkerung und substanzielle Veränderungen von Aufgabenstellung und Positionierung der Personalbereiche in Unternehmen. 


\section{Demografischer Wandel}

Seit Jahrzehnten konstant niedrige Geburtenraten und eine immer weiter steigende Lebenserwartung haben die Strukturen der Schweizer Bevölkerung stark verändert. Der Anteil der älteren Menschen hat stark zugenommen. Auf der anderen Seite ist der Anteil jüngerer Menschen erheblich gesunken. Damit verbunden ist ein wachsender Mangel an Fachkräften für die Wirtschaft. So sind in der Schweiz $41 \%$ (Deutschland $46 \%$ ) der Unternehmen bereits heute vom Fachkräftemangel betroffen. Unternehmen haben es schwer, passende Mitarbeiter zu rekrutieren. Im Jahr 2015 hat der Fachkräftemangel in der Schweiz um $8 \%$ im Vergleich zum Vorjahr zugenommen, was die Entwicklungsdynamik verdeutlicht (Tagesanzeiger 2015). Deutlich weniger kritisch als in der Schweiz ist diese Entwicklung etwa in Großbritannien und den Niederlanden, wo nur $14 \%$ der Firmen Schwierigkeiten haben, offene Stellen zu besetzen. Eine Reihe Schweizer Unternehmen muss immer wieder Kundenaufträge ablehnen, weil die nötigen Fachkräfte fehlen (Cole 2015, S. 38). Der demografische Wandel unterstützt also die Tendenz, dass junge Arbeitskräfte sich ihren Arbeitgeber aussuchen können, was die Unternehmen dazu zwingt, Employer Branding auch auf digitalen Kanälen zu betreiben, die durch potenzielle Fachkräfte privat genutzt werden (z. B. Social-Media-Plattformen).

\section{Veränderung des HRM-Aufgabenfelds durch den technologischen Wandel}

Die Personalabteilungen beschäftigen sich heute immer noch mehrheitlich mit traditionellen Aufgaben der Administration, Stammdatenpflege oder Rekrutierungsprozessen (Cachelin 2014b, S. 22). Zunehmend werden diese Aufgaben aber durch den Einsatz von Personalinformations- und -verwaltungssystemen obsolet. Eine wichtige Rolle spielen dabei Self Service-Lösungen für die Mitarbeitenden, beispielsweise bei der Spesenerfassung. IT-bedingte Aufgabenverschiebungen und Effizienzsteigerungen stellen in Verbindung mit der zunehmenden Bedeutung des Produktionsfaktors Personal im positiven Sinne eine Chance zur Neupositionierung des HRM dar. Die Trendstudie von Cachelin (2013b, S. 16) unterstützt diese Vermutung und identifiziert die Notwendigkeit zur Bildung neuer HRM-Geschäftsmodelle. Im Kontext des Digitalisierungstrends scheinen für die künftige Neuausrichtung von HR-Abteilungen insbesondere folgende Aufgabenstellungen prioritär (absteigende Wichtigkeit) (Cachelin 2013b, S. 15) (Reihenfolge absteigend):

- Talentmanagement

- Strategische Personalplanung

- HR Big-Data-Management

- HR IT-Management

- Führungskräfteentwicklung

- Mitarbeiterengagement.

Im Vordergrund steht zukünftig eine stärkere Wertschöpfung des HRM und deren Transparentmachung. Um dies zu erreichen, muss das HRM stärker über agile Strukturen in die Geschäftsprozesse des Unternehmens eingebunden werden (ebd.). 


\subsubsection{Anbieter und Nachfrager von HRM-Digitalisierungslösungen in der Schweiz}

Die derzeit besonders relevanten Anbieter von HRM-Softwarelösungen in der Schweiz werden in Anlehnung an den HR-Software-Report 2013 (HRM.ch 2013) unter Einbindung von Angaben der Firmenwebsites kurz vorgestellt. Der HR-Software-Report basiert auf einer Befragung von Personalexperten aus 138 Unternehmen in Deutschland, Österreich und der Schweiz. Die alphabetisch angeführten Unternehmen sind durchgängig mit einem Firmensitz in der Schweiz vertreten.

Das Schweizer Softwareunternehmen ABACUS beschäftigt 270 Mitarbeitende. Mit mehr als 100.000 verkauften Softwaremodulen und über 41.000 Kunden ist ABACUS der größte unabhängige Schweizer Anbieter von Business Software und konzentriert sich auf kleine und mittlere Unternehmen (KMU). Das Unternehmen ABACUS bietet zwei Hauptmodule für das HRM an: die Lohnbuchhaltung und ein HR-Gesamtpaket, das das Gros der Personalprozesse abdeckt. Schweizer Referenz-KMU sind u. a. die Herzog Haustechnik AG oder die Dr. Vollenweider AG Geotechnik.

Das amerikanische Unternehmen ADP ist seit über 60 Jahren weltweiter Führer im Bereich Gehalts- und HR-Management. Das in 135 Ländern aktive Unternehmen hat weltweit 637.000 Kunden. Das Unternehmen ADP ist in der Schweiz seit 30 Jahren vertreten und bedient über 250 Kunden in allen Geschäftsbereichen. Das Softwareangebot fokussiert auf Personalbeschaffung, -auswahl, -einsatz und -beurteilung. Ein bekannter Schweizer Referenzkunde ist die Syngenta AG.

Die deutsche Firma ATOSS ist über 25 Jahre am Markt für Workforce Management tätig. Das Unternehmen ist in mehr als 40 Ländern vertreten und gehört zu den Key Playern und Trendsettern in diesem Markt. Die Lösungen für nahezu alle Personalprozesse sind mittlerweile in acht Sprachen erhältlich. ATOSS-Lösungen sind exemplarisch in der Schweiz bei der Credit Suisse und bei Coop im Einsatz.

MICROSOFT ist weltweit einer der größten und bekanntesten Softwarehersteller und beschäftigt in der Schweiz ca. 620 Mitarbeitende. Microsoft ist nicht spezialisiert auf Personalinformationssysteme, in der von Microsoft angebotenen ERP-Lösung ist das Personalwesen aber ein integrierter Bestandteil. Referenzkunden im HR-Bereich sind zum Beispiel die deutschen Unternehmen Würth oder New Yorker.

ORACLE gehört ebenfalls zu den führenden Softwareherstellern weltweit. Mit seinen 420.000 Kunden in mehr als 145 Ländern bietet das Unternehmen eine umfassende Palette an Softwarelösungen an. Weltweit beschäftigt das Unternehmen mehr als 130.000 Mitarbeiter. Auch ORACLE deckt mit seinem HR-Softwareangebot nahezu alle Personalprozesse umfassend ab. Bekannte Schweizer Referenzkunden sind die Baloise Group oder das CERN.

Das im Jahre 2000 entstandene deutsche Unternehmen REXX SYSTEMS ist mittlerweile in über 20 Ländern vertreten und bietet ausschließlich HR-Lösungen an. 700 Kunden weltweit nutzen die einfach zu bedienende und hochskalierbare mehrsprachig verfügbare HR-Software. Schweizer Kunden sind u. a. die Helvetia und der Flughafen Zürich. 
Das von ehemaligen IBM-Mitarbeitern gegründete deutsche Unternehmen SAP sammelt seit mehr als 40 Jahren Erfahrungen im Bereich der Unternehmenssoftware. Mit über 310.000 Kunden in mehr als 190 Ländern ist die SAP im Bereich Unternehmenssoftware der umsatzstärkste Anbieter von Software und Softwareservices. Mittlerweile beschäftigt die SAP weltweit mehr als 78.000 Mitarbeitenden in 130 Ländern. Die HR-Lösungen sind modular und prozessorientiert aufgebaut. Vor allem Schweizer Großunternehmen wie SwissRe, Schweizerische Post, ABB oder die SBB setzen SAP-Systeme ein.

Als potenzielle Nachfrager von Digitalisierungsangeboten im Bereich HRM kommen alle Unternehmen, Organisationen und Institutionen infrage, die über ein kritisches Volumen an Personal(-bedarfen) verfügen. Da in der Literatur ein HRM-Digitalisierungsnachfragepotenzial eher unspezifisch thematisiert wird, kann ein auf die Digitalisierung generell abhebende Untersuchung zum Entwicklungsstand der Digitalisierung in der Schweiz, das Projekt digital.swiss, als Indikationserhebung für die weitere Entwicklung auch im HRM herangezogen werden. Die Studie leitet eine gesamtschweizerische Kennzahl ab, die den Fortschritt der Digitalisierung in der Schweiz vor dem Hintergrund des derzeit technisch Möglichen aufzeigt. Sie kommt zum Schluss, dass die Schweiz etwa die Hälfte des Wegs $(51 \%)$ hin zu einer digitalisierten Gesellschaft gegangen ist (ICT Switzerland 2016). Das höchste Entwicklungspotenzial weist das Thema „Industrie 4.0“ (Digitalisierungsscore von $18 \%$ ) auf. Am weitesten ist der Entwicklungsstand der Schweizer Basisinfrastrukturen mit einem Wert von $95 \%$. Der HR-seitig besonders interessante Wert für den Themenkreis „Fachkräfte \& Bildung“ liegt mit $56 \%$ leicht über dem Durchschnittswert. Das Nachfragepotenzial für Digitalisierungslösungen ist also auch hier noch sehr groß. Weitere branchenbezogene Untersuchungen von Ernst \& Young für die Bankenbranche und KMU (Computerworld 2016a, b) oder die breitere Studie von Accenture (2015) sehen ebenfalls noch erhebliches Wachstumspotenzial für Digitalisierungslösungen in der Schweiz, die derzeit vor allem im Kundenkontext zum Einsatz kommen. Generell am weitesten entwickelt scheinen Unternehmen im IT-, Kommunikations- und Medienbereich sowie die Banken.

\subsubsection{Problemfelder der Digitalisierung im Personalbereich}

Die Einführung von Digitalisierungsanwendungen im HR-Bereich ist nicht nur mit Vorteilen hinsichtlich der Prozesseffizienz und personalwirtschaftlicher Entscheidungen verbunden, sondern birgt auch eine Reihe potenzieller Problemfelder und Risiken.

Die Digitalisierung und die mit ihr verbundenen Möglichkeiten können für HR-Mitarbeitende Überforderungs- und Existenzängste nach sich ziehen, da insbesondere im administrativen Bereich erhebliche Einsparungen im Sinne einer besseren Wertschöpfung zu erwarten sind, die auch mit Personalreduzierungen einhergehen können, sofern die freiwerdenden Ressourcen nicht anderweitig eingesetzt werden. Positiv betrachtet heißt dies, dass Personalmitarbeitende zunehmend von Routinetätigkeiten entlastet und für anspruchsvollere und stärker kundenorientierte HR-Aufgaben frei werden. 
Eine wichtige Voraussetzung, um potenzielle Widerstände einer Digitalisierung des Personalwesens zu begegnen ist die Qualifizierung der betroffenen Personalmitarbeitenden. Das IT-/Computer-Basiswissen ist schweizweit generell bei den Berufstätigen noch unzureichend ausgeprägt, auch wenn dies individuell oft nicht so wahrgenommen wird. So überschätzen derzeit ca. $78 \%$ der Schweizer Bevölkerung ihre Computerkenntnisse teils erheblich. Dies ergab ein jüngst durchgeführter umfassender Wahrnehmungs- und Ist-Abgleich der ECDL Switzerland AG (2015). Eine fundierte Qualifizierung der HR-Mitarbeitenden im Umgang mit neuen Digitalisierungstools ist auch vor diesem Hintergrund zwingend. Gleiches gilt auch für benötigte Kompetenzen zum digitalen Wandel, die über die technische Basisqualifizierung der Personalmitarbeitenden hinausgehen. Eine neuere globale Befragung von 81 Unternehmen (Hoberg et al. 2015) hat ergeben, dass insbesondere grundlegende digitale Kompetenzen in den Bereichen Digitale Sicherheit, Business Change Management und Business Networks für eine erfolgreiche digitale Transformation in Unternehmen von Bedeutung sind. Dies sollte generell auch bei Neurekrutierungen außerhalb des Personalbereichs berücksichtigt werden.

Die Anwendung von HR-Digitalisierungslösungen birgt das Risiko, dass digitalisierte Prozessabwicklungen und Entscheidungsfundierungen zunehmend nicht mehr kritisch hinterfragt werden. Cachelin (2016b) spricht in dem Zusammenhang von sogenannten „Filter Bubbles“, wenn zum Beispiel eine Big-Data-basierte Personalvorauswahl dazu führt, dass nur noch gleichbleibende Mitarbeitendentypen rekrutiert werden, was die Innovationskraft von Unternehmen hemmen kann.

Die durch die Digitalisierung geförderte jederzeitige Datenverfügbarkeit geht auch mit einem wachsenden Risiko von Cyberattacken einher (Cachelin 2016b). Sensible Personaldaten sind hier in besonderem Maße gefährdet und zwingend bestmöglich vor Missbrauchszugriffen zu schützen. Eine aktuelle deutsche Unternehmensstudie zu IT-Sicherheit und Datenschutz (Schwartz und Muhle 2016) hat ergeben, dass für KMU insbesondere der Schutz von Software und Backup-Lösungen höchste Priorität haben und dass die Sicherheitsmaßnahmen bei allen Unternehmen umso größer ausfallen, je höher der technische Stand und der Vernetzungsgrad sind. Kaum ausgeprägt hingegen sind Maßnahmen im Bereich der Mitarbeitendenqualifizierung für Verhaltensänderungen und die Berücksichtigung von Sicherheitsaspekten bei der Rekrutierung neuer Mitarbeitender. Beides sind Bereiche, in denen die Personalabteilungen einen eigenen Beitrag für einen verbesserten Datenschutz leisten können. Ein besonders wichtiger Punkt bei der Diskussion des Datenschutzes in Verbindung mit einer Digitalisierung des HRM sind Cloud-Anwendungen, bei denen es vor allem auch darum geht, dass Informationen zu Logins, Datenbanken und weiteren sensiblen Informationen, die über das Internet zugänglich gemacht werden, nicht durch den Zugriff über ungesicherte Notebooks oder Mobilgeräte gefährdet werden. Darüber hinaus sind die Datenschutzmaßnahmen der Cloud-Anbieter durchaus nicht durchgängig zufriedenstellend (Cloudcomputing-insider 2016). Bei der Einführung von HRM-Cloud-Lösungen hat dieser Aspekt eine sehr hohe Relevanz. 


\subsubsection{Reifegradmodell}

In Anlehnung an Berghaus et al. (2015, S. 10 ff.) kann ein auf den Digitalisierungskontext des HRM abhebendes Reifegradmodell abgeleitet werden, das zur Systematisierung der eigenen empirischen Erhebung herangezogen wurde. Der St. Galler-Business-Engineering-Framework-Ansatz mit den Ebenen „Strategie“, ,Prozesse/ Organisation“ und „Technologie“ wird um die gleichsam relevanten HRM-Digitalisierungsbezugsebenen „Aktuelle und potenzielle Mitarbeitende“ (Kundenperspektive), „Kultur und Führung“ (Werteperspektive) sowie „HR-Services“ (Leistungsperspektive) erweitert und explizit in den HRM-Kontext gestellt. Ferner wird die Technologieebene durch die vier identifizierten technologischen HRM-Hauptentwicklungsfelder „Social Media“, „Data Mining“, „Cloud-Lösungen“ und „Mobility“ weiter ausdifferenziert, sodass das Modell aus neun Bewertungsfeldern mit unmittelbarem HRM-Bezug besteht (vgl. dazu Abb. 8.2). Auf die Bewertungsfelder werden fünf definierte Reifegradstufen angewendet, die für eine Gesamteinschätzung des Entwicklungsstands relevant sind. Anhand des Reifegradmodells soll somit differenziert und summarisch die Frage beantwortbar sein, wie der derzeitige Stand der HRM-Digitalisierung in der Schweiz zu bewerten ist.

Die fünf Reifegradstufen werden auf Basis einer Ist-Erhebung der Ausprägungen der Bewertungsfelder für ein Unternehmen ermittelt. Eine inhaltliche Präzisierung der Reifegradstufen findet sich in Tab. 8.2.

Die Charakterisierung der im HRM-Kontext zugrunde liegenden neun Untersuchungsdimensionen des Reifegradmodells erfolgt unter Bezugnahme auf eine Optimalausprägung eines digitalisierten Unternehmens (Digital Company) gemäß der definierten Reifegrade in Tab. 8.3.

Abb. 8.2 Reifegradmodell zur Digitalisierung des HRM in der Schweiz. (Eigene Darstellung in Anlehnung an Berghaus et al. 2015, S. 14)

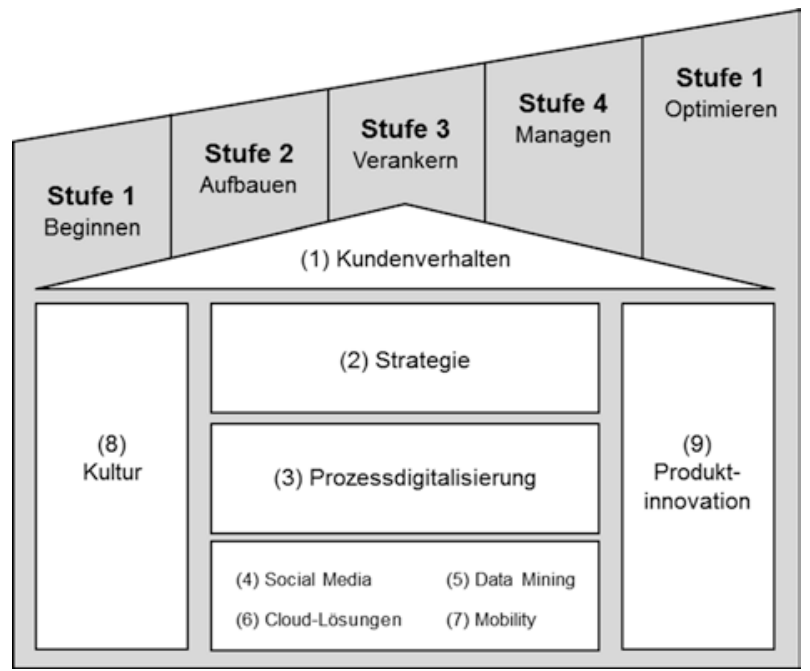


Tab. 8.2 Reifegrade zur Kennzeichnung des Digitalisierungsstands. (Quelle: Eigene Darstellung)

\begin{tabular}{l|l}
\hline Stufe & Beschreibung \\
\hline (1) Beginnen & $\begin{array}{l}\text { Prozesse sind noch nicht digitalisiert. Der Handlungsbedarf wird jedoch erkannt } \\
\text { und erste Ansätze für eine digitale Transformation sind erkennbar }\end{array}$ \\
\hline (2) Aufbauen & $\begin{array}{l}\text { Digitale Projekte werden priorisiert und vorangetrieben. Digitale Kompetenzen } \\
\text { sind bei der Rekrutierung von Mitarbeitenden eine wichtige Selektionsbasis }\end{array}$ \\
\hline (3) Verankern & $\begin{array}{l}\text { Das Thema „Digitalisierung“ ist auf strategischer Ebene verankert. Es wird als } \\
\text { Change-Projekt kontinuierlich und systematisch verfolgt. Digitale Schlüssel- } \\
\text { technologien für das Unternehmen sind definiert. Mobile Kanäle sind in allen } \\
\text { Leistungsfeldern integriert }\end{array}$ \\
\hline (4) Managen & $\begin{array}{l}\text { Neue digitale Technologien werden schnell aufgegriffen und überprüft. Neue } \\
\text { digitale Schlüsseltechnologien werden auf ihre strategische Bedeutung hin } \\
\text { analysiert. Die Automatisierung von Prozessen schreitet voran. Analytics-Tools } \\
\text { werden zur Optimierung eingesetzt und ausgebaut }\end{array}$ \\
\hline (5) Optimieren & $\begin{array}{l}\text { Der Digitalisierungsprozess wird systematisch und fortlaufend anhand von } \\
\text { Steuerungsindikatoren optimiert. Der digitale Entwicklungsstand in den Kern- } \\
\text { prozessen ist auf Best-Practice-Niveau }\end{array}$ \\
\hline
\end{tabular}

\subsection{Empirische Erhebung - Digital HRM in der Schweiz}

Das entwickelte Reifegradmodell dient als systematischer Dreh- und Angelpunkt zur Bewertung des Digitalisierungsgrades im HRM von Schweizer Unternehmen. Nachfolgend werden die Zielsetzung der empirischen Untersuchung, die methodischen Parameter und ihre Ergebnisse vorgestellt und kritisch reflektiert.

\subsubsection{Zielsetzung und Methodik}

Durch die Befragung von HRM-Experten Schweizer Unternehmen, die digitale HRM-Anwendungen einsetzen oder anbieten, soll ein (nicht repräsentativer) qualitativer Eindruck vom aktuellen Entwicklungsstand und bedeutsamen Trends bei technischen Lösungen für das HRM und deren Einsatz und Konsequenzen in der Praxis gewonnen werden. Darüber hinaus sollen Chancen und Problemfelder einer Digitalisierung im HRM erkannt und gestaltungsbezogene Empfehlungen abgeleitet werden.

Im Rahmen des gewählten induktiven qualitativen Forschungszugangs soll das „Wie“ des Beziehungsgefüges sowie dessen innere Struktur aus der Perspektive der Untersuchungsobjekte geklärt werden (Kiefl und Lamnek 1984, S. 474). Für Schweizer Unternehmen gibt es bis dato nur geringe Erkenntnisse zur Qualität und zum Ausmaß der Digitalisierung im HRM-Bereich. Grundlegend für die Konzipierung des Gesamtsettings der Erhebung war die Strukturempfehlung für empirische sozialwissenschaftliche Forschungsprozesse nach Gläser und Laudel (2006, S. 32). Das Forschungsziel 
Tab. 8.3 HRM-Dimensionen des Reifegradmodells am Beispiel einer Digital Company. (Eigene Darstellung)

\begin{tabular}{|c|c|}
\hline Dimension & Beschreibung \\
\hline $\begin{array}{l}\text { (1) Kundenver- } \\
\text { halten }\end{array}$ & $\begin{array}{l}\text { Digital Natives orientieren und bewegen sich in der Arbeitswelt bevor- } \\
\text { zugt auf digitalen Kanälen. Das HRM einer Digital Company trägt dem } \\
\text { dadurch Rechnung, dass Informationen zu gegenwärtigen und zukünftigen } \\
\text { Beschäftigten (Kunden) fortlaufend aus verschiedenen digitalen Quellen } \\
\text { generiert und zur Entscheidungsoptimierung eingesetzt werden (Verhaltens- } \\
\text { prognosen). Die Kommunikation mit aktuellen und potenziellen Mit- } \\
\text { arbeitenden erfolgt nahtlos und konsistent über alle digitalen Kanäle (z. B. } \\
\text { Self Service-Intranetplattformen). Gesellschaftliche und demografische } \\
\text { Veränderungen schlagen sich in einer kundenorientierten digitalen HR-Infra- } \\
\text { struktur nieder (hohe Usability und User Experience als Ziel) }\end{array}$ \\
\hline (2) Strategie & $\begin{array}{l}\text { In digitalisierten Unternehmen ist die Digitalisierung ein zentraler Bau- } \\
\text { stein von Geschäftsmodell und -strategie und auch der Personalstrategie. } \\
\text { Dies schlägt sich in optimierten Stellen- und Strukturlösungen für das } \\
\text { HRM und auf Mobilität, Flexibilität und Vernetzung ausgerichtete ganzheit- } \\
\text { liche Systemlösungen für HR-Manager und die Beschäftigen nieder (z. B. } \\
\text { Homeoffice-Angebot) }\end{array}$ \\
\hline $\begin{array}{l}\text { (3) Prozess- } \\
\text { digitalisierung }\end{array}$ & $\begin{array}{l}\text { In Digital Companies sind die internen HR-Prozesse durchgängig digitali- } \\
\text { siert, integriert und auf Prozesseffizienz und Kundenorientierung ausgerichtet } \\
\text { (z. B. Einsatz einer umfassenden HRM-Softwarelösung wie HCM Suite von } \\
\text { SAP) }\end{array}$ \\
\hline (4) Social Media & $\begin{array}{l}\text { Digitalisierte Unternehmen setzen in der Interaktion mit Menschen sehr } \\
\text { stark auf die sozialen Medien (YouTube, Xing, LinkedIn, Facebook etc.) } \\
\text { und tragen damit dem veränderten Kommunikationsverhalten relevanter } \\
\text { Zielgruppen Rechnung. Die breite professionelle Nutzung in verschiedenen } \\
\text { HR-Gestaltungsbereichen geht mit einer hohen Sensibilität in Bezug auf } \\
\text { mögliche Problembereiche einher (z. B. Erreichbarkeit, Qualität und Aktuali- } \\
\text { tät der Inhalte, Umgang mit Negativ- und Falschinformationen etc.) }\end{array}$ \\
\hline (5) Data Mining & $\begin{array}{l}\text { Digital Companies betreiben aktives und systematisches Data Mining und } \\
\text { verwandeln damit früher brachliegende Informationen über aktuelle und } \\
\text { potenzielle Mitarbeitende in wertgenerierendes Wissen. Der Einsatz von } \\
\text { Big Data-Applikationen und Business Intelligence-Lösungen für das HRM } \\
\text { ist selbstverständlich (z. B. Social Media-basierte Employer Branding-Ana- } \\
\text { lysen) }\end{array}$ \\
\hline $\begin{array}{l}\text { (6) Cloud- } \\
\text { Lösungen }\end{array}$ & $\begin{array}{l}\text { Cloud-Tools und -Systeme für den HR-Bereich (z. B. PeopleDoc) sind in } \\
\text { Digital Companies etabliert und stehen für innovative, flexible und kosten- } \\
\text { günstigere Anwendungsmöglichkeiten. Jeder Berechtigte kann von überall, } \\
\text { respektive von jedem internetfähigen Gerät auf HR-Daten zugreifen. }\end{array}$ \\
\hline (7) Mobility & $\begin{array}{l}\text { Mobile Geräte werden zunehmend auch zu universellen Arbeitsgeräten die } \\
\text { mit einer wachsenden Vernetzung aller Lebenssphären einhergehen. Digi- } \\
\text { talisierte Unternehmen tragen dem Rechnung, indem alle digitalen Lösun- } \\
\text { gen im HRM-Bereich auf eine sinnvolle mobile Nutzung hin geprüft und } \\
\text { entsprechend implementiert werden (z. B. Gehaltsabrechnung ist auf dem } \\
\text { Handy einsehbar). Responsive designte Applikationen sind Standard }\end{array}$ \\
\hline
\end{tabular}


Tab. 8.3 (Fortsetzung)

\begin{tabular}{l|l}
\hline Dimension & Beschreibung \\
\hline (8) Kultur & $\begin{array}{l}\text { Damit die Digitalisierung im HRM die intendierten Erfolgswirkungen } \\
\text { entfalten kann, ist eine große Offenheit der HR- und aller anderen Mit- } \\
\text { arbeitenden gegenüber neuen digitalen Technologien und der damit einher- } \\
\text { gehenden dynamischen Veränderungen erforderlich. Digitalisierung wird } \\
\text { in Digital Companies visionär, ,gelebt“ und ist als wichtiges Kulturelement } \\
\text { weitgehend etabliert (Digital Culture) }\end{array}$ \\
\hline $\begin{array}{l}\text { (9) Produkt- } \\
\text { innovationen }\end{array}$ & $\begin{array}{l}\text { Digitalisierte Unternehmen profilieren sich mit technologiebasierten Innova- } \\
\text { tionen bei HR-Dienstleistungen. Dies gilt auch und insbesondere in Bezug } \\
\text { auf die Anbieter von HR-Digitalisierungslösungen, die als Innovationstreiber } \\
\text { im HRM-Segment agieren (z. B. ADP, Atoss, Oracle, SAP etc.) }\end{array}$ \\
\hline
\end{tabular}

soll auf der Grundlage theoretisch definierter Vorabfestlegungen und einer resultierenden Samplebestimmung (Heister 2006) durch 17 leitfadengestützte Interviews von HR-Fachkräften und ein perspektivenweitendes und abrundendes Interview eines HR-Softwareexperten auf der Anbieterseite erreicht werden. Alle befragten Unternehmen sind in der Deutschschweiz ansässig. Bei der Auswahl der Unternehmen wurde wegen der hohen Zukunftsgerichtetheit der Themenstellung ein Fokus auf Großunternehmen gelegt, da diese aufgrund der Erkenntnisse der Voruntersuchung erheblich weiter bei der HRM-Digitalisierung fortgeschritten sind als das Gros der klein- und mittelständischen Unternehmen. Ausgangspunkt der Selektion geeigneter Partner war die Segmentas/ Handelszeitung-Top 500-Liste der größten Unternehmen der Schweiz mit mindestens 180 Mio. Franken Umsatz (Segmentas 2016). Bei der Auswahl der Probanden wurde auf ein möglichst breites Branchenspektrum geachtet. In Bezug auf die Anbieterseite konnte mit der SAP (Schweiz) AG einer der Marktführer für HRM-Digitalisierungslösungen gewonnen werden. Die Aussagen dieses Interviewpartners dienen primär der Validierung und Perspektivenweitung bei der Analyse der unternehmensbezogenen Aussagen auf der Nachfragerseite. Die teilnehmenden Unternehmen sind, mit Ausnahme von drei anonymisierten Großunternehmen, Tab. 8.4 entnehmbar. Für die Nachfrager- und Anbieterseite wurde auf Basis der Struktur des Reifegradmodells jeweils ein bewusst sehr offen gehaltener Fragebogen konzipiert und vorgängig getestet. Die Experteninterviews wurden im Zeitraum Juli bis November 2016 durchgeführt. Die aufgezeichneten Interviews wurden anschließend sinngemäß transkribiert, strukturell codiert und mit Microsoft Excel ausgewertet.

\subsubsection{Ergebnisse}

Nachfolgend werden die wichtigsten Ergebnisse der Erhebung in Anlehnung an die zugrunde liegende Struktur und Nummerierung des Reifegradanalysemodells dargelegt. 
Tab. 8.4 Befragte Schweizer Großunternehmen. (Eigene Darstellung)

\begin{tabular}{|c|c|c|}
\hline Unternehmen & Branche & Experte \\
\hline BKW AG & Energieversorgung/-verteilung & $\begin{array}{l}\text { Leitungsperson } \\
\text { HR }\end{array}$ \\
\hline BLS AG & Bahnen/Öffentlicher Verkehr & $\begin{array}{l}\text { HR-Expertin und } \\
\text { HR-Business } \\
\text { Partner }\end{array}$ \\
\hline Bystronic Laser AG & Maschinenindustrie & $\begin{array}{l}\text { Leitungsperson } \\
\text { HR }\end{array}$ \\
\hline Calida AG & Bekleidungsindustrie & $\begin{array}{l}\text { Zwei Leitungs- } \\
\text { personen HR }\end{array}$ \\
\hline Frutiger AG & Bau & $\begin{array}{l}\text { Leitungsperson } \\
\text { HR }\end{array}$ \\
\hline Genossenschaft Migros Aare & Detailhandel & $\begin{array}{l}\text { Leitungsperson } \\
\text { HR }\end{array}$ \\
\hline SAP (Schweiz) AG ${ }^{a}$ & Computer/Informatik & $\begin{array}{l}\text { Anonym (mit } \\
\text { HRM-Markt- } \\
\text { kenntnis) }\end{array}$ \\
\hline Schweizerische Mobiliar Holding AG & Versicherungen und Vorsorge & $\begin{array}{l}\text { Leitungsperson } \\
\text { HR }\end{array}$ \\
\hline Schweizerische Post AG & Dienstleistungen & $\begin{array}{l}\text { Leitungsperson } \\
\text { HR }\end{array}$ \\
\hline $\begin{array}{l}\text { Schweizerische Radio- und Fernseh- } \\
\text { gesellschaft (SRG SSR) }\end{array}$ & Mediendienstleistungen & $\begin{array}{l}\text { Leitungsperson } \\
\text { HR }\end{array}$ \\
\hline Schweizerische Bundesbahnen (SBB) & Bahnen/Öffentlicher Verkehr & HR-Experte \\
\hline Swisscom AG & Telekommunikation & $\begin{array}{l}\text { Leitungsperson } \\
\text { HR }\end{array}$ \\
\hline Valiant Bank AG & Banken & $\begin{array}{l}\text { Leitungsperson } \\
\text { HR }\end{array}$ \\
\hline Ypsomed AG & Medizinaltechnik & $\begin{array}{l}\text { Leitungsperson } \\
\text { HR }\end{array}$ \\
\hline $\begin{array}{l}\text { Unternehmen Automobil- und Motor- } \\
\text { fahrzeuggewerbe }\end{array}$ & $\begin{array}{l}\text { Automobil-/Motorfahrzeug- } \\
\text { gewerbe }\end{array}$ & $\begin{array}{l}\text { Leitungsperson } \\
\text { HR }\end{array}$ \\
\hline Bundesbehörde (im 3. Kreis) $^{\text {b }}$ & Öffentlicher Dienst & $\begin{array}{l}\text { Leitungsperson } \\
\text { HR }\end{array}$ \\
\hline Unternehmen Rüstungsgüterindustrie & Rüstungsgüterindustrie & $\begin{array}{l}\text { Leitungsperson } \\
\text { HR }\end{array}$ \\
\hline
\end{tabular}

${ }^{a}$ Anbieter von HRM-Digitalisierungslösungen (alle anderen Unternehmen sind Nachfrager)

${ }^{\mathrm{b}}$ Anstalt/Betrieb mit eigener Rechtspersönlichkeit, der zu $100 \%$ im Besitz des Bundes ist 


\section{(1) Kundenverhalten}

In Bezug auf die Analyse des Kundenverhaltens kann für nahezu alle untersuchten Unternehmen der Nachfragerseite festgestellt werden, dass die HR-Abteilungen bislang noch keine größeren Aktivitäten zur Erhebung der Bedürfnisse von aktuellen und potenziellen Mitarbeitenden hinsichtlich der Digitalisierung ergriffen haben. Digitale Innovationen im HRM werden eher technikgetrieben von außen und von internen Experten proaktiv auf der Grundlage von Annahmen zur Effizienzsteigerung angestoßen und gehen weniger von konkret erfragten Bedürfnislagen der Mitarbeitenden aus. Vereinzelt finden sich entsprechende Fragestellungen in Mitarbeitendenbefragungen wieder, in der Regel sind diese aber nicht sehr prominent lanciert. Losgelöst vom Digitalisierungsaspekt erfolgen allerdings in nahezu allen Unternehmen regelmäßige (Online-)Mitarbeitendenbefragungen und -gespräche. Ein kleiner Teil der Unternehmen verzichtet jedoch bewusst ganz auf Mitarbeitendenbefragungen oder ist im Zeitablauf von diesen aufgrund eines geringen Erkenntnisgewinns wieder weggekommen. Einen in technischer und inhaltlicher Hinsicht innovativen Ansatz verfolgt ein Unternehmen mittels eines webbasierten Tools, das es den Mitarbeitenden erlaubt über kurze Statements zu vordefinierten Themen Stimmungen wiederzugeben und an Entscheidungen mitwirken zu können.

In Bezug auf verhaltensbezogene Aspekte des demografischen Wandels im Kontext der Digitalisierung gibt es keine durchgängig einheitliche Einschätzung der Befragten. Der zumeist unterstellte Digitalisierungsvorsprung von jüngeren Mitarbeitenden wird von wenigen Experten sogar grundsätzlich infrage gestellt: Vereinzelt seien ältere Mitarbeitende deutlich technisch affiner eingestellt als jüngere. Das Gros der Befragten bestätigt aber grundsätzlich die Existenz größerer Wissens- und Erfahrungslücken zwischen älteren und jüngeren Generationen. Junge Mitarbeitende seien in der Regel wesentlich fundierter und vertrauter im Umgang mit neueren digitalen Entwicklungen und agieren oft als Treiber für digitale Innovationen in den Unternehmen. Ansatzpunkte für eine Angleichung der Digitalisierungsniveaus können Jung-Alt-Mentoring- oder Coaching-Modelle sein. Neben dem Alter kommen aber eine Reihe anderer Rahmensetzungen zum Tragen, die auf die Grundhaltung der Mitarbeitenden und deren Umgang mit der Digitalisierung einwirken wie etwa das Berufsfeld, die digitale Alltagsrelevanz oder die Dauer der Betriebszugehörigkeit und -prägung.

Die HR-Abteilungen der befragten Unternehmen haben den Digitalisierungstrend im Grundsatz zwar erkannt, bei einigen Unternehmen besteht aber offenbar noch ein spürbarer Nachholbedarf beim Einsatz von kundenorientierten digitalen HR-Lösungen. Insgesamt stehen die untersuchten Schweizer Unternehmen erst am Anfang, was die eingehende digitalisierungsbezogene Bedarfs- und Verhaltensanalyse von Mitarbeitenden angeht (Stufe 1 gemäß Reifegradmodell).

\section{(2) Strategie}

Die strategische Verankerung der Digitalisierung im HRM ist eine wichtige Voraussetzung für den Erfolg digitaler Transformationsprozesse. Für die meisten Befragten 
stellt die Digitalisierung einen unumgehbaren Megatrend dar (,vierte industrielle Revolution"), der die zukünftige Strategie und den nachhaltigen Erfolg der Organisation mitbestimmen wird. Gemäß der Einschätzung nahezu aller Befragter stehen die Unternehmen diesbezüglich aber erst am Anfang von sehr weitgehenden Veränderungen in der Arbeitswelt, aus denen neue Berufsbilder und Arbeitsformen hervorgehen werden. Erste größere strategische Digitalisierungsprojekte werden auf Ebene Gesamtunternehmen und im HR-Bereich auf den Weg gebracht. Ein Beispiel ist die Realisierung eines strategischen Omni-Channel-Projekts in einem Unternehmen, das zum Ziel hat, alle im Kontakt mit Bewerbenden und Mitarbeitenden eingesetzten Kanäle miteinander zu verbinden. Neben dem großen Potenzial für neue Geschäftsmodelle auf Gesamtunternehmensebene werden für den HR-Bereich vor allem Neugestaltungs- und Beschleunigungsoptionen für Personalprozesse als wichtig erachtet. Ein negativer Aspekt, der in dem Zusammenhang wiederholt genannt wird, ist die vermutete deutliche Einschränkung von künftigen Gestaltungsspielräumen für HR-Mitarbeitende.

Im strategischen Denken der HR-Probanden ist die Digitalisierung als strategisches Handlungsfeld durchgängig erkannt worden. Einzelne Unternehmen haben bereits HR-Projektexperten mit ausschließlichem Digitalisierungsfokus im Einsatz. Schon umgesetzte strategische Maßnahmen in Bezug auf die Digitalisierung des HRM sind allerdings derzeit eher noch die Ausnahme. Eine tendenziell vorsichtige, reaktive Grundhaltung der Probanden ist erkennbar. Auffallend ist allerdings, dass die großen staatsnahen Betriebe im Themengebiet in der Tendenz etwas progressiver unterwegs sind. Im Vergleich zur ersten analysierten Dimension kann für die Strategiedimension pauschal eine fortgeschrittenere Entwicklungsstufe attestiert werden. Die strategische Dimension der HRM-Digitalisierung befindet sich bei den untersuchten Großunternehmen im Aufbau (Stufe 2 gemäss Reifegradmodell).

\section{(3) Prozessdigitalisierung}

Bei der Analyse der dritten Dimension „Prozessdigitalisierung“ wird hinterfragt, in welchem Ausmaß interne HR-Prozesse durch die befragten Unternehmen bereits digitalisiert wurden.

Unabhängig von der Branche, der Unternehmensgröße oder der Rechtsform der untersuchten Unternehmen zeigt sich, dass das papierlose Büro im HR-Bereich seit einigen Jahren weitgehend realisiert ist. Eine Prozessdigitalisierung hat bei sämtlichen der befragten Unternehmungen mehrheitlich in denselben HR-Funktionsfeldern (Recruiting, Lohnabrechnung, Zeiterfassung) stattgefunden. Die Bereitschaft, Prozesse zu digitalisieren, steigt dabei mit der Höhe des Administrationsgrades des Prozesses an. Prozesse, welche einen großen administrativen Aufwand mit sich bringen, haben somit eine höhere Tendenz digitalisiert zu werden. Bei einer Detailbetrachtung der Umsetzungstiefe und -breite der Prozessdigitalisierung sind im Einzelvergleich signifikante Unterschiede erkennbar. Die Bandbreite reicht hier beispielhaft von eher traditionellen Standardumsetzungen im Bereich Online-Stellenausschreibungen und Bewerbermanagement über den Einsatz von Matching-Software im Recruiting und elektronische Personaldossiers bis 
hin zur toolbasierten Einbindung der Mitarbeitenden in das Employer Branding und hiermit verbundener Prozesse (Employer Advocacy). Eine Folge der relativ fortgeschrittenen Digitalisierung von HR-Standardprozessen ist die zunehmende Verlagerung von administrativen Arbeitsschritten auf die Kunden. Dies entspricht zumeist dem Bedürfnis der Mitarbeitenden nach selbstorganisierten HR-Services, kann aber auch negativ im Sinne einer Arbeitsverlagerung wahrgenommen werden. Wichtig für eine positive Wahrnehmung ist, dass die Prozessverbesserungen mit spürbaren Vereinfachungen einhergehen. So setzt ein Unternehmen beispielsweise bei der Erfassung von Arbeitszeiten bei externen Projekten Tablets ein, über die auch jederzeit die Lohnabrechnung abgerufen werden kann.

Dass die Prozessdigitalisierung das Personalwesen stark verändert, ist für alle Experten ein Faktum. In welchem Ausmaß dies der Fall ist, wird unterschiedlich eingeschätzt. Vielfach findet durch die Digitalisierung von HR-Prozessen lediglich eine Arbeitsveränderung und keine Arbeitseinsparung statt. Eingesparte Zeiten (z. B. durch das nicht mehr erforderliche Einscannen von Bewerbungsunterlagen) können nun durch HR-Mitarbeitende anders und produktiver genutzt werden. Darüber hinaus wird von einer erhöhten Dynamik, Vernetzung und Transparenz der Prozesse ausgegangen, was mit neuen, zumeist höheren Anforderungen an HR-Mitarbeitende einhergeht.

In Bezug auf die zeitliche Staffelung von Prozessdigitalisierungen wurden und werden offenbar administrative Prozesse wie die Arbeitsvertragsabwicklung, die Spesenabrechnung, die Lohnabrechnung und die Zeiterfassung tendenziell vor den restlichen HR-Prozessen digitalisiert. Kostenintensivere Tools mit nicht eindeutigem Mehrwert (z. B. ein Zeugnisgenerator) kommen eher nicht zum Einsatz. Nahezu alle HR-Bereiche sind bemüht, das Employer Branding und den Rekrutierungsprozess so digital wie möglich zu gestalten. Lediglich eines der untersuchten Unternehmen hat quasi sämtliche digitalisierbaren HR-Prozesse von der Rekrutierung bis zum Unternehmensaustritt von Mitarbeitenden auch digitalisiert (HR-Dokumentenplattform und Personendatenpflege, Zeiterfassungssystem mit Genehmigungsworkflows, elektronische Mitarbeiterbeurteilung, Aus- und Weiterbildungstool, Zeugniserstellungstool, Pensionskassenschnittstelle etc.). Vereinzelt werden die prozessbasierten HR-Services konzentriert in Shared Services-Centern bearbeitet.

Zusammenfassend kann festgehalten werden, dass die HR-Abteilungen der befragten Schweizer Unternehmen eine Reihe von Ansätzen der Prozessdigitalisierung bereits umgesetzt haben und hier Chancen für das HRM sehen. Der Entwicklungsstand ist bei einigen Unternehmen, die ganze Prozessketten umfassend abdecken, sehr hoch, bei anderen noch auf der Stufe von Standardprozessautomatisierungen verblieben und auf isolierte Prozessoptimierungen ausgerichtet. Im internationalen Vergleich zum Stand der Prozessdigitalisierung etwa mit nordamerikanischen Großunternehmen hinken die Schweizer Unternehmen zumeist noch hinterher. Über alle betrachteten Unternehmen hinweg kann summarisch von einer Prozessdigitalisierung im fortgeschritteneren Aufbau gesprochen werden (Stufe 2 gemäß Reifegradmodell). 


\section{(4) Social Media}

Die Analyse der vierten Dimension „Social Media“ befasst sich mit Art und Ausmaß der Einbindung von sozialen Netzwerken in das HRM der befragten Großunternehmen.

Die meisten HR-Experten sehen Social Media für die Zukunft als tragenden Baustein eines digitalisierten HRM, vor allem für die Rekrutierung und das Employer Branding. Im Umfeld der sozialen Medien geht es für die Unternehmen darum, sich positiv zu präsentieren und von der Konkurrenz abzuheben, aber auch um die Beschaffung von Informationen über ihre Zielgruppen am Arbeitsmarkt. Insbesondere die öffentlich zugänglichen Business-Plattformen Xing und LinkedIn werden von den HR-Experten als hierfür geeignet angesehen. Die HR-Social-Media-Auftritte werden zumeist aber nicht sehr aktiv betreut und befinden sich bei den meisten Unternehmen noch in der Auf- und Ausbauphase. Einzelne Unternehmen weichen auch hier positiv vom Gros des Samples $\mathrm{ab}$ und rekrutieren bereits intensiv, zum Teil via Active Sourcing, über Business-Plattformen. Private Netzwerke wie Facebook und Instagram werden HR-seitig bislang offenbar nur rudimentär von den Unternehmen genutzt und gepflegt. In Bezug auf die im Internet präsenten Bewertungsplattformen, wie zum Beispiel Kununu, gibt es kein einheitliches Bild. Die Bewertungen dieser Plattformen durch die HR-Experten divergieren stark. Einige Unternehmen agieren proaktiv andere reaktiv im Umgang mit den Plattformen und weitere Unternehmen ignorieren sie völlig, beziehungsweise sehen diese kritisch und scheuen den erforderlichen Zeitaufwand für eine Plattformbetreuung. Snapchat und Twitter, die von vielen Mitarbeitenden privat genutzt werden, werden bis anhin als Plattform für HR-Aktivitäten nur minimal oder gar nicht genutzt.

Alle Unternehmen sind sich darin einig, dass Social Media-Aktivitäten HR-seitig zunehmend bedeutsamer werden, und dass eine professionelle Nutzung von interaktiven Social-Media-Kanälen, neben der Notwendigkeit eines entsprechenden Know-hows, vor allem mit hohem zeitlichen Ressourceneinsatz verbunden ist. Aktuell befindet sich die Mehrheit der befragten Unternehmen noch in einer frühen Phase einer HR-Social Media-Erschließung. Integrative Gesamtlösungen mit breit vernetzten und anderen digitalen HR-Aktivitäten abgestimmten HR-Social Media-Aktivitäten sind derzeit bei den Unternehmen noch nicht oder nur ansatzweise im Einsatz. Die Social Media-Aktivitäten der untersuchten Unternehmen werden vor diesem Hintergrund summarisch noch dem Aufbaustadium zugeordnet (Stufe 2 gemäß Reifegradmodell).

\section{(5) Data Mining}

Nur etwa ein Fünftel der befragten Unternehmen investieren eher geringe finanzielle Mittel in den Auf- und Ausbau von Data Mining-Aktivitäten im HRM. Die Thematik wird als sehr populär betrachtet, konkrete Nutzungsoptionen sieht man aber eher in der Zukunft. Nur drei der interviewten Experten messen der Analyse von Big Data einen tiefen Stellenwert bei, alle anderen halten die Entwicklungen in diesem Bereich jedoch, insbesondere in Großunternehmen, für relevant und wichtig für das HRM (der Zukunft). Ein befragtes Unternehmen untersucht derzeit die Interaktionen und die Kommunikation von freiwillig mitwirkenden Mitarbeitenden, um Muster zu identifizieren, 
aus denen eventuelle mehrwertschaffende Maßnahmen abgeleitet werden können. Ein anderes Unternehmen setzt eine Absenzenmanagementanwendung ein, die bei Auffälligkeiten Führungskräfte automatisch per Mail informiert. Beide Beispiele stehen für eher rudimentäre Data Mining-Anwendungen mit Ausbaupotenzial in den befragten Unternehmen. Die im HR-Kontext besonders wichtige Anforderung der jederzeitigen Gewährleistung des Datenschutzes und der Einhaltung von gesetzlichen Vorgaben im Umgang mit Mitarbeitendendaten beim Einsatz von HR-Data Mining wird von allen Experten so betont. Die Gefahren, die mit dem Einsatz von HR-Data Mining verbunden sind, werden von nahezu allen Befragten gesehen. Neben der Gefahr des Datenmissbrauchs und von „Big Brother“-Ängsten in der Belegschaft sind dies vor allem auch Risiken der Fehlinterpretation aufgrund von veralteten oder qualitativ schlechten Daten oder die Entstehung von unproduktiven Datenfriedhöfen und der fachlichen Überforderung von HR-Mitarbeitenden, die in der Regel weder Mathematiker noch Statistiker sind. Etwa die Hälfte der Experten, vor allem der größeren Unternehmen, betont aber die Chancen für Verbesserungen von Entscheidungen im HRM durch die Analyse von HR-Big Data.

Erste HR Business Intelligence-Lösungen werden von etwas mehr als einem Drittel der untersuchten Unternehmen aktuell eingesetzt. Ein weiteres Fünftel prüft auf analytischer Ebene eine Einführung möglicher Anwendungen. Ein in diesem Themenfeld aktives Unternehmen führt beispielsweise momentan ein neues HR BI-Tool ein, das stark auf Visualisierung von Sachverhalten für die Linienführungskräfte abhebt und die Mitarbeitendenportfolios mit einer Fülle an Detailinformationen zu den einzelnen Mitarbeitenden jederzeit einsehbar macht. Ein weiteres Unternehmen setzt vermehrt auf die Vernetzung von Nicht-HR- mit den HR-Daten auf einer Plattform mit dem Hauptziel der Optimierung von Arbeitsabläufen. Die in der Literatur oft hervorgehobene zunehmende Relevanz von Prognoseoptionen und der Möglichkeit zur Trendidentifikation kann durch die Befragungsergebnisse nicht bestätigt werden. Abschließend kann für den Themenkreis HR Data Mining, respektive HR Big Data und HR Business Intelligence aggregiert ein Entwicklungsstand bestätigt werden, der erst am Anfang zu stehen scheint (Stufe 1 gemäß Reifegradmodell).

\section{(6) Cloud-Lösungen}

Die sechste Dimension des Reifegradmodells umfasst den Entwicklungsstand der Unternehmen in Bezug auf die Realisierung von HR-Cloud-Lösungen. Alle HR-Experten betonen die zunehmende Wichtigkeit, von überall und in verschiedensten Arbeitsprozessen auf die HR-Daten zugreifen zu können, um den gestiegenen Ansprüchen der Mitarbeitenden und Führungskräfte in Bezug auf Mobilität und Flexibilität genüge zu leisten, etwa wenn Tochtergesellschaften enger in das Unternehmensnetzwerk der Mutter eingebunden werden sollen. Darüber hinaus werden Cloud-Lösungen auch nicht unbeträchtliche Kostensenkungspotenziale zugewiesen, die relativ schnell und mit deutlicher Entlastung der unternehmensinternen kostenträchtigeren IT- und Supportstrukturen realisierbar sind. Von einigen Experten wird die in Verbindung mit den angeführten Eigenschaften verbundene hohe Einfachheit von Cloud-Lösungen ferner als große 
Chance zur Optimierung von HR-Prozessen durch Standardisierung gesehen. Nahezu alle Befragten bestätigen vor diesem Hintergrund eine hohe Wichtigkeit entsprechender Digitalisierungsoptionen. Die HR-Cloud gehört heute schon, und in Zukunft wohl noch in vermehrtem Umfang, zum Geschäftsalltag des Personalmanagements. Das Gros der untersuchten Unternehmen greift also bereits auf entsprechende Digitalisierungslösungen zurück. Diejenigen Unternehmen, die bis dato noch ohne Cloud-Einsatz agieren, sind in ihrem diesbezüglichen Entscheidungsraum eingeschränkt, zum Beispiel durch rechtliche Restriktionen bei öffentlichen Unternehmen (aktuell wird deshalb auf Bundesebene eine „Bundes-Cloud“ für öffentliche Institutionen diskutiert), aus Sicherheitsgründen in einem sensiblen Branchenumfeld (Hack-Gefahr) oder aufgrund anderweitiger technischer Lösungsansätze wie Corporate W-LAN. In funktionaler Hinsicht werden hauptsächlich Prozesse wie das Rekrutierungs- und Bewerbungsmanagement, die Lohnabwicklung, die Zeiterfassung und das HR Performance Management in die Cloud ausgelagert. Nur bei wenigen Unternehmungen werden allerdings sämtliche Prozesse in einer einheitlichen Cloud-Lösung integriert.

Die befragten Experten stehen in ihrem privaten Umfeld Cloud-Lösungen positiv gegenüber. Familienfotos, Videos, persönliche Daten etc. werden von ihnen in die Cloud ohne größere Bedenken hochgeladen. Eine gewisse Grundskepsis kann hingegen bei fast der Hälfte der HR-Manager festgestellt werden, wenn es um sensible Personaldaten eines Unternehmens geht. Von Gesetzes wegen herrschen in der Schweiz eindeutige Restriktionen, wie Personaldaten behandelt werden müssen, und welche Daten eine Unternehmung für ihre Zwecke analysieren darf. Solche sensiblen Daten dann noch in eine Cloud auszulagern, verbreitet, bei aller Technikaffinität, eine gewisse Unsicherheit bei den HR-Verantwortlichen. Alle Befragten sind sich einig, dass Datenschutz und Datensicherheit hier einen zentralen Stellenwert einnehmen. Dies wird auch von der Anbieterseite bestätigt, die diesem Kundenbedürfnis mit umfassenden Authentifizierungs-, Sicherheits- und Backup-Systemen nachkommt. Eine hundertprozentig sichere Lösung gibt es allerdings in der heutigen Zeit nicht mehr. Das gilt jedoch auch für die in der Regel noch stärker gefährdeten eigenen Nicht-Cloud-Systemlösungen der Unternehmen. In dem Zusammenhang spielt der Standort der Personaldaten-Server eine wichtige Rolle. Die meisten Unternehmen haben Regelungen festgelegt, in welchem Land die Daten physisch gespeichert werden dürfen. Die Schweiz wird hier als vertrauenswürdigstes Land genannt. Die USA stehen hingegen zunehmend in kritischer Diskussion.

Ein losgelöst von den technisch-strukturellen Aspekten von HR-Clouds resultierendes Gefahrenfeld für die Mitarbeitenden wird von einigen Experten für das Gesundheitsmanagement gesehen. Die mit den Clouds verbundene Zeit- und Ortsungebundenheit erhöht das Risiko einer unerwünschten zu hohen Überschneidung von Berufs- und Privatleben mit negativen Konsequenzen für die Work-Life-Balance von oftmals besonders leistungswilligen Mitarbeitenden (Erhöhung des Burn-out-Risikos).

Insgesamt kommen HR-Cloud-Lösungen aus Sicht der Befragten also eine hohe aktuelle und zukünftige Bedeutung zu. Das HR-Traktandum wird durchgängig als wichtig betrachtet, es besteht aber noch eine Reihe von Unsicherheiten bezüglich 
der Anwendung, vor allem in Bezug auf die Datensicherheit. Breite und Tiefe der HR-Prozessabdeckung in den Unternehmen ist aber offenbar noch signifikant ausbaubar. Auch fehlt es augenscheinlich noch an einer umfassenden strategischen Einbettung von Cloud-Ansätzen im HRM. Trotzdem kann angesichts der bereits starken Verbreitung und Implementierungsoffenheit von einer gewissen ,Verankerung“ von HR-Cloud-Lösungen in den untersuchten Schweizer Unternehmen gesprochen werden (Stufe 3 gemäß Reifegradmodell).

\section{(7) Mobility}

Die Grundhaltung der befragten Schweizer Unternehmen gegenüber Mobility-Ansätzen kann durchweg als positiv bezeichnet werden. Vor allem die betrachteten Unternehmen mit öffentlich-rechtlichem Hintergrund scheinen hier eine Vorreiterrolle einzunehmen. Fünf der untersuchten Firmen gehören zu den Unterzeichnern der „Work Smart Charta“, die flexible, ortsunabhängige Arbeitsformen fördern möchten, um motivierende Rahmenbedingungen für ihre Mitarbeitenden zu schaffen, den Arbeitsmarkt besser erschließen zu können und die zur Verfügung stehenden Ressourcen sowie die Infrastruktur ,smarter“ zu nutzen (Work Smart 2016). Mobility und flexible Arbeitsmodelle gehen allerdings nicht zwingend in allen Bereichen einher. Unternehmen mit einem hohen Anteil von „Blue Collar“-Mitarbeitenden sind hier deutlich eingeschränkter als etwa IT-Dienstleistungsunternehmen mit größtenteils hochqualifizierten Mitarbeitenden. Mobility kann in diesem Kontext auch mit der Wahrnehmung einer Privilegierung der „WhiteCollar"-Mitarbeitenden einhergehen. Dies schafft neue, transparenzfördernde Regelungsbedarfe. Die in Verbindung mit den Cloud-Lösungen angeführte Gefahr von psychischen Problemen und Erschöpfungszuständen wird auch in Verbindung mit den MobilityLösungen gesehen. Darüber hinaus besteht hier aber auch ein größeres Missbrauchspotenzial in anderer Hinsicht, wenn Mobility dazu führt, dass die intendierte Flexibilität in Arbeitsvermeidung mündet.

Auf der Modell- und Instrumenteebene steht Mobility für die zunehmende Bedeutung von Gleit-, Jahresarbeitszeit und Teilzeitansätzen sowie Desk Sharing. Außerdem befassen sich die Unternehmen vermehrt mit Homeoffice-Konzepten. Die grundlegende Offenheit für die angeführten Modelle ist bei allen Unternehmen vorhanden, es bestehen jedoch deutliche Unterschiede, was die Regelungstiefe der konkreten Umsetzungen angeht. Die Bandbreite reicht hier aus Sicht der betroffenen Mitarbeitenden von ,sehr autonom“ bis zu ,sehr restriktiv“. Wie bei den Cloud-Lösungen fehlt auch in diesem Themenkreis oft eine personalstrategische Verankerung, was Interpretationsspielräume öffnet und in Verbindung mit fehlendem Effizienzreporting Spannungen im Arbeitsumfeld mobiler Mitarbeitender wahrscheinlicher macht.

Bezüglich der Umsetzung von standortbezogenen aktivitätsorientierten Arbeitsplatzkonzepten zeichnet sich für die befragten Unternehmen ein stark fragmentiertes Bild ab. So hat beispielsweise ein Dienstleistungsunternehmen an seinem Hauptstandort eine ,inspirierende Arbeitswelt“ für seine Mitarbeitenden geschaffen. Im Gegensatz zum Arbeiten an anderen Standorten kann dort die Arbeitszeit durch die Mitarbeitenden 
vollkommen eigenverantwortlich organisiert werden. Mit den flexiblen Arbeitsmodellen einher geht eine offenbar zunehmende Bedeutung des „Third-Place“-Konzepts (zum Beispiel flexible Nutzung von kurzfristig anmietbaren Büroräumlichkeiten an Bahnhöfen), das eine Mobilitätslücke zwischen Büro und Homeoffice schließt. Sofern es die architektonische Gestaltung der Räumlichkeiten zulässt, hat ein Teil der untersuchten Unternehmen das „Open Space“-Büro mit unpersönlichen Arbeitslandschaften, Meetingzonen und Wechselarbeitsplätzen eingeführt. Digitale Mobilität geht also tendenziell einher mit Flexibilisierung der Arbeitszeitmodelle und der Arbeitsräume. In Bezug auf die drei HR-Handlungsfelder kann aufgrund der Befragungsresultate von einer wechselseitigen Verstärkung ausgegangen werden.

Die Effizienz dezentraler, technikunterstützter flexibler Arbeitsmodelle hängt aus Sicht der Befragten offenbar stark von der Existenz einer Grundvertrauenskultur ab. Bedürfnisbezogene Mobilitätslösungen für Mitarbeitende wie Homeoffice, die sorgfältig auf die arbeitsorganisatorischen Erfordernisse abgestimmt sind, können aus Sicht der HR-Experten die Produktivität, Motivation und Mitarbeiterbindung spürbar erhöhen und auch zu einer Verbesserung der Soft Skills von Mitarbeitenden führen. Dies ist aber aus Sicht einiger Experten nicht für jedes Unternehmen und jede Mitarbeiterkategorie gleichermaßen realisierbar. Aus der Perspektive der HRM-Mitarbeitenden eröffnen sich außerdem neue Wege, einen intensiveren Kontakt zu den Linienführungskräften und -Mitarbeitenden zu pflegen.

Neben der Notwendigkeit einer „On-Off-Kompetenz“ mobiler Mitarbeitender besteht eine besondere Herausforderung neuer mobiler Konzepte in den veränderten sozialen Strukturen: Kommunikation und Information innerhalb eines Teams können „,verwässert“ werden. Es können sich unerwünscht homogene Gruppen bilden und Koordinationsbrüche entstehen. Vertrauen kann missbraucht werden und der Teamzusammenhalt schwinden. Dem muss durch organisatorische Maßnahmen wie fixe Arbeitstage mit kompletter Teampräsenz entgegengewirkt werden. Außerdem sind auch mobile Arbeitsplatzlösungen mit Sicherheitsproblemen verbunden, und es können Innovationsprozesse gehemmt werden. Die potenziellen Probleme werden von den befragten HR-Experten also durchaus erkannt.

Über alle untersuchten Unternehmen hinweg kann festgehalten werden, dass digitalisierungsbasierte Mobility-Lösungen in stark unterschiedlichem Ausmaß implementiert sind. Einige Unternehmen haben schon beachtliche Anstrengungen zur Mobilitätsförderung ihrer Mitarbeitenden unternommen, andere streben dies vertieft erst in der Zukunft an. Auch hier kann, analog zur Verbreitung von Cloud-Lösungen, den Unternehmen übergreifend eine gewissen „Verankerung“ von Mobility-Ansätzen attestiert werden (Stufe 3 gemäß Reifegradmodell).

\section{(8) Kultur}

Digitalisierung ist immer mit strukturellen und kulturellen Veränderungen in den Unternehmen verbunden. Dem trägt die achte Dimension des Reifegradmodells Rechnung, die die „softe“ Seite des digitalen Wandels HR-seitig beleuchtet. Seitens der befragten 
HR-Experten wird eine offene und dynamische Unternehmenskultur wiederholt als Voraussetzung für den digitalen Wandel genannt. Auf der Ebene von Subkulturen wird in Abhängigkeit vom Ausmaß der Betroffenheit von Mitarbeitenden durch den digitalen Wandel jedoch auf eine Differenzierungserfordernis hingewiesen. Die Digitalisierung wird als ein kulturrelevanter Aspekt unter einer Reihe anderer Unternehmensfaktoren wie die Technologieorientierung, Größe, Branche, regionale Verankerung oder die Firmenstruktur gesehen. Wie bei allen unternehmenskulturellen Phänomenen auch spielen das Verhalten des Topmanagements und der nachgelagerten Führungsebenen im Kontext der Digitalisierung eine wichtige Rolle. In allen befragten Unternehmen wird versucht, die kulturellen Voraussetzungen für den digitalen Wandel zu fördern, sei dies durch Schulungen und die Kommunikation bei Anlässen, inspirierende Arbeitswelten oder etwa durch den Einsatz von technischen Hilfsmitteln wie Tablets bei den Vorstellungs- und Mitarbeitendengesprächen oder Self Service-Angeboten für eine stärkere Einbindung der Mitarbeitenden in Personalprozesse. In Bezug auf wandelbegleitende Kulturmaßnahmen herrscht aber nachvollziehbar eine sehr große Heterogenität mit hoher Unternehmensspezifität vor. Ein wichtiger kultureller Stellhebel ist allerdings für alle Unternehmen die Rekrutierung von neuen Mitarbeitenden mit erwünschten digitalen Kompetenzprofilen und die Einbindung aller Mitarbeitenden bei großen Digitalisierungsprojekten. Vorhandene Mitarbeitende, die sich aus Angst oder mangelnden Fähigkeiten dem digitalen Wandel verschließen, geraten in Gefahr, nicht mehr wettbewerbs-, beziehungsweise arbeitsmarktfähig zu werden. Dem sollte das HRM durch die angeführten und gleichgerichteten Maßnahmen entgegenwirken.

In Bezug auf die HR-Mitarbeitenden schlagen sich neue digitalisierungsbedingte Stellenprofile (eHR-Experten) und die Auslagerung und Vereinfachung administrativer Prozesse in einigen Unternehmen in einem von der Literatur an sich schon lange postulierten, nun aber offenbar auch breiter real stattfindenden Paradigmenwechsel für das HRM-Personal nieder, der weg von administrativen Aufgaben hin zum qualifizierten Coaching und der Beratung der Linie durch die HR-Mitarbeitenden führt. Die wertschöpfende Ausrichtung der Personalabteilungen schreitet also weiter voran und macht in Bezug auf dazu stimmige Verhaltens- und Werteanpassungen auch vor den HR-Managern und -Mitarbeitenden nicht halt. Kompetenzen wie Abstraktions- und analytisches Denkvermögen werden damit einhergehend zunehmend wichtiger. Insgesamt gehen die Befragten eher von einer Verschiebung und einer qualitativen Veränderung von HR-Aufgaben als von einem kompletten Wegfallen von HR-Arbeit aus, auch wenn stellenbezogene Einsparpotenziale für das HRM noch gesehen werden.

In allen Unternehmen wird also die hohe Relevanz der Unternehmenskultur für eine erfolgreiche Digitalisierung erkannt und dem durch unterschiedliche HR-induzierte oder -unterstützte Maßnahmen entsprochen. Tendenziell scheint sich in den Unternehmen die Unternehmenskultur eher in der Folge als vorausgehend zur Digitalisierung zu verändern. Die Einbindung von wertrelevanten Aspekten der Digitalisierung wie Flexibilität, Mobilität oder die Wertschätzung digitaler Kompetenzen befindet sich summarisch über alle analysierten Unternehmen hinweg noch in der Aufbauphase (Stufe 2 gemäß Reifegradmodell). 


\section{(9) Produktinnovation}

Die Analyse der neunten Dimension des Reifegradmodells befasst sich mit der Entwicklung und Verwendung digitaler Produktinnovationen im HRM. Aus Anbietersicht scheinen für die Zukunft prozessbezogen vor allem in den Bereichen Employer Branding, Recruiting, Learning, HR Services und Talent-/Careermanagement noch Entwicklungsmöglichkeiten vorhanden zu sein. Diese Wahrnehmung deckt sich mit Eindrücken aufseiten der nachfragenden Unternehmen. Aus Sicht der HR-Experten kommen innovativen kundenorientierten und kostensparenden Self Service-Angeboten künftig eine noch wichtigere Rolle zu. Bei deren Einsatz gibt es aktuell in Bezug auf Breite und Intensität noch große Unterschiede zwischen den Unternehmen. Self Service-Lösungen kommen momentan vor allem in der Spesenabrechnung, Weiterbildung, bei Personalgewinnungsanträgen, der Lohnabrechnung, der Zeit- und Absenzenerfassung sowie der Personaladministration zum Tragen. Ausgebaute HR-Lösungen auf der Grundlage von künstlicher Intelligenz sind Stand heute noch in keinem der befragten Unternehmen im Einsatz, man verspricht sich diesbezüglich aber, bei aller Unsicherheit und Ungewissheit, für die Zukunft viel, zunächst wohl im Bereich der Rekrutierung oder beim Talentmanagement. HR Data Analytics-Ansätze werden zwar auf persönlicher Ebene von den HR-Experten zumeist eher zurückhaltend bewertet, für die Unternehmen werden aber in Zukunft eine weitere Intensivierung der Aktivitäten und damit verbundene Regelungsbedarfe (Persönlichkeitsrechte) gesehen. Als Gegenposition zum Big Data-Trend wird von einzelnen Experten für die Zukunft eine Entwicklung in Richtung „Small Data“ im Sinne von „Zurück zum Individuum“ und zum Faktor „Mensch“ in Unternehmen ausgemacht. Weitere potenzielle Trendbereiche einer Digitalisierung des HRM sehen einzelne Befragte im Bereich Virtual Reality, etwa über virtuelle Einblicke in Arbeitsabläufe oder ganze Arbeitswelten. Auch dem „Internet of Things“ wird ein gewisses Potenzial für das HRM beigemessen, wenngleich die Erwartungen hier noch wenig konkret sind.

Die neunte Dimension des Reifegradmodells setzt sowohl an der Angebots- als auch an der Nachfragerseite an. Auf der einen Seite gehen digitale Produktinnovationen in der Regel von den Anbietern neuer digitaler HRM-Systemlösungen aus, auf der anderen Seite muss für diese Angebote seitens der Nachfrager eine hinreichende Offenheit und Investitionsbereitschaft bestehen. Die Angebotssituation auf dem Schweizer Markt für HR-Digitalisierungslösungen kann durchaus als innovativ und zukunftsorientiert gekennzeichnet werden, unter anderem, da in dem Bereich über international agierende IT-Unternehmen ein hoher internationaler Diffusionsgrad besteht. Auch eine große, wenngleich nicht unkritische Offenheit und Aufgeschlossenheit der Personalmanager und der hinter ihnen stehenden Unternehmen für HR-Digitalisierungsinnovationen ist gegeben. Eine Reihe von innovativen Ansätzen kommt, auch unter Einbindung der Resultate der vorgängig betrachteten Dimensionen, zur Anwendung, wenngleich einige davon sehr unternehmensspezifisch ausfallen. Übergreifend kann in Bezug auf das Innovationspotenzial und die -bereitschaft der analysierten Schweizer Großunternehmen von einem ausgeprägten Gestaltungswillen ausgegangen werden, der über eine 
bloße Verankerung von Einzelinnovationen etwas hinausgeht: Man will Digitalisierungsinnovationen im HRM managen und tut dies auch (Stufe 4 gemäß Reifegradmodell).

Abschließend kann der Stand der Digitalisierung des HRM der betrachteten 16 Schweizer Großunternehmen über alle Dimensionen und alle Unternehmen hinweg als „fortgeschritten im Aufbau“ charakterisiert werden (Stufe 2 gemäß Reifegradmodell) (vgl. dazu Abb. 8.3). Eine Tendenz in Richtung „Verankerung“ der Digitalisierung ist summarisch betrachtet erkennbar. Einige Vorreiterunternehmen haben diesen fortgeschritteneren Reifegrad bereits erreicht oder sogar bereits überschritten.

Eine aggregierte Beantwortung der eingangs gestellten fünf Forschungsfragen erfolgt in der Übersicht in Tab. 8.5.

Die untersuchten Schweizer Unternehmen bewegen und verändern sich also digitalisierungsbedingt in allen identifizierten Trendbereichen, wenngleich beim Umsetzungsstand der Digitalisierung eine große Bandbreite ausgemacht werden konnte. Vor allem die untersuchten staatlichen oder staatsnahen Betriebe sind im HR-Digitalisierungsprozess schon recht weit vorangeschritten. Dies hängt vermutlich aber primär mit deren überdurchschnittlicher Größe, techniknahen Geschäftsfeldern und hohen administrativen Anteilen zusammen und weniger mit ihrer Rechtspersönlichkeit. Im administrativen Bereich steigt der Digitalisierungsgrad tendenziell bei hoher Verwaltungslastigkeit der HR-Prozesse. Der damit verbundene Aufgabenwandel in Personalabteilungen fördert die Entwicklung des HR-Bereichs in Richtung wertschaffende Service- und Beratungseinheit für die Linie. Wertschöpfung heißt dabei im Kontext Digitalisierung nicht nur Kostensenkung, sondern auch eine Effektivitätsund Effizienzsteigerung, eine bessere Wettbewerbspositionierung, Attraktivitätsverbesserungen und eine Erhöhung des Flexibilitätspotenzials im Unternehmen.

Abb. 8.3 Stand der HR-Digitalisierung in Schweizer Großunternehmen. (Eigene Darstellung)

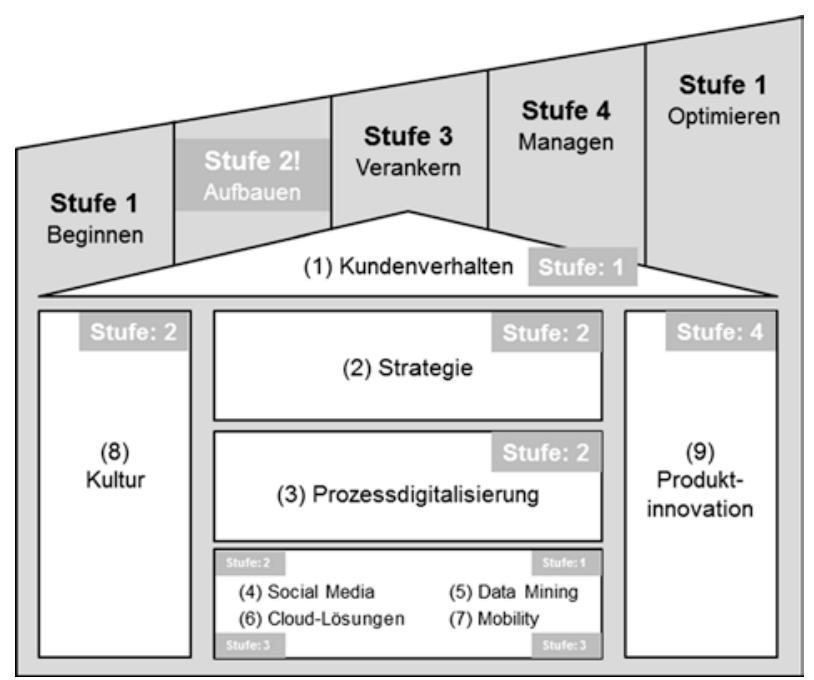


Tab. 8.5 Kernaussagen zu den fünf Forschungsfragen. (Eigene Darstellung)

\begin{tabular}{|c|c|}
\hline Forschungsfrage & Kernaussagen \\
\hline $\begin{array}{l}\text { Welche unter dem } \\
\text { Begriff der Digitalisie- } \\
\text { rung subsumierbaren } \\
\text { Trends sind für das } \\
\text { HRM weshalb von } \\
\text { Bedeutung? }\end{array}$ & 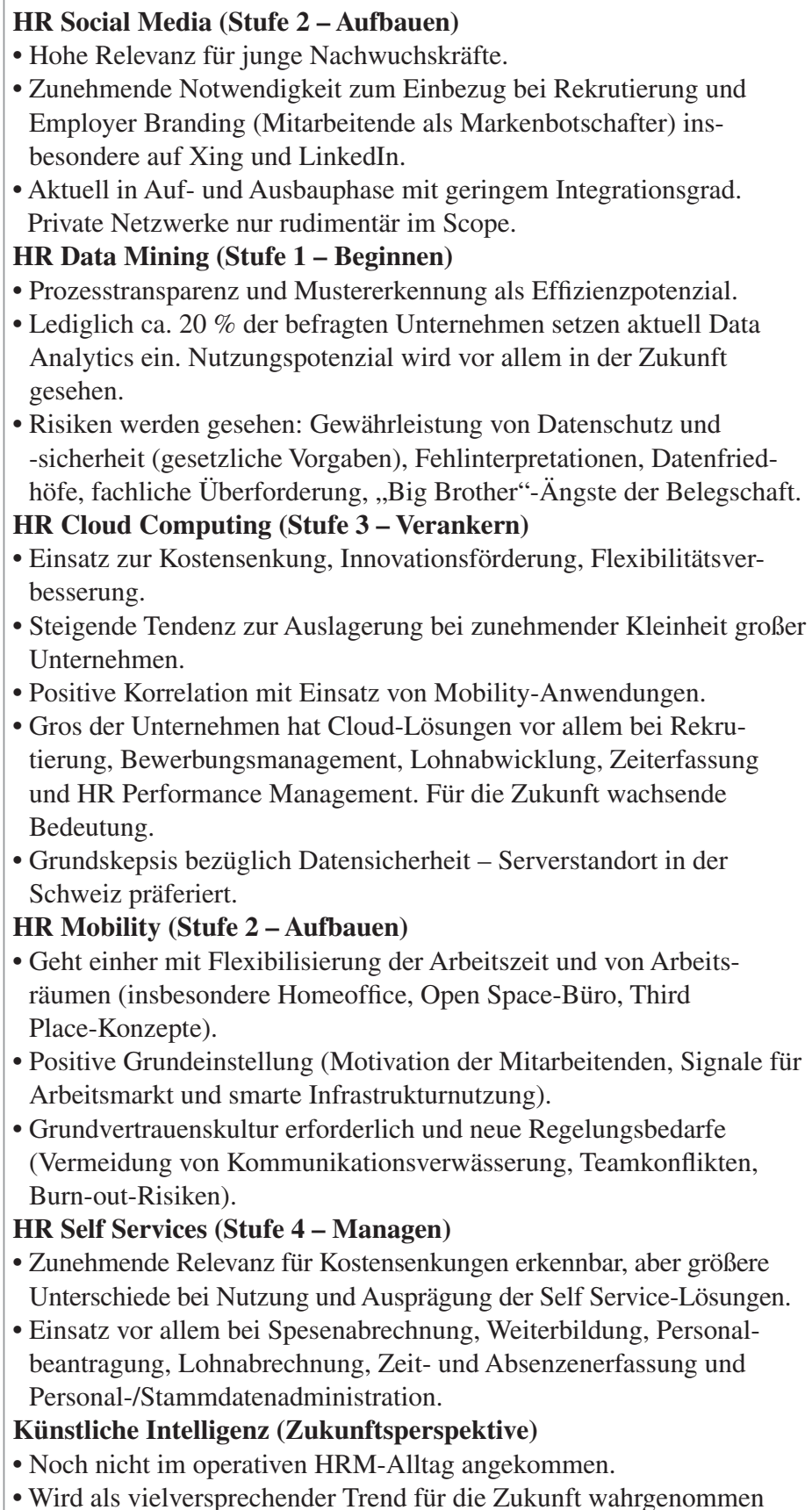 \\
\hline
\end{tabular}




\begin{tabular}{|c|c|}
\hline Forschungsfrage & Kernaussagen \\
\hline $\begin{array}{l}\text { Was sind in personaler } \\
\text { Hinsicht zentrale Chan- } \\
\text { cen und Problemfelder } \\
\text { der Digitalisierung? }\end{array}$ & $\begin{array}{l}\text { Chancen für das HRM } \\
\text { - Kostensenkungen und Qualitätsverbesserungen durch Prozess- } \\
\text { optimierung und Entscheidungsunterstützung. } \\
\text { - Unterstützt Wertschöpfung und Serviceorientierung im HRM: } \\
\text { Beratung statt Administration; strategische statt operative Personal- } \\
\text { arbeit. } \\
\text { Problemfelder für das HRM } \\
\text { - Datenschutz und Datensicherheit. } \\
\text { - Akzeptanz bei Mitarbeitenden (Self Services, „Big Brother“). } \\
\text { - Entgrenzung von Privat- und Berufswelt. } \\
\text { - Fachliche Überforderung von HR-Mitarbeitenden. } \\
\text { - Wachsende Abhängigkeit von Systemanbietern mit Folgekosten. }\end{array}$ \\
\hline $\begin{array}{l}\text { Welche Konsequenzen } \\
\text { und Gestaltungs- } \\
\text { empfehlungen lassen } \\
\text { sich aus den wich- } \\
\text { tigsten Trends für die } \\
\text { einzelnen Politikfelder } \\
\text { des HRM ableiten? }\end{array}$ & $\begin{array}{l}\text { - Trends beeinflussen alle Funktionsfelder, bzw. HR-Prozesse in mehr } \\
\text { oder minder großem Umfang. } \\
\text { - Positive Digitalisierungskultur und Neueinstellungen als wichtige } \\
\text { Stellhebel für die Zukunftsfähigkeit der Human Resources und des } \\
\text { HRM. } \\
\text { - Förderung von digitalen und von Beratungskompetenzen für HR-Mit- } \\
\text { arbeitende - Digital Change als Dauerzustand. } \\
\text { - Digital HR-Experte als neues Berufsprofil im HRM. } \\
\text { - Stärkere Zusammenarbeit zwischen IT- Bereichen und HRM zur } \\
\text { gemeinsamen Change-Bewältigung. } \\
\text { - Stärkere Bedarfsorientierung bei Digitalisierung im HR-Bereich (nicht } \\
\text { alles, was technisch möglich ist, ist auch gut für die „Kunden“ in der } \\
\text { Linie). }\end{array}$ \\
\hline $\begin{array}{l}\text { Wie sind einzelne } \\
\text { Digitalisierungstrends } \\
\text { aktuell im HRM } \\
\text { Schweizer Unter- } \\
\text { nehmen eingegangen? }\end{array}$ & $\begin{array}{l}\text { - Anstöße für neue HR-Digitalisierungslösungen kommen entweder } \\
\text { von internen Experten oder von externer Anbieterseite (Spezialisten- } \\
\text { lösungen mit eher gering konkretisierter Bedarfsorientierung in Bezug } \\
\text { auf Mitarbeitende und Führungskräfte als HR Service-Kunden). } \\
\text { - Kostensenkung, Arbeitgeberattraktivität und damit verbundene } \\
\text { Wettbewerbsfähigkeit am Arbeitsmarkt als kausale Haupttreiber der } \\
\text { Digitalisierung. }\end{array}$ \\
\hline $\begin{array}{l}\text { Wie, bzw. in welche } \\
\text { Richtung entwickelt } \\
\text { sich das HRM Schwei- } \\
\text { zer Unternehmen im } \\
\text { Kontext der Digitali- } \\
\text { sierung in den nächsten } \\
\text { Jahren weiter? }\end{array}$ & $\begin{array}{l}\text { - Wachstumstendenzen in allen identifizierten Trendbereichen. } \\
\text { Geschwindigkeit der Umwälzungen ist ungewiss, aber tendenziell } \\
\text { hoch. } \\
\text { - Weitere forcierte Fortführung von HR-Prozessautomatisierungen. } \\
\text { - HRM als wertschöpfungsgenerierendes internes Service-Center mit } \\
\text { hohem Digitalisierungsgrad. } \\
\text { - Digitalisierungsbedingte Profilveränderungen für HR-Mitarbeitende. }\end{array}$ \\
\hline
\end{tabular}




\subsection{Diskussion und Forschungsperspektiven}

Die theoretische und empirische Analyse der Digitalisierung im Kontext des HRM Schweizer Großunternehmen hat großteils Übereinstimmungen, aber auch einige Differenzen und Pointierungen hervorgebracht. So kann etwa der hohe Stellenwert und die teils enthusiastische Bewertung von HR Data Analytics in früheren Studien durch die eigene Untersuchung nicht bestätigt werden. Die Befragten sehen diesen Entwicklungstrend für die Zukunft zwar als sehr relevant an, erkennen aber durchaus auch kritische Aspekte dieses Themenkreises, die die weitere Entwicklung beeinträchtigen können. Eine hohe Konvergenz der theoretischen und der eigenen empirischen Perspektive konnte in Bezug auf die Notwendigkeit einer strategischen Verankerung der Digitalisierung festgestellt werden, wenn mit ihr die postulierten positiven Effektivitäts- und Effizienzeffekte realisiert werden sollen. In der Realität ist diese Verankerung aber zum gegenwärtigen Zeitpunkt eher noch eingeschränkt gegeben. Fehlende Digitalisierungsstrategien gehen mit Defiziten und Unsicherheiten bei der Implementierung von HR-Digitalisierungslösungen einher. Diesbezüglich sind sich die HR-Experten auf einer übergreifenden Argumentationsebene einig. Es mangelt bei einigen Unternehmen offenbar noch an einer ganzheitlichen Sicht auf die HR-Digitalisierung, die auch strukturelle und kulturelle Aspekte mit einbezieht (perspektivische Triade: Digital Strategy - Digital Structure - Digital Culture). Im Vergleich mit früheren Studien fällt auf, dass die Bedeutung der Social Media-Nutzung offenbar markant zugenommen hat (HR Social Media Multichannel Management). Prioritäre Nutzungsbereiche (Rekrutierung und Employer Branding) und relevante Plattformen (Xing, LinkedIn, Facebook) früherer Analysen konnten bestätigt werden. Die Plattform YouTube scheint aber vor dem Hintergrund der eigenen Erhebung in vorangehenden Untersuchungen hinsichtlich der untersuchten Firmengruppe überbewertet zu sein. In Ergänzung zu den in der Theorie vorgefundenen kritischen und unkritischen Beiträgen in Bezug auf die Verwässerung von Arbeitszeit und Freizeit kann für die befragten HR-Experten ein durchaus vorhandenes kritisches Grundbewusstsein attestiert werden, das gesundheitliche Überlegungen mit einbezieht. Die durch die Digitalisierung geschaffenen Möglichkeiten sollen demnach so genutzt werden, dass die Mitarbeitenden dies als motivationserhöhenden Zugewinn an Arbeits- und Lebensqualität empfinden und nicht als arbeitsbelasteten Zugriff auf die Privatsphäre. Flexibilität der Mitarbeitenden darf in langfristiger Sicht auch im Sinne der Unternehmen nicht zum Selbstausbeutungsmodell besonders engagierter Mitarbeitender verkommen. Der vielfach postulierte hohe Entwicklungsstand der Digitalisierung konnte insgesamt für den HR-Bereich nur eingeschränkt bestätigt werden. Hier ist nach wie vor auch bei Großunternehmen von einem hohen Entwicklungspotenzial auszugehen, das bei klein- und mittelständischen Unternehmen wohl noch um ein Vielfaches höher liegen dürfte. 
In Bezug auf die angewandte Forschungsmethodik ist auf eine Reihe potenziell kritischer Aspekte hinzuweisen. Das qualitative Forschungssetting mit einer relativ geringen Samplegröße und der Konzentration auf einen bestimmten (Deutsch-)Schweizer Unternehmenstypus schränkt die Repräsentativität und Generalisierbarkeit der Aussagen stark ein. Diese Defizite sind der Preis für eine größere inhaltliche Substanz und Breite der gemachten Aussagen im Sinne einer differenzierten aktuellen Momentaufnahme mit hohem deskriptivem Gehalt. Die methodisch fundierte Umsetzung der Interviewführung und -aufbereitung (Transkription und Codierung) mittels der Systematisierungsstruktur des entwickelten Reifegradmodells spricht ferner für eine gewisse Güte der analytischen Ergebnisse (Verfahrensdokumentation) (siehe hierzu und zur nachfolgenden Argumentationsführung auch Mayring 2002). Eine argumentative Interpretationsabsicherung fand durch einen kontinuierlichen Abgleich theoretischer und empirisch gewonnener Erkenntnisse und der empirischen Aussagen untereinander statt. Der Forschungsprozess erfolgte ferner durch Anlehnung an das Prozessmodell von Gläser und Laudel (2006, S. 32) weitgehend regelgeleitet. Die Nähe zum Forschungsgegenstand sollte durch die Befragung von ausgewählten Fachexperten vor Ort im unmittelbaren Arbeitsumfeld der Probanden und durch eine transparente Darlegung von Forschungsinhalt und Forschungszielen ihnen gegenüber (Forschung für die Betroffenen) hergestellt werden. Die Forschungsergebnisse wurden nach Abschluss der Analyse den teilnehmenden Unternehmen zugestellt, ohne dass grundlegende Korrekturen vorgenommen werden mussten (kommunikative Validierung). Die Kombination der gewählten Methodik mit einer breiteren quantitativen Erhebung könnte zur weiteren Fundierung der gemachten Aussagen sicher spürbar beitragen (Triangulation). Eine weitere Option zur qualitativen Verbesserung der Analyse wäre die Einbindung einer größeren Zahl von Unternehmen der Anbieterseite gewesen, die ursprünglich vorgesehen, aber beim gegebenen zeitlichen Rahmen nicht realisierbar war. Deshalb wurden die Anbieteraussagen nur zur Abrundung, Validierung und Interpretationsabsicherung hinsichtlich der befragten HR-Experten der Nachfragerseite verwendet.

Die Ergebnisse der vorliegenden empirischen Erhebung können als Basis für zukünftige weitere Untersuchungen im ausgewählten Themengebiet herangezogen werden. Angesichts der hohen Dynamik des Forschungsfelds und der hohen Praxisrelevanz wären tiefer gehendere qualitative und quantitative Analysen für die verschiedenen Funktionsfelder des HRM, für die Produktlösungen und -innovationen auf Anbieterseite, für umgesetzte Digital Change-Konzepte (,Wie“), für die quantitative Operationalisierung realisierter Einsparungen und Qualitätsverbesserungen oder für die Entwicklung integrativer Implementierungsmodelle (Digital HRM System Fit) wünschenswert. Auch in Bezug auf das zugrunde gelegte eingeschränkte Sample wäre eine Ausweitung des Befragungsscopes auf alle (Groß-)Unternehmen der Schweiz, inklusive Tessin und Romandie oder auf andere Unternehmensgrößen (KMU) von Interesse. 


\section{Literatur}

Accenture (Hrsg.). (2015). Digital Index Switzerland 2015. Biel: SAP AG intern (unveröffentlicht). Bankl, M. (2014). Lebensphasenorientierte Personalarbeit - Ein nachhaltiger Ansatz. In B. Rosenberger (Hrsg.), Modernes Personalmanagement: Strategisch-operativ-systemisch (S. 306-314). Wiesbaden: Gabler.

Beck, C. (2008). Personalmarketing 2.0: Vom Employer Branding zum Recruiting. Köln: Luchterhand.

Berghaus, S., Back, A., \& Kaltenrieder, B. (2015). Digital Transformation Report. St. Gallen: Institut für Wirtschaftsinformatik, Universität St. Gallen.

Botfrei Blog. (2012). Cloud-Computing - Oder was is teigentlich die Cloud? https://blog.botfrei. de/2012/12/cloud-computing-oder-was-ist-eigentlich-die-cloud/. Zugegriffen: 25. Mai 2016.

Cachelin, J. L. (2013a). Digitalisierung als Reifeprozess. Personalmagazin, 11(13), 51-53. https:// www.wissensfabrik.ch/digitalisierung-als-reifeprozess/. Zugegriffen: 25. Apr. 2016.

Cachelin, J. L. (2013b). HRM Trendstudie 2013. https://www.wissensfabrik.ch/hrmtrend2013/. Zugegriffen: 25. Mai 2016.

Cachelin, J. L. (2013c). Big Data Mining im HRM. https://www.wissensfabrik.ch/hr-big-data/. Zugegriffen: 25. Mai 2016.

Cachelin, J. L. (2014a). 5 Arbeitsfelder für das HR im digitalen Transformationsprozess. HR Today (3). https://www.wissensfabrik.ch/anleitungdigital/. Zugegriffen: 25. Apr. 2016.

Cachelin, J. L. (2014b). HR-Kompetenzen in einer digitalen Welt. Personal Manager, 2(2014), 22-24.

Cachelin, J. L. (2015). HRM Trendstudie. Risiken digitaler Arbeitswelten. https://www.wissensfabrik.ch/hrmtrend2014/. Zugegriffen: 25. Apr. 2016.

Cachelin, J. L. (2016b). Wissensfabrik. Risiken der digitalen Arbeitsumgebung. https://www. wissensfabrik.ch/digitalerisiken/\#software. Zugegriffen: 21. Mai 2016.

Cantin, F. (1999). Die innerbetriebliche Kommunikation verstehen und gestalten. Ein konstruktivistischer Ansatz und eine qualitative Analyse. Bern: Haupt.

Cloudcomputing-insider. (2016). Die 10 grössten Gefahren beim Einsatz von Cloud-Infrastrukturen. http://www.cloudcomputing-insider.de/die-10-groessten-gefahren-beim-einsatz-von-cloud-infrastrukturen-a-517146/. Zugegriffen: 28. Mai 2016.

Cole, T. (2015). Digitale Transformation. Warum die deutsche Wirtschaft gerade die digitale Zukunft verschläft und was jetzt getan werden muss!. München: Vahlen.

Computerworld. (2016a). Schweizer Banken: Digitalisierung erst am Anfang. http://www.computerworld.ch/news/it-branche/artikel/schweizer-banken-digitalisierung-erst-am-anfang-69399/. Zugegriffen: 9. Mai 2016.

Computerworld. (2016b). Schweizer KMU: Digitalisierung startet beim Kunden. http://www.computerworld.ch/marktanalysen/studien/artikel/schweizer-kmu-digitalisierung-startet-beim-kunden-69563/. Zugegriffen: 9. Mai 2016.

ECDL Switzerland AG (Hrsg.). (2015). Selbsteinschätzung und effektive PC-Kenntnisse der Schweizer Bevölkerung. Basel: ECDL Switzerland AG.

Eilers, S., Möckel, K., Rump, J., \& Schabel, F. (2016). HR-Report 2015/2016. Schwerpunt Unternehmenskultur. Bern: Hays AG.

Ernst \& Young (Hrsg.). (2016). Digitalisierung bei mittelständischen Unternehmen. Ergebnisse Kurzumfrage. http://www.ey.com/Publication/vwLUAssets/EY_Umfrage_Digitalisierung_mittelstaendische_Unternehmen_Schweiz/\$FILE/EY-Ergebnisse-Umfrage-Digitalisierung-2016.pdf. Zugegriffen: 25. Apr. 2016. 
Focus (2010). Was ist eigentlich Cloud Computing?. http://www.focus.de/digital/computer/kurzerklaert-was-ist-eigentlich-cloud-computing_aid_559544.html. Zugegriffen: 2. Juni 2016.

Gläser, J., \& Laudel, G. (2006). Experteninterviews und qualitative Inhaltsanalyse als Instrumente rekonstruierender Untersuchungen (2., durchgesehene Aufl.). Wiesbaden: VS Verlag.

Gora, W. (2016). Personalarbeit im Wandel: Digitalisierung als Chance, Personalpraxis24.de. http:// www.personalpraxis24.de/aktuelles/thema-der-woche/archiv-themen-der-woche/personalarbeitim-wandel-digitalisierung-als-chance/. Zugegriffen: 25. Apr. 2016.

Hackl, B., \& Gerpott, F. (2014). HR 2020 - Personalmanagement der Zukunft. München: Vahlen.

Haubrock, A. (2004). Personalmanagement. Stuttgart: Kohlhammer.

Heister, A. (2006). Qualitative Interviews - Ein Leitfaden zu Vorbereitung und Durchführung inklusive einiger theoretischer Anmerkungen. http://www.univie.ac.at/igl.geschichte/kaller-dietrich/WS\%2006-07/MEXEX_06/061102Durchf\%FChrung\%20von\%20Interviews.pdf. Zugegriffen: 30. Apr. 2016.

Heymann, B. (2009). Das Personalmanagement in mittelständischen Unternehmen. Hamburg: Igel.

Hoberg, P., Krcmar, H., Oswald, G., \& Welz, B. (2015). IDT Survey 2015 - Skills for digital transformation. Biel: SAP AG intern (unveröffentlicht).

Holtbrügge, D. (2015). Personalmanagement (6., neubearb. u. erw. Aufl.). Berlin: Springer.

HRM.ch (2013). HR-Software Report 2013 - die Ergebnisse. http://www.hrm.ch/fachartikel/ hr-software-report-2013-die-ergebnisse-10827. Zugegriffen: 27. Apr. 2016.

Huck-Sandhu, S. (Hrsg.). (2015). Interne Kommunikation im Wandel: Theoretische Konzepte und empirische Befunde. Heidelberg: Springer.

HWZ (Hrsg.). (2015). Über 50 Prozent der Unternehmen sind digitale Dinosaurier. http://www. fh-hwz.ch/de/news/news-detail/studie-digitale-transformation-2015.htm. Zugegriffen: 25. Apr. 2016.

ICT Switzerland (2016). Scorcard. http://digital.ictswitzerland.ch/. Zugegriffen: 9. Mai 2016.

Jäger, W., \& Petry, T. (2012). Entreprise 2.0 - Die digitale Revolution der Unternehmenskultur. Warum Personalmanager jetzt gefordert sind. Köln: Wolters Kluwer.

Karaus, S. (2003). Vergleich ausgewählter Anwendungsmöglichkeiten zur Nutzung des Elecetronic Human Resources Management in Unternehmen. Hamburg: Diplomica.

Kiefl, W., \& Lamnek, S. (1984). Qualitative Methoden in der Marktforschung. Planung und Analyse, 11(12), 474-480.

Kienbaum (Hrsg.). (2015). Ergebnisbericht HR-Trendstudie 2015. http://www.kienbaum.com/ Portaldata/1/Resources/downloads/brochures/Kienbaum_HR-Trendstudie_FINAL.pdf. Zugegriffen: 25. Apr. 2016.

Kolb, M. (2008). Personalmanagement. Grundlagen. Konzepte - Praxis. Wiesbaden: Gabler.

Kolb, M. (2010). Personalmanagement. Grundlagen und Praxis des Human Resources Management (2. Aufl.). Wiesbaden: Gabler.

Mayring, P. (2002). Qualitative Sozialforschung (5. Aufl.). Weinheim: Verlagsgruppe Beltz.

Nicolai, Ch. (2009). Personalmanagement (2. Aufl.). Stuttgart: Lucius \& Lucius.

Olsok, A., Kühn, T., Schulz, A., Bruhn, H., \& Kirch, J. (2016). Datenschutz im Personalmanagement. Gemeinschaftsstudie.. Berlin: Bitkom.

Pentaho (Hrsg.). (2012). BI4HR - Flexible Analyse und Reporting von Personaldaten. http://www. bi4hr.ch/download/BI4HR\%20Casestudy.pdf. Zugegriffen: 25. Mai 2016.

PwC (Hrsg.). (2013). HR und Social Media: Hier klickt der Zeitgeist. http://www.pwc.ch/user_ content/editor/files/publ_hrs/pwc_hr_und_social_media_d.pdf. Zugegriffen: 30. Mai 2016.

PwC (Hrsg.). (2014). Moving HR to the cloud? https://www.pwc.ch/user_content/editor/files/publ_ hrs/pwc_moving_hr_to_the_cloud_e.pdf. Zugegriffen: 25. Mai 2016.

Scherm, E., \& Süss, S. (Hrsg.). (2011). Personalmanagement. München: Vahlen. 
Scherm, E., \& Süss, S. (Hrsg.). (2013). Personalmanagement. München: Vahlen.

Schwarb, T., \& Vecchia, M. D. (2016). HRM - 4.0 Digitales Human Resource Management. Rheinfelden: BPX-Edition.

Schwartz M., \& Muhle, A. (2016). Chancen der Digitalisierung nutzen: Datenschutz und IT-Sicherheit gehören dazu. KfW Research Magazin, 117(8), Februar 2016. Frankfurt: KfW Research.

Segmentas (Hrsg.). (2016). TOP 500 Handelszeitung. Die grössten Unternehmen der Schweiz. http://www.segmentas.ch/top500. Zugegriffen: 13. Dez. 2016.

Selke, M. (2012). Personalmanagement. In C. Thielscher (Hrsg.), Medizinökonomie (S. 197-241). Wiesbaden: Gabler.

Spachmann, K., \& Huck-Sandhu, S. (2013). Interne Kommunikation - Stellenwert und Neuausrichtung. In Bentele, G., Piwinger, M., \& Schonborn, G. (Hrsg.), Loseblattwerk Kommunikationsmanagement (Beitrag 3.96). Neuwied: Luchterhand.

Spachmann, K., \& Huck-Sandhu, S. (2015). Zwischen Beteiligung und Dialog: Social Media in der internen Kommunikation. Ergebnisbericht zur Umfrage 2014. Pforzheim/Stuttgart: Hochschule Pforzheim/Universität Hohenheim. http://www.ik-trends.de/wp-content/ uploads/2015/07/IK2014_Social-Media.pdf. Zugegriffen: 27. Apr. 2016.

Stock-Homburg, R. (2008). Personalmanagement. Theorien, Konzepte, Instrumente. Wiesbaden: Gabler.

Tagesanzeiger. (2015). Hälfte der Firmen fehlen geeignete Arbeitskräfte. Tagesanzeiger Online, 17.08.2015. http://www.tagesanzeiger.ch/olympia2012/switzerland/haelfte-der-firmen-fehlengeeignetearbeitskraefte/story/16550916?track. Zugegriffen: 14. Apr. 2016.

Tetzel, T. (2016). Cloud Computing: SaaS, PaaS \& IaaS einfach erklärt. https://www.datenschutzbeauftragter-info.de/die-cloud-saas-paas-und-iaas-einfach-erklaert/. Zugegriffen: 25. Mai 2016.

Thom, N. (2001). Personalmanagement - Überblick und Entwicklungstendenzen. In N. Thom, \& R. J. Zaugg (Hrsg.), Excellence durch Personal- und Organisationskompetenz (S. 117-131). Bern: Haupt.

Wachter, T. (2015, August 18). Personalentwicklung: Das sind die Ziele. http://www.weka.ch/ themen/personal/personal-fuehrung-und-personalentwicklung/personalentwicklung/article/ personalentwicklung-das-sind-die-ziele/. Zugegriffen: 28 Apr. 2016.

Wagner AG (Hrsg.). (2016). BI/ Analytics. KMU Lösungen für BI und Analytics. http://www.wagner.ch/loesungen/bi-analytics.html. Zugegriffen: 25. Mai 2016.

Westerman, G., Bonnet, D., \& McAfee, A. (2014). Leading digital: Turning technology into business transformation. Harvard: Harvard Business Press.

Winkler, S. (2016). Was HR-Profis beschäftigt. HR-Today, 27.01.2016. https://www.hrtoday.ch/ article/was-hr-profis-beschäftigt. Zugegriffen: 26. Apr. 2016.

Work Smart. (2016). Was ist die Charta? http://work-smart-initiative.ch/de/überuns/charta-unterzeichnen/. Zugegriffen: 18. Dez. 2016.

Schellinger, Jochen (Prof. Dr./jochen.schellinger@bfh.ch) Studiengangsleiter Master of Science in Business Administration an der Berner Fachhochschule. Lehr- und Forschungstätigkeiten in den Bereichen Strategisches Management, Marketing und Personalmanagement. Davor 15 Jahre Praxistätigkeit bei der Deutschen Sparkassenorganisation, im Haniel-Konzern, an der Universität Tübingen sowie in der Daimler AG.

Goedermans, Marlies (M.Sc. BA/marlies.g@bluewin.ch) Abgeschlossenes konsekutives Masterstudium in Betriebsökonomie mit Vertiefung Geschäfts- und Unternehmensentwicklung an der Berner Fachhochschule. Derzeit als Finance Operations Specialist bei der SAP Schweiz beschäftigt. 
Kolb, Lars (M.Sc. BA/lars.kolb@gmx.ch) Abgeschlossenes konsekutives Masterstudium in Betriebsökonomie mit Vertiefung Geschäfts- und Unternehmensentwicklung an der Berner Fachhochschule. Derzeit als Product Manager mit Kaderfunktion bei Interdiscount - eine Division der Coop Genossenschaft - beschäftigt.

Sebai, Yassin (M.Sc. BA/yaessu@msn.com) Abgeschlossenes konsekutives Masterstudium in Betriebsökonomie mit Vertiefung Geschäfts- und Unternehmensentwicklung an der Berner Fachhochschule. Derzeit als Projektleiter Sponsoring \& Events bei der Schweizerischen Mobiliar Versicherungsgesellschaft beschäftigt.

Open Access Dieses Kapitel wird unter der Creative Commons Namensnennung 4.0 International Lizenz (http://creativecommons.org/licenses/by/4.0/deed.de) veröffentlicht, welche die Nutzung, Vervielfältigung, Bearbeitung, Verbreitung und Wiedergabe in jeglichem Medium und Format erlaubt, sofern Sie den/die ursprünglichen Autor(en) und die Quelle ordnungsgemäß nennen, einen Link zur Creative Commons Lizenz beifügen und angeben, ob Änderungen vorgenommen wurden.

Die in diesem Kapitel enthaltenen Bilder und sonstiges Drittmaterial unterliegen ebenfalls der genannten Creative Commons Lizenz, sofern sich aus der Abbildungslegende nichts anderes ergibt. Sofern das betreffende Material nicht unter der genannten Creative Commons Lizenz steht und die betreffende Handlung nicht nach gesetzlichen Vorschriften erlaubt ist, ist für die oben aufgeführten Weiterverwendungen des Materials die Einwilligung des jeweiligen Rechteinhabers einzuholen.

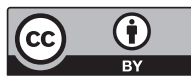




\title{
Big Data Analytics
}

\section{Analyse der prädiktiven Fähigkeit von \\ Twitter-Sentiments auf die Entwicklung \\ des Börsenkurses von Technologieunternehmen}

\author{
Flurina Fiona Baumann, Nadine Belinda Brunner \\ und Kim Oliver Tokarski
}

\begin{abstract}
Zusammenfassung
Die Datenmengen vervielfachen sich in der heutigen Zeit konstant, was zum Begriff Big Data geführt hat. Durch diese Datenmengen entsteht ein neues Potenzial, Fragen $\mathrm{zu}$ beantworten. Eine dieser Fragestellungen, welche mithilfe von Big Data untersucht werden kann, ist, inwiefern die Social-Media-Daten die Veränderung von Börsenkursen voraussagen können. Diese Studie untersucht die prädiktive Fähigkeit von Twitter-Nachrichten im Zusammenhang mit einem Technologieunternehmen und dessen Börsenkurs anhand von zwei Anwendungsfällen. Konkret wird anhand der Twitter-Nachrichten mithilfe einer Sentimentanalyse die Stimmung der Twitter-Nutzer mit den Veränderungen des Börsenkurses verglichen. Diese Analyse wird anhand der Technologieunternehmen Facebook und Amazon vorgenommen. In einem ersten Schritt wird untersucht, ob eine Beziehung zwischen den Twitter-Sentiments und dem Börsenkurs besteht. In einem zweiten Schritt, ob die Twitter-Sentiments eine Voraussagekraft für die Veränderung des Börsenkurses haben. Die Auswertung zeigt bei beiden Unternehmen eine positive Korrelation der Twitter-Sentiments und des Börsenkurses auf. Weiter konnte mithilfe der Granger-Analyse eine signifikante Voraussagekraft der Twitter-Sentiments für die Börsenkurse beider Unternehmen ermittelt werden. Die Twitter-Sentiments können die Börsenkurse 13 h voraussagen.
\end{abstract}

F. F. Baumann $(\bowtie)$

Bern, Schweiz

N. B. Brunner

Muttenz, Schweiz

K. O. Tokarski

Departement Wirtschaft, Berner Fachhochschule, Bern, Schweiz

E-Mail: kim.tokarski@bfh.ch

J. Schellinger et al. (Hrsg.), Digitale Transformation und Unternehmensführung,

https://doi.org/10.1007/978-3-658-26960-9_9 


\subsection{Ausgangslage und Problemstellung}

„Knowledge is Power“ diese Aussage ist in der heutigen Zeit sehr treffend (Mayer-Schönberger und Cukier 2013). Der Gesellschaft und somit auch den Unternehmen stehen immer mehr Daten zur Verfügung (Wrobel, Voss et al. 2015). Das durchschnittliche Unternehmen hatte bereits 2014 circa 427-mal so viele Daten wie jemals in der US Kongressbibliothek gespeichert wurde (Davenport und Paulus 2014). Davon haben $25 \%$ der Daten einen potenziellen Wert für die Gesellschaft und die Unternehmen (Davenport und Paulus 2014).

Seit 2011 hat das Interesse der Gesellschaft an Big Data zugenommen (Ward und Barker 2013). Aufgrund der Allgegenwärtigkeit des Begriffs und somit der Herkunft aus den Gebieten Wissenschaft, Industrie und Medien gibt es laut Ward und Barker (2013) keine klare Definition. Die gängigsten Definitionen beinhalten jedoch die sogenannten vier V's: Volume, Velocity, Variety und Veracity. Dabei sind die am häufigsten in der Literatur genannten Eigenschaften von Big Data Volume, Variety und Velocity (Bendler et al. 2018; Dijcks 2013; Dorschel 2015; Gluchowski und Chamoni 2016; IBM 2017; Inmon und Linstedt 2015; Mayer-Schönberger und Cukier 2013; Wrobel et al. 2015). Die Eigenschaft „Veracity“ wurde aufgrund der stetig wachsenden Social-Media-Daten erst später hinzugefügt. Diese Social-Media-Daten sind benutzergeneriert und verlangen deshalb eine weitere Eigenschaft der Begrifflichkeit „Big Data“ (Bendler et al. 2018). Die Eigenschaft „Volume“ sollte dabei nicht als notwendige Eigenschaft angesehen werden. Wird aufgrund einer Spezialisierung eines Themas nur ein geringes Datenvolumen erreicht, handelt es sich dann trotzdem noch um Big Data. Jedoch gilt die Verwaltung von einem hohen Datenvolumen mit strukturierten Daten in einer relationalen Datenbank nicht als Big-Data-Anwendung (Dorschel 2015).

Im Bereich Big Data Analytics gibt es bereits viele Anwendungsmöglichkeiten. Im Speziellen wird Big Data Analytics bereits oft für unternehmerische Fragestellungen verwendet. Beispielsweise kann das Cross-Selling und Up-Selling mithilfe von Reputationssystemen erhöht oder logistische Fragestellungen können anhand von Verkehrs- und Fahrzeugdaten besser beantwortet werden (Davenport und Paulus 2014; EMC und Greenplum 2012; Marr 2016; Dorschel 2015). Es gibt aber auch im gesellschaftlichen Bereich Anwendungsmöglichkeiten. Neben der Möglichkeit, mit Big Data Analytics beispielsweise Ausbrüche von Epidemien besser vorauszusagen, um dementsprechend zu reagieren, kann diese auch in der Planung und Bewirtschaftung der öffentlichen Infrastruktur helfen (King und Hajnal 2014; Manyika et al. 2018). Wie das Beispiel zeigt, wird Big Data Analytics oft zur Voraussage von Ereignissen genutzt.

Gerade im Bereich von prädiktiven Fragestellungen gibt es einen Anwendungsfall, welcher sowohl für Unternehmen wie auch für Privatpersonen genutzt werden kann. Dieser Anwendungsfall steht in Verbindung mit der Theorie des Homo Oeconomicus. Gemäß dieser Theorie strebt jeder Marktteilnehmer u. a. eine Nutzenmaximierung an (Mankiw 2004). Dies betrifft insbesondere auch die Akteure am Aktienmarkt. Bei einer Investition im Aktienmarkt stellt sich seit jeher die Frage, wie sich die entsprechenden Aktien entwickeln und ob sich eine Investition lohnt oder nicht (Scherbaum 2017). 
Bisher mussten sich die Investoren mehrheitlich auf die Unternehmensinformationen und Ratingagenturen verlassen, wenn sie die Investitionsentscheidungen trafen (Scherbaum 2017). Der Aktienkurs wird jedoch aus dem Zusammenspiel von Angebot und Nachfrage eines Aktientitels gebildet. Diese Kauf- und Verkaufsentscheidung der Anleger basieren wiederum auf deren Erwartungen, welche unter anderem anhand der gesellschaftlichen Stimmung gebildet werden (Nguyen et al. 2015; Scherbaum 2017). Aus diesem Grund ist es bei der Fragestellung nach der Entwicklung eines Aktienkurses besonders wichtig die Erwartungen der Anleger zu verstehen.

Bereits mehrere Studien haben sich mit der Fragestellung befasst, welche Daten diese Erwartungen der Anleger beziehungsweise die allgemeine Stimmung im Zusammenhang mit dem Unternehmen voraussagen. Dabei haben sich Social-Media-Daten als erfolgreiche Barometer herausgestellt. In der Mehrheit der betreffenden Studien konnte einen Zusammenhang oder sogar eine prädiktive Aussagekraft von Social-Media-Daten für die Aktienkurse festgestellt werden. Die bisher untersuchten Studien beschäftigen sich jedoch mehrheitlich mit spezifischen Fragestellungen in diesem Bereich. Aus diesem Grund ist für das Verständnis der Voraussagekraft von Social-Media-Daten für Börsendaten noch immer ein großes Potenzial vorhanden.

Die vorliegende Studie soll einen weiteren Einblick in die Möglichkeiten von Big Data Analytics im Zusammenhang mit der Voraussage von Aktienkursen geben und dabei aufzeigen, dass eine Analyse von Big Data ohne ein großes Budget möglich ist.

\subsection{Stand der Forschung}

Bereits seit den späten 1990er-Jahren haben sich Forschende mit dem Zusammenhang beziehungsweise der prädiktiven Fähigkeit von Social-Media-Kanälen und der Veränderung von Börsenkursen beschäftigt. Im folgenden Abschnitt wird der Stand der Forschung anhand von drei Kriterien verglichen:

- Form der Analyse der Social Media Daten

- Unterschied im gewählten Kommunikationskanal

- Art der untersuchten Börsentitel

Dabei wird jeweils untersucht, ob die Studien einen signifikanten Zusammenhang der beiden Datenquellen nachweisen können.

\subsubsection{Analyse Social Media}

Der Zusammenhang zwischen den Nachrichten auf Social Media oder anderen ähnlichen Kanälen und den Veränderungen an der Börse wurde bereits in verschiedenen Formen erforscht. Als einer der ersten Forscher hat sich Wysocki (1998) mit diesem Thema 
auseinandergesetzt. Dabei hat er sich im Gegensatz zu späteren Forschungsarbeiten vor allem auf die Anzahl der Nachrichten beschränkt, ohne dabei den Informationsgehalt der Nachrichten zu berücksichtigen. Neben Wysocki (1998) haben auch noch Reed (2016) und Mao et al. (2012) sich mit der Anzahl der Nachrichten als Variable befasst. Reed (2016) hat herausgefunden, dass die Intensität der Nachrichten mit wirtschaftlichem Inhalt einen signifikant negativen Effekt auf die Börsenkurse hat. Mao, Wei et al. (2012) haben bei der Analyse der Nachrichtenvolumen nur die Nachrichten evaluiert, welche inhaltlich einen Bezug zur Börse hatten. Viele der neueren Forschungsarbeiten haben sich jedoch bei der Analyse der Nachrichten nicht auf die Anzahl der Nachrichten beschränkt, sondern auch auf den emotionalen Inhalt der Nachrichten. Dabei gab es nur wenige Forschungsarbeiten, welche keinen signifikanten Zusammenhang zwischen den öffentlichen Nachrichten und der Veränderung an der Börse aufzeigten. Vu et al. (2012) konnten beispielsweise durch die Reaktion beziehungsweise Stimmung auf Twitter-Nachrichten mit einer Wahrscheinlichkeit von $75 \%$ bis $83 \%$ die Börsenkurse von vier ausgewählten Technologieaktien voraussagen. Dabei haben die Forscher Nachrichten mit bestimmten Schlüsselwörtern zur Marke oder deren Produkten erfasst und als positiv (bullish), negativ (bearish) oder neutral eingestuft. Auch alle anderen Forschungsarbeiten mit einer Sentimentanalyse haben die Klassifizierung der Nachrichten in diese drei Gruppen genutzt. Gesamthaft haben 17 Forschungsarbeiten für die Analyse der Social-Media-Nachrichten die Methode der Sentimentanalyse verwendet. Davon konnten 14 Studien einen signifikanten Zusammenhang zwischen den Sentiments der SocialMedia-Nachrichten und der Veränderung der Börsenkurse feststellen (Bollen et al. 2011; Antweiler und Frank 2004; Sprenger und Welpe 2010; Oh und Sheng 2011; Liew und Wang 2016; Zhang et al. 2011; Forbergskog und Blom 2014; Vu et al. 2012; Rao und Srivastava 2012; Ranco et al. 2015; Zheludev et al. 2014; Mao et al. 2011; Souza et al. 2015; Nguyen et al. 2015). Drei weitere Forschungsarbeiten konnten keinen signifikanten Zusammenhang ermitteln (Brown 2012; Das und Chen 2007; Das et al. 2005). Vier der bisherigen Forschungsarbeiten haben für die Analyse der Social-Media-Nachrichten keine Sentimentanalyse verwendet, sondern lediglich die Anzahl der Nachrichten gemessen. Davon konnten drei Studien wiederum einen signifikanten Zusammenhang zwischen der Anzahl von Social-Media-Nachrichten und der Veränderung des Börsenkurses feststellen (Wysocki 1998; Reed 2016; Mao et al. 2012, währen eine Studie kein signifikantes Ergebnis ausweisen konnte (Tumarkin und Whitelaw 2001). Die Abb. 9.1 zeigt die Analyse der Social-Media-Nachrichten im Zusammenhang mit dem Forschungsergebnis. Wie der Übersicht zu entnehmen ist, dominieren die Forschungsarbeiten, welche einen signifikanten Zusammenhang nachweisen konnten, stark.

\subsubsection{Kommunikationskanal}

Bei den bisherigen Studien wurde meist Twitter als Bezugsquelle für die Voraussage der Veränderung an der Börse verwendet. Bei den 13 Arbeiten, welche Twitter-Daten 
Abb. 9.1 Übersicht Forschungsergebnisse Analyse Social Media. (Eigene Darstellung)

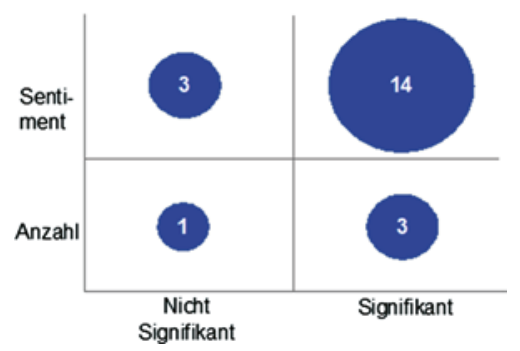

verwendet haben, konnte der Zusammenhang auch in fast allen Fällen nachgewiesen werden (Bollen et al. 2011; Reed 2016; Liew und Wang 2016; Zhang et al. 2011; Forbergskog und Blom 2014; Vu et al. 2012; Rao und Srivastava 2012; Ranco et al. 2015; Zheludev et al. 2014; Mao et al. 2012; Mao et al. 2011; Souza et al. 2015). Nur die Ergebnisse von Brown (2012) waren nicht aussagekräftig. Wobei hier auch zu erwähnen ist, dass es sich nur um ein Testdataset handelte. Als Börsenforum wurden verschiedene Kanäle verwendet. Beispielsweise haben Tumarkin und Whitelaw (2001) und Antweiler und Frank (2004) Nachrichten des Börsenforums RangingBull.com analysiert. Auch MessageBoard (Nguyen et al. 2015) und Stocktwits.com (Oh und Sheng 2011; Sprenger und Welpe 2010) wurden als Nachrichtenforum mit Börsenbezug genannt. Gesamthaft haben acht Studien die Social Media Daten von Börsenforen bezogen. Dabei konnten fünf der Studien einen signifikanten Zusammenhang zwischen den Social-Media-Nachrichten und den Veränderungen der Börsenkurse aufweisen (Wysocki 1998; Antweiler und Frank 2004; Oh und Sheng 2011; Nguyen et al. 2015; Sprenger und Welpe 2010). Drei weitere Studien konnten keine Signifikanz feststellen (Tumarkin und Whitelaw 2001; Das und Chen 2007; Das et al. 2005).

Neben den börsenbezogenen Nachrichtenforen und Twitter wurde kaum ein anderer Social-Media-Kanal für den Bezug der Stimmungsdaten genannt. Twitter ist, wie auch der Auflistung von erfolgreichen Forschungsarbeiten in der Abb. 9.2 entnommen werden kann, ein gutes Instrument für die Voraussage der Veränderung an der Börse. Microblogging ist aufgrund des hohen Volumens, der Echtzeitanalyse und der Kürze der Nachrichten besonders für eine starke Voraussagekraft der Veränderungen an der Börse

Abb. 9.2 Übersicht Forschungsergebnisse Kommunikationskanal. (Eigene Darstellung)

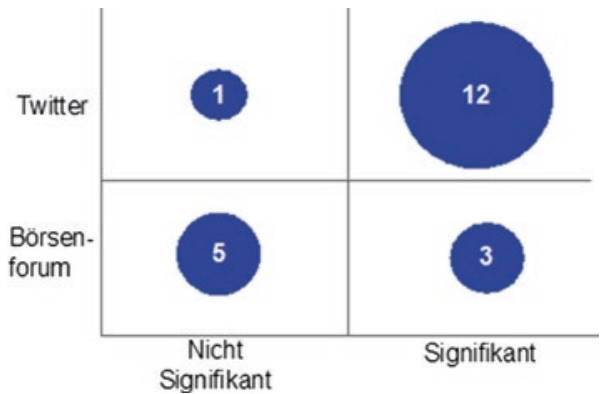


geeignet (Oh und Sheng 2011). Insbesondere ist Twitter ein Informationsinstrument, welches nicht nur die großen Geschichten beinhaltet, sondern auch sehr regionale Informationen abbildet. Diese Informationen sind auf Twitter meist bereits vor der Veröffentlichung auf den offiziellen Nachrichtenportalen vorhanden (Souza, Kolchyna et al. 2015). Twitter hat auch gemäß Souza Kolchyna et al. (2015) nachweislich eine höhere Aussagekraft bezüglich der Veränderungen an der Börse als herkömmliche Nachrichten.

\subsubsection{Börsentitel}

Für die beobachteten Werte an der Börse wurden bisher sowohl Indizes wie auch einzelne Wertschriftentitel verwendet. Als Indizes wurde oft der Dow Jones Industrial Average (DJIA) untersucht (u. a. Bollen et al. 2011; Reed 2016; Rao und Srivastava 2012). Daneben wurde auch der Index Standard \& Poors 500 (S\&P 500) oft als Messgröße eingesetzt (u. a. Sprenger und Welpe 2010; Zhang et al. 2011; Mao 2012). Wenn in den Forschungsarbeiten einzelne Aktientitel beobachtet wurden, handelt es sich meistens um eine eher größere Anzahl von Aktien. Dabei wurden oft Aktien von größeren und bekannteren Unternehmen miteinbezogen. Beispielsweise wurde bei Souza et al. (2015) unter anderem die Veränderung der Aktien von Abercrombie \& Fitch Co. und Nike Inc. miteinbezogen, aber nie als Fokus der Untersuchung betrachtet. Besonders oft wurden auch Technologieunternehmen in die Beobachtung miteinbezogen ( Vu et al. 2012; Das et al. 2005; Tumarkin und Whitelaw 2001; Das und Chen 2007; Liew und Wang 2016; Rao und Srivastava 2012). Der Abb. 9.3 kann entnommen werden, dass eine sich eine größere Anzahl von Untersuchungen mit Indizes als Dateninput für die Veränderung der Börsenkurse beschäftigt hat.

Von insgesamt 18 Studien, welche einen Indizes untersucht haben, konnten auch 16 Studien einen signifikanten Zusammenhang aufweisen (Bollen et al. 2011; Sprenger und Welpe 2010; Oh und Sheng 2011; Reed 2016; Zhang et al. 2011; Forbergskog und Blom 2014; Rao und Srivastava 2012; Ranco et al. 2015; Zheludev et al. 2014; Mao et al. 2012; Mao et al. 2011). Nur zwei weitere Studien konnten keinen signifikanten Zusammenhang ermitteln (Brown 2012; Das und Chen 2007). Zehn weitere Forschungsarbeiten haben

Abb. 9.3 Übersicht Forschungsergebnisse Index. (Eigene Darstellung)

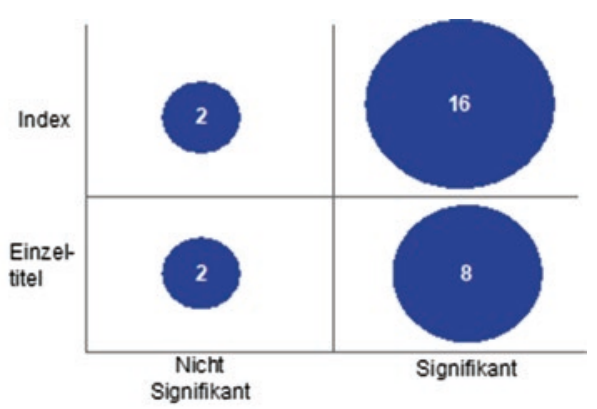


einzelne Wertschriftentitel als Vergleichswert herangezogen. Davon konnten auch acht Studien einen signifikanten Zusammenhang aufweisen (Wysocki 1998; Antweiler und Frank 2004; Liew und Wang 2016; Vu et al. 2012; Rao und Srivastava 2012; Mao et al. 2012; Souza et al. 2015; Nguyen et al. 2015). Zwei weitere Studien konnten wiederum keinen signifikanten Zusammenhang ermitteln (Tumarkin und Whitelaw 2001; Das et al. 2005).

\subsubsection{Erkenntnisse aus der Theorie und Forschungslücke}

Die bisherigen Forschungsarbeiten zeigen auf, dass es mehrheitlich einen Zusammenhang zwischen den Social-Media-Daten und den Veränderungen der Börsenkurse gibt. Die Sentimentanalyse als Stimmungsbarometer spiegelt sich in den Ergebnissen als besonders aussagekräftig wider. Aufgrund der neuen Möglichkeiten durch die Sentimentanalyse wurden in den letzten Jahren kaum mehr nur die Anzahl der Nachrichten analysiert. Die Sentimentanalyse ist somit ein empfehlenswertes Instrument zur Voraussage der Börsenkurse anhand von Social-Media-Nachrichten. Weiter wurde der Social-Media-Kanal Twitter mehrfach erfolgreich für ähnliche Studien verwendet und eignet sich aufgrund der Eigenschaft des Microbloggings besonders für die Analyse. Bisher haben sich die meisten Forschungsarbeiten mit der Voraussage der Veränderung von ganzen Indizes oder Branchen befasst. Die Forschungsarbeiten, welche sich einzelnen, ausgewählten Aktientitel widmen, haben meist große international tätige Unternehmen gewählt. Dabei wurden nur selten individuelle Aktien von Technologieunternehmen untersucht. Da die Aktien von Technologieunternehmen aufgrund von hohen Renditepotenzialen und anderseits auch hohen Risiken starken Schwankungen unterworfen sind, eigenen sich die Aktientitel jedoch besonders für die Analyse (Ciolli 2017; Investopedia 2018).

\subsubsection{Hypothesen und theoretisches Modell}

Bisher gab es keine Studie, welche sich mit der Frage beschäftigt, ob die Stimmung gegenüber spezifischen Technologieunternehmen auf Social Media einen Zusammenhang mit den Veränderungen der Börsenkurse dieser spezifischen Technologieunternehmen aufweist bzw. eine Voraussagekraft für die Entwicklung der Börsenkurse hat. Die Resultate der Studien zu Technologie-Indizes und einzelner Börsentitel anderer Branchen weisen stark darauf hin, dass die Stimmung auf Social Media einen Zusammenhang und sogar eine Voraussagekraft für die einzelnen Börsentitel von Technologieunternehmen haben. Das Twitter als Social-Media-Plattform für Börsenthemen bereits mehrmals erfolgreich in ähnlichen Studien genutzt wurde, zeigt, dass Twitter eine angemessene Plattform für die vorliegende Studie ist. Aufgrund der oben 


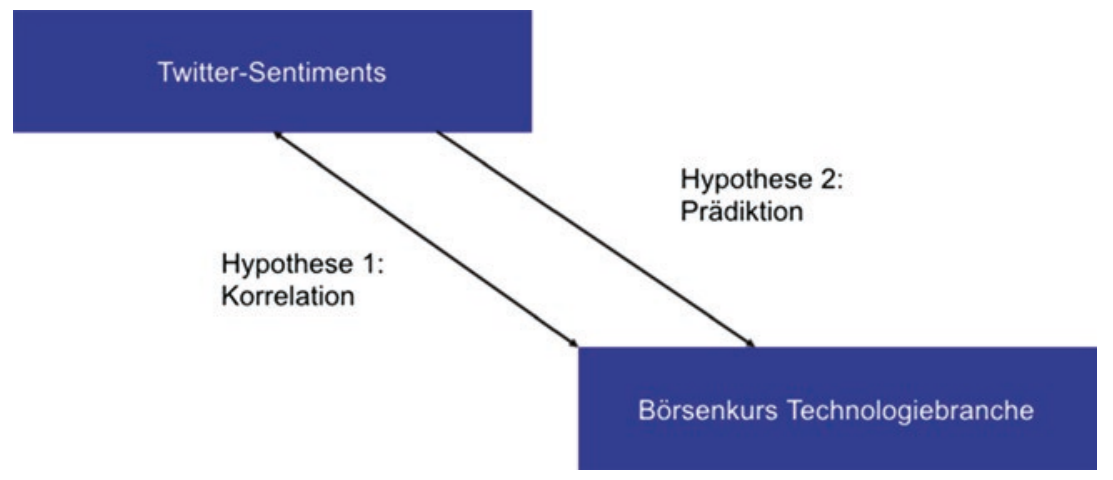

Abb. 9.4 Theoretisches Modell. (Eigene Darstellung)

erwähnten Begründungen, basierend auf bisherigen Forschungsergebnissen, werden folgende Hypothesen aufgestellt:

\section{- Hypothese 1}

Die Twitter-Sentiments im Zusammenhang mit einzelnen Technologieunternehmen weisen eine Korrelation mit der Entwicklung des Börsenkurses dieser Unternehmen auf.

\section{- Hypothese 2}

Die Twitter-Sentiments im Zusammenhang mit einzelnen Technologieunternehmen haben eine Voraussagekraft für die Entwicklung des Börsenkurses dieser Unternehmen.

Aus den oben aufgeführten Hypothesen ergibt sich für den Zusammenhang der Datensätze Twitter-Sentiments zu den einzelnen Technologieunternehmen und der Entwicklung der Börsenkurse derselben Unternehmen das theoretische Modell gemäß Abb. 9.4.

\subsection{Methodisches Vorgehen}

\subsubsection{Rahmenbedingungen und Plattform}

Die Definition der Rahmenbedingungen bezüglich der Plattform und der Unternehmen dient als Leitplanke für die Umsetzung.

In der vorliegenden Arbeit wurden die Sentiments der Tweets von der Twitter-Plattform verwendet. Dies aufgrund der größten Akzeptanz dieser Plattform in der Finanzbranche und der bereits erfolgreichen Nutzung in vielen Forschungsbereichen (Sprenger und Welpe 2010). 
Nach (Nguyen et al. 2015) gibt es zwei verschiedene Arten von Tweets auf Twitter. Eine Art Tweet beginnt ein neues Thema die zweite Art Tweets, ein sogenannter Retweet, antwortet auf ein Thema (Nguyen et al. 2015). Die verschiedenen Arten von Tweets führen zu einem komplizierten Kommunikationsnetzwerk. In der vorliegenden Arbeit wurde die Art der Tweets vernachlässigt und unabhängig verwendet.

\subsubsection{Unternehmen}

Für diese Studie wurden drei Technologieunternehmen gewählt, da diese Branche an der Börse besonders interessant im Sinne des Wachstums sowie Entwicklungspotenzials ist (Ciolli 2017; Investopedia 2018). Aus den Technologieunternehmen wurden Tesla, Facebook und Amazon als Studienobjekte ausgewählt. Dies weil diese drei Unternehmen gemäß (Forbes Technology Council 2018) in den Top fünf der interessantesten Technologieaktien vom Jahr 2018 vertreten sind und aktuell in den Medien besonders präsent (Kawa 2018). Ein weiterer Punkt für die Auswahl ist der Name der Unternehmen, respektive deren Produkt oder Service. Das Produkt beziehungsweise der Service ist direkt mit dem Unternehmensnamen verbunden. Die Tweets können deshalb direkt mit dem Unternehmensnamen Tesla, Amazon oder Facebook gefiltert werden (Nguyen et al. 2015). Da die Unternehmenssprache Englisch ist, werden für die Studie nur englischsprachige Tweets berücksichtigt (Kroker 2013).

Neben der oben aufgeführten Rahmenbedingung soll die Umsetzung kostenlos sein. Dies wurde in der Umsetzung respektive in der Auswahl der Tools berücksichtigt.

\subsubsection{Datenpipeline}

Um die beschriebenen Hypothesen zu testen, wird dem Ablauf gemäß Abb. 9.5 gefolgt. In einem ersten Schritt wird das Datenformat definiert, welches aus strukturierten oder unstrukturierten Daten bestehen kann. In einem zweiten Schritt müssen Entscheidungen zu den Bezugsplattformen und den Möglichkeiten zum Bezug der Daten getroffen

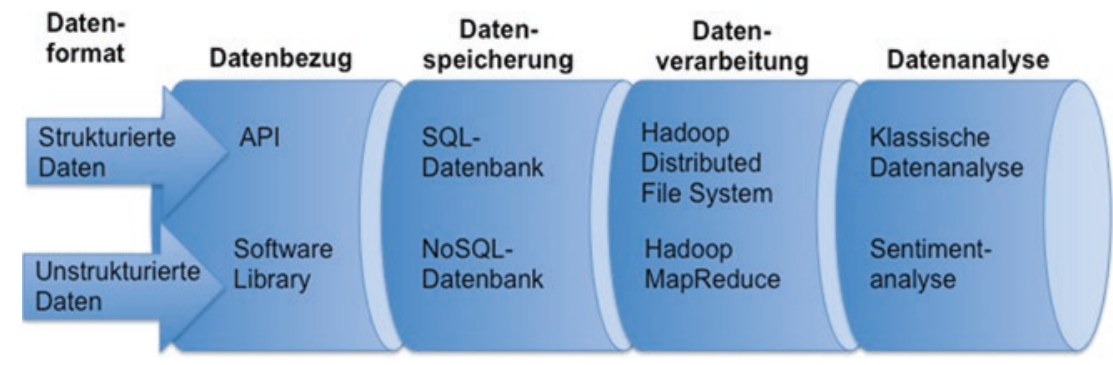

Abb. 9.5 Datenpipeline. (Eigene Darstellung) 
werden. Weiter müssen die bezogenen Daten in einer Datenbank gespeichert werden, welche den gewählten Inputdaten entspricht. Bei einer großen Datenmenge kann außerdem ein Datenverarbeitungssystem miteinbezogen werden, um die Datenmenge verarbeitbar zu machen. Als letzter Schritt müssen die analysiert und interpretiert werden, um die Fragestellung zu beantworten.

Nachstehend wird vertieft auf die Schritte der Datenpipeline eingegangen. Die einzelnen Schritte beinhalten nur die für diese Forschungsarbeit notwendigen Überlegungen und sind daher nicht abschließend zu betrachten.

\subsubsection{Datenformat}

Daten können in zwei verschiedene Typen eingeteilt werden (Inmon und Linstedt 2015). Einerseits gibt es die strukturierten Daten, welche ein klares und gleichbleibendes Format aufweisen. Auf der anderen Seite gibt es die unstrukturierten Daten, welche keiner standardisierten Struktur folgen und dementsprechend auch mit Maschinen nicht einfach $\mathrm{zu}$ verarbeiten sind (Inmon und Linstedt 2015). In der vorliegenden Arbeit wurden beide Datenformate verwendet. Die Twitter-Daten sind aufgrund des freien Textformats als unstrukturierte Daten zu betrachten. Die Börsendaten sind in der Länge und Form klar definiert und folgen daher einer klaren Struktur.

\subsubsection{Datenbezug}

Für den Datenbezug der Twitter- und Börsendaten wurden verschiedene Anwendungen evaluiert. Die Abb. 9.6 zeigt als Übersicht auf, welche Anwendungen für den Bezug der zwei Twitter-Daten und der Börsendaten gewählt werden.

Damit Tweets von Twitter und der Börse gesammelt werden können, wird eine API (Mao et al. 2012; Ranco et al. 2015) und eine Library (Baumann 2018) benötigt. Twitter bietet die Standard-API, die Premium-API und die Enterprise-API an. Die Enterprise-API sowie die Premium-API sind kostenpflichtig, während die Standard-API kostenlos ist. Aus diesem Grund wird in der vorliegenden Arbeit die Standard-API verwendet (Twitter 2018). Innerhalb der Standard-API kann die Streaming-API oder die Searching-API verwendet werden (Twitter 2018). Die Streaming-API erlaubt die Tweets in Echtzeit herunterzuladen, jedoch nicht in der Vergangenheit zu suchen. Für eine Voraussage des Aktienkurses wird normalerweise die Streaming-API von Twitter verwendet ( Vu et al. 2012). Allerdings ist für eine Streaming-API eine permanent laufende Infrastruktur mit Servern notwendig. In der vorliegenden Arbeit wird aufgrund der fehlenden kostenintensiven Infrastruktur die Searching-API verwendet. Diese API kann für Suchanfragen verwendet werden (Rao und Srivastava 2012; Ranco et al. 2015). Die Tweets können bis zu sieben Tage in die Vergangenheit bezogen werden. Die Library, als zweite Komponente für den Datenbezug, übernimmt die Kommunikation mit 


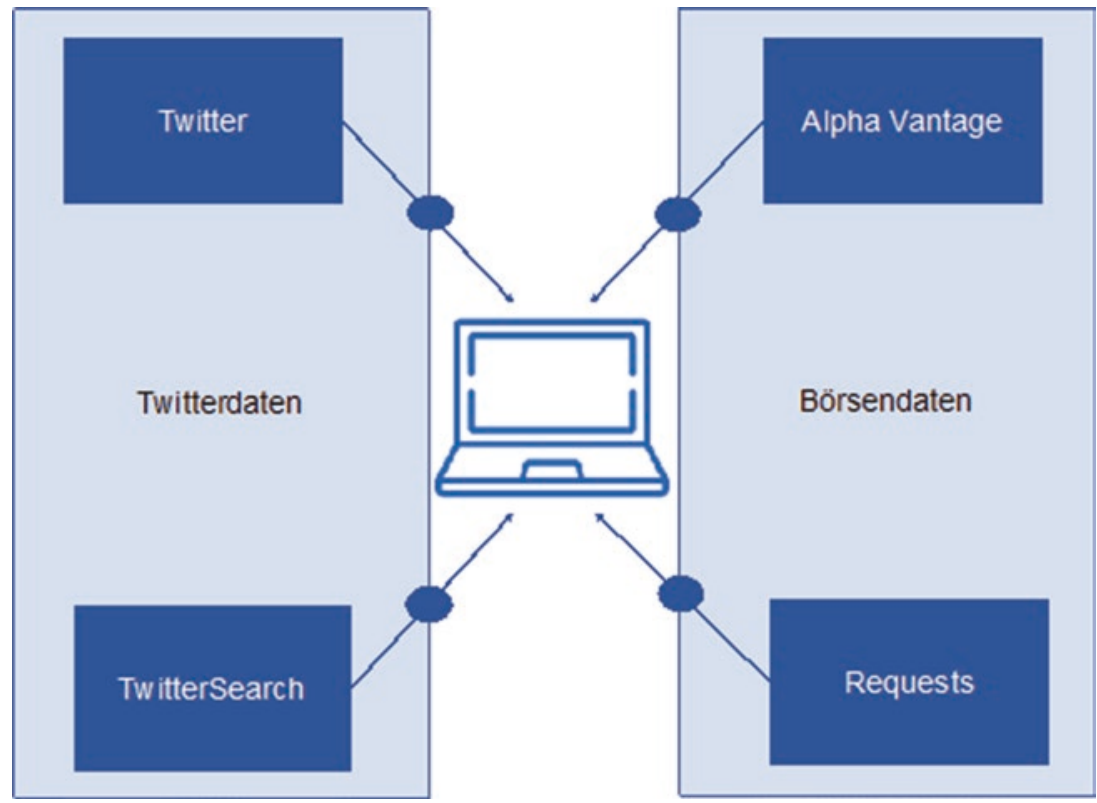

Abb. 9.6 Datenbezug. (Eigene Darstellung)

der Twitter-API anhand öffentlich verfügbarer Funktionen (educalingo 2018; Technopedia 2018). Grundsätzlich wurden Libraries für die Programmiersprache Python mit einer Internetrecherche evaluiert und auf die Eigenschaften kostenlos, installierbar, funktionsfähig respektive mit einer Lösung der Paginierung für Suchresultate, getestet. Die TwitterSearch Library, von der Technischen Universität München (Koepp 2018) beinhaltet nach einer Fehlerkorrektur der Paginierung alle Eigenschaften und wird deshalb als geeignet betrachtet und in dieser Studie verwendet (Baumann 2018).

Die Börsenkurse werden mittels Python-Skript von der Plattform Alpha Vantage bezogen (Alpha Vantage 2018). Diese Plattform bietet die Börsendaten bereits in Stundenintervallen an und die Daten sind in die Vergangenheit beziehbar. Die Anforderungen an diese Library sind geringer als bei der Twitter-API, daher fand hier keine Evaluation der Library statt und es wurde die populäre Python Library „requests“ verwendet (Reitz 2018).

\subsubsection{Datenspeicherung}

Damit die Daten, welche von Twitter bezogen wurden, verarbeitet werden können, müssen die Daten in einer Datenbank gespeichert werden (Baumann 2018). Dafür gibt es unter anderem SQL- und NoSQL-Datenbanken. Die SQL eignet sich für strukturierte Daten während NoSQL vor allem für die Speicherung von unstrukturierten Daten 
Abb. 9.7 Datenbanken. (Eigene Darstellung)
SQL NOSQL

Strukturierte Daten

Unstrukturierte Daten

auszeichnet (Moniruzzaman und Hossain 2013; Litzel 2017). Die Datenbanken können wie in Abb. 9.7 aufgezeigt verwendet werden.

Da das Datenformat der beiden Datenströme sowohl strukturiert wie auch unstrukturiert ist, wird in der vorliegenden Studie eine NoSQL-Datenbank benötigt. Da die Tweets von der Twitter-API im Format JSON geliefert werden, wird eine Datenbank gewählt, welche das JSON-Format unterstützt. Dies soll einen möglichst robusten und einfachen Import ermöglichen. Weiter ist bei der Verwendung einer Datenbank für Big Data besonders wichtig, dass genügend Platz vorhanden ist und Abfragen gemacht werden können. Dafür eignen sich die NoSQL-Datenbanken CoucheDB oder MongoDB. Die CouchDB vom Entwicklungsteam des Apache-Projekts erfüllt diese Bedingungen. Da sie außerdem eine Library für die Programmiersprache Python zur Verfügung stellt, wird die CouchDB für diese Studie verwendet (Apache CouchDB 2018).

\subsubsection{Datenverarbeitung}

Die herkömmliche Datenverarbeitung kann aufgrund des großen Datenvolumens bei Big Data nicht effizient und schnell genug arbeiten. Aus diesem Grund gibt es das Datenverarbeitungssystem Hadoop (Marz und Warren 2015). Hadoop ist eine Open-Source-Plattform, welche aus mehreren Komponenten besteht. Unter anderem beinhaltet Hadoop ein Software-Framework für Speicheroptimierung und eine Verarbeitungskomponente für Big Data (Gang-Hoon et al. 2014). Da die Datenmenge in dieser Studie noch schnell genug verarbeitet werden kann, wird auf ein Datenverarbeitungssystem verzichtet.

\subsubsection{Datenanalyse}

Damit die Daten verarbeitet und analysiert werden können, müssen unstrukturierte Twitter-Daten zu strukturierten Daten, wie in Abb. 9.8 aufgezeigt, umgewandelt werden. Dies erfolgt mithilfe einer Sentimentanalyse. Die Sentimentanalyse untersucht die Stimmung in einem Tweet. Diese Stimmung wird als Zahl zwischen 1 und -1 repräsentiert. Eine Sentimentanalyse wird oft mittels „Machine Learning“ vortrainiert (Baumann 2018; Graf 2018). In dieser Studie wird das bereits bestehende Natural Language Toolkit (NLTK) verwendet (Bird et al. 2015). Zum NLTK gehört das Text-Analyse-Tool „Valence Aware Dictionary and sentiment Reasoner" (Vader), welches vortrainiert ist, um die Stimmung von Tweets in englischer Sprache zu ermitteln (NLTK 2017). Das Text-Analyse-Tool „Vader“ ist speziell für die Analyse von Stimmungen in sozialen 


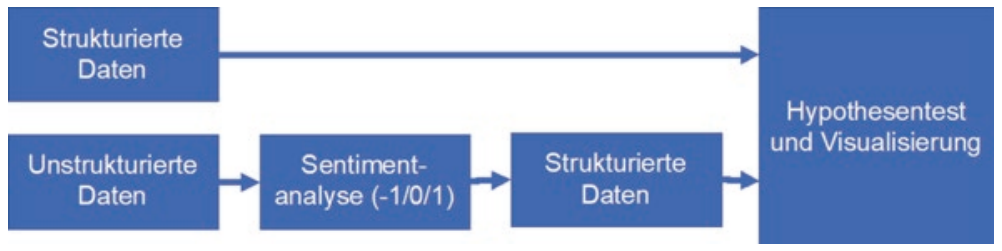

Abb. 9.8 Datenanalyse. (Eigene Darstellung)

Medien programmiert (Hutto 2018). Auch (Brown 2012) verwendet für die Sentimentanalyse erfolgreich das Natural Language Processing Toolkit in Python. Die Sentimentanalyse wurde mittels eines Skripts für alle gesammelten Tweets angewendet. Dabei wurden alle Tweets aus der CouchDB geladen, analysiert und mit dem eruierten Sentiment-Wert wieder in der CouchDB gespeichert. In einem nächsten Schritt werden für die Analysen der Daten, die Sentiments der einzelnen Tweets mittels arithmetischem Mittel pro Stunde gruppiert.

Die Börsenkurse werden in einem regelmäßigen Intervall von einer Stunde gespeichert, um eine zeitnahe Veränderung beobachten zu können. Da die Börse nicht durchgehend geöffnet ist, gibt es Lücken in den Datensätzen. Diese Lücken werden mit der Python-Library „pandas“ überbrückt (pandas 2018; Baumann 2018). Die Lücken wurden jeweils mit dem letzten bekannten Börsenkurs gefüllt.

Um die Hypothesen zu testen, wurden im letzten Schritt der Abb. 9.8 zwei verschiedene Analysen durchgeführt. Für die Hypothese 1 wurde untersucht, ob einen Zusammenhang zwischen den Twitterdaten und den Börsendaten desselben Unternehmens besteht. Aus diesem Grund wird eine Korrelationsanalyse durchgeführt. Die Hypothese 2 wird anhand der Granger-Analyse getestet. Die Granger-Analyse untersucht, ob die Twitter-Daten die Börsenkurse voraussagen können und welches Timelag (Zeitdifferenz) dazwischen liegt.

\subsection{Ergebnisse}

\subsubsection{Beschreibung des Datensatzes}

Daten der Tweets und der Börsenkurse wurden in einem Zeitraum vom 25. März 2018 bis zum 20. Mai 2018 bezogen. Für den Datenbezug wurde zur Optimierung der Tweets nicht nur die Unternehmensbezeichnung verwendet. Beim Unternehmen wurde beispielsweise Facebook zusätzlich zum Begriff Facebook auch der Hashtag \#facebook eingeschlossen. Als erster Schritt wurde der Datenbezug der Twitter-Daten zum Unternehmen Tesla angestoßen. Nach einer Fehlerbehebung folgten dann am 30. April 2018 die Einstellungen für die Unternehmen Facebook und Amazon. Aufgrund eines Funktionsfehlers ist der Zeitraum vom 16. April bis zum 29. April nicht brauchbar. 
Tab. 9.1 Zeiträume. (Eigene Darstellung)

\begin{tabular}{|c|c|c|c|c|c|}
\hline \multirow[t]{2}{*}{ Unternehmen } & \multicolumn{2}{|l|}{ Zeitraum } & \multicolumn{2}{|c|}{$\begin{array}{l}\text { Zeitraum mit } \\
\text { Funktionsfehler }\end{array}$} & \multirow[t]{2}{*}{$\begin{array}{l}\text { Anzahl verwendete } \\
\text { Tage }\end{array}$} \\
\hline & Von & Bis & Von & Bis & \\
\hline Tesla & 25.03 .2018 & 20.05.2018 & 16.04 .2018 & 29.04.2018 & 21 \\
\hline Facebook & 30.04 .2018 & 20.05.2018 & & & 21 \\
\hline Amazon & 30.04 .2018 & 20.05.2018 & & & 21 \\
\hline
\end{tabular}

Dies betrifft jedoch nur das Unternehmen Tesla, welches nun in der Mitte der Daten einen Zeitraum von 14 Tagen aufweist, welcher nicht genutzt werden kann. Eine Übersicht der Zeiträume ist der Tab. 9.1 zu entnehmen.

Innerhalb der Zeiträume, welche in der Tab. 9.1 aufgeführt sind, konnte je nach Unternehmen eine unterschiedliche Anzahl Tweets bezogen werden. Die Abb. 9.9 zeigt die bezogenen Tweets pro Unternehmen.

Wie der Abb. 9.9 zu entnehmen ist, konnten von Tesla über den gesamten Zeitraum, im Verhältnis zu Facebook und Amazon, nur wenige Daten gespeichert werden. Dabei ist noch zu bedenken, dass sich diese Anzahl Tweets von Tesla auf zwei verschiedene Zeiträume, mit einer Lücke von 14 Tagen dazwischen, beziehen. Daher ist für den durchgehenden Zeitraum vom 30. April 2018 bis zum 20. Mai 2018 nur noch etwa die Hälfte der in der Abb. 9.9 verwendeten Tweets nutzbar. Aufgrund der geringen Anzahl an brauchbaren Daten von Tesla und des großen Unterschieds der Anzahl Daten zu den zwei anderen Unternehmen, wurde Tesla als Untersuchungsobjekt ausgeschlossen. Zukünftige Forschungsarbeiten könnten das Unternehmen als Untersuchungsobjekt integrieren. Im vorliegenden Fall wurden nun aber lediglich Amazon und Facebook im Rahmen der Studie analysiert.

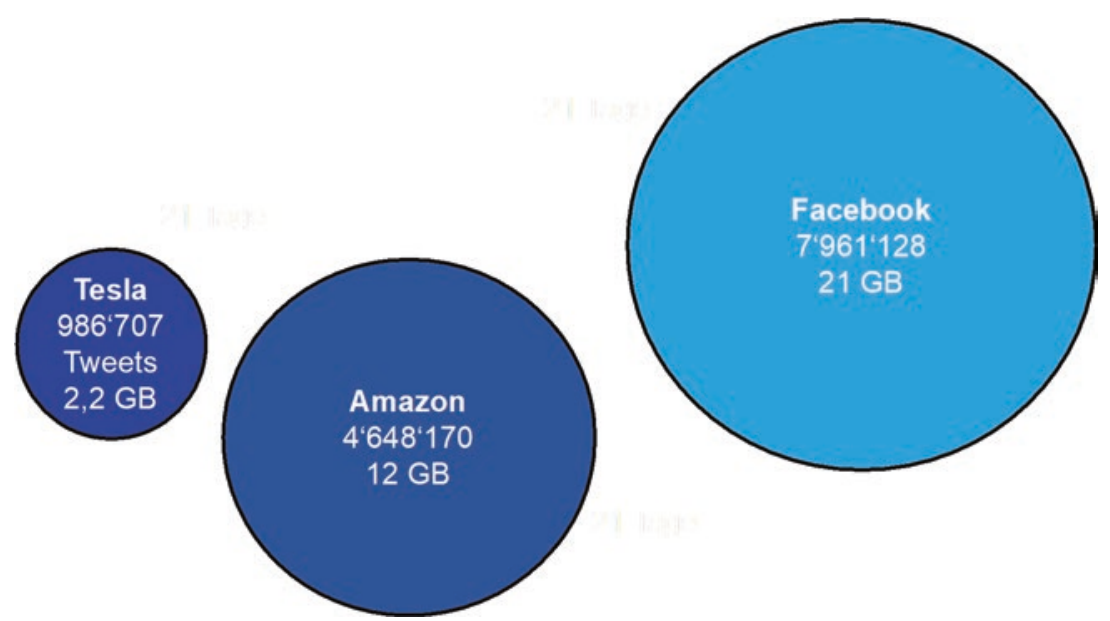

Abb. 9.9 Bezogene Tweets. (Eigene Darstellung) 


\section{Spitzenwerte}

Bereits bei der ersten Betrachtung des Verlaufs der Anzahl Tweets, der Twitter-Sentiments und der Veränderung der Börsendaten kann bei beiden Unternehmen ein leichtes Muster erkannt werden. Die Abb. 9.10 und die Abb. 9.11 zeigen jeweils den Verlauf dieser drei Werte für das Unternehmen Facebook und Amazon auf.

Im Folgenden sind die Amazon-Daten dargestellt.

Wie Abb. 9.10 und Abb. $9.11 \mathrm{zu}$ entnehmen ist, gibt es zum Teil starke Bewegungen und trotz der kurzen Zeitdauer der Beobachtung einige Spitzenwerte. Wie in der Tab. 9.2 entnommen werden kann, sind diese Spitzenwerte auch tatsächlich einem Ereignis mit Zusammenhang zum Unternehmen zuzuordnen.

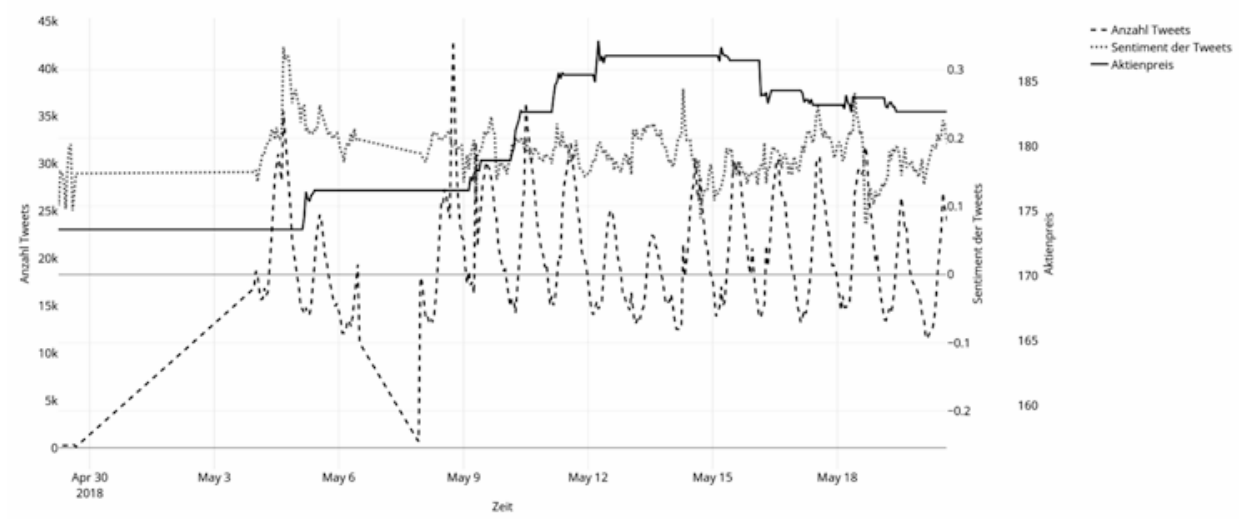

Abb.9.10 Facebook Daten. (Eigene Darstellung)

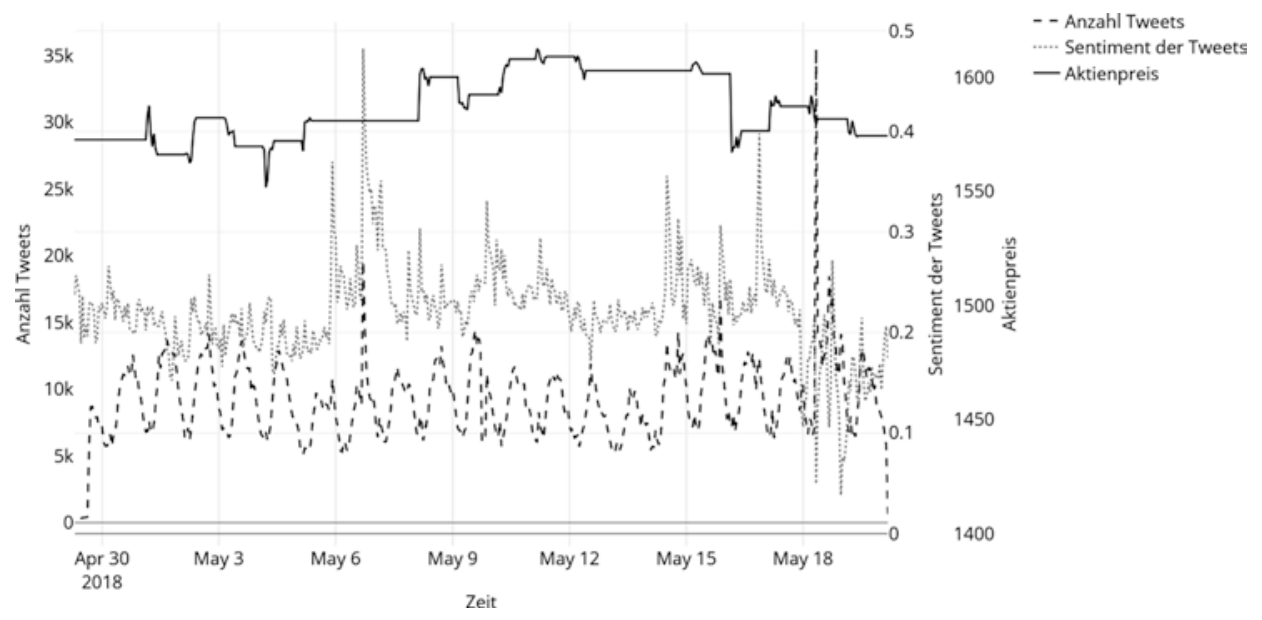

Abb. 9.11 Amazon Daten. (Eigene Darstellung) 
Tab. 9.2 Spitzenwerte. (Eigene Darstellung)

\begin{tabular}{|c|c|c|c|}
\hline \multirow[t]{6}{*}{ Facebook } & 04.05 .2018 & 0,333 & $\begin{array}{l}\text { Entlassung Mitarbeiter wegen Prahlerei mit Nutzerdaten } \\
\text { (Redaktion 2018) }\end{array}$ \\
\hline & 14.05 .2018 & 0,272 & Eigene Kryptowährung für Facebook (Bluewin 2018b) \\
\hline & 18.05 .2018 & 0,266 & $\begin{array}{l}\text { Partnerschaft mit Thinktank (Möller 2018) } \\
\text { Facebook startet Werbeeinblendungen in Stories } \\
\text { (Kroll 2018) }\end{array}$ \\
\hline & 09.05 .2018 & 0,106 & Führungsumbau (Neue Zürcher Zeitung 2018) \\
\hline & 14.05.2018 & 0,082 & $\begin{array}{l}\text { Facebook äußert Verdacht über Missbrauch von Nutzerdaten } \\
\text { durch diverse Apps (finanzen.ch 2018a) }\end{array}$ \\
\hline & 18.05.2018 & 0,076 & $\begin{array}{l}\text { Partnerschaft mit Thinktank (Möller 2018) } \\
\text { Facebook startet Werbeeinblendungen in Stories } \\
\text { (Kroll 2018) }\end{array}$ \\
\hline \multirow[t]{4}{*}{ Amazon } & 06.05 .2018 & 0,482 & Amazon prüft Einstieg ins Bankgeschäft (Prior 2018) \\
\hline & 16.05 .2018 & 0,398 & $\begin{array}{l}\text { Streik bei Amazon (finanzen.ch 2018b) } \\
\text { Neugestaltung der Lebensmittelläden (Wingfield 2018) }\end{array}$ \\
\hline & 05.05 .2018 & 0,369 & $\begin{array}{l}\text { Gewinnmaschine Amazon (Schürmann 2018) } \\
\text { Amazon-Chef hebt ab (Beglinger und Beglinger 2018) }\end{array}$ \\
\hline & 18.05.2018 & 0,185 & $\begin{array}{l}\text { Wachstum der Amazon-Aktie (Sasse 2018) } \\
\text { Amazon Prime Erhöhung der Preise (Rauffmann 2018) } \\
\text { Mögliche Einmischung der Politik bei Amazon führt zu } \\
\text { Verlierern auf beiden Seiten (Mullainathan 2018) }\end{array}$ \\
\hline
\end{tabular}

\subsubsection{Bezug zu Big Data}

Die Definition von Big Data erfolgt, wie in der Einleitung erwähnt, anhand der vier Eigenschaften Datenvolumen, Datenvielfalt, Datengeschwindigkeit und Richtigkeit. Der verwendete Datensatz weist diese Eigenschaften gemäß der Tab. 9.3 erwähnten Form auf.

Das totale verwendete Datenvolumen überschreitet bereits die Kapazitäten von bspw. dem Programm Excel (Microsoft 2018). Auch das Kriterium der Datenvielfalt wurde in dieser Studie erfüllt, da es sich sowohl um strukturierte wie auch unstrukturierte Daten handelt. Durch die sich ständig verändernden Twitter- und Börsendaten wurde die Datengeschwindigkeit ebenfalls erreicht. Das Kriterium Richtigkeit wurde aufgrund der direkt vom User generierten Daten ebenfalls erfüllt. Ein möglicher Unsicherheitsfaktor dabei ist jedoch die Sentimentanalyse. Da der Algorithmus jedoch bereits mehrmals erfolgreich getestet wurde, kann von einer „korrekten“ Analyse ausgegangen werden. Veränderungen und Weiterentwicklungen sind im Rahmen des technologischen Fortschritts möglich. Auch andere Algorithmen im genannten Kontext könnten das Untersuchungsergebnis verändern. Für die Zeitpunkte der Untersuchung gelten die zuvor dargestellten Aussagen.

Im Folgenden wird nun auf den Test der Hypothesen eingegangen. 
Tab. 9.3 Big-Data-Eigenschaften. (Eigene Darstellung)

\begin{tabular}{|c|c|c|}
\hline Eigenschaften & Inhalt & Verwendeter Datensatz \\
\hline Datenvolumen/Volume & Anzahl und Größe von Daten & - 32,6 Gigabyte \\
\hline Datenvielfalt/Variety & Quellen und Arten von Daten & $\begin{array}{l}\text { - Quellen: Twitter und Alpha } \\
\text { Vantage } \\
\text { - Arten: strukturiert und } \\
\text { unstrukturiert }\end{array}$ \\
\hline Datengeschwindigkeit/Velocity & $\begin{array}{l}\text { Datengenerierung in hoher } \\
\text { Geschwindigkeit }\end{array}$ & $\begin{array}{l}\text { - 32,6 Gigabyte innerhalb } \\
\text { 21 Tagen } \\
\text { • Stetige Veränderung }\end{array}$ \\
\hline Richtigkeit/Veracity & $\begin{array}{l}\text { Vollständigkeit und Verläss- } \\
\text { lichkeit der Dateninhalte }\end{array}$ & $\begin{array}{l}\text { - User-generierte Textdaten } \\
\text { vorhanden }\end{array}$ \\
\hline
\end{tabular}

\subsubsection{Hypothesentest}

\subsubsection{Korrelationsanalyse}

\subsection{Facebook}

Das Streudiagramm der Abb. 9.12 zeigt die Beziehungspunkte zwischen den Twitter-Sentiments im Zusammenhang mit Facebook (x-Achse) und dem Börsenkurs von Facebook (y-Achse). Die meisten Datenpunkte befinden sich auch hier eher bei der Orientierungslinie. Fast alle Punkte befinden sich außerdem im positiven Bereich des Streudiagramms. Der Spearman's Korrelationskoeffizient zeigt ebenfalls eine eher starke positive Beziehung mit dem Wert $r=0,557$. Die Signifikanz ist mit dem Wert von $\mathrm{p}=0,00 \mathrm{sehr}$ hoch. Diese Analyse zeigt, dass die Twitter-Sentiments zu Facebook und der Börsenkurs von Facebook sich zyklisch bewegen und einen starken Zusammenhang aufweisen.

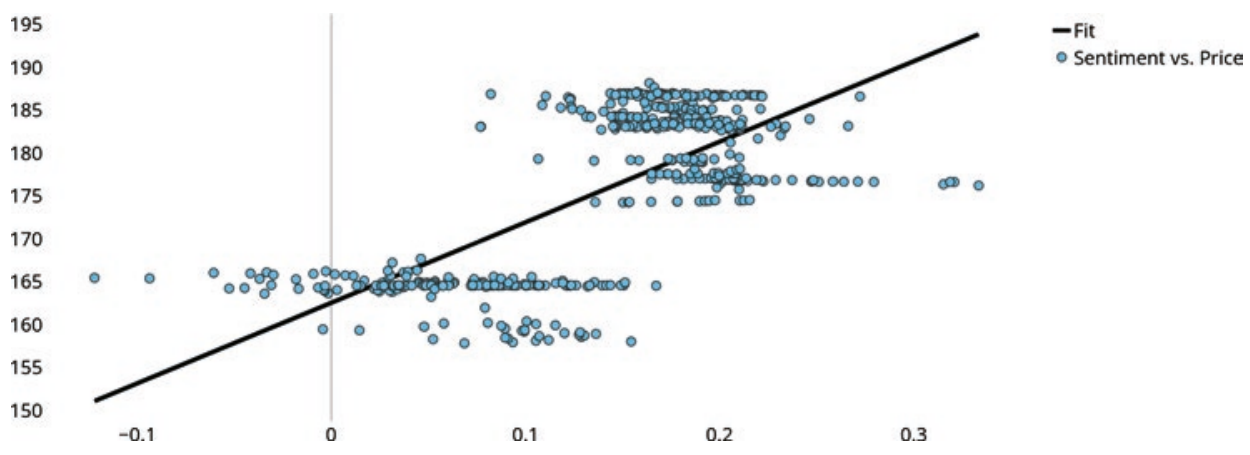

Abb. 9.12 Streudiagramm - Facebook. (Eigene Darstellung) 


\subsection{Amazon}

Das Streudiagramm in Abb. 9.13 zeigt die Beziehung der Twitter-Sentiments zu Amazon und der Börsenkurse von Amazon. Wie das Streudiagramm zeigt, liegen die Punkt weit auseinander und weniger um die Orientierungslinie herum. Auch der Spearman's Korrelationskoeffizient zeigt mit einem Wert von $\mathrm{r}=0,319$ nur einen moderaten Zusammenhang auf. Die Signifikanz für diesen Wert ist mit $p=0,00$ ebenfalls gegeben und weist auf, dass die gemessenen Daten aussagekräftig sind. Daher kann davon ausgegangen werden, dass zwischen den Twitter-Sentiments zu Amazon und den Börsenkursen von Amazon eine moderate positive Beziehung besteht.

\subsection{Schlussfolgerung}

Die Hypothese 1 lautete:

- „Die Twitter-Sentiments im Zusammenhang mit einzelnen Technologieunternehmen weisen eine Korrelation mit der Entwicklung des Börsenkurses dieser Unternehmen auf."

Wie die Analysen der Spearman's Korrelationskoeffizienten zeigen, ist die Korrelation zwischen den Twitter-Sentiments eines spezifischen Technologieunternehmens und dem Börsenkurs desselben Technologieunternehmens bei diesen zwei Beispielen gegeben. Es weisen beide Analysen einen Zusammenhang auf und somit ist die Hypothese 1 angenommen.

Im Folgenden werden nun die Ergebnisse der Analysen im Kontext der Prädikation dargestellt.

\subsubsection{Granger-Analyse}

\subsection{Facebook}

In der Abb. 9.14 ist die Wahrscheinlichkeit einer Voraussage der Börsenkurse von Facebook anhand der Twitter-Sentiments zu Facebook dargestellt. Es wurde hier wieder ein

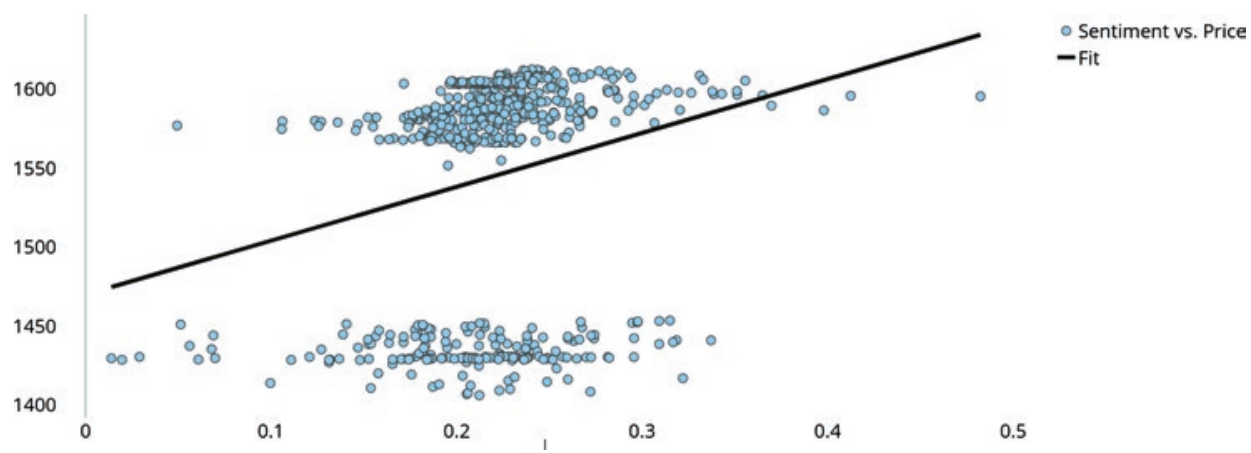

Abb. 9.13 Streudiagramm - Amazon. (Eigene Darstellung) 


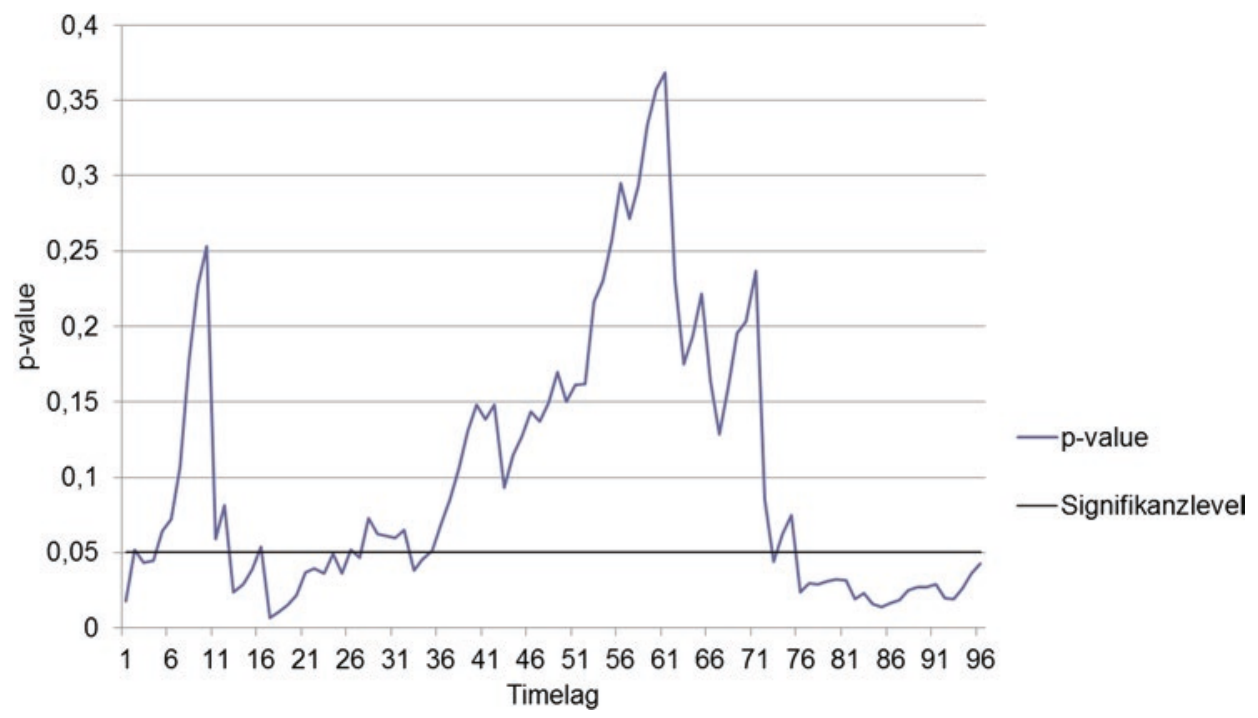

Abb. 9.14 Granger-Analyse - Facebook. (Eigene Darstellung)

Timelag von 1 bis $96 \mathrm{~h}$ analysiert. Der p-Wert in der Abb. 9.14 fällt mehrfach unter den Grenzwert von $\mathrm{p}=0,05$. Das erste Mal ist der Grenzwert bei Stunde 13 unterschritten. Bei der Stunde 28 steigt der p-Wert wieder über den Grenzwert und ab Stunde 76 liegt der p-Wert wieder unter dem geforderten Grenzwert. Somit ist anzunehmen, dass die Twitter-Sentiments zu Facebook 13 im Voraus Börsenkurs voraussagen können.

\subsection{Amazon}

Die Abb. 9.15 zeigt den Wert der Wahrscheinlichkeit, dass die Twitter-Sentiments zu Amazon die Börsenkurse von Amazon voraussagen können. Der p-Wert liegt auch bei Amazon bei der Stunde 13 erstmals unter der minimalen Grenze von $p=0,05$ bei $p=0,006$. Dies bedeutet, dass eine Wahrscheinlichkeit von mehr als $99 \%$ besteht, dass die Twitter-Sentiments zu Amazon die Börsenkurse von Amazon voraussagen. Der Börsenkurs kann folglich auch hier durch die Twitter-Daten $13 \mathrm{~h}$ im Voraus eruiert werden.

\subsection{Schlussfolgerung}

Die Hypothese 2 lautete:

- „Die Twitter-Sentiments im Zusammenhang mit einzelnen Technologieunternehmen haben eine Voraussagekraft für die Entwicklung des Börsenkurses dieser Unternehmen.“

Die Hypothese 2 kann anhand der getesteten zwei Unternehmen angenommen werden. Die Granger-Analyse bei Facebook und Amazon hat einen signifikanten Wert und ist somit erfolgreich für die Voraussage der Börsenkurse anhand von Twitter-Sentiments. 


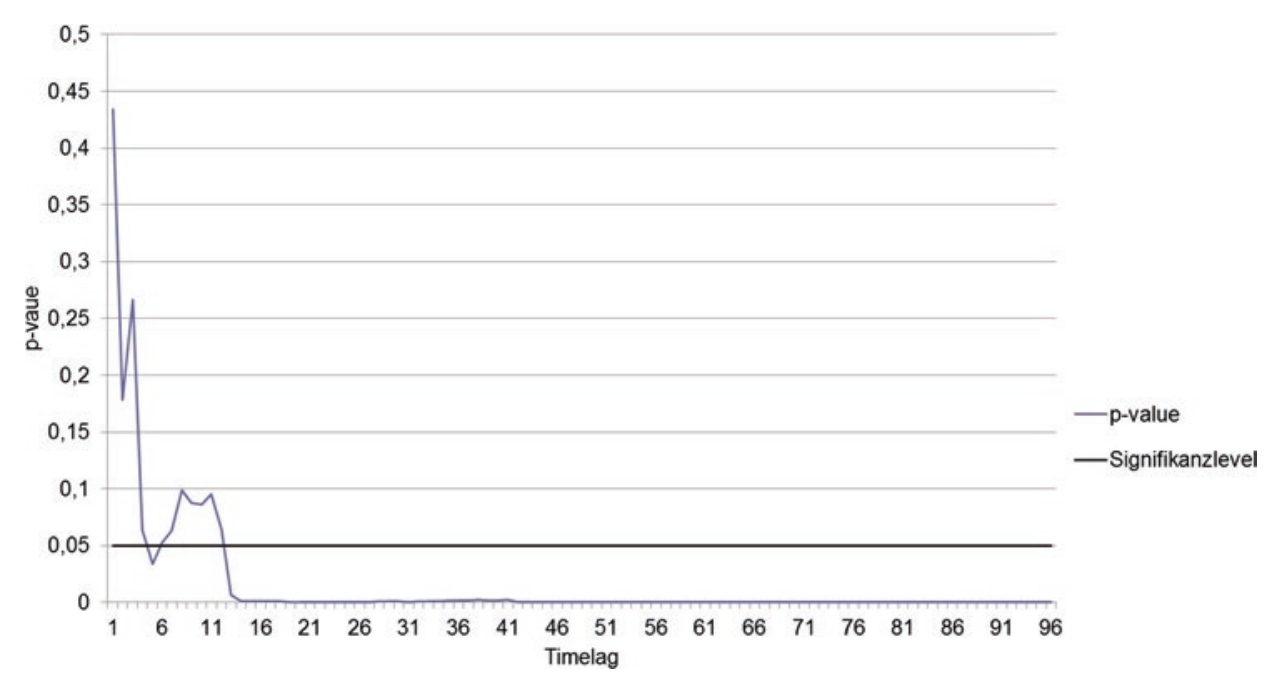

Abb. 9.15 Granger-Analyse - Amazon. (Eigene Darstellung)

Die Hypothese 2 ist somit angenommen. Bei $13 \mathrm{~h}$ Timelag weisen beide Unternehmen einen signifikanten p-Wert auf. Somit können die Daten von Twitter anhand der Sentiments die Bewegung der Börsenkurse $13 \mathrm{~h}$ im Voraus voraussagen.

\subsection{Fazit und kritische Diskussion}

Die verwendeten Daten in der vorliegenden Studie entsprechen grundsätzlich der Definition von Big Data. Somit kann sichergestellt werden, dass die Analysemethoden aus dem Bereich Big Data Analytics in dieser Studie angewendet werden können. Die Spearman's Korrelationsanalyse der Twitter-Sentiments und Börsenkurse zeigt bei beiden Unternehmen eine positive Korrelation. Auch wenn die Korrelation bei Amazon nur moderat ist. Somit ist die Hypothese 1 angenommen. Die Granger-Analyse weist ebenfalls einen signifikanten Wert aus und zeigt somit, dass die Börsenkurse durch mit Sentiment analysierte Twitter-Daten vorausgesagt werden können. Dabei ist herauszuheben, dass das Timelag bei beiden Unternehmen einen Wert von $13 \mathrm{~h}$ anzeigt. Somit zeigen die verarbeiteten Twitter-Daten ab 13 h im Voraus die Veränderung der Börsenkurse an.

\subsubsection{Methodisches Vorgehen}

Das methodische Vorgehen beinhaltete mehrere Entscheidungen, welche das Resultat möglicherweise beeinflusst haben. Ein umfassender Einflussfaktor war die Zeitdauer der Studie. Die Forschungsarbeit wurde in rund drei Monaten realisiert. Da keine 
historischen Tweets bezogen werden konnten, war der Zeitraum des Datenbezugs eingeschränkt. Ein längerer bzw. veränderter Zeitraum könnte zu Veränderungen des Ergebnisses führen. Hier sei auf eine Replikation mit einem veränderten Zeitraum für zukünftige Studien hingewiesen.

Beim Bezug der Twitter-Daten gab es ebenfalls einige Entscheidungen, welche Einfluss auf das Ergebnis genommen haben könnten. Beispielsweise wurde die Sprache der Tweets auf Englisch eingeschränkt. Diese Entscheidung war darauf zurückzuführen, dass die Sentimentanalyse anhand von bestehenden Bausteinen durchgeführt werden musste. Diese Bausteine waren sprachlich oft eingeschränkt, was dazu führte, dass für jede Sprache ein eigener Baustein hätte eingebaut werden müssen. Daher wurde die Sprache auf die gängigste Sprache von Tweets beziehungsweise auf die Unternehmenssprache der Unternehmen reduziert. Zu bedenken ist aber, dass an der Börse nicht nur Anleger der englischen Sprache teilnehmen. Somit wurde im Datensatz der Tweets und der Anleger ein unterschiedlicher Teil der Population untersucht. Diese Tatsache könnte durchaus einen Einfluss auf die Resultate haben. Ein weiterer Punkt, welcher teilweise auch mit den verschiedenen Sprachregionen zusammenhängt, ist das Problem der Zeitzonen. Da in der Granger-Analyse der Unterschied der Stunden analysiert wird, könnte eine Einbindung von anderen Zeitzonen das Resultat verändern. Neben der Einbindung von anderen Sprachen und dementsprechend auch Regionen, wäre auch ein Optimierungspotenzial beim Befehl des Datenbezugs möglich. Bei dieser Studie wurden nur Tweets berücksichtig, welche den Unternehmensnamen enthielten. Möglicherweise sind durch diese eingeschränkten Suchparameter Tweets nicht erfasst worden, welche im Kontext zum Unternehmen stehen, aber dieses nicht explizit erwähnen oder ausschreiben. Ebenfalls ist die Auswahl der Unternehmen kritisch zu hinterfragen. Obwohl diese zwei Unternehmen zu den bekanntesten Technologieunternehmen gehören, würde doch die Analyse von weiteren Unternehmen ein deutlicheres Bild der Thematik darstellen.

Neben den oben erwähnten Punkten gibt es noch weitere Überlegungen, welche in dieser Ausarbeitung unklar sind und weiter untersucht werden müssten. Beispielsweise wäre es möglich, dass neben den Tweets noch weitere Einflussfaktoren miteinbezogen werden müssten, um die Voraussage zu verbessern. Weiter ist mit dem gewählten Timelag von 1 bis 96 bei der Granger-Analyse nicht ganz klar, was nach 96 h passiert.

\subsubsection{Handlungsempfehlungen}

Für weitere Forschungsarbeiten in diesem Gebiet könnten gemäß den Erläuterungen in den vorherigen Kapiteln folgende Punkte berücksichtigt werden:

- Zeitdauer der Studie

- Sprache der Tweets und Zeitzonen

- Begriffssuche der Tweets

- Auswahl und Anzahl der analysierten Technologieunternehmen 
- Einbindung weiterer Einflussfaktoren

- Erweiterung der Timelags

- Machine Learning für Sentimentanalyse

Abschließend wird von den Autoren empfohlen, diese Studie zur Validierung zu replizieren und anhand der Handlungsempfehlungen zu optimieren.

\section{Literatur}

Alpha Vantage. (2018). Alpha Vantage - Free APIs for Realtime and Historical Financial Data, Technical Analysis, Charting, and More! Alpha Vantage. https://www.alphavantage.co/. Zugegriffen: 27. Mai 2018.

Antweiler, W., \& Frank, M. Z. (2004). Is all that talk just noise? The information content of internet stock message boards. The Journal of Finance, 59(3), 1259-1294. https://onlinelibrary. wiley.com/doi/abs/10.1111/j.1540-6261.2004.00662.x. Zugegriffen: 16. Apr. 2018.

Apache CouchDB. (2018). http://couchdb.apache.org/. Zugegriffen: 27. Mai 2018.

Baumann, J. (2018). Technische Erarbeitung Umsetzung. Expertengespräch geführt im Rahmen der Studie.

Beglinger, M. (2018). Amazon-Chef Jeff Bezos hebt ab I NZZ Neue Zürcher Zeitung. https://www. nzz.ch/gesellschaft/amazon-chef-jeff-bezos-hebt-ab-ld.1390402. Zugegriffen: 3. Juni 2018.

Bendler, J., Wagner, S., Brandt, T., \& Neumann, D. (2018). Informationsunschärfe in Big Data springerprofessional.de. https://www.springerprofessional.de/informationsunschaerfe-in-big-data/3424280?fulltextView=true. Zugegriffen: 22. Apr. 2018.

Bird, S., Klein, E., \& Loper, E. (2015). NLTK Book. http://www.nltk.org/book/. Zugegriffen: 12. Mai 2018.

Bluewin. (2018). Arbeitet Facebook an einer eigenen Kryptowährung? https://www.bluewin.ch/de/ digital/arbeitet-facebook-an-eigener-kryptowaehrung-100140.html. Zugegriffen: 3. Juni 2018.

Bollen, J., Mao, H., \& Zeng, X. (2011). Twitter mood predicts the stock market. Journal of Computational Science, 2(1), 1-8.

Brown, E. D. (2012). Will twitter make you a better investor? A look at sentiment, user reputation and their effect on the stock market. Proc. of SAIS, 7.

Ciolli, J. (2017). Stock market volatility is back - And tech stocks are taking a beating business insider. http://uk.businessinsider.com/stock-market-news-volatility-is-back-tech-stocks-takingbeating-2017-12. Zugegriffen: 16. Mai 2018.

Das, S., Martínez-Jerez, A., \& Tufano, P. (2005). eInformation: A clinical study of investor discussion and sentiment. Financial Management, 34(3), 103-137.

Das, S. R., \& Chen, M. Y. (2007). Yahoo! for Amazon: Sentiment extraction from small talk on the web. Management Science, 53(9), 1375-1388.

Davenport, T. H., \& Paulus, P. (2014). Big data @ work: Chancen erkennen, Risiken verstehen. München: Vahlen.

Dijcks, J.-P. (2013) Oracle: Big data for the enterprise. http://www.oracle.com/us/products/database/big-data-for-enterprise-519135.pdf. Zugegriffen: 31. Dez. 2018.

Dorschel, J. (2015). Praxishandbuch Big Data. Wiesbaden: Springer Fachmedien.

educalingo. (2018). Programmbibliothek. https://educalingo.com/de/dic-de/programmbibliothek. Zugegriffen: 20. Mai 2018. 
EMC und Greenplum. (2012). Ten Practical Big Data Benefits I Stories I Data Science Series Data Science Series. http://datascienceseries.com/stories/ten-practical-big-data-benefits. Zugegriffen: 16. Mai 2018.

finanzen.ch. (2018a). Facebook klemmt rund 200 Apps nach Datenskandal ab | 14.05.18 finanzen. ch. https://www.finanzen.ch/nachrichten/aktien/Facebook-klemmt-rund-200-Apps-nach-Datenskandal-ab-1024442857. Zugegriffen: 3. Juni 2018.

finanzen.ch. (2018b). Wieder Streik bei Amazon angekündigt | 16.05 .18 finanzen.ch. https:// www.finanzen.ch/nachrichten/aktien/Wieder-Streik-bei-Amazon-angekuendigt-1024660684. Zugegriffen: 3. Juni 2018.

Forbergskog, J.-O., \& Blom, C. R. (2014). Twitter and stock returns. https://brage.bibsys.no/xmlui/ handle/11250/94935. Zugegriffen: 21. Apr. 2018.

Forbes Technology Council. (2018). 12 Tech companies to watch in 2018 forbes. https:// www.forbes.com/sites/forbestechcouncil/2018/02/12/12-critical-tech-stocks-to-watch-in2018/\#fc354cc7d783. Zugegriffen: 16. Mai 2018.

Gang-Hoon, K., Trimi, S., \& Ji-Hyong, C. (2014). Big-data applications in the government sector. Communications Of The ACM, 57(3), 78-85.

Gluchowski, P., \& Chamoni, P. (Hrsg.). (2016). Analytische Informationssysteme: Business Intelligence-Technologien und-Anwendungen (5., vollständig überarbeitete Aufl.). Berlin: Springer Gabler.

Graf, L. (2018). Technische Erarbeitung und Umsetzung. Expertengespräch geführt im Rahmen der Studie.

Hutto, C. J. (2018). vaderSentiment: VADER Sentiment Analysis. https://github.com/cjhutto/vaderSentiment. Zugegriffen: 1. Juni 2018.

IBM. (2017). Big Data Analytics I IBM Analytics IBM Analytics. https://www.ibm.com/analytics/ hadoop/big-data-analytics. Zugegriffen: 31. Dez. 2017.

Inmon, W. H., \& Linstedt, D. (2015). Data Architecture: A primer for the data scientist - Big data, data warehouse and data vault. Waltham: Elsevier Inc.

Investopedia. (2018). Technology Sector Investopedia. https://www.investopedia.com/terms/t/ technology_sector.asp. Zugegriffen: 16. Mai 2018.

Kawa, L. (2018). Tech stocks fear premium just jumped to a 13-year high. In: Bloomberg.com, 28.03.2018. https://www.bloomberg.com/news/articles/2018-03-28/tech-fear-premium-jumpsto-13-year-high-on-regulatory-anxiety. Zugegriffen: 15. Mai 2018.

King, S., \& Hajnal, I. (2014). Big Data: Potential und Barrieren der Nutzung im Unternehmenskontext. Wiesbaden: Springer VS.

Koepp, Christian (2018) TwitterSearch: A Python library to easily iterate tweets found by the Twitter Search API. https://github.com/ckoepp/TwitterSearch. Zugegriffen: 27. Mai 2018.

Kroker, M. (2013). Häufigste Sprachen auf Twitter: Englisch vor Japanisch \& Spanisch - Deutsch nicht in Top-10. Kroker's Look@IT. http://blog.wiwo.de/look-at-it/2013/12/16/haufigste-sprachen-auf-twitter-englisch-vor-japanisch-spanisch-deutsch-nicht-in-top-10/. Zugegriffen: 2. Juni 2018.

Kroll, S. (2018). Facebook startet Werbeeinblendungen in Stories INTERNET WORLD Business. https://www.internetworld.de/online-marketing/facebook-marketing/facebook-startet-werbeeinblendungen-in-stories-1539373.html. Zugegriffen: 3. Juni 2018.

Liew, J. K.-S., \& Wang, G. Z. (2016). Twitter sentiment and IPO performance: A cross-sectional examination. Journal of Portfolio Management, 42(4), 129-135.

Litzel, N. (2017). Was ist NoSQL? https://www.bigdata-insider.de/was-ist-nosql-a-615718/.

Mankiw, N. G. (2004). Grundzüge der Volkswirtschaftslehre (3., überarb. Aufl.). Stuttgart: Schäffer-Poeschel. 
Manyika, J., Chui, M., Brown, B., Bughin, J., Dobbs, R., Roxburgh, C., \& Byers, A. H. (2018). Big data: The next frontier for innovation, competition, and productivity I McKinsey \& Company. https://www.mckinsey.com/business-functions/digital-mckinsey/our-insights/big-datathe-next-frontier-for-innovation. Zugegriffen: 1. Apr. 2018.

Mao, H., Counts, S., \& Bollen, J. (2011). Predicting financial markets: Comparing survey, news, twitter and search engine data. arXiv:1112.1051 [physics, q-fin].

Mao, Y., Wei, W., Wang, B., \& Liu, B. (2012). Correlating S\&P500 Stocks with Twitter Data. In: Proceedings of the First ACM International Workshop on Hot Topics on Interdisciplinary Social Networks Research. New York, NY, USA: ACM. S. 69-72.

Marr, B. (2016). Big data in practice: How 45 successful companies used big data analytics to deliver extraordinary results. Hoboken: Wiley.

Marz, N., \& Warren, J. (2015). Big Data-Principles and best practice of scalable real-time data systems. New York: Manning Publications Co.

Mayer-Schönberger, V., \& Cukier, K. (2013). Big data: A revolution that will transform how we live, work and think. London: John Murray.

Microsoft. (2018). Spezifikationen und Beschränkungen in Excel - Excel. https://support.office. com/de-de/article/Spezifikationen-und-Beschr\%C3\%A4nkungen-in-Excel-1672b34d-7043467e-8e27-269d656771c3. Zugegriffen: 2. Juni 2018.

Möller, M. (2018). Facebook geht Partnerschaft mit Thinktank ein. In: Tages-Anzeiger, 18.05.2018. https://www.tagesanzeiger.ch/digital/social-media/facebook-geht-partnerschaftmit-thinktank-ein/story/14515281. Zugegriffen: 3. Juni 2018.

Moniruzzaman, A. B. M., \& Hossain, S. A. (2013). NoSQL database: New era of databases for big data analytics - Classification, characteristics and comparison. International Journal of Database Theory and Application, 6(4), 14.

Mullainathan, S. (20. Mai 2018). When the President takes On Amazon, nobody wins. The New York Times. https://www.nytimes.com/2018/05/18/business/trump-amazon-economy.html (03.06.2018).

Neue Zürcher Zeitung. (9. Mai 2018). Facebook ordnet die Führung wichtiger Produkte neu I NZZ. Neue Zürcher Zeitung. https://www.nzz.ch/wirtschaft/facebook-ordnet-die-fuehrung-wichtiger-produkte-neu-ld.1384417. Zugegriffen: 3. Juni 2018.

Nguyen, T. H., Shirai, K., \& Velcin, J. (2015). Sentiment analysis on social media for stock movement prediction. Expert Systems with Applications, 42(24), 9603-9611.

NLTK. (2017). nltk.sentiment.vader — NLTK 3.3 documentation. https://www.nltk.org/_modules/ nltk/sentiment/vader.html. Zugegriffen: 2. Juni 2018.

Oh, C., \& Sheng, O. (2011). Investigating Predictive Power of Stock Micro Blog Sentiment in Forecasting Future Stock Price Directional Movement. Icis. Citeseer. S. 1-19.

pandas. (2018). PyPI. https://pypi.org/project/pandas/. Zugegriffen: 1. Juni 2018.

Prior, G. (2018). Amazon - Droht jetzt auch Gefahr für Banken? finanzen.net. http://www.finanzen.net/nachricht/aktien/euro-am-sonntag-meinung-amazon-droht-jetzt-auch-gefahr-fuer-banken-6139808. Zugegriffen: 3. Juni 2018.

Ranco, G., Aleksovski, D., Caldarelli, G., Grčar, M., \& Mozetic, I. (2015). The effects of twitter sentiment on stock price returns. PloS one, 10, e0138441.

Rao, T., \& Srivastava, S. (2012). Analyzing Stock Market Movements Using Twitter Sentiment Analysis. In: Proceedings of the 2012 International Conference on Advances in Social Networks Analysis and Mining (ASONAM 2012). Washington, DC, USA: IEEE Computer Society. S. 119-123.

Rauffmann, T. (2018). Amazon hebt Preise für Amazon Prime an finanzen.net. http://www.finanzen.net/nachricht/aktien/bald-auch-in-deutschland-amazon-hebt-preise-fuer-amazon-prime-an-6142378. Zugegriffen: 3. Juni 2018. 
Redaktion. (2018). 04.05.2018 - Prahlerei mit Nutzerdaten: Facebook feuert Mitarbeiter I Home24 fährt weiter Verluste ein I Instagram integriert Bezahlfunktion https://onlinehaendler-news. de/. https://www.onlinehaendler-news.de/e-commerce-tipp/31589-prahlerei-nutzerdaten-facebook-feuert-mitarbeiter-home24-verluste-instagram-bezahlfunktion.html. Zugegriffen: 3. Juni 2018.

Reed, M. (2016). A study of social network effects on the stock market. Journal of Behavioral Finance, 17(4), 342-351.

Reitz, K. (2018). Requests: HTTP for Humans - Requests 2.18.4 documentation Requests: HTTP for Humans. http://docs.python-requests.org/en/master/. Zugegriffen: 27. Mai 2018.

Sasse, R. (2018). Amazon Aktie Kursziel Finanztrends. https://www.finanztrends.info/amazonaktie-kursziel/. Zugegriffen: 3. Juni 2018.

Scherbaum, C. A. (2017). So funktioniert die Börse (3. Aufl.). Freiburg: Haufe Lexware.

Schürmann, L. (2018). Amazon Web Services: Der Aufstieg von Jeff Bezos' Gewinnmaschine manager magazin. http://www.manager-magazin.de/unternehmen/handel/amazon-web-services-der-aufstieg-von-jeff-bezos-gewinnmaschine-a-1206196.html. Zugegriffen: 3. Juni 2018.

Souza, T. T. P., Kolchyna, O., Treleaven, P. C., \& Aste, T. (2015). Twitter sentiment analysis applied to finance: A case study in the retail industry. https://arxiv.org/pdf/1507.00784.pdf.

Sprenger, T. O., \& Welpe, I. M. (2010) Tweets and trades: The information content of stock microblogs. Rochester, NY: Social Science Research Network, SSRN Scholarly Paper No. ID 1702854.

Technopedia. (2018). Software Library Techopedia.com. https://www.techopedia.com/definition/3828/software-library. Zugegriffen: 20. Mai 2018.

Tumarkin, R., \& Whitelaw, R. F. (2001). News or noise? Internet postings and stock prices. Financial Analysts Journal, 57(3), 41-51.

Twitter. (2018). Twitter developer platform developer. https://developer.twitter.com/content/developer-twitter/en.html. Zugegriffen: 27. Mai 2018.

Vu, T.-T., Chang, S., Ha, Q. T., \& Collier, N. (2012). An experiment in integrating sentiment features for tech stock prediction in Twitter. In: Proceedings of the Workshop on Information Extraction and Entity Analytics on Social Media Data. Gehalten auf der Workshop on Information Extraction and Entity Analytics on Social Media Data, Mumbai, India: The COLING 2012 Organizing Committee. S. 23-38.

Ward, J. S., \& Barker, A. (2013). Undefined by data: A survey of big data definitions. University of St Andrews, UK, pp. 1-2. https://arxiv.org/pdf/1309.5821.pdf.

Wingfield, N. (17. Mai 2018). Next up at Amazon-run whole foods: Half-priced Halibut steaks. The New York Times. https://www.nytimes.com/2018/05/16/technology/whole-foods-discountamazon-prime.html. Zugegriffen: 3. Juni 2018.

Wrobel, S., Voss, H., Köhler, J., Beyer, U., \& Auer, S. (2015). Big data, big opportunities. Informatik-Spektrum, 38(5), 370-378.

Wysocki, P. D. (1998). Cheap talk on the web: The determinants of postings on stock message boards. Rochester, NY: Social Science Research Network, SSRN Scholarly Paper. https:// papers.ssrn.com/abstract=160170. Zugegriffen: 16. Apr. 2018.

Zhang, X., Fuehres, H., \& Gloor, P. A. (2011). Predicting stock market indicators through Twitter "I hope it is not as bad as I fear". Procedia - Social and Behavioral Sciences, 26, 55-62.

Zheludev, I., Smith, R., \& Aste, T. (2014). When Can Social Media Lead Financial Markets? In: Scientific Report no. 4, pp. 1-12. https://doi.org/10.1038/srep04213. 
Baumann, Flurina (M.Sc. BA/flurina.baumann@gmail.com) Berufsbegleitendes konsekutives Masterstudium in Business Administration mit Vertiefung Business Development an der Berner Fachhochschule. Mehrjährige Tätigkeit im Bankensektor, Grosshandel und Industrie u. a. als Marketing Manager und ICT Business Analyst. Aktuell Führungsfunktion in der IT eines internationalen Industrieunternehmens.

Brunner, Nadine Belinda (M.Sc. BA/nadinebelinda.brunner@gmail.com) Berufsbegleitendes konsekutives Masterstudium in Business Administration mit Vertiefung Business Development an der Berner Fachhochschule. Mehrjährige Tätigkeit im Immobilien und Non-Profit Sektor, u. a. als Betriebswirtschaftliche Leitung. Aktuell Führung eines Standorts eines der grössten und führenden medizinischen Unternehmungen in der Schweiz.

Tokarski, Kim Oliver (Prof. Dr./kim.tokarski@bfh.ch) Professor für Entrepreneurship und Unternehmensführung und Leiter der Abteilung Weiterbildung am Departement Wirtschaft der Berner Fachhochschule. Er ist Gastprofessor an der Wirtschaftsuniversität Bukarest (ASE Bukarest), Rumänien. Seine Forschungs- und Lehrtätigkeiten liegen in den Bereichen Entrepreneurship, Social Entrepreneurship, Innovation, Digitalisierung, Strategie sowie Leadership. Er ist Autor unterschiedlicher Publikationen in den genannten Themenkontexten.

Open Access Dieses Kapitel wird unter der Creative Commons Namensnennung 4.0 International Lizenz (http://creativecommons.org/licenses/by/4.0/deed.de) veröffentlicht, welche die Nutzung, Vervielfältigung, Bearbeitung, Verbreitung und Wiedergabe in jeglichem Medium und Format erlaubt, sofern Sie den/die ursprünglichen Autor(en) und die Quelle ordnungsgemäß nennen, einen Link zur Creative Commons Lizenz beifügen und angeben, ob Änderungen vorgenommen wurden.

Die in diesem Kapitel enthaltenen Bilder und sonstiges Drittmaterial unterliegen ebenfalls der genannten Creative Commons Lizenz, sofern sich aus der Abbildungslegende nichts anderes ergibt. Sofern das betreffende Material nicht unter der genannten Creative Commons Lizenz steht und die betreffende Handlung nicht nach gesetzlichen Vorschriften erlaubt ist, ist für die oben aufgeführten Weiterverwendungen des Materials die Einwilligung des jeweiligen Rechteinhabers einzuholen.

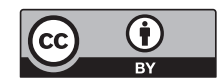




\title{
Kryptowährungen
}

\section{Eine empirisch-qualitative Analyse von Kryptowährungen gegenüber dem traditionellen Währungssystem}

\author{
Tobias Wenger und Kim Oliver Tokarski
}

\section{Zusammenfassung}

Sowohl die Finanzkrise als auch die zunehmende Digitalisierung können als zwei zentrale Haupttreiber des „,neuen“ Phänomens der Kryptowährungen angesehen werden. Kryptowährungen sollen sowohl eine Alternative zu dem traditionellen Währungssystem bieten als auch den Zahlungsverkehr vereinfachen. Das vorliegende Kapitel erarbeitet einen Vergleich von Kryptowährungen gegenüber dem traditionellen Währungssystem. Dabei liegt der Fokus auf der Kryptowährung Bitcoin und dem Wirtschaftsstandort Schweiz. Die empirischen Untersuchungen wurden mittels qualitativer Forschung durch Experteninterviews aus dem Bereich des Geldsystems und des Zahlungsverkehrs realisiert. In der Empirie wurde insbesondere der Aspekt des Vertrauens, der Resilienz wie auch der Vereinfachung von Zahlungstransaktionen analysiert.

\subsection{Einleitung, Ausgangslage und Vorgehensweise}

„Es ist und bleibt unbestritten, dass ein funktionierendes Finanzsystem von entscheidender Bedeutung für die Entwicklung und das Wachstum einer Volkswirtschaft ist“" (Sixt 2017, S. 75). Dabei zeigte insbesondere die ab dem Sommer 2007 ausgelöste Banken- und

T. Wenger $(\bowtie)$

Bern, Schweiz

E-Mail: wenger.tobias@bluemail.ch

K. O. Tokarski

Departement Wirtschaft, Berner Fachhochschule, Bern, Schweiz

E-Mail: kim.tokarski@bfh.ch

J. Schellinger et al. (Hrsg.), Digitale Transformation und Unternehmensführung, https://doi.org/10.1007/978-3-658-26960-9_10 
Finanzkrise gewisse Probleme dieses Systems auf (Deutsche Bundesbank 2017, S. 107). Die Ursachen, Faktoren und Verursacher dieser Krise sind vielfältig und konnten bis heute nicht abschließend eruiert werden (Stijin und Ayhan 2013, S. 34 ff.; The Economist 2013). Gleichwohl kam die Financial Crisis Inquiry Comission zum Ergebnis, dass die Krise vermeidbar gewesen wäre, denn die Krise sei durch „menschliche Taten und Tatenlosigkeit“ verursacht worden. Die Ursache sei ein „enormes Versagen“ von Regierung und Finanzaufsicht sowie ein ,rücksichtsloses Risikomanagement“ der Geldindustrie gewesen (Financial Crisis Inquiry Commission 2011, S. xvii-3). Um derartige Krisen zukünftig zu vermeiden, wurden unterschiedliche Regulierungsmaßnahmen, wie bspw. Basel III, eingeführt. Trotz dieser Reformen wird weiterhin bemängelt, dass das heutige System genauso instabil sei wie vor der Krise (Admati und Hellwig 2014, S. XII). Es wird argumentiert, dass die wiederkehrende Instabilität nicht behoben werden könne, solange nicht grundlegend etwas an der Ausgestaltung des Geldsystems verändert wird (Benes und Kumhof 2012; Fisher 2007; Huber 2013; Huber und Robertson 2008). Das traditionelle Geldsystem zeichnet sich dabei durch dessen zentralistische Strukturen aus, in denen die Zentralbanken, in der Schweiz ist dies die Schweizerische Nationalbank (SNB), und Geschäftsbanken wichtige Funktionen einnehmen (Deutsche Bundesbank 2017, S. 87, 125; The Corner 2017). Diese Abhängigkeit benötigt ein hohes Maß an Vertrauen der beteiligten Akteure des (Banken-, Finanz-, Währungs- bzw. Geld-)Systems (Tonkiss 2009, S. 1). Dieses Vertrauen wurde auch schon vor der Finanzkrise oftmals auf die Probe gestellt. So wurden zwischen 1970 und 2007 weltweit insgesamt 147 Bankenkrisen, 218 Währungskrisen und 66 Staatsverschuldungskrisen gezählt (Laeven und Valencia 2012, S. 3-13).

Vertrauen in das System und seine Akteure, u. a. Nationalbank, Geschäftsbanken, Staat etc., ist dabei ein essenzieller Faktor für dessen fortwährenden Erhalt und Weiterentwicklung. Ein mangelndes Vertrauen führt zu instabilen Systemen, aber durchaus auch zu einer Suche nach und Entwicklung von Alternativen. Eine Alternative sind dabei bspw. Kryptowährungen. Die Wichtigkeit des Vertrauens in das System, wird durch den unter dem Pseudonym Satoshi Nakamoto bekannten „Erfinder“ der ersten Kryptowährung Bitcoin veranschaulicht. Er steht den staatlichen Währungen und dem gängigen Vertrauenskonzept im Geldsystem kritisch gegenüber, wie das nachfolgende Zitat verdeutlicht:

The root problem with conventional currency is all the trust that's required to make it work. The central bank must be trusted not to debase the currency, but the history of fiat currencies is full of breaches of that trust. Banks must be trusted to hold our money and transfer it electronically, but they lend it out in waves of credit bubbles with barely a fraction in reserve (P2P-Foundation 2018).

Viele Kryptowährungsenthusiasten sehen in der Veröffentlichung seines Whitepapers mit dem Titel: Bitcoin: A Peer-to-Peer Electronic Cash System (Nakamoto 2008) Ende Oktober 2008, mitten in der Krise, keinen Zufall (Thiele und Diehl 2017, S. 1). Das Whitepaper verdeutlicht, wie durch die Verknüpfung von Informatik und Kryptografie das bisherige Vertrauen in die zentralen Instanzen bzw. Akteure (bspw. Nationalbanken, 
Geschäftsbanken etc.) durch einen Algorithmus und somit in die Gesetzte der Mathematik ersetzt werden kann.

Eine theoretische Aufarbeitung des Untersuchungskontextes erfolgt in Abschn. 10.2. Im Abschn. 10.4.1 sowie 10.4.2 werden die Detailergebnisse der empirisch-qualitativen Analyse zum Themenbereich des Geldsystems sowie des Zahlungsverkehrs der beiden Währungssysteme dargestellt.

Was für eine Rolle das Vertrauen im traditionellen Geldsystem gegenüber dem Kryptowährungssystem spielt, soll in der vorliegenden Ausarbeitung dieses Kapitels unter der Hypothese 1 im Abschn. 10.4.3 genauer betrachtet werden. Zudem soll in der Hypothese 2 im Abschn. 10.4.4 genauer untersucht werden, ob das Kryptowährungssystem gegenüber dem traditionellen Geldsystem eine höhere Resilienz aufweist.

Nebst der Kritik an der Ausgestaltung des traditionellen Geldsystems bildet die zunehmende Digitalisierung einen zweiten, zentralen Treiber des Aufkommens von Kryptowährungen. Der technologische Fortschritt hat dazu geführt, das Fintech-Unternehmen im Bereich des Zahlungsverkehrs innovative Zahlungsinstrumente als Alternative zu den klassischen Zahlungslösungen bieten (Robleh Ali et al. 2014, S. 4). Durch Innovationen, wie bspw. mobiles Bezahlen und Peer-to-Peer-Zahlungslösungen (P2P) hat sich ein verstärkter Wettbewerb unter den Zahlungsverkehrsanbietern entwickelt (Deloitte 2017, S. 27 ff.). Zu diesen Innovationen gehören auch die Kryptowährungen (Robleh Ali et al. 2014, S. 3). Welche Kryptowährungen gegenüber den traditionellen Zahlungsinstrumenten einen Mehrwert bieten und somit die Umsetzung von Zahlungstransaktionen vereinfachen, soll durch die Hypothese 3 im Abschn. 10.4.5 genauer betrachtet werden. Die Ausarbeitung schließt im Abschn. 10.5 mit einem Fazit zum Untersuchungskontext.

\subsection{Theorie}

\subsubsection{Kurzdarstellung und Vergleich der Geldsysteme}

In der Literatur besteht keine allgemeingültige Definition des Begriffs der Währung und des Geldes. Aus diesem Grund werden einige ausgewählte Definitionen beispielhaft in Tab. 10.1 angeführt.

Bei der Betrachtung der Definition einer Währung fällt auf, dass der Begriff eng an den Begriff des Geldes geknüpft ist und Währungen ein zeitlich sowie geografisch beschränktes System bilden. Eine Währung beschreibt also die gesetzlichen Rahmenbedingungen, in denen das Geldwesen definiert ist. Geld wird hingegen als generell akzeptierte Einheit bezeichnet, welche als Zahlungsmittel verwendet wird und somit einen gewissen Wert aufweist (vgl. Solit Gruppe 2018). Die European Central Bank (ECB) nimmt mit der Geld-Matrix in Abb. 10.1 die Abgrenzungen von reguliertem Geld vor. 
Tab. 10.1 Definitionen Währung und Geld. (Eigene Darstellung)

\begin{tabular}{l|l}
\hline Währung & Geld \\
\hline $\begin{array}{l}\text { „A system of money in general use in a parti- } \\
\text { cular country. “ (Oxford Dictionaries 2018) }\end{array}$ & $\begin{array}{l}\text { „A generally accepted medium for the } \\
\text { exchange of goods and services, for measuring } \\
\text { value, or for making payments“ (Financial } \\
\text { Dictionary 2018) }\end{array}$ \\
$\begin{array}{l}\text { "The money that is used in a particular } \\
\text { country at a particular time. "(Cambridge } \\
\text { Dictionary 2018) }\end{array}$ & $\begin{array}{l}\text { Z... offizielles und allgemein gebräuchliches } \\
\text { Zahlungsmittel in der Währung, die im Hoheits- } \\
\text { gebiet eines Staates gilt.“ (Huber 2013, S. 11) }\end{array}$ \\
\hline
\end{tabular}

\begin{tabular}{|l|c|c|c|}
\hline \multicolumn{3}{|c|}{ Geld-Matrix } \\
\hline \multirow{3}{*}{ Rechtsstatus } & \multirow{3}{*}{ Reguliert } & $\begin{array}{c}\text { Banknoten und } \\
\text { Münzen }\end{array}$ & E-Geld \\
\cline { 3 - 4 } & & Physisch & Digital \\
\cline { 3 - 4 } & \multicolumn{2}{|c|}{ Geldformat } \\
\hline
\end{tabular}

Abb. 10.1 Regulierte Geld-Matrix. (Eigene Darstellung in Anlehnung an European Central Bank 2012, S. 11)

Reguliertes Geld wird innerhalb der Geld-Matrix nach staatlich ausgegebenen Banknoten und Münzen sowie Buchgeld und E-Geld unterschieden. E-Geld ist die elektronische Repräsentation einer offiziellen Währung, welche für den elektronischen Zahlungsverkehr in offizieller Währung eingesetzt wird. An dieser Stelle ist zu betonen, dass E-Geld nicht mit Kryptowährungen zu verwechseln ist. Je nach Land ist die Einordnung von Kryptowährungen als reguliertes oder unreguliertes Geld unterschiedlich. Für eine Systematisierung kann dabei die zuvor dargestellte Geld-Matrix als Ausgangsgrundlage einer Einordnung bzw. Erweiterung dienen. Exemplarisch ist dies in Abb. 10.2 vorgenommen worden.

Um eine klare Unterscheidung zwischen Banknoten und Münzen sowie des Buchgelds vorzunehmen, ist es sinnvoll, die Geldmengendefinition der SNB (Schweizerische Nationalbank) der Tab. 10.2 zu betrachten.

Zwischen den oben aufgeführten Geldmengen bestehen rechtliche Unterschiede. In der Schweiz gelten die nachfolgenden Zahlungsmittel als gesetzliche Zahlungsmittel:

a) ,die vom Bund ausgegebenen Münzen;

b) die von der Schweizerischen Nationalbank ausgegebenen Banknoten;

c) auf Franken lautende Sichtguthaben bei der Schweizerischen Nationalbank“ (WZG, Art. 2). 


\begin{tabular}{|c|c|c|c|}
\hline \multicolumn{4}{|c|}{ Geld-Matrix } \\
\hline \multirow{4}{*}{ Rechtsstatus } & Unreguliert & $\begin{array}{l}\text { bestimmte Arten von } \\
\text { lokalen Währungen }\end{array}$ & Kryptowährungen \\
\hline & \multirow{3}{*}{ Reguliert } & \multirow{2}{*}{$\begin{array}{l}\text { Banknoten und } \\
\text { Münzen }\end{array}$} & E-Geld \\
\hline & & & Buchgeld \\
\hline & & Physisch & Digital \\
\hline & & \multicolumn{2}{|c|}{ Geldformat } \\
\hline
\end{tabular}

Abb. 10.2 Erweiterte Geld-Matrix. (Eigene Darstellung in Anlehnung an European Central Bank 2012, S. 11)

Tab. 10.2 Geldmengendefinition der SNB. (Eigene Darstellung in Anlehnung an Schweizerische Nationalbank 2016, S. 1)

\begin{tabular}{|c|c|c|}
\hline M0: & $\begin{array}{l}\text { - Notenumlauf im Publikum } \\
\text { - Zahlungsreserven der Geschäftsbanken } \\
\text { - Barreserven } \\
\text { - Unbare Überschussreserven } \\
\text { - Mindestreserven }\end{array}$ & Zentralbankgeld \\
\hline M1: & $\begin{array}{l}\text { - Bargeldumlauf im Publikum } \\
\text { - Buchgeld } \\
\text {-Sichteinlagen } \\
\text {-Transaktionskonten }\end{array}$ & \multirow[t]{3}{*}{ Publikumsgeld } \\
\hline M2: & $\begin{array}{l}\text { - Geldmenge M1 } \\
\text { - Spareinlagen }\end{array}$ & \\
\hline M3: & $\begin{array}{l}\text { - Geldmenge M2 } \\
\text { - Termineinlagen }\end{array}$ & \\
\hline
\end{tabular}

Hierbei zeigt sich, dass das Buchgeld nicht erwähnt wird und somit nicht zu den gesetzlichen Zahlungsmitteln gezählt wird. Die gesetzlichen Zahlungsmittel beschränken sich lediglich auf das Zentralbankgeld bzw. die Geldmenge M0, welche direkt von der SNB an die Geschäftsbanken herausgegeben werden und die von der eidgenössischen Münzstätte Swissmint, im Auftrag des Staates, geprägten Münzen (vgl. Huber und Robertson 2008, S. 75).

Im traditionellen Geldsystem besteht der Hauptauftrag einer Nationalbank, im vorliegenden Untersuchungskontext der SNB, darin, die Geld- und Währungspolitik im Gesamtinteresse eines Landes zu betreiben. Demgegenüber sind hingegen die Geschäftsbanken kommerziell und privatwirtschaftlich geführte Unternehmen (Huber 2013, S. 70), (NBG, Art. 5). Nebst dieser zentralistischen Ausrichtung wird das vorherrschende Zweistufen-Geldsystem als fraktionales Reservesystem mit multipler Geldschöpfung 
bezeichnet (Huber und Robertson 2008, S. 7-8). Durch diese Systemausgestaltung ist es den Geschäftsbanken durch Kreditvergabe möglich, Buchgeld zu erschaffen bzw. durch Kreditrückzahlung Buchgeld zu vernichten (Deutsche Bundesbank 2017, S. 78; McLeay et al. 2014, S. 16).

Die Geschäftsbanken betreiben aufgrund ihrer profitorientierten Ausrichtung eine prozyklische Buchgeldschöpfung. Das heißt in guten Wirtschaftszeiten werden mehr Kredite vergeben, während in wirtschaftlich schlechten Zeiten die Bereitschaft zur Kreditvergabe sinkt (Benes und Kumhof 2012, S. 5; Huber 2013, S. 70). Es wird kritisiert, dass durch dieses Verhalten die bereits vorhandenen Marktübertreibungen noch akzentuiert werden. Einerseits werden in wirtschaftlich guten Zeiten die Preisinflation und die Spekulationsblasen verstärkt. Anderseits wird in wirtschaftlich schlechten Zeiten die Deflationen und die Gefahr einer Kreditklemme forciert (Blanchard und Illing 2009, S. 659; Huber und Robertson 2008, S. 70-76).

In der Schweiz ist die SNB bspw. die einzige Instanz, welche das Recht besitzt, gesetzliche Zahlungsmittel zu erstellen (Huber und Robertson 2008, S. 75). Die Erhöhung der Geldmenge ist dabei abhängig von der Geldpolitik des Landes. Hingegen ist es den Geschäftsbanken verboten, selbst Banknoten und Münzen herzustellen. Nichtsdestotrotz erschaffen die Geschäftsbanken im traditionellen Geldsystem die Mehrheit des Geldes. Dies wird dadurch ermöglicht, das Buchgeld per Gesetz nicht als staatliches Geld gilt. Buchgeld stellt lediglich ein Anrecht auf die Auszahlung von Bargeld dar (WZG, Art. 2), (Siedenbiedel 2012, S. 1-2; Huber und Robertson 2008, S. 10). Die Geschäftsbanken sind jedoch verpflichtet das Buchgeld vollumfänglich und jederzeit in Bargeld umzutauschen (Deutsche Bundesbank 2017, S. 57; Siedenbiedel 2012, S. 2). Da in der Schweiz 88 \% der Geldmenge in Form von Buchgeld und lediglich $12 \%$ aus Zentralbankgeld besteht, kann diesem Versprechen nicht vollumfänglich nachgekommen werden (Schweizerische Nationalbank 2015, S. 26). Das bedeutet, dass die Geschäftsbanken dem Publikum rund zehnmal mehr Geld versprechen, als dass sie eigentlich besitzen. Daraus zeigt sich, dass die Geschäftsbanken im traditionellen Geldsystem ein gewisses Risiko eingehen. Die Geschäftsbanken spekulieren darauf, dass es zu keinem Vertrauensverlust des Publikums, bspw. in Form von Bank Runs, dem plötzlichen Ansturm der Kunden auf Banken mit dem Ziel ihre Einlagen abzuheben, kommt und stets nur wenige Personen gleichzeitig ihr Buchgeld in Bargeld umtauschen wollen. Derartige Bank Runs können aufgrund der starken Geschäftsbankenvernetzung im schlimmsten Fall zum Zusammenbruch des Finanzsystems führen. Dieser Umstand macht auch verständlich, warum der ehemalige Vizepräsident der SNB, Jean-Pierre Danthine die Meinung vertritt, dass das heutige Geldsystem einzig auf Vertrauen basiert (Danthine 2014, S. 4).

In der Literatur werden die nachfolgenden Hauptprobleme am traditionellen Geldsystem genannt, welche in Tab. 10.3 dargestellt sind (vgl. Benes und Kumhof 2012; Fisher 2007; Huber 2013; Pfluger 2016).

Eine neue, innovative Alternative zum traditionellen Geldsystem bildet das Geldsystem der Kryptowährungen. Insbesondere die Dezentralität spielt in dieser 
Tab. 10.3 Auswahl an Problemen des traditionellen Geldsystems. (Eigene Darstellung)

\begin{tabular}{l|l}
\hline $\begin{array}{l}\text { Kontrollverlust der Zentralbank über die Geld- } \\
\text { menge }\end{array}$ & $\begin{array}{l}\text { Zunehmende öffentliche und private Ver- } \\
\text { schuldung }\end{array}$ \\
\hline Prozyklische Buchgeldschöpfung & $\begin{array}{l}\text { Erzielung von Geldschöpfungsgewinnen durch } \\
\text { die Geschäftsbanken }\end{array}$ \\
\hline Gefahr von Bank Runs & $\begin{array}{l}\text { Geld gelangt als Schuld in den Wirtschafts- } \\
\text { kreislauf }\end{array}$ \\
\hline
\end{tabular}

Aufgrund des Umfangs und der Relevanz für diese Forschungsarbeit sowie des Umstands, dass sich einige dieser Probleme mit anderen genannten Problemen überschneiden, werden in der Ausarbeitung lediglich die in der linken Spalte der Tab. 10.3 genannten Probleme vertieft thematisiert

Systemausgestaltung eine wichtige Rolle und bildet einen entsprechenden Gegenpol zum traditionellen Geldsystem (Blocher et al. 2017, S. 369; Sixt 2017, S. 62-63; Lee 2015, S. 8). Gleichwohl gibt es zwischen den beiden Systemen auch Gemeinsamkeiten, denn beide Währungen sind im Idealfall u. a. teilbar, beweglich, schwer zu kopieren (fälschungssicher) und sie sind ein knappes Gut. Am Beispiel von Bitcoin äußert sich dies insbesondere darin, dass die Geldmenge nicht wie im traditionellen Geldsystem beliebig durch eine zentrale Instanz erhöht werden kann, sondern dass eine vordefinierte Geldmengenobergrenze von 21 Mio. Bitcoins besteht (Blocher et al. 2017, S. 365; Sixt 2017, S. 108). Dabei wird die traditionelle Geldpolitik durch einen Algorithmus im Kryptowährungscode ersetzt. Durch diese Funktionsweise kann die Gefahr einer Inflationierung der Bitcoins ausgeschlossen werden (Sixt 2017 S. 31, 108). Jedoch stellt auch die im Kryptowährungssystem vorhanden Deflation eine Gefahr dar. Dadurch, dass ein Großteil der Kryptowährungen aufbewahrt bzw. gespeichert werden, wird die Umlaufgeschwindigkeit des Gelds beeinträchtig (Cointelegraph 2014). Dies führt zu einem tiefen Liquiditätsgrad des Bitcoins und zu einem ,thin market“, was wiederum dazu führt, dass bereits kleine Änderungen des Angebots oder der Nachfrage zu einer erhöhten Kursvolatilität führen (Blockchain 2018b; Blocher et al. 2017, S. 365; Sixt 2017, S. 110). Die Funktionsweise einer Geldmengenobergrenze, der Algorithmus und die daraus folgenden Deflation und Kursvolatilität schränkt die Nutzbarkeit der Kryptowährung als Recheneinheit und Zahlungsmittel ein (Fortune 2017; Blocher et al. 2017, S. 365; Sixt 2017, S. 110).

Wie auch im traditionellen Geldsystem bestehen auch im Kryptowährungssystem profitorientierte Parteien, die sogenannten Miner (Brühl 2017, S. 137; BTC-Echo 2018; Koenig 2017, S. 84-85; Sixt 2017, S. 31). Für die zur Verfügungstellung der Rechenleistung im Sinne der Verarbeitung von Transaktionen, Absicherung und Synchronisierung von Blöcken bzw. Hashes auf der Blockchain bzw. dem Bitcoin-Netzwerk erhalten die Miner einen Block-Reward in Form von Bitcoins (Bitcoin Magazine 2018). Aufgrund der Profitmaximierung haben sich Mining Pools gebildet (Hileman und Rauchs 2017, S. 89). Dies führt dazu, dass aktuell mindestens $99 \%$ der Blöcke auf der Bitcoin-Blockchain durch Mining Pools erstellt werden (Blocher et al. 2017, 
S. 368). Die fünf größten Pools erzeugen zusammen etwa $70 \%$ der Blöcke. Vier von diesen fünf Mining Pools operieren von China aus (Blockchain 2018a). Dieses Verhalten reduziert einerseits die angestrebte Dezentralität, andererseits besteht die Gefahr einer 51-prozentigen Attacke (hierbei handelt es sich um einen Angriff auf das BitcoinNetzwerk, welcher durch die Kontrolle der Mehrheit der Mining-Power durch eine Partei realisiert werden könnte). (Bonneau et al. 2015, S. 104 ff.; Blocher et al. 2017, S. 367; Sixt 2017, S. 103).

Nebst den Minern gehören auch weitere Parteien zum Kryptowährungssystem, namentlich die Kryptowährungsdienstleister, welche Dienstleistungen in Form von Wallets (Aufbewahrung), Exchanges (Transfer/Handel/Umwandlung in andere Währungen) und Payment Solutions (Zahlung) zur Verfügung stellen (Brühl 2017, S. 138). Das bedeutet, dass wie im traditionellen Geldsystem auch im Kryptowährungssystem Intermediäre existieren. Diese Unternehmen können nicht nur potenzielle Angriffsziele in Form von Hackerangriffen werden, sondern auch selbst betrügerische Absichten in Form von Ponzi Schemes (Betrugssystem auf Basis der Generierung von Gewinnen für alte Investoren durch die Gewinnung neuer Investoren; vergleichbar bspw. mit dem Pyramidensystem des Betrugs) verfolgen (Blocher et al. 2017, S. 367; Sixt 2017, S. 92). Daher ist die von Satoshi Nakamoto gemachte Aussage, dass Kryptowährungen ohne Vertrauen auskommen können, kritisch zu hinterfragen (P2P-Foundation 2018).

Die aus der Literatur eruierten, ausgewählten Probleme der beiden Geldsysteme werden in der Abb. 10.3 zusammengefasst. Hierbei werden die Probleme den jeweiligen Ursachen und einem Oberbegriff zugeordnet.

Obwohl je nach System unterschiedliche Probleme und Ursachen existieren, bestehen dennoch gewisse Parallelen. Die Probleme der beiden Geldsysteme lassen sich auf die Funktionsweise, die finanziellen Anreize und das Vertrauenskonzept zurückführen.

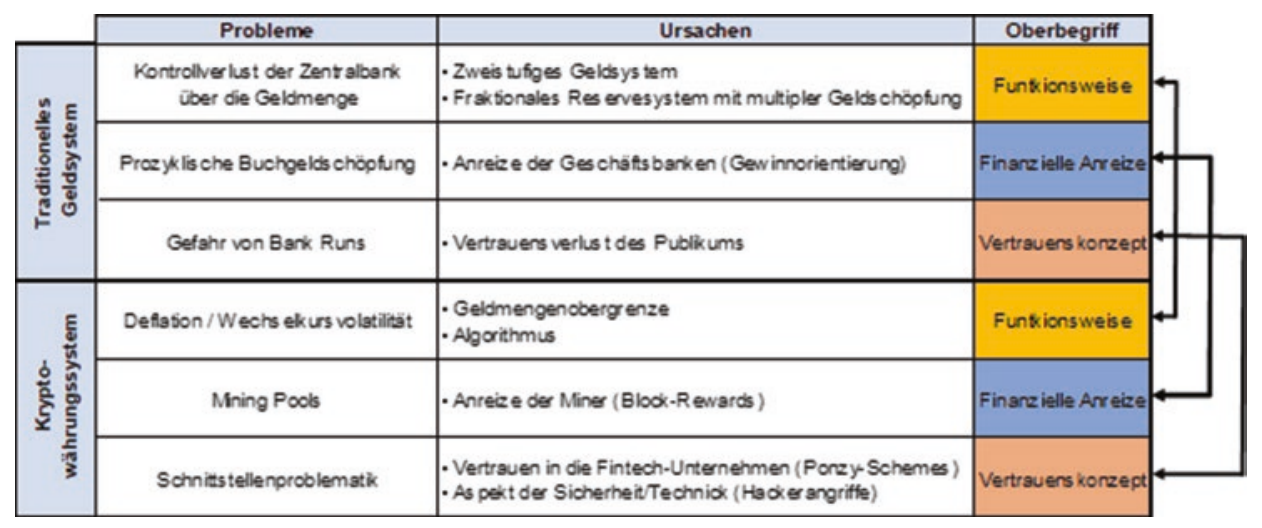

Abb. 10.3 Probleme und Ursachen der beiden Geldsysteme. (Eigene Darstellung) 


\subsubsection{Kurzdarstellung und Vergleich des Zahlungsverkehrs}

Dem Zahlungssender und dem Zahlungsempfänger stehen in der Schweiz zahlreiche unterschiedliche Zahlungsinstrumente zur Verfügung (Berner Kantonalbank 2018; Bundesamts für Kommunikation 2016, S. 14-15). Diese Vielfalt ist insbesondere auf den technologischen Fortschritt zurückzuführen (Swissbanking 2016). Einerseits wurden die klassischen Zahlungsinstrumente durch neue Zahlungsfunktionen erweitert, z. B. Debitund Kreditkarten mit kontaktlosem Bezahlen, Überweisungen, welche mittels E-Banking übermittelt werden können etc., andererseits sind auch neue Zahlungsanbieter wie bspw. Twint (www.twint.ch) und Apple Pay entstanden, welche mit neuen Zahlungsfunktionen, wie mobiles Bezahlen per Handy und P2P-Zahlungslösungen eine Alternative zu den klassischen Zahlungsanbietern darstellen (Hungerland et al. 2017, S. 23; Robleh Ali et al. 2014, S. 4).

Die Abb. 10.4 soll einen Gesamtüberblick über die in der Ausarbeitung behandelten Zahlungsinstrumente mit Einsatzgebiet in der Schweiz aufzeigen. Die Zahlungsinstrumente wurden aus Sicht des Zahlungssenders nach elf Kriterien im Vergleich zum Bargeld bewertet. Während die Farbe Grün bedeutet, dass das Kriterium erfüllt ist, bedeutet die Farbe Gelb, dass das Kriterium teilweise bzw. unter gewissen Bedingungen/ Einschränkungen geben ist. Rot bedeutet, dass das Kriterium nicht erfüllt wird. Auf eine entsprechende Feingliederung wie eine detaillierte Gebührenanalyse oder Geschwindigkeitsvergleich etc. wird an dieser Stelle verzichtet. Gleichermaßen wurden Auslandzahlungstransaktionen nicht berücksichtigt.

Die Abb. 10.4 zeigt, dass jedes Zahlungsinstrument über entsprechende Vor- und Nachteile verfügt. Grundsätzlich kann aber der Zahlungsverkehr innerhalb der Schweiz aufgrund eines gut ausgebauten SIC-Zahlungssystems (Swiss Interbank Clearing) sowie den diversen Zahlungsinstrumenten als relativ effizient eingestuft werden (SIX Interbank Clearing 2016, S. 3, 2018; SIX Group AG, 2018, S. 5). Demgegenüber bestehen jedoch gewisse Probleme bzw. Limitationen, was den internationalen Zahlungsverkehr anbelangt. Aufgrund von unterschiedlichen lokalen Gesetzen und Praktiken

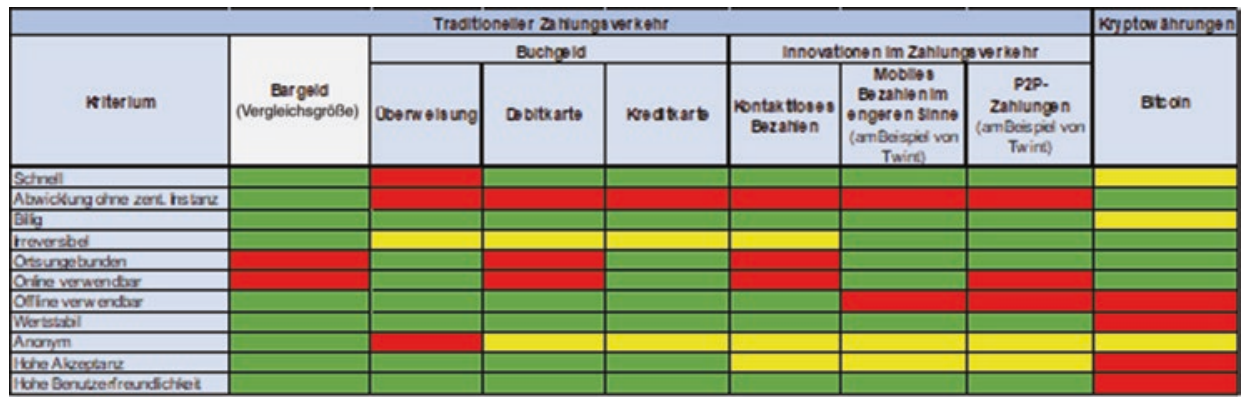

Abb. 10.4 Vergleich der Zahlungsinstrumente. (Eigene Darstellung) 
innerhalb des Banken- und Finanzsystems sowie eines fehlenden globalen Standards, können Überweisungsaufträge in Entwicklungsländern bis zu 20 Tage dauern und erhöhte Kosten verursachen (Blocher et al. 2017, S. 7; Goldman Sachs 2014, S. 19; Park 2006). Nebst dieser vergleichsweise ineffizienten internationalen Zahlungsabwicklung verfügen weltweit rund $38 \%$ der erwachsenen Bevölkerung über kein Geschäftsbankkonto (Demirguc-Kunt et al. 2014, S. 5). Als Hauptbarrieren werden Gründe, wie bspw. mangelndes Wissen über Finanzprodukte, Ausbaupotenzial von rechtlichen Rahmenbedingungen, mangelnde Förderung von neuen Kreditvergabetechnologien, hohe Kontoführungsgebühren, weite Entfernung zu einem Finanzinstitut und mangelndes Vertrauen in die Geschäftsbanken, genannt (Weltbank 2014b, S. 3-5).

Doch auch die Kryptowährungen verfügen über Probleme bzw. Limitationen. Die Hauptprobleme bestehen insbesondere in einem Zielkonflikt zwischen Skalierbarkeit und Dezentralität (Altcointoday 2018; Sixt 2017, S. 42, 96-99). Zudem gestaltet sich eine dezentrale Metakonsensfindung als schwierig (Koenig 2017, S. 89 ff.). Auch die (aktuell) tiefe Benutzerfreundlichkeit bildet ein Hemmnis, Kryptowährungen als Zahlungsmittel zu verwenden (CCN 2015; Fortune 2017; Koenig 2017, S. 60-61).

\subsection{Methodik}

Das Ziel der vorliegenden empirisch-qualitativen Analyse liegt darin, einen Vergleich zwischen Kryptowährungen und dem traditionellen Währungssystem vorzunehmen. Um dieses Ziel erreichen zu können, wurde das Währungssystem in zwei Bestandteile gegliedert. Dabei handelt es sich um das Themenfeld des Geldsystems und des Zahlungsverkehrs. Für die jeweiligen Themenfelder wurden Hypothesen ausgearbeitet. Die Abb. 10.5 visualisiert den Zusammenhang der Bestandteile des Währungssystems, die dazugehörigen Hypothesen und das Ziel der vorliegenden Ausarbeitung.

Üblicherweise werden Forschungsthesen als Grundlage von qualitativen Forschungsmodellen gebildet (Mayer 2013, S. 28). Dennoch ist es durchaus legitim, auch Hypothesen für einen qualitativen Forschungszweck aufzustellen (Mayring 2015, S. 25). Aufgrund der Neuartigkeit von Kryptowährungen und dem Fokus auf den Wirtschaftsstandort Schweiz wurde in dieser Forschungsarbeit eine empirische Studie mittels einer Expertenbefragung durchgeführt (Gardini 2007, S. 17-18). Die zu erschließenden Forschungsinhalte werden durch eine qualitative Methode ermittelt. Im Zentrum steht das Wissen eines Experten in einem bestimmten Themenbereich (Bohnsack et al. 2003, S. 57). Für die Auswahl der Erkenntnissubjekte wurden Exponenten aus den zwei für diese Forschungsarbeit relevanten Bestandteilen, namentlich dem Geldsystem und dem Zahlungsverkehr ausgewählt. Die Experten sind in relevanten hierarchisch-inhaltlichen Positionen angesiedelt und verfügen über ein umfassendes, tiefes Wissen der Materie und erfüllen den Status bzw. Anforderungen an einen Experten, wie in Abb. 10.6 dargestellt. 


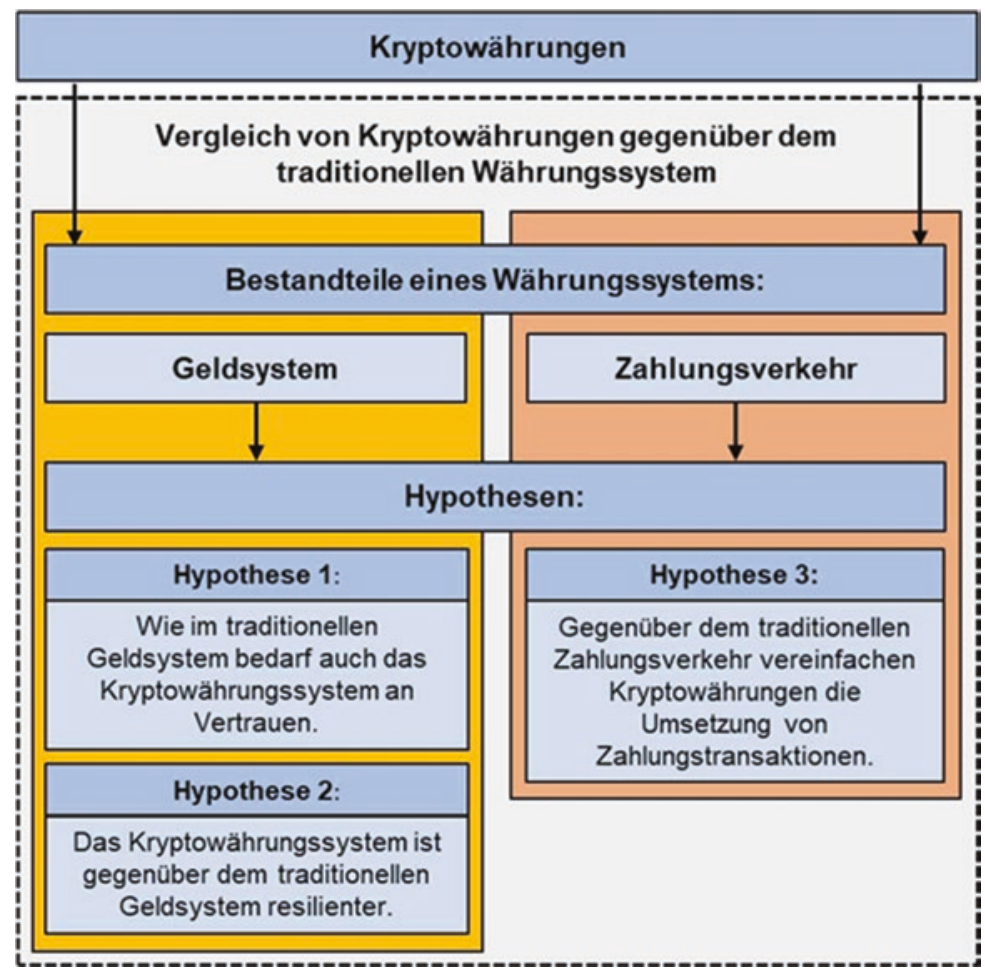

Abb. 10.5 Bestandteile, Hypothesen und Ziele der Forschungsarbeit. (Eigene Darstellung)

\begin{tabular}{|c|c|c|c|c|c|c|c|}
\hline \multirow{2}{*}{ Nr. } & \multirow{2}{*}{ Interviewpartner } & \multirow{2}{*}{ Unternehmen } & \multirow{2}{*}{ Funktion } & \multicolumn{2}{|c|}{ Trad. Wahrungssystem } & \multicolumn{2}{|c|}{ Kryptowährungen } \\
\hline & & & & Geldsystem & Zahlungsverkehr & Geldsystem & Zahlunosverkehr \\
\hline 1 & Thomas Ankenbrand & $\begin{array}{l}\text { Hochs chule Luzern } \\
\text { AVACO AG } \\
\text { Soranus AG } \\
\end{array}$ & $\begin{array}{l}\text { Leiter Competence Center Investments } \\
\text { Partner } \\
\text { VR-Präsident }\end{array}$ & & & & \\
\hline 2 & Jan Frecè & Berner Fachhochschule & Wissenschaftlicher Mitarbeiter & & & & \\
\hline 3 & Anonym & Finanzdienstleister & Head of Domain Architecture & & & & \\
\hline 4 & René Hûsler & $\begin{array}{l}\text { Hochschule Luzern } \\
\text { Crypto Valley Association }\end{array}$ & $\begin{array}{l}\text { Direltor Departement Informatik } \\
\text { Mitgründer }\end{array}$ & & & & \\
\hline 5 & Christian Jaag & $\begin{array}{l}\text { Swiss Economics } \\
\text { Cryptecon }\end{array}$ & $\begin{array}{l}\text { Managing Partner } \\
\text { Gründer und Direitor }\end{array}$ & & & & \\
\hline 6 & Thierry Kneissler & Twint & CEO & & & & \\
\hline 7 & Tobias Lehmann & SIX Groupe & Future Scenarios Lead & & & & \\
\hline \multirow[t]{2}{*}{8} & Sprock Andreas & $\begin{array}{l}\text { SDX Groupe } \\
\text { milliPay Systems AG }\end{array}$ & $\begin{array}{l}\text { Head Innovation Management } \\
\text { coO. Sales \& Marketing/Co-Founder }\end{array}$ & & & & \\
\hline & & & Total & 2 & 2 & 2 & 2 \\
\hline
\end{tabular}

Abb. 10.6 Auswahl der Erkenntnissubjekte. (Eigene Darstellung) 


\subsection{Empirie}

Im folgenden Unterkapitel wird die empirisch-qualitative Analyse im Detail beschrieben. Dabei erfolgt zunächst die Auswertung sowie Erörterung zum Themenbereich des Geldsystems. In Anschluss wird ein Vergleich des Zahlungsverkehrs der beiden Währungssysteme vorgenommen. Im Anschluss werden die drei definierten Hypothesen zusammenfassend beantwortet:

- Hypothese 1: Wie im traditionelle Geldsystem bedarf auch das Kryptowährungssystem an Vertrauen.

- Hypothese 2: Das Kryptowährungssystem ist gegenüber dem traditionellen Geldsystem resilienter.

- Hypothese 3: Gegenüber dem traditionellen Zahlungsverkehr vereinfachen Kryptowährungen die Umsetzung von Zahlungstransaktionen.

\subsubsection{Vergleich Geldsystem}

\subsubsection{Aspekt des Vertrauens}

Als Hauptinnovation von Kryptowährungen sowie als entsprechende Faszination von Bitcoin wird erwähnt, dass für die Abwicklung einer Zahlungstransaktion kein Vertrauen in eine zentrale Instanz benötigt wird (Frecè 2018, S. 50; Anonym 2018, S. 198; Hüsler 2018, S. 77, 322; Jaag 2018, S. 82). Dies ist insofern möglich, als dass innerhalb des Bitcoin-Systems eine basisdemokratische und dezentrale Buchführung ermöglicht wird. Das bedeutet, dass das Vertrauen in eine zentrale Institution durch das Vertrauen in eine Mehrheit eines Systems ersetzt wird (Anonym 2018, S. 50). Im Folgenden wird untersucht, inwiefern und wo das Vertrauen im Vergleich zum traditionellen Geldsystem auch im Kryptowährungssystem eine Rolle spielt. Grundsätzlich ist festzuhalten, dass es sich beim Vertrauen um einen relativen und subjektiven Aspekt handelt (Ankenbrand 2018, S. 95, 99; Frecè 2018, S. 90). Gerade weil es sich um eine individuelle Wahrnehmung handelt, können die nachfolgenden Vertrauensfaktoren je nach Person als unterschiedlich relevant gewichtet werden.

\subsection{Kryptowährungscode}

Im Umgang mit Kryptowährungen bedarf es Vertrauen in den Kryptowährungscode bzw. in die darin enthaltene Mathematik, dass sich darin keine Fehler befinden (Frecè 2018, S. 90; Anonym 2018, S. 193; Jaag 2018, S. 110, 258; Lehmann 2018, S. 225; Sprock 2018, S. 68). Dieser Aspekt ist insofern nicht zu vernachlässigen, da im Bereich der Softwareentwicklung im Schnitt auf 120 Zeilen-Code 7 Programmierfehler gemacht werden. Wenn nun bspw. ein Smart-Contract 60.000 Zeilen-Code aufweist, befinden sich darin im Schnitt 3500 Programmierfehler. Das bedeutet, dass oftmals zwischen der Richtigkeit des Kryptowährungscodes und der Genauigkeit der Mathematik eine Diskrepanz 
besteht (Frecè 2018, S. 50; Sprock 2018, S. 68). Da es sich bei Kryptowährungen um ein Open-Source-Konzept handelt, wird der Kryptowährungscode öffentlich zugänglich gemacht. Somit kann jede Person, die entsprechende Funktionsweise nachvollziehen und sich von der Richtigkeit des Codes selbst überzeugen. Da eine entsprechende Überprüfung ein hohes Maß an technologischem Wissen und Zeit voraussetzt, kann der Kryptowährungscode von der breiten Bevölkerung nicht selbstständig überprüft werden. Frecè (2018) argumentiert, dass es in einem solchen Fall das Vertrauen in eine zentrale, Instanz braucht, welche für die Öffentlichkeit eine Bewertung der entsprechenden Kryptowährung vornimmt. Als Beispiel wird Google angeführt, welche analog der Ratingagentur Moody's \& Fitch die im traditionellen Währungssystem Unternehmen bewertet, eine derartige Überprüfung vornehmen könnte. Hierbei stellt sich jedoch auch die Frage, ob die Bevölkerung Google vertraut und inwiefern diese bei der Überprüfung tatsächlich neutral und unabhängig bei der Bewertung vorgeht (Frecè 2018, S. 90). Nebst einer zentralen Institution argumentiert Jaag (2018), dass auch die Anzahl der in einer Kryptowährung involvierten Personen ein entsprechender Indikator für das Vertrauen in eine Kryptowährung darstellen kann. Je größer die Community, desto mehr technologisch versierte Personen haben sich mit dem Kryptowährungscode auseinandergesetzt und desto fundierter ist eine Kryptowährung. Somit kann eine Vertrauensbildung auch über eine dezentrale Community stattfinden (Jaag 2018, S. 110, 346). Diese zwei Möglichkeiten bestehen laut den Experten, um das Risiko eines Hackerangriffs auf Fehler im Kryptowährungscode zu reduzieren und dadurch Vertrauen in die Kryptowährungen zu generieren. Ein Bestandteil des Kryptowährungscodes bildet unter anderem der darin enthaltenen Algorithmus der Geldmengenschöpfung. Im Vergleich dazu besteht im traditionellen Geldsystem kein solch mathematisch verankerter Geldschöpfungsprozess. Die Geldschöpfung wird durch die Zentralbanken und die Geschäftsbanken dynamisch und aufgrund ökonomischer Einflussgrößen betrieben. Somit wird das Vertrauen in das menschliche Handeln (Geldschöpfung und Geldpolitik) im traditionellen Geldsystem durch das Vertrauen in den Kryptowährungscode bzw. in den darin enthaltenen Algorithmus im Kryptowährungssystem ersetzt (Frecè 2018, S. 66; Hüsler 2018, S. 193).

\subsection{Kryptografische Verfahren}

Wie im traditionellen Geldsystem muss auch im Kryptowährungssystem sichergestellt werden, dass das Geld nicht fälschbar ist. Im traditionellen Geldsystem wird dies bspw. durch die Verwendung von Sicherheitsmerkmalen auf Banknoten sichergestellt. Diese Sicherheitsmerkmale werden laufend dem technologischen Fortschritt angepasst. Diese Anpassung wird regelmäßig durch die Ausgabe von neuen Banknotenserien durch die Schweizerische Nationalbank durchgeführt. Im Kryptowährungssystem wird die Fälschungssicherheit, d. h. das Double-Spending-Problem, durch kryptografische Verfahren gelöst (Frecè 2018, S. 42; Anonym 2018, S. 50; Hüsler 2018, S. 264-270; Jaag 2018, S. 46). Wie im traditionellen Geldsystem besteht auch im Kryptowährungssystem die Möglichkeit, dass durch technologische Innovationen die Fälschbarkeit 
der Geldeinheiten vereinfacht werden kann. Dieses Problem äußert sich im Kryptowährungssystem bspw. durch neuartige kryptografische Verfahren oder Hochleistungsrechner. Insofern müssen Kryptowährungen fortlaufend dem technologischen Fortschritt angepasst werden. Ansonsten würde das Double-Spending-Problem, also die vereinfachte Fälschbarkeit von Kryptowährungen, zunehmen. Insofern benötigen die Systemteilnehmer ein Vertrauen in die kryptografischen Verfahren. Falls dieses Vertrauen nicht mehr vorhanden sein sollte und bekannt wird, dass innerhalb eines Kryptowährungssystems die Möglichkeit besteht, Double-Spending zu betreiben, würden sich die Systemteilnehmer aus der Kryptowährung zurückziehen. Die Kryptowährung wäre defacto unbrauchbar (Frecè 2018, S. 94; Anonym 2018, S. 82; Lehmann 2018, S. 225).

\subsection{Entwickler}

Nebst diesen technologischen Aspekten wie dem Kryptowährungscode und dem kryptografischen Verfahren muss auch den Entwicklern von Kryptowährungen vertraut werden. Diese stehen mit ihrem Ruf und ihrem Know-how für das Funktionieren einer Kryptowährung ein (Anonym 2018, S. 82; Hüsler 2018, S. 262; Jaag 2018, S. 112-124, 342). Im traditionellen Geldsystem sind diese mit einem Teilaufgabengebiet der Zentralbank zu vergleichen, welche mit ihrem Namen und ihrem Vertrauen die Funktionsfähigkeit einer traditionellen Währung gewährleistet (Anonym 2018, S. 50; Jaag 2018, S. 82).

\subsection{Kryptowährungsdienstleister}

Vertrauen muss zudem auch dem Kryptowährungsdienstleister an den Schnittstellen des Kryptowährungssystems entgegengebracht werden (Ankenbrand 2018, S. 47; Frecè 2018, S. 126; Anonym 2018, S. 46, 82-90; Hüsler 2018, S. 157, 430-434; Jaag 2018, S. 110; Lehmann 2018, S. 57-59; Sprock 2018, S. 80). Nebst Anbietern von Exchanges und Payment Solutions sind hier auch Wallet-Anbieter betroffen. Letztere sind für die Verwaltung von Kryptowährungs-Adressen und -Transaktionen wie auch für die Erstellung von privaten Schlüsseln zuständig. Diese Aufbewahrung von digitalen Gütern, wie es auch die privaten Schlüssel sind, bedarf über einen längeren Zeithorizont ein hohes Maß an Sorgfalt (Anonym 2018, S. 86). Bei einem allfälligen Verlust des Private-Schlüssels kann kein Zugriff mehr auf die entsprechenden Kryptowährungen gewährleistet werden. Diese Aufbewahrung von Kryptowährungen kann grundsätzlich auf zwei verschiedene Arten stattfinden. Die erste Option der physischen Aufbewahrung besteht darin, dass der Systemteilnehmer sich selbstständig um die Aufbewahrung der Kryptowährungen kümmert und diese auf einem physischen Ledger Wallet oder den privaten Schlüssel alternativ in Papierform an einem sicheren Ort verwahrt. Bei der Aufbewahrung des privaten Schlüssels in einem physischen Ledger Wallet muss jedoch auch ein Vertrauen in den Hersteller der Hardware, sprich dass diese richtig funktioniert und keinen technologischen Fehler aufweist, bestehen (Anonym 2018, S. 174). Ansonsten braucht es bei dieser Variante der selbstständigen, physischen Aufbewahrung kein Vertrauen mehr in eine Drittpartei. Entscheidet sich ein Kryptowährungsbenutzer für diese Lösung, dann ist dieser selbstständig für die fachgerechte Aufbewahrung verantwortlich. 
Hier muss der Anwender lediglich Vertrauen in sich selbst haben und sich selbst die Aufbewahrung selbst zumuten können. Potenzielle Risiken in der physischen Aufbewahrung bestehen darin, dass der private Schlüssel verloren gehen oder gestohlen werden könnte (Anonym 2018, S. 82, 86; Jaag 2018, S. 110, 390). Diese Option kommt im traditionellen Geldsystem der Aufbewahrung von Bargeld gleich, wo der Besitzer selbstständig dafür verantwortlich ist, die Vermögenswerte gegen Diebstahl oder Verlust zu schützen (Frecè 2018, S. 186; Hüsler 2018, S. 157). Jaag (2018) und Frecè (2018) argumentieren, dass die Option der physischen Aufbewahrung von Kryptowährungen gegenüber dem Bargeld im traditionellen Geldsystem den Vorteil hat, dass Kryptowährungen über größere Distanzen ohne die Einbindung von einem Intermediär verwendet werden können. Diese Möglichkeit besteht im traditionellen Geldsystem nicht und bildet somit eine wertvolle Option (Jaag 2018, S. 390; Frecè 2018, S. 434). Inwiefern die Zahlungstransaktion jedoch ohne die Zuhilfenahme eines Anbieters aus dem Bereich Payment Solutions, welcher de facto auch als Intermediär taxiert werden kann, initialisiert werden kann, ist kritisch zu hinterfragen. Die zweite Option besteht darin, dass man für die Verwahrung der Kryptowährungen auf einen Online-Wallet-Anbieter zurückgreift. Diese Alternative bietet sich an, wenn die Systemteilnehmer das Risiko eines physischen Verlustes nicht tragen möchten. Bei dieser Variante muss der Systemteilnehmer jedoch dem Wallet-Anbieter vertrauen, dass dieser die Kryptowährungen sorgfältig und sicher aufbewahrt, keinen Missbrauch betreibt und den Systemteilnehmern den Zugriff nicht verweigert. Ein solcher Missbrauch könnte sich bspw. in Form einer böswilligen Absicht des Wallet-Anbieters äußern, indem dieser die Kryptowährungen stiehlt (Ponzi Schemes) oder die Online-Plattform durch eine Drittpartei gehackt wird und die Kryptowährungen entwendet werden (Frecè 2018, S. 186; Anonym 2018, S. 90; Hüsler 2018, S. 157; Jaag 2018, S. 52-24; Lehman 2018, S. 225). Sprock (2018) geht jedoch davon aus, dass die Wallet-Anbieter von einer nachhaltigen Geschäftstätigkeit langfristig mehr profitieren würden als in Form eines einmaligen, kurzfristigen und böswilligen Handelns. Diese ökonomischen Anreize reduzieren die Wahrscheinlichkeit von Ponzi Schemes (Sprock 2018, S. 84). Die Funktion des Wallet-Anbieters kann im traditionellen Geldsystem mit der Führung eines Geschäftsbankkontos verglichen werden. Aufgrund dieser Analogie können sich diverse Experten vorstellen, dass das Wallet Angebot, welche sich bisher mit wenigen Ausnahmen wie Swissquote auf Fintech-Unternehmen beschränken, durchaus auch ein zukünftiges Geschäftsfeld für die Geschäftsbanken bilden könnten (Ankenbrand 2018, S. 71 ff.; Frecè 2018, S. 314; Anonym 2018, S. 174; Hüsler 2018, S. 157, 161-167; Jaag 2018, S. 110, b, 186-198). Geschäftsbanken würden sich insofern für die Aufbewahrung eignen, da diese in der Wahrnehmung der Experten in der Schweiz ein hohes Vertrauen seitens der Bevölkerung genießen. Dieses Vertrauen in die Geschäftsbanken ist jedoch im internationalen Kontext nicht in jedem Land gegeben (Frecè 2018, S. 166; Anonym 2018, S. 58; Hüsler 2018, S. 157; Jaag 2018, S. 50; Kneissler 2018, S. 192).

Bei dieser zweiten Option muss also wie auch im traditionellen Geldsystem einer zentralen Institution vertraut werden, dass diese die Vermögenswerte fachgerecht und sicher 
aufbewahrt. Kritisch zu hinterfragen ist jedoch, ob durch dieses Szenario nicht die Vision des Bitcoin-Gründers Satoshi Nakamoto verfehlt wird.

\subsection{Miner}

Einem individuellen Miner muss kein Vertrauen geschenkt werden, da dieser lediglich die Buchführung betreiben muss und als Erlös einen Block-Reward erhält (Frecè 2018, S. 98; Hüsler 2018, S. 189; Anonym 2018, S. 50). Der Vertrauensfaktor nimmt durch die Bildung von Mining-Pools zu. Dieses Vertrauen besteht darin, dass die individuellen Miner sich nicht zu einem dominanten Mining-Pool zusammenschließen. Würde ein Mining-Pool mehr als $51 \%$ der Rechenleistung verfügen, wäre das System bei einer entsprechenden Absprache der Miner fälschbar bzw. würde nicht mehr funktionieren. Die Dezentralität wäre in einem solchen Fall nicht mehr gegeben und die Buchführung könnte alleinig von einem Mining-Pool übernommen werden (Frecè 2018, S. 116-118, 234; Hüsler 2018, S. 161, 189; Jaag 2018, S. 258). In der Vergangenheit kam es bereits einmal zu einer solch dominanten Stellung eines Mining-Pools. Hierbei haben sich die Miner jedoch selbstständig in kleinere Mining-Pools umverteilt. Jaag (2018) und Anonym (2018) sehen darin einen Marktmechanismus und das Bestreben der Miner darin, das System aufrechtzuerhalten. Es wird argumentiert, dass diese aufgrund von ökonomischen Anreizen (Block-Reward und Transaktionsgebühren) ein Interesse daran haben, dass das System funktionsfähig bleibt (Anonym 2018, S. 66-74; Jaag 2018, S. 266). Im traditionellen Geldsystem wird die Buchführung durch eine Geschäftsbank, vorgenommen. Hier brauchen die Geschäftsbankkunden ein Vertrauen, dass die Buchführung der Geschäftsbanken korrekt vorgenommen wird. Diesen zentralen Institutionen muss vertraut werden, dass diese die Daten richtig verwalten, die Buchführung nicht fälschen und dass jederzeit Zugang zu den Vermögenswerten besteht und man nicht aus dem Bankensystem ausgeschlossen wird (Frecè 2018, S. 62; Jaag 2018, S. 50, 156).

\subsection{Staat}

Wie im traditionellen Geldsystem braucht es auch innerhalb des Kryptowährungssystem das Vertrauen in den Staat, dass dieser durch rechtliche Rahmenbedingungen die Funktionsweise der beiden Geldsysteme nicht einschränkt bzw. sogar verunmöglicht (Frecè 2018, S. 94; Anonym 2018, S. 54, 142; Hüsler 2018, S. 398; Lehmann 2018, S. 55). Im Fall von Kryptowährungen könnte der Staat bspw. aufgrund von gewissen Indizien besorgt sein, dass mit Kryptowährungen Geldwäscherei oder sonstige illegale Aktivitäten betrieben werden (Ankenbrand 2018, S. 53-63; Lehmann 2018, S. 239275). Ein Eingriff in das System durch den Staat ist nicht möglich. Dennoch besteht die Möglichkeit, dass dieser gewisse Restriktionen oder regulatorische Maßnahmen an den Schnittstellen (Kryptowährungsdienstleister) einführt bzw. gewisse Dienstleistungen komplett verbietet. Ein derartiger Eingriff würde den Handel mit Kryptowährungen stark einschränken und für die Kryptowährungsteilnehmer unattraktiv machen. Insbesondere eine erschwerte Konvertierung in Fiat-Geld und vice versa könnte den Gebrauch von Kryptowährungen stark einschränken. 
Obwohl der Staat nicht direkt in das Kryptowährungssystem eingreifen bzw. dieses regulieren kann, muss dem Staat jedoch vertraut werden, dass dieser den Handel mit Kryptowährungen an den Schnittstellen nicht einschränkt oder verbietet (Ankenbrand 2018, S. 95-99; Jaag 2018, S. 372-386; Lehmann 2018, S. 237).

\subsubsection{Aspekt der Resilienz}

Wie in der Literatur bestätigen auch die Experten, dass Kryptowährungen als Gegenpol zum traditionellen Geldsystem entwickelt wurden. Als Auslöser und Notwendigkeit eines solchen Kryptowährungssystems werden die bestehenden Probleme des traditionellen Geldsystems genannt, welche zu der Krise ab 2007 geführt haben. Von dieser Alternative zum traditionellen Geldsystem erhofft man sich eine höhere Resilienz (Ankenbrand 2018, S. 67; Anonym 2018, S. 58; Jaag 2018, S. 68-70; Sprock 2018, S. 48). Dabei stellt sich die Fragen, inwiefern man im Geldsystem überhaupt von einer Krise sprechen kann (Frecè 2018, S. 246; Hüsler 2018, S. 282, 310; Jaag 2018, S. 394). Es wird argumentiert, dass es defacto keine Geldkrise geben kann, sondern dass eine realwirtschaftliche Krise, wie bspw. die Vergabe von faulen Krediten, analog der Subprime-Krise im Jahr 2007, einen Einfluss auf die Geldwertstabilität hat (Hüsler 2018, S. 338). Da es unterschiedliche Arten, Ausprägungen und Ursprünge von Krisen geben kann, wurde sich bei der Befragung zum Aspekt der Resilienz an der Finanzkrise ab dem Jahr 2007 orientiert (vgl. Abschn. 1.1). Dabei wird insbesondere auf die in der Literatur ausgearbeiteten Probleme des traditionellen Geldsystems in der Schweiz eingegangen und untersucht, inwiefern ein Kryptowährungssystem diese reduzieren bzw. verhindern kann. Trotz dieser in der Literatur beschriebenen Probleme verfügt in der Wahrnehmung der Experten die Schweiz über ein stabiles Geldsystem, eine solide Geldpolitik und über vertrauensvolle Geschäftsbanken. Insofern wird der potenzielle Mehrwert von Kryptowährungen auf die Resilienz des Geldsystems in der Schweiz als marginal eingestuft (Ankenbrand 2018, S. 47; Frecè 2018, S. 166-170; Anonym 2018, S. 58; Jaag 2018, S. 56-62, S. 68-70; Kneissler 2018, S. 88, 216; Lehmann 2018, S. 41-47). Anders gestaltet sich der potenzielle Mehrwert von Kryptowährungen laut einigen Experten im internationalen Kontext. Entgegen der Schweiz herrscht in diversen Ländern, insbesondere in Drittweltländern, ein dysfunktionales Geldsystem. Als Beispiele werden Länder wie Venezuela und Malawi genannt. In diesem Zusammenhang wird argumentiert, dass Kryptowährungen für die Bevölkerung eine Alternative zu den lokalen Fiat-Währungen bilden könnten, um sich dem dysfunktionalen Geldsystem entziehen zu können. Hier könnte eine algorithmisch festgelegte Geldpolitik gegenüber dem durch Menschen erzeugte dysfunktionale Geldpolitik einen Mehrwert erzeugen (Frecè 2018, S. 74, 166; Hüsler 2018, S. 380-382; Jaag 2018, S. 62; Lehmann 2018, S. 93-95).

Bevor man sich der Frage stellen kann, welches Geldsystem denn nun eine höhere Resilienz aufweist, gilt es vorgängig festzuhalten, wie ein solches Kryptowährungssystem denn zukünftig ausgestaltet sein wird. Dabei besteht die Möglichkeit, dass Kryptowährungen das traditionelle Geldsystem ergänzen oder ersetzen werden. Die Mehrheit der Experten geht davon aus, dass die Kryptowährungen die staatlichen 
Fiat-Währungen zukünftig nicht ersetzten, sondern als Parallelwährung ergänzen werden (Frecè 2018, S. 368; Anonym 2018, S. 174; Hüsler 2018, S. 342; Jaag 2018, S. 82, 152-154, 394; Kniessler 2018, S. 202-204; Sprock 2018, S. 60). Unter Berücksichtigung dieser Ausgangslage werden diverse akademische Diskussionen geführt, ob die SNB eine Kryptowährung (z. B. SNB-Coin) herausgegeben soll und inwiefern dieser gegenüber dem traditionellen Geldsystem eine erhöhte Resilienz aufweist (Jaag 2018, S. 102-130; Frecè 2018, S. 314; Anonym 2018, S. 168-174; Hüsler 2018, S. 390). Der Grund, weshalb gerade eine Zentralbank eine Kryptowährung ausgeben könnte liegt gemäß Jaag (2018) darin, dass für die Sicherstellung einer nachhaltigen gesamtwirtschaftlichen Funktionsweise, die Kryptowährung mit entsprechenden Aktiva als Reserve hinterlegt werden müsste. Nur so kann sichergestellt werden, dass eine Kryptowährung auch werthaltig ist. $\mathrm{Zu}$ solch einer Hinterlegung wäre eine Nationalbank, wie bspw. die SNB in der Lage (Jaag 2018, S. 104-106, 126). Welche Probleme des traditionellen Geldsystems wie der Kontrollverlust der Zentralbank über die Geldmenge, die prozyklische Buchgeldschöpfung und die Gefahr von Bank Runs laut den Experten durch die Emission eines SNB-Coins bestehen bleiben bzw. sich reduzieren oder beseitigt werden, wird nachfolgenden kritisch diskutiert.

\subsection{Kontrollverlust der Zentralbank über die Geldmenge}

Bis dato bleibt unklar, wie ein solcher SNB-Coin technologisch, wie auch organisatorisch ausgestaltet sein könnte. Es sind von über einer fixen Geldmengenobergrenze bis hin zu einer variablen Geldmenge zahlreiche Modelle denkbar. Aufgrund der hohen Komplexität und der Neuartigkeit sollte man sich vor der Ausgabe eines SNB-Coin ausreichend Gedanken über deren Wirkung machen (Anonym 2018, S. 78, 142, 170-174; Hüsler 2018, S. 340-342; Sprock 2018, S. 108). Aufgrund dieser Ausgangslage stehen den Experten nicht ausreichende Informationen zur Verfügung, um eine abschließende Antwort darauf geben zu können, ob ein SNB-Coin das Problem eines Kontrollverlustes der Zentralbank über die Geldmenge löst. Trotzdem wird bzgl. einer potenziellen Ausgestaltung eines SNB-Coins im weiteren Verlauf von einer fixen Geldmengenobergrenze analog dem Bitcoin ausgegangen. Die Geldschöpfung erfolgt in diesem Szenario direkt durch und an das Publikum. In diesem Kryptowährungssystem kämen die traditionellen Funktionsweisen wie das zweistufige Geldsystem und das fraktionale Reservesystem mit multipler Geldschöpfung nicht zum Tragen. Der SNB-Coin würde direkt an das Publikum und nicht wie im traditionellen Geldsystem an die Geschäftsbanken ausgegeben werden. In einem solchen Szenario würde aufgrund der festgelegten Geldmengenobergrenze für die SNB kein Handlungsspielraum mehr bestehen, über diese Obergrenze hinaus weitere Geldeinheiten zu erstellen. Jaag (2018) argumentiert, dass durch eine solche Systemausgestaltung entgegen dem traditionellen Geldsystem von der SNB ausgelöste Angebotsschocks ausgeschlossen werden können. Das Risiko von Nachfrageschocks würden jedoch weiterhin bestehen bleiben (Jaag 2018, S. 400-402). Nichtsdestotrotz müsste gemäß Hüsler (2018) im laufenden Handel nebst der Wertstabilität des CHF auch jene des SNB-Coins durch eine zentrale Instanz sichergestellt werden. Insofern können 
Angebotsschocks nicht mehr direkt durch die SNB ausgelöst werden, aber es existiert immer noch die Möglichkeit, dass aufgrund einer Marktverunsicherung oder Marktpanik durch die Systemteilnehmer ein großes Verkaufsvolumen zu einem Angebotsschock führen kann (Hüsler 2018, S. 131-153). Trotz dem durch die Geldmengenobergrenze limitierten Handlungsspielraum der SNB im Kryptowährungssystem würde die SNB nebst der Ausgabe von Bargeld (M0) ein weiteres Geldmengensteuerungsinstrument zur Verfügung haben. Basierend auf diesem Szenario würde das Problem des Kontrollverlustes der Zentralbank über die Geldmenge reduziert werden. Dennoch handelt es sich hierbei um eine Annahme und muss an dieser Stelle aufgrund der fehlenden Informationen über die Systemausgestaltung und das Fehlen von praktischen Erfahrungen als unklar taxiert werden. Auch unklar ist, wie sich eine Gesellschaft unter einem anhaltenden deflationären Geldsystem, wie dies bei dem SNB-Coin der Fall wäre, verhalten würde (Ankenbrand 2018, S. 91; Jagg 2018, S. 62). Nebst dem potenziellen Ansatz von Kryptowährungen, das Geldsystem durch Dezentralisierung resilienter auszugestalten, findet in der Schweiz auf politischer Ebene eine Diskussion über die Vollgeldinitiative statt. Interessanterweise verspricht die Vollgeldinitiative eine höhere Resilienz des Geldsystems durch Zentralisierung, also indem die SNB mehr Macht und Kontrolle über die Steuerung der Geldmenge erhält (Frecè 2018, S. 138; Anonym 2018, S. 170; Hüsler 2018, S. 69; Lehmann 2018, S. 49-55). Insofern besteht zwischen den unterschiedlichen Vertretern von Kryptowährungen und den Vertretern der Vollgeldinitiative eine grundsätzliche Diskrepanz darüber, ob die Resilienz des Geldsystems durch Dezentralisierung oder Zentralisierung verbessert werden kann.

\subsection{Prozyklische Buchgeldschöpfung}

Anders gestaltet sich jedoch die Situation, wenn es darum geht, das Problem der prozyklischen Buchgeldschöpfung zu diskutieren. Wie auch im traditionellen Geldsystem existiert im Kryptowährungssystem die Möglichkeit, Kredite zu vergeben. Insofern besteht auch im Kryptowährungssystem die Gefahr, dass ein Kreditnehmer den aufgenommenen Kredit nicht zurückbezahlen kann. Ein wesentlicher Unterschied besteht jedoch darin, dass eine Kreditvergabe im Kryptowährungssystem entgegen dem traditionellen Geldsystem keinen Einfluss auf die Ausweitung der Geldmenge zur Folge hat. Der Grund liegt darin, dass dem Kryptowährungssystem eine andere Funktionsweise als das fraktionale Reservesystem mit multipler Geldschöpfung zugrunde liegt. Im Kryptowährungssystem erfolgt die Geldschöpfung ausschließlich aufgrund des im Kryptowährungscode festgehaltenen Algorithmus und wird durch die Miner geschöpft. Als Konsequenz davon besteht auch kein Geldschöpfungsmultiplikator. Das bedeutet, dass im Vergleich zum traditionellen Geldsystem im Kryptowährungssystem keine Möglichkeit existiert „Kryptowährungs-Buchgeld“ zu erzeugen. Infolgedessen muss im Kryptowährungssystem keine Unterscheidung zwischen den unterschiedlichen Geldmengenkategorien (M0, M1, M2, M3) vorgenommen werden. Dennoch bleibt das Risiko, dass ein Kredit nicht zurückbezahlt werden kann auch im Kryptowährungssystem bestehen. Die Folge eines solchen Kreditausfalls wird jedoch insofern reduziert, 
als dass aufgrund des Fehlens eines Geldschöpfungsmultiplikators im Kryptowährungssystem die sich daraus entstehenden Folgen nicht potenzieren (Frecè 2018, S. 136-162; Anonym 2018, S. 54, 94-108; Hüsler 2018, S. 171-185, 284-290; Jaag 2018, S. 120134, S. 394).

Aufgrund dieser Systemausgestaltung besteht das im traditionellen Geldsystem inhärente Problem der prozyklischen Buchgeldschöpfung der Geschäftsbanken nicht. Obwohl dieses Problem im Kryptowährungssystem nicht existiert, basiert die Funktionalität des Kryptowährungssystems wie das traditionelle Geldsystem auf finanziellen Anreizen. Insofern sind in beiden Geldsystemen auf Wettbewerb ausgerichtete ökonomische Prinzipien vorhanden (Anonym 2018, S. 74, 206; Hüsler 2018, S. 77, 211-213; Jaag 2018b, S. 266, 326). Während interessanterweise in der Literatur diese ökonomischen Prinzipien im traditionellen Geldsystem zu der prozyklischen Buchgeldschöpfung führen und entsprechend als Problem taxiert werden, wird in der Praxis argumentiert, dass im Kryptowährungssystem durch diese ökonomischen Anreize (Block-Rewards) die Funktionalität und die Sicherheit gewährleistet wird (Jaag 2018b, S. 266, 326).

\subsection{Bank Runs}

Wie im traditionellen Geldsystem besteht auch im Kryptowährungssystem die Gefahr von Bank Runs. Die Theorie zeigt, dass Banks Runs insbesondere dann entstehen, wenn ein Vertrauensverlust des Publikums in die Währung oder in die Geschäftsbank eintritt. In der Praxis wurde ersichtlich, dass auch im Kryptowährungssystem der Aspekt des Vertrauens eine wichtige Rolle spielt. Dabei muss im Kryptowährungssystem im Vergleich zum traditionellen Geldsystem anderen Parteien und technologischen Komponenten vertraut werden. Insofern können die durch einen Vertrauensverlust ausgelösten Bank Runs auch im Kryptowährungssystem nicht vermieden werden. Ein Sonderfall bildet hierbei jedoch eine potenzielle Ausgabe einer staatlichen Kryptowährung, wie bspw. einem SNB-Coin. Entgegen dem Buchgeld im traditionellen Geldsystem würde der SNB-Coin in der Schweiz als gesetzliches Zahlungsmittel taxiert werden. Dies führt dazu, dass eine gesetzliche Pflicht zur Annahme eines SNB-Coins anfällt. Wenn der SNB-Coin auf einem physischen Ledger Wallet aufbewahrt wird, trägt der Kryptowährungsbenutzer in diesem Fall lediglich das makroökonomische Risiko (Kaufkraftrückgang). Dies ist dem Bargeld in der Schweiz gleichzusetzen. Wird der SNB-Coin jedoch bei einem Online-Wallet-Anbieter aufbewahrt, so trägt der Kryptowährungsbesitzer noch immer das spezifische, mikroökonomische Risiko eines Kreditausfalls des Online-Wallet Anbieters. Somit wäre bei einem Misstrauen gegenüber dem Kryptowährungsdienstleister auch in diesem Fall ein Bank Run auf die Online-Wallet-Anbieter nicht $\mathrm{zu}$ verhindern. Aus dieser Konstellation mit einer staatlich anerkannten Kryptowährung würde sich eine bisher unbekannte Option eröffnen. Im traditionellen Geldsystem war man bis anhin für das Bezahlen von größeren Geldbeträgen entweder auf nicht gesetzliches Buchgeld angewiesen oder man ließ sich den Betrag in gesetzlichem Bargeld auszahlen. Die zweite Option kann jedoch zu Mehrkosten in Bezug 
auf die Transportsicherheit führen. Mit einer staatlichen Kryptowährung würde neu die Möglichkeit bestehen, dass digitales Geld, den rechtlichen Status als gesetzliches Zahlungsmittel erhält. Damit bestünde neu die Möglichkeit, ein gesetzliches Zahlungsmittel elektronisch aufzubewahren und zu versenden (Anonym 2018, S. 58, 166; Hüsler 2018, S. 292-310; Jaag 2018, S. 134, 394). Was für Herausforderungen und Barrieren sowohl in der Schweiz als auch im internationalen Kontext im Umgang mit Kryptowährungen im Zahlungsverkehr bestehen, werden im nachfolgenden Kapitel kritisch diskutiert.

\subsubsection{Vergleich Zahlungsverkehr}

\subsubsection{Aspekt der Vereinfachung von Zahlungstransaktionen}

In der Wahrnehmung der Experten verfügt die Schweiz im Zahlungsverkehr über eine sehr leistungsfähige, durch die SIX betriebene, Finanzmarktinfrastruktur. Des Weiteren steht den Konsumenten in der Schweiz eine Vielzahl an Zahlungsinstrumenten zur Auswahl. Aufgrund dieser Ausgangslage spielen Kryptowährungen in der Wahrnehmung der Experten in der Schweiz aktuell im Zahlungsverkehr eine untergeordnete Rolle. Der Grund für diese Einschätzung liegt darin, dass Kryptowährungen gegenüber den traditionellen Zahlungsinstrumenten aktuell keinen wesentlichen Mehrwert bieten. Zudem befindet sich der Schweizer Zahlungsverkehr in einem sehr kompetitiven Umfeld, was die Eintrittsbarrieren für eine nachhaltige Etablierung von neuen Zahlungsinstrumenten stark erschwert (Frecè 2018, S. 292-294; Anonym 2018, S. 58; Hüsler 2018, S. 380382; Kneissler 2018, S. 88, 180, 216; Lehmann 2018, S. 219-225; Sprock 2018, S. 190192). Im Zahlungsverkehr spielt insbesondere der Netzwerkeffekt eine entscheidende Rolle. Je mehr Personen Kryptowährungen akzeptieren und je vielfältiger dies einsetzbar sind, desto wertvoller werden sie als Zahlungsmittel (Ankenbrand 2018, S. 77-79; Anonym 2018, S. 160-166; Kneissler 2018, S. 40-44). Diese kritische Größe was die Akzeptanz anbelangt, ist heute noch nicht gegeben. Dies hat auch damit zu tun, dass das Ausrollen einer flächendeckenden Hardwareinfrastruktur wie Kryptowährungsautomaten oder Bezahlterminals am Point-of-Sale sehr kostenintensiv ist. Es wird sogar davon ausgegangen, dass sich aufgrund der tiefen Transaktionsgebühren, welche die Kryptowährungen aufweisen müssten, keine Erträge damit erzielen lassen, sondern ein Verlustgeschäft darstellen würden (Hüsler 2018, S. 402; Sprock 2018, S. 150-152; Kneissler 2018, S. 42-44). Nebst dieser wirtschaftlichen Hürde verfügen die Kryptowährungen, wie bspw. Bitcoin, über diverse Probleme bzw. Limitationen. Trotz dieser Probleme bzw. Limitationen herrscht bei den Experten eine positive Grundstimmung was neue technologische Verfahren anbelangt, um diese bestehenden Probleme bzw. Limitationen zu lösen. Es wird argumentiert, dass es sich bei Bitcoin um einen Testversuch handelt und das darauf aufbauend durchaus das Potenzial besteht, dass bessere und anwendungs- und zielgerichtetere Kryptowährungen entstehen werden (Frecè 2018, S. 166, 174-178, 394; Anonym 2018, S. 36-38; Hüsler 2018, S. 386). 
Wenn davon ausgegangen werden kann, dass sich die Kryptowährungen technologisch weiterentwickeln werden, so sieht Anonym (2018) trotz einer gut ausgebauten Finanzmarktinfrastruktur und der Vielzahl an vorhandenen Zahlungsinstrumenten, für Kryptowährungen einen Anwendungsfall in der Schweiz im Bereich des Micropayments. Er geht davon aus, dass sich die Art und Weise, wie heute Rechnungen gestellt werden, zukünftig verändern wird. Als Beispiel nennt er Dienstleistungen im Internet wie die Verwendung von Webcams in einem Skigebiet und Temperatur- und Wetterabfragen. Diese Dienstleistungen werden aktuell noch kostenlos zur Verfügung gestellt. Er kann sich vorstellen, dass derartige kostenlose Dienstleistungen durch die zukünftige Möglichkeit, Kryptowährungen Micropayments durchzuführen, kostenpflichtig gemacht werden könnten. So würde man für den Gebrauch solcher Dienstleistungen bspw. 1 Rappen pro Minute bezahlen. Nebst der Möglichkeit, bisher kostenlos zur Verfügung gestellte Dienstleistungen neu kostenpflichtig auszugestalten, würde auch die Möglichkeit bestehen, bereits bestehende kostenpflichtige Angebote kostentechnisch anders zu strukturieren. Als Beispiel könnte man sich vorstellen, dass zukünftig nicht mehr eine ganze Zeitung gekauft werden muss, sondern dass man die Möglichkeit hätte, spezifische Zeitungsartikel zu lesen und lediglich für diesen Artikel anstelle für die ganze Zeitung zu bezahlen. Auch müssten bspw. die Parkplatzgebühren nicht mehr halbstündlich bezahlt werden, sondern könnten zukünftig nach dem effektiven Gebrauch abgerechnet werden. Zudem werden Anwendungsbeispiele in der Musikbranche angeführt, wo mittels Kryptowährungen die Möglichkeit bestehen würde, die Künstler direkt zu bezahlen ohne dass dabei ein Intermediär, wie aktuell bspw. Apple oder Spotify, zwischengeschaltet werden müsste. Somit könnte sichergestellt werden, dass die Bezahlung direkt an die Künstler erfolgt und dass von diesen Gebühren kein Teilbetrag mehr an die erwähnten Dienstleister abgetreten werden müsste. Derartige Zahlungslösungen könnten mit Kryptowährungen sehr einfach implementiert werden (Anonym 2018, S. 182). Bisher kostenlose Dienstleitungen zukünftig kostenpflichtig auszugestalten, dem steht der ehemalige COO/Mitgründer des Micropayment Unternehmens milliPay Systems AG Sprock (2018) kritisch gegenüber. Dabei ist er mit Anonym (2018) einig, dass eine technologische Implementierung eines Mircopayment-Systems kein Problem darstellt. Hingegen sieht er viel mehr ein Problem im Geschäftsmodell. Als Beispiel wird die Einführung einer Plastiktütengebühr der Detailhändler von 5 Rappen erwähnt. Nach der Einführung dieser Gebühr sei der Bezug der Plastiktüten stark zurückgegangen. Genau diesen Fall habe er beim Start-up milliPay Systems AG auch erlebt. Die Tatsache, dass der Preis einer Dienstleistung oder eines Gutes von kostenlos auf 1 Rappen oder einen Bruchteil eines Rappens erhöht wird, wird in der subjektiven Wahrnehmung des Konsumenten maximal teurer, sodass oftmals gänzlich darauf verzichtet wird und eine Verhaltensänderung (man bringt seine eigene Plastiktüte mit) bevorzugt wird. Deshalb ist er bzgl. des Erfolgs eines Micropayment-Systems, welches darauf abzielt, bisher kostenlos zur Verfügung gestellte Dienstleistungen neu kostenpflichtig auszugestalten, skeptisch gegenüber eingestellt (Sprock 2018, S. 116). Nebst diesem potenziellen Anwendungsfeld des Micropayments wurden von den Experten keine weiteren Einsatzgebiete im Zahlungsverkehr von Kryptowährungen in der Schweiz genannt. 


\subsubsection{Grenzüberschreitender Zahlungsverkehr}

Nebst der Auffassung der Experten, dass Kryptowährungen in der Zahlungslandschaft Schweiz einen untergeordneten Stellenwert einnehmen bzw. einnehmen werden, sind die Experten der Auffassung, dass im internationalen Kontext ein Anwendungsfall für die Vereinfachung von Zahlungstransaktionen besteht. Wie in der Theorie wird auch in der Praxis der internationale Zahlungsverkehr, insbesondere außerhalb von Europa und in Drittweltländern, als langsam und kostenintensiv klassifiziert (Frecè 2018, S. 282-290; Anonym 2018, S. 150; Jaag 2018, S. 74; Kneissler 2018, S. 100-104, 192; Lehmann 2018, S. 69-71).

Entgegen dem nationalen, besteht im internationalen, Zahlungsverkehr nach Kneissler (2018), ein konkretes Kundenbedürfnis, diese Zahlungen effizienter auszugestalten. Dabei wird kritisch hinterfragt, ob Kryptowährungen für diese Problemlösung tatsächlich das richtige Instrument darstellen oder diese das bestehende Problem bzw. die Limitation nicht allenfalls sogar noch verkomplizieren (Kneissler 2018, S. 104, 176). Dem stimmt Sprock (2018) zu, denn auch er ist sich nicht sicher, ob Kryptowährungen im internationalen Zahlungsverkehr tatsächlich das richtige Problem bzw. die Limitation adressieren (Sprock 2018, S. 140). Auch Lehmann (2018) und Frecè (2018) zweifeln daran, ob Kryptowährungen eine geeignete Lösung für das Problem bzw. die Limitation darstellen. In ihrer Wahrnehmung wurden die internationalen Zahlungsverkehrsanbieter bisher noch nicht von neuen Anbietern herausgefordert. Entsprechend habe man sich im traditionellen Markt unter den bestehenden internationalen Zahlungsanbietern arrangiert. Sie gehen davon aus, dass, wenn ein substanzieller Teil der Auslandzahlungen über Kryptowährungen durchgeführt werden würde, durchaus Handlungsspielraum bei den traditionellen Anbietern bestehen würde, um sowohl die Kosten wie auch die Geschwindigkeit von internationalen Zahlungstransaktionen zu reduzieren. Diese vorhandenen Probleme bzw. Limitationen im traditionellen internationalen Zahlungsverkehr würden sich ihrer Auffassung nach nicht auf technologische Limitationen beziehen, sondern auf das kommerzielle Eigeninteresse der Zahlungsanbieter. Ihrer Meinung nach sei dieser Handlungsspielraum noch so groß, dass die potenziell vorhandenen Kosten- und Geschwindigkeitsvorteile von Kryptowährungen wieder aufgehoben werden könnten. Insofern gehen sie nicht davon aus, dass Kryptowährungen einen nachhaltigen und substanziellen Marktanteil im internationalen Zahlungsverkehr erreichen können. Der Vorteil von Kryptowährungen bestehe jedoch darin, dass die traditionellen Zahlungsanbieter herausgefordert werden würden und diese dazu gezwungen wären, ihr Geschäftsmodell zu überdenken bzw. ihre Dienstleistungen effizienter und kostengünstiger auszugestalten (Lehmann 2018, S. 69; Frecè 2018, S. 294). Ein Beispiel dafür ist, dass Western Union auf die Kryptowährungen reagiert hat, indem sie die Preise für ihren Service angepasst hat (Frecè 2018, S. 134, 270-278).

Trotz dieser Bedenken wird argumentiert, dass Kryptowährungen insbesondere den Vorteil bieten, dass die Kryptowährungszahlungen auch tatsächlich beim Endbegünstigten ankommen (Anonym 2018, S. 150; Kneissler 2018, S. 126-128). Um eine Kryptowährungszahlung $\mathrm{zu}$ empfangen, werden lediglich ein Mobiltelefon mit 
Internetzugang sowie eine Online-Wallet benötigt. Dieser Zugang sei bei einem Großteil der Bevölkerung, auch in Drittweltländern, gegeben. Dabei hat sich insbesondere anhand der Einführung der Mobile-Payment-Lösung M-Pesa in Kenia gezeigt, dass in Drittweltländern durchaus mit den neuen Technologien umgegangen werden kann (Ankenbrand 2018, S. 135; Hüsler 2018, S. 412-418; Jaag 2018, S. 164-168, 222-258; Kneissler 2018, S. 134-136; Sprock 2018, S. 138-140). Insofern wird das Problem der Experten in diesem Anwendungsfall weniger im Umgang mit Kryptowährungen gesehen, sondern vielmehr wie in der Schweiz in der mangelnden Akzeptanz und der fehlenden Hardware-Infrastruktur. Die Problematik besteht darin, dass wenn der Begünstige in einem Drittweltland die Kryptowährungen empfangen hat, er diese aufgrund des Fehlens eines Geschäftsbankenkontos nicht in lokales Fiat-Geld umtauschen kann. Zudem besteht aktuell aufgrund der fehlenden Hardware-Infrastruktur am Pointof-Sale auch die die Möglichkeit, mit den Kryptowährungen Waren einzukaufen. Das heißt, der Umtausch von Kryptowährungen in reale Werte gestaltet sich in Drittweltländern sehr schwierig (Hüsler 2018, S. 428-430; Jaag 2018, S. 176-186; Kneissler 2018, S. 104; Sprock 2018, S. 152, 160). Als denkbarer Lösungsansatz wird erwähnt, dass ein Institut mit einem flächendenkenden Filialnetz einen kostenpflichtigen Service anbieten könnte, Kryptowährungen in Fiat-Währungen und vice versa zu tauschen. Als denkbare Beispiele werden Western Union oder das Filialnetz eines Detailhändlers (Kioskreihe) vorgeschlagen. Bei einem solchen Szenario wäre durch das Filialnetz ein großer Skalierungseffekt vorhanden, was für eine breitere Akzeptanz sorgen könnte (Jaag 2018, S. 242; Hüsler 2018, S. 432-434; Frecè 2018, S. 294).

\subsubsection{Unbanked People}

Nebst dem Vorteil, dass Kryptowährungen Menschen in Drittweltländern ohne Geschäftsbankenkonto die Möglichkeit gibt, Kryptowährungszahlungen zu senden und zu empfangen, wird als zusätzlicher Nutzen auch die Wertaufbewahrung erwähnt. Da die Unbanked People über kein Geschäftsbankenkonto verfügen, wird deren Vermögen entweder in Form von realen Gütern oder in Form von Bargeld aufbewahrt. Es wird argumentiert, dass bei einem solchen Verhalten ein erhöhtes Diebstahlrisiko besteht und dass die Wertaufbewahrung in Form von Kryptowährungen dieses reduzieren würde, (Frecè 2018, S. 62; Hüsler 2018, S. 75-77; Jaag 2018, S. 222, 236-242). In der Wahrnehmung von Lehmann (2018) wird durch die Sicherheitsvorkehrung eines privaten Schlüssels seiner Meinung nach das Diebstahlrisiko gegenüber dem Bargeld reduziert. Er gibt jedoch zu bedenken, dass auch bei Kryptowährungen nebst dem Risiko, dass jemand sich durch Erpressung oder eine sonstige Bedrohung Zugang zu den Kryptowährungen verschaffen könne, zusätzlich noch das Diebstahlrisiko durch Hackerangriffe auf den Online-Wallet-Anbieter hinzukommt (Lehmann 2018, S. 83 ff.). Nichtsdestotrotz bieten Kryptowährungen abseits des traditionellen Geldsystems die Möglichkeit, ohne regulatorische Anforderungen wie Ausweisdokumente etc. die finanzielle Inklusion zu erhöhen und sich zudem einem dysfunktionalen Geldsystem zu entziehen Als Beispiele werden Länder wie Venezuela und Malawi genannt. Hier könnte eine algorithmisch festgelegte 
Geldpolitik gegenüber der durch Menschen erzeugten, dysfunktionalen Geldpolitik einen Mehrwert erzeugen (Frecè 2018, S. 74, 166; Hüsler 2018, S. 380-382; Jaag 2018, S. 62; Lehmann 2018, S. 93-95).

Inwiefern der Staat eine solche Koexistenz neben der lokalen Fiat-Währung toleriert, ist kritisch zu hinterfragen. Auch wenn die Kryptowährung per se nicht reguliert werden kann, kann der Staat den Schnittstellen Restriktionen auferlegen. Eine solche Handhabung wäre durchaus denkbar, denn es bestehen oftmals auch Restriktionen, was den Besitz von anderen Fiat-Währungen, abgesehen von der lokalen Fiat-Währung anbelangt (Kneissler 2018, S. 168-172). Uneinigkeit herrscht jedoch unter den Experten in der Frage, ob durch eine derartige finanzielle Inklusion, wie in der Theorie beschrieben, auch die Wohlfahrt eines Landes erhöht wird. Sowohl Kneissler (2018) wie auch Jaag (2018) argumentieren, dass sich durch die Möglichkeit am Handel teilzunehmen, die Wohlfahrt erhöhen würde (Kneissler 2018, S. 174, 176; Jaag 2018, S. 162, 434-442). Hüsler (2018) kann sich nicht vorstellen, dass durch die finanzielle Inklusion der Handel erhöht wird. Er gibt zu bedenken, dass die entsprechenden Realgüter immer noch ausgetauscht werden müssen. Insofern sieht er keine direkte Beziehung zwischen finanzieller Inklusion und einer Wohlfahrtssteigerung (Hüsler 2018, S. 436-458). Nebst der Verwendung von Kryptowährungen in Drittweltländern gibt Frecè (2018) zu bedenken, dass auch in den anderen Ländern in Europa gewisse Minderheiten keinen Zugang zu einem Geschäftsbankenkonto erhalten. Dabei wird das Beispiel genannt, dass es aktuell für einen in der Schweiz lebenden Amerikaner, aus regulatorischen Gründen, sehr umständlich ist, ein Geschäftsbankenkonto in der Schweiz zu eröffnen. Derartige Restriktionen gestalten die Abwicklung von Zahlungstransaktionen, wie bspw. das Bezahlen der Miete, die Krankenkassenzahlungen oder den Erhalt der Lohnzahlungen, als sehr umständlich (Frecè 2018, S. 58, 322-338). Trotz dieser genannten potenziellen Anwendungsfälle im nationalen und internationalen Kontext gilt es an dieser Stelle nochmals zu erwähnen, dass aktuell immer noch zahlreichen Probleme bzw. Limitationen, wie bspw. eine erhöhte Wechselkursvolatilität, Skalierungsprobleme, tiefe Benutzerfreundlichkeit etc., bestehen. Bevor diese Probleme nicht gelöst werden, sind sämtliche erwähnten Anwendungsfälle in der Praxis zurzeit nicht anwendbar.

Im Folgenden werden die zentralen drei Hypothesen bzw. Forschungsfragen auf Basis der Analyse beantwortet.

\subsubsection{Hypothese 1: Wie im traditionelle Geldsystem bedarf auch das Kryptowährungssystem an Vertrauen}

Bereits in der Theorie kann erahnt werden, dass die vom Bitcoin-Erfinder Satoshi Nakamoto gemachte Aussage, dass Kryptowährungen ohne Vertrauen auskommen, relativiert werden muss (P2P-Foundation 2018).

Im Rahmen der vorliegenden empirisch-qualitativen Studie bestätigen sämtliche befragten Experten, dass auch im Kryptowährungssystem genauso wie im traditionellen 
Geldsystem, Vertrauen benötigt wird (Ankenbrand 2018; Frecè 2018; Anonym 2018; Hüsler 2018; Jaag 2018; Kneissler 2018; Lehmann 2018; Sprock 2018). Die gewonnenen Erkenntnisse zum Thema Vertrauen wurden systematisiert und in einem Vergleich in der Abb. 10.7 dargestellt.

Es wird deutlich, dass das Kryptowährungssystem im Vergleich zum traditionellen Geldsystem einer neuen Vertrauensebene bedarf. Die Aufgabe der Zentralbank (1), Geldpolitik zu betreiben, die Fälschungssicherheit der Banknoten zu gewährleisten und mit ihrem Ruf für das Funktionieren der Fiat-Währung einzustehen, wird im Kryptowährungssystem durch den Kryptowährungscode, das kryptografische Verfahren und die Entwickler ersetzt (1). Somit wird im Kryptowährungssystem ein Teil des sozialen Vertrauens in die Ebene des technologischen Vertrauens verschoben. Die Rolle der Geschäftsbanken (2), die Buchführung durchzuführen und unter anderem das Vermögen sicher aufzubewahren, wird im Kryptowährungssystem von den Minern und den Kryptowährungsdienstleistern übernommen (2). Lediglich die Rolle des Staates bleibt in beiden Geldsystemen bestehen (3). Im traditionellen Geldsystem muss insgesamt drei Akteuren vertraut werden, im Kryptowährungssystem verschiebt sich dies aufgrund der gewünschten Dezentralität auf insgesamt vier Akteure und zwei technologische Komponenten. Somit lässt sich die Hypothese wie folgt beantworten:

\section{Hypothese 1: Wie im traditionellen Geldsystem bedarf auch das Kryptowährungs- system an Vertrauen}

Wie das traditionelle Geldsystem bedarf auch das Kryptowährungssystem an Vertrauen. Das Vertrauen im Kryptowährungssystem verschiebt sich im Vergleich zum traditionellen Geldsystem auf andere Akteure und auf eine technologische Ebene.
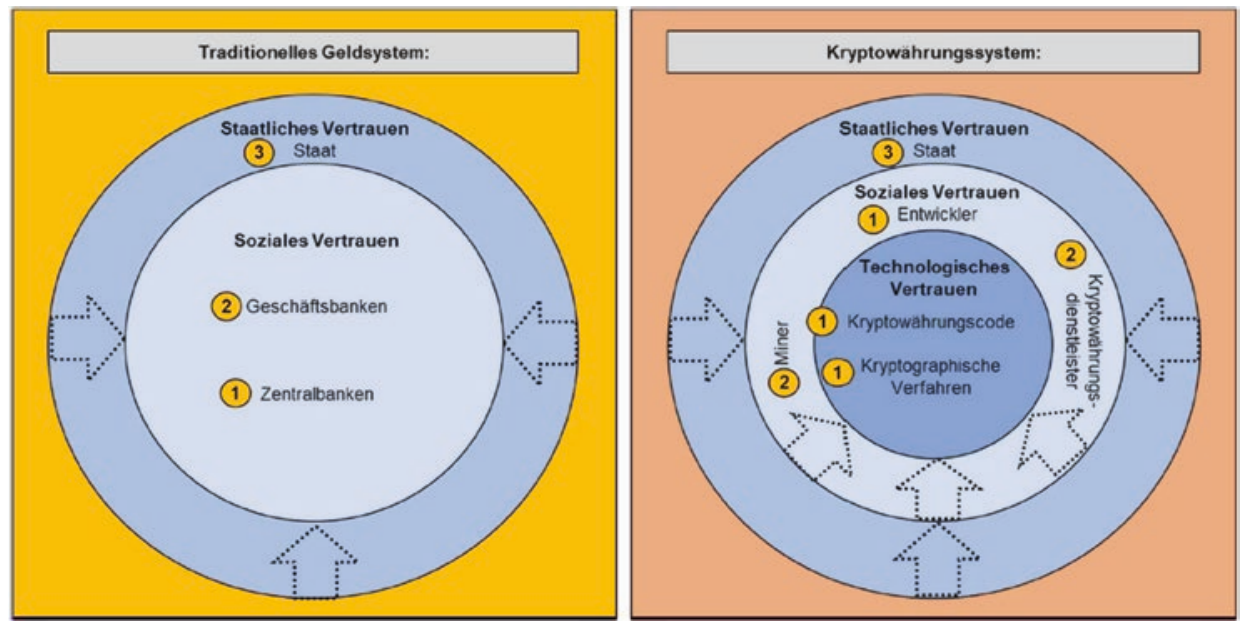

Abb. 10.7 Vertrauensaspekte der Geldsysteme. (Eigene Darstellung) 
Ob nun das traditionelle Geldsystem oder das Kryptowährungssystem das vertrauensvollere System darstellt, kann aufgrund der subjektiven Komponenten nicht abschließend festgehalten werden. Dieser Fragestellung könnte in weiterführenden quantitativen Forschungen, speziell der Nutzer bzw. der Bevölkerung, genauer nachgegangen werden. Eine mögliche Hypothese könnte lauten:

Das traditionelle Geldsystem stellt im Vergleich zum Kryptowährungssystem das vertrauensvollere Geldsystem dar.

\subsubsection{Hypothese 2: Das Kryptowährungssystem ist gegenüber dem traditionellen Geldsystem resilienter}

Durch die Literaturanalyse ließen sich die Probleme im traditionellen Geldsystem in drei Hauptprobleme unterteilen. Dies sind namentlich der Kontrollverlust der Zentralbank über die Geldmenge, die prozyklische Buchgeldschöpfung und die Gefahr von Bank Runs.

Durch die geführten Experteninterviews sollte herausgefunden werden, ob die genannten Probleme durch ein Kryptowährungssystem gelöst werden und dadurch im Vergleich zum traditionellen Geldsystem resilienter sind. Die Abb. 10.8 zeigt, welche Probleme im traditionellen Geldsystem, bspw. durch die Emission einer staatlichen Kryptowährung (z. B. SNB-Coin), gelöst werden könnten.

Es hat sich gezeigt, dass sowohl das traditionelle Geldsystem wie auch das Kryptowährungssystem eines Vertrauenskonzeptes bedürfen. Dies führt dazu, dass im Kryptowährungssystem die Gefahr von Bank Runs bestehen bleibt. Bei einem allfälligen Vertrauensverlust werden die Kryptowährungen verkauft und in eine andere, vertrauenswürdigere Währung konvertiert. Im Gegensatz dazu kann im Kryptowährungssystem das Problem der prozyklischen Buchgeldschöpfung gelöst werden. Wie im traditionellen Geldsystem basiert die Durchführung der Geldschöpfung auch im Kryptowährungssystem auf finanziellen Anreizen. Der Unterschied besteht darin, dass das Kryptowährungssystem nach einer anderen Funktionsweise als das fraktionale Reservesystem mit multipler Geldschöpfung funktioniert. Demnach besteht im Kryptowährungssystem keine Möglichkeit, die Geldmenge durch Kreditvergabe über die im

\begin{tabular}{|c|c|c|c|c|}
\hline & Probleme & Ursachen & Oberbegriff & Problemlösung \\
\hline \multirow{2}{*}{ 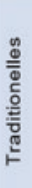 } & $\begin{array}{l}\text { Kontrollverlust der } \\
\text { Zentralbank über die } \\
\text { Geldmenge }\end{array}$ & $\begin{array}{l}\text { - Zweistufiges Geldsystem } \\
\text { - Fraktionales Reservesystem mit multipler } \\
\text { Geldschőpfung }\end{array}$ & Funktionsweise & Unklar \\
\hline & $\begin{array}{l}\text { Prozyklische } \\
\text { Buchgeldschöpfung }\end{array}$ & - Anreize der Geschäftsbanken (Gewinnorientierung) & Finanzielle Anreize & Ja \\
\hline
\end{tabular}

Abb. 10.8 Problemlösung durch ein Kryptowährungssystem. (Eigene Darstellung) 
Kryptowährungscode vorgesehene Anzahl der Geldeinheiten zu erhöhen. Eine Möglichkeit der Behebung des Kontrollverlustes der Nationalbank wäre die Ausgabe einer eigenen Kryptowährung (bspw. SNB-Coin). Unklar bleibt dabei aber, inwiefern die Zentralbank durch die Emission eines SNB-Coins den Kontrollverlust über die Geldmenge reduzieren kann. Fakt ist, dass diese nebst der Ausgabe von Bargeld ein weiteres Instrument zur Steuerung der Geldmenge M0 erhält. Aufgrund der Neuartigkeit, fehlender Informationen und Praxiserfahrung, können die makroökonomischen Folgen einer Emission eines SNB-Coins zum aktuellen Zeitpunkt nur sehr schwer abgeschätzt werden. Insbesondere ist auch kritisch zu betrachten, dass zwischen den unterschiedlichen Vertretern von Kryptowährungen und bspw. der Vollgeldinitiative eine grundsätzliche Diskrepanz in der Frage besteht, ob die Resilienz des Geldsystems durch Dezentralisierung oder Zentralisierung verbessert werden kann.

Trotz dieser in der Literatur erwähnten Probleme im Geldsystem, verfügt die Schweiz in der Wahrnehmung der Experten über ein stabiles Geldsystem, eine solide Geldpolitik und über vertrauenswürdige Geschäftsbanken. Aufgrund dieser Ausgangslage wird in der Praxiswahrnehmung davon ausgegangen, dass Kryptowährungen für die Erhöhung der Resilienz des Geldsystems in der Schweiz eine untergeordnete Rolle einnehmen werden. Nichtsdestotrotz scheint es durchaus vorstellbar, dass eine staatliche Kryptowährung ein Teil der in der Literatur angesprochenen Probleme im traditionellen Geldsystem lösen könnte. Dennoch besteht durch ein derartiges Experiment die Gefahr, dass durch die Lösung eines bestehenden Problems neue Probleme entstehen. Aus diesem Grund muss die Hypothese 2 wie folgt beantwortet werden:

\section{Hypothese 2: Das Kryptowährungssystem ist gegenüber dem traditionellen Geld- system resilienter}

Für eine Beantwortung der Hypothese 2 fehlen klare Kriterien und eine nachvollziehbare Abgrenzung zum Thema Krise in einem Geldsystem. Zudem müsste genauer untersucht werden, wie die Ausgestaltung einer staatlichen Kryptowährung sowohl technologisch als auch organisatorisch funktionieren würde. Aufgrund der Neuartigkeit und der fehlenden Informationen und Praxiserfahrung kann die Hypothese nicht abschließend beantwortet werden.

Um den Grad der Unsicherheit reduzieren zu können, sollten vorgängig in einer weiterführenden Forschung bspw. die folgenden Hypothesen beantwortet werden:

- Eine Geldsystemkrise beruht immer auf realwirtschaftlichen Faktoren.

- Die Resilienz eines Geldsystems wird durch Dezentralität und nicht durch Zentralität erhöht.

- Die Ausgabe eines resilienten SNB-Coins wird nicht durch einen Algorithmus definiert.

- Ein resilienter SNB-Coin wird durch eine Private-Blockchain gewährleistet. 
Des Weiteren könnte aufgrund der thematischen Abgrenzung dieser Ausarbeitung auf den Wirtschaftsstandort Schweiz sowie der Einschätzung der Experten eine genauere Betrachtung der Auswirkung von Kryptowährungen auf Länder mit einem dysfunktionalen Geldsystem interessant sein. Eine mögliche Hypothese für weiterführende Forschungen könnte sein:

Kryptowährungen erzielen in Ländern mit einem dysfunktionalen Geldsystem einen Mehrwert für die Einwohner.

\subsubsection{Hypothese 3: Gegenüber dem traditionellen Zahlungsverkehr vereinfachen Kryptowährungen die Umsetzung von Zahlungstransaktionen}

Wie in der Literatur bestätigten auch die Experten, dass der schweizerische Zahlungsverkehr sehr effizient ausgestaltet ist. Dies wird insbesondere auf die sehr leistungsfähige Finanzmarktinfrastruktur sowie auf die große Auswahl an Zahlungsinstrumenten zurückgeführt. Viele Handlungsfelder werden bereits sehr gut durch Bargeld, Debit-/ Kreditkarte, E-Banking, Mobile Payment und P2P-Zahlungen abgedeckt. Aufgrund dieser Ausgangslage wird in der Expertenwahrnehmung keine Notwendigkeit bzw. kein Bedürfnis nach einem zusätzlichen Zahlungsinstrument in der Schweiz gesehen. Zusätzlich zum kompetitiven Umfeld kommt bei den Kryptowährungen hinzu, dass zahlreiche Probleme bzw. Limitationen bestehen. Aufgrund dieser Probleme bzw. Limitationen im Zahlungsverkehr werden Kryptowährungen primär als Spekulationsobjekt eingesetzt. Deshalb wird zum aktuellen Zeitpunkt in der Expertenwahrnehmung die Umsetzung von Zahlungstransaktionen durch Kryptowährungen nicht vereinfacht.

Die Mehrheit der Experten ist jedoch optimistisch gestimmt, dass diese Probleme zukünftig gelöst werden können. Wenn dieses Szenario eintreten würde und alle Probleme bzw. Limitationen gelöst werden könnten, dann wird der Bereich des Micropayments als einzig mögliches zukünftiges Einsatzgebiet von Kryptowährungen genannt. Ansonsten werden von den interviewten Experten keine weiteren mehrwertschaffenden Einsatzmöglichkeiten von Kryptowährungen in der Schweiz erwähnt.

Anders gestaltet sich die Situation im internationalen Kontext. Die Experten sehen Potenzial darin, dass Kryptowährungen dazu beitragen könnten, den internationalen Zahlungsverkehr zu vereinfachen. Der Grund liegt darin, dass entgegen dem nationalen Zahlungsverkehr der internationale Zahlungsverkehr sowohl was die Transferkosten wie auch die Transfergeschwindigkeit anbelangt, ineffizient ausgestaltet ist. Hier besteht im Vergleich zum nationalen Zahlungsverkehr ein konkretes Bedürfnis. Dabei bestehen die Ineffizienzen laut den Experten nicht aus technologischen Problemen bzw. Limitationen, sondern aus ökonomischen Gründen ist seitens der Zahlungsanbieter kein Anreiz vorhanden, diesen Service kundenfreundlicher auszugestalten. Hier besteht die Möglichkeit, dass Kryptowährungen als Konkurrenz zu den bestehenden internationalen 
Zahlungsanbietern zukünftig zur Lösung dieser Ineffizienzen beitragen könnten. Laut den Experten wurden die internationalen Zahlungsverkehrsanbieter in diesem Geschäftsfeld nur selten von neuen Anbietern herausgefordert.

In diesem Zusammenhang könnten Kryptowährungen dazu beitragen, dass die traditionellen Zahlungsdienstleister ihre Services anpassen werden, sodass der durch Kryptowährungen potenziell erzielte Geschwindigkeits- und/oder Kostenvorteil wieder kompensiert werden könnte. Als erste Reaktion auf die Kryptowährungen und als Anzeichen für diesen Handlungsspielraum wird die Gebührenreduktion von Western Union erwähnt.

Ein weiteres Szenario könnte sein, dass Kryptowährungen für Unbanked People die Option darstellen, überhaupt an einem Zahlungsverkehrssystem teilzunehmen. Auch hier könnten Kryptowährungen zukünftig einen entsprechenden Mehrwert bieten. Basierend auf diesen Erkenntnissen kann die Hypothese 3 wie folgt beantwortet werden:

\section{Hypothese 3: Gegenüber dem traditionellen Zahlungsverkehr vereinfachen Krypto- währungen die Umsetzung von Zahlungstransaktionen}

Gegenüber dem traditionellen Zahlungsverkehr vereinfachen Kryptowährungen die Umsetzung von Zahlungstransaktionen zum aktuellen Zeitpunkt in der Schweiz nicht. Dennoch verfügen Kryptowährungen im Bereich des Micropayments im internationalen Zahlungsverkehr und bei den Unbanked People über das Potenzial, zukünftig die Umsetzung von Zahlungstransaktionen zu vereinfachen.

Aufgrund des Hauptfokus dieser Ausarbeitung auf den Wirtschaftsstandort Schweiz wäre es interessant herauszufinden, ob Kryptowährungen bereits heute in Ländern mit einer schlechter ausgebauten Finanzmarktinfrastruktur, wo viele Unbanked People existieren, einen Mehrwert erzielen können. Somit könnte eine weiterführende Hypothese wie folgt lauten:

Kryptowährungen erzielen in Drittweltländern einen höheren Mehrwert als in Industrieländern.

\subsection{Fazit}

Seit der Erfindung von Bitcoin am 31.Oktober 2008 hat sich der Kurs um ein Vielfaches erhöht, allerdings bei einer hohen Volatilität. Diese hohe Kursvolatilität, das politische Image und das Disruptionspotenzial haben zu einem erhöhten Interesse der Medien und der Bevölkerung geführt. Diese anhaltende Euphorie bringt insofern einen Nachteil mit sich, als dass eine Diskrepanz zwischen Hype und Realität von Kryptowährungen besteht. Aus diesem Grund wurde zusammenfassend, basierend auf der Theorie und der Empirie, ein Vergleich der Vor- und Nachteile von Kryptowährungen gegenüber dem traditionellen Währungssystem mit Fokus auf den Wirtschaftsstandort Schweiz erstellt, welcher in Abb. 10.9 dargestellt ist. 


\begin{tabular}{|c|c|c|c|}
\hline & & Traditionelles Währungssystem & Kryptowährungen \\
\hline \multirow{2}{*}{ 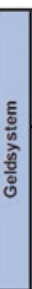 } & $\begin{array}{l}\overline{\overline{0}} \\
\text { के }\end{array}$ & $\begin{array}{l}\text { - Praxiserfahrung des fraktionalen Geldsystems mit multipler } \\
\text { Gelschopfung ( } 500 \text { Jahre) } \\
\text { - Solide Geldpolitik der SNB } \\
\text { - Stabiler Schweizer Franken } \\
\text { - Vertrauenswurdige Geschaftsbanken }\end{array}$ & $\begin{array}{l}\text { - Keine Prozykdische Buchgeldschópfung möglich } \\
\text { - Geldmenge kann nicht beliebig erhöhtwerden } \\
\text { - Dezentralisierung der Macht (Basis dekokratischer Ansatz) } \\
\text { - Moglichkeit eines digitalen gesetzichen Zahlungsmittels" }\end{array}$ \\
\hline & 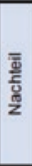 & $\begin{array}{l}\text { - Bedarf an staatlichem und sozalen Vertrauen } \\
\text { - Kontrollverlust der Zentralbank uber die Geldmenge } \\
\text { - Prozyklische Buchgeldschöpfung } \\
\text { - Zentralisierung der Macht } \\
\text { - Gefahr von Bank Runs }\end{array}$ & $\begin{array}{l}\text { - Bedarf an staatlichem, technologischem und sozialem Vertrauen } \\
\text { - Beschränkte Praxiserfahrung (Auswirkungen einer staatlichen } \\
\text { Kryptowahrung auf die Geldpolitik ist weitgehends unerforscht) } \\
\text { - Deflation / Wechselkurs volablitatt } \\
\text { - Gefahr von Mining-Pools } \\
\text { - Schnitts tellenproblematik } \\
\text { - Gefahr von Bank Runs }\end{array}$ \\
\hline \multirow{2}{*}{ 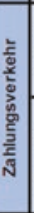 } & $\begin{array}{l}\overline{\bar{\pi}} \\
\text { 홍 }\end{array}$ & $\begin{array}{l}\text { - Effiziente nationale Finanzmarktinfrastruktur } \\
\text { - Große Auswahl an Zahlungsinstrumenten } \\
\text { - Klassische Zahlungsinstrumente verfugen aber eine hohe Akzeptanz } \\
\text { - Hohe Benutzerfreundlichkeit }\end{array}$ & $\begin{array}{l}\text { - Abwicklung einer dezentralen Zahlungstrans aktion } \\
\text { - Potenzal Micropayments effizienter durchzufuhren" } \\
\text { - Potenzell Mehrwert im grenzaberschreitenden Zahlungsverkehr" } \\
\text { - Potenzal der finanzellen Inklusion der Unbanked People*" }\end{array}$ \\
\hline & $\begin{array}{l}\frac{\overline{0}}{\frac{2}{2}} \\
\frac{\text { II }}{2}\end{array}$ & $\begin{array}{l}\text { - Hohe Eintrittsbarrieren fur Innovationen } \\
\text { - Ineffizenzen im internationalen Zahlungsverkehr } \\
\text { - Fur die Durchfuhrung einer Zahlungstransaktion (außer Bargeld) } \\
\text { braucht es ein Geschaftsbankkonto }\end{array}$ & $\begin{array}{l}\text { - Skalierungsproblem } \\
\text { - Abnehmende Dezentralitat } \\
\text { - Metakonsensfindung } \\
\text { - Tiefe Benutzerfreundlichkeit } \\
\text { - Hoher Ressourcenverbrauch }\end{array}$ \\
\hline
\end{tabular}

Abb. 10.9 Vergleich der beiden Währungssysteme in der Schweiz. (Eigene Darstellung)

Die Empirie hat gezeigt, dass die von Satoshi Nakamoto gemachte Aussage, dass Kryptowährungen ohne Vertrauen auskommen, hinterfragt werden muss. Die Experteninterviews haben gezeigt, dass beide Geldsysteme Vertrauen brauchen. Das Vertrauen kann dabei in staatliches, soziales und technologisches Vertrauen unterteilt werden. Der Hauptunterschied besteht darin, dass ein Teil des Vertrauens in das menschliche Handeln (soziales Vertrauen) im traditionellen Geldsystem durch die Gesetze der Mathematik (technologisches Vertrauen) im Kryptowährungssystem ersetzt wird. Konkret wird die Aufgabe der Zentralbank, im traditionellen Geldsystem Geldpolitik zu betreiben und die Fälschungssicherheit der Banknoten zu gewährleisten, im Kryptowährungssystem durch den Kryptowährungscode und das kryptografische Verfahren ersetzt. Dies führt dazu, dass das Kryptowährungssystem im Gegensatz zum traditionellen Geldsystem über eine zusätzliche Vertrauensebene, namentlich dem technologischen Vertrauen verfügt. Des Weiteren verschiebt sich das Vertrauen innerhalb des sozialen Vertrauens auf andere Akteure. So wird die Rolle der Geschäftsbanken, die Buchführung und u. a. das Vermögen aufzubewahren, im Kryptowährungssystem durch die Miner und die Kryptowährungsdienstleister ersetzt. Des Weiteren verschiebt sich der Ruf der Zentralbank, für die Fiat-Währung einzustehen, im Kryptowährungssystem auf die Entwickler.

Die Vorteile des Kryptowährungssystems gegenüber dem traditionellen Geldsystem liegen darin, dass aufgrund der Funktionsweise und der Geldmengenobergrenze keine Möglichkeit zur prozyklischen Buchgeldschöpfung besteht und die Geldmenge nicht beliebig erhöht werden kann. In der Wahrnehmung der Experten verfügt die Schweiz dank einer soliden Geldpolitik und vertrauenswürdigen Geschäftsbanken über ein stabiles Geldsystem. Aufgrund dieser Wahrnehmung wird der potenzielle Mehrwert einer staatlichen Kryptowährung (z. B. SNB-Coin) zur Erhöhung der Resilienz in der Schweiz als marginal eingestuft. Auch wenn durch ein SNB-Coin gewisse Probleme des traditionellen Geldsystems gelöst werden könnten, besteht in einem solchen Experiment eine 
hohe Unsicherheit darin, ob durch die Lösung eines Problems nicht neuartige Probleme entstehen und dadurch allenfalls die Krisenanfälligkeit nicht sogar noch erhöht wird. Aufgrund der Neuartigkeit von Kryptowährungen und den beschränkten Praxiserfahrungen kann an dieser Stelle nicht abschließend festgehalten werden, ob das Kryptowährungssystem oder das traditionelle Geldsystem resilienter ist.

Ein Vorteil von Kryptowährungen im Zahlungsverkehr liegt darin, dass eine Zahlungstransaktion dezentral abgewickelt werden kann. Dennoch muss für eine Initialisierung und das Empfangen einer Zahlungstransaktion wieder auf einen Kryptowährungsdienstleister zurückgegriffen werden. Aufgrund der vertrauenswürdigen Geschäftsbanken wird der Mehrwert einer solchen dezentralen Zahlungstransaktion in der Schweiz als marginal eingestuft. Nebst diesem Vorteil verfügen die Kryptowährungen jedoch über diverse Probleme bzw. Limitationen, welche die Verwendung als Zahlungsmittel stark erschweren. Aus diesem Grund vereinfachen Kryptowährungen die Abwicklung von Zahlungstransaktionen in der Schweiz zum aktuellen Zeitpunkt nicht. Die Experten sind jedoch zuversichtlich, dass die Mehrheit dieser Probleme bzw. Limitationen durch neue technologische oder organisatorische Verfahren gelöst werden können. Trotzdem sieht die Mehrheit der Experten in der Schweiz aufgrund der effizienten Finanzmarktinfrastruktur und der Vielfalt an Zahlungsinstrumenten kein großes Bedürfnis nach einer weiteren Zahlungslösung. In einem solchen Szenario besteht dennoch das Potenzial, dass Micropayments effizienter durchgeführt und im internationalen Zahlungsverkehr die grenzüberschreitenden Zahlungstransaktionen vereinfacht sowie die finanzielle Inklusion der Unbanked People erhöht werden könnte.

Abschließend kann festgehalten werden, dass Kryptowährungen in der Schweiz aktuell primär als Spekulationsobjekt und weniger als Zahlungsmittel verwendet werden. Gerade dieser Spekulationsgedanke und das Disruptionspotenzial der zugrunde liegenden Blockchain-Technologie führen zu einer Diskrepanz zwischen Hype und Realität. Es ist kritisch anzumerken, dass hohe Erwartungen an die Kryptowährungen bestehen, jedoch noch kaum vorzeigbare Lösungen vorliegen. Zudem stellt sich die Frage ob Kryptowährungen für die obengenannten potenziellen Einsatzgebiete die zielführendste Lösung bilden oder die Probleme nicht mit einem anderen Lösungsansatz sogar noch zielgerichteter gelöst werden könnten. Die zukünftige Hauptherausforderung von Kryptowährungen liegt darin, ein tatsächliches Problem zu lösen und sich von einem Disruptionspotenzial hin zu einer nachhaltigen und mehrwertsteigernden Innovation zu entwickeln.

\section{Literatur}

Admati, A., \& Hellwig, M. (2014). The bankers' new clothes. Princeton: Princeton University Press.

Altcointoday. (2018). Bitcoin and Ethereum vs Visa and PayPal - Transactions per second. https:// altcointoday.com/bitcoin-ethereum-vs-visa-paypal-transactions-per-second/. Zugegriffen: 9. Juni 2018. 
Ankenbrand, T. (2018). Befragter Experte im Rahmen der durchgeführten qualitativen Analyse. Anonym. (2018). Befragter Experte im Rahmen der durchgeführten qualitativen Analyse.

Benes, J., \& Kumhof, M. (2012). The Chicago plan revisited. Washington: International Monetary Fund. https://www.imf.org/external/pubs/ft/wp/2012/wp12202.pdf. Zugegriffen: 9. Juni 2018.

Berner Kantonalbank. (2018). Privatkonto. https://www.bekb.ch/de/privatkunden/zahlen/zahlungskonten/privatkonto. Zugegriffen: 9. Juni 2018.

Bitcoin Magazine. (2018). Bitcoins made in China. https://bitcoinmagazine.com/articles/bitcoins-made-in-china-1399943910/. Zugegriffen: 9. Juni 2018.

Blanchard, O., \& Illing, G. (2009). Makroökonomie (5. Aufl.). München: Pearson Education Deutschland.

Blocher, W., Hanl, A., \& Michaelis, J. (2017). Revolutionieren Kryptowährungen die Zahlungssysteme? https://www.researchgate.net/publication/322951497. Zugegriffen: 9. Juni 2018.

Blockchain. (2018a). Hashrate Verteilung. https://blockchain.info/de/pools. Zugegriffen: 9. Juni 2018.

Blockchain. (2018b). Market price. https://blockchain.info/de/charts/market-price. Zugegriffen: 9. Juni 2018.

Bohnsack, R., Marotzki, W., \& Meuser, M. (2003). Hauptbegriffe Qualitative Sozialforschung; Ein Wörterbuch. Wiesbaden: VS Verlag.

Bonneau, J., et. al. (2015). SoK: Research perspectives and challenges for Bitcoin and cryptocurrencies. https://www.eecis.udel.edu/ ruizhang/CISC859/S17/Paper/survey1.pdf. Zugegriffen: 9. Juni 2018.

Brühl, V. (2017). Bitcoins, Blockchain und Distributed Ledgers, Funktionsweise, Marktentwicklungen und Zukunftsperspektiven. https://link.springer.com/content/pdf/10.1007\%2Fs10273-017-2096-3. pdf. Zugegriffen: 9. Juni 2018.

BTC-Echo. (2018). Wie funktioniert Bitcoin-Mining? https://www.btc-echo.de/tutorial/wie-kannich-bitcoins-minen/\#howto. Zugegriffen: 9. Juni 2018.

Bundesamts für Kommunikation. (2016). Analyse des Schweizer Zahlungsverkehrs. https://www. bakom.admin.ch/dam/bakom/de/dokumente/tc/Post\%20und\%20Presse/BAKBASEL\%20 Analyse_des_Schweizer_Zahlungsverkehr\%20.pdf.download.pdf/BAKBASEL\%20Analyse_ des_Schweizer_Zahlungsverkehr\%20vom\%2001.02.2016\%20mit\%20Korrektur\%20auf\%20 Seite\%2015.pdf. Zugegriffen: 9. Juni 2018.

Cambridge Dictionary. (2018). Currency. https://dictionary.cambridge.org/dictionary/english/currency. Zugegriffen: 9. Juni 2018.

CCN. (2015). Huobi accidentally sends 920 BTC and 8100 LTC to wrong account. https://www. cryptocoinsnews.com/huobi-accidentally-sends-920-btc-8100-ltc-wrong-account/. Zugegriffen: 9. Juni 2018.

Cointelegraph. (2014). Is Tim Swanson right that Bitcoin hoarding is bad? https://cointelegraph. com/news/is-tim-swanson-right-that-bitcoin-hoarding-is-bad. Zugegriffen: 9. Juni 2018.

Danthine, J. (2014). Hat Bargeld eine Zukunft? https://www.snb.ch/de/mmr/speeches/id/ ref_20140508_jpd/source/ref_20140508_jpd.de.pdf. Zugegriffen: 9. Juni 2018.

Deloitte. (2017). Mobile Payment, Kein Aufwärtstrend beim mobilen Bezahlen. https://www2. deloitte.com/de/de/pages/technology-media-and-telecommunications/articles/mobile-payment. html. Zugegriffen: 9. Juni 2018.

Demirguc-Kunt A., et. al. (2014). The global findex database 2014, measuring financial inclusion around the world. http://documents.worldbank.org/curated/en/187761468179367706/pdf/ WPS7255.pdf. Zugegriffen: 9. Juni 2018.

Deutsche Bundesbank. (2017). Geld und Geldpolitik. https://www.bundesbank.de/Redaktion/DE/ Downloads/Veroeffentlichungen/Schule_und_Bildung/geld_und_geldpolitik.pdf?_blob=publicationFile. Zugegriffen: 9. Juni 2018. 
European Central Bank. (2012). Virtual currency schemes. https://www.ecb.europa.eu/pub/pdf/ other/virtualcurrencyschemes201210en.pdf. Zugegriffen: 9. Juni 2018.

Financial Crisis Inquiry Commission. (2011). Final report of the national commission on the causes of the financial and economic crisis in the United States. http://www.bu.edu/econ/ files/2012/01/fcic_final_report_full.pdf.ZZugegriffen: 9. Juni 2018.

Financial Dictionary. (2018). Money. https://financial-dictionary.thefreedictionary.com/money. Zugegriffen: 9. Juni 2018.

Fisher, I. (2007). 100\% Geld. Kiel: Gauke.

Fortune. (2017). Bernanke thinks Bitcoin will fail (but he likes blockchain). http://fortune. com/2017/10/16/bitcoin-bernanke/. Zugegriffen: 9. Juni 2018.

Gardini, M. A. (2007). Einführung in das Marketing-Management: Ein Überblick in Schaubildform. München: Oldenbourg.

Frecè, J. (2018). Befragter Experte im Rahmen der durchgeführten qualitativen Analyse.

Goldman Sachs. (2014). All about Bitcoin. https://www.paymentlawadvisor.com/files/2014/01/ GoldmanSachs-Bit-Coin.pdf. Zugegriffen: 9. Juni 2018.

Hileman, G., \& Rauchs, M. (2017). Global cryptocurrency benchmarking study. https://www.jbs. cam.ac.uk/fileadmin/user_upload/research/centres/alternative-finance/downloads/2017-global-cryptocurrency-benchmarking-study.pdf. Zugegriffen: 9. Juni 2018.

Huber, J. (2013). Monetäre Modernisierung. Zur Zukunft der Geldordnung: Vollgeld und Monetative (3. Aufl.). Marburg: Metropolis.

Huber, J., \& Robertson, J. (2008). Geldschöpfung in öffentlicher Hand: Weg zu einer gerechten Geldordnung im Informationszeitalter. Kiel: Gauke.

Hungerland F., et. al. (2017). Die Zukunft des Geldes: Das Geld der Zukunft. https://www.econstor.eu/bitstream/10419/162390/1/890366063.pdf. Zugegriffen: 9. Juni 2018.

Hüsler, R. (2018). Befragter Experte im Rahmen der durchgeführten qualitativen Analyse.

Jaag, C. (2018). Befragter Experte im Rahmen der durchgeführten qualitativen Analyse.

Kneissler, T. (2018). Befragter Experte im Rahmen der durchgeführten qualitativen Analyse.

Koenig, A. (2017). Crypto Coins, Investieren in digitale Währungen. Münche: FinanzBuch.

Laeven, L., \& Valencia, F. (2012). Systemic banking crises database: An update. https://www. imf.org/en/Publications/WP/Issues/2016/12/31/Systemic-Banking-Crises-Database-AnUpdate-26015. Zugegriffen: 9. Juni 2018.

Lee, D. (2015). Handbook of digital currency, Bitcoin, innovation, financial instruments and big data. Amsterdam: Elsevier \& AP. http://aksitha.com/Big\%20Data\%20Technologies/ Big\%20Data/HANDBOOK\%20OF\%20DIGITAL\%20CURRENCY\%20-\%20Bitcoin, \%20 Innovation, \%20Financial\%20Instruments, \%20and\%20Big\%20Data\%20\%5B2015\%5D.pdf. Zugegriffen: 9. Juni 2018.

Lehmann, T. (2018). Befragter Experte im Rahmen der durchgeführten qualitativen Analyse.

Mayer, H. (2013). Interview und schriftliche Befragung, Grundlagen und Methoden empirischer Sozialforschung (6. Aufl.). München: Oldenbourg Wissenschaftsverlag.

Mayring, P. (2015). Qualitative Inhaltsanalyse, Grundlagen und Techniken (12. Aufl.). Weinheim: Beltz.

McLeay, M., Radla, A., \& Ryland, T. (2014). Money creation in the modern economy. Bank of England. https://www.bankofengland.co.uk/-/media/boe/files/quarterly-bulletin/2014/moneycreation-in-the-modern-economy.pdf. Zugegriffen: 9. Juni 2018.

Nakamoto, S. (2008). Bitcoin: A peer-to-peer electronic cash system. https://bitcoin.org/bitcoin. pdf. Zugegriffen: 9. Juni 2018.

NBG. Bundesgesetz über die Schweizerische Nationalbank vom 01.05.2004. https://www.admin. ch/opc/de/classified-compilation/20021117/index.html. Zugegriffen: 9. Juni 2018.

Oxford Dictionaries (2018). Currency. https://en.oxforddictionaries.com/definition/currency. Zugegriffen: 9. Juni 2018. 
P2P-Foundation. (2018). Bitcoin. https://wiki.p2pfoundation.net/bitcoin. Zugegriffen: 9. Juni 2018.

Park, Y. S. (2006). The inefficiencies of cross-border payments: How current forces are shaping the future. http://euro.ecom.cmu.edu/resources/elibrary/epay/crossborder.pdf. Zugegriffen: 9. Juni 2018.

Robleh Ali et. al. (2014). Innovations in payment technologies and the emergence of digital currencies. https://poseidon01.ssrn.com/delivery.php?ID $=40507210300309308809409108508612$ 70980180090660120370921231211130890290820731150981081101010570261231240541 1802910612401410209605800503704106001300703110107608210102704201000710611 71200930850300160881211150070031 . Zugegriffen: 9. Juni 2018.

Schweizerische Nationalbank. (2015). Statistisches Monatsheft. https://www.snb.ch/de/mmr/reference/stat_monatsheft_2015_08/source/stat_monatsheft_2015_08.de.pdf. Zugegriffen: 9. Juni 2018.

Schweizerische Nationalbank. (2016). Geldmengendefinition. https://www.snb.ch/de/mmr/reference/monpol_monstat_definition/source/monpol_monstat_definition.de.pdf. Zugegriffen: 9 . Juni 2018.

Siedenbiedel, C. (2012). Wie kommt Geld in die Welt? http://www.faz.net/aktuell/wirtschaft/wirtschaftswissen/geldschoepfung-wie-kommt-geld-in-die-welt-11637825.html. Zugegriffen: 9. Juni 2018.

SIX Group AG. (2018). Die Swiss Value Chain. https://www.six-group.com/dam/download/publications/swiss-value-chain-de.pdf. Zugegriffen: 9. Juni 2018.

SIX Interbank Clearing. (2016). Geschäftsbericht 2016. https://www.six-interbank-clearing.com/ dam/downloads/de/annual-report-2016.pdf. Zugegriffen: 9. Juni 2018.

SIX Interbank Clearing. (2018). Das Zahlungssystem SIC. https://www.six-interbank-clearing. com/de/home/payment-services/sic/sic-transaction-prices.html. Zugegriffen: 9. Juni 2018.

Sixt, E. (2017). Bitcoins und andere dezentrale Transaktionssysteme. Blockchain als Basis einer Kryptoökonomie. Wiesbaden: Springer Gabler. https://link.springer.com/content/ pdf/10.1007\%2F978-3-658-02844-2.pdf. Zugegriffen: 9. Juni 2018.

Solit Gruppe. (2018). https://www.solit-kapital.de/geld-ist-nicht-gleich-waehrung. Zugegriffen: 9. Juni 2018.

Sprock, A. (2018). Befragter Experte im Rahmen der durchgeführten qualitativen Analyse.

Stijin, C., \& Ayhan, K. (2013). International Monetary Fund: Financial crises explanations, types, and implications. https://www.imf.org/en/Publications/WP/Issues/2016/12/31/Financial-Crises-Explanations-Types-and-Implications-40283. Zugegriffen: 9. Juni 2018.

Swissbanking. (2016). Zahlungssysteme im Wandel. http://www.swissbanking.org/de/services/ insight/insight-3.16/zahlungssysteme-im-wandel. Zugegriffen: 9. Juni 2018.

The Corner. (2017). The huge fallacy of the modern monetary theory: Money is not free. http:// thecorner.eu/world-economy/the-huge-fallacy-of-the-modern-monetary-theory-money-is-notfree/62858/. Zugegriffen: 9. Juni 2018.

The Economist. (2013). https://www.economist.com/news/schoolsbrief/21584534-effects-financial-crisis-are-still-being-felt-five-years-article. Zugegriffen: 9. Juni 2018.

Thiele, C., \& Diehl, M. (2017). Kryptowährung Bitcoin: Währungswettbewerb oder Spekulationsobjekt, Welche Konsequenzen sind für das aktuelle Geldsystem zu erwarten? https://www. bundesbank.de/Redaktion/DE/Downloads/Presse/kryptowaehrung_bitcoin.pdf?__blob=publicationFile. Zugegriffen: 9. Juni 2018.

Tonkiss, F. (2009). Trust, confidence and economic crisis. https://www.ceps.eu/system/files/article/2009/09/196-202-Tonkiss.pdf. Zugegriffen: 9. Juni 2018.

Weltbank. (2014b). Financial inclusion. http://siteresources.worldbank.org/EXTGLOBALFINREPORT/Resources/8816096-1361888425203/9062080-1364927957721/GFDR-2014_Complete_ Report.pdf. Zugegriffen: 9. Juni 2018.

WZG. Bundesgesetz über die Währung und die Zahlungsmittel vom 22.12.1999. https://www. admin.ch/opc/de/classified-compilation/19994336/index.html. Zugegriffen: 9. Juni 2018. 
Wenger, Tobias (M.Sc. BA/wenger.tobias@bluemail.ch) Master of Business Administration (MBA) an der University of the Sunshine Coast, Australien und Master of Science (MSc) in Business Administration an der Berner Fachhochschule mit Vertiefung Corporate and Business Development. Langjährige Bankerfahrung. Aktuell als Vermögensberater Private Banking bei der Berner Kantonalbank AG beschäftigt.

Tokarski, Kim Oliver (Prof. Dr./kim.tokarski@bfh.ch) Professor für Entrepreneurship und Unternehmensführung und Leiter der Abteilung Weiterbildung am Departement Wirtschaft der Berner Fachhochschule. Er ist Gastprofessor an der Wirtschaftsuniversität Bukarest (ASE Bukarest), Rumänien. Seine Forschungs- und Lehrtätigkeiten liegen in den Bereichen Entrepreneurship, Social Entrepreneurship, Innovation, Digitalisierung, Strategie sowie Leadership. Er ist Autor unterschiedlicher Publikationen in den genannten Themenkontexten.

Open Access Dieses Kapitel wird unter der Creative Commons Namensnennung 4.0 International Lizenz (http://creativecommons.org/licenses/by/4.0/deed.de) veröffentlicht, welche die Nutzung, Vervielfältigung, Bearbeitung, Verbreitung und Wiedergabe in jeglichem Medium und Format erlaubt, sofern Sie den/die ursprünglichen Autor(en) und die Quelle ordnungsgemäß nennen, einen Link zur Creative Commons Lizenz beifügen und angeben, ob Änderungen vorgenommen wurden.

Die in diesem Kapitel enthaltenen Bilder und sonstiges Drittmaterial unterliegen ebenfalls der genannten Creative Commons Lizenz, sofern sich aus der Abbildungslegende nichts anderes ergibt. Sofern das betreffende Material nicht unter der genannten Creative Commons Lizenz steht und die betreffende Handlung nicht nach gesetzlichen Vorschriften erlaubt ist, ist für die oben aufgeführten Weiterverwendungen des Materials die Einwilligung des jeweiligen Rechteinhabers einzuholen.

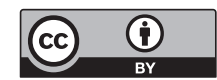




\title{
Das Potenzial von Smart Contracts an einem Beispiel aus der Finanzbranche - Smart Rating
}

\author{
Vincent Furrer und Klaus-Georg Deck
}

\begin{abstract}
Zusammenfassung
Dieser Beitrag stellt die Grundsätze von auf Smart Contracts und der Blockchain-Technologie basierenden Rating-Verfahren zur Bonitätsbewertung vor. Es werden die technologischen Grundlagen von Blockchain und Smart Contracts erläutert und diskutiert, inwieweit die Finanzbranche im Bereich des Ratings von dieser Technologie profitieren kann. Zunächst werden die Probleme traditioneller Ratingverfahren und deren Akteure thematisiert und anschließend Lösungsansätze aufgezeigt, um diese zu überwinden. Damit zeigt dieser außerhalb der traditionellen Bankdienstleistungen befindliche Anwendungsfall, dass mit der Blockchain-Technologie neue Potenziale für Geschäftsmodelle entstehen, die etablierte Prozesse grundlegend verändern und damit ein weiteres Mosaik zur Disruption der Finanzbranche darstellen können.
\end{abstract}

\section{$11.1 \quad$ Einleitung}

Die hohen Kursschwankungen der Kryptowährungen sind unverkennbar. Sie können als Symptom für eine indifferente und uneinheitliche Bewertung der zugrunde liegenden Blockchain-Technologie gewertet werden. Einige sehen in ihr ein außerordentlich hohes Innovationspotenzial, insbesondere bei der schnelleren Abwicklung von Prozessen

V. Furrer $(\bowtie)$

Bern, Schweiz

K.-G. Deck

Berner Fachhochschule Wirtschaft, Bern, Schweiz

E-Mail: klaus-georg.deck@bfh.ch 
bei gleichzeitig tiefen Transaktionskosten, andere dagegen warnen vor schwer kalkulierbaren Risiken und grundsätzlich vor zu hohen Erwartungen.

Ein sehr großes Potenzial wird in den sogenannten Smart Contracts gesehen (Jakob et al. 2018, S. 1). Diese ,intelligenten Verträge“ werden auf einer Blockchain definiert und garantieren die Einhaltung ihrer Bedingungen sowie die Ausführung der hinterlegten Konsequenzen als Folgeprozesse. Aufgrund der automatisierten Verarbeitung werden Transaktionskosten reduziert und gleichzeitig die Vertragssicherheit erhöht. Auf der anderen Seite sind die Vertragsteilnehmer den in einer Software codierten Bedingungen ausgeliefert. Hier besteht die Gefahr einer technisch fehlerhaften Programmierung, aber auch, dass die Vertragsbedingungen nicht den Erwartungen der Beteiligten entsprechen.

Auf Smart Contracts basierende Anwendungsszenarien existieren mittlerweile für so gut wie alle Branchen, insbesondere in der Industrie, der Logistik und der Finanzbranche. Unternehmen schließen sich zu Konsortien zusammen, um auf eigenen Blockchain-Plattformen bestehende Prozesse abzubilden und neue vielversprechende Geschäftsmodelle zu etablieren.

Anhand eines auf Smart Contracts und der Blockchain-Technologie basierenden Rating-Verfahrens zur Bonitätsbewertung soll das Potenzial dieser Technologie veranschaulicht und auf die sich stellenden Herausforderungen aufmerksam gemacht werden. Es wird eine dezentrale autonome Organisation entwickelt, welche mithilfe von Smart Contracts ein automatisiertes Emittenten-Rating für ein multinationales Unternehmen generiert. Das dargestellte Verfahren beseitigt das mit dem Paradigma ,der Emittent bezahlt" bezeichnete Grundproblem des traditionellen Geschäftsmodells groBer Rating-Agenturen, und damit auch die damit verbundenen Implikationen. Zudem zeigt sich an diesem Beispiel, wie in Zukunft ähnliche Geschäftsmodelle und Geschäftsprozesse gestaltet und implementiert werden können.

Im nächsten Kapitel werden die technologischen und begrifflichen Grundlagen zu Blockchain und Smart Contracts dargestellt und anschließend die traditionellen Ratingprozesse in der Finanzbranche sowie deren Bedeutung für die Wirtschaft und die Probleme dieses Geschäftsmodells beschrieben. Im Hauptteil wird das Smart-Rating-Szenario entwickelt und erläutert, wie eine dezentrale autonome Organisation mit verschiedenen Netzwerkteilnehmern strukturiert sein könnte und welche Rahmenbedingungen erfüllt sein müssen, um einen solchen Rating-Prozess zu realisieren. Abschließend folgen das Fazit und ein Ausblick in mögliche Szenarien von blockchainund smart-contract-basierten Anwendungen im Finanzumfeld.

\subsection{Technologische Grundlagen}

In diesem Kapitel werden die technologischen Grundlagen dargestellt und einige Fachbegriffe expliziert, soweit sie zum Verständnis von smart-contract-basierten Szenarien benötigt werden. 


\subsubsection{Blockchain-Technologie}

Bei einer Blockchain handelt es sich um eine dezentrale, verteilte Datenbank innerhalb eines Peer-to-Peer-Netzwerks, die von vielen gleichberechtigt genutzt werden kann. Informationen über mehrere Transaktionen werden in Einheiten (Blöcken) zusammengefasst, die unter anderem einen kryptografischen Schlüssel über die Daten des aktuellen Blocks und des Schlüssels des Vorgängerblocks enthalten. Da also jede Information eines Blocks über den Schlüssel in den nachfolgenden Block eingeht, entsteht dadurch eine unveränderliche, manipulationssichere Kette von Informationsblöcken (Blockchain). Sollte jemand eine Transaktion verändern wollen, müssten mit dem Block, der diese Transaktionsinformation enthält, alle Nachfolgerblöcke angepasst werden, was wegen der redundant im Netzwerk verteilten Information so gut wie unmöglich ist.

Eine Blockchain garantiert sowohl die Authentizität der Benutzer als auch die Unveränderbarkeit der Daten. Im Gegensatz zu klassischen Systemen (etwa einem Datenbank-System oder einem ERP-System) gibt es keine zentrale Instanz, die diese Eigenschaften als Intermediär sicherstellt, sondern die Teilnehmer der Blockchain agieren gleichzeitig als deren Betreiber. Die Übereinstimmung innerhalb des Netzwerkes und damit das Vertrauen unter den Teilnehmenden wird durch einen für jede einzelne Blockchain fest integrierten Konsensmechanismus festgestellt (Jakob et al. 2018, S. 2 ff.).

Je nach intendiertem Anwendungsszenario wird zwischen öffentlichen und privaten Blockchains unterschieden. Öffentliche Blockchains bilden häufig die technologische Basis für Kryptowährungen und sind grundsätzlich für jeden offen. Das unter dem Pseudonym Satoshi Nakamoto (Nakamoto 2008) als Reaktion auf die Bankenkrise veröffentlichte Dokument „Bitcoin: A Peer-to-Peer Electronic Cash System“ und die kurze Zeit später bereitgestellte öffentliche Bitcoin-Implementierung gelten als Ursprung aller Kryptowährungen und der Blockchain-Ideologie überhaupt.

Private Blockchains dagegen werden primär im unternehmerischen und industriellen Bereich verwendet, wobei der Kreis der Teilnehmenden von vornherein auf die am jeweiligen Geschäftsszenario beteiligten Akteure beschränkt wird. Typische Anwendungsfelder sind im Supply Chain Management genauso zu finden wie in der Versicherungsbranche oder im Kreditgeschäft.

\subsubsection{Smart Contracts}

Unter Smart Contracts versteht man in Software realisierte, ausführbare Programme oder Module, welche die Logik vertraglicher Bedingungen in einer Wenn-Dann-Struktur technisch realisieren und beim Eintreten der relevanten Ereignisse definierte Aktivitäten automatisiert auslösen.

Nick Szabos Beispiel eines Getränkeautomaten, der bei hinreichendem Geldbetrag die Wasserflasche unwiderruflich bereitstellt und einen eventuellen Restbetrag automatisiert ausbezahlt, verdeutlicht plakativ die Funktionsweise eines solchen Smart 
Contract (vgl. Diedrich 2016, S. 166; Jakob et al. 2018, S. 4). Denkbar sind aber auch umfassendere Szenarien, etwa in der Versicherungsbranche, wenn sich ein Kfz-Versicherungsnehmer einer vordefinierten Abhängigkeit zwischen Versicherungsprämie und Nutzungsdaten (Nutzungsdauer und Kilometerleistung) unterwirft. Einem in dieser Weise realisierten Smart Contract würden die erforderlichen Informationen über eine Schnittstelle verfügbar gemacht und die dynamisch ermittelte Prämie würde zwischen den Konten der Beteiligten automatisch verbucht, im Idealfall als Payment-Service innerhalb der gleichen Blockchain.

Werden Smart Contracts innerhalb einer Blockchain realisiert, entfällt auch die Rolle des Intermediärs (z. B. Banken, Notare), da das Vertrauen auf den Sicherheitsund Konsensmechanismen der Blockchain-Technologie basiert. Aufgrund der Automatisierung und der Reduktion des Verwaltungs- und Bearbeitungsaufwandes führt dies zu deutlich schnelleren Ausführungszeiten und kostengünstigeren Transaktionen. Notwendige Voraussetzung sind jedoch die digitale Repräsentierbarkeit der beteiligten Ereignisse sowie die Verfügbarkeit der entsprechenden Daten über Schnittstellen (Jakob et al. 2018, S. 6).

\subsubsection{Blockchain Oracle}

Smart Contracts sind vielfach auf Informationen über die reale Welt angewiesen, etwa über die Wettersituation bei Schadenereignissen, über Zugausfälle und Flugverspätungen, bis hin zu Marktdaten und Informationen über die Finanzwelt. Da eine Blockchain ein in sich geschlossenes System bildet und somit kein direkter Zugriff aus ihr auf externe Daten möglich ist, werden diese durch sogenannte Oracles verfügbar gemacht. Ziel und Aufgabe eines Oracles ist einerseits die Datenbeschaffung, -bewertung und -aggregation sowie deren Bereitstellung für eine Blockchain unter Wahrung von Datensicherheit und Datenauthentizität (Diedrich 2016, S. 187).

\subsubsection{Dezentralisierte Automatische Organisation - DAO}

Eine dezentralisierte autonome Organisation (DAO) ist eine selbstgesteuerte Organisation, die auf der Blockchain-Technologie und Smart Contracts beruht. Die Organisation wird durch die vorprogrammierten Regeln und Bedingungen der Smart Contracts gesteuert und bestimmt. Aktivitäten einer DAO können nicht gestoppt werden und es ist schwierig, eine bereits implementierte Funktionalität zu ändern. Ein bekanntes Beispiel einer selbstgesteuerten Organisation ist „The DAO“, welche auf der Ethereum-Blockchain beruht. An diesem Projekt zeigten sich eindrucksvoll die Schwachstellen eines DAO, indem Angreifer in kleinen Einheiten Beträge (der Kryptowährung Ether) entnommen haben. Auch wenn dies nicht unbeobachtet geschah, konnte niemand etwas dagegen tun; der Angreifer hatte sich einen Programmierfehler zunutze gemacht, welcher 
es ihm erlaubte, diese Beträge gemäß den formal - wenn auch nicht semantisch - korrekten - Regeln des DAO abzuziehen (Hertig 2018).

\subsubsection{Dezentralisierte Applikation - DApp}

Bei einer dezentralisierten Applikation (DApp) handelt es sich um eine verteilte OpenSource-Anwendung, die weder von einem Einzelnen entwickelt noch betrieben wird. Sie basiert auf einer Blockchain, über die verschlüsselte Token erzeugt und angeboten werden. Damit spiegeln DApps das Blockchain-Paradigma eines verteilten, dezentralen Systems ohne Intermediär wider (Diedrich 2016, S. 164 f.). DApps gelten als sehr vertrauenswürdig, da ihr Coding als Open-Source-Software in der Blockchain sicher verwahrt wird.

\subsubsection{Initial Coin Offerings - ICO}

Initial Coin Offering (ICO) stellt eine Methode der Kapitalaufnahme dar, mit der typischerweise Start-Ups oder Projekte im Blockchain-Umfeld finanziert werden. Investoren erhalten im Gegenzug digitale Coins oder Token, die später entweder zur Nutzung der neu entwickelten Softwarelösung verwendet werden können oder an einer digitalen Börse getauscht werden. Der Unterschied zwischen beiden liegt darin, dass Coins auf einer eigenen Blockchain basieren, wogegen sich Token auf eine andere, bereits bestehende Blockchain, beziehen. Im Jahr 2017 brachten 430 ICOs insgesamt 4,6 Mrd. US\$ ein und haben sich damit zur beliebtesten Projekt- und Start-upFinanzierungsmethode im Blockchain-Umfeld entwickelt (Torcasso 2018).

\subsection{Rating-Prozesse in der Finanzbranche}

Nachfolgend wird die Bedeutung von Ratings für die Wirtschaft erläutert. Ein möglicher Rating-Prozess einer Rating-Agentur wird dargestellt, Rating-Kriterien und die Einordnungen von Ratings in Rating-Klassen werden aufgezeigt. Anschließend erfolgt eine Beschreibung der Problemstellungen des traditionellen Geschäftsmodells von Rating-Agenturen und abschließend wird die Regulierung von Rating-Agenturen erläutert.

\subsubsection{Bedeutung für die Wirtschaft}

Anfang der 1970er-Jahre, als Kreditrisiken vermehrt wahrgenommen wurden, setzte das internationale Wachstum der drei großen Rating-Agenturen Moody's, Standard \& Poor's und Fitch Ratings ein, zudem entstanden seither zahlreiche neue Agenturen (Buschmeier 2011, S. 154 f.). 
Ratings sollen einen Indikator für die Kreditwürdigkeit einer Institution darstellen und die Bonität des potenziellen Schuldners sowie das Ausfallrisiko eines Kredits repräsentieren. Ein potenzieller Kreditnehmer mit einem guten Rating wird zu besseren Bedingungen Fremdkapital beziehen können als ein Interessent mit schlechter Bonität. Viele Unternehmen, Institution und Staaten sind daher auf ein gutes Rating anerkannter Rating-Agenturen angewiesen, denn ohne oder mit eher schlechten Ratings werden sie von Kapitalgebern nicht oder kaum beachtet.

Kredit-Ratings werden wie folgt differenziert (vgl. Ott 2011, S. 5):

Beurteilungsgegenstand: Emission/Emittent

Zeitlicher Bezug: Langfristig/Kurzfristig

Währung: Landeswährung/Fremdwährung

Beauftragung: Beauftragtes Rating/Public-Information-Rating/Unsolicited Rating

\subsubsection{Rating-Prozess}

Die detaillierten Rating-Verfahren werden von den Rating-Agenturen als Geschäftsgeheimnis betrachtet und nicht offengelegt. Aus diesem Grund werden nachfolgend nur die Schritte eines beauftragten öffentlich einsehbaren Ratings aufgeführt.

In einer ersten Phase wird das auftraggebende Unternehmen über das anzuwendende Rating-Verfahren informiert. Danach sammeln Analysten aus einem zusammengestellten Team interne und externe Informationen. Die benötigten internen Informationen zu den Finanzen und zur allgemeinen Natur des Unternehmens müssen offengelegt werden. In weiteren Diskussionsrunden mit Unternehmensvertretern werden die Stärken und Schwächen des Unternehmens, die allgemeine Situation der entsprechenden Branche und diverse andere Aspekte thematisiert. Nach weiteren Abklärungen und der abgeschlossenen Informationssammlung erfolgt mithilfe einer branchenspezifischen Rating-Methodik eine Erstanalyse. In einem nächsten Schritt wird der führende Analyst dem Rating-Komitee eine Rating-Empfehlung abgeben. Den Entscheid über das Rating fällt die Mehrheit dieses Komitees. Das auftraggebende Unternehmen wird über den Entschluss des Rating-Komitees informiert und das Rating wird auf der Webseite der jeweiligen Rating-Agentur und in der Finanzpresse publiziert. Unternehmens-Ratings und Wertschriften-Ratings werden danach mindestens alle 12 Monate überprüft, Staats-Ratings alle 6 Monate (vgl. Moody's 2018).

\subsubsection{Rating-Kriterien}

Gemäß Six Swiss Exchange (Six Swiss Exchange 2018) wird ein Rating in vier verschiedene Kategorien von Faktoren unterteilt. Dies sind quantitative, qualitative, Erfahrungs- und Umweltfaktoren. Je nach Branche oder Industrie werden andere Faktoren 
oder Kriterien zusätzlich berücksichtigt. Ebenfalls kann die Gewichtung dieser Faktoren und Kriterien unterschiedlich ausfallen. Buschmeier (2011, S. 150 ff.) führt als Beispiele von quantitativen Faktoren die Eigenkapitalquote, den Cashflow, die Kapitalstruktur, die Liquidität, die Eigenkapitalrendite und weitere auf. Als qualitative Faktoren werden dort unter anderen die Branchenstellung, die Produktstellung, die Marktstellung, das Management, die Strategie, die interne Wertschöpfung, die Planung, die Finanzpolitik genannt. Erfahrungsfaktoren können beispielsweise die Einhaltung von Verträgen, die termingerechte Zahlung von Zinsen oder die Lieferanten- und Kundenbeziehungen beinhalten. Schließlich können Faktoren wie die Branchenentwicklung oder die Standortbestimmung unter den Umweltfaktoren für die Ermittlung eines Ratings mit einbezogen werden.

\subsubsection{Rating-Klassen}

Die Tab. 11.1 zeigt die Rating-Klassen nach Standard \& Poor's und Moody's und die Unterteilung dieser Klassen in Investment Grade und Speculative Grade Ratings.

\subsubsection{Probleme des Geschäftsmodells}

Fehlerhafte oder unangemessene Ratings können verheerende Folgen haben, wie etwa die Bankrotte von Enron und WorldCom zeigen. Enron ist von den drei großen Rating-Agenturen erst viel zu kurz vor dem Bankrott von einem „Investment Grade“ zu „Speculative Grade“ herabgestuft worden. Vor dem Ausbruch der Finanzkrise sind hypothekarisch gesicherte Wertpapiere zu gut bewertet worden. In diesen Fällen wogen sich viele Anleger aufgrund der zu guten Ratings in einer unangebrachten Sicherheit (Mathis et al. 2009, S. 657 ff.). Im prominenten Fall Lehman Brothers wurde dieses Unternehmen bis zum Tag der Eröffnung des Gläubigerschutzverfahrens hervorragend

Tab. 11.1 Rating-Klassen. (Eigene Darstellung in Anlehnung an Boerse Stuttgart GmbH 2018)

\begin{tabular}{|c|c|c|c|}
\hline & S\&P & Moody's & Klassenbeschreibung \\
\hline \multirow[t]{4}{*}{ Investment Grade } & AAA & Aaa & Sehr gute Bonität \\
\hline & $\mathrm{AA}+; \mathrm{AA} ; \mathrm{AA}-$ & Aa1; Aa2; Aa3 & Sehr gute bis gute Bonität \\
\hline & $\mathrm{A}+; \mathrm{A} ; \mathrm{A}-$ & $\mathrm{A} 1 ; \mathrm{A} 2 ; \mathrm{A} 3$ & $\begin{array}{l}\text { Gute bis befriedigende Boni- } \\
\text { tät }\end{array}$ \\
\hline & $\mathrm{BBB}+$; BBB; BBB- & Baa1; Baa2; Baa3 & Befriedigende Bonität \\
\hline \multirow[t]{4}{*}{ Speculative Grade } & $\mathrm{BB}+; \mathrm{BB} ; \mathrm{BB}-$ & $\mathrm{Ba} 1 ; \mathrm{Ba} 2 ; \mathrm{Ba} 3$ & Ausreichende Bonität \\
\hline & $\mathrm{B}+; \mathrm{B} ; \mathrm{B}-$ & B1; B2; B3 & Mangelhafte Bonität \\
\hline & $\mathrm{CCC}+$; $\mathrm{CCC}$; $\mathrm{CCC}-$ & Caa1; Caa2; Caa3 & Ungenügende Bonität \\
\hline & D & D & Fehlende Bonität \\
\hline
\end{tabular}


bewertet (Standard \& Poor's: A, Moody's: A2, Fitch: A+), obwohl es schon einige Monate zuvor deutliche Anzeichen von Problemen im Unternehmen gab (Wildmoser et al. 2009, S. 660).

Ein weiteres Problem betrifft das Phänomen einer selbsterfüllenden Prophezeiung. Wird ein Emittent schlecht bewertet, sinkt seine Reputation und es steigt seine Zinslast, was sich negativ auf sein Geschäftsergebnis und damit auf rating-relevante Kennzahlen auswirkt. Gärtner und Griesbach (2012) gehen sogar so weit, dass sie diesen Effekt als zentralen Auslöser der europäischen Schuldenkrise ausmachen.

Nachfolgend werden einige der in der Literatur aufgeführten Problemstellungen zum Geschäftsmodell der Ratingagenturen aufgeführt, die später bei der Ausarbeitung des auf Smart Contracts basierten Rating-Prozesses vermieden werden sollen.

\section{Die Macht der Rating-Agenturen in der Wirtschaft}

Das Rating einiger europäischer Staaten wurde in den letzten Jahren herabgestuft. Ein sogenanntes Downgrade impliziert eine gesunkene Kreditwürdigkeit, was dazu führt, dass dem Kreditgeber für das gestiegene Risiko mehr Zinsen bezahlt werden müssen. Ein weiterer Aspekt, welcher die Macht der Rating-Agenturen zu Auge führt, besteht darin, dass etwa Pensionskassen und institutionelle Anleger nur Papiere eines Schuldners mit einem Investmentgrade halten dürfen. Durch ein Downgrade einer Rating-Agentur können zahlreiche Anleger nahezu gleichzeitig gezwungen werden, bestimmte Wertpapiere verkaufen zu müssen, was auf dem Markt zu einem Überangebot führt (NZZ 2016). Es wird eine Abwärtsspirale in Gang gesetzt, welche nur schwer aufzuhalten ist.

\section{Oligopolistischer Markt}

Zurzeit sind lediglich zehn Rating-Agenturen in den USA (SEC 2017) und fünf in der Schweiz national anerkannt (FINMA o. D.). In der EU sind 26 Rating-Agenturen anerkannt (ESMA 2017). Gemessen am Umsatz teilen sich die drei großen Rating-Agenturen, Moody's, Standard \& Poor's und Fitch Ratings, einen Marktanteil von 93 \% in Europa. In Amerika beträgt der Marktanteil dieser Rating-Agenturen sogar $96 \%$ gemessen an der Anzahl Ratings (SEC 2017).

\section{Interessenskonflikte bei den beauftragten Ratings}

Das verbreitetste Geschäftsmodell der Rating-Agenturen, ,,der Emittent bezahlt“, bringt Interessenskonflikte mit sich. Der Emittent wählt selber die Rating-Agentur aus, von der er geratet werden wird und bezahlt diese für das Rating. Nach White (2010) liegt der Interessenskonflikt bei einer Rating-Agentur darin, dass aus Reputationsgründen der Rating-Agentur ein qualitativ adäquates Rating ermittelt werden soll, aber gleichzeitig der Kunden zufrieden gestellt werden muss, der an einer hohen Bonität interessiert ist.

\section{Asymmetrische Information}

Dem Emittenten stehen mehr Informationen über sein Unternehmen zur Verfügung als dem Investor. Die Behebung dieser Informationsasymmetrie wird häufig als Argument 
gesehen, wieso es überhaupt Ratingagenturen gibt. Nach Rhee (2015) besteht die Aufgabe von Ratingagenturen jedoch weniger in der Beschaffung und Veröffentlichung neuer Informationen als in der Ordnung, Sortierung und Aggregation der großen Menge an bereits öffentlich zugänglichen Daten. Wenn Emittenten gegenüber der Rating-Agentur interne Informationen offen legen, dann - aus verständlichen Gründen - besonders solche, die zu einer guten Bewertung führen. Daher ist davon auszugehen, dass durch diese Bewertungspraxis die Informationsasymmetrie nicht beseitigt wird.

\section{Reine Meinungsäußerung}

Die Rating-Vergabe gilt als reine Meinungsäußerung. Alle drei großen Rating-Agenturen, Moody's (2018), Standard und Poor's (2018) und Fitch Ratings (2018) bekräftigen das auf ihren Webseiten. Aus diesem Grund müssen Rating-Agenturen nur bei extremen Fällen für fehlerhafte Ratings haften (Buschmeier 2011, S. 174). Nach Däubler (2012, S. 18) hat ein positives Rating auch für die US-Gerichtsbarkeit keine größere Bedeutung als die Würdigung einer Stadt oder Region in einem Reiseführer.

\section{Mangelnde Transparenz}

Die großen drei Rating-Agenturen haben keine detaillierten Angaben über ihre Rating-Prozesse veröffentlicht. Daher ist der Öffentlichkeit nicht bekannt, mithilfe welcher Methoden ein Rating zustande kommt. So verursachte etwa eine Herabstufung von Thyssen-Krupp durch Standard \& Poor's aufgrund einer nicht nachvollziehbaren Neubewertung von Pensionsverpflichtungen zusätzliche Zinskosten für das Unternehmen in Höhe von 30 Mio. EUR pro Jahr (Däubler 2012, S. 19).

\subsubsection{Aufsicht und Regulierung}

In diesem Abschnitt werden einige ausgewählte Regulatoren genannt und die wichtigsten Regelwerke für die Regulierung von Ratingagenturen vorgestellt. Dabei soll dargestellt werden, dass weltweit tätige Ratingagenturen mit unterschiedlichen Regulatoren und Regelwerken konfrontiert sind.

Bei der eidgenössischen Finanzmarktaufsicht (FINMA 2018) und der U. S. Securities and Exchange Commission (SEC 2018) handelt es sich um nationale Aufsichtsbehörden, die Wertpapier- und Marktaufsichtbehörde (ESMA 2018) ist die Aufsichtsbehörde der EU. Die Anerkennung durch die jeweilige Aufsichtsbehörde ist für eine Rating-Agentur wichtig, denn Ratings von anerkannten Rating-Agenturen werden oft für regulatorische Zwecke verwendet (Buschmeier 2011, S. 181).

Der Verhaltenskodex der IOSCO (2015) stellt ein internationales Regelwerk und einen Leitfaden für die Rating-Agenturen dar, wobei es sich um einen freiwilligen Rahmen handelt und keine automatischen Sanktionen zu befürchten sind, sollte sich eine Rating-Agentur nicht daran halten (Buschmeier 2011, S. 183). Bei den Grundsätzen des Verhaltenskodex handelt es sich um folgende Aspekte: 
- Qualität und Integrität des Kreditrating-Prozesses

- Unabhängigkeit und Freiheit von Interessenskonflikten

- Transparenz und Offenlegung betreffend die Rating-Aktivität

- Vertrauliche Behandlung der zur Verfügung gestellten Informationen

Das Financial Stability Board (FSB 2018), eingerichtet von den G20-Staaten, ist eine internationale Organisation, welche das globale Finanzsystem überwacht und Empfehlungen abgibt.

Die Regelwerke Basel II und III des Basler Ausschusses für Bankenaufsicht (BIS 2018) sind ebenfalls relevant für Rating-Agenturen. Bei Basel II werden unter anderem die externen Ratings von Rating-Agenturen für die Bestimmung der Kreditrisiken berücksichtigt. Bei Basel III wird unter anderem festgehalten, dass man sich weniger auf externe Ratings abstützen will.

\subsection{Smart Rating}

\subsubsection{Einführung}

Bei Smart Rating handelt es sich um eine DAO, welche ein stark automatisiertes Rating-Verfahren ermöglicht. Es handelt sich um ein beauftragtes Emittenten-Rating für multinationale Unternehmen. Bei der Erläuterung des Beispiels wird das Potenzial von Smart Contracts und deren Rolle in diesem Anwendungsfall in den Vordergrund gestellt. Zudem wird auf die Ausgestaltung eines möglichen Netzwerkes Bezug genommen. Das erarbeitete Beispiel basiert auf einer tiefgreifenden Literaturrecherche und Gesprächen mit potenziellen Anspruchsgruppen. Einige Inputs aus den Gesprächen sind in das Beispiel eingeflossen, manch andere konnten dagegen nicht berücksichtigt werden. Bei der Ausgestaltung von Smart Rating sind verschiedenen Gruppen von Netzwerkteilnehmern und äußere Rahmenbedingungen definiert worden. Zudem wurde ein mögliches Anreizsystem beschrieben. Schließlich werden die Vorteile von Smart Rating gegenüber einem klassischen Emittenten-Rating aufgeführt sowie die Herausforderungen, welche sich bei einer Umsetzung ergeben würden.

\subsubsection{Netzwerkteilnehmer}

Nachfolgend werden die verschiedenen Gruppen von Netzwerkteilnehmern vorgestellt.

Experten

Eine Gruppe von Branchen- und Rating-Experten wird nach vordefinierten Kriterien ausgewählt und mit der Erarbeitung der Rating-Vorlage beauftragt. Diese natürlichen Personen bestimmen, welche quantitativen Kennzahlen, qualitative Kriterien, Erfahrungswerte oder Umweltfaktoren für ein Rating berücksichtigt und wie 
diese gewichtet werden sollen. Sie werden für die Mitarbeit entlohnt und können ein Rating gegen Bezahlung abonnieren.

Rater

Rater, natürliche Personen mit Branchenkenntnissen, werden nach vordefinierten Kriterien für die Beurteilung der qualitativen Faktoren ausgewählt. Die Netzwerkreputation eines Raters entscheidet über die Gewichtung einer Beurteilung und die Entschädigung für eine Abgabe einer Beurteilung. Diese Gruppe kann zudem Ratings entgeltlich abonnieren.

Emittent Der Emittent ist eine juristische Person und der Auftraggeber eines Ratings. Bei der Auftragsabgabe, muss er die für das Rating relevanten Unternehmensdaten über seine blockchain-basierte digitale Unternehmens-ID freigeben und einen bestimmten Betrag an Rating-Coins hinterlegen. Nachdem ein Rating-Prozess durchgeführt werden konnte, wird das in Auftrag gegebene Rating automatisch dem Emittenten zugeordnet.

Reine Abonnenten Die reinen Abonnenten abonnieren - wie der Name schon sagt lediglich die Ratings. Sie sind natürliche oder juristische Personen, in der Regel private oder institutionelle Anleger

\subsubsection{Rahmenbedingungen}

In diesem Abschnitt werden die Rahmenbedingungen für eine mögliche Realisierung von Smart Rating festgelegt.

\section{Smart Rating DApp}

Die DApp ist die Schnittstelle zwischen den Netzwerkteilnehmern und dem Smart Rating DAO. Sie greift automatisch auf die notwendigen Informationen der Netzwerkteilnehmer zu, insbesondere auf Bilanzen, Erfolgsrechnungen, Geldflussrechnungen, Zahlungsverläufe, Umweltfaktoren, digitale Lebensläufe etc. Zudem können die Netzwerkteilnehmer über neue Aufträge und Änderungen bei abonnierten Ratings informiert werden. Um mit dem Smart-Rating-DAO interagieren zu können, muss jeder Netzwerkteilnehmer die DApp herunterladen.

\section{Blockchain-basierte digitale Identität}

Für die Erarbeitung von Smart Rating ist die blockchain-basierte digitale ID aus zwei Gründen essenziell. Zum einen muss jeder Emittent, der geratet werden soll, eine Unternehmens-ID hinterlegen, der die relevanten unternehmensspezifischen Kennzahlen (Bilanzen, Erfolgsrechnungen, Geldflussrechnungen etc.) hinzugefügt werden. Damit die Korrektheit dieser Finanzzahlen gewährleistet ist, muss eine anerkannte Institution, wie beispielsweise eine Revisionsstelle, dies mit ihrer digitalen Signatur bestätigen. Der zweite Grund liegt darin, dass mithilfe der blockchain-basierten digitalen ID passende Experten und Rater für ein Emittenten-Rating ermittelt werden können. 


\title{
Oracle
}

Die Informationen $\mathrm{zu}$ den definierten Umweltfaktoren werden über geeignete Oracles gewonnen. Ein solches Oracle könnte beispielsweise Informationen über einen bestimmten Branchenindex beinhalten oder über die konjunkturelle Lage oder die Inflationsrate eines Landes.

\section{ICO}

Das Smart-Rating-DAO soll mithilfe eines ICO finanziert werden. Ein Teil der Rating-Coins würde im Rahmen des ICO öffentlich verteilt und ein anderer Teil würde dem DAO zugeschrieben werden. Die nötigen Werbeausgaben, um eine kritische Masse zu erreichen, sowie die Löhne der Programmierer, welche die Codes des Smart RatingDAO schreiben, werden damit beglichen. Zudem benötigt der Emittent Rating-Coins, um einen Rating-Auftrag aufzugeben. Rater und Experten benötigen Rating-Coins, damit sie von ihrem Stimmrecht Gebrauch machen können und eine Beurteilung zu den qualitativen Kriterien abgeben können.

\subsubsection{Rating-Prozess}

Die Abb. 11.1 und 11.2 stellen den Ratingprozess eines in Auftrag gegebenen Emittenten-Rating für ein multinationales Unternehmen dar.

\begin{abstract}
Aktualisierungsprozess
Um die Aktualität eines Ratings sicherzustellen, ist das Verfahren regelmäßig, im Idealfall täglich, durchzuführen. Die Aktualisierung der quantitativen Faktoren sowie der Erfahrungsfaktoren erfolgt über die blockchain-basierte digitale Unternehmens-ID. Die Aktualisierung der Umweltfaktoren erfolgt ebenfalls täglich mithilfe branchenspezifischer Oracles. In einer späteren Ausbaustufe des Prozesses wäre auch eine automatisierte, ereignisgesteuerte Aktualisierung über digitale Agenten denkbar. Bei den qualitativen Faktoren kommt es in größeren Zeitperioden (etwa halbjährlich) zu einer Neubewertung. Indem die quantitativen sowie die Erfahrungs- und Umweltfaktoren täglich aktualisiert werden, verfügt ein Investor bereits früh über Indikatoren bezüglich einer möglichen Veränderung des Ratings.
\end{abstract}

\subsubsection{Anreizsystem}

Smart Rating benötigt ein Anreizsystem, bestehend aus einem Reputationssystem und einem Vergütungssystem, damit genügend Experten für die Erarbeitung einer Vorlage zur Verfügung stehen und genügend Rater bei der Bewertung der qualitativen Faktoren eines Emittenten teilnehmen. 


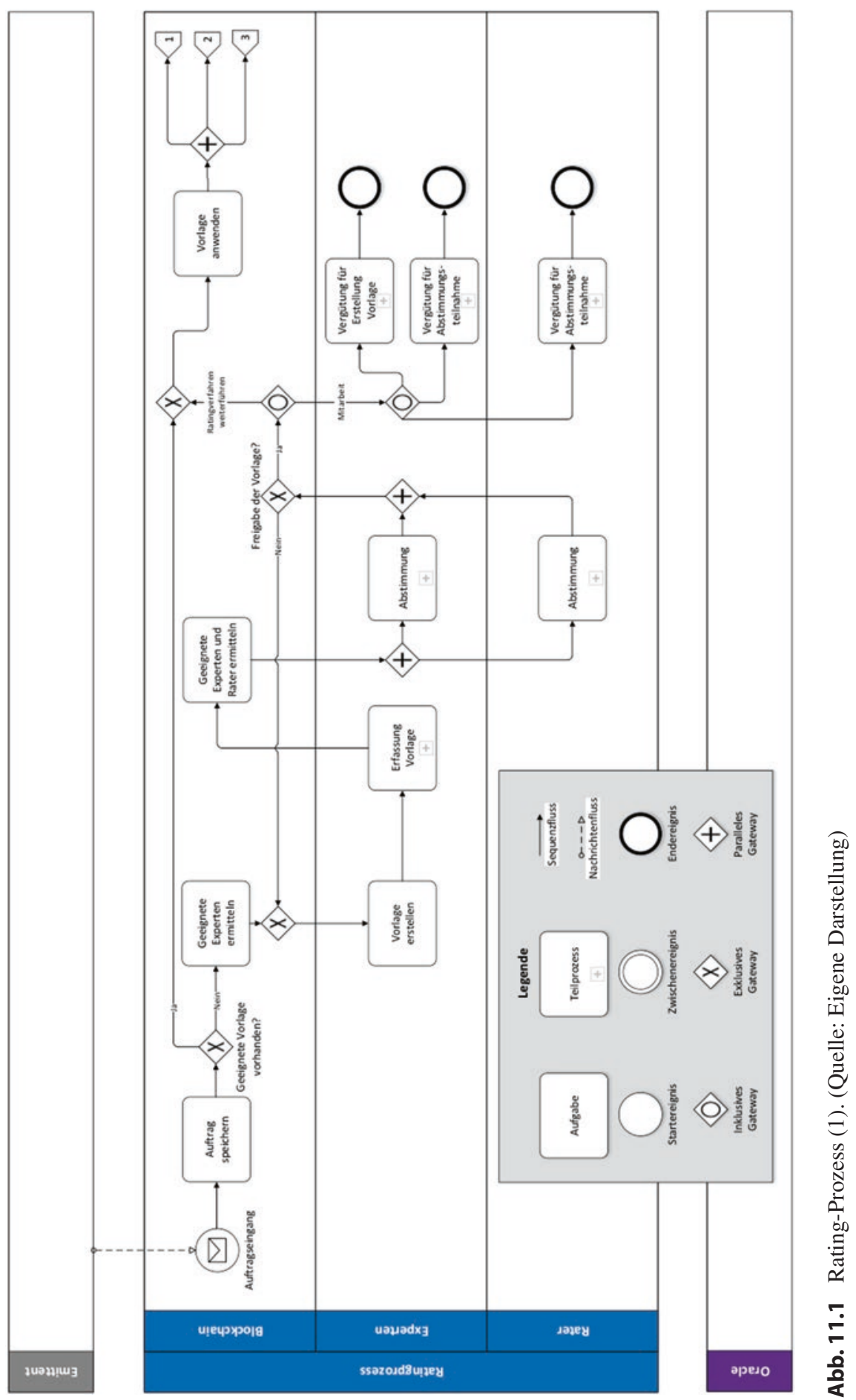




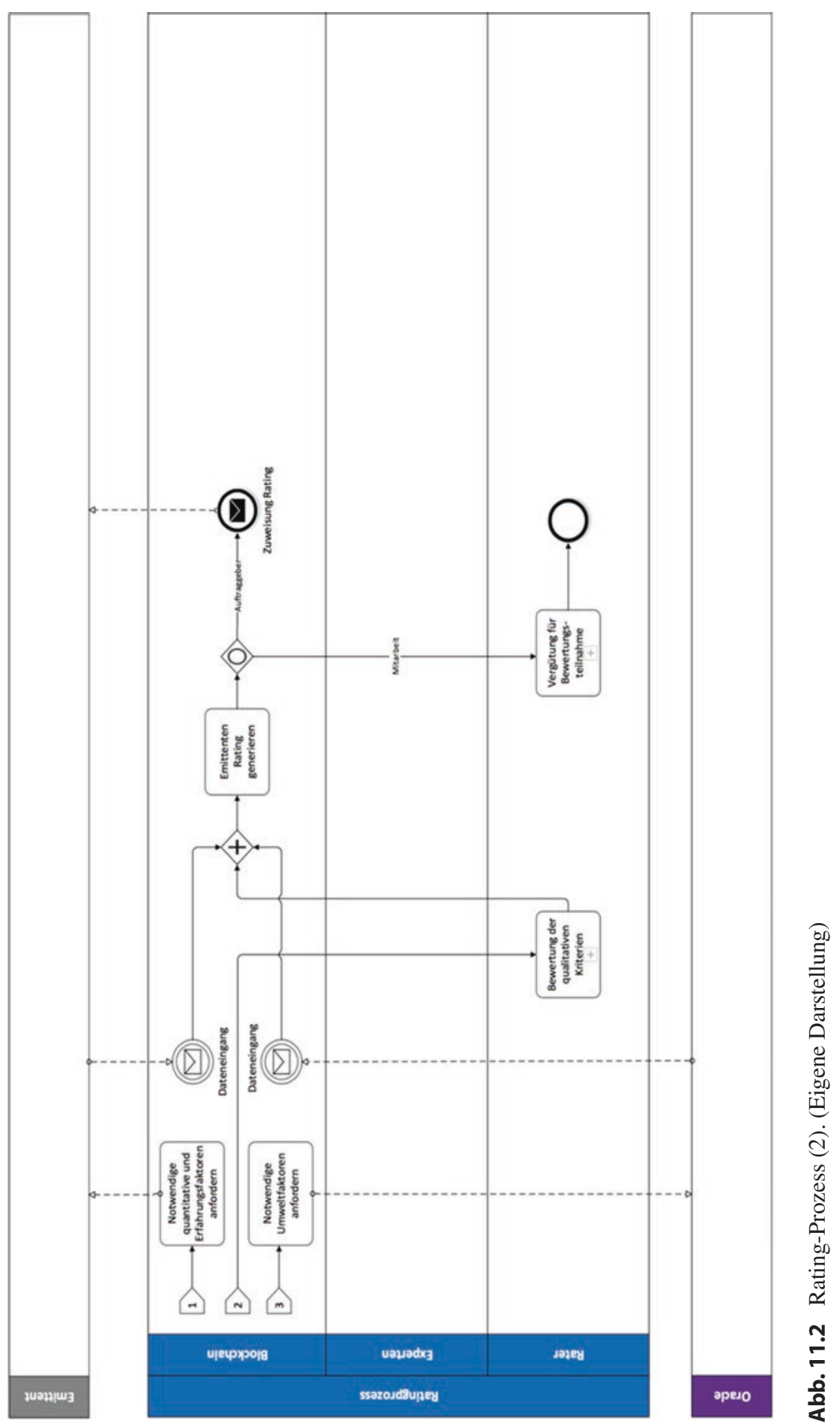


Die Höhe der Vergütung ist abhängig vom Arbeitsaufwand und von der Netzwerkreputation. Zudem sind die Treiber der Netzwerkreputation bei der Vergütung relevant. Die Experten werden für die Erstellung einer Vorlage und für die Abstimmungsteilnahmen vergütet und die Rater für die Bewertungs- sowie für die Abstimmungsteilnahmen. Bei der Vergütung der Rater könnte die Anwendung einer Bonus-Malus-Regelung (Sicking 2011) sinnvoll sein, um absichtliche Fehlbewertungen zu vermeiden. Die Rater werden im Normalfall für ihre Bewertungsteilnahme belohnt. Erkennt Smart Rating allerdings, dass mehrere Bewertungen eines Raters stark von der Median-Bewertung abweichen, so wird dieser dafür bestraft. In diesem Fall wird ihm ein Teil oder der Gesamtbetrag seiner zuvor hinterlegten Rating-Coins abgezogen und er erhält keine Vergütung für die Bewertungsteilnahme.

Die Reputation eines Raters oder eines Experten misst den Einfluss, den ein Teilnehmer bei einer Abstimmung hat und weist auf die Aktivität im Netzwerk und die Qualität seiner Arbeit hin.

\section{Veränderung der Netzwerkreputation des Experten}

Die Netzwerkreputation des Experten steigt nach der Erstellung einer qualitativ guten Vorlage und mit der Teilnahme an Abstimmungen zur Freigabe von Vorlagen, an denen der Experte nicht gearbeitet hat. Die Reputation sinkt, falls der Experte über längere Zeit inaktiv ist.

\section{Veränderung der Netzwerkreputation des Raters}

Die Netzwerkreputation des Raters steigt mit der Teilnahme an Abstimmungen zur Freigabe von Vorlagen und mit einer genauen Bewertung der qualitativen Faktoren eines Emittenten-Ratings. Die Reputation sinkt, wenn die Bewertung der qualitativen Faktoren bei mehreren Punkten stark vom Median abweicht. Zudem sinkt die Reputation, wenn der Rater über längere Zeit inaktiv ist.

\subsubsection{Vorteile gegenüber dem klassischen Rating-Verfahren}

Das Rating-Verfahren von Smart Rating ist transparenter. Es ist klar, nach welchen Kriterien und mit welcher Gewichtung ein Rating zustande gekommen ist. Zudem stellt die tägliche Aktualisierung einzelner Faktoren des Ratings einen Vorteil dar, indem bereits früh Tendenzen sichtbar werden.

Der Interessenskonflikt beim Geschäftsmodell „,der Emittent bezahlt“ wird mithilfe der Blockchain-Technologie und den Smart Contracts gelöst. Aufgrund der Tatsache, dass sich der Emittent niemals mit dem Experten oder den Ratern direkt trifft, wird eine Distanz zwischen dem Auftraggeber eines Ratings und den restlichen Netzwerkteilnehmern geschaffen. Zudem fließen viele Daten (quantitative, Erfahrungs- und Umweltdaten) automatisch in das Rating ein. 
Ein Rating würde bei Smart Rating ebenfalls nur eine reine Meinungsäußerung darstellen. Da jedoch die Meinung vieler Teilnehmer berücksichtigt wird, ist anzunehmen, dass eine solches Rating ausgewogener ist als das eines einzelnen Akteurs.

Da die am Rating-Prozess und an dessen Ergebnis Beteiligten nicht direkt vom Emittenten bezahlt werden, entschärft dies deutlich die oben genannten Interessenskonflikte, was zu einer Objektivierung des Ratings beiträgt.

Das Problem der asymmetrischen Informationsverteilung könnte von Smart Rating zumindest teilweise gelöst werden. Unternehmensinterne für die Öffentlichkeit kritische Informationen könnten einem standardisierten und automatisierten Rating-Prozess verfügbar gemacht werden, ohne dass diese Daten direkt veröffentlicht werden müssten. Deren Korrektheit kann zwar nicht hundertprozentig garantiert werden, es wäre jedoch denkbar, diese etwa durch Vergleich zu anderen Kennzahlen (Bilanz, Umsatz etc.) automatisiert auf Plausibilität zu prüfen.

Die Beschaffung und Verarbeitung der Daten würde einen großen Vorteil zur heutigen Situation darstellen, da diese zum Großteil automatisiert stattfinden könnte. Damit sinkt nicht nur der Aufwand für einen Rating-Prozess, sondern es steigt auch die Qualität. Übertragungsfehler werden minimiert und die Homogenität der Daten über verschiedene Rating-Prozesse hinweg ist gewährleistet.

\subsubsection{Herausforderungen}

Damit ein Smart-Rating-DAO überhaupt funktionieren kann, müssen zunächst noch einige Hürden überwunden werden. Auf der technologischen Ebene sind weder die Blockchain-Technologie noch die Smart Contracts vollständig ausgereift. Viele Blockchains haben Probleme mit der Skalierbarkeit und können nur wenige Transaktionen pro Sekunden ausführen. Bei einem komplexen Smart Contract wie bei Smart Rating hat dies eine deutlich längere Ausführungszeit zur Folge. Ein weiterer Punkt sind die Kosten. Jede Ausführung einer Funktion eines Smart Contracts löst eine eigene Transaktion auf der Blockchain aus, was bei einer automatischen Organisation des kompletten SmartRating-DAO zu vielen Transaktionen und entsprechenden Kosten führt. Nicht zu vernachlässigen sind die softwaretechnischen Herausforderungen, die bei der Entwicklung und Implementierung einer hoch komplexen DAO entstehen. In diesem Bereich sind - im Gegensatz zu etablierten Szenarien wie etwa in der Software-Entwicklung von ERP-Systemen - wenig Erfahrungen vorhanden, auf die man sich stützen könnte.

Außerdem muss berücksichtigt werden, dass es Herausforderungen gibt, welche nichts mit der Technologie zu tun haben. Die Ausgestaltung des Anreizsystems wird als einer der kritischen Erfolgsfaktoren von Smart Rating erachtet. Damit Smart Rating die aufgeführten Vorteile gegenüber einem klassischen Rating erreichen kann, muss eine kritische Masse an Personen und Unternehmen für das Projekt begeistert werden. Netzwerkteilnehmer müssen in einem genügenden Maße für eine gute und ehrliche Arbeit entlohnt werden. 
Ebenso wichtig wird es sein, die Regulatoren von diesem Beispiel überzeugen zu können. Viele der potenziellen Abonnenten sind darauf angewiesen, dass ihre Ratings von einer anerkannten Rating-Agentur stammen. Daher hängt die Akzeptanz von Smart Rating bei den Investoren stark davon ab, ob es von den globalen Regulatoren anerkannt wird. Bei einer internationalen Ausrichtung von Smart Rating würde man mit den drei großen Rating-Agenturen Moody's, Standard \& Poor's und Fitch Ratings konkurrieren. Daher müsste sich Smart Rating als eine günstigere, bessere und anerkannte Lösung etablieren. Bei einer internationalen Ausrichtung müssten zudem die quantitativen Daten der verschiedenen Buchführungsgrundsätze in eine einheitliche Form gebracht werden.

\subsection{Fazit und Ausblick}

Mithilfe des ausgearbeiteten Smart-Rating-Beispiels konnte dargelegt werden, dass zukünftig ganze Geschäftsmodelle und Organisationen auf Smart Contracts basieren könnten. Sowohl die Vorteile eines dezentralen und automatisierten Emittenten-Ratings gegenüber einem klassischen Rating sind aufgezeigt worden als auch die Grenzen der Smart Contracts und die Herausforderungen, welche Smart Rating zu bewältigen hat.

Die Erarbeitung des Beispiels erwies sich als eher schwierig, weil zwei komplexe Themen, Smart Contracts und Rating-Agenturen, kombiniert worden sind. Da es vor allem darum ging, das Potenzial von Smart Contracts aufzuzeigen, konnte nicht auf alle Details eingegangen werden. Unter Berücksichtigung vieler Aspekte, wie der blockchain-basierten digitalen ID, der Smart Rating DApp und der automatischen Generierung eines Ratings, konnte klar aufgezeigt werden, dass Smart Contracts in diversen Bereichen eingesetzt werden können.

Abschließend ist es wichtig, Smart Rating realistisch einzuordnen. Ein solcher Rating-Prozess könnte in der Tat zur Steigerung der Transparenz, zur Verringerung von Interessenskonflikten und zur Abstützung auf die Meinung einer breiteren Personengruppe beitragen. Es ist allerdings zu beachten, dass bislang noch kein annähernd komplexes Szenario realisiert wurde. Gründe dafür sind die Skalierbarkeit, die Latenzzeiten und die zurzeit noch hohen Transaktionskosten. Zudem muss erwähnt werden, dass man von einer Anerkennung durch die Regulatoren noch weit entfernt ist. Weiter werden sich DAOs, welche auf der Blockchain-Technologie beruhen, zunächst einmal in anderen Branchen beweisen müssen, bevor eine DAO in der stark regulierten Finanzbranche anerkannt wird. Schließlich wird es nicht einfach sein, die marktbeherrschende Position der etablierten Rating-Agenturen zu durchbrechen, zumal diese selbst das geringste Interesse haben, das aus ihrer Sicht gut funktionierende Geschäftsmodell aufzugeben.

Die Entwicklung der Blockchain-Technologie und der Smart Contracts wird weiterhin interessant zu beobachten sein. Die Unternehmen und Communities, welche sich zum Ziel gesetzt haben, Geschäftsprozesse und Geschäftsmodelle digitaler und effizienter zu gestalten, eine lückenlose Nachverfolgbarkeit von Verträgen zu gewährleisten und Mittelsmänner zu eliminieren, werden diese Technologie stetig weiterentwickeln. 
Vielleicht wird es einige der heute etablierten Unternehmen in naher Zukunft nicht mehr geben. Möglicherweise wird der Kern der Organisationen der Zukunft aus Programmcode bestehen und Geschäftsprozesse werden mithilfe definierter Regeln gesteuert und automatisiert ausgeführt.

\section{Literatur}

BIS. (2018). Bank für internationalen Zahlungsausgleich. https://www.bis.org. Zugegriffen: 19. Jan. 2019.

Boerse Stuttgart GmbH. (2018). Ratings. https://www.boerse-stuttgart.de/de/boersenportal/segmente-und-initiativen/bondm/ratings/. Zugegriffen: 19. Jan. 2019.

Buschmeier, A. (2011). Ratingagenturen: Wettbewerb und Transparenz auf dem Ratingmarkt. Wiesbaden: Gabler \& Springer Fachmedien.

Däubler, W. (2012). Rechtskontrolle von Ratingagenturen? Kritische Justiz 2012, 18-25. https:// doi.org/10.5771/0023-4834-2012-1-18. Zugegriffen: 21. Febr. 2019.

Diedrich, H. (2016). Ethereum. blockchains, digital assets, smart contracts, decentralized autonomous organizations. Bristol: Wildfire.

ESMA. (2017). Report on CRA market share calculation. European securities and markets authority, publiziert am 20.12.2017, https://www.esma.europa.eu/sites/default/files/library/esma33-5209_-_cra_market_share_calculation_2017.pdf. Zugegriffen: 19. Jan. 2019.

ESMA. (2018). Credit rating agencies. European Securities and Markets Authority. https://www. esma.europa.eu/regulation/credit-rating-agencies. Zugegriffen: 19. Jan. 2019.

FINMA. (2018). Anerkennung von Ratingagenturen. https://www.finma.ch/de/bewilligung/ratingagenturen/. Zugegriffen: 19. Jan. 2019.

FINMA. (o. D.). Anerkannte Ratingagenturen. https://www.finma.ch/de/ /media/finma/dokumente/finmapublic/bewilligungstraeger/ratingagenturen.pdf?la=de. Zugegriffen: 19. Jan. 2019.

Fitch. (2018). Rating Definitions. https://www.fitchratings.com/site/definitions. Zugegriffen: 19. Jan. 2019.

FSB. (2018). Financial stability board. http://www.fsb.org/about/. Zugegriffen: 19. Jan. 2019.

Gärtner, M., \& Griesbach, B. (2012). Rating agencies, self-fulfilling prophecy and mulitple equilibra? An empirical model of the European sovereign debt crisis 2009-2011. Discussion paper no. 201215. University of St. Gallen. https://ideas.repec.org/p/usg/econwp/201215.html. Zugegriffen: 11. Febr. 2019.

Hertig, A. (2018). What is a DAO? https://www.coindesk.com/information/what-is-a-dao-ethereum/. Zugegriffen: 28. Febr. 2019.

IOSCO. (2015). Code of conduct fundamentals for credit rating agencies. International Organization of Securities Commissions. http://www.iosco.org/library/pubdocs/pdf/IOSCOPD482.pdf. Zugegriffen: 19. Jan. 2019.

Jakob, S., Schulte, A. T., Sparer, D., Koller, R., \& Henke, M. (2018). Blockchain und Smart Contracts. Effiziente und sichere Wertschöpfungsnetzwerke. (Whitepaper Fraunhofer IML). http://s. fhg.de/Hsd. Zugegriffen: 21. Febr. 2019.

Mathis, J., McAndrews, J., \& Rochet, J. C. (2009). Rating the raters: Are reputation concerns powerful enough to discipline rating agencies? Journal of Monetary Economics, 56(5), 657674.

Moody's. (2018). Issuer/Investor requested ratings process detail. https://www.moodys.com/sites/ products/ProductAttachments/SP32056_MIS_Ratings\%20Process\%20Diagram_v13.pdf. Zugegriffen: 19. Jan. 2019. 
Nakamoto, S. (2008). A peer-to-peer electronic cash system. https://bitcoin.org/bitcoin.pdf. Zugegriffen: 2. Jan. 2019.

NZZ. (2016). 7 Antworten zu Rating-Agenturen. Neue Zürcher Zeitung. https://www.nzz.ch/ finanzen/fonds/rating-gesellschaften-im-ueberblick-die-macht-der-agenturen-ld.103325. Zugegriffen: 19. Jan. 2019.

Ott, C. (2011). Der Informationsgehalt von Credit Ratings am deutschen Aktienmarkt, Eine empirische Untersuchung (1. Aufl.). Wiesbaden: Gabler Verlag \& Springer Fachmedien.

Rhee, R. J. (2015). Why credit rating agencies exist. Economic notes by Banca Monte die Paschi di Siena SpA 2 (Bd. 44, S. 161-175). https://onlinelibrary.wiley.com/doi/pdf/10.1111/ ecno.12034. Zugegriffen: 19. Jan. 2019.

SEC. (2017). Annual report on nationally recognized statistical rating organisations. U.S. securities and exchange commission. https://www.sec.gov/ocr/reportspubs/annual-reports/2017-annual-report-on-nrsros.pdf. Zugegriffen: 19. Jan. 2019.

SEC. (2018). Office of credit ratings. U.S. securities and exchange commission. https://www.sec. gov/page/ocr-section-landing. Zugegriffen: 19. Jan. 2019.

Sicking, M. (2011). Bonus-Malus-Regelung im Einkauf und im Projekt, Wie man mit Anreiz- und Sanktionssystemen zu zuverlässigen Lieferungen kommt. heise online. https://www.heise.de/ resale/artikel/Bonus-Malus-Regelung-im-Einkauf-und-im-Projekt-1208406.html. Zugegriffen: 19. Jan. 2019.

Six Swiss Exchange. (2018). Rating. https://www.six-swiss-exchange.com/knowhow/popup/glossary_de.html?id=Rating. Zugegriffen: 19. Jan. 2019.

Standard \& Poor's. (2018). Understanding ratings. What are credit ratings. https://www.spratings. com/en_US/understanding-ratings. Zugegriffen: 19. Jan. 2019.

Torcasso, D. (2018). Die grössten ICOs kommen aus der Schweiz. Handelszeitung (19.01.2018). https://www.handelszeitung.ch/digital-switzerland/kryptowahrungen-die-grossten-icos-kommen-aus-der-schweiz. Zugegriffen: 2. Febr. 2019.

White, L. J. (2010). Markets: The credit ratings agencies. Journal of economic perspectives, 24(2), 211-226. https://pubs.aeaweb.org/doi/pdfplus/10.1257/jep.24.2.211. Zugegriffen: 19. Jan. 2019.

Wildmoser, G., Schiffer, K. J., \& Langoth, B. (2009). Haftung von Ratingagenturen gegenüber Anlegern? Recht der Internationalen Wirtschaft. RIW, 2009(10), 657-668. https://www.schiffer. de/fileadmin/sci-templates/downloads/RIW1009_pdf_Rating.pdf. Zugegriffen: 21. Febr. 2019.

Furrer, Vincent (B.Sc BA/vincent.furrer94@gmail.com) Vollzeit Bachelorstudium in Betriebsökonomie an der Berner Fachhochschule mit Vertiefung Banking \& Finance. Aktuell beim Amt für Informatik und Organisation des Kantons Bern als Praktikant in den Bereichen ICT-Beschaffung und ICT-Projektmanagement angestellt.

Deck, Klaus-Georg (Prof. Dr./klaus-georg.deck@bfh.ch) Studium der Mathematik, Philosophie und Informatik, Visiting Scholar am Massachusetts Institute of Technology (MIT), langjährige Berufstätigkeit unter anderem bei der SAP AG, Abraxas Informatik AG und Helvetia Versicherungen in den Bereichen ERP-Systeme, Business Intelligence und Data Science. Professor für Wirtschaftsinformatik an der Dualen Hochschule Baden-Württemberg und Dozent an der Berner Fachhochschule. Forschungsschwerpunkte: Algorithmen, Neue Technologien und Digitalität in Wirtschaft, Wissenschaft und Gesellschaft. 
Open Access Dieses Kapitel wird unter der Creative Commons Namensnennung 4.0 International Lizenz (http://creativecommons.org/licenses/by/4.0/deed.de) veröffentlicht, welche die Nutzung, Vervielfältigung, Bearbeitung, Verbreitung und Wiedergabe in jeglichem Medium und Format erlaubt, sofern Sie den/die ursprünglichen Autor(en) und die Quelle ordnungsgemäß nennen, einen Link zur Creative Commons Lizenz beifügen und angeben, ob Änderungen vorgenommen wurden.

Die in diesem Kapitel enthaltenen Bilder und sonstiges Drittmaterial unterliegen ebenfalls der genannten Creative Commons Lizenz, sofern sich aus der Abbildungslegende nichts anderes ergibt. Sofern das betreffende Material nicht unter der genannten Creative Commons Lizenz steht und die betreffende Handlung nicht nach gesetzlichen Vorschriften erlaubt ist, ist für die oben aufgeführten Weiterverwendungen des Materials die Einwilligung des jeweiligen Rechteinhabers einzuholen.

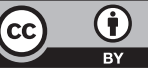




\title{
Erfolgsfaktoren für die Kundengewinnung mit digitalem Marketing
}

\section{Am Beispiel des Online Marketing Tools Google AdWords}

\author{
Seraina Ulber und Etienne Rumo
}

\begin{abstract}
Zusammenfassung
Den Absatz zu steigern, ist das übergeordnete Ziel von effizientem Marketing. Die Digitalisierung begründet die Umsetzung vieler Online-Marketingaktivitäten. Ein wichtiger Player im digitalen Marketing ist Google AdWords, ein Tool, welches es Unternehmen ermöglicht, dass deren Produkte und Dienstleistungen von potenziellen Kunden gefunden werden, wenn auf der Suchmaschine von Google danach gesucht wird. Die Identifikation von Erfolgsfaktoren bei der Arbeit mit Google AdWords ist aufgrund der Schnelllebigkeit des Themenfelds sowie des komplexen AdWords-Auktionssystems eine Herausforderung. Eine Fallstudienanalyse, in deren Rahmen erfahrene Unternehmungen beim Marketing mit Google AdWords sowie Fachexperten befragt werden, deckt die Erfolgsfaktoren für Marketingmaßnahmen mit Google AdWords auf. Eine moderne und nutzerfreundliche Webseite mit relevanten Inhalten sowie eine hohe Relevanz zwischen Keyword, Werbeanzeige und Zielseite sind essenzielle Voraussetzungen für erfolgreiche Google-AdWords-Kampagnen.
\end{abstract}

\footnotetext{
S. Ulber $(\bowtie)$

Chur, Schweiz

E-Mail: seraina.ulber@bluemail.ch

E. Rumo
}

Berner Fachhochschule Wirtschaft, Bern, Schweiz

E-Mail: etienne.rumo@bfh.ch 


\subsection{Einleitung}

Nachdem Edmund Jerome McCarthy (1960) als Erster auf die vier Hauptachsen des Marketings einging und die Verflechtung dieser Komponenten den Marketing-Mix nannte, welcher die weltbekannten 4 P's Product (Produkt), Place (Vertrieb), Promotion (Kommunikation) und Price (Preis) beinhaltet und seit jeher die Basis von jeder Marketingstrategie stellt, wurden die vier P's bald auch im digitalen Marketing unentbehrlich. Der Megatrend rund um die Digitalisierung und damit einhergehend die Erfindung des World Wide Web im Jahr 1989 haben die Anforderungen an Marketingstrategien nachhaltig geprägt. Der Einbezug von Online-Marketing stellt heute einen gleichwertigen oder sogar überwiegenden Aspekt der Marketingmaßnahmen von Unternehmungen dar. Beim Online-Marketing werden die Planung sowie die Umsetzung von Marketingaktivitäten unter Verwendung von digitalen Systemen beschrieben (Wendeler o. J.). Ein bedeutender Player im Zeitalter der Digitalisierung ist die Unternehmung Google LLC, welche im Jahr 2000 den Ableger Google AdWords lanciert hat. Das Tool beschäftigt sich damit, Usern, die mit der Suchmaschine von Google nach etwas Bestimmtem suchen, zielgerichtete und relevante Werbung zu schalten (Weller und Calcott 2012). „Reach your customers in the moment that matters with the relevant information“ (Leu 2018a), lautet der Slogan, den Google im Rahmen der Vermarktung seiner AdWords verwendet. Google ist bestrebt, sich ständig weiterzuentwickeln, damit sie ihrem Credo gerecht wird und am Puls der Zeit ist. Die Suchmaschine von Google ist weltweit die mit großem Abstand Bedeutendste. Google hatte im Jahr 2017 einen Marktanteil von über $92 \%$ inne, während der erste Verfolger Bing einen Marktanteil von weniger als $3 \%$ aufwies (Lunapark 2017). Zudem bleibt Google der Spitzenreiter bei der Frage, wer den größten Anteil am globalen digitalen Werbemarkt besitzt. Der Suchmaschinengigant liegt mit einem geschätzten Anteil von 31,2 \% im Jahr 2019 deutlich vorne (Internet World Business 2018).

Für die erfolgreiche und effiziente Arbeit mit Google AdWords sind die Erfolgsfaktoren von Unternehmung zu Unternehmung verschieden und stark von der Sortimentsvielfalt und der Branche abhängig. Die Identifikation von Erfolgsfaktoren für das Marketing mit Google AdWords stellt sich daher als sehr vielfältig und herausfordernd heraus. Trotzdem gibt es grundlegende, allgemeingültige Faktoren, welche berücksichtigt werden sollten, um mit Google AdWords erfolgreiche Marketingmaßnahmen zu implementieren. Eine weitere Challenge des Marketings mit Google AdWords besteht darin, der sich ständig ändernden Dynamik des digitalen Marketings gerecht zu werden. Was heute allgemeingültig scheint, kann bereits morgen veraltet sein. Die Funktionsweise von Marketing mit Google AdWords steht in starker Verbundenheit mit dem Google Algorithmus, der darüber entscheidet, welche Anzeige von welchem Anbieter wann ausgespielt wird. Aufgrund der Tatsache, dass der Google-Algorithmus ein einmaliges Phänomen in Sachen Komplexität und Genialität ist und im Hintergrund Auktionen zur Ausspielung 
jeder einzelnen Werbeanzeige durchführt, ist das Marketing mit Google AdWords teilweise unberechenbar (VCS 1 2018; VCS 2 2018; VCS 4 2018; VCS 5 2018).

Der Output dieses Beitrags deckt Erfolgsfaktoren des Marketings mit Google AdWords im Rahmen einer empirischen Untersuchung auf. Dabei wird eine Art Anleitung für erfolgreiche Google-AdWords-Strategien aufgezeigt. Die Identifikation der Erfolgsfaktoren beim Marketing mit Google AdWords dient als Ausgangslage für Unternehmungen, welche in der Digitalisierung einen Schritt vorankommen möchten und in Zukunft anhand von Google AdWords Werbung platzieren oder deren Skills zur Optimierung von bestehenden AdWords-Kampagnen verbessern möchten.

\subsection{Online-Marketing}

\subsubsection{Bedeutung und Einordnung}

Die Planung, Koordination und Kontrolle der auf die relevanten Märkte ausgerichteten Aktivitäten einer Firma zur Erreichung der Unternehmensziele werden als Marketing beschrieben. Dazu gehören auch absatzorientierte Instrumente wie Werbung, wobei die funktionale Perspektive von Marketing weit darüber hinausgeht. Die Bedürfnisse der adressierten Akteure und letztendlich deren Befriedigung sind ebenso Teil des Marketings. Direkte Verkaufsförderung oder die Steigerung der Bekanntschaft von Marken sind weitere entscheidende Faktoren im Marketingprozess. Online-Marketing im Speziellen ermöglicht komplett neue Marketingalternativen und stellt nicht nur einen weiteren Kanal für die Umsetzung der klassischen Produkt-, Preis-, Distributions- und Kommunikationsstrategien dar, sondern kann als grundlegender Innovationsprozess verstanden werden (Griesbaum 2012).

Die datengetriebene Automatisierung des Marketings ist seit Jahren weltweit ein nachhaltiges und stets aktuelles Thema. Die Rede ist dabei vom digitalen bzw. OnlineMarketing. Eine sehr pragmatische Definition dieser Art von Marketing besagt, dass es die Umsetzung des Marketings in Online-Medien bedeutet und der Fokus häufig auf der Kommunikationspolitik (Promotion) liegt (Busch 2014). Das Online-Marketing ist ein wichtiger Bestandteil des Digital Business und für die heutige Unternehmensführung ein unentbehrlicher Player. Es fasst alle Marketingaktivitäten zusammen, die auf der Grundlage von internetbasierten Technologien durchgeführt werden (Griesbaum 2012). In den letzten zwei Jahrzehnten hat sich die digitale Welt rasant entwickelt und ist heute für einen Großteil der Konsumenten nicht mehr aus dem Alltag wegzudenken (Dänzler und Heun 2014). Die weltweiten Ausgaben für Werbung im Internet überstiegen im Jahr 2017 gemäß Herrmann (2017) die Grenze von 200 Mrd. US\$. Eine zentrale Rolle in diesem Umfeld hat die Unternehmung Google LLC inne. Google ist die meist besuchte Webseite der Welt, Marktführer bei weltweiten Suchanfragen und somit ein prägender Player des digitalen bzw. Online-Marketings (Weller und Calcott 2012). 
Bereits um die Jahrtausendwende und somit nach gut 10 Jahren Existenz des Internets wurde deutlich, dass die bestehenden Marktstrukturen tiefgreifende Änderungen durchgehen und sich dies in Zukunft weiter verstärken wird. Link (2000) hielt zudem fest, dass das Online-Marketing essenzielle Weiterentwicklungen mit sich bringen wird, die für das Offline-Marketing nicht relevant waren. Dabei handelt es sich beispielsweise um Sicherheitstechnologien und damit einhergehend um die Internetkriminalität, welche eng verknüpft ist mit der Datenschutzproblematik. Eine Studie hat ergeben, dass für mehr als $50 \%$ der deutschen Bevölkerung eine steigende Digitalisierung dazu führt, dass deren Angst vor Internetkriminalität ebenfalls steigt (Hamburg 2017). Das Misstrauen, das Skeptiker dem Internetmedium entgegenbringen, basiert gemäß Frost (2013) auf der Sorge um die eigenen Daten und anderen Sicherheitsbedenken. Obschon das World Wide Web gemäß BFS (2017) von $79 \%$ der europäischen Bevölkerung und $88 \%$ der schweizerischen Bevölkerung wöchentlich genutzt wird und der Anteil an Internet-Nutzern somit immens groß ist, bestehen Hürden für die Nutzung. Die Datenschutzproblematik ist zweifelslos eine dieser Hürden.

Das Europäische Parlament (2018) hat festgehalten, dass $37 \%$ der Konsumenten fast ausschließlich online shoppen. Zum anderen ist das Internet für $57 \%$ der Konsumenten die erste Quelle, um sich vor dem Entscheid über den Kauf oder eines Produkts zu informieren (Hohlstein 2016). Ersteres Statement deutet den anhaltenden Wandel in Richtung Online-Shopping an, während letztere Aussage aufzeigt, wie wichtig es für Unternehmungen ist, mit den eigenen Produkten und/oder Dienstleistungen online im World Wide Web präsent zu sein. Der Trend der Digitalisierung und damit derjenige des Wandels vom Offline- hin zum Online-Marketing schlägt sich unter anderem bei den Werbeausgaben durch. Während die Prozentzahlen der globalen Werbeausgaben für die Medien Zeitungen, Magazine, TV und Radio seit 2012 stets sinken, erhöhten sich die digitalen, prozentualen Werbeausgaben von 18,3\% im Jahr 2012 zu geschätzten $27 \%$ im Jahr 2019 (Stone 2014; Herrmann 2017).

\subsubsection{Echtzeit- und crossmediales Marketing}

Ein wichtiger Ableger des Online-Marketings ist das Realtime-Advertising-Prinzip, welches den Verkauf von Online-Werbeplätzen in Echtzeit beschreibt. Im Gegensatz zum traditionellen Display-Markt, in dem Werbeplätze für eine bestimmte Zeitspanne fest erworben werden, werden diese beim Realtime Advertising in Echtzeit versteigert und ausschließlich für passende Nutzer angezeigt (Advidera o. J.). Zusammengefasst dreht sich alles um die intensivere Nutzung von Daten, Technik und künstlicher Intelligenz im Marketing, mit dem übergeordneten Ziel einer Verbesserung der Marketingeffizienz in Echtzeit (Busch 2014). Die zunehmende Digitalisierung ermöglicht unmittelbare Feedbacks und ungeahnte Optimierungsmöglichkeiten. Werbung kann exakt passend gesteuert, Motive und Botschaften abhängig vom Individuum und der Situation augenblicklich angepasst werden. Ressourcenintensive Arbeiten wie die Mediaplanung nach 
Umfeld sowie psychografische Zielgruppenmodelle erübrigen sich, denn das Marketing kann Individuen erkennen, diese ansprechen und den Erfolg sofort messen. Diese Vorteile bringt das Echtzeitmarketing, das passend mit den Begriffen Geschwindigkeit, Reiz und Reaktion beschrieben werden kann (Engel 2015). Obschon das Echtzeitmarketing einige grundlegende Prinzipien des Marketings über den Haufen wirft, hält Busch (2014) fest, dass es sich beim Realtime Advertising nicht um einen plötzlich aufgetretenen Hype handelt, sondern sich dieses über einen Zeitraum von etwas mehr als fünf Jahren entwickelte. Dabei waren verschiedene Entwicklungsstränge ausschlaggebend, welche im Endeffekt und in Kombination eine hohe Relevanz erfahren haben. Diese Entwicklungen stellen zusammen die Möglichkeiten dar, welche die Marketingbranche derzeit in der Praxis bei der Lösung von Problemstellungen exploriert. Das Interesse des Werbemarkts für derartige Technologie und Wissenschaft setzt enorme finanzielle Mittel frei, die eine zusätzliche Beschleunigung des bisherigen Weges ermöglichen. Realtime Advertising ist ein neues, wertschöpfendes Prinzip im Umgang mit digitaler Werbung, in welchem der unaufhaltsame Prozess der Digitalisierung von Werbung und Marketing stets in Betracht gezogen werden muss.

Im Zuge der rasanten Entwicklung des Online-Marketings darf nicht vergessen werden, dass die Wichtigkeit des Offline-Marketings weiterhin bestehen bleibt. Die Kombination der Marketingzweige online und offline wird crossmediales oder auch integriertes Marketing genannt und stellt für Unternehmungen ein hochspannendes Tool dar, um sich Wettbewerbsvorteile zu verschaffen, wenn die Integration und Verflechtung beider Zweige erfolgreich gestaltet werden. Der Prozess des crossmedialen Marketings bezeichnet gemäß der Autorin Stich (2017) die Verknüpfung der Online-Werbemaßnahmen mit der Offline-Werbung. Das crossmediale Marketing wird hauptsächlich der Kommunikationspolitik zugeordnet. Die integrierte Marketingkommunikation beabsichtigt einen abgestimmten Einsatz der unterschiedlichen Kommunikationsinstrumente, damit schlüssige, und überzeugende Aussagen über Produkte, Dienstleistungen und Unternehmen kommuniziert werden können. Dabei muss ersichtlich sein, dass anhand von verschiedenen Tools identische Aussagen verbreitet werden. Diese Messages werden parallel zu und schlüssig mit den verschiedenen Kommunikationsinstrumenten in einer ganz bestimmten Zeitperiode gestreut (Rumo 2017).

\subsection{Google AdWords}

Google AdWords ist ein im Oktober 2000 lanciertes Programm der Firma Google LLC, welches aus dem Online-Marketing-Mix nicht mehr wegzudenken ist. Es handelt sind dabei um die Werbeanzeigen auf den Ergebnisseiten von Google. Gemäß Google AdWords (o. J.) soll es für Unternehmungen möglich sein, von potenziellen Kunden immer dann gefunden zu werden, wenn bei Google gezielt nach Produkten oder Dienstleistungen gesucht wird. Google AdWords ist jedoch nicht nur für die werbenden Händler interessant und vielversprechend, sondern auch für die Gründerfirma lukrativ. 
Das AdWords-Programm hat das gesamte Unternehmen verändert und Google endlich eine richtige Einnahmequelle beschert. Seit der Einführung des Tools ist AdWords die Haupteinnahmequelle des Unternehmens und auch heute noch für fast $90 \%$ des Umsatzes verantwortlich (GoogleWatchBlog 2015).

Ein Erfolgsfaktor für die Allgegenwärtigkeit von Google AdWords im OnlineMarketing ist deren Kostenmodell, Pay per Click genannt. Dieses ist so strukturiert, dass Werbende nicht für Einblendungen der Anzeigen, sondern lediglich für Klicks auf die Werbeanzeigen bezahlen. Google AdWords stellt somit eine der effektivsten Werbemöglichkeiten im Internet dar und ist weltweit die bekannteste Werbealternative (Google AdWords o. J.; Michnew 2015). Bis zu 90 \% der Konsumenten, welche eine Kaufabsicht haben, recherchieren vor dem Kauf in Suchmaschinen (DIM Deutsches Institut für Marketing GmbH o. J.). Gemäß Beck (2011) werden weltweit täglich 3,6 Mrd. Suchanfragen auf Google gestellt. Diese Fakten beweisen, dass es sich heutzutage kaum ein Unternehmen leisten kann, auf die Präsenz auf Suchmaschinen zu verzichten. Bei jeder Suchanfrage haben Unternehmungen jeglicher Branchen die Möglichkeit, sich selbst und ihre Webseite zu präsentieren, damit Neukunden anzuwerben und Umsatz zu generieren. Studers (2008) Google-AdWords-Studie hat überdies ergeben, dass Unternehmungen dank Google AdWords nicht lediglich den Absatz fördern und Leads generieren können, sondern auch, dass die bloße Einblendung von AdWords-Anzeigen ebenfalls einen deutlich nachweisbaren Branding-Effekt hat. Dieser äußerst begehrte Effekt wurde Google AdWords lange Zeit abgesprochen, konnte durch die Google-Studie „Markenbildung mit AdWords“ aber bestätigt werden. Eine Marke steigert seine Bekanntheit dank Google AdWords im Schnitt um $21 \%$ (Busch 2014).

Der Nutzen von Google AdWords lässt sich dadurch bestätigen, dass Menschen sich online über ein Produkt oder eine Dienstleistung informieren, für das bzw. die sie sich interessieren. Suchmaschinen sind dafür das meistgenutzte Medium. Aufgrund dessen kann festgehalten werden, dass die Suchmaschinenwerbung eine enorm hohe Reichweite hat. Da die Zielgruppe den Werber erst bei konkretem Bedarf sucht, findet und kontaktiert, lässt sich die Höhe an Einblendungen der Anzeigen sehr gezielt steuern, wenn strategisch gute Keywords eingebucht werden. Da die Suchmaschinenwerbung den Nutzer im entscheidenden Moment erreicht - eben erst und nur dann, wenn der User aktiv danach sucht - ist sie äußerst relevant und der generierte Traffic auf der Webseite des Werbenden sehr hochwertig (Yapi 2016).

\subsubsection{AdWords-Grundlagen}

Im Gegensatz zur Suchmaschinenoptimierung (Search Engine Optimization SEO), bei welcher technische und inhaltliche Maßnahmen ergriffen werden, damit die eigene Webseite möglichst weit oben in den natürlichen Suchergebnissen positioniert werden kann, geht es bei der Suchmaschinenwerbung (Search Engine Advertising SEA) um kostenpflichtige Textanzeigen, die aufgrund eines gewählten Keywords geschaltet werden. 
Aufgrund von zwei unterschiedlichen, voneinander unabhängigen Systemen erfolgt die Schaltung der natürlichen bzw. der bezahlten Anzeigen. Die beiden Systeme beeinflussen einander nicht (Yapi 2016).

Der grundsätzliche Ablauf von Google AdWords ist denkbar simpel und pragmatisch zu erklären. Der erste Schritt, welcher Google AdWords ins Spiel bringen, ist die Suchanfrage eines Nutzers auf der Google Webseite. Als Ergebnis erhält der User verschiedene Vorschläge, die organischer (SEO) sowie auch bezahlter (SEA) Natur sind. Bezahlte Suchergebnisse sind oben links unter dem Titel mit „Anzeige“ gekennzeichnet. Das Wort „Anzeige“ ist dabei umrahmt (vgl. „Schritt 2“ in Abb. 12.1). Wenn der Nutzer auf eine bezahlte Anzeige klickt, kommt er auf die relevante Landingpage der Webseite, die verlinkt ist. Findet er das passende Produkt und bestellt dieses, löst er eine Conversion aus und der Umsatz wird dem Marketing mit Google AdWords zugeschrieben (Traffic3 o. J.). Abb. 12.1 stellt diesen Ablauf dar. Dass der Kunde ein Produkt kauft und somit eine Conversion auslöst zeigt den Idealfall. Der User könnte die Webseite nach einigen Sekunden oder Minuten auch wieder verlassen, ohne eine Bestellung zu tätigen.

Das Beispiel in Abb. 12.1 basiert auf der Suchanfrage „Kinderfahrrad online kaufen“ und zeigt auf, welche Schritte ein User mindestens durchläuft, bevor er eine Conversion tätigt.

\subsubsection{AdWords Begrifflichkeiten}

Das Verständnis einiger AdWords-Schlagwörter ist essenziell für das Marketing mit Google AdWords. Die Beschreibung der folgenden Begriffe soll helfen, sich einen Überblick über das Themengebiet zu verschaffen.

Impressionen Impressionen bedeuten Einblendungen auf den Google-Ergebnisseiten. Jedes Mal, wenn eine Anzeige bei einer Suchanfrage eines Users erscheint, gilt dies als Impression bzw. Einblendung. Impressionen sind im Suchmaschinenmarketing von Google AdWords kostenlos.

Klicks Klicks entstehen, wenn auf eine Impression auf den Google-Ergebnisseiten geklickt wird. Die Anzahl Klicks gibt an, wie oft Suchende auf ausgespielte Anzeigen geklickt haben. Nach dem Klick landet der User auf der vom Werbenden verlinkten Landingpage. Klicks sind im Suchmaschinenmarketing von Google AdWords kostenpflichtig.

Click through Rate (CTR) Die Click through Rate ist die Kennzahl, welche die Anzahl der Klicks auf Online-Werbeanzeigen im Verhältnis zu der Anzahl der insgesamt erschienenen Impressionen darstellt. Die CTR ist eine wichtige Kennzahl für die Auswertung und Analyse von Google-AdWords-Kampagnen. Gemäß Leu (2018b) wird grundsätzlich eine CTR von $5 \%$ oder höher als erfolgreich angesehen. 


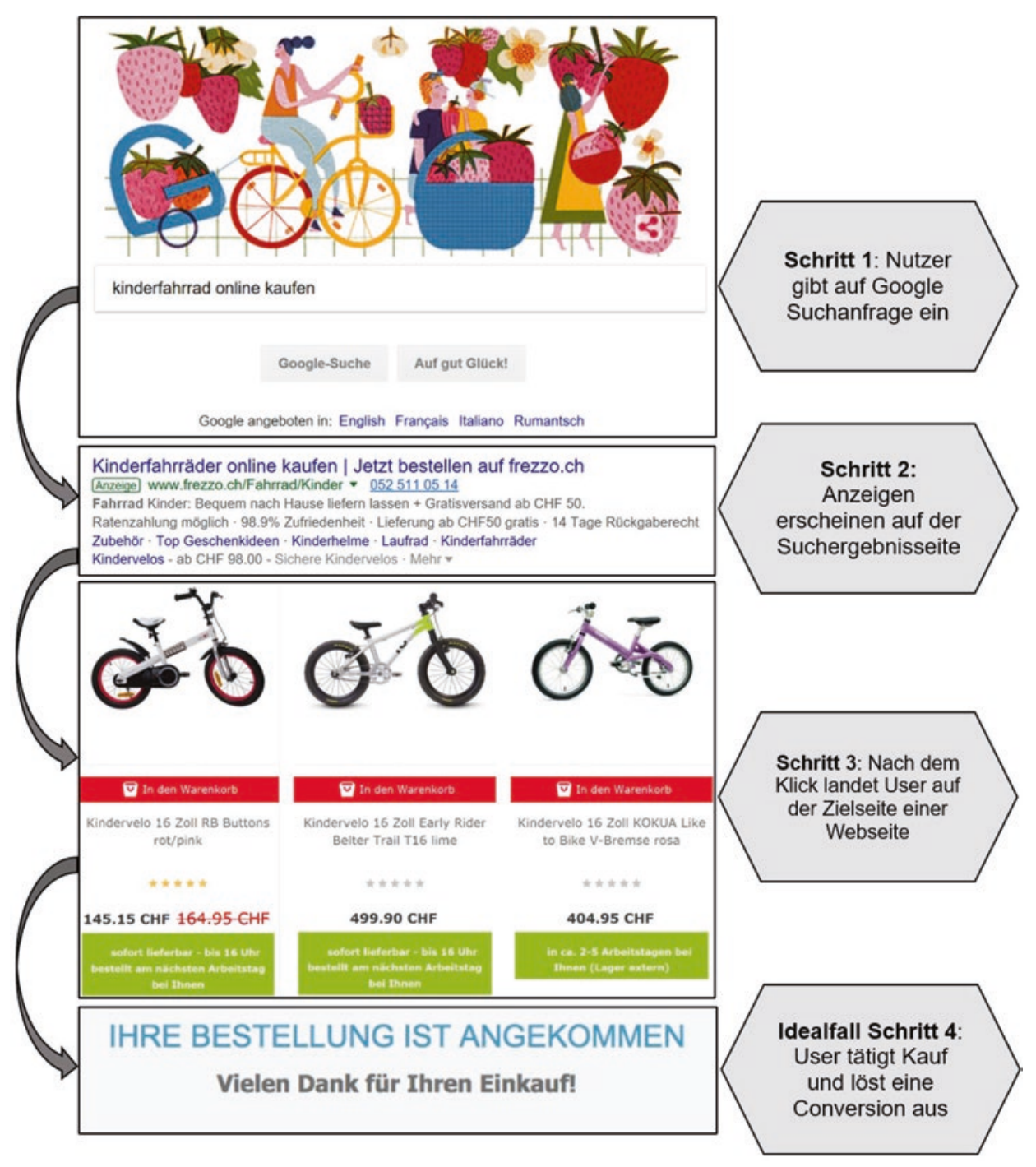

Abb. 12.1 Ablauf einer Google AdWords Conversion. (Eigene Darstellung)

Quality Score Stellt den Qualitätsfaktor für Keywords oder Anzeigen dar. Der Qualitätsfaktor resultiert einerseits aus der Keyword Performance, welche aus den drei Faktoren der historischen Klickrate, der Relevanz für den Anzeigentext sowie der Kontoleistung auf Google besteht. Des Weiteren ist die Webseitenqualität entscheidend, womit Komponenten wie die Mobilgerätefreundlichkeit oder eine einfache Navigation gemeint sind (Leu 2018b). Die Relevanz zwischen Keyword, Anzeige und Landingpage ist eine wichtige Voraussetzung für die Erreichung eines hohen Qualitätsfaktors und beeinflusst 
die Kosten per Klick und den Anzeigerang wesentlich. Der Quality Score kann zwischen 1 und 10 liegen, wobei 1 die schlechteste und 10 die beste Einstufung bedeutet.

Anzeigenrang Der Anzeigenrang resultiert aus der Multiplikation des maximalen CPC(Cost per Click)-Gebots mit dem Quality Score. Das CPC-Gebot entsteht, indem der Werber vorab für jedes Keyword einen maximalen Preis festlegt, den er für einen Klick auf eine seiner Anzeigen zu diesem Keyword zu bezahlen bereit ist (Yapi 2016). Der Anzeigenrang beschreibt die Position, auf welcher die Anzeige auf den Google-Ergebnisseiten eingeblendet wird.

Anzeigenrang $=$ Maximales CPC-Gebot * Quality Score

Auktion Für jede einzelne Suchanfrage gibt es im Hintergrund eine Auktion, in welcher der Google-Algorithmus maßgebend ist und entscheidet, welche Anzeigen eingeblendet werden. Bevor die Auktion extern geht, gibt es vorerst eine interne Auktion, d. h. die eigenen Anzeigen im AdWords-Konto werden gegeneinander geprüft. Pro Suchanfrage geht nämlich jeweils nur ein Keyword mit einer Anzeige ins Rennen, damit der Werber sich nicht selbst konkurrenziert. Das Keyword mit dem höchsten Anzeigenrang wird ins Rennen geschickt (Leu 2018a).

Effektiver CPC Ist der effektive Preis, welcher für einen Klick bezahlt wird. Die Berechnung des effektiven CPC's setzt sich aus der Division des Anzeigenrangs der nachfolgenden Anzeige mit dem eigenen Qualitätsfaktor plus 0,01 CHF zusammen (Leu 2018b).

-Effektiver $\quad \mathrm{CPC}=$ Anzeigenrang nachfolgende Anzeige/eigener Qualitätsfaktor +0,01 CHF

\subsubsection{Kostenmodell}

Die Schaltung von bezahlten Textanzeigen auf der Google Suchmaschine und anderen Suchmaschinen sowie Webseiten, die zum Google-Werbenetzwerk gehören, werden pro Klick auf eine Anzeige abgerechnet, also über einen Klickpreis. Die Werbeform wird auch Pay per Click (PPC) genannt. Dank diesem erfolgsorientierten Kostenmodell zahlt der Werber nur für den Besuch auf der auf einer Anzeige verlinkten Landingpage. Der Klickpreis wird dabei in einem Auktionsverfahren für jedes eingebuchte Keyword und bei jeder Eingabe eines Suchbegriffs von neuem ermittelt (Abschn. 12.3.1.1, „Anzeigenrang“ und „effektiver CPC“) (Yapi 2016). Um zu verhindern, dass ausschließlich diejenigen Anzeigen gute Anzeigepositionen erreichen können, die mit großem Budget gesegnet sind, funktioniert Google AdWords mit der Formel für den Anzeigerang, welche die Relevanz zwischen Keyword, Anzeige und Landingpage miteinbezieht und nicht lediglich das Gebot für den Klick berücksichtigt (Abschn. 12.3.1.1, Anzeigenrang). Google schützt sein Programm damit nicht nur davor, dass ausschließlich Werbende mit hohen Marketingbudgets den Vorrang erhalten und es somit unattraktiv für Unternehmungen mit kleineren Werbebudgets wird, sondern hat auch ein großes Interesse 
daran, seinen Nutzern möglichst relevante Ergebnisse anzuzeigen (traffic3 o. J.). Dies ist eine wichtige Maßnahme, um sicherzustellen, dass die Google User mit den angezeigten Suchergebnissen zufriedengestellt werden.

\subsubsection{Zielsetzung}

Der Inhalt der Webseite, die beworben werden soll, ist Ausgangspunkt für die Festlegung der Werbeziele, die mit Google AdWords verfolgt werden. Eine konkretisierte Zielsetzung ist gemäß der Autorin Dörr von Maydell (2016) wichtig, damit eine Strategie aufgebaut werden kann. Nur so kann die Performance des Marketings mit Google AdWords effektiv ausgewertet werden. Die Zielsetzung soll formuliert werden, bevor das Google-AdWords-Konto eingerichtet wird.

Yapi (2016) betont, dass für die Suchmaschinenwerbung die komplette Marketingplanung (online sowie offline) in Betracht gezogen werden muss. Komponenten wie das Budget, die Laufzeit oder verwendete Formate sollten so weit wie möglich auf andere Werbemaßnahmen abgestimmt sein. Diese Haltung ist damit verknüpft, dass klassische Medien das Interesse für ein Produkt oder eine Dienstleistung wecken sollen. Wer das erweckte Interesse für seine AdWords-Kampagnen nutzen kann, erhöht seine Chancen auf Erfolg. Google unterscheidet im Grundsatz zwischen den drei folgenden Kampagnenzielen.

1. Branding-Kampagne: Steigerung der Markenbekanntheit als Ziel

2. Traffic-Kampagne: Nutzung der Webseiteninhalte als Ziel

3. Direct-Response-Kampagne: Abschließen einer Aktion auf der Webseite als Ziel

\subsubsection{Kontenstruktur}

Ein AdWords Konto besteht aus Kampagnen und Anzeigegruppen, in denen sich die Keywords und Anzeigen befinden. Auf der Ebene des Kontos werden grundsätzliche Komponenten wie Zugriffsrechte, Zeitzone, Währung und Rechnungseinstellungen bestimmt. Auf Kampagnenebene befindet man sich bereits im Detail. Ein weiteres Level darunter befinden sich die Anzeigegruppen, welche die Arbeit mit CPC's für die gesamte Anzeigegruppe, Keywords mit teilweise separaten CPC's, Anzeigentexte und das demografische Targeting beinhalten (Leu 2018a). Auf Kampagnenebene wird zudem der Teil des Google-Werbenetzwerks ausgesucht, in dem die Anzeigen erscheinen sollen. Dabei haben Werbende die Wahl zwischen folgenden Strategien (Yapi 2016):

\section{Suchnetzwerk}

Nach der Eingabe von Begriffen im Google Suchfeld werden über, unter oder neben den organischen Suchergebnissen bezahlte Anzeigen geschaltet (Yapi 2016). Die Voraussetzungen, dass die Anzeigen in den Suchergebnissen erscheinen, sind die Relevanz der Anzeige auf die Suchanfrage sowie ein aktives AdWords-Konto mit einem bestehenden Budget (Learnwithgoolede 2018). Bei der Suchnetzwerkwerbung erscheinen die Anzeigen nur dann, wenn jemand spezifisch und gezielt nach Begriffen 
sucht, welche die werbende Unternehmung in einer Kampagne oder einer Anzeigegruppe als Keyword hinterlegt hat.

\section{Display-Netzwerk}

Anzeigen unterschiedlicher Formate werden auf Webseiten, die entweder ausgewählt werden oder thematisch zu gewissen Keywords passen, platziert. Zudem erscheinen die Anzeigen auf Google-Produkten (Yapi 2016). Mit dem Display-Netzwerk können Werbende ihre Anzeigen auf ausgewählten Websites schalten und dazu auch Fotos oder Videos benutzen. Das Google-Display-Netzwerk umfasst gemäß Learnwithgooglede (2014) über eine Million Webseiten, Smartphone Apps, Videos, Blogs und andere Online-Plattformen, in denen AdWords-Anzeigen erscheinen können. Mit dieser Art von Werbung können über $80 \%$ der globalen Internetnutzer erreicht werden und der Werbende erhält damit eine immense Streumöglichkeit. Im Gegensatz zur Alternative „Suchnetzwerk“ gibt es beim Display-Netzwerk keine vorausgegangene Suchanfrage, welche dazu führt, dass eine relevante Anzeige geschaltet wird. Der Streuverlust ist bei dieser Art von Werbung somit höher.

Werbende können die beiden Werbearten abhängig von den Zielen der AdWords-Kampagnen einzeln benutzen oder kombinieren. Entscheidend ist das Bewusstsein für die Feinstrukturierung des AdWords-Kontos. Je besser die Strukturen gestaltet sind, desto effizienter sind Keywords, Anzeigen und Zielseiten aufeinander abgestimmt. Damit wird nicht nur eine höhere Qualität erreicht, sondern es ist auch möglich, das Budget besser zu kontrollieren (Yapi 2016). Die Kontenstruktur wird gemäß Leu (2018a) im Optimalfall an der Struktur der Webseite ausgerichtet, damit die Abgrenzung der Keyword-Listen und deren Anzeigen simpel getätigt werden kann.

\subsubsection{Keywords, Anzeigegruppen und Anzeigen}

\section{Keywords}

Ein einflussreicher Faktor des Marketings mit Google AdWords sind die Keywords. Die Wahl und Platzierung dieser ist für den Erfolg einer Google-AdWords-Kampagne maßgebend. Denn die Anzeigen erscheinen in den Suchergebnissen nur dann, wenn die Keywords zur Suchanfrage des Nutzers passen. Gemäß Yapi (2016) ist der Ausgangspunkt für eine effiziente Auswahl von Keywords zudem der Inhalt der beworbenen Seite sowie die sprachliche Sichtweise der Besucher.

Bei der Generierung der Keywords ist es zunächst wichtig, Begriffe zu sammeln, die den Inhalt der zu bewerbenden Webseite wiedergeben. Einzahl- und Mehrzahlformen, unterschiedliche Schreibweisen, Synonyme und Worte aus der Alltagssprache sind dabei genauso wichtig wie Fachbegriffe. Grundsätzlich lassen sich Keywords in drei verschiedene Typen unterteilen: 
1. Generische Begriffe: Beschreiben ein Thema, eine Branche oder einen Bereich von Produkten oder Dienstleistungen allgemein, ohne dabei ins Detail zu gehen (z. B. Reisen, Ferien).

2. Spezifische Begriffe: Beschreiben konkrete Unterbereiche, Produkte oder Angebote und sind detaillierter als generische Begriffe (z. B. Städtereise Paris buchen, Ferien Italien All Inclusive).

3. Markenbegriffe: Brand-Keywords bezeichnen ein Unternehmen oder ein Produkt (z. B. Iberostar, Iberostar Hotel Kroatien).

Um zu definieren, welche Suchanfragen die Schaltung einer Anzeige auslösen sollen, bestehen sogenannte Matchtypes, auch Keyword-Optionen genannt (Yapi 2016). Die Funktionsweise dieser ist eine wesentliche Komponente beim Google-AdWords-Marketing. Dem Werbenden stehen fünf Keyword-Optionen zur Auswahl, die unterschiedliche Voraussetzungen für die Ausspielung von Anzeigen bei Suchanfragen des Nutzers aufweisen (Leu 2018b). Die Zuteilung der verschiedenen Keyword-Optionen wird im AdWords-Konto anhand von bestimmten Zeichen ausgeführt. Tab. 12.1 zeigt die Optionen, welche für die Keywords gewählt werden können, inklusive der verwendeten Zeichen.

Tab. 12.1 Übersicht Keyword-Option. (In Anlehnung an Leu 2018b)

\begin{tabular}{l|l|l}
\hline Keyword-Option und Keyword & Anzeige kann erscheinen bei & Anzeige erscheint nicht bei \\
\hline $\begin{array}{l}\text { Weitgehend passend (engl. } \\
\text { bead) }\end{array}$ & $\begin{array}{l}\text { Laptop } \\
\text { Laptop online bestellen } \\
\text { MacBook bestellen } \\
\text { Leistungsstarker PC } \\
\text { Dell Laptop gratis }\end{array}$ & \\
\hline $\begin{array}{l}\text { Weitgehend passend+(engl. } \\
\text { broad match modifier) } \\
\text { Keyword: +Laptop +kaufen }\end{array}$ & $\begin{array}{l}\text { Laptop online kaufen } \\
\text { Laptop kaufen heute } \\
\text { HP Laptop kaufen } \\
\text { Kaufen Laptop }\end{array}$ & $\begin{array}{l}\text { Laptop bestellen } \\
\text { Laptop günstig } \\
\text { Laptop Gewinnspiel }\end{array}$ \\
\hline $\begin{array}{l}\text { Passende Wortgruppe (engl. } \\
\text { phrase) } \\
\text { Keyword: „Laptop kaufen“ }\end{array}$ & $\begin{array}{l}\text { Laptop kaufen heute } \\
\text { Laptop kaufen online } \\
\text { Shop Laptop kaufen }\end{array}$ & $\begin{array}{l}\text { Kaufen Laptop } \\
\text { Laptop online kaufen } \\
\text { Laptop heute kaufen } \\
\text { Laptop günstig }\end{array}$ \\
\hline $\begin{array}{l}\text { Genau passend (engl. exact) } \\
\text { Keyword: [Laptop kaufen] }\end{array}$ & Laptop kaufen & $\begin{array}{l}\text { Laptop bestellen } \\
\text { Jetzt Laptop kaufen } \\
\text { Laptop }\end{array}$ \\
\hline $\begin{array}{l}\text { Ausschließend (engl. negative) } \\
\text { Keyword: -Gebrauchsan- } \\
\text { weisung }\end{array}$ & $\begin{array}{l}\text { Laptop kaufen } \\
\text { Laptop } \\
\text { Günstiger Laptop kaufen }\end{array}$ & $\begin{array}{l}\text { Laptop Gebrauchsanweisung } \\
\text { Gebrauchsanweisung Laptop } \\
\text { Laptop kaufen mit Gebrauchs- } \\
\text { anweisung }\end{array}$ \\
\hline
\end{tabular}


Die fünf Keyword-Optionen funktionieren trichterartig und werden von oben nach unten immer spezifischer. Bei der generischen Option ,weitgehend passend“ sind Anzeigenschaltungen auch bei ähnlichen Wortgruppen oder Synonymen möglich. Diese Option wird für jene Keywords verwendet, die ein geringes Suchvolumen und wenig Markenbegriffe aufweisen (Yapi 2016). Während ein Vorteil dieses Matchtypes darin besteht, dass mehr Impressionen und Klicks generiert werden können, liegen die Nachteile darin, dass die Anzeige möglicherweise für irrelevante Suchanfragen geschaltet wird und somit Klicks entstehen, die nicht nachhaltig sind. Bei dieser Keyword-Option sollen auszuschließende Keywords verwendet und laufend aktualisiert werden (Leu 2018b). Am Ende des Trichters befindet sich der Matchtype „genau passend“, welcher Anzeigen nur dann ausspielt, wenn die Suchanfrage ausschließlich aus dieser Wortgruppe besteht (Yapi 2016). Diese Option erlaubt es, genau zu kontrollieren, für welche Suchanfragen die Anzeigen geschaltet werden. Ausschließende Keywords erübrigen sich bei dieser Option (Leu 2018b). Negative Keywords stellen jene Begriffe dar, zu denen die werbende Unternehmung auf keinen Fall seine Anzeigen schalten möchte.

\section{Anzeigegruppen}

Eine AdWords-Kampagne kann aus einer einzelnen oder mehreren Anzeigegruppen bestehen. Anzeigegruppen sind ein Unterelement der Kampagnen. Es ist pro Keyword auch möglich, in der Anzeigegruppe einzelne maximale CPC-Gebote festzulegen. Mithilfe der Anzeigegruppen können die Anzeigen thematisch sortiert und unterschiedliche Produkte und Dienstleistungen getrennt werden. Die Anzeigegruppen sind ein Strukturelement, um die Keywords und die Anzeigentexte zusammenzufassen. Damit möglichst viele Nutzer angesprochen werden, ist es sinnvoll, für jede Anzeigegruppe mehrere verschiedene Textanzeigen einzupflegen und mit passenden Keywords zu kombinieren (Yapi 2016). Zwei Anzeigen pro Anzeigegruppe sollten mindestens erstellt werden, damit die Erfahrung zeigen kann, welche Anzeige erfolgreicher ist, indem sie gegeneinander getestet werden (Aufgesang GmbH o. J.a).

\section{Anzeigen}

Die AdWords-Anzeigen sind die Werbungen, die den Nutzern auf den Ergebnisseiten angezeigt werden. Die Anzeigen werden in der jeweiligen Anzeigegruppe erstellt und sollten möglichst gut auf die darin enthaltenen Keywords abgestimmt sein. Zusätzlich muss sich die Anzeige auf den Inhalt der beworbenen Seite beziehen, was bedeutet, dass das Angebot des Werbenden die konkreten Vorteile für den Nutzer deutlich darstellen (Yapi 2016). Um eine positive Nutzererfahrung mit den Anzeigen zu erreichen, müssen zwei Kriterien erfüllt sein. Einerseits müssen die Anzeigen relevant, andererseits zutreffend und ehrlich sein. Für die Aufsetzung von Anzeigen bestehen wichtige Richtlinien, die eingehalten werden müssen, damit die Werbeanzeigen ausgespielt werden. Superlativen, die Werbende für sich selbst verwenden, müssen von Dritten bestätigt werden. Das Markenrecht darf nicht verletzt werden und somit ist es nicht erwünscht, 
fremde Marken als Keywords im Anzeigentext zu gebrauchen (Leu 2018b). Die Sorgfalt bei der Erstellung der Textanzeigen ist äußerst wichtig und kontinuierlich zu überprüfen.

Abb. 12.2 zeigt eine Google-AdWords-Werbeanzeige. Die zwei Slogans der ersten Zeile sind die Titelzeilen, die zweite Zeile besteht aus der Anzeige-URL und dem Hinweis, dass es sich um eine bezahlte Werbung (umrahmtes Wort „Anzeige“) hält. Der dritten Zeile kann die Beschreibung des Produkts oder der Dienstleistung entnommen werden.

\subsubsection{AdWords-Vertiefung}

Nachdem Grundbegriffe, wichtige Einzelheiten und die Funktionsweise von Google AdWords im Generellen verstanden sind, bietet eine Vertiefung im Fachgebiet die Chance, sich von der Konkurrenz abzuheben und das Marketing mit Google AdWords zu optimieren. Es bestehen Tools, welche teilweise direkt im AdWords-Konto integriert sind, während andere zusätzlich heruntergeladen werden können.

\subsubsection{Google Analytics}

Google Analytics ist ein Ableger der Unternehmung Google LLC und dient der Datenverkehrsanalyse. Die Haupteigenschaft von Google Analytics besteht demnach aus der Webanalyse und ist ein wichtiges Trackingtool für Unternehmungen, die einen Online-Auftritt haben und diesen optimieren möchten. Das Tool ist eigenständig und somit nicht im Google-AdWords-Konto integriert. Google Analytics erlaubt es, die Entwicklung der Webseite auf einen Blick zu erkennen und die Performance der Webseite zu kontrollieren, optimieren und verbessern.

Gemäß SEO Küche (o. J.a) und Unic (o. J.) sind die Hauptaspekte von Google Analytics folgende:

- Information bezüglich Besucheranzahl, Verweildauer und Verhalten der Besucher.

- Kenntnis der Suchbegriffe, über welche Besucher die Webseite gefunden haben.

- Demografische Merkmale wie Sprache und Standort der Besucher sind bekannt.

- Kenntnis darüber, welche Inhalte der Webseite sich die Besucher angesehen haben.

- Die Devices, welche die Besucher verwenden, sind bekannt.

- Google Analytics hat mehrere bahnbrechende Neuerungen implementiert.

- Die Verknüpfung mit dem Google-AdWords-Konto ist problemlos möglich.

- Die Einbindung von sozialen Netzwerken steht zur Verfügung.

Olivenöl Online Bestellen | Neue Ernte November 2017 Anzeige www.olio-nobile.ch/Bio-Öle -

Handgelesen, direkt vom Baum auf Ihren Tisch. Wir kennen unsere Produzenten.

Abb. 12.2 Grafik einer Werbeanzeige. (Eigene Darstellung) 
- Es können individuelle Ziele definiert und gemessen werden.

- Marketingaktivitäten können gezielt gemessen werden.

Als Nachteil des Tools muss genannt werden, dass Google durch die Einbindung von Google Analytics Zugang zu einer Vielzahl von internen Daten erhält. Es herrscht Unbekanntheit darüber, wie weit Google die gesammelten Daten zur Erstellung von umfassenden Benutzerprofilen nutzt und zukünftig für Marketingzwecke verwenden wird.

\subsubsection{Anzeigenerweiterungen}

Die Anzeigenerweiterungen dienen gemäß Google-AdWords-Schulung (Leu 2018a) einerseits dazu, die Anzeigen optisch größer zu machen und somit mehr Visibilität zu erhalten. Andererseits können die Werbenden die Anzeige mit relevanten Informationen erweitern, um die Attraktivität für den Nutzer zu erhöhen. Überdies sind die Erweiterungen wichtig, um auf den Ergebnisseiten vor der Konkurrenz zu erscheinen. Denn Werbende, welche die Anzeigenerweiterungen nutzen, haben eine erhöhte Chance, einen verbesserten Anzeigenrang zu erreichen, weil seit dem Jahr 2013 die Verwendung von Anzeigenerweiterungen in die Berechnung des Rankings im Google-Algorithmus einfließt und damit die Performance optimieren kann. Google steuert die Inhalte und die Schaltung der Anzeigenerweiterungen Mal für Mal. Es kann auch sein, dass keine Erweiterungen ausgespielt werden, sondern lediglich die bloße, ursprüngliche Anzeige (Yapi 2016). Die Anzeigenerweiterungen sind ein AdWords-internes Tool und der Zugriff läuft direkt in den Kampagnen ab.

Während die klassische Anzeige mit Titel, Anzeige-URL und 80-Zeichen-Beschreibung grundlegende Informationen liefert, geben Anzeigenerweiterungen ausführlichere Inputs bezüglich Produkt, Dienstleistung und/oder Unternehmung. Werbende Unternehmen haben dank den Anzeigenerweiterungen die Möglichkeit, potenziellen Kunden aufzuzeigen, was sie ergänzend zum Produkt oder der Dienstleistung, nach welcher aktuell gerade gesucht wird, noch Zusätzliches zu bieten haben. Zudem beinhalten die Anzeigenerweiterungen sogenannte „Click-to-Call-Buttons“, welche die User dazu aufrufen, eine Aktion zu tätigen, auf eine Anzeige zu klicken, sich den Webseiteninhalt anzuschauen oder Kontakt mit dem Werbenden aufzunehmen (Leu 2018a). In Abschn. „Anzeigenerweiterungen einpflegen“ finden sich Beschreibungen der Anzeigenerweiterungen, welche für das Liveprojekt benutzt wurden. Abb. 12.3 stellt eine Werbeanzeige auf Google AdWords dar, welche mit der Anzeigenerweiterung vom Typ „Sitelink“ ausgestattet ist.

\subsubsection{Search Console}

Die Search Console ist wie Google Analytics ein externes Tool, kann aber ebenfalls mit dem Google-AdWords-Konto verknüpft werden. Bei der Search Console geht es um die grundlegende Thematik, im Web gefunden zu werden. Mithilfe der Search Console können Werbende beobachten, wie die eigene Webseite bei den Suchanfragen abschneidet und anhand davon Optimierungen vornehmen. Mit der Search Console kann 


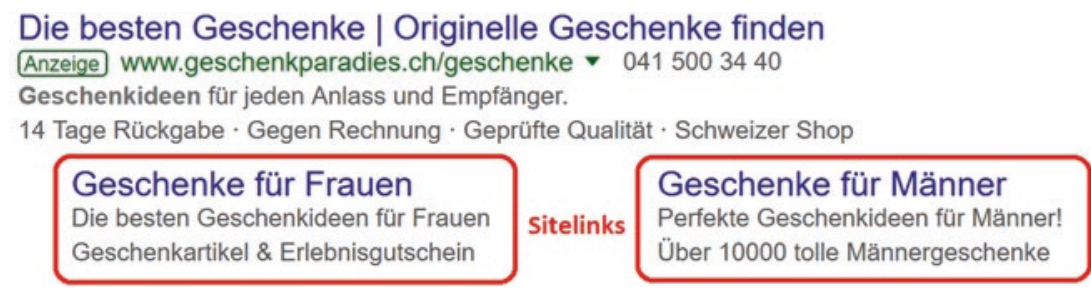

Abb. 12.3 Beispiel für Sitelink-Erweiterungen. (Eigene Darstellung)

sichergestellt werden, dass Google die eigene Webseite auf dem Radar hat und diese den Suchenden bei relevanten Suchanfragen auch einblendet. Ein weiterer Vorteil der Search Console besteht darin, dass das Tool regelmäßig nach Fehlern und Unregelmäßigkeiten sucht und diese meldet, falls negative Aspekte aufgefallen sind. Bei der Erstellung einer neuen Unter- oder Produkteseite, kann dank des Tools nachvollzogen werden, welche Begriffe die Suchenden auf die betroffenen Unterseiten gebracht hat. Somit erhält der Werbende die Möglichkeit, Outperformer zu pushen und diese für andere Unterseiten anzupassen und schlechte Performer zu eliminieren (Google Webmasters 2015). Die Google Search Console ist für die Suchmaschinenoptimierung von großem Wert (SEO Küche o. J.b).

\subsubsection{AdWords-Editor}

Der AdWords-Editor ist ein externes Tool, welches unterstützend für die Arbeit mit Google AdWords eingesetzt und kostenlos heruntergeladen werden kann. Der Editor dient hauptsächlich dazu, große Kampagnen zu bearbeiten. Zudem fungiert das Tool auch als Back-up, denn die Daten müssen vom Editor ins AdWords-Konto kopiert werden. Somit können Tests durchgeführt werden, für welche beispielsweise 100 neue Keywords implementiert und dafür 100 andere Keywords pausiert werden. Nach einiger Zeit können Schlüsse bezüglich Effizienz gezogen und die besser performenden Keywords beibehalten werden. Ein weiterer Vorzug des Tools ist, dass Keywords-Duplikate erkannt und bereinigt werden können (Leu 2018a). Als wichtigste Vorteile des AdWords-Editors können gemäß Google (o. J.) die Aspekte genannt werden, dass das Tool es erlaubt, Änderungen offline vorzunehmen und diese danach ins AdWords-Konto zu übertragen.

\subsubsection{Keyword-Platzhalter}

Dank dem Keyword-Platzhalter kann auf eine hilfreiche Funktion zurückgegriffen werden, welche es möglich macht, das Keyword, welches zur Anzeigenschaltung geführt hat, in einen entsprechenden Platzhalter einfließen zu lassen. Der Keyword-Platzhalter kann für jede beliebige Textanzeige verwendet werden, wobei der Keyword-Platzhalter-Code entweder im Anzeigentitel, in der Beschreibung oder im der angezeigten URL eingebaut wird (Aufgesang GmbH o. J.b). Bei jeder Suchanfrage wird jenes Keyword, welches zur Anzeigenschaltung geführt hat, automatisch in den Platzhalter eingefügt. 
Der Sinn hinter der Idee ist, dass der User in den Suchergebnissen das von ihm eingegebene Keyword eins zu eins in der Anzeige sieht und diese für ihn somit an Relevanz gewinnt. Eine Problematik des Keyword-Platzhalters stellen Falschschreibweisen dar, die in die Anzeige übernommen werden können, falls der Suchende den eingegebenen Begriff falsch buchstabiert. Das Tool ist in Google AdWords integriert und kann bei Bedarf für jede einzelne Anzeige benutzt werden (Leu 2018a).

Die vier beschriebenen Tools sind zwar die bekanntesten und einflussreichsten, sie sind aber nicht abschließend. Google AdWords bietet in weiteren Tools Funktionen und Maßnahmen an, um das Marketing mit Google AdWords zu verbessern. Alle zur Verfügung stehenden Tools oder Funktionen sind darauf ausgelegt, das Marketing mit Google AdWords zu vereinfachen und zu optimieren.

\subsubsection{Optimierungsmöglichkeiten}

$15 \%$ aller täglichen Suchanfragen wurden vorher noch nie genau so verwendet (Leu 2018a). Dieser Fakt verdeutlicht, dass Google AdWords sich in einem äußerst dynamischen Umfeld bewegt. Aufgrund dessen ist es essenziell, dass Werbende am Ball bleiben, mit den Anpassungen, Veränderungen und Neuheiten von Google mitgehen und bestrebt sind, deren AdWords-Konto laufend zu optimieren. Google AdWords bietet dafür Optimierungsmöglichkeiten anhand von verschiedenen Aktionen. Mit diesen Werkzeugen soll es den Werbenden möglich sein, dass wichtige Aspekte nicht vergessen gehen und die Kampagnen komplett sowie ausführlich sind. Ein grundlegender Optimierungstipp von AdWords besagt, dass mit optimalen Anzeigentexten die CTR und somit die gesamte Kampagnenperformance verbessert werden kann. Die Webanalyse ist dabei bei eher tiefen Budgets entscheidend. Beispielsweise kann mit den Webanalysetools die Segmentierung der Präsenz bezogen auf Wochentage oder Tageszeiten ins Auge gefasst und somit starke Wochen- oder Tageszeiten gepusht werden. Erfahrungswerte sind beim Marketing mit Google AdWords äußerst wichtig, da keine allgemeingültigen Aussagen für Kampagnen gemacht werden können und jedes Konto eine eigene Performance mit unterschiedlichen Auffälligkeiten aufweist (Leu 2018a, b).

Um die eigenen Kampagnen ständig zu optimieren und die Detailarbeit so auszuführen, dass das Maximum aus SEA-Maßnahmen herausgeholt werden kann, müssen sich Werbende bei der Auswahl der Keywords des Prozesses zwischen Bewusstsein, Einsatz und Leistung bewusst sein. Damit wird der Online-Kaufprozess vereinfacht abgebildet. Wer diesen Prozess ganzheitlich versteht und seine Keyword-Gruppen sowie Anzeigen daran ausrichtet, kann den potenziellen Kunden leiten und beeinflussen und das Marketing mit Google AdWords dadurch optimieren. Für Werbende ist es von hoher Wichtigkeit, dass die optimale Mischung zwischen generischen und spezifischen Keywords gefunden werden kann und dass sie sich bewusst sind, wonach die Nutzer in den jeweiligen Prozessschritten suchen. Gemäß Google-AdWords-Schulungen (Leu 2018a, b) ist es überdies sinnvoll, den größten Teil des Budgets für spezifische Suchanfragen auszugeben, 
damit die Relevanz der Werbung hoch ist. Gleichzeitig ist es wichtig, dass der ganze Prozess abgedeckt ist und Werbende auch in generische Keywords investieren. Zudem wird zur Optimierung empfohlen, in den Werbeanzeigen mit Saisonalität zu arbeiten. Auch die persönliche Ansprache der Kunden sowie die sogenannten „Call to Action Buttons“ werden als erfolgsversprechend angesehen. Zu guter Letzt wird betont, dass Spezialangebote bestens ankommen, da alle Menschen im Grundsatz Schnäppchenjäger sind.

\subsubsection{Relevanz zwischen Keyword, Anzeige \& Zielseite}

Das meistgenannte Argument für die Erreichung einer guten Performance im Google-AdWords-Konto ist die Relevanz zwischen Keyword, Anzeige und Zielseite. Wenn die eingeblendete Anzeige nicht auf die Suchanfrage des Users passt, wird er nicht darauf klicken, weil die Relevanz nicht gegeben ist. Wenn die Anzeige für die Suchanfrage zwar relevant ist und der Nutzer aus diesem Grund auf die Anzeige klickt, die verlinkte Landingpage am Ende dann aber nicht die gewünschten Informationen bzw. Angebote liefert, ist der User sofort wieder weg. Der Klick hat den Werber dann Geld gekostet, aber der Nutzer wurde nicht befriedigt und dem Werbenden ist es nicht gelungen, gute Werbung zu machen. Diese Entwicklung des Suchanfrageprozesses ist nicht erwünscht. Es ist essenziell, dass die Zielseite genau mit der Anzeige und dem, was darin versprochen wird, übereinstimmt (Leu 2018b). Nebst der Relevanz ist es entscheidend, dass der Inhalt der Landingpage eigenständig und originell ist. Der Text muss authentisch, konsistent und transparent sein. Zudem ist zu beachten, dass die gelieferten Informationen auf der Landingpage prägnant sind und keine langen Textwüsten beinhalten (Leu 2018a). Die Aussage bezüglich Kürze und Prägnanz wird von Oettrich (2016) widerlegt, indem empfohlen wird, lieber mehr als weniger zu schreiben, mindestens aber 300 Wörter. Übergreifend passt der Slogan „Content is King“ ausgezeichnet, wobei für Google entscheidend ist, dass ausreichend Text vorhanden ist, um diesen im Hintergrund durch die Funktionsweise des Google-Algorithmus einzustufen.

\subsection{Methodisches Vorgehen}

In diesem Kapitel werden die empirische Herangehensweise dieses Beitrags detailliert beschrieben und die Instrumente der Erkenntnisgewinnung dargestellt. Auf die Methode der Datenerhebung wird vertieft eingegangen. Zudem wird die Aufsetzung eines Pilotprojekts dargestellt.

Jonker und Pennink (2010, S. 23) empfehlen eine 4-stufige Forschungspyramide als hilfreiches Instrument für die Erarbeitung einer wissenschaftlichen Arbeit. Das Forschungsparadigma, die Forschungsmethodologie, die Forschungsmethode und die Forschungstechnik bilden diese vier Level. Auf allen vier Ebenen werden Entscheidungen getroffen. Die vier Level bestimmen die wissenschaftliche Herangehensweise und die Art der Umsetzung des Praxisprojekts. Die Methodologie dieses Beitrags wird zudem durch die wissenschaftliche Fragestellung beeinflusst. Die Forschungsfrage 
„Welches sind die Erfolgsfaktoren für die Kundengewinnung mit Google AdWords? Ein Pilotprojekt in Zusammenarbeit mit der Hofbauer $\mathrm{GmbH}^{\text {“ }}$ zeichnet sich zum einen durch eine offene Fragestellung aus. Zum anderen zeigt die Forschungsfrage auf, dass ein Pilotprojekt live durchgeführt wird, um die identifizierten Erfolgsfaktoren zu testen. Das Ziel richtet sich demnach deutlich auf die Identifikation von Erfolgsfaktoren beim Marketing mit Google AdWords aus, welche anhand von einer Case Study Analysis aufgedeckt werden sollen. Für die Bestätigung oder Verwerfung der Erfolgsfaktoren dient das Pilotprojekt und für ein fundiertes Vorwissen bezüglich der Themenfelder Online-Marketing und Google AdWords wird eine ausführliche Literaturrecherche vorausgesetzt. Für die Aufdeckung von Erfolgsfaktoren für das Marketing mit Google AdWords eignet sich eine qualitative explorative Erhebung mittels Experteninterviews ausgezeichnet. Dabei wurden einerseits drei Google-AdWords-Experten befragt, um Einblicke in die Welt des Online-Marketings im Generellen und Google AdWords im Spezifischen zu erhalten. Die drei Experten sind bei einem offiziellen Google Partner angestellt, der AdWords-Marketing als Dienstleistung anbietet. Andererseits wurden Experten aus sieben verschiedenen Unternehmen unterschiedlichster Größen und Branchen zu derer Arbeit und Erfahrungen mit Google AdWords interviewt. Die Methodologie ist demnach stark qualitativ geprägt. Diese Herangehensweise wird von Gläser und Laudel (2009, S. 25-29) gestützt. Genanntes Vorgehen erlaubt ein offener Forschungsprozess, was dem noch eher neuartigen und vor allem sehr dynamischen Themengebiet dieses Projekts entspricht.

\subsubsection{Datenerhebung}

Um die qualitative Datenerhebung sorgfältig durchzuführen, orientiert sich die Abwicklung inklusive Vorbereitung und Auswertung an dem von Gläser und Laudel (2010, S. 203) vorgeschlagenen Ablauf. Dieser startet mit theoretischen Vorüberlegungen, welche die Formulierung der Forschungsfrage und die theoretische Analyse und Bestimmung von Variablen beinhaltet, gefolgt von der Extraktions-Vorbereitung, welche die Fixierung des Materials, die Aufnahme von aus der Theorie bekannten Ausprägungen der Variablen sowie der Bestimmung von Indikatoren ins Auge fasst. Der Hauptaspekt dieses Zwischenschritts ist die Festlegung des Samples. Gefolgt wird dies von der Extraktion, welche sich um die Formulierung von Extraktionsregeln, die Aufnahme neuer Ausprägungen, die allfällige Veränderung existierender oder die Konstruktion neuer Variablen und die Aufnahme neuer Indikatoren kümmert. Dieser Schritt wird grob auch Materialdurchlauf genannt und beschreibt die Durchführung der Experteninterviews. Anschließend erfolgen die Aufbereitung der Daten und somit die Sortierung nach zeitlichen oder sachlichen Aspekten, die Zusammenfassung bedeutungsgleicher Informationen sowie die Beseitigung elementarer Fehler. Danach wird die Datenerhebung ausgewertet und dabei fallübergreifende Zusammenhänge und Fälle analysiert (Gläser und Laudel 2010). Nachfolgend wird auf die einzelnen Phasen eingegangen. 


\section{Theoretische Vorüberlegungen}

Die Forschungsfrage „Welches sind die Erfolgsfaktoren für die Kundengewinnung mit Google AdWords? Ein Pilotprojekt in Zusammenarbeit mit der Hofbauer GmbH“ wurde im Rahmen der Vorstudie bestimmt. Ebenso erfolgte in dieser Phase die Erhebung des aktuellen Forschungsstands anhand von Sekundärliteratur. Damit konnten die Forschungslücke sowie die Zielformulierung deutlich abgesteckt werden. Die zur Beantwortung der Forschungsfrage notwendigen Angaben wurden anhand von Experteninterviews sowie der Teilnahme an Google-Kursen eruiert. Damit wurde sichergestellt, dass einzelne Variablen in Abhängigkeit zueinander schließlich die Erfolgsfaktoren des SEA mit Google AdWords identifizieren können. Dank des durchgeführten Pilotprojekts konnten die Variablen geprüft werden. Die qualitative Datenerhebung wurde im Stil einer Case Study durchgeführt.

\section{Vorbereitung Extraktion: Beschreibung des Samples}

Die Literaturrecherche, die Gespräche mit Google AdWords Experten sowie die Teilnahme an Google AdWords Kursen haben aufgezeigt, welche Variablen bereits erhoben wurden und zu welchen Variablen bereits Studien bestehen. Da Online-Marketing und damit einhergehend Google AdWords äußerst aktuelle und populäre Themen sind, bestehen dazu viele Studien und Theorien. Dennoch ist zu betonen, dass keine ganzheitlichen Konzepte für eine erfolgreiche Implementierung eines Google-AdWords-Kontos bestehen.

Bei der Definition des Samples wurde darauf geachtet, dass die Vertreter der Case Study aus verschiedenen Branchen und unterschiedlich großen Unternehmungen stammen, damit möglichst vielfältige Ansichten gewonnen werden können. Zudem wurde das Sample bewusst so erstellt, dass Unternehmungen dabei sind, welche mit Google AdWords ihren Onlineshop bewerben sowie auch Firmen, welche den Fokus nicht auf dem Onlineshop haben oder gar keinen solchen betreiben. Dies weil die Vorstudie ergeben hat, dass die Arbeit mit Google AdWords wesentlich davon abhängt, ob Werbende einen Onlineshop haben und somit direkt Produkte und/oder Dienstleistungen verkaufen oder eben nicht. Um eine zweite Sicht auf das Thema zu erhalten, wurden nebst den Vertretern der Fallstudie drei Google-AdWords-Experten befragt. Aufgrund der unterschiedlichen Problemfelder und Informationsquellen wurden zwei halbstrukturierte Leitfäden erarbeitet, je einen für die Google-AdWords-Experten und einen für die Vertreter der Case Study. Die Leitfäden wurden vorgängig getestet und während der Erhebungsphase laufend angepasst. Außerdem wurden sie kontinuierlich auf dessen Validität geprüft, damit die Resultate zuverlässig erhoben werden konnten. Den Vertretern der Case Study wurde im Vorfeld ein Fragebogen zum biografischen Hintergrund sowie eine auszufüllende Tabelle mit Tools und Eigenschaften von Google AdWords zugestellt, damit eine optimale Vorbereitung auf das Gespräch möglich war und Einblicke in die AdWords-Arbeitsweise der Fallstudienvertreter gewonnen werden konnte. Die Arbeitgeber der Befragten werden aufgrund der Vertraulichkeit der Daten anonymisiert. Der Arbeitgeber der Google-AdWords-Experten wurde umschrieben, 
aber auch anonymisiert. In Tab. 12.2 werden die Google-AdWords-Experten, welche in explorativen Interviews befragt wurden, beschrieben.

In Tab. 12.3 werden die anonymisierten Unternehmungen mit einigen Eckpfeilern beschrieben und die Position des befragten Experten im Unternehmen angegeben. Das Sample besteht aus Unternehmungen aus verschiedenen Regionen der Deutschschweiz.

Tab. 12.2 Interviewpartner - Google-AdWords-Experten. (Eigene Darstellung)

\begin{tabular}{l|l|l}
\hline Name & Arbeitgeber & Position \\
\hline Jeannine Künzler & Offizieller Google Partner, Chur & Leiterin Performance Marketing \\
\hline Tamara Ulber & Offizieller Google Partner, Chur & Medienberaterin \\
\hline Thomas Zimmermann & Offizieller Google Partner, Chur & Online Campaign Manager \\
\hline
\end{tabular}

Tab. 12.3 Interviewpartner - Vertreter Case Study. (Eigene Darstellung)

\begin{tabular}{|c|c|c|}
\hline Name & Unternehmensdetails & Position des Befragten \\
\hline Vertreter Case Study 1 & $\begin{array}{l}\text { Branche: Versicherungen \& Vorsorge } \\
\text { Anzahl MA: >4000 } \\
\text { Gründung: vor } 1850 \\
\text { Jahresumsatz, grob: } 3,5 \text { Mio. CHF }\end{array}$ & Online-Marketing-Manager \\
\hline Vertreter Case Study 2 & $\begin{array}{l}\text { Branche: E-Commerce (Generalist) } \\
\text { Anzahl MA: }>40 \\
\text { Gründung: } 2007 \\
\text { Jahresumsatz, grob: } 28 \text { Mio. CHF }\end{array}$ & Geschäftsführer \\
\hline Vertreter Case Study 3 & $\begin{array}{l}\text { Branche: öffentlicher Verkehr } \\
\text { Anzahl MA: >36 } \\
\text { Gründung: vor } 1950 \\
\text { Jahresumsatz, grob: } 650.000 \mathrm{CHF}\end{array}$ & $\begin{array}{l}\text { Marketing-Kommunikation, } \\
\text { AdWords-Verantwortlicher }\end{array}$ \\
\hline Vertreter Case Study 4 & $\begin{array}{l}\text { Branche: E-Commerce (Kinderfahr- } \\
\text { zeuge) } \\
\text { Anzahl MA: }<8 \\
\text { Gründung: } 2014 \\
\text { Jahresumsatz, grob: } 1 \text { Mio. CHF }\end{array}$ & Geschäftsführer \\
\hline Vertreter Case Study 5 & $\begin{array}{l}\text { Branche: E-Commerce (Generalist) } \\
\text { Anzahl MA: >100 } \\
\text { Gründung: } 2009 \\
\text { Jahresumsatz, grob: } 42 \text { Mio. CHF }\end{array}$ & AdWords-Verantwortlicher \\
\hline Vertreter Case Study 6 & $\begin{array}{l}\text { Branche: E-Commerce (Generalist) } \\
\text { Anzahl MA: >1000 } \\
\text { Gründung: } 2001 \\
\text { Jahresumsatz, grob: } 830 \text { Mio. CHF }\end{array}$ & $\begin{array}{l}\text { Leiter Digital Marketing \& } \\
\text { Social Media }\end{array}$ \\
\hline Vertreter Case Study 7 & $\begin{array}{l}\text { Branche: Sicherheitstechnik } \\
\text { Anzahl MA: <8 } \\
\text { Gründung: } 2014 \\
\text { Jahresumsatz, grob: } 450.000 \mathrm{CHF}\end{array}$ & Geschäftsführer \\
\hline
\end{tabular}




\section{Extraktion: Durchführung der qualitativen Datenerhebung}

Die Durchführung der Experteninterviews, welche jeweils am Sitz des Unternehmens durchgeführt wurden, dauerte zwischen 40 und 80 min. Während der Extraktion wurden Variablen neu aufgenommen oder verändert und kontinuierlich neue Indikatoren gewonnen. Diese wurden für die darauffolgenden Interviews miteinbezogen.

\section{Aufbereitung}

Um eine systematische und übersichtliche Analyse der qualitativen Datenerhebung sicherzustellen, wurde im Bereich der Experteninterviews mit dem Auswertungstool Atlas.ti gearbeitet. Die Benutzung dieses Tools hat die Erstellung von Codes und Codefamilien ermöglicht, welche aus den Interviewgesprächen extrahiert wurden. Um die Codes zu clustern, wurden Codefamilien gegründet, welche Zitate enthalten, deren verschiedene Codes in einem direkten Zusammenhang zueinanderstehen. Damit eine saubere Codierung gewährleistet werden konnte, wurden alle 10 Interviews in WordDateien transkribiert und die Textdateien mit Zeilennummern versehen, was die exakte Angabe von Quellen vereinfacht. Um die Anonymität der befragten Unternehmen nicht zu gefährden, wurden die Unternehmensnamen in den Transkriptionen mit Codes überschrieben.

\section{Auswertung}

Ein wichtiger Schritt der Auswertung ist die Datenauswertung mittels qualitativer Inhaltsanalyse. Das übergeordnete Ziel dieser Auswertung ist die Erreichung einer Auflistung von Google-AdWords-Erfolgsfaktoren. Um Erfolgsfaktoren aufzudecken, werden die Unternehmungen der Befragten klassifiziert. Als Erfolgs- bzw. Misserfolgsindikator für die befragten Unternehmungen gelten die Zahlen der Umsatzentwicklung des jeweiligen Unternehmens, anhand welcher erklärt werden kann, ob es sich bei der jeweiligen Firma um einen Outperformer oder einen Under-Performer handelt. Die identifizierten Faktoren bei Outperformern geben gemäß der Klassifizierung Anlass, dass es sich um generelle Erfolgsfaktoren beim Marketing mit Google AdWords handelt. Die Interpretation der Ergebnisse aus der Primärforschung erfolgt auch mittels Gegenüberstellung der Erkenntnisse aus der Sekundärforschung, die in der Vorstudie gewonnen werden konnte. Zum Abschluss des Berichts wird der erarbeitete Forschungsstand dargestellt.

\subsubsection{Pilotprojekt}

Aufgrund des starken Praxisbezugs dieses Themenfelds wurde entschieden, ein Liveprojekt durchzuführen, um die gewonnen Erkenntnisse anhand davon zu überprüfen, und damit zu bestätigen oder zu verwerfen. Der Partner für die Implementierung des Pilotprojekts ist ein KMU aus der Region Bern mit der Organisationsform einer GmbH, welches bisher kein Augenmerk auf das SEA mit Google AdWords gesetzt hatte, das 
Potenzial dieses Marketingtools aber als sehr hoch einstuft. Der Partner des Liveprojekts wird in Abschn. 12.6.1 beschrieben.

Die gewonnen Erkenntnisse aus den Gesprächen mit Google-AdWords-Experten, aus Besuchen der beiden Kurse „Learn with Google - Suchmaschinenmarketing mit Google AdWords“ und aus der qualitativen Datenerhebung konnten dank des Pilotprojekts getestet werden. Für die Durchführung des Pilotprojekts wurde vom Geschäftsführer der Partnerfirma ein SEA-Budget von $1000 \mathrm{CHF}$ gesprochen. AdWords arbeitet mit monatlichen Budgets, welche aufgrund der budgetierten $1000 \mathrm{CHF}$ auf $500 \mathrm{CHF}$ pro Monat gesetzt wurde. Da die Kampagnen mit Tagesbudgets ausgestattet werden müssen, wurde das tägliche Budget zwischen $16 \mathrm{CHF}$ und $17 \mathrm{CHF}$ festgelegt und jeweils über die drei bzw. vier laufenden Kampagnen aufgeteilt. Genaueres bezüglich Tagesbudgets und Kampagnen ist in Abschn. 12.6 nachzulesen. Ebenfalls in Abschn. 12.6 finden sich Informationen bezüglich Eröffnung des AdWords-Kontos, Generierung von Keywords, Aufsetzung von Anzeigegruppen sowie Anzeigen und weitere Aufwendungen bezüglich AdWords-Kampagnenerstellung. Die Liveschaltung des Pilotprojekts erfolgte am 6. April 2018 und dauerte knapp zwei Monate. Eine ständige Überwachung und Optimierung sollten in diesem Arbeitsschritt gewährleistet werden. Die Dynamik und Schnelllebigkeit des Online-Marketings stellt eine große Herausforderung in Sachen Aktualität dar. Die laufende Einbringung von gewonnen Erkenntnissen in das Pilotprojekt wurde sichergestellt. Wichtige Einsichten und Meinungen, die aus den durchgeführten Interviews gewonnen werden konnten, wurden kontinuierlich im Pilotprojekt eingebettet. Weitere Kampagnen-Optimierungen wurden mithilfe der Leiterin des Performance-Marketings eines offiziellen Google-Partners aus Chur besprochen und implementiert.

\subsection{Resultate}

Dieses Kapitel rekonstruiert die gewonnen Erkenntnisse aus der qualitativen Datenerhebung mittels Experteninterviews. Das Hauptaugenmerk liegt dabei auf der Darstellung von Erfolgsfaktoren für das Marketing mit Google AdWords.

\subsubsection{Klassifizierung des Samples}

Damit die von den befragten Experten als erfolgsversprechende Aktivitäten für das Marketing mit Google AdWords benannten Aspekte tatsächlich als Erfolgsfaktoren bestätigt werden können, wurde die Umsatzentwicklung der sieben Unternehmungen über die letzten Jahre in Betracht gezogen. Die Unternehmungen wurden anhand dieser Kennzahl sowie dem Einbezug des Gründungsjahrs und der schweizweiten Bekanntheit in die zwei Klassen „Outperformer“ und „moderate Performer“ eingeteilt. Die Datenverfügbarkeit für diese Kennzahl wurde von den interviewten Personen im Nachgang auf Anfrage 
per E-Mail zur Verfügung gestellt. Da die Entwicklung aller befragten Unternehmen als positiv eingestuft werden kann, gilt für diese Forschungsarbeit die Annahme, dass die von den Experten als erfolgsversprechend ausgewiesenen Aspekte tatsächlich Erfolgsfaktoren fürs Marketing mit Google AdWords darstellen. Tab. 12.4 zeigt die Klassifizierung der Arbeitgeber der Interviewpartner.

Die Zahlen 1 bis 7 der Case Study sind für die Arbeitgeber mit der gleichen Ziffer versehen wie in Tab. 12.3. Die Klassifizierung hat ergeben, dass die befragten Unternehmen mit einer Ausnahme durchwegs positive Entwicklungen aufzeigen. Dies beweist, dass die Fallstudie ausnahmslos florierende Unternehmen beinhaltet. Infolgedessen gibt es im Sample keine schwachen Performer, sondern ausschließlich moderate Performer und Outperformer. Die einzige negative Umsatzentwicklung der Unternehmung 3 der Case Study wurde vom befragten Experten relativiert und mit einer prozentualen Steigerung von buchenden Personen (PAX) um 17,5 \% im Jahr 2017 gegenüber 2016 untermauert (Branche von Case Study 3: Öffentlicher Verkehr, vgl. Tab. 12.3).

\subsubsection{Moderate Performer}

Die Arbeitgeber der Vertreter der Case Study mit den Nummern 3, 4, 5 und 7 wurden als moderat erfolgreiche Unternehmen eingestuft. Es ist zu betonen, dass dies keineswegs ein schlechtes Zeugnis ist, denn die Kennzahlen und Entwicklungen der befragten

Tab. 12.4 Klassifizierung des Samples. (Eigene Darstellung)

\begin{tabular}{|c|c|c|}
\hline Unternehmung & Umsatzentwicklung in \% & Klassifizierung \\
\hline Case Study 1 & $\begin{array}{l}2015:+0,72 \% \\
2016:+3,71 \% \\
2017:+3,99 \%\end{array}$ & Outperformer \\
\hline Case Study 2 & $\begin{array}{l}2015:+ \text { ca. } 25 \% \\
2016:+ \text { ca. } 25 \% \\
2017:+ \text { ca. } 25 \%\end{array}$ & Outperformer \\
\hline Case Study 3 & $\begin{array}{l}\text { 2015: keine Angabe } \\
\text { 2016: }+12,75 \% \\
\text { 2017: }-11,16 \%\end{array}$ & Moderate Performer \\
\hline Case Study 4 & $\begin{array}{l}\text { 2015: + } 133 \% \text { (Gründung 2014) } \\
2016:+194 \% \\
2017:+42 \%\end{array}$ & Moderate Performer \\
\hline Case Study 5 & Keine Angaben (Jahresumsatz ca. 42 Mio. CHF) & Moderate Performer \\
\hline Case Study 6 & $\begin{array}{l}\text { 2015: keine Angabe } \\
\text { 2016: +12,65\% } \\
\text { 2017: +18,47\% }\end{array}$ & Outperformer \\
\hline Case Study 7 & $\begin{array}{l}\text { 2015: Gründung } \\
\text { 2016: }+733,3 \% \\
\text { 2017: }+276 \%\end{array}$ & Moderate Performer \\
\hline
\end{tabular}


Unternehmen lassen sich sehen und sprechen eine hoffnungsvolle Sprache für die Zukunft. Die vier Unternehmungen wurden aufgrund dessen als moderater Performer eingestuft, weil sie zum Teil noch sehr jung sind (Case Study 4 und 7) und die äußerst hohen Umsatzentwicklungszahlen der ersten Jahre noch nicht überbewertet werden dürfen. Die als moderate Performer eingestuften Unternehmungen konnten die prozentuale Umsatzsteigerung von 2015 auf 2016 für das Folgejahr 2017 nicht halten. Die beiden Case Studies 3 und 5 wurden als moderat eingestuft, weil deren Entwicklung gemäß Medien eher stagnieren und auf dem aktuellen Level bestehen bleiben. Wenn diese beiden Unternehmungen ihr Niveau halten können, kann dies jedoch als befriedigend eingestuft werden, denn beide sind im Markt stark etabliert und bewegen sich zudem in einem Marktumfeld, das aktuell nicht stark expandiert.

\subsubsection{Outperformer}

Die Arbeitgeber der Vertreter der Case Study mit den Nummern 1, 2 und 6 wurden als Outperformer eingestuft, was bedeutet, dass sie eine außerordentlich positive Umsatzsteigerung und weitere äußerst nachhaltige Kennzahlen ausweisen können. Zwei der drei klassifizierten Outperformer (Case Studies 1 und 6) sind schweizweit bekannt und dürfen sich selbst in ihren Branchen als Vorzeigeunternehmung betiteln. Es handelt sich dabei um einzigartig erfolgreiche Firmen, die in Wirtschaft und Gesellschaft stark verankert sind. Die Unternehmung hinter Case Study 2 ist jünger, aber sehr dynamisch und ambitiös. Die Firma zeichnet sich seit der Gründung vor gut 10 Jahren durch stetiges und starkes Wachstum (ca. 25 \% Umsatzsteigerung pro Jahr) aus. Die Unternehmen, die als Outperformer eingestuft wurden, konnten ihre prozentuale Umsatzsteigerung über die letzten Jahre halten oder sogar weiter verbessern.

Da alle befragten Unternehmen positive Entwicklungen und Zukunftsaussichten ausweisen, wird in diesem Beitrag davon ausgegangen, dass deren Informationen bezüglich des Marketings mit Google AdWords glaubwürdig und repräsentativ sind. Alle befragten Personen sind ausgewiesene Fachleute im Bereich Online-Marketing und arbeiten seit längerer Zeit, teilweise sogar seit bald einem Jahrzehnt, mit Google AdWords.

\subsubsection{Qualitative Datenauswertung}

Die sieben befragten Personen aus der Fallstudie sowie einer der drei befragten Google-AdWords-Experten wurden im Vorfeld des Interviewgesprächs gebeten, einen Fragebogen zum biografischen Hintergrund, welcher zusätzlich zwei Tabellen bezüglich Arbeitsweisen mit Google AdWords und dessen Tools und Funktionen beinhielten, auszufüllen. Dadurch konnte eruiert werden, wie viele der acht befragten Experten die verschiedenen Tools und Eigenschaften von Google (Abschn. 12.3.2) mit welcher Frequenz benutzen. Auffallend ist dabei, dass sieben Experten angegeben haben, dass sie Anzeigenerweiterungen immer benutzen. Auch die Nutzung von Google Analytics wurde von je vier Befragten mit ,immer“ bzw. „manchmal“ beantwortet. Die 
Auswertung der Fragebögen hat ergeben, dass die Anzeigenerweiterungen sowie Google Analytics eine deutliche Wirkungsweise haben.

Die Analyse der primären Datenforschung hat aufgezeigt, dass durch ein nachhaltiges Marketing mit Google AdWords auch die organischen Suchergebnisse verbessert werden können. Dies stellt damit ein doppelter Vorteil von Google AdWords dar, denn Werbende haben damit die Chance, die Visibilität mit SEA sowie auch SEO zu optimieren (VCS 2 2018 und VCS 3 2018).

\subsubsection{Die Macht von Google AdWords}

Die Auswertung der Datenerhebung hat ergeben, dass ein wichtiger Faktor für die enorm hohe Priorität, welches das Online-Marketing-Tool Google AdWords genießt, vom wichtigen Status der Firma Google gegeben ist. Die Suchmaschine von Google, welche gemäß Lunapark (2017) weltweit einen Marktanteil von $90 \%$ hat, ist ein wichtiges Zugpferd des SEA-Tools Google AdWords. Google stellt jene Plattform zur Verfügung, auf welcher der größte Anteil an Internetusern nach Produkten und Dienstleistungen sucht. Abgesehen davon, ob AdWords ein nutzerfreundliches und ausgereiftes Tool ist, ist es für Werbende wichtig, dort präsent zu sein, wo die Masse sucht, und aus diesem Grund kommen Werbende, Stand heute, nicht an Google und seinen AdWords vorbei, wenn sie SEA betreiben möchten. Dieses Statement wurde von allen 10 befragten Experten abgegeben (Ulber 2018; VCS 1 2018; VCS 2 2018; VCS 3 2018; VCS 4 2018; VCS 5 2018; VCS 6 2018; VCS 7 2018; Zimmermann 2018). Google ist es gelungen, eine einzigartige Suchmaschine mit einem derart ausgereiften Algorithmus zu erschaffen, der schlicht und einfach unersetzbar ist (VCS 1 2018; VCS 4 2018; VCS 7 2018). Der Status einer so klaren, weltweiten Nummer 1 ist aus Werbetreibender Sicht auf der einen Seite sicherlich interessant, da Werbende sich nicht mit mehreren Anbietern auseinandersetzen müssen (VCS 6 2018). Trotzdem muss erwähnt werden, dass ein gewisses Maß an Klumpenrisiko besteht.

\subsubsection{Eigenschaften des Online-Marketings}

Das Nutzerverhalten von Online-Usern ist anders als das Verhalten von Offline-Kunden, und diesen Unterschieden müssen Werbende sich bewusst sein, damit Online-Marketing erfolgreich betrieben werden kann. Gemäß Künzler (2018) benötigt es bei der OnlineWerbung durchschnittlich sieben Kontakte eines Users, bis sich dieser für einen Kauf oder eine andere Aktion (z. B. Ausfüllen eines Kontaktformulars) überzeugen lässt. Es ist für Werbende also wichtig, dass sie Content bieten, dank welchem sich Kunden informieren können. Wenn sie zu einem späteren Zeitpunkt dann bereit sind für den Kauf oder eine Aktion ist die Chance groß, dass sie sich an den Anbieter des Contents erinnern und für die Conversion bzw. den Kauf wieder die Webseite jenes Anbieters aufsuchen (VCS 12018 und VCS 3 2018). Eine andere Eigenschaft des Online-Marketings und seiner Nutzer ist der Fakt, dass das Umfeld sehr schnelllebig und dynamisch ist und alles unmittelbar geschieht (Ulber 2018). Außerdem muss beachtet werden, dass ein Großteil der Kunden hybride Eigenschaften aufweisen. Das heißt es gibt Leute, die Produkte 
oder Dienstleistungen online suchen und sie danach offline im Laden oder Geschäft kaufen und umgekehrt (VCS 1 2018). Die Grundtendenz der Population geht immer mehr dahin, dass sich das Nutzerverhalten stärker auf Online-Tools konzentriert. Die Affinität der Leute für Online-Aktivitäten steigt (VCS 2 2018).

\subsubsection{Chancen des Online-Marketings}

Die Vorzüge des Online-Marketings sind sehr vielfältig. Zusammenfassend setzen sich gemäß Primärforschung folgende Aspekte als entscheidende Vorteile des OnlineMarketings durch:

- Messbarkeit des Erfolgs und damit einhergehend eine saubere Analyse des OnlineMarketings (Ulber 2018; VCS 1 2018; VCS 2 2018; VCS 3 2018; VCS 7 2018; Zimmermann 2018).

- Die Nachvollziehbarkeit von Aktionen dank der Analyse des Nutzerverhaltens ermöglicht die Aufdeckung der Intention von Internetusern. Wenn Werbetreibende es schaffen, sich in den Kunden hineinzuversetzen und Online-Werbung in Bezug darauf zu schalten, kann dies einen sehr wichtigen Wettbewerbsvorteil generieren (Ulber 2018; VCS 1 2018; VCS 3 2018; VCS 6 2018; VCS 7 2018).

- Unmittelbarkeit und Flexibilität, die es ermöglichen, Anpassungen oder Neuheiten sofort zu implementieren und somit kurze Reaktionszeiten aufweisen (VCS 12018 ; VCS 6 2018).

- Zielgerichtete Werbung mit wenig Streuverlust und direkter Ansprache lässt eine Budgetoptimierung zu und sorgt somit für effizientes Online-Marketing (Ulber 2018; VCS 3 2018; VCS 4 2018; VCS 6 2018).

- Die unmittelbare Vergleichbarkeit mit Mitbewerbern und die sofort mögliche Steuerung von verschiedenen Aktionen schaffen Spielräume um sich von den Mitbewerbern abzuheben (VCS 2 2018; VCS 3 2018).

- Im Vergleich zum Offline-Marketing findet im Online-Marketing meistens kein Medienbruch statt, was die Customer Journey optimiert (VCS 6 2018; VCS 7 2018).

Die meistgenannte Chance des Online-Marketings ist die Messbarkeit. Diese ist durch die Einbettung von Trackingcodes möglich, welche aufzeigen, wie ein User sich auf der Webseite verhält, bis er zum Abschluss kommt. Die Abschlüsse oder Käufe werden Conversions genannt und erlauben es, den Erfolg des Online-Marketings zu messen (VCS 1 2018; VCS 3 2018; Zimmermann 2018). Obwohl die Messbarkeit oft als Vorteil des Online-Marketings angeschaut wird, ist die Erfolgsmessung teilweise sehr schwierig, gerade bei Werbetreibenden ohne Onlineshop. Wenn kein Onlineshop vorhanden ist, wo User den Kauf direkt tätigen können, fällt die Messung des Erfolgs schwer. Schließlich kann es sein, dass ein Internetnutzer ein Angebot online gefunden hat, den Abschluss dann aber im Offline-Store vor Ort tätigt. Der Kauf müsste in dem Fall der Online-Werbung zugeschrieben werden. Natürlich ist auch die umgekehrte Option möglich, dass ein Produkt im Offline-Store entdeckt und dann online bestellt wird. Die 
stets korrekte Zuteilung der Werbeeinnahmen ist beinahe unmöglich (VCS 1 2018; VCS 2 2018; Zimmermann 2018). Eine weitere Hürde für die Erfolgsmessung ist die Nichteinbindung von Google Analytics (Abschn. 12.3.2) (VCS 3 2018). Zudem müssen bei der Erfolgsmessung die Werbeausgaben in Betracht gezogen werden, da die simple Betrachtung einer allfälligen Umsatzsteigerung, ohne Kenntnis der Werbekosten, nicht ausreicht. Marge ist dabei das Zauberwort. Diese wird gemäß Marketingexperten (VCS 2 2018; VCS 3 2018; VCS 4 2018; VCS 5 2018; VCS 6 2018) anhand der Kennzahlen KUR, ROI oder ROAS überwacht und optimiert.

\subsubsection{Integrierte Marketingkommunikation}

Die Primärforschung hat aufgezeigt, dass es möglich ist, den Fokus ausschließlich auf einen Marketing-Kanal zu legen (Ulber 2018). Trotzdem machen die Aussagen der Befragten deutlich, dass die Ausführungen von Rumo (2017) bezüglich Einbindung von crossmedialem Marketing im Regelfall die optimale Lösung für werbende Unternehmungen darstellt. Das Angebot eines crossmedialen Marketing-Mixes ist nachhaltig und kann einen Erfolgsfaktor für die Performance einer ganzheitlichen Marketingstrategie darstellen. Mit crossmedialem Marketing ist einerseits gemeint, dass bestenfalls offline sowie online Werbung getätigt wird und andererseits, dass innerhalb der beiden Stränge wiederum verschiedene Kanäle benutzt werden (Ulber 2018; VCS 1 2018; VCS 3 2018; VCS 5 2018; VCS 6 2018; VCS 7 2018; Zimmermann 2018). Im Bereich des Online-Marketings haben sich hauptsächlich Aktivitäten im Remarketing, welche versuchen Leute zurückzuholen, die früher mal auf der Webseite waren, und im Displaynetzwerk, bei welchem Visibilität und somit Brand-Awareness erreicht werden möchte, als effizient herauskristallisiert (VCS 1 2018; VCS 2 2018; VCS 3 2018; VCS 6 2018; VCS 7 2018). Das zur Verfügung stehende Budget spielt beim Implementieren einer integrierten Marketingkommunikation eine zentrale Rolle. Wenn das Budget ausreichend ist, wird der crossmediale Mix unerlässlich, um einen möglichst großen Teil der Leute zu erreichen und somit Kunden zu gewinnen (Ulber 2018; VCS 6 2018; Zimmermann 2018).

\subsubsection{Auffälligkeiten Google AdWords}

Die Datenerhebung hat ergeben, dass die Eintrittsbarrieren beim Marketing mit Google AdWords relativ tief sind. Eine unumgängliche Voraussetzung ist - wie es für Marketingmaßnahmen üblich ist - die Bereitschaft, Budget für eine AdWords-Kampagne zu sprechen (Ulber 2018). Bezogen auf nötige Fachkompetenzen ist die Marketingaktivität mit relativ wenig Vorwissen machbar (VCS 6 2018). Trotzdem ist es von entscheidendem Vorteil, wenn die für AdWords verantwortlichen Personen eine grundlegende technische Affinität aufweisen, analytisch denken und eine Kampagnenperformance auswerten können (VCS 7 2018; Zimmermann 2018). Im großen Ganzen sind AdWords-Kampagnen ein Abbild der Realität, was bedeutet, dass das AdWords-Marketing nur so gut sein kann, wie es die ganzheitliche Leistung der werbenden Unternehmung im Allgemeinen ist (VCS 6 2018). Eine Restriktion von Google AdWords 
liegt in der Bewerbung von neuen, unbekannten Produkten oder Dienstleistungen, die schlichtweg nicht auf dem Netz gesucht und somit auch nicht gefunden werden (VCS 2 2018; VCS 4 2018; VCS 6 2018). Daher macht es keinen Sinn, Werbebudgets für Anzeigen zu sprechen, die Keywords hinterlegt haben, nach denen kein Mensch sucht. Ein Beispiel für einen solchen Fall stellten vor einigen Jahren Laufräder für Kinder dar. Das Produkt hatte sich noch nicht etabliert, das Wort „Laufrad“ war unbekannt und hatte daher kein Suchvolumen (VCS 4 2018).

Funktionsweisen und Eigenheiten des Tools, welche den befragten Experten beim Marketing mit Google AdWords auffallen, sind unter anderem das komplexe Auktionssystem und der damit verbundene stetige Wettbewerb, den aufgrund des äußerst ausgewieften Suchalgorithmus von Google niemand vollständig unter Kontrolle haben kann (VCS 4 2018). Daraus resultiert die Herausforderung, die optimale Strategie zu finden (VCS 2 2018). Die ausgeprägte Dynamik und Schnelllebigkeit des Themenfelds, welche Vergleiche nicht ganz simpel anstellen lässt, sowie die Leitzielsetzung von Google, mit AdWords ein Tool anzubieten, das Internetnutzern relevante Werbung anzeigt, beeinflussen die Funktionsweise von AdWords maßgeblich (VCS 1 2018; VCS 2 2018; VCS 4 2018; VCS 5 2018).

Bezüglich den für Google AdWords ausschlaggebenden Kennzahlen fällt auf, dass die CTR, also das Verhältnis zwischen Impressionen und Klicks, bei Unternehmungen ohne Onlineshop als aussagekräftiger und wichtiger betrachtet wird als bei Werbenden mit Onlineshop. Die befragten Personen der Fallstudie, die mit AdWords hauptsächlich einen Onlineshop bewerben, haben angegeben, dass die Kennzahl der CTR zweitrangig ist. Die Erklärungen dafür lauteten, dass die CTR zwar aufzeigt, ob die geschalteten Anzeigen bei den Usern gut ankommen und gute CTR-Histories in den Auktionsverfahren von Google miteinbezogen werden, Kennzahlen wie die Kosten pro Conversion, also die Kosten pro Abschluss, die Kosten-Umsatz-Relation (KUR) oder der Return on Advertising Spend (ROAS) jedoch bedeutungsvoller sind (VCS 4 2018; VCS 5 2018; VCS 6 2018). Dies hat damit zu tun, dass Werbende mit Onlineshop dank des direkten Kaufs der Kunden eine detailliertere Erhebung von Werbeeinnahmen erhalten und diese dann mit den Werbeausgaben vergleichen können, um die Kampagne als erfolgreich oder weniger erfolgreich einzustufen. Für Produkte oder Dienstleistungen, die nicht mit einem Klick im Internet bestellt werden können, ist es komplizierter, die Einnahmen und damit den Erfolg der Werbung genau zu messen. Daher wird eher auf Kennzahlen wie die CTR fokussiert. Google gibt zur Orientierung vor, dass für eine erfolgreiche Werbung eine CTR von $5 \%$ anzustreben ist (Leu 2018b).

\subsubsection{Chancen des Marketings mit Google AdWords}

Ein Großteil der in Abschn. 12.5.2.3 genannten Chancen des Online-Marketings lässt sich auf Google AdWords übertragen. Dies aus dem simplen Grund, dass AdWords ein Strang im Online-Marketing ist und übergeordnet dieselben Eigenschaften und Vorzüge aufweist wie andere Online-Marketing-Maßnahmen. Diese Aspekte werden in diesem 
Kapitel nicht wiederholt niedergeschrieben. Der Fokus liegt auf spezifischen Vorteilen, welche sich werbende Unternehmungen dank Google AdWords erarbeiten können.

Das Erkennen der Intentionen von Internetnutzern erfährt bei Google AdWords eine vertiefte Ausführung. Aufgrund dessen, dass beim Marketing mit Google AdWords vorausgesetzt wird, dass ein User eine Suchanfrage auf Google startet, bevor die eigene Anzeige geschaltet wird, ist die Relevanz der Werbung höher als beim Gießkannenprinzip. Es werden ausschließlich jene Leute angesprochen, bei denen das Interesse für das beworbene Produkt oder die beworbene Dienstleistung bereits geweckt ist und der Streuverlust daher äußerst gering ausfällt (VCS 6 2018). Man ist im Verkaufsprozess bereits einen Schritt weiter und der Kunde wird bei seinem Interesse abgeholt und begleitet, was innerhalb des Online-Marketings eine einmalige Ausgangslage darstellt (Ulber 2018; VCS 6 2018).

In der Schweiz ist Google AdWords heute teilweise noch in der Findungs- und Implementierungsphase. Das Potenzial zur Ausbaufähigkeit ist in den meisten Regionen groß. Urbane Gebiete wie Zürich oder Bern sind einen langen Schritt weiter als andere Kantone und Regionen (Ulber 2018). Dieser Zustand stellt für Werbende eine Chance dar, da die Konkurrenz überschaubar ist. Andererseits bedeuten im Suchalgorithmus von Google weniger Suchanfragen günstigere Klicks und somit tiefere Kosten für die die werbenden Parteien (Ulber 2018; VCS 7 2018; Zimmermann 2018).

Obschon einige Argumente gegen den Einsatz von Brandkampagnen (Abschn. 12.3.1.3) existieren, hat die Primärforschung ergeben, dass die Bewerbung des eigenen Brands durchaus sinnvoll ist. Ein Grund, sich gegen Brandkampagnen zu entscheiden, ist die Verlinkung der Werbeanzeige mit der Startseite und nicht auf eine spezifische Unterseite. Dies kann die Relevanz der Anzeige negativ beeinträchtigen (VCS 6 2018). Des Weiteren scheinen Brandkampagnen für Unternehmungen, welche in ihrer Branche die Nummer 1 sind, nicht zielführend zu sein (VCS 6 2018). Zu guter Letzt wird bei Verzicht auf Brandkampagnen die zu geringe Bekanntheit des Brands angegeben, was dazu führt, dass keine Suchanfragen dazu stattfinden (VCS 2 2018). Die Argumente, welche für eine Durchführung von Brandkampagnen sprechen, sind dennoch deutlich ausgeprägter. Im Grundsatz ist Visibilität für die Brand-Awareness äußerst wichtig, welche durch Brandkampagnen gewährleistet wird. Zudem wird den Usern Online-Präsenz bewiesen, was eine positive Auswirkung auf den Eindruck der User hat. Brandkampagnen ermöglichen mehr Klicks und im Algorithmus von Google wird die Relevanz der Unternehmen, die Brandkampagnen betreiben, verbessert (VCS 1 2018; VCS 4 2018; Zimmermann 2018). Dies wirkt sich wiederum auf die Auktionen und die bezahlten Klickpreise aus. Als weiterer Schlüsselfaktor für Brandkampagnen gelten die günstigen Klickpreise, welche durch die hohe Relevanz bei der Bewerbung des eigenen Brands entstehen (Ulber 2018; VCS 1 2018; VCS 2 2018; VCS 4 2018; VCS 72018 ; Zimmermann 2018).

Eine weitere Chance des Marketings mit Google AdWords ist die Möglichkeit, für Zielpersonen sehr relevante und zielgerichtete Werbung zu schalten (VCS 1 2018; VCS 3 2018; VCS 4 2018). Zudem ist es dank dem AdWords-Kostenmodell (Abschn. 12.3.1.2) 
mit kleinen Budgets möglich, bei relevanten Suchanfragen Visibilität zu erhalten und gegen die monetäre Übermacht der großen Player auf dem Markt zu konkurrenzieren (Ulber 2018; VCS 2 2018). Weiter besteht bei Google AdWords die Option, sofortige Aktionen bzw. Reaktionen durchzuführen, welche innerhalb von Sekunden angepasst und live geschaltet werden können (VCS 1 2018; VCS 6 2018).

\subsubsection{Google AdWords Erfolgsfaktoren}

In diesem Kapitel werden die wichtigsten Erfolgsfaktoren, die sich bei der Analyse der Datenerhebung herauskristallisiert haben, erklärt. Es ist essenziell, dass Werbende sich nicht ausschließlich auf die Integration der nachfolgenden Erfolgsfaktoren fokussieren, sondern sich den in diesem Kapitel bisher beschrieben Eigenschaften, Chancen und Auffälligkeiten des Online-Marketings sowie von Google AdWords (Abschn. 12.5.2.2, 12.5.2.3 und 12.5.2.5) bewusst sind und diese Faktoren in die Kampagne einfließen lassen.

\subsubsection{Top-Erfolgsfaktor 1: Darstellung und Aufbau der Webseite}

Einer der meistgenannten Faktoren für ein zielführendes Marketing mit Google AdWords ist die Struktur der Webseite, auf welcher die Produkte oder Dienstleistungen beworben werden. Die Qualität der Webseite ist sowohl für Google und die damit verbundene Einstufung der Qualitätsfaktoren als auch für die Internetuser entscheidend, denn diese müssen sich auf der Webseite wohl fühlen und zurechtkommen, ansonsten sind sie sofort wieder weg. In die Struktur der Webseite fließen Faktoren wie die Benutzerfreundlichkeit und der Page Speed ein. Ersteres verlangt eine simple und logische Usability, letzteres ist deshalb wichtig, weil die Nutzer nicht lange Geduld haben und die Webseite sofort verlassen, wenn zu lange Ladezeiten bestehen (Ulber 2018; VCS 1 2018; VCS 7 2018). Die Webseite muss zwingend responsive, d. h. mobile optimiert sein, da ein Großteil der Suchanfragen (ca. $80 \%$ ) über Smartphones statt stationären Desktops gestartet werden (Ulber 2018; Zimmermann 2018). Nebst der Struktur der Webseite ist der darauf präsentierte Inhalt mitentscheidend für die Performance der AdWords-Maßnahmen. Es muss gewährleistet werden, dass die zur Verfügung gestellten Informationen für die User relevant und übersichtlich sind. Mit optimalem Content kann erreicht werden, dass die Leute auf der Webseite verbleiben und nicht gleich wieder abspringen, was im Endeffekt den Qualitätsfaktor erhöht und somit Einfluss nimmt auf den CPC (VCS 2 2018; VCS 3 2018; VCS 4 2018; VCS 5 2018; VCS 6 2018; Zimmermann 2018). Die Webseite fungiert heutzutage als eine Art Visitenkarte, welche einer Unternehmung Glaubwürdigkeit bescheren kann, sofern diese benutzerfreundlich, aktuell und modern daherkommt (Zimmermann 2018).

Äußerungen von interviewten Experten, welche indizieren, dass bei der Werbung mit Google AdWords nicht entscheidend ist, ob der User tatsächlich das beworbene Produkt kauft oder er sich eben für ein anderes entscheidet, beweist, dass die Aufgabe von 
Google AdWords darin besteht, die Leute auf die Webseite des Werbenden zu locken, danach aber der Auftritt der Webseite entscheidend ist für den Erfolg der Kampagnen. Die Customer Journey muss zufriedenstellend sein und diese kann durch die positive Erfahrung auf der Webseite beeinflusst werden (VCS 5 2018; VCS 7 2018). Zusammenfassend kann festgehalten werden, dass der Auftritt und die Gestaltung der Webseite ausgeprägt wichtige Erfolgsfaktoren für das Marketing mit Google AdWords sind.

\subsubsection{Top-Erfolgsfaktor 2: Relevanz zwischen Keyword, Anzeige und Zielseite}

Eng verknüpft mit dem oben dargestellten Erfolgsfaktor ist auch der in diesem Kapitel beschriebene, dank der Datenerhebung identifizierte, Erfolgsfaktor. Es geht im Grundsatz darum, hochrelevante Werbung zu schalten (VCS 3 2018; VCS 7 2018). Für die Erreichung hoher Relevanz beim Marketing mit Google AdWords wird die Dreifaltigkeit zwischen gesuchtem Keyword, geschalteter Anzeige und verlinkter Landingpage erfordert (VCS 1 2018). Die Dreifaltigkeit zwischen Keyword, Anzeige und Landingpage sorgt dafür, dass Internetuser Werbung angezeigt bekommen, die für sie genau passend und somit hochrelevant ist. Dies führt im AdWords-Konto zu einem höheren Qualitätsfaktor (Abschn. 12.3.1.1) und damit zu einer besseren Performance und tieferen Kosten (Ulber 2018; VCS 3 2018; VCS 5 2018; VCS 7 2018; Zimmermann 2018).

Um die Relevanz der Werbung hoch zu halten, ist unter anderem die Verlinkung der korrekten Landingpage in der Anzeige entscheidend. Es ist essenziell, dass Internetnutzer nach dem Klick auf die Anzeige sofort auf der passenden Unterseite der Website landen und relevante Informationen vor sich haben, ohne dass sie sich durch die Webseite klicken müssen (Ulber 2018; VCS 3 2018; VCS 4 2018; VCS 5 2018; VCS 6 2018; VCS 7 2018). Zudem erscheint im Optimalfall das vom User eingegebene Keyword eins zu eins in der ausgespielten Anzeige, um die Relevanz für den potenziellen Kunden zu erhöhen (VCS 6 2018; VCS 7 2018). Ein Instrument, welches dafür sorgt, dass die vom User eingetippte Suchanfrage in der Anzeige ersichtlich ist, ist der Gebrauch des Keyword-Platzhalters (Abschn. 12.3.2) (Zimmermann 2018). Die Primärforschung hat überdies ergeben, dass das Bewerben fremder Brands eine nicht nachhaltige und zu teure Strategie ist. Beim Bewerben von fremden Brands werben Unternehmen mit Namen von sehr bekannten Marken oder Unternehmen (z. B. Digitec Galaxus im E-Commerce oder Zürich Insurance Group in der Versicherungsbranche), um einen Teil der Suchabfragen abzufangen, die spezifisch mit dem Namen des Brands bzw. Players gesucht werden. Dazu buchen Werbetreibende den Brandnamen als Keyword ein, sodass sie bei einer allfälligen Suchanfrage in die Auktion gelangen. Aufgrund dessen, dass mit dieser Strategie Anzeigen ausgespielt werden, die nicht mit der Suchanfrage der User übereinstimmen, ist die Relevanz dieser Anzeigen nicht ausreichend hoch, der Qualitätsfaktor entsprechend niedrig und die Werbekosten daher beträchtlich (VCS 3 2018; VCS 4 2018; VCS 5 2018; VCS 6 2018). 
Google AdWords ermöglicht eine äußerst relevante und spezifische Bewerbung von Produkten oder Dienstleistungen für User, deren Interesse für das Produkt oder die Dienstleistung bereits vorhanden ist. Um eine möglichst hohe Relevanz zu erhalten, ist es wichtig, sich auf die spezifische Schiene der Werbung zu konzentrieren, statt auf die breite Masse abzuzielen (Zimmermann 2018).

\subsubsection{Top Erfolgsfaktor 3: Verknüpfung mit Google Analytics}

Um langfristig von den gespeicherten Daten der AdWords-Aktivitäten zu profitieren und eine saubere, kontinuierliche Analyse zu erhalten, welcher wichtige Informationen zum Nutzerverhalten entnommen werden können, bedarf es der Verknüpfung des AdWords-Kontos mit dem Auswertungstool Google Analytics. Die Datenerhebung hat ergeben, dass Erfahrungswerte der eigenen AdWords-Kampagnen sehr vielsagend sind und tiefgehende Analysen sowie Optimierungspotenzial aufzeigen (VCS 2 2018; VCS 3 2018; VCS 4 2018; VCS 52018 und VCS 7 2018). Obschon oberflächliche Analysen auch im AdWords-Konto herausgezogen werden können, stellt der Erhalt von Zusatzinformationen durch die Einbindung von Google Analytics einen Erfolgsfaktor dar (VCS 2 2018; VCS 5 2018). Unter anderem zeigt Google Analytics detailliert an, welches Verhalten der User nach dem Klick auf die Anzeige an den Tag legt, wie lange er auf der Landingpage der Anzeige und auf der Website im Allgemeinen verweilt (Ulber 2018). Die Kampagnen-Optimierung ist durch Google Analytics zudem möglich, da in Google Analytics Sicht auf das große Ganze gewährt wird und die Daten von Google AdWords nicht separat, sondern in der gesamten Kette des Online-Marketings inklusive Kostendaten aufgezeigt werden (VCS 1 2018; VCS 6 2018). Eine weitere Voraussetzung für eine weiterführende Analyse mit Google Analytics ist das Einbauen von Tracking Codes von AdWords in Analytics (VCS 1 2018), was datenschutztechnisch zwar als problematisch angesehen werden kann. Trotzdem wird die Einbindung von Google Analytics im Generellen und Tracking Codes im Speziellen bei Marketingaktivitäten mit Google AdWords dringend empfohlen.

\subsubsection{Top-Erfolgsfaktor 4: Anzeigenerweiterungen einpflegen}

Ein wichtiger Erfolgsfaktor, welcher in der AdWords-Schulung betont wurde, hat sich mit der Auswertung der Datenerhebung bestätigt. Es handelt sich dabei um die hohe Priorität der Anzeigenerweiterungen (Abschn. 12.3.2.2) (Leu 2018a). Die Nutzung von Anzeigenerweiterungen führt dazu, dass der Werbende seinen potenziellen Kunden sowie auch Google zusätzliche Informationen bietet. Google stuft die Anzeigen anhand davon höher ein und für die User bedeutet dies, dass sie detailliertere Informationen erhalten können und die Relevanz der Anzeige damit erhöht werden kann (VCS 1 2018; VCS 2 2018; VCS 4 2018; VCS 6 2018; Zimmermann 2018). Mit den Anzeigenerweiterungen heben Werbende sich zudem von den Mitbewerbern, welche diese Tools nicht nutzen, ab und können sich somit einen Vorteil erwirtschaften (VCS 2 2018; VCS 6 2018). Des Weiteren vergrößert die Nutzung von Anzeigenerweiterungen die geschalteten Anzeigen, weil die zur Verfügung gestellten Informationen platziert wer- 
den müssen. Dadurch erhalten Werbetreibende mehr Werbeplatz und Visibilität (VCS 5 2018; Zimmermann 2018). Die Anzeigeerweiterungen senden Signale. Eine aktivierte Anruferweiterung z. B. kann Erreichbarkeit signalisieren und Internetnutzer zu einer Handlung auffordern (VCS 3 2018; VCS 4 2018). Zusammenfassend bieten die Anzeigenerweiterungen mit der angebotenen Vielzahl von Erweiterungen eine breite Palette an abwechslungsreichen Optionen, die Werbende für eine größere Anzeige und damit mehr Visibilität sowie für höhere Relevanz und damit einen verbesserten Qualitätsfaktor nutzen können.

\subsubsection{Weitere Erfolgsfaktoren}

Für ein nachhaltiges und effizientes Marketing mit Google AdWords bedarf es nebst den vier Top-Erfolgsfaktoren der Implementierung weiterer Aspekte, um eine ganzheitlich erfolgreiche Kampagne zu erreichen. Zum einen spielen der Qualitätsfaktor und der Anzeigenrang eine zentrale Rolle, denn diese entscheiden, wie viel der Werbende für den Klick bezahlt. Der Qualitätsfaktor ist stark von den beiden Top-Erfolgsfaktoren 1 und 2 abhängig, denn mit einer optimalen Website und relevanten Anzeigen sind wichtige Voraussetzungen für einen hohen Qualitätsfaktor gegeben (Zimmermann 2018). Mit einem hohen Qualitätsfaktor kann eine Budgetoptimierung erreicht werden, weil die Kosten pro Klick sinken (Ulber 2018; VCS 1 2018; VCS 5 2018; VCS 7 2018). Obschon mit exakten und wahrheitsgetreuen Angaben auf der Website und einer hohen Relevanz zwischen Keyword, Anzeige und Landingpage wichtige Beiträge zur Budgetoptimierung geleistet und somit Kosten gesenkt werden können, ist nicht wegzureden, dass ein höheres Werbebudget vielfältigere Optionen bietet (Ulber 2018; VCS 3 2018; VCS 6 2018; VCS 7 2018).

Um AdWords-Kampagnen sauber auswerten zu können ist es wichtig, dass von Anfang an Zielsetzungen getätigt werden. Es muss klar definiert werden, was mit der Kampagne erreicht werden und worauf der Fokus liegen soll (VCS 1 2018; VCS 3 2018; VCS 5 2018; VCS 6 2018). Die Primärforschung hat ergeben, dass Werbende von der Marketingstrategie ableiten sollen, wie sie in welchen Kanälen präsent sein wollen (VCS 3 2018; VCS 6 2018). Zudem ist auch eine deutliche Abgrenzung der verschiedenen Kampagnen innerhalb des AdWords-Kontos wichtig. Damit kann verhindert werden, dass Werbende sich selbst konkurrenzieren (VCS 1 2018). Bezüglich Optimierung und Anpassung von Kampagneninhalten sind sich die befragten Experten einig, dass eigene Erfahrungen gemacht und verschiedene Dinge getestet werden müssen, um langfristig eine verbesserte Performance hinzukriegen (Ulber 2018; VCS 2 2018; VSC 6 2018; VCS 7 2018). Trial \& Error-Analysen sind für die ausgesprochen hohe Komplexität von Google AdWords wichtig und erfolgsversprechend für die laufende Optimierung der Kampagnen (VCS 4 2018). Nebst dem Testen verschiedener Aspekte sprechen sich die Experten dafür aus, kleinere Anpassungen sofort und kontinuierlich anzubringen (VCS 2 2018; VCS 7 2018). Größere, kampagnenübergreifende Optimierungen hingegen sollen erst nach einer Zeitspanne von ca. 2-3 Monaten vorgenommen werden, da sich eingepflegte Aspekte im Google-Algorithmus zuerst 
einpendeln müssen, bevor sie ausgewertet werden können (Ulber 2018; VCS 7 2018; Zimmermann 2018).

\subsubsection{Zwischenfazit}

Nach ausführlicher Gestaltung der Resultate aus der qualitativen Erhebung kann die Forschungsfrage ,Welches sind die Erfolgsfaktoren für die Kundengewinnung mit Google AdWords? Ein Pilotprojekt in Zusammenarbeit mit der Hofbauer GmbH“ umfassend beantwortet werden. Die Experten konnten mit Ausführungen zu ihrer Arbeit und den Erfahrungen beim Marketing mit Google AdWords wichtige Anhaltspunkte darüber liefern, welche Aspekte zwingend beachtet werden müssen, um mit AdWords Erfolg zu haben. Es hat sich herausgestellt, dass Relevanz das bedeutende Stichwort ist für eine effiziente Arbeit mit Google AdWords.

Für den gesamten Auftritt des Online-Marketings ist es unabdingbar, dass die User Experience positiv und zielführend ist. Der Internetnutzer soll sich durch OnlineMarketing-Aktivitäten angesprochen fühlen und zu einer Handlung bewegt werden. Es soll jedoch eine angenehme Erfahrung sein, ohne negativ geprägten Handlungsdruck. Die werbende Unternehmung muss einen guten Eindruck hinterlassen und transparent aufzeigen, was sie anbietet, um sich von der Konkurrenz abzuheben. Dazu ist das Aufzeigen von Unique Selling Propositions (USP), Key Performance Indicators (KPI) und damit einhergehend Wettbewerbsvorteilen grundlegend. Ein konsistentes Gesamtpaket des Online-Auftritts führt dazu, dass der Customer Journey wohlwollend ist und die Kunden damit nachhaltig an die Unternehmung gebunden werden können.

\subsubsection{Handlungsempfehlungen}

Nach Abschluss der Datenanalyse werden folgende Handlungsempfehlungen für das Marketing mit Google AdWords abgegeben.

\section{Top-Erfolgsfaktoren implementieren und pflegen}

Die vier Top-Erfolgsfaktoren (Abschn. 12.5.3.1, 12.5.3.2, 12.5.3.3 und 12.5.3.4) werden mit einigen wenigen Ausnahmen allesamt von allen 10 befragten Experten empfohlen, was die Dringlichkeit dieser Maßnahmen unterstreicht. Aufgrund dessen ist es unentbehrlich, dass Werbende sich ausführlich mit den Erfolgsfaktoren auseinandersetzen und Fokus darauf legen. Wenn diese Aspekte nachhaltig eingepflegt sind, ermöglicht dies optimale Voraussetzungen für eine ausgezeichnete Basis, um mit Google AdWords Erfolg zu haben. Der Schnelllebigkeit dieses Business muss jedoch Rechnung getragen werden, was eine kontinuierliche und anhaltende Optimierung und Anpassung erfordert.

Die Implementierung der vier Top-Erfolgsfaktoren erhöht die Relevanz des Marketings aus Sicht der potenziellen Kunden sowie auch aus Sicht des Anbieters Google LLC, welche beide eine tragende Rolle spielen, wenn es um den Erfolg mit Google AdWords geht. Eine kampagnenübergreifend hohe Relevanz ist als Erfolgsgarant anzuschauen und dazu ist eine Stringenz zwischen Keyword, Anzeige und Landingpage ausschlaggebend. 


\section{Chancen des Online-Marketings sowie von Google AdWords beachten}

Das Marketing in Online-Kanälen unterscheidet sich wesentlich zum Marketing in Offline-Kanälen. Dieser Fakt muss beachtet werden und gerade ein hohes Bewusstsein bezüglich Chancen des Online-Marketings kann einen wichtigen Wettbewerbsvorteil bringen. Einer der auffälligsten Aspekte des Online Marketings ist die Unmittelbarkeit, welche die stetige Aufrechterhaltung des aktuellen Stands erfordert und Marketing in Echtzeit ermöglicht (Abschn. 12.2.2). Kurze Reaktionszeiten in diesem schnelllebigen Umfeld bedeuten einen Vorteil gegenüber der Konkurrenz zu haben und von der Dynamik der Branche profitieren zu können. Dies setzt Flexibilität und Agilität voraus. Sich in die User hineinzuversetzen, um zu erfahren, wie das Nutzerverhalten in spezifischen Situation aussehen könnte und die User dadurch bewusst zu lenken und steuern, hat ebenfalls positive Einflüsse auf das Marketing mit Google AdWords (Abschn. 12.5.2.2 und 12.5.2.3).

\section{Eigenen Weg gehen, Erfahrungen sammeln und davon profitieren}

Jede werbende Unternehmung hat aufgrund des beworbenen Angebots, der Finanzkraft, der Bekanntheit, des Know-hows, der Erfahrung und der Möglichkeiten sehr viele, individuelle Eigenheiten, die sich von allen anderen Mitbewerbern unterscheiden. Dies führt dazu, dass jede AdWords-Kampagne einmalig ist und spezielle Eigenschaften aufweist. Aufgrund dessen ist es essenziell, dass Unternehmungen, welche mit Google AdWords werben wollen, ihre eigenen Erfahrungen sammeln, diese analysieren, auswerten und anhand davon laufende Verbesserungen erzielen können.

\subsection{Pilotprojekt}

In diesem Kapitel werden die Erfahrungen des Liveprojekts dokumentiert. Sie sollen Einblicke in den Aufbau der Kampagne geben, die identifizierten Erfolgsfaktoren - die laufend berücksichtigt wurden - erkennen lassen, Herausforderungen aufzeigen und das Fazit des Liveprojekts darstellen.

\subsubsection{Projektpartner}

Die Hofbauer GmbH mit Sitz in Jegenstorf BE wurde im Jahr 2013 gegründet und bewegt sich in verschiedenen Bereichen der Sportbranche. Einerseits bietet die Unternehmung individuelle Arbeits- und Vereinsbekleidung an, welche nach Wunsch bedruckt und bestickt werden kann. Ein Teil stellt dabei auch das Merchandising dar, indem Vereinsartikel, wie z. B. Minishirts, Baseball Caps, Bodenteppiche etc., individuell gestaltet und bedruckt werden können. Clubs und Vereine gehören damit zu den wichtigsten Zielgruppen. Das zweite Standbein der Firma sind Events und Camps. Da die Gründer der Firma, zwei Brüder, langjährige Unihockey-Nationalspieler sind, geben sie ihre 
Erfahrung und ihr Know-how in Unihockey Camps, Trainings und Trainingstagen an die Jugend und andere Wissbegierige weiter und sorgen so für eine nachhaltige Entwicklung der Sportart Unihockey. Aktuell arbeiten vier Mitarbeitende für die Unternehmung, davon ist jedoch nur der Geschäftsführer in Vollzeit beschäftigt. Der Bekanntheitsgrad der vor knapp sechs Jahren gegründeten Unternehmung hat Steigerungspotenzial. Anhand des Liveprojekts wagte sich die Unternehmung zum ersten Mal an das Thema Google AdWords heran. Dieser Beitrag wertet die Zahlen und Ergebnisse des GoogleAdWords-Marketings der Firma Hofbauer GmbH für eine Zeitspanne von knapp zwei Monaten aus.

\subsubsection{Aufbau Pilotprojekt}

Nachdem die Gespräche mit AdWords Experten, die Teilnahme an Google-Kursen und der Theorieteil dieses Beitrags dazu führten, dass wichtiges Know-how bezüglich Google AdWords gewonnen wurde, wurde die Befragung der Fallstudienvertreter durchgeführt, um das erarbeitete Know-how weiter zu vertiefen. Auf dieser Basis konnte das Pilotprojekt, welches täglich überwacht und optimiert wurde, live geschaltet werden. In diesem Kapitel wird detailliert beschrieben, wie das Google-AdWords-Konto der Firma Hofbauer GmbH aufgebaut wurde.

\subsubsection{Erstellung AdWords Konto \& Kampagnen}

Bevor das Marketing mit Google AdWords live geschaltet werden konnte, wurde ein AdWords-Konto erstellt und bestmöglich vorbereitet. Das Pilotprojekt hat am 6. April 2018 effektiv gestartet und endete am 2. Juni 2018. Somit dauerte es etwas mehr als acht Wochen und weist für die Analyse 58 aktive Tage auf. Bevor das AdWords-Konto eröffnet und strukturiert wurde, wurden an der bestehenden Webseite einige Komponenten angepasst. Beispielsweise mussten die Begriffe für die Menüpunkte optimiert werden, damit diese eine sinnvolle Unterteilung der AdWords-Kampagnen zulassen. Die Begriffe in der Menüleiste lauten seit der Anpassung „Home“, „Bekleidung“, ,Vereinsartikel“ und „Unihockey“, welche die Hauptgeschäftsfelder der Unternehmung darstellen. Zudem wurden allgemeine Informationen wie die Rubrik „Über uns“ ausgebaut, damit den Webseitenbesuchern ausführlichere Angaben über die Firma sowie den Personen dahinter gegeben wird. Nach der Anmeldung des Google Kontos wurden im AdWords-Tool vorerst die verschiedenen Kampagnen definiert und erstellt. Insgesamt wurden vier Kampagnen definiert, welche gemäß Tipp der Google-AdWords-Grundlagenschulung (Leu 2018b) auf die Menüleiste der bereits bestehenden Webseite ausgerichtet wurde (Abschn. 12.3.1.4).

Nebst der Brandkampagne, die „Brand“ genannt wurde, wurden anhand der Menüleiste aus Abb. 12.4 folgende drei Kampagnen aufgesetzt: „Bekleidung“, „Vereinsartikel“ und „Unihockey“. Zusätzlich wurde die Brandkampagne implementiert, da die Datenerhebung ergeben hat, dass diese Art von Kampagnen für die Steigerung des 


\section{h图fbauer}

Home Bekleidung $\vee$ Vereinsartikel $\vee$ Unihockey

Abb. 12.4 Menüleiste der Webseite. (Eigene Darstellung)

Bekanntheitsgrads wichtig ist. Die Kosten dafür halten sich in Grenzen und somit wird für niedrige Klickpreise Visibilität gewährleistet, wenn User nach dem Brand suchen (Abschn. 12.3.1.3 und 12.5.2.6).

Bei der Aufsetzung der vier Kampagnen wurden nachstehende Eigenschaften gewählt, die zu Beginn jeder Kampagnenimplementierung festgelegt werden müssen:

1. Kampagnennamen: „Brand“, „Bekleidung“, „Vereinsartikel“, „Unihockey“

2. Typ: Nur Suchnetzwerk. Der Typ Suchnetzwerk wurde gewählt, damit vorerst ausschließlich Leute angesprochen werden, die aktiv auf Google nach den beworbenen Produkten oder Dienstleistungen suchen und diese effektiv bei Google im Suchfeld eingeben. Somit wurde auf Einblendungen ohne vorausgehende Suchanfragen verzichtet, die ansonsten auf im Voraus bestimmten Webseiten ausgespielt werden (Displaynetzwerk). Hauptgründe dafür waren die begrenzten Ressourcen an Zeit und Geld für das Pilotprojekt.

3. Standorte: Variante „Ich möchte selbst wählen“ ausgewählt. Dann wurden Kantone der Deutschschweiz angewählt. Der Kanton Fribourg wurde miteinbezogen, da er zweisprachig ist und in der Nähe des Unternehmenssitzes der Hofbauer GmbH liegt.

4. Gebotsstrategie: Variante „manueller CPC“ ausgewählt. Damit sind Anpassungen der Gebote für den Cost per Click für jedes Keyword einzeln und manuell möglich.

5. Budget: $17 \mathrm{CHF}$ pro Tag. Google rechnet mit 30 Tagen pro Monat. 30 Tage à $17 \mathrm{CHF}$ ergeben $510 \mathrm{CHF}$ pro Monat, die in dieser Zeitspanne maximal ausgegeben werden durften (Abschn. 12.3.1.2). Das Tagesbudget wurde auf die verschiedenen Kampagnen aufgeteilt. Dieses wurde anhand von Prioritätsangaben des Partners für das Pilotprojekt vergeben. Dabei wurde entschieden, dass 1 CHF pro Tag für die Branding-Kampagne, 8 CHF pro Tag für die Bekleidungskampagne und je 4 CHF pro Tag für die Vereinsartikel- und Unihockey-Kampagne investiert werden soll (Tab. 12.5).

\section{Anzeigengruppen}

Gemäß Leu (2018b) wird sinnvollerweise für jede Produktegruppe eine Anzeigegruppe erstellt und für jede Anzeigegruppe danach eine spezifische Keyword-Liste hinterlegt (Abschn. 12.3.1.5). Dabei sollten für die Einhaltung der Einfachheit und Nutzerfreundlichkeit die Anzeigegruppen an die Unterpunkte der Menüleiste angeglichen werden. Diese Vorgaben wurden eingehalten. 
Tab. 12.5 Strukturierung der Anzeigegruppen. (Eigene Darstellung)

\begin{tabular}{l|l|l}
\hline Kampagne & Anzeigegruppen & Tagesbudget \\
\hline Brand & 1. Hofbauers.ch & $1,00 \mathrm{CHF}$ \\
\hline Bekleidung & $\begin{array}{l}\text { 1. Individuelle Vereinsbekleidung } \\
\text { 2. Freizeit- \& Arbeitsbekleidung } \\
\text { 3. Kollektion Kempa/Uhlsport }\end{array}$ & $8,00 \mathrm{CHF}$ \\
\hline Vereinsartikel & $\begin{array}{l}\text { 1. Duschtïcher } \\
\text { 2. Flexfit-Caps } \\
\text { 3. Minishirts }\end{array}$ & \\
\hline Unihockey & $\begin{array}{l}\text { 1. Camps } \\
\text { 2. Trainingstage }\end{array}$ & \\
\hline
\end{tabular}

\section{Anzeigen und Anzeigentexte}

Nachdem die Kampagnen sowie die Anzeigegruppen definiert waren, ging es darum, die Werbeanzeigen zu erstellen. Diese fungieren als Bindeglied zwischen den hinterlegten Keywords und der verlinkten Landingpage der Webseite. Bei der Anzeigenerstellung wurden jeweils folgende Punkte durchlaufen:

1. Anzeigetyp: Textanzeige. Alle anderen Typen wurden in diesem Forschungsprojekt nicht benötigt, da diese über den Kampagnentyp „Nur Suchnetzwerk“ hinausgehen.

2. Finale URL: Für jede Anzeige muss die passende Unterseite der Website verlinkt sein. Es ist wichtig, dass User nicht auf der Home-Seite landen, sondern direkt an relevante Informationen auf der gewünschten Landingpage gelangen (Leu 2018b).

- Beispiel Pilotprojekt: https://www.hofbauers.ch/vereinsbekleidung/wunschdesign/für Anzeigen, welche die Anzeigegruppe „Individuelle Vereinsbekleidung“ betreffen.

3. Erster und zweiter Anzeigentitel: Dafür stehen je 30 Zeichen zur Verfügung. Die beiden Titel werden mit einem Trennstrich separiert und sollen anzeigen, was mit der spezifischen Anzeige angeboten wird.

- Beispiel Pilotprojekt: 1. Titel: Individuelle Vereinsbekleidung, 2. Titel: Turnvereine \& Indoorsportarten

4. Pfad: Stellt die gewünschte URL dar, die in der Anzeige eingeblendet wird. Diese beginnt mit der gewöhnlichen URL der Startseite und kann danach mit $2 \times 15$ Zeichen, getrennt durch ein Slash, nach eigenen Vorstellungen ergänzt werden.

- Beispiel Pilotprojekt: hofbauers.ch/bekleidung/bedrucken

5. Beschreibung: Für die Umschreibung des Angebots stehen maximal 80 Zeichen zur Verfügung. Mit diesen 80 Zeichen erhält der Werbende die Möglichkeit, sein Angebot zu spezifizieren und das Interesse der User zu wecken.

- Beispiel Pilotprojekt: Die beste Adresse für Sublimationsdruck

Abb. 12.5 packt die beschriebenen Komponenten 1 bis 5 in eine Anzeige, welche in abgebildetem Format in den Google Suchergebnissen eingeblendet wird, wenn der Algorithmus errechnet hat, dass die Anzeige ausreichend relevant ist, um ausgespielt zu werden. 
Abb. 12.5 Beispiel-Anzeige. (Eigene Darstellung in Anlehnung an Leu 2018b, S. 65)
Individuelle Vereinsbekleidung - Turnvereine \& Indoorsportarten

Anzeige hofbauers.ch/bekleidung/bedrucken

Die beste Adresse für Sublimationsdruck.

\section{Keywords}

Das Herzstück von Google AdWords sind die in den Anzeigegruppen hinterlegten Keywords. Die Keywords bestehen aus den Angeboten an Produkten und Dienstleistungen, welche die werbende Unternehmung bewerben möchte. Die Auswahl der hinterlegten Keywords entscheidet, ob die Anzeigen eines Werbenden bei einer Suchanfrage eines Nutzers in die interne sowie externe Auktion (Abschn. 12.3.1.1) gehen und somit die Chance haben, eingeblendet und angeklickt zu werden. Vorgängig wurde die Applikation „PPC Keyword Generator“ heruntergeladen. Diese sorgt dafür, dass die bestimmten Keywords auf alle möglichen Weisen kombiniert und somit eine große Anzahl an Keywords generiert wird, die in die jeweils passende Anzeigegruppe eingebettet werden. Insgesamt konnten somit 11.155 Keywords erstellt werden, von denen viele wie erwartet ein geringes Suchvolumen aufweisen und nicht relevant sind. Auf diesem vorerst breit gefächerten Weg können unwichtige Keywords laufend deaktiviert werden und die Möglichkeit besteht, verschiedene Kombinationen zu testen, um zu sehen, welche Keywords am effektivsten sind. Jene Keywords, nach denen nicht gesucht wird, werden nicht ausgespielt und somit auch nicht angeklickt und generieren daher keine Kosten. Dieses Vorgehen wurde von Jeannine Künzler empfohlen, die eine fundierte Expertise mit dem Aufsetzen von AdWords-Kampagnen aufweist. Überdies hat auch die Fallstudienanalyse ergeben, dass Keywords vorerst breit gestreut werden, um danach trichtermäßig Einschränkungen einfließen zu lassen (VCS 1 2018).

\subsubsection{Dokumentation Pilotprojekt}

In diesem Kapitel soll verdeutlicht werden, welche spezifischen Handlungen für das Pilotprojekt durchgeführt wurden, was diese gebracht haben und wie sich die implementierten Erfolgsfaktoren im Liveprojekt eingespielt haben. Die Click through Rate, also das Verhältnis zwischen Einblendungen und Klicks, fungiert im Pilotprojekt als maßgebende Kennzahl für den Erfolg bzw. Misserfolg der AdWords-Kampagne des Pilotprojekts.

\subsubsection{Implementierung der identifizierten Erfolgsfaktoren}

Die Handlungsempfehlungen (Abschn. 12.5.3.7), die sich aus der Fallstudienanalyse sowie den Gesprächen mit AdWords-Experten ergeben haben, wurden laufend integriert. 


\section{Darstellung und Aufbau Website}

Die von Google vergebenen, dynamischen Qualitätsfaktoren hängen stark mit der Qualität von Webseiteninhalten der verlinkten Landingpages zusammen (Abschn. 12.3.1.1 und 12.5.3.1). Um die Qualitätsfaktoren der Keywords zu verbessern, wurden Bemühungen zur Optimierung des Inhalts der verlinkten Unterseiten unternommen. Dabei wurde der in Abschn. 12.3.3.1 beschriebenen optimalen Textlänge von mindestens 300 Wörtern pro Unterseite Beachtung geschenkt. Zusätzlich wurden die hinterlegten Keywords bewusst im Inhalt der Unterseite eingebaut, um die Relevanz der Werbung zu erhöhen. Diese Maßnahmen führten zum Erfolg, welcher anhand der verbesserten Qualitätsfaktoren im Google-AdWords-Konto belegt werden kann. Als Beispiel hierfür wird die Optimierung der verlinkten Unterseite der Anzeigegruppe ,individuelle Vereinsbekleidung“ (Tab. 12.5) aufgeführt. Der Text der Landingpage wurde am 13. April 2018 von 183 auf 475 Wörter erweitert und die im AdWords eingebuchten Keywords wurden bewusst im Text miteinbezogen. Diese Maßnahmen führten dazu, dass sich die Aufteilung der Qualitätsfaktoren von Keywords dieser Anzeigegruppe gemäß Tab. 12.6 veränderte. Die Angabe nach dem Multiplikationszeichen zeigen den erreichten Qualitätsfaktor.

Gemäß Leu (2018c) und VCS 7 (2018) ist es erstrebenswert, Qualitätsfaktoren von 6 oder mehr zu erreichen. Tab. 12.6 zeigt eine deutliche Verbesserung des Verhältnisses der Qualitätsfaktoren im Verlauf der Kampagne auf. Der Page Speed sowie die Auflösung der Bilder auf der Website wurden kontrolliert. Diese Anforderungen sind erfüllt, ebenso wie die Anforderung einer mobile optimierten Webseite. Diese Voraussetzungen sind unterstützend für die Erreichung von hohen Qualitätsfaktoren.

\section{Relevanz zwischen Keyword, Anzeige und Zielseite}

Um die Dreifaltigkeit der Relevanz zwischen Keyword, Anzeige und Zielseite zu erfüllen, wurden im Pilotprojekt verschiedene Maßnahmen ergriffen. Von Beginn weg wurde darauf fokussiert, dass die eingebuchten Keywords mit der aufgesetzten Anzeige stark korrelieren und die Anzeige ausschließlich Informationen bietet, welche sich auf der in der Anzeige verlinkten Zielseite in ausführlicher Form wiederfinden. Dadurch sollte sichergestellt werden, dass die Relevanz der AdWords-Aktivitäten hoch ist.

Ein Indikator für die Relevanz einer Kampagne ist die CTR. Zwar kann diese Kennzahl die Relevanz zwischen Anzeige und der verlinkten Unterseite nur beschränkt

Tab. 12.6 Veränderung des Qualitätsfaktors nach Optimierung der Website. (Eigene Darstellung)

\begin{tabular}{l|l}
\hline Qualitätsfaktoren per 13.04.2018 & Qualitätsfaktoren per 02.06.2018 \\
\hline $0 \times 10 / 10$ & $1 \times 10 / 10$ \\
\hline $1 \times 8 / 10$ & $6 \times 8 / 10$ \\
\hline $1 \times 7 / 10$ & $17 \times 7 / 10$ \\
\hline $19 \times 6 / 10$ & $32 \times 6 / 10$ \\
\hline
\end{tabular}


bestätigen. Dafür zeigt das Verhältnis zwischen Impressionen und Klicks deutlich auf, ob die Relevanz zwischen den eingebuchten Keywords und den ausgespielten Anzeigen gegeben ist. Wenn User auf die ausgespielte Anzeige klicken ist dies ein Indiz dafür, dass ihnen die Anzeige die für sie relevanten Informationen bietet. Gemäß den Aussagen von befragten AdWords-Experten (Ulber 2018; Zimmermann 2018) ist die CTR ab $5 \%$ als sehr gut einzustufen. Das Pilotprojekt erreichte über die Laufzeit von knapp zwei Monaten eine CTR von 9,32\% und übertrifft die Anforderungen für eine Einstufung „CTR ist gut" deutlich. Die gute Performance der CTR ist teilweise auf den Fokus Relevanz zurückzuführen, welcher sich als wichtiger Erfolgsfaktor herauskristallisiert hat und während des Pilotprojekts durchgehend berücksichtigt wurde.

Eine weitere Optimierung ist gemäß Künzler (2018) und VCS 6 (2018) der Ausschluss von negativen Keywords (Tab. 12.1 und Abschn. 12.3.1.5). Dadurch kann verhindert werden, dass bei bestimmten, für den Werbenden irrelevanten Suchanfragen die eigenen Werbeanzeigen ausgespielt und Kosten für Klicks generiert werden. Hauptsächlich bei der Branding Kampagne, welche den Nachnamen Hofbauer beinhaltet, wurden im AdWords regelmäßig die Suchanfragen überwacht, welche zu Klicks geführt hatten. Wenn die Suchanfragen offensichtlich nichts mit der Hofbauer GmbH zu tun hatten, wurden die Wörter als auszuschließendes Keyword erfasst.

\section{Auswertungen mit Google Analytics}

Die Verknüpfung des AdWords-Kontos mit Google Analytics wurde gleichzeitig mit der Implementierung der Kampagne durchgeführt. Die Auswertungen in Google Analytics wurden jedoch aufgrund von Zeitmangel nicht detailliert analysiert und allfällige daraus gewonnene Erkenntnisse daher nicht in das Projekt eingepflegt. Diese Aktivitäten hätten den Rahmen dieses Beitrags gesprengt. Es wird der Hofbauer $\mathrm{GmbH}$ für die zukünftige Arbeit mit Google AdWords jedoch empfohlen, das Nutzerverhalten der Webseitenbesucher vertieft zu analysieren und die eigene Kampagne anhand davon zu optimieren. Nichtsdestotrotz stellt die Verknüpfung des AdWords-Kontos mit Google Analytics einen wichtigen Faktor für das Liveprojekt dar. Es bietet nämlich interessante Informationen zur Performance der Kampagne. Vorerst wurde die Entwicklung der Besucherzahlen auf der Webseite der Hofbauer GmbH angeschaut. Dabei wird der Zeitraum zwischen dem Kampagnenstart am 6. April 2018 bis zum Ende des Pilotprojekts am 2. Juni 2018 mit dem gleichen Zeitraum unmittelbar davor verglichen. Diese Analyse hat ergeben, dass dank des Google-AdWords-Projekts für die Hofbauer GmbH eine Steigerung von 21,8 \% auf 1476 Webseitenbesucher in der Zeitspanne des Projekts erzielt werden konnte. Diese Zahl darf als äußerst positiv gewertet werden, da zusätzlicher Traffic auf der Website von gut einem Fünftel eine beträchtliche Anzahl an potenziellen Neukunden mit sich bringt.

\section{Anzeigenerweiterungen einpflegen}

Aufgrund der hohen Wichtigkeit der Nutzung von Anzeigenerweiterungen, welche sich während der Datenerhebung herauskristallisiert hat (Abschn. 12.5.3.4), wurde klar, dass 
die Erweiterungen für das Liveprojekt genutzt und ausprobiert werden müssen. Der Fokus wurde auf die Anzeigenerweiterungen, genannt Snippet-, Sitelink- \& Anruferweiterung, sowie der Erweiterung mit Zusatzinformationen gelegt. Letztere werden auch Callouts genannt und finden sich in der geschalteten Anzeige als zusätzliche Informationen zum Unternehmen. Nachfolgend werden die Performances der genutzten Anzeigenerweiterungen während des Pilotprojekts dargestellt:

Callouts sind Erweiterungen mit Zusatzinformationen und finden sich in der geschalteten Anzeige als zusätzliche Informationen zum Unternehmen. Im Liveprojekt ist auffällig, dass die CTR aller Callouts über $10 \%$ und somit über dem durchschnittlichen CTR des Projekts (9,32\%) liegen.

Die Snippets, welche ähnlich wie die Callouts als Zusatzinformationen in der Anzeige fungieren sind im Gegensatz zu den Callouts mit zur Verfügung stehenden Kategorien vorgegeben. Für das Pilotprojekt wurden die Kategorien „Marken“ und „Dienstleistungen“" auserwählt und das Angebot der Hofbauer GmbH anhand davon beschrieben. Die Snippet-Erweiterungen liegen mit einer durchschnittlicher CTR von 10,14\% ebenfalls über der CTR des gesamten Projekts (9,32\%).

Kleine, separate Anzeigen innerhalb der geschalteten Anzeige werden Sitelinks genannt (vgl. Abb. 12.3). Die Sitelinks zeigen den Usern Angebote an, welche das werbende Unternehmen nebst dem aktuell gesuchten Produkt oder der Dienstleistung zusätzlich anbietet. Sitelinks sorgen zudem dafür, dass die Anzeige größer wird und mehr Werbeplatz einnimmt. Auch die Sitelink-Erweiterungen weisen im Liveprojekt mit einer durchschnittlicher CTR von 10,43\% einen überdurchschnittlichen Wert im Vergleich zur CTR des gesamten Liveprojekts $(9,32 \%)$ auf.

Die vierte genutzte Erweiterung ist die Anruferweiterung, bei der über die geschalteten Anzeigen die Telefonnummer der Hofbauer GmbH angezeigt wird und auf die bei der Suche mit einem Smartphone geklickt werden kann, um den Anruf direkt einzuleiten. Die Performance der Anruferweiterung mit 1095 Einblendungen und 124 dazugehörigen Klicks ergibt eine CTR von 11,32\%, was im Allgemeinen einen starken und fürs Pilotprojekt höheren Wert als die durchschnittlichen 9,32\% darstellt.

Die bei allen implementierten Anzeigenerweiterungen höheren CTR als die durchschnittliche CTR von 9,32\% geben Aufschluss darüber, dass die Anzeigen erfolgreicher waren, wenn sie mit den in den einzelnen Kampagnen eingebetteten Erweiterungen ausgespielt wurden, als wenn sie in der Anzeige nicht ersichtlich waren. Daraus wird geschlossen, dass die Relevanz der Anzeigen bei Ausspielung der Anzeigeerweiterungen höher ist als bei Nichteinblendung der Anzeigenerweiterungen. Abschn. 12.5.3.4 hält fest, dass Werbende sich mit der Nutzung von Anzeigenerweiterungen von der Konkurrenz abheben und sich somit einen Wettbewerbsvorteil verschaffen können. Die Kennzahlen der Anzeigenerweiterungen für das Pilotprojekt lassen den Schluss zu, dass die Performance dank der Implementierung von Callouts, Snippets, Sitelinks und der Anruferweiterung verbessert wurde. Der Top-Erfolgsfaktor 4 (Abschn. 12.5.3.4) kann somit durch das Liveprojekt bestätigt werden. 


\subsection{Diskussion und Fazit}

Google AdWords bietet eine effiziente Plattform für Online-Marketing. Der Beitrag hat aufgedeckt, dass die Relevanz, welche sich wie ein roter Faden durch die Marketingmaßnahmen ziehen soll, das magische Wort beim Marketing mit Google AdWords ist, um eine positive Auswertung der Ergebnisse zu erlangen. Gemäß Erkenntnissen der Datenerhebung und dank der Durchführung des Pilotprojekts kann die folgende Hypothese gewagt werden: effizientes Marketing mit Google AdWords erfordert als Basis ein attraktives Angebot an Produkten und/oder Dienstleistungen, eine moderne und nutzerfreundliche Webseite, eine sorgfältige Stringenz zwischen Werbung und Angebot und somit eine hohe Relevanz zwischen eingebuchten Keywords, geschalteten Anzeigen und verlinkten Zielseiten sowie eine fortlaufende Überwachung und Optimierung des Google-AdWords-Kontos mit Einbezug von spezifischen Eigenheiten, welche jede einzelne werbende Unternehmung aufweist, und Erfahrungen, die im Laufe der Zeit gewonnen werden.

Die Datenerhebung hat ergeben, dass die Schnelllebigkeit und Dynamik des Themenfelds Online-Marketing und Google AdWords (Abschn. 12.5.2.5) sowie die individuellen Eigenheiten und Funktionsweisen der werbenden Unternehmungen dazu führen, dass es kein allgemeingültiges Rezept für erfolgreiche AdWords-Strategien gibt. Die Einhaltung der Top-Erfolgsfaktoren (Abschn. 12.5.3) bietet jedoch eine wertvolle Grundlage für effizientes Google-AdWords-Marketing.

Die Auswertung des Liveprojekts bestätigt die anhand der Datenerhebung aufgedeckten Erfolgsfaktoren. Obschon keine detaillierte Beschreibung der Zielsetzung für das Pilotprojekt ausgearbeitet wurde und somit eine saubere Auswertung des Liveprojekts erschwert wird, darf die AdWords-Kampagne der Hofbauer $\mathrm{GmbH}$ als erfolgreich gewertet werden. Von den in Abschn. 12.3.1.3 dargestellten Kampagnentypen können ausschließlich die zwei Typen Branding- sowie Traffic-Kampagnen für die Hofbauer GmbH relevant sein, da der dritte Typ, Direct-Response-Kampagnen, auf Conversions und somit Abschlüsse zielen. Deren Messung ist nur für Werbende mit Onlineshop, die Verkäufe sofort eins zu eins nachvollziehen können, sinnvoll. Die Branding-Kampagne hat eine Steigerung der Markenbekanntheit als Ziel, was für die junge Partnerfirma des Liveprojekts einen wichtigen Aspekt darstellt (Abschn. 12.6.1). Für Branding-Kampagnen gelten Impressionen als Messwert für den Erfolg. Während der AdWords-Kampagne wurden die Werbeanzeigen des Pilotprojekts 7040 Mal eingeblendet, was einem Tagesdurchschnitt von 120 Impressionen entspricht. Obwohl unbekannt bleibt, wie viele User die Anzeigen tatsächlich wahrgenommen bzw. angeschaut haben darf festgehalten werden, dass der Brand der Unternehmung gegen außen getragen und von der Öffentlichkeit wahrgenommen wurde.

Das Pilotprojekt liegt mit einer durchschnittlichen CTR von 9,32\% deutlich über der CTR von $5 \%$, welche als Grenzwert für die Einstufung als ,gute Performance“ gilt. Der zweite Kampagnentyp, welcher für Unternehmen ohne Onlineshop messbar ist, 
sogenannte Traffic-Kampagnen (Abschn. 12.3.1.3), haben die Nutzung von Webseiteninhalten als Ziel und deren Erfolg misst sich an Klicks auf die Werbeanzeigen, die auf eine Zielseite der Webseite führen. Durch die Livekampagne haben 656 Klicks auf die Website der Partnerfirma geführt und die Besucherzahl (direkte, organische und bezahlte Zugriffe) ist um 21,8\% im Vergleich zur gleichen, vorangehenden Zeitspanne auf 1476 Nutzer angestiegen. Dank des erhöhten Traffic verfügt die Hofbauer GmbH heute über eine deutlich höhere Anzahl an potenziellen Kunden. Die Aussage des Geschäftsführers der Hofbauer $\mathrm{GmbH}$ darüber, dass Kontakt- und Offertanfragen seit Projektstart zugenommen haben, deckt sich mit dem Aspekt, dass während des Pilotprojekts sechs Conversions gemessen wurden. Dabei haben User, welche über AdWords auf die Webseite gelangt sind, unmittelbar nach dem Klick auf die Werbeanzeige das Kontaktformular auf der Webseite ausgefüllt. Aktionen, bei denen User über die bezahlte Suche auf die Webseite gelangt sind, danach aber eine E-Mail über den herkömmlichen Weg gesendet haben oder später über den direkten oder organischen Zugriff auf die Seite zurückgekommen sind und eine Aktion getätigt haben, werden im AdWords nicht als Conversion gemessen, was eine Schwierigkeit der Messbarkeit des Online-Marketings darstellt (Abschn. 12.5.2.3).

Dieser Beitrag bestätigt, dass Google AdWords ein effizientes Tool des Online Marketings darstellt und vielen Unternehmen eine geeignete und sinnvoll einsetzbare Plattform bietet.

\section{Literatur}

Advidera. (о. J.). Real time advertising. https://www.advidera.com/glossar/real-timeadvertising/. Zugegriffen: 14. Mai 2018.

Aufgesang GmbH. (o. J.a). AdWords Anzeigengruppe Definition. https://www.semdeutschland.de/ adwords-agentur/adwords-glossar/adwords-anzeigengruppe-definition/. Zugegriffen: 16. Mai 2018.

Aufgesang GmbH. (o. J.b). Dynamic Keyword Insertion (Keyword-Platzhalter) Definition \& Info. https://www.sem-deutschland.de/adwords-agentur/adwords-glossar/dynamic-keyword-platzhalter/. Zugegriffen: 17. Mai 2018.

Beck, A. (2011). Google AdWords (3. Aufl.) Heidelberg: eine Verlagsgruppe der Hüthig Jehle Rehm GmbH.

Bundesamt für Statistik (BFS). (2017). Internetnutzung. https://www.bfs.admin.ch/bfs/de/home/ statistiken/kultur-medien-informationsgesellschaftsport/informationsgesellschaft/gesamtindikatoren/haushaltebevoelkerung/internetnutzung.html. Zugegriffen: 14. Mai 2018.

Busch, O. (Hrsg.). (2014). Realtime Advertising. Digitales Marketing in Echtzeit: Strategien, Konzepte und Perspektiven. Wiesbaden: Springer Gabler.

Dänzler, S., \& Heun, T. (2014). Marke und digitale Medien. Der Wandel des Markenkonzepts im 21. Jahrhundert. Wiesbaden: Springer Gabler.

DIM Deutsches Institut für Marketing GmbH, (o. J.). Google AdWords Seminar, Köln. https:// www.marketinginstitut.biz/seminare/online-marketing/google-adwords-seminar/. Zugegriffen: 29. Dez. 2017.

Dörr von Maydell, H. (2016). Google AdWords-Tipp: Setzen Sie sich Ziele. https://www.heise-regioconcept.de/google/google-adwords-ziele. Zugegriffen: 14. Mai 2018. 
Engel, D. (2015). Ist Realtime die Echte Zeit? Springer Professional. 2015. https://www.springerprofessional.de/marketing-+-vertrieb/markenstrategie/ist-realtime-dieechte-zeit/6599162. Zugegriffen: 14. Mai 2018.

Europäisches Parlament. (2018). Online-Shopping für alle: Ende von Geoblocking. http:// www.europarl.europa.eu/news/de/headlines/economy/20180126STO94142/onlineshopping-fur-alle-ende-von-geoblocking. Zugegriffen: 13. Mai 2018.

Frost, S. (2013). Datenschutz ist größtes Hindernis. https://www.tagesspiegel.de/wirtschaft/nonliner-atlas-2013-datenschutz-ist-groessteshindernis/8105722.html. Zugegriffen: 14. Mai 2018.

Gläser, J., \& Laudel, G. (2009). Experteninterviews und qualitative Inhaltsanalyse. Als Instrumente rekonstruierender Untersuchungen (3. Aufl.). Wiesbaden: VS Verlag.

Gläser, J., \& Laudel, G. (2010). Experteninterviews und qualitative Inhaltsanalyse. Als Instrumente rekonstruierender Untersuchungen (4. Aufl.). Wiesbaden: VS Verlag.

Google. (o. J.). AdWords Editor Hilfe. https://support.google.com/adwords/editor/answer/2484521?hl=de\&ref_topic $=3290839$. Zugegriffen: 17. Mai 2018.

Google AdWords. (o. J.). Schalten Sie Anzeigen bei Google. http://www.google.ch/adwords/?channel=ha\&subid=ch-de-ha-aw-aw-cb1_xx_txx_xx_xx_bau-non!o2 118682095-138815685732kwd-19087281652. Zugegriffen: 30. Dez. 2017.

Google Webmasters. (2015). Google search console. https://www.youtube.com/watch?v=SoxU5kz15Kc. Zugegriffen: 17. Mai 2018.

GoogleWatchBlog. (2015). 15 Jahre AdWords: Infografik zeigt Statistiken rund um Googles Gelddruckmaschine. https://www.googlewatchblog.de/2015/10/jahre-adwordsinfografik-statistiken/. Zugegriffen: 11. Mai 2018.

Griesbaum, J. (2012). Online marketing. https://www.unihildesheim.de/media/fb3/informationswissenschaft/IIM_IW/griesbaum/GdPIuD_C4.pdf. Zugegriffen: 13. Mai 2018.

Hamburg, I. (2017). Jeder Zweite hat Angst vor Cyberkriminalität. https://www.marketing-boerse. de/News/details/1719-Jeder-Zweite-hat-Angst-vor-Cyberkriminalitaet/138539. Zugegriffen: 14. Mai 2018.

Herrmann, S. (2017). Werbemarkt-Studie von Zenith: Werbung im Social Web überholt Print. https:// www.wuv.de/medien/werbung_im_social_web_ueberholt_print. Zugegriffen: 12. Febr. 2019.

Hohlstein, K. (2016). Die Bedeutung des Online Marketings wächst. https://www.billomat.com/ magazin/die-bedeutung-des-online-marketings-waechst/\#. Zugegriffen: 13. Mai 2018.

Internet World Business. (2018). Diese Player dominieren den weltweiten digitalen Werbemarkt. https://www.internetworld.de/onlinemarketing/google/playerdominieren-weltweiten-digitalenwerbemarkt-1466688.html. Zugegriffen: 12. Febr. 2019.

Jonker, J., \& Pennink, B. W. (2010). The essence of research methodology. A concise guide for master and PhD students in management science. Berlin: Springer.

Learnwithgooglede. (2014). Was ist das Google Display-Netzwerk? https://www.youtube.com/ watch?time_continue $=20 \& v=d r Y Z b 2 g U p E 0$. Zugegriffen: 16. Mai 2018.

Learnwithgooglede. (2018). Einstieg in Google AdWords: Was sind Suchnetzwerk-Anzeigen? https://www.youtube.com/watch?v=t6Uj_eDQpbs. Zugegriffen: 16. Mai 2018.

Leu, E. (2018a). Skript zum Modul "Learn with Google: Suchmaschinenmarketing mit Google AdWords - Vertiefung». Zürich: Google Switzerland.

Leu, E. (2018b). Skript zum Modul "Learn with Google: Suchmaschinenmarketing mit Google AdWords - Grundlagen». Zürich: Google Switzerland.

Leu, E. (2018c). Skript zum Modul «Learn with Google: Suchmaschinenmarketing mit Google AdWords - Vertiefung». Zürich: Google Switzerland.

Link, J. (2000). Wettbewerbsvorteile durch Online Marketing. Die strategischen Perspektiven elektronischer Märkte (2. Aufl.). Berlin: Springer.

Lunapark. (2017). Suchmaschinenmarktanteile weltweit 2017. https://www.lunapark.de/ blog/9907-suchmaschinen-marktanteile-weltweit-2014/. Zugegriffen: 19. Mai 2018. 
McCarthy, E. J. (1960). Basic Marketing. A Managerial Approach. Homewood: Indiana University \& Richard D. Irwin, Inc.

Michnew, E. (2015). Suchmaschinenmarketing abseits von Google AdWords. https://www.amazeemetrics.com/de/blog/suchmaschinenmarketing-abseits-von-googleadwords\#. Zugegriffen: 14. Mai 2018.

Oettrich, S. (2016). 7 Tipps, wie Ihr KMU ein Top-Ranking auf Google erhält. https://www.bag.ch/ blog/27360-2268/7_Tipps_wie_Ihr_KMU_ein_Top-Ranking_auf_Google_erhaelt.html?s=58. Zugegriffen: 20. Mai 2018.

Rumo, E. (2017). Kommunikationsmanagement und Social Media Marketingkommunikation. Skript zum Modul RSSM. Bern: Berner Fachhochschule.

SEO Küche. (o. J.a). Was ist Google analytics? https://www.seokueche.de/lexikon/google-analytics/. Zugegriffen: 16. Mai 2018.

SEO Küche. (o. J.b). Was sind die Google Webmaster Tools? https://www.seo-kueche.de/lexikon/ google-webmaster-tools/. Zugegriffen: 17. Mai 2018.

Stich, K. (2017). Mit dem crossmedialen Marketing Online- und Offline-Marketing verbinden. http://cmm-magazine.ch/mit-dem-crossmedialen-marketing-online-undoffline-marketing-verbinden/. Zugegriffen: 14. Mai 2018.

Stone, M. (2014). Share of global adspend, by medium, 2012 and 2015. https://newsbizblog. org/2014/07/18/share-of-global-adspend-by-medium-2012-and-2015/. Zugegriffen: 14. Mai 2018.

Studer, L. (2008). Studie: Google AdWords und ihr Branding-Effekt, Zürich: Yourposition AG. https://www.yourposition.ch/de/blog/studie-google-adwords-und-ihr-brandingeffekt. Zugegriffen: 2. Jan. 2018.

Traffic3. (о. J.). Wie funktionieren AdWods? https://traffic3.net/wissen/adwords/adwords-ablauf. Zugegriffen: 15. Mai 2018.

Ulber, T. [Medienberaterin] (2018). Identifikation von Erfolgsfaktoren für die Kundengewinnung mit Google AdWords, Interview mit Seraina Ulber vom 23.03.2018.

Unic. (o. J.) Webanalyse: Besser gratis? - Warum für ein Tool zahlen, wenn es Google Analytics gibt? https://www.unic.com/de/kompetenzen/expertenblog/2014/webanalyse-besser-gratis-warum-fuer-ein-tool-zahlen-wenn-es-googleanalytics-gibt. Zugegriffen: 16. Mai 2018.

Vertreter Case Study (VCS) 1 [Online Marketing Manager Unternehmen 1]. (2018). Identifikation von Erfolgsfaktoren für die Kundengewinnung mit Google AdWords, Interview mit Seraina Ulber vom 27.03.2018.

Vertreter Case Study (VCS) 2 [Geschäftsführer Unternehmen 2]. (2018). Identifikation von Erfolgsfaktoren für die Kundengewinnung mit Google AdWords, Interview mit Seraina Ulber vom 28.03.2018.

Vertreter Case Study (VCS) 3 [Marketing-Kommunikation, Google AdWords Verantwortlicher Unternehmen 3]. (2018). Identifikation von Erfolgsfaktoren für die Kundengewinnung mit Google AdWords, Interview mit Seraina Ulber vom 29.03.2018.

Vertreter Case Study (VCS) 4 [Geschäftsführer Unternehmen 4]. (2018). Identifikation von Erfolgsfaktoren für die Kundengewinnung mit Google AdWords, Interview mit Seraina Ulber vom 03.04.2018.

Vertreter Case Study (VCS) 5 [Google AdWords Verantwortlicher Unternehmen 5]. (2018). Identifikation von Erfolgsfaktoren für die Kundengewinnung mit Google AdWords, Interview mit Seraina Ulber vom 03.04.2018.

Vertreter Case Study (VCS) 6 [Leiter digital Marketing \& social Media Unternehmen 6]. (2018). Identifikation von Erfolgsfaktoren für die Kundengewinnung mit Google AdWords, Interview mit Seraina Ulber vom 04.04.2018.

Vertreter Case Study (VCS) 7 [Geschäftsführer Unternehmen 7]. (2018). Identifikation von Erfolgsfaktoren für die Kundengewinnung mit Google AdWords, Interview mit Seraina Ulber vom 06.04.2018. 
Weller, B., \& Calcott, L. (2012). The definitive guide to Google AdWords. Create versatile and powerful marketing and advertising campaigns. New York: Springer.

Wendeler, V. (o. J.). Digitales Marketing - Eine neue Ära für Wachstum. Köln: Wendeler Marketing Consulting. https://www.wemarcon.de/de/marketing/digitales-marketing/. Zugegriffen: 30. Dez.r 2017.

Yapi, L. (2016). Die ganze Welt des Online-Marketings. Google AdWords, Zürich: Swiss Marketing.

Zimmermann, T. [Online Campaign Manager] (2018). Identifikation von Erfolgsfaktoren für die Kundengewinnung mit Google AdWords, Interview mit Seraina Ulber vom 23.03.2018.

Ulber, Seraina (M.Sc. BA/seraina.ulber@bluemail.ch) Masterstudium in Betriebswirtschaft an der Berner Fachhochschule mit Vertiefung Corporate / Business Development. Langjährige Tätigkeit als Kundenbetreuerin im Aussendienst bei Optitrade \& Service AG, einem Importeur und Distributor, der den Schweizer Gross- und Fachhandel beliefert. Ab Juni 2019 bei der Firma Coldtec AG als Key Account Managerin angestellt.

Rumo, Etienne J. (Dr./etienne.rumo@hefr.ch) ist assoziierter Professor für Marketing und Sales Management an der Hochschule für Wirtschaft in Freiburg und Dozent am konsekutiven Masterstudiengang der Berner Fachhochschule mit Studien (MBA) an der University of Edinburgh (UK) und $(\mathrm{PhD})$ der Université de Lorraine (France). Seine Forschungsaktivitäten konzentrieren sich auf Sales Management und International Entrepreneurship. Seit über 10 Jahren begleitet er als Berater KMU's und Grossbetriebe in den Bereichen Verkauf, Vertrieb und International Business Development. 
Open Access Dieses Kapitel wird unter der Creative Commons Namensnennung 4.0 International Lizenz (http://creativecommons.org/licenses/by/4.0/deed.de) veröffentlicht, welche die Nutzung, Vervielfältigung, Bearbeitung, Verbreitung und Wiedergabe in jeglichem Medium und Format erlaubt, sofern Sie den/die ursprünglichen Autor(en) und die Quelle ordnungsgemäß nennen, einen Link zur Creative Commons Lizenz beifügen und angeben, ob Änderungen vorgenommen wurden.

Die in diesem Kapitel enthaltenen Bilder und sonstiges Drittmaterial unterliegen ebenfalls der genannten Creative Commons Lizenz, sofern sich aus der Abbildungslegende nichts anderes ergibt. Sofern das betreffende Material nicht unter der genannten Creative Commons Lizenz steht und die betreffende Handlung nicht nach gesetzlichen Vorschriften erlaubt ist, ist für die oben aufgeführten Weiterverwendungen des Materials die Einwilligung des jeweiligen Rechteinhabers einzuholen.

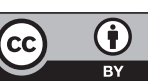




\title{
Augmented Reality als Entscheidungshilfe beim Möbelkauf
}

\section{David Aemmer, Jonas Bigler, Marco Birkhofer, Marie Brechbühler Pešková und Deane Harder}

\begin{abstract}
Zusammenfassung
Diese Studie untersucht den Einfluss von Augmented Reality (AR) auf den Kaufentscheidungsprozess beim Möbelkauf. Ziel der Studie waren konkrete, handlungsleitende und wissenschaftlich fundierte Erkenntnisse für die Praxis im Umgang mit der digitalen Transformation. Dafür wurde der Einfluss einer AR-App in der Möbelbranche untersucht. Es konnte gezeigt werden, dass die AR-App ihren Nutzern eine wertvolle Unterstützung im Kaufentscheidungsprozess bietet. Die App funktioniert jedoch nicht als eigenständiger Kanal, sondern entfaltet ihren Nutzen nur im Zusammenspiel mit den bisherigen Kanälen und Hilfsmitteln. Insbesondere der Austausch mit Dritten bietet deutliches Entwicklungspotenzial und Chancen, die
\end{abstract}

D. Aemmer $(\square)$

Kerzers, Schweiz

E-Mail: david_aemmer@gmx.ch

J. Bigler

Bern, Schweiz

E-Mail: jonas.bigler@gmx.ch

M. Birkhofer

Port, Schweiz

E-Mail: marco.birkhofer@bluewin.ch

M. Brechbühler Pešková

Institut Unternehmensentwicklung, Berner Fachhochschule Wirtschaft, Bern, Schweiz

E-Mail: marie.brechbuehler@bfh.ch

D. Harder

Berner Fachhochschule Wirtschaft, Bern, Schweiz

E-Mail: deane.harder@bfh.ch 
Customer Experience weiter zu verbessern. Einen klaren Kundenmehrwert würde eine händlerübergreifende AR-App bieten. Weiterer Forschungsbedarf ergibt sich aus den Rückkoppelungseffekten zwischen veränderter Kundeninteraktion und Technologieentwicklung.

\subsection{Einleitung}

Diese Studie untersucht den Einfluss von Augmented Reality (AR) auf den Kaufentscheidungsprozess beim Möbelkauf. Die Untersuchung beschränkt sich dabei auf bestimmte Phasen des Entscheidungsprozesses. Mit diesem Forschungsfokus sollen konkrete, handlungsanleitende Erkenntnisse für die Praxis zur digitalen Transformation gewonnen werden.

Die Geschwindigkeit der Technologieentwicklung nimmt im Zeitalter der digitalen Transformation gefühlt exponentiell zu. Dadurch erfahren Wirtschaft und Gesellschaft einen einschneidenden, andauernden Wandel. Der Schweizer Möbelmarkt gehört im europäischen Vergleich zu den umsatzschwächeren Märkten, was aber angesichts der Population wenig erstaunt. Europäisch führend sind Deutschland mit EUR 22 Mrd., Italien mit EUR 20,9 Mrd. sowie Großbritannien mit EUR $11 \mathrm{Mrd}$. Umsatz im Jahr 2015 (PwC 2017). In der Schweiz war der Umsatz für Wohnungseinrichtung im selben Jahr CHF 3,85 Mrd. Werden dabei nur die Möbel betrachtet, lag der Umsatz bei CHF 2,7 Mrd. (Verband möbelschweiz 2017). Für das Jahr 2018 geht die Schweizer Möbelindustrie von einer stagnierenden Entwicklung aus, gleichzeitig herrscht weiterhin rege Bautätigkeit in der Schweiz, was die Nachfrage anregt (Verband möbelschweiz 2017). Durch die Marktdurchdringung von Smartphones und der damit ermöglichten mobilen Vernetzung haben Customer $^{1}$ einen ständigen Zugang zu einer großen Anzahl an Gütern. So haben sich die Einkaufsgewohnheiten in den letzten Jahren verändert und zu einer Verschiebung vom stationären in den Online-Handel bewirkt. Damit verändern sich die Anforderungen der Customers an das Einkaufserlebnis. Gleichzeitig bieten sich durch den Einsatz von digitalen Instrumenten für Anbieter neue Möglichkeiten, mit ihren Customers zu interagieren. Die Vernetzung schwappt nun auf weitere Bereiche über, so wird im Zuge der digitalen Transformation von Trends, wie bspw. Big Data, Cloud Computing, Internet of Things, Internet of Everything, Machine Learning, Augmented Intelligence oder Super Intelligence, gesprochen. Die durch die zahlreichen Trends bedingten Chancen und Risiken für Unternehmen sind vielfältig. Sie verlangen von Unternehmen allgemein und damit auch in der Möbelbranche eine strategisch verankerte Veränderungsbereitschaft, Innovationskraft und konsequente Kundenorientierung. Hier knüpft die Studie an.

\footnotetext{
${ }^{1}$ Zur besseren Lesbarkeit werden nachfolgend Kundinnen und Kunden bzw. Konsumentinnen und Konsumenten unter dem englischen Begriff Customers subsumiert.
} 


\subsubsection{Digitale Transformation und Augmented Reality (AR)}

Eine Transformation beschreibt eine tief greifende Veränderung auf vielen verschiedenen Ebenen. Die mit der Digitalisierung entstehenden Möglichkeiten führen zu einem weitreichenden Wandel, der die Wirtschaft, wie auch die ganze Gesellschaft betrifft (Peter 2017) und dadurch unser Alltagsleben verändert (Pousttchi 2017) (Abb. 13.1). Entsprechend definiert das MIT Center for Digital Business die digitale Transformation als „,den Einsatz neuer digitaler Technologien (Social Media, Smartphone, Analysen und integrierte Geräte) um wesentliche Verbesserungen im Geschäftsumfeld zu ermöglichen (beispielsweise die Customer Experience verbessern, den Betrieb verschlanken oder neue Geschäftsmodelle kreieren)“ (Fitzgerald et al. 2013). Für die rein unternehmensbezogene Betrachtung wird auch der Begriff „Digital Business Transformation“ mit beispielsweise nachstehender Definition des Global Centers for Business Transformation verwendet: „Die digitale Business Transformation ist ein Wandel in Organisationen, der digitale Technologien und Geschäftsmodelle nutzt, um die Leistungsfähigkeiten zu steigern“ (Wade 2015). In diesem Kontext wird zwischen den drei Wirkungsdimensionen Leistungserstellung, Geschäftsmodelle und Kundeninteraktion bzw. Kundenerlebnis unterschieden (KPMG 2014; Pousttchi 2017). Im Rahmen dieser Studie werden die Auswirkungen auf die Dimension Kundeninteraktion anhand einer App untersucht, die Augmented Reality (AR) zur Auswahl von Möbelstücken nutzt (im Folgenden FURNIT-AR genannt).

Die Grenze zwischen Augmented Reality (AR) und Virtual Reality (VR) ist fließend: das Kontinuum beginnt bei der realen Umgebung ohne virtuelle Bestandteile, bei AR ist die Realität bereits mit virtuellen Inhalten ergänzt, der reale Anteil überwiegt jedoch noch, bei der Augmented Virtuality ist der virtuelle Anteil höher (Mehler-Bicher und Steiger 2014, S. 10) und schließlich endet das Kontinuum bei der virtuellen Umgebung bzw. VR. Unter $V R$ wird eine computergenerierte, virtuelle Umgebung verstanden, die von Menschen in einer gewissen Weise über die Sinne als real wahrgenommen werden kann (Henneberg 2017, S. 20; Mehler-Bicher und Steiger 2014, S. 9). In der Literatur

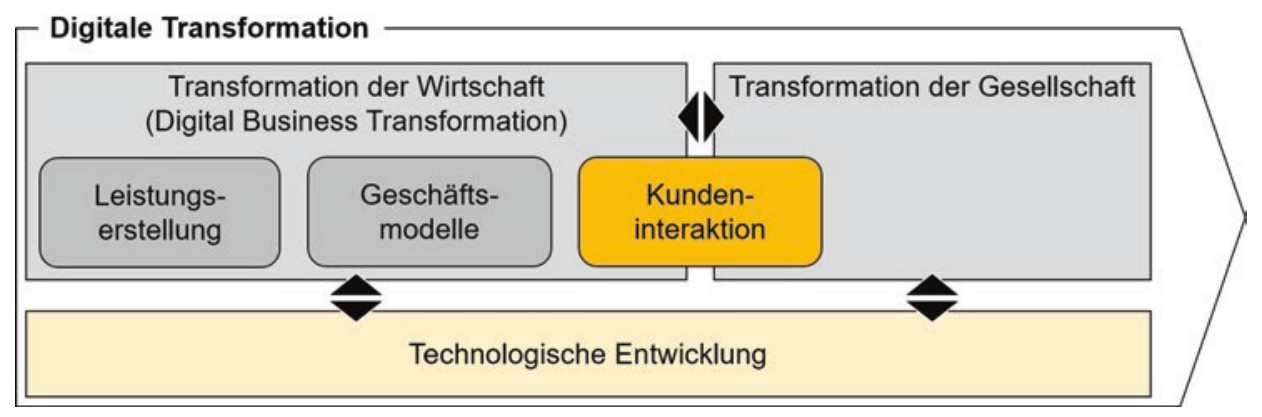

Abb. 13.1 Modell der digitalen Transformation. (Eigene Darstellung) 
spricht man oft vom Reality-Virtuality Continuum (Milgram et al. 1994, S. 283). Innerhalb dieses Kontinuums konzentriert sich die vorliegende Studie auf AR und ihrer Anwendung auf Smartphones.

Mit der im September 2017 lancierten App FURNIT-AR sucht der Möbelhändler $F_{U R N I T^{2}}$ mithilfe einer AR-Anwendung den Weg über die Smartphones direkt in die Haushalte der Customers (Rode 2017). Die App basiert auf der Technologie „ARKit“ von Apple (Lüft 2018), die es erlaubt, über die Smartphone-Kamera Möbelstücke in den eigenen vier Wänden zu platzierten. Zudem können bestehende Einrichtungselemente über die Smartphone-Kamera analysiert werden, worauf die App ähnliche Möbel aus dem Sortiment von FURNIT vorschlägt. Die App soll Customers beim Kaufentscheid begleiten, unterstützen und Sicherheit vermitteln, die richtige Entscheidung zu fällen. Insgesamt beabsichtigt FURNIT den Customers ein positiveres, erinnerungswürdigeres Einkaufserlebnis zu bieten (Husic 2018). Wie einleitend beschrieben, geht die vorliegende Studie davon aus, dass die App auch dem eigentlichen Kauf vorgelagerte Schritte des Kaufentscheidungsprozesses beeinflussen kann. Es wird angenommen, dass sich FURNIT durch die App zusätzliche Möglichkeiten zur Interaktion mit Customers erschließt, zugleich stellt die Applikation eine Quelle für interessante Nutzerdaten dar (cebit.de 2017).

\subsubsection{Kaufentscheidungsprozess}

Die konsequente Ausrichtung von Unternehmen auf Customers gewinnt mit den durch die digitale Transformation getriebenen Neuerungen an Bedeutung. Dieses Kapitel gibt Definitionen sowie Hintergrundinformationen zum Kaufentscheidungsprozess bzw. der Customer Experience als wichtige Grundlage der Studie. Dabei werden die für die Untersuchung relevantesten Phasen im ausgewählten Modell genauer beleuchtet.

Ein Konsument ist eine Person, die mindestens einmal bei einem Anbieter gekauft bzw. konsumiert hat (Zaharia 2006, S. 35). Nach Kotler et al. (2016, S. 990) handelt es sich bei Konsumenten um ,Einzelpersonen und Haushalte, die Güter und Dienstleistungen zum persönlichen Gebrauch kaufen“. Winters ergänzt, dass Konsumenten meist die Möglichkeit haben, unter verschiedenen Produkten und Lieferanten zu wählen (Winters 2016, S. 22). Die vorliegende Studie konzentriert sich auf das Verhalten und die Prozesse von Customers im Sinne der Endverbrauchenden, weil diese die Kaufentscheidungen für Möbel treffen.

Zur Einordnung des Untersuchungsbereiches der vorliegenden Studie wird die Definition von Holland (2018) herangezogen: „Die Customer Experience umfasst die Gesamtheit aller Eindrücke, die ein Kunde während der gesamten Dauer einer Kundenbeziehung von einem Unternehmen erhält. Sie umfasst sämtliche individuellen Wahrnehmungen

\footnotetext{
${ }^{2}$ Aus Gründen der Vertraulichkeit wurden sämtlichen Klarnamen anonymisiert.
} 
und Interaktionen des Kunden an den verschiedenen Kontaktpunkten (sog. „Touchpoints“) mit einem Unternehmen. Die Customer Experience stellt ein holistisches Konstrukt dar, das mehrere Prozessphasen umschließt und als vorgelagertes Konstrukt zur Kundenbindung betrachtet wird.“ Die Customer Experience beinhaltet dementsprechend die Interaktion zwischen einer Unternehmung und ihren Customers sowohl vor, während sowie nach allen Kaufentscheidungsprozessen (Berry et al. 2002).

Unter dem Kaufentscheidungsprozess wird der gesamte Prozess von der Produktwahrnehmung bis zur Produktauswahl bzw. im erweiterten Sinne das Zustandekommen des Kaufentschlusses verstanden (Kirchgeorg 2018). Typischerweise wird dieser Prozess in einzelne Phasen unterteilt und abhängig vom Kaufobjekt zwischen voneinander abweichenden Arten von Kaufentscheidungsprozessen unterschieden. Die in der Literatur beschriebenen Modelle zum Kaufentscheidungsprozess haben gemeinsam, dass sie nicht nur die Kaufentscheidung an sich beinhalten, sondern auch die Phasen vor und nach dem Kauf beleuchten (z. B. Kotler et al. 2016; Peter und Olson 2010; Rutschmann und Belz 2014; Winters 2016). So lässt sich der idealtypische Ablauf eines Kaufentscheidungsprozesses auf die Vorkaufphase, die Kaufphase sowie die Nachkaufphase abstrahieren (Griese und Bröring 2011, S. 37). Diese drei Phasen werden zum besseren Verständnis des Vorgangs in der Literatur häufig in fünf (z. B. Gerth 2015, S. 72; Kotler et al. 2016, S. 287), sechs (z. B. Kirchgeorg 2018) oder noch mehr Schritte unterteilt (z. B. Peter und Olson 2010, S. 193 f.; Winters 2016, S. 221 f.). Obwohl die Teilschritte oftmals ähnlich bezeichnet und beschrieben werden, ist die Zuordnung in die übergeordnete Vorkaufsphase oder Kaufphase nicht immer einheitlich gewählt. Dies könnte darauf hindeuten, dass die Phasen und Ablaufschritte in der Realität nicht linear oder sequenziell ablaufen und die Phasenübergänge fließend erfolgen. Dem wird in der Diskussion des alternativen Ansatzes zum Kaufentscheidungsprozess von Rutschmann und Belz (2014) sowie bei der unten beschriebenen Modellkombination Rechnung getragen.

Nachfolgend wird auf die fünf Phasen nach Kotler et al. (2016) Bezug genommen, weil sich damit trotz der stark vereinfachten Darstellung des Kaufentscheidungsprozesses der untersuchte Einfluss von AR in ausreichender Form einordnen lässt:

1. Problemerkennung bzw. Wahrnehmung des Bedarfs

2. Informationssuche

3. Bildung und Bewertung von Alternativen

4. Kaufentscheidung inkl. Kaufabschluss

5. Bewertung der Kaufentscheidung und Verhalten in der Nachkaufphase

Im Zuge der digitalen Transformation gilt es, dieses Modell zum Kaufentscheidungsprozess kritisch zu hinterfragen. So lösen sich Rutschmann und Belz (2014) zuweilen stark von diesem etablierten Modell und gehen aufgrund der digitalen Transformation von einer Tendenz zu neuen Verhaltensprinzipien aus. Diese Verhaltensprinzipien erzeugen das neue Kaufmuster „Gewohnheit und Opportunismus“ (Rutschmann und Belz 2014, S. 44 ff.). In diesem Zusammenhang empfehlen sie, die Kundenprozesse zu 
analysieren und dabei auf die Handlungen sowie situativen äußeren Einflüsse vor dem effektiven Kaufabschluss zu fokussieren (Rutschmann und Belz 2014, S. 23). Abgeleitet von den dadurch gewonnenen Analyseresultaten sollen die Einflusspunkte im komplexen Kaufentscheidungsprozess identifiziert werden. Besonders interessant erscheint dabei der aus dem zweiten Verhaltensprinzip („Handlungen erfolgen beiläufig“) abgeleitete Rückschluss, wonach Customers im Kaufentscheidungsprozess nicht mehr ein Fernziel (den Produktkauf), sondern ein Nahziel (den Lustgewinn beim unmittelbaren nächsten Handlungsschritt) vor Augen haben (Rutschmann und Belz 2014, S. 47 f.). Diese Aspekte werden für den empirischen Teil übernommen: Es wird z. B. untersucht, ob mit dem Einsatz von AR eine sogenannte Instant Gratification (Patel 2014) entlang des Kaufentscheidungsprozesses erreicht werden kann.

Die vorliegende Studie basiert auf dem Modell von Kotler et al. (2016), ergänzt um Aspekte von Rutschmann und Belz (2014) (Abb. 13.2). Damit wird v. a. das „,komplexe Kaufverhalten“ berücksichtigt, insbesondere wird der Vermutung Rechnung getragen, dass Kaufentscheidungsprozesse eher selten linear oder sequenziell ablaufen. Zudem wird anerkannt, dass zeitnahe Belohnungen für Customers auf dem Weg zum Kaufentscheid für Unternehmen einen wesentlichen Erfolgsfaktor darstellen können. Es wird weiterhin angenommen, dass mit gezielt herbeigeführten Instant-Gratification-Momenten in den einzelnen Kundenhandlungsschritten die übergeordnete Costumer Experience optimiert werden kann. Dies wiederum kann dabei helfen, die Kundenzufriedenheit $\mathrm{zu}$ erhöhen, die Kundenbindung zu verstärken und somit die Unternehmensziele zu erreichen. Die vorliegende Studie geht davon aus, dass mit der AR-Anwendung FUR$N I T-A R$ an mehreren Stellen in diesem Modell angeknüpft werden kann. Die Wirkung von AR soll entsprechend anhand dieses kombinierten Modells untersucht werden.

Der grundlegende Prozess der Kundenentscheidung sowie die übergeordnete Customer Experience ist mittlerweile gut erforscht (vgl. Klaus 2015, S. 5). Auch im Bereich der Verhaltensforschung liegen zahlreiche Studien vor - wenn auch der Bezug zum Kaufverhalten oftmals nicht hergestellt wird (Rutschmann und Belz 2014, S. 9). Welche konkreten und allenfalls einschneidenden Einflüsse die neuen Technologien und Anwendungen im Zuge der digitalen Transformation aufweisen, ist jedoch kaum erforscht. Zwar werden Tendenzen zu neuen Kaufmustern und Verhaltensprinzipien vermutet, jedoch sind diese oftmals allgemein und global gehalten, sodass diese der Praxis kaum konkrete, handlungsauslösende Anknüpfungspunkte bieten. Ein empirischer Nachweis der vermuteten Veränderungen fehlt.

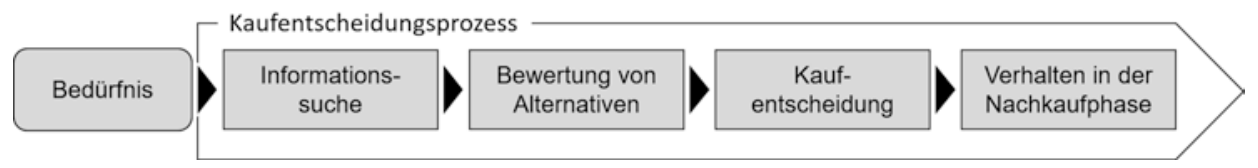

Abb. 13.2 Kaufentscheidungsprozess. (Eigene Darstellung, nach Kotler et al. 2016) 
Weitere Forschungslücken bestehen im Entscheidungsprozess spezifisch für den Möbelkauf. In der Literatur herrscht wenig Konsens $\mathrm{zu}$ dessen Zuteilung in eine bestimmte Form des Kaufentscheidungsprozesses. Mit den neuen digitalen Möglichkeiten sowie der stark zunehmenden Anzahl an Daten, die Customers bezüglich ihres Verhaltens in der digitalen Welt hinterlassen, ergibt sich außerdem ein kontinuierlicher Bedarf für Validierungen in der Praxis. Augmented Reality wird zwar in den Rankings zu den wichtigsten Trends oft ganz oben geführt und den Technologien wird auch in der Möbelbranche großes Potenzial zugeschrieben. Gleichzeitig scheint jedoch noch nicht klar zu sein, welchen Nutzen die Technologien tatsächlich bieten werden. Es fehlt größtenteils an empirischen Untersuchungen des effektiven Einflusses der Technologien - gerade auch im Zusammenhang mit dem Kaufentscheidungsprozess. Dies liegt vermutlich daran, dass noch wenig markttaugliche Anwendungen lanciert wurden und die zugrunde liegende Technologie selbst noch laufend fundamentale Entwicklungsschritte erlebt. Mit der vorliegenden Studie sollen nun erste Erkenntnisse im Bereich der Dimension der Kundeninteraktion erarbeitet werden. Die Lücke bezüglich des Einflusses neuer Technologien auf den Kaufentscheidungsprozess wird anhand der AR-Beispielanwendung FURNIT-AR fokussiert auf die Möbelbranche untersucht. Zudem werden empirische Erkenntnisse zu den Eigenschaften des Kaufentscheidungsprozesses beim Möbelkauf insgesamt sowie bezüglich spezifischer Handlungsschritte innerhalb des Kaufentscheidungsprozesses gewonnen.

\subsection{Forschungsfrage}

Die zentrale Forschungsfrage der Studie lautete: Wie beeinflusst AR den Kaufentscheidungsprozess beim Möbelkauf?

Der Fokus wird dabei auf die Phasen vor dem eigentlichen Kaufabschluss gelegt. Diese Fokussierung ist darin begründet, dass der Einfluss von AR in der Nachkaufphase nur schwierig untersucht werden kann. Um die Forschungsfrage beantworten zu können, wurden aus der Theorie zum Kaufentscheidungsprozess sowie den in der Literatur angegebenen Auswirkungen der digitalen Transformation drei Thesen zum Einfluss von AR formuliert:

\section{AR animieren potenzielle Kunden, entlang des Kaufentscheidungsprozesses mit Anbietern und deren Produkten zu interagieren.}

Es wird davon ausgegangen, dass im Vergleich zu den heutigen Möglichkeiten zur Informationssuche sowie Bewertung von Alternativen (auch über den Online-Kanal) ein wesentlicher Zusatznutzen durch AR entsteht. So wird vermutet, dass Customers mit einem Bedarf sich über das interaktive Hilfsmittel AR während den genannten Phasen vermehrt und/oder intensiver mit den Produkten des Anbieters auseinandersetzen. Eine niederschwellige Initiierung der Interaktion hat dabei hohe Relevanz. So kann mit der App über die Kamera des Smartphones ein Möbelstück markiert werden und anschließend 
werden ähnliche Möbelstücke angezeigt. Durch die vermehrte bzw. intensivierte Interaktion wird erwartet, dass die Wahrscheinlichkeit eines Kaufabschlusses beim Anbieter der AR-Anwendung steigt (Rutschmann und Belz 2014).

\section{AR vermag die Konversion potenzieller Customers auf dem Weg zum Kaufent- scheid zu fördern, u. a. durch Effekte zur Instant Happiness.}

Weil mit AR die Informationssuche sowie die Bewertung von Alternativen mit einem gewissen Spaßfaktor verbunden sind und über die Anwendung die Möglichkeit entsteht, Customers für das vom Anbieter gewünschtes Voranschreiten im Prozess zum nächsten Teilschritt (nicht zwingend bis zur nächsten Phase) zu belohnen, wird von einer konversionsförderlichen Wirkung ausgegangen. Dies insbesondere auch durch die Möglichkeit, anhand eines Fotos des virtuellen Gegenstandes in der Bestimmungsumgebung auf einfache Art und Weise die Meinung anderer abzuholen (Patel 2014).

\section{Augmented Reality (AR) erhöht die Sicherheit von Customers, das ,richtige“6 Möbelstück zu kaufen.}

Weil das Möbelstück durch AR in der Bestimmungsumgebung angezeigt werden kann, wird angenommen, dass Customers besser beurteilen können, ob das Möbelstück ihren Wünschen und Vorstellungen entspricht. Dieser Effekt wird im Modell anhand eines Verlaufes der Sicherheit entlang des Kaufentscheidungsprozesses dargestellt. Der mit AR erreichte Sicherheitsgrad wird dabei anderen Hilfsmitteln gegenübergestellt. Gerade im Vergleich mit dem Online-Handel wird diesbezüglich eine maßgebliche Erhöhung der Sicherheit erwartet. Dies könnte dazu beitragen, dass die vorhandene Hemmschwelle beim Onlinekauf reduziert wird und die Bedeutung des stationären Handels abnimmt (Rutschmann und Belz 2014).

\subsection{Methoden}

Die Forschungsfrage wurde mithilfe von Leitfadeninterviews mit zwei relevanten Zielgruppen bearbeitet, Customers und Experts. Als Einstieg in das Forschungsfeld wurden diesen Leitfadeninterviews ethnografische Beobachtungen vorangestellt (Schulz 2014, S. 225). Mit einer unstrukturierten, explorativen Beobachtung (Halbmayer und Salat 2011) der Phasen Informationssuche sowie Bewertung von Alternativen des Kaufentscheidungsprozesses in einem Möbelhaus wurde ein erster grober Eindruck des Untersuchungsbereichs erhalten. Die Beobachtungen wurden mittels Fotos bzw. Filmaufnahmen dokumentiert und dienten zur Ermittlung von relevanten Faktoren im Kaufentscheidungsprozess.

Die Forschungsfrage wurde im Rahmen von teilstrukturierten Leitfadeninterviews mit Customers im und außerhalb eines Möbelgeschäfts von FURNIT sowie Verkaufspersonal von FURNIT, NITU und Möbel ITURE untersucht. Dafür wurden die Thesen operationalisiert, wobei vier Analysedimensionen definiert und je Zielgruppe konkrete Fragen pro 
Dimension erarbeitet wurden. Der entstandene Leitfaden für die Interviews wurde vor Durchführung der Interviews einem Vortest unterzogen. Um eine gute Vergleichbarkeit der Aussagen der Interviewpartner zu erreichen, wurden neben den qualitativen Fragen zusätzlich ergänzende Fragen mit Antwortskalen (1-10) verwendet. Die interviewten Personen wurden während der Interviews mit der AR-App konfrontiert. Sie wurden aufgefordert diese zu testen. Danach erfolgten weitere Fragen zur Nutzung der AR-App.

Insgesamt wurden 24 Interviews zu je 15-20 min geführt. Es wurden 18 Interviews mit Customers durchgeführt, davon 15 im Möbelgeschäft, da dort Customers in tatsächlichen Kaufentscheidungsprozessen und mit einem realen Bedarf anzutreffen sind. Bezüglich Geschlecht und Alter konnte eine hohe Durchmischung erreicht werden. Von den 15 befragten Customers von FURNIT waren acht weiblich und sieben männlich. Die befragten Kundinnen waren zwischen 18 und 62 und durchschnittlich etwas über 35 Jahren alt. Das Alter der befragten Kunden bewegte sich zwischen 20 und 51 Jahren mit einem Durchschnitt von knapp 35 Jahren. Customers wurden zufällig ausgewählt und im Möbelhaus in der Nähe von Produkten angesprochen, die ein relativ hohes Involvement vermuten ließen (eher höhere Preisklasse sowie hohe Visibilität in der Bestimmungsumgebung, z. B. Esstisch). Wichtig war, dass die im Interesse stehenden Produkte oder ähnliche in der App FURNIT-AR vorhanden waren, sodass die Zielpersonen im Rahmen der Befragung mit der Anwendung der App konfrontiert werden konnten. Drei Interviews wurden außerhalb des Möbelhauses durchgeführt. Damit sollte festgestellt werden, inwiefern die Antworten beim Einsatz außerhalb des stationären Handels ausfallen. Die Interviewpersonen sollten dabei eine gewisse Affinität zum Online-Handel aufweisen. Ergänzend wurden sechs weitere Interviews mit Verkaufsmitarbeitenden der Möbelbranche geführt $\left(\right.$ Expert $\left.^{3}\right)$. Da die Haltung gegenüber der App FURNIT-AR bei FURNIT-Experts von Experts von Mitbewerbern abweichen könnte (erhöhte Identifikation mit der App, da vom eigenen Unternehmen angeboten oder übermäßige Ablehnung der App, da eine direkte Bedrohung in der Anwendung gesehen wird), wurden drei Interviews mit FURNIT-Experts und drei Interviews mit Experts des Mitbewerbers Möbel ITURE durchgeführt.

Zunächst wurde versucht, die in der Literatur beschriebenen Muster im Kaufentscheidungsprozess zu validieren. Dafür wurden Customers sowie Experts befragt, wie ein typischer Möbelkauf eines relativ teuren Möbels typischerweise abläuft. Zu beachten galt es dabei, dass der Besuch im Möbelhaus in unterschiedlichen Phasen im Kaufentscheidungsprozess erfolgen kann und nur anhand der Beobachtungen nicht festzustellen ist, welche Schritte und Abklärungen die Customers bereits vor dem Besuch des Möbelhauses durchlaufen hatten. Obwohl es folglich nicht eindeutig zu erkennen war, in welcher Phase sich die beobachteten Customers genau befanden, konnten vier grundsätzlich unterschiedliche Verhaltensweisen identifiziert werden:

\footnotetext{
${ }^{3}$ Zur besseren Lesbarkeit werden nachfolgend Expertinnen und Experten unter dem englischen Begriff Experts subsumiert.
} 
1. Customers passierten die entsprechenden Bereiche relativ rasch, ohne den einzelnen Möbelstücken größere Beachtung zu schenken. Daraus wurde geschlossen, dass diese Customers kein Bedürfnis nach einem Möbelstück im entsprechenden Verkaufsbereich hatten.

2. Customers schlenderten langsamer durch den Bereich, stoppten, sahen sich einzelne Möbelstücke genauer an, tauschten sich mit der Begleitperson aus, blätterten Stoffmuster in unterschiedlichen Farbvarianten durch und gingen danach weiter. Die Beobachtungen wurden dahingehend interpretiert, dass diese Customers dabei waren, sich Informationen zu beschaffen und Auswahlalternativen zu bilden.

3. Customers nahmen sich bei einzelnen Möbelstücken deutlich mehr Zeit, testeten das entsprechende Möbelstück aus, diskutierten mit der Begleitperson, ließen sich beraten und machten teilweise Fotos von den Möbelstücken. Daraus wurde gefolgert, dass diese Customers bereits ins Bewerten von Alternativen übergegangen waren.

4. Customers notierten sich den Lagerstandort eines Möbelstücks auf einem dafür vorgesehenen Notizzettel, fotografierten diesen oder lösten (voraussichtlich) beim Kundenberatenden eine Bestellung aus. Diese Customers hatten sich (zumindest vorübergehend) für den Kauf entschieden.

Die Grenze zwischen den Phasen Informationssuche und Bewertung von Alternativen oder auch der Übergang zur Phase Kaufentscheidung waren aus den Beobachtungen nicht eindeutig zu identifizieren. Dies wurde als Hinweis gewertet, dass diese Phasen nicht linear, sondern mehrfach und iterativ durchlaufen werden. Gleichzeitig wurden die Hauptphasen des Kaufentscheidungsprozesses wiedererkannt, trotz gewisser Überschneidungen. Das zugrunde liegende Modell wurde deshalb als angemessen erachtet.

Alle durchgeführten Interviews wurden transkribiert, kodiert und ausgewertet. Die Kodierung wurde nach dem Gesprächsleitfaden strukturiert, abgeleitet von den Thesen zur Forschungsfrage. Es wurden fünf Code-Familien gebildet: 1) Aussagen bezüglich der aktuellen Wohn- und Einrichtungssituation und 2) Kanäle für die Informationsbeschaffung, 3) Aussagen zur Überprüfung der drei Thesen, 4) Aussagen zum Kaufentscheidungsprozess und schließlich wurden 5) im Rahmen eines zweiten Kodierungsdurchlaufs (Second Cycle) relevante Aussagen identifiziert, die einen Rückschluss auf die App als Kanal, auf den Einfluss des aktuellen Entwicklungsstandes der Technologie und auf eine mögliche Weiterentwicklung der App schließen ließen. Diesen Code-Familien wurden 31 Codes zugeordnet. Insgesamt wurden 323 Aussagen aus den Interviews analysiert.

\subsection{Ergebnisse}

Nachfolgend werden die Ergebnisse der empirischen Erhebung erläutert. 


\subsubsection{Analyse des Kaufentscheidungsprozesses}

Die im Modell zum Kaufentscheidungsprozess definierten Phasen konnten in den Beobachtungen und Antworten der Interviewten nachvollzogen werden. So gab beispielsweise eine 21-jährige Kundin an: „Ich habe das Sofa zuerst im Internet ausgesucht, dann hier vor Ort ausprobiert und fotografiert. Damit ich es zuhause auch nochmal ansehen kann, um zu sehen, ob es mir morgen immer noch gefällt und vielleicht noch ein zweites Mal vorbeigehen, um das Möbelstück anzusehen und dann noch Vergleiche machen kann mit anderen." Ein Verkaufsexperte bei FURNIT bestätigt folgenden Ablauf: „Sie suchen zuerst im Internet und sehen sich den Katalog an. Dann kommen sie zu uns. Sie machen auch Fotos oder Videos." Damit wurden die in der Theorie beschriebenen Phasen des Kaufentscheidungsprozesses bestätigt.

Dass der Prozess iterativ anstatt linear abläuft sowie teilweise lange dauert (Abb. 13.3), konnte von verschiedenen Antworten abgeleitet werden. So erläuterte eine 47-jährige Kundin: „Ich überlege lange vor dem Kauf, sehe mir die Internetseite an und stelle das Zimmer um und habe gemerkt, ich kann es nicht so kaufen, wie ich es will, und habe es nochmals umgeändert. Also dreimal habe ich es umgeändert und dann konnte ich sagen, ja so stimmt's, und dann kaufe ich es." Eine Expertin von Möbel ITURE gab weiter an, dass die Customers gerade bei teureren Möbeln üblicherweise mehrmals vorbeikommen, dass der effektive Entscheid dann aber relativ rasch getroffen wird. Auffallend ist zudem, dass die Phase Informationssuche bei vielen mit dem Ausmessen eines Zimmers beginnt. Danach dauern die Phasen Informationssuche und Bewertung typischerweise relativ lange, bevor der definitive Entscheid gefällt.

Während den Beobachtungen fiel auf, wie intensiv Customers das Smartphone während des Einkaufs einsetzten. Möbelstücke wurden fotografiert und es wurden Informationen mit dem Smartphone gesucht, mitgebracht oder geteilt (Abb. 13.4). Es konnte

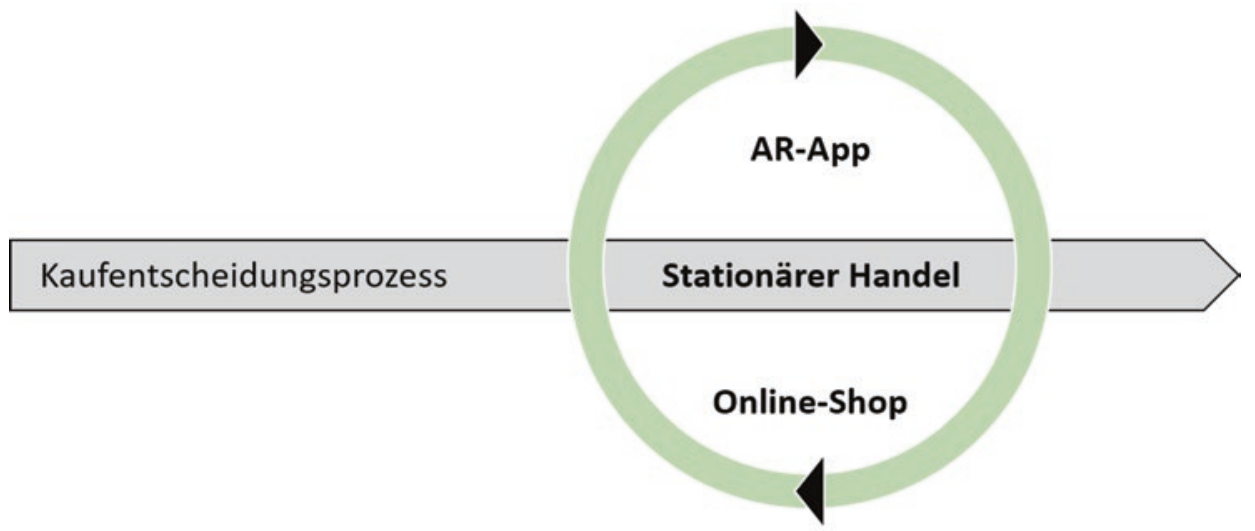

Abb. 13.3 Iterativer Kaufentscheidungsprozess unter Einbindung verschiedener Kanäle. (Eigene Darstellung) 

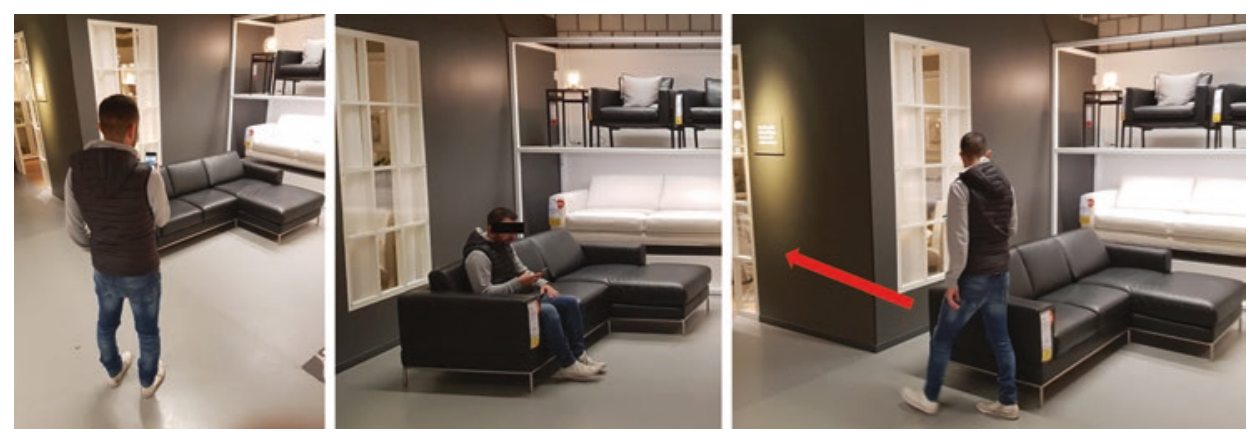

Abb. 13.4 Ethnografische Beobachtung von Kunden und deren intensive Verwendung des Smartphones. (Eigene Darstellung)

vielfach beobachtet werden, wie über das Smartphone Anrufe getätigt wurden. Das den Telefongesprächen vor- und nachgelagerte Verhalten ließ darauf schließen, dass dabei eine gemeinsame Abwägung von Alternativen stattfand oder eine mögliche Kaufentscheidung abgestimmt wurde. In der Phase Kaufentscheidung inkl. Kaufabschluss wurden also Dritte miteinbezogen. Jedoch wurde diesem Aspekt lediglich am Rande der These 2 eine weiterreichende Bedeutung beigemessen. Konkret die Möglichkeit zur Sammlung bzw. Speicherung von Informationen, dem Teilen dieser Informationen mit weiteren Entscheidern, dem daraus abgeleiteten Bilden und Vergleichen von Alternativen sowie dem Abstimmen eines Kaufentscheides wurden als potenziell maßgeblich für die Beurteilung des möglichen Einflusses von AR auf den Kaufentscheid erachtet und in den Kundeninterviews berücksichtigt.

\subsubsection{These 1: Förderung der Interaktion mit dem Anbieter}

Um herauszufinden, ob über die AR-App FURNIT-AR potenzielle Customers animiert werden, entlang des Kaufentscheidungsprozesses mit den Anbietern zu interagieren, sollten Customers auf einer Skala von 1-10 angeben, wie sehr ihnen das Ausprobieren der App Spaß gemacht hat. Die kundenberatenden Experts wiederum wurden direkt gefragt, in welchem Ausmaß eine solche App die Interaktion der Customers mit den Anbietern und deren Produkten fördere. Die quantitativen Erhebungen wurden durch Aussagen in den Interviews ergänzt.

Von den 18 befragten Customers haben 16 mit einem Wert von 7 oder höher geantwortet. Mit einem Mittelwert von 7,8 lässt sich festhalten, dass das Ausprobieren der App den meisten Customers einen gewissen Spaß bereitet hat. Die Wahrscheinlichkeit einer zukünftigen Nutzung der App wurde mit einem Mittelwert von 7,6 ähnlich hoch angegeben. Besonders auffällig ist, dass ein Drittel der Befragten $(n=6)$ angaben, die App zukünftig ganz sicher (Skalenwert 10) zu nutzen und dass die Kunden, die außerhalb 
des Möbelgeschäfts befragt wurden, mit Abstand die tiefsten Wahrscheinlichkeiten für eine zukünftige Nutzung angaben. Daraus lässt sich schließen, dass die App erst ab einem gewissen Punkt im Kaufentscheidungsprozess als hilfreich eingeschätzt wird.

Von den sechs befragten Experts nannten fünf bei der Frage zur Förderung der Interaktion mit den Anbietern einen Wert von 7 oder höher (Mittelwert 6,8). Einzig eine Kundenberaterin bei FURNIT gab bei dieser Frage einen sehr tiefen Wert an. Bei der Erklärung des tiefen Wertes wies die Kundenberaterin jedoch darauf hin, dass es sehr auf die jeweiligen Customers ankommt und gerade bei der jüngeren Generation eine ansteigende Interaktion über die App zu erwarten sei: „Gewisse Kunden würden uns nicht mehr brauchen für Anfragen zu Größe oder Farbe oder was jetzt passen würde. Die Kunden, die noch persönliche Beratung wünschen, aber eher nicht; das ist relativ schwierig. Aktuell wünschen noch viele Kunden eine Beratung. Bei der zukünftigen Generation, die mit Computern aufgewachsen ist, wird die Nutzung aber sicher noch zunehmen." Mit einem Mittelwert von 7,3 lässt sich deshalb festhalten, dass die Experts der App eine anregende Wirkung in Bezug auf Interaktion der Customers mit den Anbietern zusprechen. Interessant ist, dass die Experts von Möbel ITURE mit zweimal einer 8 sowie einmal mit einer 10 diesen Punkt als besonders relevant einschätzen. Die Kundenberaterin, die den maximalen Wert angegeben hat, erklärt zusätzlich: „Kunden sind immer selbstständiger. Sie möchten den Entscheid immer mehr in den eigenen Händen haben oder einen Vorentscheid fällen. Ich finde eine solche App sehr einladend zum Einkaufen, denn es erfüllt den Wunsch nach einem sofortigen Ereignis.“

\subsubsection{These 2: Begünstigung der Konversion}

Um herauszufinden, ob die AR-App FURNIT-AR eine Steigerung an Kaufabschlüssen bewirkt, sollten Customers auf einer Skala von 1-10 angeben, wie nützlich die App für sie beim Kaufentscheidungsprozess war. Die kundenberatenden Experts wiederum wurden gefragt, in welchem Ausmaß eine solche App den Kaufentscheidungsprozess in Richtung Kaufabschluss unterstützt. Die quantitativen Erhebungen wurden durch Aussagen in den Interviews ergänzt.

Customers schätzen den Nutzen der AR-App beim Vergleichen mit anderen Möbelstücken mit einem Mittelwert von 7,9 als besonders hilfreich ein. Dieser Schritt im Kaufentscheidungsprozess ist der Phase Bewertung von Alternativen zuzuordnen. Der hohe Nutzen wird von den interviewten Customers damit begründet, dass mit der App verschiedene infrage kommende Möbelstücke am Bestimmungsort platziert und an Ort und Stelle miteinander verglichen werden können. So gibt eine FURNIT-Kundin prägnant und treffend an: „Das hilft sicher sehr gut. Dann kann man alles reinstellen und sehen, welches am besten in den Raum passt.“ Ein 51-jähriger Kunde führt aus: „Es hilft natürlich, dass man zeigen kann, wie es aussieht und wie es wirkt. Also es hilft sicher besser als ein Foto, weil man es im Prinzip so wahrnimmt, wie es sich im Raum darstellt, wie man es sieht.“ 
Customers bescheinigen der App einen hohen Nutzen beim Einbezug von Dritten mit einem Mittelwert von 7,6. Zu beachten gilt es, dass bei dieser Frage lediglich sieben Customers angegeben haben, dass sie Dritte bei der Kaufentscheidung miteinbeziehen. Die angegebenen Werte wurden zusätzlich kommentiert. So führt eine 18-jährige FURNIT-Kundin aus: „Man kann es sich einfach besser vorstellen statt, wenn ich jetzt ein Foto von dem schicken würde.“

Mit jeweils Mittelwerten von 6,9 bei der Informationssuche und 7,3 beim effektiven Kaufentscheid wird der Nutzen der App leicht tiefer eingeschätzt. Im Fall der Informationssuche erklärte dazu ein 26-jähriger FURNIT-Kunde: „Also, ich glaube, ich würde trotzdem zuerst im Internet schauen, weil es ja nicht Sinn macht 100 verschiedene Möbel hochzuladen“. Eine 21-jährige FURNIT-Kundin weist darauf hin, dass sie gewisse, für sie wichtige Informationen nur im stationären Handel findet: „,[Die App] ist gut, aber wie gesagt, man kann die Möbel nicht berühren." Auch hinsichtlich des Nutzens beim Kaufentscheid wird wiederum auf das nötige Zusammenspiel mit den weiteren Kanälen hingewiesen. In diesem Fall wird vor allem auf die hohe Relevanz des stationären Handels bei der Entscheidungsfindung hingewiesen. So sagte beispielsweise eine 49-jährige Kundin: „Ich glaube die Entscheidung mache ich nicht aufgrund der App. Die fälle ich hier im Laden beim Anschauen. Sie hilft natürlich, weil man die Möbelstücke umstellen kann.“ Ein 26-jähriger Kunde bemerkte dazu: „Ich habe eher das Gefühl, ich würde es am Schluss nutzen, um zu sehen wie es in der Wohnung steht. Denn den Hauptentscheid, denke ich, habe ich schon vorher gefällt. Das wäre dann eher noch um zu sehen, ob es gut in der Wohnung steht.“ Aus dieser Aussage lässt sich neben der Relevanz weiterer Kanäle ein Indiz für den nichtlinearen Kaufentscheidungsprozess herauslesen. So scheint dieser Kunde die bereits ausgewählte Alternative im Nachhinein nochmals absichern zu wollen. Dies deutet auf ein Zutreffen der These 3 hin. Bezüglich des Nutzens der App bei der Informationssuche sowie dem Kaufentscheid lässt sich zusammenfassen, dass die Customers der App zwar durchaus einen Mehrwert attestieren, sich jedoch nicht eine isolierte Verwendung vorstellen können. Onlineshops vornehmlich für die Informationssuche sowie der stationäre Handel für die Informationssuche wie auch für die Entscheidungsfindung werden trotz den Möglichkeiten der App als unabdingbar gewertet.

Die Experts erachteten die App ebenfalls beim Vergleichen von Möbelstücken mit einem Mittelwert von 7,8 als am hilfreichsten. Den Nutzen beim Entscheiden wie auch bei der Informationssuche schätzen die Experts leicht tiefer ein als die Customers. Dabei wird wiederum mehrfach auf die Relevanz des stationären Handels hingewiesen. So meint eine Expertin von Möbel ITURE in Bezug auf den Kaufentscheid: „Es ist zwar sehr cool, um sich das Ganze bildlich vorstellen zu können, aber schlussendlich müssen die Leute auch einmal draufsitzen können." Noch deutlicher drückt sich eine weitere Expertin bei Möbel ITURE aus: „Nicht relevant. So eine App ist nicht relevant bei einem teuren Möbelkauf. ... So eine App hilft vielleicht, aber beim Entscheid halte ich es für nicht relevant." Bei der Informationssuche äußert sich die gleiche Kundenberaterin ähnlich kritisch: „Nach Informationen? Ich habe keine Informationen erhalten. 
Enttäuschend.“ Andere Experts schätzten den Nutzen der App deutlich höher ein und alle Experts antworteten bei der abschließenden Frage, ob die App ihrer Ansicht nach den Kaufentscheidungsprozess unterstützt, mit „Ja“.

\subsubsection{These 3: Erhöhung der Wahlsicherheit}

Um herauszufinden, ob über die AR-App FURNIT-AR die Sicherheit bei der Auswahl der Möbelstücke erhöht wird, sollten Customers auf einer Skala von 1-10 angeben, wie sie den Einfluss der App auf ihre Wahlsicherheit wahrnahmen. Die kundenberatenden Experts wurden gefragt, in welchem Ausmaß eine solche App den Customers Wahlsicherheit vermittelt. Die quantitativen Erhebungen wurden durch Aussagen in den Interviews ergänzt.

Um einen Effekt auf die Sicherheit abzuschätzen, das ,richtige“ Möbelstück zu kaufen, wurden die Customers gefragt, wie sicher sie beim letzten Möbelkauf bezüglich ihrer Entscheidungen waren. Hierbei haben von 18 befragten Customers 12 angegeben, sicher gewesen zu sein. Die 49-jährige FURNIT-Kundin beschreibt dies folgendermaßen: „Da war ich schon überzeugt, sonst hätte ich es nicht gekauft.“ Einige Customers haben als Antwort gegeben, dass sie $100 \%$ sicher waren, womit eine Erhöhung der Wahlsicherheit durch zusätzliche Hilfsmittel obsolet wird. Etwas offener formulierte dies ein 49-jähriger Kunde: „Sehr sicher, weil ich lange überlege. Wobei mit dieser Applikation ist es natürlich ja sehr hilfreich, weil man sich das dann halt direkt anschauen kann." Von den restlichen sechs befragten Customers waren drei mäßig sicher und zwei unsicher. Ein 20-jähriger FURNIT-Kunde erklärt: „Ja, denn man hat immer so ein bisschen Zweifel, dass es dann doch nicht reinpasst.“ Eine 23-jährige FURNIT-Kundin beschreibt: „Also beim letzten Kauf hatten wir nur Fotos und es kam dann gut, aber es hätte natürlich auch sehr schiefgehen können. Also wir hatten halt nicht immer die gleichen Informationen und waren uns etwa $60 \%$ sicher." Customers sind in den meisten Fällen überzeugt, das Richtige zu kaufen. Trotzdem gab mindestens ein Drittel der Customers an, dass bezüglich Sicherheit die Customer Experience gesteigert werden könnte (mäßig sicher oder unsicher).

Ebenfalls befragt wurden die Customers, wie sie prüfen, ob die Möbelstücke in die Bestimmungsumgebung passen. Dabei erwähnten zahlreiche Customers, dass sie zuerst den Raum ausmessen um sicherzustellen, dass das entsprechende Möbel von der Größe her in den Bestimmungsort passt. Andere erklärten, dass sie sich die Möbel in der Bestimmungsumgebung vorstellen. Ein 49-jähriger FURNIT-Kunde erläuterte: „Ich versuche mir das vor dem geistigen Auge vorzustellen, im Grunde, das, was man halt damit (die App) macht. Passt der Farbton, die Größe, das Ausmessen. Diese Richtung.“ Es wurde somit von den Customers bestätigt, dass es eine gewisse Herausforderung ist, zu prüfen, ob ein Möbelstück in die Bestimmungsumgebung passt. Ebenfalls kann von den Aussagen abgeleitet werden, dass die App zur Unterstützung der Vorstellungskraft als hilfreich eingeschätzt wurde. 
Bei der Einschätzung zum Einfluss der AR-App FURNIT-AR auf die Wahlsicherheit haben die Customers mit einem Mittelwert von 6,9 geantwortet. Im Vergleich zu anderen Fragen zum Nutzen der App war dies ein eher tiefer Wert. Daraus wird geschlossen, dass die Erhöhung der Wahlsicherheit durch die App nicht als Hauptnutzen eingeschätzt wird. Die Aussage einer 47-jährigen Kundin, die den Wert 1 angegeben hatte, verdeutlicht dies: „Die Applikation gibt mir hier keine Sicherheit.“ Trotzdem geben immerhin elf Customers einen Wert von 7 oder höher an. Dies lässt vermuten, dass die App zumindest eine absichernde Wirkung beim Entscheid vermittelt.

Die Experts wurden gefragt, welche Gründe es für die Unsicherheit bei Customers gibt. Häufig wurde dabei genannt, dass die Customers nicht wüssten, ob es Zuhause passe. So führte eine Expertin von FURNIT aus: „Was sicher eine Rolle spielt ist, wie passt es Zuhause in den Raum. Wir haben oft auch Kunden, die Fotos von einem Raum zeigen und fragen, ob das Möbelstück da jetzt hineinpasst. Und das andere ist sicher meist die Größe, dass sie nicht sicher sind wegen den Maßen und Zuhause nicht gemessen haben. Was man auch immer noch hat ist, dass sie bei der Konkurrenz etwas gesehen haben und noch abwägen. “ Ein Experte von Möbel ITURE wiederum bestätigt den Grund der fehlenden Fähigkeit sich das Möbelstück in der Bestimmungsumgebung vorstellen zu können. Interessant erscheint zudem die Annahme einer Expertin von Möbel ITURE bezüglich der mangelnden Vorstellungskraft der jüngeren Generation: „,... und leider, dass muss ich betonen, leider verliert die junge Generation durch die Nutzung solcher Apps das Vorstellungsvermögen, das visuelle Vorstellungsvermögen. Generationen sind mit Cartoons im Fernsehen aufgewachsen und dass fördert sicher nicht das Vorstellungsvermögen. Und so braucht diese Generation die Unterstützung einer solchen App." Neben weiteren Gründen für die Unsicherheit (z. B. Widerstandsfähigkeit des Materials, Qual der Wahl etc.) gehen die Experts folglich davon aus, dass nicht immer das nötige Vorstellungsvermögen vorhanden ist, um bei der Kaufentscheidung sicher zu sein.

Auch die Experts wurden gefragt, wie die Customers überprüfen, ob die infrage kommenden Möbelstücke in die Bestimmungsumgebung passen. Dies erachteten die Experts als Herausforderung. Ein Beispiel wird von einer Expertin von Möbel ITURE ausgeführt: „Das ist schwierig, hier braucht man Vorstellungsvermögen. Typischerweise nimmt der Kunde den Katalog, steht in seinem Zimmer und dann probiert er, sich ein neues Sofa vorzustellen." Sowohl Möbel ITURE wie auch FURNIT bieten dafür Lösungen an. So erklärt ein Experte von Möbel ITURE: „Wir haben die Möglichkeit, dem Kunden Möbel mitzugeben, damit er diese ausprobieren kann.“ Außerdem besteht ein Rückgaberecht, sollte das Möbelstück nicht in die Bestimmungsumgebung passen.

Schließlich wurden auch die Experts gebeten einzuschätzen, wie gut AR-Apps den Customers Sicherheit geben, das ,richtige“ Möbelstück zu kaufen. Wiederum schätzten die Experts den Effekt der App mit einem Mittelwert von 6,9 ähnlich ein wie die Customers. Das Resultat wird durch Aussagen eines Experts plausibilisiert: „Also ich denke, wenn er sich das hier jetzt anschaut oder Zuhause mit der App, kann er dadurch einfach noch einen Impuls mehr erhalten um zu merken: Doch das passt jetzt!“ Auch hier ist entscheidend, dass die App eine zusätzliche Unterstützung darstellt und den Nutzen nicht als alleinstehender Kanal entfalten kann. 


\subsection{Diskussion}

Die Studie hatte zum Ziel, den Einfluss von AR auf den Kaufentscheidungsprozess beim Möbelkauf zu beleuchten. Insgesamt lässt sich feststellen, dass AR den Kaufentscheidungsprozess beim Möbelkauf unterstützen kann. Die Erkenntnisse aus den Interviews zum Kaufentscheidungsprozess können wie folgt zusammengefasst werden: Die Phasen im Prozess werden zwar beibehalten, jedoch ist die Dauer der Phasen Informationssuche und Bewertung von Alternativen undefiniert und eine Abgrenzung in der Praxis oft nicht möglich. Die von den Customers sowie Experts angegebenen Hilfsmittel sowie die Erkenntnisse aus den ethnografischen Beobachtungen ergänzen den Entscheidungsprozess. Zuerst werden das Internet und die dort verfügbaren Onlineshops konsultiert. Teilweise werden für die Informationssuche Kataloge genutzt und (selten) bei Freunden und Bekannten nach Inspiration gesucht. Für die Customer Experience gewinnt der stationäre Handel an Bedeutung. Interessante Möbelstücke werden fotografiert oder gefilmt. Freunde, Bekannte, Familienmitglieder oder Partner werden für die Entscheidungsfindung einbezogen. Schließlich wird ein Kaufentscheid gefällt und das Möbelstück online oder in einem Möbelgeschäft geordert.

Auffällig ist, dass alle Interviewten angegeben haben, sich im Internet zu informieren. Viele Befragte blättern zudem ganz zu Beginn Kataloge zur Ideenfindung durch. Dies geschieht teilweise auch, weil im richtigen Moment ein entsprechender Katalog Zuhause im Briefkasten landet. Die Experts bei FURNIT sowie Möbel ITURE geben an, dass immer mehr Customers vorinformiert im stationären Handel erscheinen und schon in etwa wüssten, was sie suchen. Dies wird von den Händlern ebenfalls bestätigt. So geben NITU und Möbel ITURE in einem aktuellen Artikel an, dass $50 \%$ der Customers bei NITU und $80 \%$ der Customers bei Möbel ITURE bereits vor dem Besuch im Möbelgeschäft online nach Produkten suchen (Felber 2018). Zu Beginn der Informationssuche kann somit das Internet mit den Onlineshops als wichtigstes Hilfsmittel bezeichnet werden. Kataloge werden teilweise ebenfalls konsultiert. Seltener stellen Möbelstücke bei Freunden und Bekannten eine Inspiration dar.

In einem zweiten Schritt geben die meisten Customers an, dass sie weiterführende Informationen im stationären Handel suchen. Auffällig war, wie häufig während den Interviews darauf hingewiesen wurde, dass es bei Möbeln wichtig sei, dass man sie mit den eigenen Augen vor Ort sieht, anfassen und ausprobieren kann. Daraus wird gefolgert, dass im stationären Handel konkretere Alternativen für den Möbelkauf gebildet werden und der Übergang zur Phase Bewertung von Alternativen fließend erfolgt. Wichtig erscheint aber, dass die Interviewten häufig angaben, nicht direkt beim Besuch im Möbelhaus zu entscheiden, sondern sich später erneut ins Möbelhaus zu begeben. Daraus lässt sich ableiten, dass weder die Phase Informationssuche noch die Phase Bewertung der Alternativen vollständig abgeschlossen sind.

Um die am Ende der Phase Informationssuche gebildeten Alternativen zu bewerten, begeben sich die meisten befragten Customers in ein Möbelgeschäft. Bei den ausgewählten Möbelstücken mit einem komplexen Kaufentscheidungsprozess, konkret 


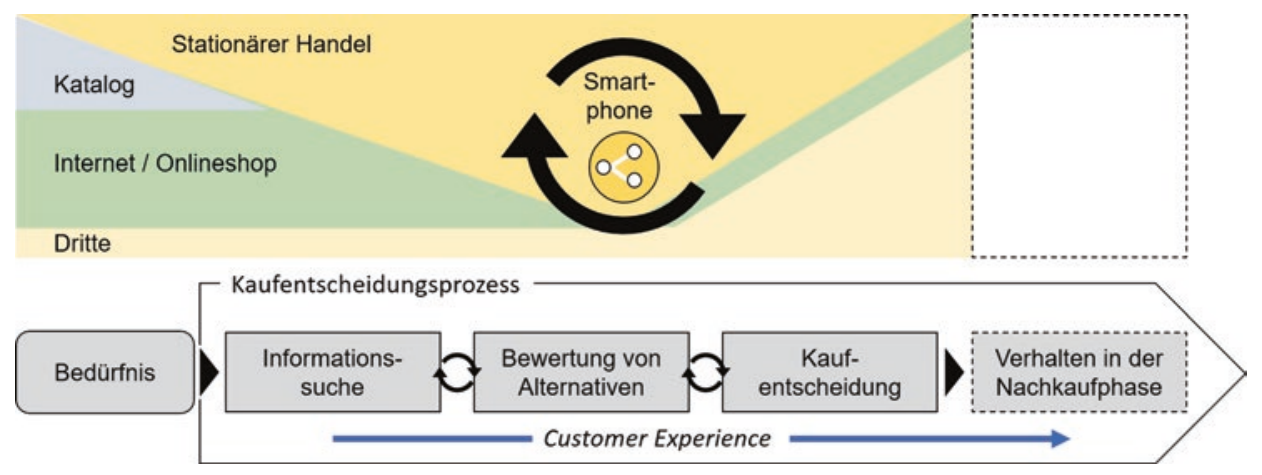

Abb. 13.5 Übersicht über die Bedeutung verschiedener Kanäle im Kaufentscheidungsprozess

den Sofas oder Schränken, ist es vielen Customers wichtig, sich Zeit zu lassen und die Möbelstücke vor Ort anzusehen. Im Zusammenhang mit dem Einfluss der AR-App FURNIT-AR ist besonders erwähnenswert, dass Customers oft Fotos oder Videos von potenziellen Möbelstücken machten. Besonders gut widerspiegelt sich dies in der nachfolgenden Aussage eines Kundenberaters bei FURNIT: „Wir haben schon Einzelpersonen, es ist da aber so, dass die Personen vermehrt Zuhause anrufen, um sich mit dem Partner oder wem auch immer abzusprechen. Was man mittlerweile auch sieht ist, dass Kunden Fotos machen und senden, gleichzeitig am Telefon sind.“ Diese Aufnahmen dienen also dem nachgelagerten Bewerten der Alternativen, aber auch dem gezielten Einbezug von Dritten (Abb. 13.5).

\subsubsection{These 1: Förderung der Interaktion mit dem Anbieter}

Aus den hohen Werten beim Spaßfaktor und der Wahrscheinlichkeit einer zukünftigen Nutzung bei den Customers sowie den übereinstimmenden Aussagen der Experts kann geschlossen werden, dass die App die Interaktion mit den Anbietern solcher Apps anregt. Aufgrund der tieferen Werte durch die außerhalb des Möbelgeschäfts befragten Customers kann von einer hohen Herausforderung bezüglich rechtzeitiger Zurverfügungstellung der App ausgegangen werden. Kritisch lässt sich sicherlich hinterfragen, ob die App auch nach einem tatsächlichen Anwendungsversuch als noch so stimulierend für die Interaktion eingeschätzt werden würde. Trotz gewisser Vorbehalte wird - auch aufgrund einer erwarteten Weiterentwicklung der Technologie und deshalb einer besseren Funktionalität - davon ausgegangen, dass die These 1 zutrifft und durch AR potenzielle Customers animiert werden, entlang des Kaufentscheidungsprozesses vermehrt mit Anbietern und deren Produkten zu interagieren.

Die Customers schätzen die Wahrscheinlichkeit für eine zukünftige Nutzung der App FURNIT-AR als relativ hoch ein. Ein Drittel der Befragten war sich sogar sicher und gab den höchstmöglichen Wert an. Deshalb erstaunt es, dass die App nicht bereits bekannter 
und deren Nutzung verbreiteter ist. Ein Grund dafür ist sicherlich, dass FURNIT die App (noch) nicht aktiv bewirbt und auch nicht mit den bestehenden Kanälen verbunden hat. In der Filiale in der Region Bern konnten weder visuelle Hinweise auf die App gefunden werden, noch war die App den befragten Verkaufs-Experts bekannt. Aufgrund der Reaktionen der Interviewten kann gefolgert werden, dass gerade Customers mit einem unmittelbar vorhandenen Bedürfnis zum Möbelkauf Gefallen an der App finden könnten. Wenn auch noch nicht alle Funktionen in überzeugender Qualität umgesetzt sind, könnte die App einen Begeisterungsfaktor im Einkaufserlebnis bei FURNIT darstellen. Da weitere Anbieter an vergleichbaren Anwendungen arbeiten oder bereits veröffentlicht haben, sollte nicht lange mit der Umsetzung der untenstehenden Handlungsempfehlungen abgewartet werden, um den Charakter eines Begeisterungsfaktors nutzen zu können.

\subsubsection{These 2: Begünstigung der Konversion}

Abgeleitet von den Ergebnissen wird gefolgert, dass die AR-App FURNIT-AR einen begünstigenden Effekt auf die Konversion bewirkt. Dies nicht unbedingt aufgrund von Effekten der Instant Happiness - zumindest konnte dies nicht im Rahmen der Studie festgestellt werden -, sondern weil die App als besonders nützliches Hilfsmittel für das Bewerten von Alternativen wahrgenommen wird. Dieser Nutzen wurde sowohl von Customers als auch Experts angegeben. Die dadurch begründete Begünstigung der Konversion funktioniert hauptsächlich im Zusammenspiel mit weiteren Kanälen, vornehmlich den Onlineshops und dem stationären Handel. Da die Phasen Informationssuche und Bewertung von Alternativen (bis hin zur Kaufentscheidung) voraussichtlich iterativ, sich überschneidend und nicht sequenziell ablaufen, kann geschlossen werden, dass dank der App zu jedem Zeitpunkt im Kaufentscheidungsprozess bequem eine aussagekräftige Bewertung einer Alternative ermöglicht wird. Mit der digitalen Transformation kann eine Entwicklung hin zu einer verstärkten Interaktion zwischen Individuen über digitale Kanäle beobachtet werden. In diesem Zusammenhang kann davon ausgegangen werden, dass die App gerade in Situationen mit mehreren Entscheidern einen hohen Mehrwert bieten kann. Entsprechend positiv waren die Aussagen zur Bewertung von Alternativen sowie den Einbezug von Dritten für die Entscheidungsfindung. So lässt sich zu jeder Zeit im Kaufentscheidungsprozess die Haltung Dritter abholen und dadurch kann Sicherheit beim Ausschließen oder Favorisieren von Alternativen gewonnen werden. Es findet somit eine zeitliche und personelle Entkopplung im Kaufentscheidungsprozess statt, wodurch eine Konversion begünstigt wird.

\subsubsection{These 3: Erhöhung der Wahlsicherheit}

Von Customers und Experts wird der AR-App ein positiver, beitragender Effekt bezüglich der Sicherheit bestätigt, das ,richtige“ Möbelstück zu kaufen. Indem Möbelstücke 
über die AR-App in der Bestimmungsumgebung angezeigt werden können, vermögen Customers besser zu beurteilen, ob das Möbelstück in die Bestimmungsumgebung passt. Aus diesem Grund wurde erwartet, dass im Vergleich zu den bisher zur Verfügung stehenden Kanälen und Hilfsmitteln die Sicherheit steigt, das ,richtige“ Möbelstück zu kaufen. Aufgrund des Zusammenspiels verschiedener Kanäle im Kaufentscheidungsprozess muss diese These in der ursprünglichen Form verworfen werden, insbesondere, wenn die App als isolierter Kanal betrachtet wird. Vielmehr ist anzunehmen, dass das für den Kaufentscheid notwendige Maß an Sicherheit bequemer sowie eventuell früher erreicht wird. Ohne die App wäre häufig ein erneuter Besuch von Möbelhäusern bzw. Onlineshops nötig. Die Customer Experience wird durch die Möglichkeiten der AR-App also verbessert, jedoch ist es unklar, ob der Grad an Sicherheit im Kaufentscheidungsprozess erhöht wird. Es stellt sich mit Blick in die Zukunft die Frage, ob allenfalls bei besserer Qualität und Funktionalität sowie positiven Erfahrungen bei der Nutzung der App die Sicherheit erhöht und ein Kanal oder auch mehrere Kanäle teilweise substituiert werden können. Dass jedoch, wie bei der ursprünglichen Beschreibung der These angenommen, aufgrund der App der stationäre Handel an Bedeutung verliert, konnte anhand der Empirie nicht belegt werden.

\subsubsection{Kritische Aussagen zum Nutzen der AR-App}

Neben den unterstützenden Wirkungen der AR-App wurden auch diverse eher kritische bzw. den Nutzen der App relativierende Aussagen geäußert. Neben dem Einfluss der Technologie wird diesbezüglich vor allem das Zusammenspiel mit bestehenden Kanälen thematisiert.

Sehr kritisch zu beurteilen ist die aktuelle Qualität der Anwendungen. So wurde bei den Interviews mehrfach darauf hingewiesen, dass die App nur Erfolgschancen hat, wenn sie der erwarteten Funktionalität und Qualität entspricht. Beim Einsatz der App im Rahmen der Studie fiel auf, dass je nach Bedingungen (z. B. Lichtverhältnissen) die Resultate eine ungenügende Qualität aufwiesen (z. B. nicht realitätsnah im Raum einfügt). In einem aktuellen Bericht über den Entwicklungsstand der AR-Technologie wird die Grafikqualität der bestehenden AR-Apps der Möbelbranche als bescheiden beurteilt (Settele 2018). Dies bestätigten auch Customers und äußerten sich teilweise skeptisch zu den Ergebnissen der App. Die aktuelle Qualität führt zu Zweifeln, was den Mehrwert im Kaufentscheidungsprozess schmälert.

Wie bereits erwähnt wurde die AR-App lediglich als unterstützendes Hilfsmittel wahrgenommen. Der Kaufentscheidungsprozess wird deshalb durch AR nicht grundlegend verändert. Die App stellt ein Bindeglied zwischen den aktuellen Kanälen dar und eignet sich vermutlich in erster Linie für das Bewerten von Alternativen inklusive des Einbezugs von Dritten. Nur wenn die App darauf ausgerichtet ist, die erwartete Funktionalität liefert und auch in den bestehenden Kanälen am richtigen Ort zum richtigen Zeitpunkt auf das zusätzliche Hilfsmittel aufmerksam gemacht wird, kann sich der 
effektive Nutzen der App entfalten. Besonders wichtig deshalb, dass Customers überall dort auf die App aufmerksam gemacht werden, wo zur Bildung der Alternativen Informationen gesucht werden. Eine Fokussierung sollte dabei auf Möbelstücke mit einem komplexen Kaufentscheidungsprozess bzw. High Involvement geschehen. Da aktuell kaum Verknüpfungen zwischen den Kanälen vorhanden sind, kann nicht davon ausgegangen werden, dass der in den vorhergehenden Kapiteln beschriebene Nutzen bereits heute eine breite Kundengruppe erreicht hat.

Es ist durchaus denkbar, dass zukünftig zusätzliche Hilfestellungen, beispielsweise in Form von Bewertungen von Produkten durch Customers, über digitale Kanäle verfügbar gemacht und auch in den Kaufentscheidungsprozess einbezogen werden. Dies kann zu einer Stärkung der digitalen Kanäle führen. Bei teuren Möbelstücken, die für längere Zeit in Verwendung bleiben und eine hohe Sichtbarkeit in den Räumlichkeiten der Customers aufweisen, wird aber voraussichtlich noch längere Zeit vorausgesetzt, die Möbel vor dem Kauf ausprobieren und mit eigenen Augen in der Realität anschauen zu können. Die wohl andauernde hohe Relevanz des stationären Handels schränkt jedoch den Nutzen von AR nicht ein. Weil sich Customers im Möbelhaus an unterschiedlichen Punkten im Kaufentscheidungsprozess befinden, bietet sich die Chance AR als besonders wertvoll eingeschätztes Hilfsmittel zur Verfügung zu stellen.

\subsection{Handlungsempfehlungen}

Der Zusatznutzen von AR im Kaufentscheidungsprozess wird im Zusammenspiel mit den bestehenden Kanälen als hoch eingeschätzt. Die Möbelbranche ist damit in der digitalen Transformation auf einem strategisch sinnvollen Weg. Ein Großteil der befragten Personen gab als sehr wahrscheinlich an, dass sie die App nach dem Kennenlernen weiterhin nutzen werden. Die im Rahmen der Studie gemachten Beobachtungen legen jedoch nahe, dass durch die fehlende Verknüpfung der Kanäle viele Customers gar nicht auf die App aufmerksam werden - zumindest nicht zum richtigen Zeitpunkt. Damit sich der Nutzen von AR entfalten kann, sollte im richtigen Moment am richtigen Ort auf die App aufmerksam gemacht werden. Weder bei den ethnografischen Beobachtungen, noch bei den Interviews in der FURNIT Filiale, noch bei den darauffolgenden Nachforschungen konnte eine gezielte Verknüpfung von bestehenden Kanälen mit der AR-App festgestellt werden. Dies gilt gleichermaßen für den Onlineshop, inklusive der mobilen Version, den aktuellen Katalog sowie für den stationären Handel. Nachfolgend sind deshalb einige konkrete Verbesserungsvorschläge für eine gezielte Verknüpfung der jeweiligen Hilfsmittel aufgeführt.

\section{Digitale Kanäle}

Der Kaufentscheidungsprozess beginnt mit der Informationssuche. Um sich eine erste Übersicht zu verschaffen, nutzen viele Customers das Internet bzw. Onlineshops. Obwohl dort die gleichen Produkte präsentiert werden, die auch in der App FURNIT-AR 
enthalten sind, gibt es keine direkten Hinweise auf die App. Sollen Customers direkt bei der Informationssuche eine erste Bewertung von potenziellen Möbeln vornehmen können, sollten sie unmittelbar beim Produkt auf die Möglichkeit aufmerksam gemacht werden. Zudem sollte der darauffolgende Prozess - sprich der Download der App oder, falls bereits vorhanden, die Suche nach den entsprechenden Möbelstücken - sehr einfach möglich sein. Dies könnte allenfalls mit einem Link auf den App-Store oder mit QR-Codes gelöst werden. Erfolgt die Informationssuche über ein mobiles, App-fähiges Gerät, sollte ein direkter Weg in FURNIT-AR angestrebt werden.

\section{Verknüpfung mit dem Katalog}

Beim aktuellen Katalog findet sich am Ende ein kurzer Hinweis auf die App. Damit auch Customers, die den Katalog zur Informationssuche nutzen, von den Vorteilen der AR-App profitieren können, sollten auch im Katalog möglichst in der Nähe der relevanten Produkte Hinweise auf die App enthalten sein. Damit nicht der ganze Katalog mit QR-Codes gefüllt werden muss, sind die relevantesten Möbel mit einem entsprechend komplexen Kaufprozess (Sofas, Esstische, Schränke) für eine Verknüpfung auszuwählen.

\section{Verknüpfung mit dem stationären Handel}

Aufgrund der hohen Relevanz des stationären Handels sind die Hinweise auf die AR-App hier besonders wichtig. Gerade wenn Customers sich noch in einer relativ frühen Phase des Kaufentscheidungsprozesses befinden, wäre es wertvoll, wenn die Möbel direkt in der geplanten Wohnumgebung visuell beurteilt werden könnten. Die dafür notwendige „Infrastruktur“ bringen Customers selbst mit: das Smartphone, das schon aktuell als Hilfsmittel beim Einkaufen eingesetzt wird.

Noch fast relevanter als die visuellen Hinweise auf die App ist, dass die Experts die App gezielt in Gesprächen als Hilfsmittel einbringen können. Dafür wäre es wichtig, dass die Kenntnisse zur App erhöht werden. Bei den Interviews wurde festgestellt, dass die Experts aktuell keine oder kaum Kenntnisse der App aufweisen. Gerade noch unentschlossenen Customers könnte die AR-App jedoch mit in die nächsten Phasen des Kaufentscheidungsprozesses gegeben werden. Zur Verbesserung der Customer Experience ist außerdem notwendig, dass Experts über ein gewisses Anwendungswissen verfügen, um Customers auch bezüglich der richtigen Handhabung des Hilfsmittels beraten zu können.

\section{Einbezug von Dritten}

Eine wertvolle Funktion der App stellt die Möglichkeit dar, Dritte im Kaufentscheidungsprozess miteinbeziehen zu können. Aktuell ist jedoch in der App FURNIT-AR ein Bruch vorhanden. So muss die Screenshot-Funktion am Smartphone verwendet werden, um eine Einrichtungssituation weiterleiten zu können. Ein direktes Teilen aus der App heraus ist bislang nicht möglich. So könnte beim direkten Teilen aus der App heraus zusätzlich gefragt werden, ob die aktuelle Einrichtungssituation geteilt oder nur das Möbelstück an Dritte weitergeschickt werden soll. Gerade Bewohner von Wohngemeinschaften oder auch Paare, bei denen ein gemeinsamer Besuch des Möbelgeschäfts 
zeitlich nicht möglich ist, könnten davon profitieren. Zusätzlich würde diese Funktion eine Chance zur weiteren Verbreitung der App bieten. Bei einem Versand eines Möbelstückes an Customers, die nicht über die App verfügen, könnte eine Einladung zum Download der App geschickt werden. Ähnlich wie es beispielsweise bei WhatsApp oder auch Twint gelöst ist, wenn eine Nachricht bzw. Geld an Leute ohne App verschickt werden soll.

\section{Anzeigequalität}

Um den Mehrwert der App zu steigern, wird eine Verbesserung der Anzeigequalität empfohlen. Die App wird bei schlechter Anzeigequalität kaum großen Anklang finden. Aktuell herausfordernd ist, dass die Möbelstücke auch in Wände hineingezogen werden können und deshalb die Größenanzeige im Raum nicht mehr realistisch ist. Zwar kann man mit sorgfältigem Scannen und Platzieren der Möbelstücke Abhilfe verschaffen, jedoch ist das relativ aufwendig und nicht benutzerfreundlich. Wünschenswert wäre, wenn die App gerade Wände erkennen und dem Benutzer die real vorhandenen Grenzen auch in der App als Rahmen vorgeben würde. Schwierig zu lösen ist, dass teilweise Möbel im Raum stehen, die von den Customers tatsächlich ,übereinandergestellt“ werden möchten. Die Umsetzung der genannten Verbesserung bei der Anzeigequalität erfordert voraussichtlich anspruchsvolle technische Lösungen. Dies könnte bedeuten, dass allenfalls hohe Kosten mit der Umsetzung dieser Anforderungen verbunden sind. Es ist abzuwägen, ob der durch die bessere Anzeigequalität entstehende Zusatznutzen die Kosten rechtfertigt.

\section{Anleitung für die richtige Anwendung}

Nach dem Download der App FURNIT-AR werden die Customers kurz in die Funktionen der App eingeleitet. Danach sind jedoch die Hilfestellungen in der App nicht mehr auffindbar und die Funktionalitäten sind nicht immer selbsterklärend. Sicherlich gibt es weitere Tipps und Tricks, die für eine Optimierung der Customer Experience nützlich wären. Deshalb wäre es wichtig, dass auch nach der Anleitung zu den ersten Schritten, direkt nach dem Download, ein Menüpunkt mit Hilfestellungen zur Verfügung steht.

\section{Anbieterunabhängige App}

Obwohl der Nutzen durch die Customers beim Bewerten von Alternativen als sehr hoch eingeschätzt wird, verbirgt sich bei diesem Punkt auch eine große Herausforderung. So entwickeln zurzeit diverse Anbieter eigene AR-Applikationen. Es ist jedoch naheliegend, dass die Customers sich nicht nur Möbelstücke eines Anbieters anschauen. Deshalb wäre eine Anzeige von Möbelstücken unabhängig vom Anbieter eine zusätzliche Erleichterung für die Customers. In verschiedenen Branchen haben sich in den letzten Jahren Plattformen etabliert, die Angebote mehrerer Markteilnehmer einschließen (z. B. Airbnb, Uber, Alibaba, Amazon etc.). Es gilt jedoch festzuhalten, dass auch ohne eine Weiterentwicklung der App in diese Richtung voraussichtlich ein Nutzen bezüglich des Vergleichens von Alternativen für die Customers entsteht. So lassen sich zumindest 
die in der App verfügbaren Möbelstücke besser beurteilen. Da beim Bilden sowie dem Bewerten von Alternativen mehrere Hilfsmittel verwendet werden, besteht die Möglichkeit aus den bestehenden Kanälen eine Verknüpfung zur App herzustellen, um die Customers auf diese aufmerksam zu machen.

\subsection{Fazit}

Die Ausgangslage dieser Studie war die Marktdurchdringung des Smartphones und die damit ermöglichte mobile Vernetzung als Treiber sich ändernden Einkaufsgewohnheiten. Wie bei den ethnografischen Beobachtungen gesehen und im Anschluss bei den Interviews validiert, machten Customers Fotos oder Videos, um zu einem späteren Zeitpunkt Möbelstücke miteinander zu vergleichen sowie um Dritte in den Kaufentscheidungsprozess miteinzubeziehen. Der Entscheidungsprozess beim Möbelkauf weist klare Muster auf. So beginnen die meisten Customers mit der Suche nach Informationen im Internet oder konsultieren Kataloge. Nach einer ersten Vorauswahl wird für weitere Informationen der stationäre Handel besucht. Danach folgen potenziell weitere Internetrecherchen sowie, vor allem bei teureren Möbelstücken, oftmals mehrfache Besuche in den Möbelhäusern. Vielfach werden die Entscheide zwischen mehreren Personen abgestimmt oder Dritte in den Kaufentscheidungsprozess einbezogen. Die aus der Literaturrecherche abgeleiteten Phasen des Kaufentscheidungsprozesses konnten wiedererkannt werden. Jedoch scheinen gerade die Phasen Informationssuche und Bewertung von Alternativen aufgrund der neuen Möglichkeiten, sich orts- und zeitunabhängig mit dem Einkauf auseinanderzusetzen, stärker miteinander zu verschmelzen. So scheinen die Phasen teilweise parallel stattzufinden und sich mehrfach zu wiederholen. Auf die bei Customers sowie den meisten Experts vor den Interviews noch unbekannte App FURNIT-AR wurde mit sichtbarem Erstaunen reagiert. Das erstmalige Ausprobieren bereitete den Customers Spaß und die Möglichkeiten der App wurden als hilfreich für den Kaufentscheidungsprozess eingeschätzt. Jedoch nicht als alleinstehendes Hilfsmittel, sondern als Bindeglied zwischen den aktuellen Kanälen. Gerade der stationäre Handel nimmt eine unverändert hohe Relevanz im Entscheidungsprozess beim Möbelkauf ein dies zumindest bei den Möbelstücken mit einem komplexen Kaufprozess bzw. hohem Involvement. Besonders für die Bewertung von Alternativen eignet sich AR laut Angabe der Customers. Beim immer wichtiger werdenden Einbezug von Dritten bietet die App zusätzliche Möglichkeiten bzw. Entwicklungspotenzial. Die Sicherheit, das richtige Möbelstück zu kaufen, erhöht die untersuchte AR-App FURNIT-AR nicht unbedingt. Jedoch kann die App helfen, Kaufintentionen schneller und einfacher abzusichern. Insgesamt zeigte sich in den Erhebungen, dass die AR-App einen wichtigen Stellenwert im orts- und zeitunabhängigeren Kaufentscheidungsprozess einnehmen kann. Zusammenfassend lässt sich feststellen, dass AR den Kaufentscheidungsprozess beim Möbelkauf unterstützen kann und gezielt weiterentwickelt werden sollte. Mit den empirischen Ergebnissen und den davon abgeleiteten Handlungsempfehlungen leistet die vorliegende 
Studie einen Beitrag zum besseren Verständnis der mit der digitalen Transformation einhergehenden Veränderungen und zeigt sinnvolle Entwicklungsrichtungen für die Möbelbranche auf.

\section{Literatur}

Berry, L., et al. (2002). Managing the total customer experience. MIT Sloan Management Review, 43(3), 85-90.

cebit.de. (2017). „Virtuelle Vielfalt: Das sind die Business Use-Cases für VR - Augmented \& Virtual Reality“. CEBIT. https://www.cebit.de/de/news-trends/news/virtuelle-vielfalt-das-sind-diebusiness-use-cases-fuer-vr-319. Zugegriffen: 12. Juni 2018.

Felber, P. (2018). „Die Migros-Tochter Interio rüstet sich mit einem neuen Ladenkonzept für die digitale Zukunft“. az Aargauer Zeitung. https://www.aargauerzeitung.ch/wirtschaft/diemigros-tochter-interio-ruestet-sich-mit-einem-neuen-ladenkonzept-fuer-die-digitale-zukunft-132983314. Zugegriffen: 4. Jan. 2019.

Fitzgerald, M., Kruschwitz, N., Bonnet, D., \& Welch, M. (2014). Embracing digital technology: A new strategic imperative. MIT Sloan Management Review, 55(2), 1.

Gerth, N. (2015). IT-Marketing: Produkte anders denken - Denn nichts ist, wie es scheint (2., aktualisierte, überarbeitete u. erweiterte Aufl.). Berlin: Springer Gabler.

Griese, K.-M., \& Bröring, S. (2011). Marketing-Grundlagen: Eine fallstudienbasierte Einführung (1. Aufl). Wiesbaden: Gabler (Lehrbuch).

Halbmayer, E., \& Salat, J. (2011). „Strukturierung qualitativer Interviews, Institut für Kultur- und Sozialanthropologie, Universität Wien“. Qualitative Methoden der Kultur- und Sozialanthropologie. http://www.univie.ac.at/ksa/elearning/cp/qualitative/qualitative-31.html. Zugegriffen: 15. Juni 2018.

Henneberg, S. (Hrsg.). (2017). Virtual reality (1. Aufl.). New York: Greenhaven Publishing (Opposing viewpoints).

Holland, H. (2018). „Definition: Customer experience management“. Gabler Wirtschaftslexikon Online. https://wirtschaftslexikon.gabler.de/definition/customer-experience-management-54478/version-277507. Zugegriffen: 14. Juni 2018.

Husic, S. (2018). „Die Realität ist nicht genug“. Creditreform. (Report Bewegbild), vernetzt Augmented Reality (06), 50.

Kirchgeorg, M. (2018). „Definition: Involvement“. Gabler Wirtschaftslexikon Online. https://wirtschaftslexikon.gabler.de/definition/involvement-39554. Zugegriffen: 16. Juni 2018.

Klaus, P. (2015). Measuring customer experience: How to develop and execute the most profitable customer experience strategies. New York: Palgrave Macmillan.

Kotler, P., Armstrong, G., Harris, L. C., et al. (2016). Grundlagen des Marketing (6., aktualisierte Aufl.). Hallbergmoos: Pearson Deutschland (wi - Wirtschaft).

KPMG. (2014). Digital transformation in switzerland. Report.

Lüft, A. L. (2018). „Diesmal gilt's“. Horizont (Report Bewegtbild), 12, 40.

Mehler-Bicher, A., \& Steiger, L. (2014). Augmented Reality: Theorie und Praxis (2., überarbeitete Aufl.). München: De Gruyter.

Milgram, P., Takemura, H., Utsumi, A., et al. (1994). „Augmented reality: A class of displays on the reality-virtuality continuum“. In H. Das (Hrsg.), (S. 282-292). https://doi.org/10.1117/12.197321.

Patel, N. (2014). ,The psychology of instant gratification and how it will revolutionize your marketing approach“. Entrepreneur. https://www.entrepreneur.com/article/235088. Zugegriffen: 16. Juni 2018. 
Peter, J. P., \& Olson, J. C. (2010). Consumer behavior \& marketing strategy (9. Aufl.). internat. ed. Boston: McGraw-Hill.

Peter, M. K. (2017). KMU-Transformation: Als KMU die Digitale Transformation erfolgreich umsetzen: Forschungsresultate und Praxisleitfaden. Olten: FHNW.

Pousttchi, K. (2017). „Digitale Transformation“. Enzyklopaedie der Wirtschaftsinformatik. http:// www.enzyklopaedie-der-wirtschaftsinformatik.de/lexikon/technologien-methoden/InformatikGrundlagen/digitalisierung/digitale-transformation. Zugegriffen: 1. Juni 2018.

PwC. (2017). Die deutsche Möbelbranche Struktur, Trends und Herausforderungen. (Branchenanalyse). Report.

Rode, J. (2017). „Künstliche Welten“. Lebensmittel Zeitung 52, 20.

Rutschmann, M., \& Belz, C. (2014). Reales Marketing: Kunden zum Kauf führen; Kaufprozesse, Kaufhandlung, Erfolg. Stuttgart: Schäffer-Poeschel.

Schulz, M. (2014). „Ethnografische Beobachtung“. In: A. Tillmann, S. Fleischer, \& K.-U. Hugger (Hrsg.), Handbuch Kinder und Medien (S. 225-235). Wiesbaden: Springer VS (Digitale Kultur und Kommunikation). https://doi.org/10.1007/978-3-531-18997-0_17.

Settele, C. (2018). „Google und Apple: Handys mit Augmented Reality“. NZZ am Sonntag. https:// nzzas.nzz.ch/wissen/google-und-apple-handys-mit-augmented-reality-ld.1444968. Zugegriffen: 27. Dez. 2018.

Wade, M. R. (2015). „Digital Business Transformation“. IMD business school. www.dbt/reports/ framework/. Zugegriffen: 3. Juni 2018.

Winters, Phil. (2016). Customer Strategy: Aus Kundensicht denken und handeln (2. Aufl.). Freiburg: Haufe Gruppe.

Zaharia, S. (2006): Multi-Channel-Retailing und Kundenverhalten: Wie sich Kunden informieren und wie sie einkaufen (1. Aufl.). Lohmar: Eul (Reihe: Kundenorientierte Unternehmensführung).

Aemmer, David (M.Sc. BA/david_aemmer@gmx.ch) Konsekutives Masterstudium in Betriebsökonomie (Vertiefung in Corporate/Business Development) an der Berner Fachhochschule. Langjährige Erfahrung im Qualitäts-, Prozess- und Projektmanagement. Aktuell bei PostNetz tätig als Projektleiter. Unter anderem Leiter des Großprojekts „Filiale der Zukunft“ und dabei verantwortlich für die Entwicklung sowie den Rollout des neuen Konzepts für die eigenbetriebenen Filialen der Post an über 300 Standorten.

Bigler, Jonas (M.Sc. BA/jonas.bigler@gmx.ch) Konsekutives Masterstudium in Betriebsökonomie (Vertiefung in Corporate / Business Development) an der Berner Fachhochschule mit Stipendium der Hirschmann Stiftung. Langjährige Erfahrung als Markt- und Produktmanager von digitalen und physischen Finanzdienstleistungen im B2B- und B2C-Bereich. Leidenschaftlich interessiert am Internet mit eigenen Projekten bezüglich eSports und Online-Kleinanzeigen.

Birkhofer, Marco (M.Sc. BA/marco.birkhofer@bluewin.ch) Masterstudium in Betriebswirtschaft an der Berner Fachhochschule mit Vertiefung Corporate and Business Development und davor Bachelorstudium in Business Administration mit Vertiefung Strategy ebenfalls an der Berner Fachhochschule. Derzeit als Berater Privat- und Geschäftskunden sowie stellvertretender Geschäftsstellenleiter bei der Valiant Bank AG beschäftigt.

Brechbühler Pešková, Marie (Prof. Dr./marie.brechbuehler@bfh.ch) ist in der Lehre und Forschung an der Berner Fachhochschule, Departement Wirtschaft, tätig. Sie unterrichtet insbesondere Module in den Bereichen „Nachhaltige Wirtschaft, Strategie und Innovation und Inter- 
national Business. Sie leitet der Forschungsschwerpunkt „Nachhaltigkeit“ und widmet sich in ihrer angewandten und praxisorientierten Forschungstätigkeit den Themen „Transformation zur nachhaltigen Gesellschaft und Wirtschaft“, „Nachhaltige Kreisläufe: Sustainable Consumption, Circular Economy, nachhaltige Geschäftsmodelle, Recycling“ sowie „Corporate Sustainability/ CSR/social Entrepreneurship“.

Harder, Deane (Prof. Dr./deane.harder@bfh.ch) Tätigkeit in Lehre und Forschung an der Berner Fachhochschule, Departement Wirtschaft. Schwerpunkte sind Innovation, Verhaltensökonomie und Systemik im Bereich Nachhaltigkeit. Vorher 10 Jahre Praxiserfahrung in der Unternehmensberatung und -entwicklung. Studium und Promotion in Biologie an den Universitäten Freiburg i. Br. (Deutschland) und Otago University (Neuseeland).

Open Access Dieses Kapitel wird unter der Creative Commons Namensnennung 4.0 International Lizenz (http://creativecommons.org/licenses/by/4.0/deed.de) veröffentlicht, welche die Nutzung, Vervielfältigung, Bearbeitung, Verbreitung und Wiedergabe in jeglichem Medium und Format erlaubt, sofern Sie den/die ursprünglichen Autor(en) und die Quelle ordnungsgemäß nennen, einen Link zur Creative Commons Lizenz beifügen und angeben, ob Änderungen vorgenommen wurden.

Die in diesem Kapitel enthaltenen Bilder und sonstiges Drittmaterial unterliegen ebenfalls der genannten Creative Commons Lizenz, sofern sich aus der Abbildungslegende nichts anderes ergibt. Sofern das betreffende Material nicht unter der genannten Creative Commons Lizenz steht und die betreffende Handlung nicht nach gesetzlichen Vorschriften erlaubt ist, ist für die oben aufgeführten Weiterverwendungen des Materials die Einwilligung des jeweiligen Rechteinhabers einzuholen.

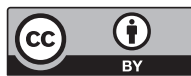




\title{
Online-Abonnement-Vermarktung
}

\section{Erfolgreicher Marketingmix für die Abonnement- Vermarktung von Konsumgütern}

\author{
Romain Descloux und Etienne Rumo
}

\begin{abstract}
Zusammenfassung
Die Online-Abonnement(Abo)-Vermarktung für Konsumgüter gilt als lukrativer Nischenmarkt im E-Commerce. Obwohl etablierte Firmen sowie Start-ups neue Konzepte erarbeiten, bleibt der Erfolg von Unternehmen eine Unbekannte. Davon ausgehend wurden im Rahmen des vorliegenden Beitrags die Schlüsselfaktoren des kuratierten Online-Abo-Marktes untersucht, die den Erfolg fördern. Bisher wurden keine branchenspezifischen Analysen durchgeführt, welche sich auf den Marketingmix im Online-Abo-Bereich beziehen. Aufgrund der identifizierten Lücken wird der Frage nachgegangen, wie Unternehmen anhand des Marketingmix-Ansatzes eine erfolgreiche Online-Abo-Vermarktung für Konsumgüter gestalten können. Im Mittelpunkt steht daher der kuratierte Online-Abomarkt. Im ersten Teil dieses Beitrages werden die Themen ,Subscription economy“ und der Marketingmix behandelt. Im zweiten Teil des Beitrages folgt eine qualitative Bestandsaufnahme von vier untersuchten Fallstudien. Anhand einer Cross-Case-Untersuchung mit Bezug auf prozentuale Umsatz- und Abonnentenentwicklungen werden Schlüsselfaktoren für eine erfolgreiche Abo-Vermarktung herauskristallisiert. Die Kombination von konzeptioneller und empirischer Analyse erlaubt es, wichtige Schlüsselfaktoren für eine erfolgreiche Online-Abo-Vermarktung aufzuzeigen, welche auch für andere Online-Abo-Anbieter relevant sein dürften.
\end{abstract}

R. Descloux $(\bowtie)$

Bern, Schweiz

E. Rumo

Berner Fachhochschule Wirtschaft, Bern, Schweiz

E-Mail: etienne.rumo@bfh.ch 


\subsection{Einleitung}

\subsubsection{Ausgangslage und Problemstellung}

Neue Technologien haben zu einer Veränderung des Konsumverhaltens geführt und bieten für Unternehmen neues Wachstumspotenzial im Onlinebereich. Im Moment ist ein bekanntes Geschäftsmodell dabei, den Detailhandel zu verändern, denn Abonnements erleben eine Wiedergeburt im E-Commerce (Rudolph et al. 2017, S. 889). In den USA hat dieses Phänomen bereits einen Namen: Es wird von „Subscription Economy“ gesprochen (Maier 2015). Diese Abo-Geschäftsmodelle heben sich vom konventionellen E-Commerce ab und gelten als lukrativer Nischenmarkt (Elaguizy 2014). Vor kurzem sind diese Geschäftsmodelle auch auf dem Markt für materielle Güter angekommen. Der Erfolg ist in den USA, im Vereinigten Königreichen und in Australien bereits sichtbar. In Europa nimmt die Bedeutung dieses Themas stark zu (Connexity 2016, S. 4).

Der Abo-Trend, der von den Unternehmen wahrgenommen wird, führt in vielen Organisationen $\mathrm{zu}$ einem Umdenken. Etablierte Firmen sowie Start-ups explorieren neue Marketingkonzepte, um vom Abo-System zu profitieren (CB Insight 2016). Es ist nicht überraschend, dass das Abo-Business von Online-Marketing-Experten als ,the new black of internet marketing“ bezeichnet wird (Patel 2015). Mit der „Subscription Economy“ verschiebt sich der Fokus auf die Kundenbeziehung (Zuora 2017). Das Ziel ist, die Abonnenten möglichst lange zu binden, um wiederkehrende Umsätze sicherzustellen.

Inzwischen hat auch die Suche nach Erfolgsfaktoren begonnen, die entscheidend sind, um dieses vielversprechende Potenzial zu nutzen (Elaguizy 2014). Dementsprechend wurde der lukrative Markt von mehreren Autoren untersucht (Baxter 2015; Rudolph et al. 2017; Savage und Hanser 2016; Woo und Ramkumar 2018). Die Forschung hat den Fokus bis jetzt jedoch hauptsächlich auf Abo-Geschäftsmodelle gelegt, die intangible Dienstleistungen wie Handy- und Fernsehabos betreffen. Abo-Dienste für materielle Güter wurden in akademischen Studien dagegen bisher kaum untersucht. Dies eröffnet ein spannendes Forschungsfeld. Der vorliegende Beitrag soll aufdecken, wie anhand des Marketingmix-Ansatzes ein solches Geschäftsmodell trotz der Komplexität der verschiedenen Branchen und Produktkategorien aufgebaut werden kann, wobei den Besonderheiten des Online-Konsumgütermarktes Rechnung getragen wird.

\subsubsection{Ziele}

Dieser Artikel beschäftigt sich mit der Vermarktung von kuratierten Online-Abos für Konsumgüter. Dieser Markt wächst schnell und verspricht lukrative Ertragsaussichten. Doch warum sind manche Unternehmen mit ihrem Abo-System erfolgreich und andere nicht? Für materielle Güter besteht bei dieser Frage eine Forschungslücke (Rudolph et al. 2017, S. 890). Ziel ist es, anhand von Case Studies herauszufinden, welche Erfolgsfaktoren im Marketingmix relevant für die erfolgreiche Online-Abo-Vermarktung sind. 
Für die Untersuchung wurden vier heterogene KMU ausgewählt, die Online-Abos für Kosmetik, Tiernahrung und Lebensmittel vermarkten. Alle vier Firmen bieten OnlineAbos an, die sich dem kuratierten Modell annähern. Diese Organisationen werden anhand der sieben klassischen Instrumente des Marketingmixes untersucht und verglichen. Bei der empirischen Untersuchung wurde bewusst auf möglichst viele Gemeinsamkeiten geachtet, sodass die Erkenntnisse auf unterschiedliche Branchen zutreffen und dort eine erfolgreiche Marketinggestaltung unterstützen können.

\subsection{Theoretische Grundlagen}

\subsubsection{Subscription Economy}

Die Digitalisierung hat frischen Wind in das Abo-Geschäftsmodell gebracht: Zuerst war dies bei digitalen Produkten wie Handys zu beobachten und inzwischen insbesondere bei Multimedia-Streaming-Dienstleistungen wie Netflix und Spotify. Das Abomodell ist kein vorübergehender Trend. Die Prognose für das Streaming-Portal Netflix geht bis zum Jahr 2020 von einem immensen Sprung von derzeit rund 500.000 auf über 11 Mio. Abonnenten aus (Maier 2015). Vor kurzem ist dieses Geschäftsmodell auch auf dem Markt für materielle Güter angekommen. „Birchbox“, tätig auf dem Abomarkt für Kosmetik, hat diesen Wandel im Jahr 2010 initiiert (Schifrin 2016, S. 1). Das Unternehmen begann zunächst nur mit Geschenk-Abos für Make-up, aber bis heute hat sich das Angebot stark weiterentwickelt (Saffron 2017). „CrateJoy“, der weltweit erste Abo-Online-Marktplatz, bietet mehr als 1300 Abos von Büchern bis zu Lebensmitteln (CrateJoy 2018) an. Darunter sind Abos für Konsumgüter wie Kosmetik oder Lebensmittel, die sehr geschätzt sind (Elaguizy 2014).

Abos erleben somit eine Wiedergeburt im E-Commerce (Rudolph et al. 2017, S. 889). In den USA hat dieses Phänomen bereits einen Namen und es ist von einer ,Subscription Economy“ die Rede (Maier 2015). Die Anzahl an Besuchern auf der Webseite von Abobox-Anbietern ist in den USA zwischen 2013 und 2016 um circa $3000 \%$ gestiegen (Fetto 2016, S. 3). Im Vergleich dazu sind die Besuche auf Online-Retail-Webseiten nur um $168 \%$ gestiegen (Fetto 2016, S. 3). Dieses Wachstum zeigt auf, wie sich das Konsumverhalten durch dieses neue digitalisierte Angebot verändert und $\mathrm{zu}$ einem Umdenken in der Kundenbeziehung führt. Vor ein paar Jahren waren die obersten Ziele die Kundenakquirierung und der Versand von Gütern. Mit der „Subscription Economy“ liegt der Fokus nun auf der Kundenbeziehung (Zuora 2017).

\subsubsection{Begriffsdefinition}

Abonnements sind definiert als „eine Vereinbarung zwischen Verbraucher und Unternehmen über wiederkehrende Käufe eines Produkts oder einer Dienstleistung. Sie zielen darauf $\mathrm{ab}$, die technologischen Möglichkeiten des eCommerce zu nutzen, um 
vorhersehbarere und kostengünstigere Modelle zur Generierung von Kundennutzen zu ermöglichen“ (Rudolph et al. 2017, zit. n. Baxter 2015 S. 890). Das Streben nach langfristigen Kundenbeziehungen und wiederkehrenden Umsätzen verlangt nach neuen Geschäftsabläufen. Online-Abo-Anbieter brauchen einen neuen Ansatz zur Pflege von Abobeziehungen. Nach dem Verkaufsabschluss spielt das Marketing eine bedeutende Rolle. Der Kunde muss überzeugt sein, dass das Abo seinen Wert hat. Kreative Vermarkter sollen Mehrwert durch Inhalte, Community und zusätzliche Services schaffen (Janzer 2015).

\subsubsection{Abomodelle}

Der Online-Abomarkt entwickelt sich ständig weiter. Jedes Jahr kommen etwa 100 neue Unternehmen auf den Markt. Diese Dynamik erschwert die Einordnung der existierenden Geschäftsmodelle (Woo und Ramkumar 2018, S. 121). Forschende der Universität St-Gallen haben eine Systematik zur Klassifizierung von Abomodellen erstellt. Dank des wissenschaftlichen Hintergrunds gilt diese Zuordnung als Grundlage für die Klassifizierung der Unternehmen, die im Rahmen dieses Artikels empirisch analysiert werden. Laut ihrer Studie können Abos in drei Gruppen gegliedert werden, wobei vordefinierte, kuratierte und überraschende Abos unterschieden werden (Rudolph et al. 2017, S. 891). Die Hauptmerkmale jedes Archetyps werden im Folgenden näher erläutert.

Vordefinierte Abos bieten dem Verbraucher alltägliche Produkte wie Rasierapparate und Lebensmittel. Für den Konsumenten ist dies bequem, da vordefinierte Abos ihre regelmäßigen Bedürfnisse erfüllen. Das Angebot ist besonders für Personen interessant, die wenig Zeit zum Einkaufen haben. Bei solch einem Modell spielt die Flexibilität eine wichtige Rolle: Anbieter sollten den Verbrauchern die Kontrolle geben, ihr Abo hinsichtlich Lieferfrequenz und Versandmenge jederzeit anpassen zu können. Dieses Modell ähnelt dem „Replenishment“-Modell bzw. dem „Regular-supplies“-Modell.

Überraschende Abos ermöglichen im Gegensatz dazu den Zugang zu Inspiration und neuesten Entwicklungen innerhalb einer Produktkategorie, ohne dass dafür der Konsument aktiv einbezogen werden muss. Statt Bequemlichkeit ist der Treiber für überraschende Abos das Erlebnis, Produkte zu entdecken. Inspiration ist sehr wichtig. Da dieses Modell keinen besonderen Bedarf erfüllt, existiert kein Sättigungspunkt, außer wenn neue Lebensprioritäten wie finanzielle Restriktionen auftauchen.

Kuratierte Abos stellen einen Kompromiss zwischen den beiden anderen Abomodellen dar. Kuratierte Händler bieten Verbrauchern eine maßgeschneiderte Einkaufsberatung, meist in Form von Einkaufsassistenten, die regelmäßig Produkte nach persönlichen Vorlieben versenden. Die Komplexität wird reduziert, da die Auswahl durch interne Experten geschieht. Bequemlichkeit und Inspiration sind hier gleich wichtig. Abb. 14.1 fasst die vorgeschlagene Taxonomie der Abomodelle zusammen. 


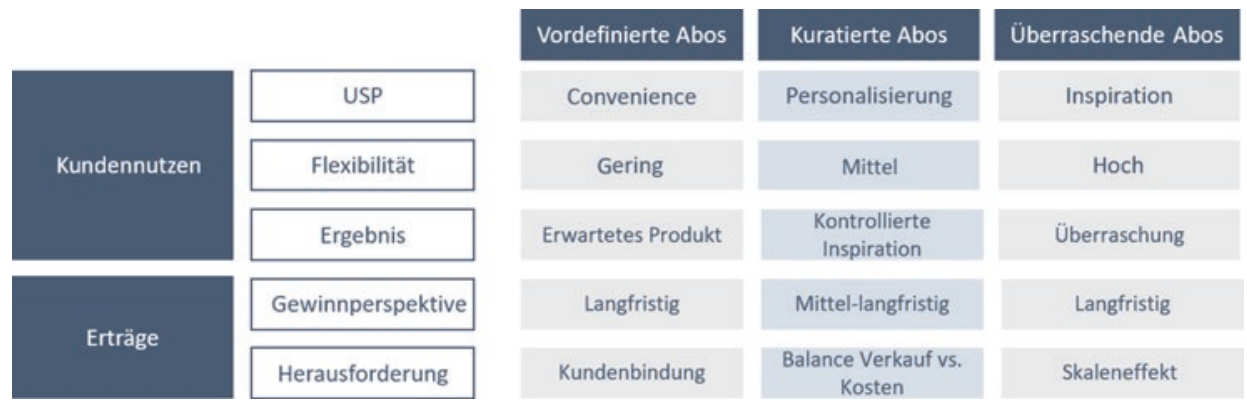

Abb. 14.1 Die drei Abomodelle. (Eigene Darstellung in Anlehnung an Rudolph et al. 2017, S. 892)

\subsubsection{Herausforderungen der Abomodelle in Bezug auf das Marketing}

Der Online-Abomarkt umfasst derzeit ungefähr 2000 verschiedene Anbieter und deckt fast jede Produktkategorie ab (DesMarais 2016). Die Branche wächst schnell und man kann geradezu zusehen, wie stetig neue Abo-Geschäftsmodelle hinzukommen (Fetto 2016). Eine ähnliche Entwicklung wird auch auf dem Abomarkt für materielle Güter beobachtet. Insgesamt 57 Unternehmen, die nur Konsumgüter anbieten, haben dazu 1,4 Mrd. US\$ in Risikokapital gesammelt (CB Insight 2016). Die Abomarktstruktur in den USA, in Großbritannien und in Australien setzt sich dabei wie folgt zusammen: Die Lebensmittel-, Tiernahrungs- und Pflegebranchen stellen $50 \%$ des Online-Aboangebotes dar, was auch die Relevanz der Beispielunternehmen des vorliegenden Beitrags verstärkt (Fetto 2016, S. 5). Das Wachstum dieses Geschäftsbereiches ist erfolgsversprechend. Jedoch haben die Verbraucher keine inhärente Vorliebe für Abos. Sie suchen nicht nach tollen Abos, sondern nach tollen Erlebnissen.

Die Überzeugungsarbeit, ein Abo anzumelden, fortzuführen stellt eine Herausforderung dar. Die Erkenntnisse bedeutender Studien und Artikel werden hier nach den 4Ps gegliedert und wiedergegeben. Dies kann dabei helfen, theoretische Lösungswege bei der Gestaltung des Marketingmixes zu finden.

In Bezug auf Promotion gelten positive Online-Rezensionen und Mundpropaganda als wichtiger Auslöser für Verbraucher, sich bei einem Aboservice anzumelden. Abonnent zu werden, bedeutet manchmal, Teil einer Gruppe oder Mitglied einer Gemeinschaft zu werden. Dadurch entsteht eine sehr starke Beziehung zwischen dem Kunden und dem Produkt (Baxter 2015, S. 12 ff.). „Etwas Neues auszuprobieren“ war dabei besonders relevant für kuratierte und überraschende Abomodelle. Das Abo-Geschäftsmodell hat nicht nur zum Ziel, das Leben der Abonnenten leichter zu machen, sondern auch Inspiration zu schaffen (Rudolph et al. 2017, S. 892). Bei der Produktpolitik stellt die Abstimmung zwischen Angebot und Nachfrage eine zentrale Herausforderung dar. Die gelieferten Produkte sollen an die tatsächlichen Kundenbedürfnisse angepasst sein. Im Fall der Unzufriedenheit mit der Produktqualität oder einer allgemeinen schlechten 
Dienstleistung machen Konsumenten kaum Kompromisse. Sie kündigen, weil das Vertrauen verloren ist und das versprochene Erlebnis nicht ihren Erwartungen entspricht. Um das Abo fortzusetzen, erwarten die Verbraucher, dass personalisierte Abos im Verlaufe der Zeit immer individueller werden. Hier ist die Personalisierung ein Schlüsselfaktor zur Kundenbindung (Chen et al. 2018). Beim Preis sind Abos attraktiver für Konsumenten, die durch finanzielle Anreize wie Rabatte motiviert werden können oder eine starke Bindung zum Produkt zeigen. Die Geldeinsparung gilt zwar als Beweggrund, um einen Vertrag abzuschließen, ist die Einsparung jedoch nicht der wichtigste Punkt (Chen et al. 2018, S. 5 f.; ING 2018, S. 33). Die Preispolitik spielt hierbei eine ausschlaggebende Rolle.

Eine Studie von Mckinsey (2016) zeigt auch auf, dass Online-Abo-Anbieter Schwierigkeiten haben, Konsumenten zu gewinnen und zu binden. Nur $53 \%$ der Befragten kannten etwa die Top-Abo-Firmen. Zudem ist die Konversionsrate schwach. Nur $55 \%$ derjenigen, die ein Online-Abo in Betracht ziehen, melden sich schließlich auch an. Der Bekanntheitsgrad ist noch gering, was die Bedeutung einer maximierenden Marketingmixgestaltung noch verstärkt.

\subsubsection{Marketingmix}

Durch Marketing kann ein Unternehmen wirtschaftlichen Erfolg erreichen. Dies erfolgt durch den Einsatz geeigneter Maßnahmen, um Kunden zu gewinnen und zu binden. Die Grundidee ist, die Bedürfnisse der Konsumenten zu identifizieren und diese durch das jeweilige Angebot auf den Zielmärkten zu erfüllen (Kotler et al. 2016, S. 39). Die Kundenakquirierung und die Kundenbindung gelten als Kernaufgabe des Marketings (Kühn 2007, S. 9).

Konkret geht es darum, Maßnahmen zur Gestaltung von Produkten und Dienstleistungen, zur Distribution sowie zur Preis- und Kommunikationspolitik zu bestimmen. Die Gesamtheit dieser Aktivitäten zum Verkauf eines Produktes oder Sortimentes wird als Marketingmix bezeichnet (Kühn 2007, S. 9).

\section{Instrumente des Marketingmixes}

Die Marketingmaßnahmen sollten harmonisch gestaltet werden und als Gesamtheit wirken, um den angestrebten Verkaufserfolg zu erreichen (Kühn 2007, S. 15). Der Kunde soll überzeugt sein, dass sein Wertgewinn tatsächlich höher ist als das, was er ausgegeben hat. Wenn die Kundenerwartungen übertroffen werden, lässt sich der Konsument langfristig binden (Fueglistaller et al. 2016, S. 202). Das wohl bekannteste Modell des Marketingmixes stammt von McCarthy (1964) und unterscheidet vier Hauptelemente, die sogenannten 4Ps „Product“, „Price“, „Promotion“ und „Place“ (McCarthy 1964).

Die Bedeutung der ,intern unterstützenden“ Marketing-Führungsinstrumente, wie z. B Marketingorganisation und Marketingplanung, bildet eine Voraussetzung für den Erfolg. In den späten 1970er-Jahren hat die Anerkennung der Marketinginfrastruktur dazu 
geführt, das Modell des Marketingmixes zu aktualisieren. Daraus sind die sogenannten 7Ps entstanden, nachdem das klassische Modell um drei neue Elemente erweitert wurde, nämlich „People“, „Processes“ und „Physical facilities“. Die 7Ps werden seit den 1980er-Jahren angewandt und gelten heute noch als wichtiges Mittel, um den Marketingmix zu gestalten.

\subsubsection{Erfolgsfaktoren für die Gestaltung des Marketingmixes}

Die Gestaltung eines erfolgreichen Marketings ist eine große Herausforderung. Dies lässt sich durch die Anzahl von Einzelmaßnahmen erklären, die im Rahmen eines Marketingmixes zusammenwirken und komplexen externen Umweltfaktoren (Wettbewerber, Konsumwandel, etc.) angepasst werden müssen. Die Erfüllung von drei Forderungen kann die Gestaltung des Marketingmix positiv beeinflussen (Kühn 2007, S. 15 ff.). Erstens ist eine harmonische Gestaltung der extern wirksamen Marketingmaßnahmen notwendig (Kühn 2007, S. 15; Homburg 2017, S. 928 ff.), zweitens eine situative Ausgestaltung der Elemente des Marketingmixes (Homburg 2017, S. 200; Kühn 2007, S. 15) und drittens ist die Intensität der Marketingmixinstrumente zu beachten (Bruhn 2016, S. 220).

Für die Gestaltung des Marketingmixes müssen also klare Prioritäten gesetzt werden. Es lohnt sich z. B., sich auf bestimmte Einsatzgebiete (Märkte, Marktsegment) und auch Einsatzzeiten zu konzentrieren (Kotler et al. 2016, S. 353 f.).

\subsubsection{Marketingsituationsanalyse}

Im Laufe der Zeit haben sich in der Forschung Ansätze eines „,modernen“ Marketings durchgesetzt. In der vorliegenden Arbeit liegt der Fokus diesbezüglich auf dem systemorientierten Ansatz, der die Erfassung und Beschreibungen komplexer Beziehungssysteme ermöglicht (Meffert et al. 2015, S. 36 f.). Von Marketingverantwortlichen, die die Marketingmixmethode anwenden, wird erwartet, dass sie spezifische Merkmale der zu bewältigenden Problemsituation wie Besonderheiten des Marktes oder der Zielgruppen in Rechnung stellen (Kühn und Pfäffli 2007; Meffert et al. 2015). Die Erarbeitung eines Marketingmixkonzeptes verlangt somit verschiedene strategische Grundsatzentscheidungen. Um sich für die optimale Lösung zu entscheiden und die Qualität des Marketingmixkonzeptes zu optimieren, ist die Erhebung, Analyse und Gewichtung der Ausgangsdaten von ausschlagender Bedeutung. Zur Marketingsituationsanalyse gehören dabei die Markt- und die Angebotsanalyse, welche die Basis für die Präzisierung der Problemsicht bilden (Kühn 2007, S. 25). Ziel ist es, relevante Einflussfaktoren herauszuarbeiten (Bruhn 2016, S. 25).

\section{Marktanalyse}

Die Marktanalyse ist eine übersichtliche Systemdarstellung, die die wichtigsten Markteilnehmer und Marktzusammenhänge aufzeigt. Als Hilfsmittel wird das „Marktgesicht“ empfohlen (Kühn 2007, S. 29). 
Die Gliederung des Marktes in Segmente und Teilmärkte ermöglicht eine differenzierte Erfassung der Marktgegebenheit. Damit kann den heterogenen Wünschen des Marktsegments durch differenzierte Marktleistungen entsprochen werden (Meffert et al. 2015, S. 174). Dank ihrer strategischen Bedeutung werden Teilmärkte ausgewählt, die als Ansatzpunkte für eine Angebotsspezialisierung dienen und sich durch eine besondere Wettbewerbssituation auszeichnen. So lässt sich zum Beispiel der Markt für kuratierte Online-Konsumgüterabos aktuell untergliedern in Lebensmittel, Tiernahrung und Kosmetik. Wenn zur Bestimmung der Marktsegmente nur wenige Informationen zur Verfügung stehen, müssen subjektive Kenntnisse und Erfahrungen externer Marktkenner genutzt werden, damit eine grobe Vorstellung entwickelt werden kann. Diese subjektive Marktinterpretation bringt jedoch viele Hindernisse mit sich (Meffert et al. 2015, S. 179)

\section{Marktspezifische Erfolgsfaktoren}

Hofer und Schendel (1978) sprechen von sogenannten „Schlüssel-Erfolgsfaktoren“ und meinen damit insbesondere auch Marketingmaßnahmen, die für die Erreichung einer vorteilhaften Marktposition sorgen und somit für den Erfolg entscheidend sind (Hofer und Schendel, 1978, S. 77 ff.). Diese marktspezifischen Faktoren sind von Markt zu Markt unterschiedlich und gelten als Ausgangpunkt, um Stärken und Schwächen beurteilen und die wichtigsten Marketinginstrumente für den Absatzerfolg besser steuern zu können. Die zentrale Aufgabe der Marketingsituationsanalyse ist die Bestimmung dieser Erfolgsfaktoren. Hierzu hat Kuhn das sogenannte Dominanz-Standard-Modell erarbeitet, welches vier Kategorien von Faktoren unterscheidet (Kühn, 2007, S. 45 ff.):

- Dominierende Faktoren sind wichtig für die Differenzierung gegenüber der Konkurrenz und den Aufbau von strategischen Wettbewerbsvorteilen.

- Standardfaktoren haben sich durch Anpassung (Imitation der Konkurrenz) oder durch technische Standards herausgebildet. Aus Sicht der Kunden müssen diese Elemente vorhanden sein.

- Komplementäre Faktoren sind eher als Unterstützung der dominierenden Faktoren zu betrachten.

- Marginale Faktoren umfassen jene Marketinginstrumente, die in einem bestimmten Markt nicht angewendet werden.

Die Abb. 14.2 zeigt das Dominanz-Standard-Modell auf.

Die zwei ersten Kategorien sind für den Absatzerfolg oder Misserfolg ausschlaggebend und stellen demgemäß Erfolgsfaktoren dar. Die komplementären und marginalen Faktoren spielen dagegen nur eine sekundäre Rolle.

Als Faktoren im Sinne des Dominanz-Standard-Modells sind sowohl die Instrumente des Marketingmixes wie Produktgestaltung als auch die Instrumentendimensionen (,Submixe“) wie etwa Qualität anzusehen. Grundlage für die Anwendung des Dominanz-Standard-Modells sind einerseits Informationen über Bedürfnisse und Kaufkriterien der Konsumenten und andererseits die Ergebnisse der Marktanalyse. 


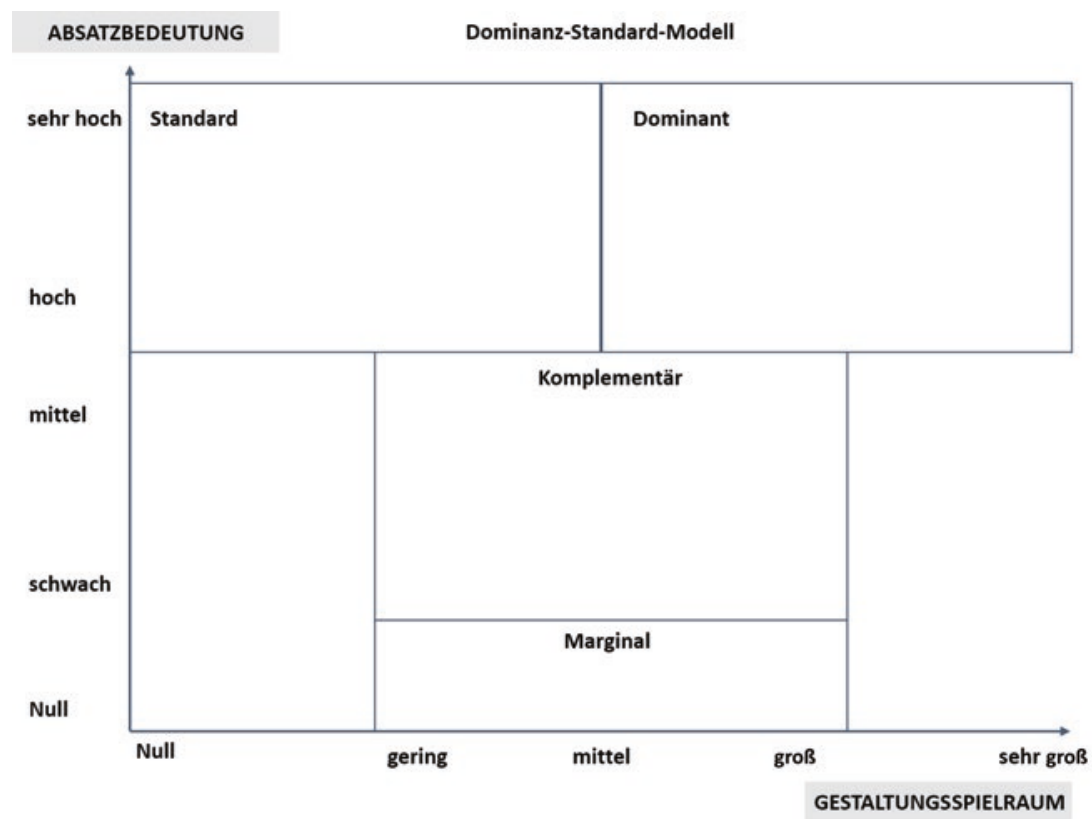

Abb. 14.2 Dominanz-Standard-Modell. (Eigene Darstellung in Anlehnung an Kühn 2007, S. 47)

Die Beurteilung soll immer aus der Marktsicht erfolgen. Dementsprechend gilt das Resultat der Erfolgsfaktorenanalyse für die aktuelle Marktsituation und alle beteiligten Anbieter. Welche Erfolgsfaktoren die betrachteten Unternehmen nutzen können, hängt von den verfügbaren Ressourcen und der strategischen Ausrichtungen ab (Kühn 2007, S. 47).

\subsubsection{Zusammenfassung - Marketingmix und Subscription Economy}

Die neuen Technologien haben den Alltag der Menschen verändert. Sie sind untereinander vernetzt, sie interagieren und ihre Wertvorstellungen wandeln sich. Die Art, ein Produkt zu verkaufen, fordert neue Ansätze und Firmen suchen immer mehr nach Innovationen, um den Kunden einen Mehrwert zu schaffen (Bruhn 2016; Homburg 2017; Kotler et al. 2017; Kühn und Pfäffli 2007). Die Literatur weist auf einige interessante Anhaltspunkte hin, die zu einer erfolgreichen Gestaltung des Marketingmixes beitragen könnten. Im Rahmen einer Online-Abo-Vermarktung von Konsumgütern könnten Kundenorientierung (Meffert et al. 2015, S. 855), Erlebnisse (Bruhn 2016, S. 41) und Engagement (Bruhn 2016, S. 60; Meffert et al. 2015, S. 855) als theoretische Stoßrichtungen besonders spannend sein. Ob sich diese zentrale Beobachtung auch in der Marketingmixgestaltung eines Abo-Unternehmens widerspiegelt, wird im empirischen Teil dieses Beitrages genauer untersucht. 


\subsection{Methodisches Vorgehen}

Der empirische Teil dieser Arbeit greift auf ein selbst erarbeitetes Modell zurück, das hauptsächlich den klassischen 7P-Ansatz berücksichtigt. Allerdings werden drei zusätzliche Hauptkomponenten ergänzt, um eine bestmögliche Angebotsanalyse im digitalen Umfeld durchführen zu können. Um aussagekräftige Erkenntnisse erlangen zu können, werden als „Key Performance Indicators“ die Entwicklung der Anzahl der Abos, die Umsatzentwicklung und die Marktanteilentwicklung betrachtet. Die Gliederung des Modells ist Abb. 14.3 zu entnehmen.

Das Ziel, Schlüsselfaktoren für die Vermarktung von Online-Abos zu eruieren, wurde aufgrund der gewonnenen Erkenntnisse der Literaturrecherche definiert. Die sichtbar gewordene Forschungslücke dient als Grundlage der Leitfadenkonzeption für die Interviews.

Um die Forschungsfrage beantworten $\mathrm{zu}$ können, wurde als zielführendes Forschungsdesign eine Multiple Case Study gewählt (Yin 2014). Die Erhebungsmethode

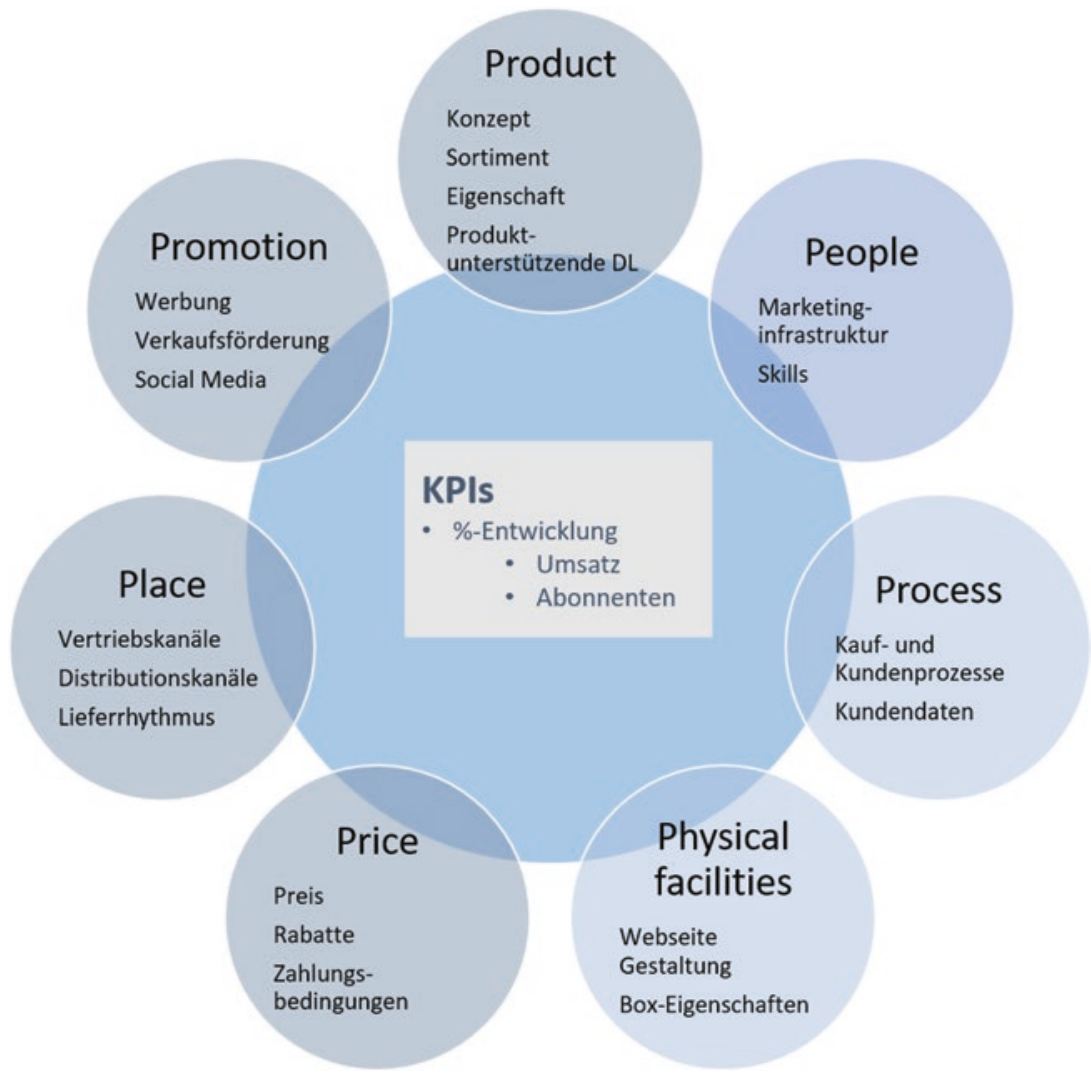

Abb. 14.3 7Ps - Modell für die Empirie. (Eigene Darstellung) 


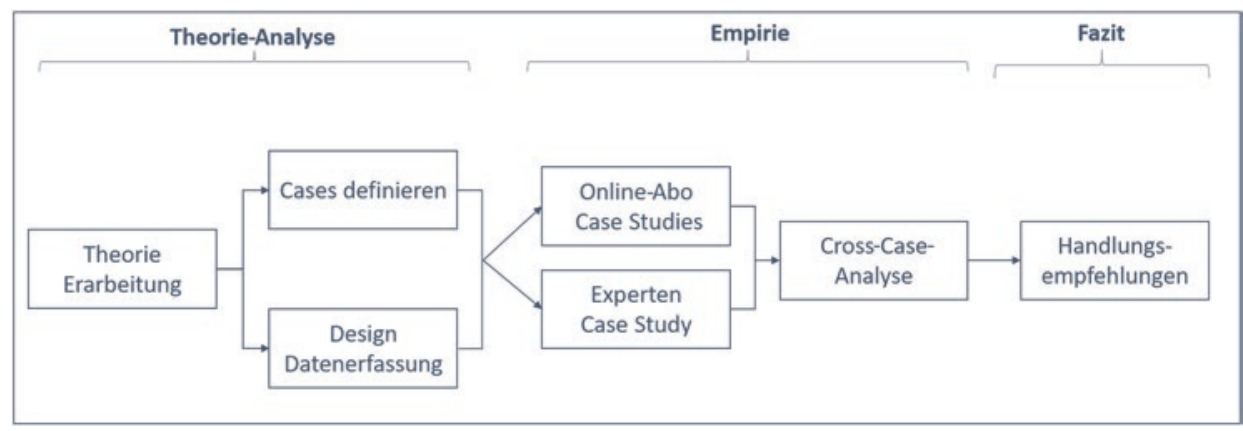

Abb. 14.4 Multiple-Case-Study-Prozess. (Eigene Darstellung in Anlehnung an Yin 2014, S. 60)

ist in Abb. 14.4 genauer dargestellt. Die Suche nach Unternehmen wurde auf Deutsch, Französisch und Englisch getätigt. Nach 26 Anfragen für ein Interview waren jedoch nur vier Ansprechpartner bereit, an der Studie teilzunehmen. Aus diesem Grund ist das Sampling sehr unterschiedlich, was die Suche nach Schlüsselfaktoren erschwert.

Es wurden vier Online-Abo-Unternehmen aus den Branchen Kosmetik, Tiernahrung und Lebensmittel selektiert. Aufgrund des Einflusses von Abomodellen auf das Wertangebot, versuchte der Autor mit Anbietern des gleichen Abotyps Kontakt aufzunehmen. Alle vier Firmen bieten Online-Abos an, die sich am kuratierten Modell orientieren. Dies ermöglicht einen besseren Vergleich. Bei der Empirie wurde bewusst auf möglichst viele Gemeinsamkeiten geachtet, sodass die Reichweite der Erkenntnisse es ermöglicht, unterschiedliche Branchen bei einer erfolgreichen Marketinggestaltung zu unterstützen.

Anhand der Case-Study-Analyse wurde nach Gemeinsamkeiten gesucht. Im Mai 2018 wurden dazu zwei Interviews auf Englisch und vier auf Deutsch durchgeführt. Die Datenanalyse nutzte drei verschiedene Auswertungsmethoden. Erstens wurden die vier Unternehmensbefragungen einzeln ausgewertet. Zweitens wurden die Experteninterviews separat untersucht. Zwei Experteninterviews dienten dabei als Bestätigung der bisherigen Interviewergebnisse. Zuletzt wurden die Unternehmen in „Under-“ und „Overperformer“ unterteilt.

\subsubsection{Datenauswertung}

Die Festlegung von Gütekriterien stellt die Qualität der Forschung sicher. Bei den qualitativen Gütekriterien orientiert sich die Arbeit an Gurtner und Clerc (2016) sowie Noppeney (2016), die Reliabilität, Validität und Glaubwürdigkeit fordern. Eine Forschungsarbeit ist reliabel, wenn ersichtlich ist, wo die Datenerhebung endet und wo die Interpretation der Forschenden beginnt. Zudem soll die Reliabilität die Vergleichbarkeit der Vorgehensweise der verschiedenen Beteiligten (Interviewende, Transkribierende, Auswertende) verbessern (Gurtner und Clerc 2016; Noppeney 2016). Dementsprechend 
wurde die prozedurale Reliabilität durch das gezielte Schaffen eines stabilen und konsistenten Vorgehens sichergestellt. Zur Sicherung der Stabilität wurden die Erhebungen zu unterschiedlichen Zeitpunkten und an unterschiedlichen Orten durchgeführt. Die Konsistenz ist durch die Verwendung eines Leitfadens gewährleistet, welcher im Vorfeld mit einem Pre-Test überprüft wurde (Clerc 2017).

\subsubsection{Qualitative Inhaltsanalyse}

Alle sechs Befragungen im Rahmen der Unternehmens- und Experteninterviews wurden entweder auf Deutsch oder auf Englisch durchgeführt und anschließend transkribiert. Zur weiteren Verarbeitung der Daten wurden die Interviews aufgenommen, wozu die Probanden vor dem Interview ihre Erlaubnis gaben. Die Interviews erfolgten telefonisch. Wichtig war, dass alle Interviewenden gleich vorgingen und die Interviewpartner in einem möglichst ähnlichen Setting befragten. Der Interviewleitfaden enthielt daher kommunikative Validierungen. Die sechs Befragungen dauerten zwischen 50 und $100 \mathrm{~min}$.

Bei der Datenanalyse werden die vorher erhobenen und erfassten Daten analysiert. Bei einem qualitativen Forschungsansatz ist dabei auch eine qualitative Analyse erforderlich. Diese kann zum einen der Interpretation, zum anderen aber auch als Grundlage für weitere Datenerhebungen dienen (Flick 2007). Das Ziel ist, die wesentlichen Inhalte beizubehalten und ein Gerüst zu schaffen, welches ein Abbild des Grundmaterials ist (Mayring 2016). Die Datenauswertung erfolgte durch Excel. Um die Aussagen aus den Interviews effizient analysieren zu können, wurden für diese Arbeit selektive Protokolle eingesetzt (Bortz und Döring 2006, S. 330).

Die Ergebnisse der Primärforschung, also der Unternehmens- und Experteninterviews wurden um Daten der Sekundärforschung, also des „Desk Research“ zu Webseiten und sozialen Netzwerken, ergänzt. Die Sammlung von Informationen aus Webseiten und sozialen Medien diente also zur Ergänzung der Primärforschung. Die Resultate des „Desk Research“ können als Grundlage für zukünftige Marketingmaßnahmen dienen (Kuss und Eisend 2010, S. 2 f.). Anhand der erhobenen Daten aus den Firmen- und Experteninterviews wurden in der Cross-Case-Analyse schließlich Under- und Overperformer klassifiziert und die Informationen entsprechend neu ausgewertet. Durch den Vergleich und die Interpretation der Excel-Auswertung soll die Cross-Analyse ermöglichen, im Rahmen der Multiple-Case-Studie Schlüsselfaktoren herauszuarbeiten.

\subsubsection{Präsentation der Stichprobe}

Die Stichprobe besteht aus vier Firmen, die kuratierte Abomodelle vermarkten. Die vier Geschäftsmodelle werden in diesem Kapitel kurz vorgestellt.

\section{ANiFit}

Seit 2001 verkauft ANiFit natürliche und gesunde Tiernahrung für Hunde und Katze. Die Produkte sind ausschließlich über Berater im Direktvertrieb erhältlich. Der direkte 
Kontakt mit dem Kunden via Vertrauensperson ist die Grundlage für den Erfolg dieses Geschäftsmodells. Um die Kundenbedürfnisse zu erfüllen, hat ANiFit in einen Onlineshop investiert. Seit einem Jahr bietet das Unternehmen die Möglichkeit, ein Abo abzuschließen.

\section{Kukimi.de}

Das 2014 gegründete Unternehmen Kukimi ist ein deutsches Start-up, das Fertiggerichte für den deutschen Markt liefert. Die Kukimi GmbH macht es möglich, sich mit minimalem Aufwand und ohne selbst zu kochen ausgewogen zu ernähren. Zielgruppe sind Menschen, die sich bewusst ernähren wollen, aber dafür wenig Zeit haben. Mehrere Investoren haben Kapital im Millionenbereich in das Unternehmen investiert, das beim „Accelerator“ von ProSiebenSat.1 an Bord war. Im Jahr 2016 ist jedoch eine Finanzierungsrunde geplatzt, was zur Insolvenz der Firma führte. Trotz dieser Schwierigkeiten wurde die Firma neu gegründet und neu gestartet, da weiterhin Potenzial gesehen wird.

\section{Pick N Dazzle}

Pick N Dazzle ist ein innovativer Player auf dem Markt für kuratierte Boxabos mit einzigartigem Konzept und Wettbewerbsvorteil. Das Start-up bietet die erste und einzige persönliche Beauty-Box mit Fullsize-Produkten an, die jeden Monat individuell für jeden Kunden kuratiert wird. Pick N Dazzle gewann 2015 in Großbritannien den „Pure-Beauty“-Award für den innovativsten Vertriebskanal.

\section{Früchtebox Express}

Die Früchtebox Express AG beschreibt sich als ,ein auf den Handel mit frischen Früchten spezialisiertes Logistik-Unternehmen“ (Fruechtebox 2018). Seit sieben Jahren bietet es kuratierte Online-Abos in der ganzen Schweiz und in Liechtenstein an. Die Gründer sahen ein Potenzial in berufstätigen Personen, die gesunde Pausen machen wollen. Aus diesem Grund hat die Firma das Angebot auf Unternehmen ausgerichtet, die für das Wohlbefinden der Mitarbeitenden sorgen wollen. Diese Geschäftsbeziehung ist dennoch als B2C einzuordnen, weil es sich um eine Gruppe von Konsumenten handelt, die im Büro bequem von leckeren Früchten profitieren wollen.

\subsection{Erkenntnisse der Cross-Case-Analyse}

\subsubsection{Angebotsanalyse - Einteilung in Under- und Overperformer}

Ziel der Cross-Analyse ist, die Unternehmen anhand der erzielten Umsatz- und Abonnentenentwicklung in Under- und Overperformer in Abb. 14.5 einzuteilen. Das Unternehmen ANiFit, welches die erwünschten Zahlen in Bezug auf das Aboangebot nicht übermitteln konnte, wurde für diese Analyse nicht beachtet. Insgesamt wurden 


\begin{tabular}{|l|l|l|l|}
\hline KPIs & Kukimi & Pick N Dazzle & Früchtebox \\
\hline \%-Umsatzentwicklung & $-30 \%$ (Fußnote) & $+20 \%$ & $30-40 \%$ \\
\hline \%-Abonnentenentwicklung & $+20 \%$ & $+10-15 \%$ & $20-25 \%$ \\
\hline Einteilung & Performer & Performer & Overperformer \\
\hline
\end{tabular}

Abb. 14.5 Einteilung Performer vs. Overperformer. (Eigene Darstellung)

somit drei Unternehmen verglichen. Da alle drei Unternehmen eine positive Entwicklung aufweisen, wird keine Firma als Underperformer beschrieben. Stattdessen wird im Laufe der Analyse von Performern und Overperformern gesprochen. Fruechtebox kann dabei als Overperformer charakterisiert werden. In der Analyse wird auf die relevanten Gemeinsamkeiten und Unterschiede der Stichprobe eingegangen, um branchenübergreifende Schlüsselfaktoren aufzeigen zu können. Die Cross-Analyse stützt sich auf die Interviews und auf die Ergebnisse der sekundären Erhebung.

\subsubsection{Vergleich zwischen Performern und Overperformern}

\section{Produkt}

Früchtebox hat ein klares Wertangebot. Das Unternehmen liefert saisonale heimische Früchte direkt ins Büro. Die Früchte sorgen für Vitamine und bringen Energie. Die Zielgruppe ist klar definiert und kann zwischen mehreren Boxen wählen. Die Früchteauswahl ermöglicht eine gewisse Abwechslung. Dadurch kann das Unternehmen den Konsumenten zudem ein wenig lenken. Die Auswahl lässt außerdem Spielraum für einen gewissen Grad an Personalisierung. Diese über die Zeit begrenzte Produktzusammenstellung ermöglicht es, den Kunden zu binden und durch persönlichen Kontakt Vertrauen zu schaffen. Dieser Aspekt ist ein USP für Früchtebox (Stahl 2018, Z411-414). Der erste persönliche Kontakt ist entscheidend, um Vertrauen zu generieren und eine Beziehung aufzubauen. Das Gespräch erfolgt schnell nach Bestelleingang und soll unkompliziert sein. Ziel ist es, den Kunden besser kennenzulernen, damit er das passende Obst erhält. Bei der Produktgestaltung steht Convenience im Vordergrund und gilt als starker Hebel für die Kundenbindung (Stahl 2018, Z176-183).

Besonders auffallend ist der Verzicht auf das Wort Abo und somit auf Verträge. Der Gründer hat dies im Interview sehr oft erwähnt. Die Firma spricht kaum von Abos, sondern betont den Aspekt ,,jederzeit künd- oder veränderbar“. Es ist die einzige Firma, die dieses Element so deutlich kommuniziert. Bei allen drei Unternehmen ist aber festzustellen, dass sie sich vom Begriff Abo distanzieren wollen. Kukimi spricht von „Programmen“ und Pick N Dazzle von einem „,curated beauty programm“. Das Wort Abo ist anscheinend negativ konnotiert und jedes Zwangsgefühl soll vermieden werden.

Im Gegensatz zu Früchtebox haben die beiden Performer-Unternehmen unterschiedliche Zielgruppen definiert. Die Organisationen befinden sich noch in der Entwicklungsphase und die Teilmärkte können noch verfeinert werden. Bei Pick N Dazzle 
scheint der persönliche Kontakt nicht von hoher Bedeutung. Die Firma vertraut der einzigartigen Empfehlungstechnologie. Die Algorithmen sollen für Kundenzufriedenheit sorgen. Es besteht jedoch die Gefahr, dass Fehler von den Konsumenten nicht leicht vergeben werden. Irren ist menschlich, aber wie sind Computerfehler zu beurteilen? Zusammenfassend ist festzustellen, dass sich drei branchenübergreifende Faktoren besonders abheben. Dabei geht es um Convenience als Grundlage der Produktpolitik, eine begrenzte personalisierte Produktzusammenstellung und persönliche produktunterstützende Dienstleistungen.

\section{Promotion}

Die Zusammenarbeit mit Google hat Früchtebox zum Durchbruch verholfen. Es wurde festgestellt, dass die Beteiligung von Google bei der Kampagnenaufstellung einen starken Einfluss auf den Erfolg hatte. Aus diesem Grund sollte nicht Google Adword für sich betrachtet werden, sondern das Gesamtpaket an Leistungen. Zum Beispiel hat Kukimi Google Adwords ausgeschaltet. Da Pick N Dazzle ab September ebenfalls von Google unterstützt wird, wird es spannend sein, zu beobachten, ob diese Marketingmaßnahme als branchenübergreifender Schlüsselfaktor betrachtet werden kann. Die marktspezifischen Erfolgsfaktoren - sprich vor allem Convenience und Flexibilität - sollten sich in der Kommunikation widerspiegeln. Diese Kernelemente deutlich zu kommunizieren, ist eine Herausforderung.

Als Overperformer macht Früchtebox dies besonders gut. Die Kommunikation begrenzt sich auf die Produkteigenschaften und den „Probier-mal“-Effekt. Alle Informationen und Botschaften sind auf der Webseite leicht zu finden und zu verstehen. Die Kommunikation von Pick N Dazzle auf der Webseite ist sehr umfangreich. Die qualitativen und tief greifenden Informationen können ein Vorteil sein (Marinova 2018, Z446-461). Sie können potenzielle Verbraucher aber auch überfordern. Die Webseite ist sehr interaktiv und fördert dadurch, dass der Besucher seine Daten eingibt. Als Kritikpunkt kann man sich fragen, ob diese Kommunikationsart das Zielpublikum, Frauen zwischen 35 und 55 Jahren, anspricht. Das Werteangebot von Kukimi wird nicht einheitlich vermarktet, was die Kunden verunsichern kann. Wichtige Informationen in Bezug auf Abos wie Zahlungsbedingungen oder Kündigungsfrist sind auf der Webseite nicht vorhanden. Dies kann die Kaufentscheidung negativ beeinflussen.

Jedes der Unternehmen betreibt Facebook-, Instagram- und Twitter-Konten. Eine Korrelation zwischen der Anzahl an Abonnenten auf Facebook und den Umsätzen konnte jedoch nicht belegt werden. Für die Kosmetikbranche ist diese Plattform besonders relevant. Onlinepräsenz gilt als Voraussetzung, um seine Produkte vorstellen zu können. Die Bedeutung der sozialen Medien wurde von alle Firmen betont, jedoch ist sie nicht entscheidend für eine erfolgreiche Vermarktung. Schließlich kann die Relevanz dreier Kommunikationsinstrumente betont werden. Dies sind der Verzicht auf den Begriff „Abo“, die Nutzung von Adwords, aber nur wenn Google eine gewisse Unterstützung bietet, und das Bestreben, „Convenience“ und „Flexibilität“" zu kommunizieren. 


\section{Place}

Die untersuchten Unternehmen vertreiben ihre Produkte alle vorwiegend über die Webseite. Die neuen Anforderungen der Konsumenten erfordern die direkte Lieferung von Produkten. Sie wollen die Boxen schnell und zu bestimmten Zeiten erhalten. Flexibilität spielt in der Distribution eine wichtige Rolle. Eine eigene Logistik zu haben, bietet Fruechtebox einen Wettbewerbsvorteil. $80 \%$ des Umsatzes erfolgt durch eigene Lieferung. Das Abomodell ermöglicht es, die Lagerhaltung zu beherrschen. Die Distribution ist heutzutage sogar so wichtig, dass Pick N Dazzle das ganzes Konzept darauf aufgebaut hat. Das Kosmetikunternehmen hofft, dass diese Effizienz für die Zukunft einen Vorteil bringt. Wegen der aktuellen Verkaufsmengen sind die Logistikkosten bei Kukimi hoch und verringern die Margen.

Vor einigen Jahren war die Suche nach Vertriebskanäle noch sehr wichtig. Mit dem digitalen Online-Zeitalter gewinnt nun die Logistik an Bedeutung. Es ist daher nicht überraschend, dass Fruechtebox sich als „spezialisiertes Logistik-Unternehmen“ beschreibt. Bei näherer Betrachtung der Distributionspolitik stellt man fest, dass dieser Element in Zukunft immer wichtiger werden wird. Als Schlüsselfaktor kann der Aufbau einer effizienten Supply Chain angesehen werden.

\section{Price}

Die Preisbestimmung erfolgt situativ je nach Markt und Produkt. Online-Abo-Konsumenten bezahlen gerne monatlich. Die Breite des Sortiments macht bei allen drei Unternehmen das Online-Aboangebot für jede potenzielle Kundengruppe zugänglich. Der Aspekt „Value for money“ ist für das kuratierte Abomodell aktuell auf jeden Fall noch relevant. Wie die Untersuchung aufgezeigt hat, verliert dieses Kriterium an Bedeutung, wenn die Produktzusammenstellung stimmt (Bishof 2018, Z453).

Interessant zu beobachten war, dass die Konsumenten noch immer die Bezahlung per Rechnung wünschen. Früchtebox bietet nur diese Möglichkeit. Kukimi hat sie sogar neu eingeführt, woraufhin die Verkaufszahlen stark gewachsen sind. Rabatte werden von allen Unternehmen genutzt. Bei Kukimi und Pick N Dazzle fungieren sie als finanzieller Anreiz, um die Kaufentscheidung zu beeinflussen. Früchtebox setzt entsprechende Instrumente ein, wenn ein Kunde bestimmte Mengen bezieht. Aber die Schweizer Firma arbeitet nicht proaktiv mit dieser Maßnahme. Der Einfluss der Preispolitik auf die Online-Abo-Vermarktung lässt sich insgesamt als nicht besonders stark einstufen. Natürlich sollen die Preise mit dem Angebot übereinstimmen, aber bestimmte Instrumente können nicht als Erfolgsfaktoren betrachtet werden.

\section{People}

Die Marketinginfrastruktur ist in den analysierten Firmen klein gehalten. Im Durchschnitt kümmern sich zwei Personen um die Marketingaktivitäten. Jedoch verfügen diese Firmen über eine sehr gute Fachkompetenz. Alle Interviewten haben eine wirtschaftliche Ausbildung absolviert. Daneben werden sie von Investoren oder von verschiedenen Anspruchsgruppen unterstützt. Die Firma Früchtebox hat von der Erfahrung von Herrn 
Liechti, Gründer des seit zwanzig Jahren erfolgreichen Online-Socken-Abos „Black Socks“, profitiert. Die Firmen sind einfach organisiert. Sie reagieren schnell und können sich rasch anpassen. Im Vergleich zu größeren Online-Abo-Unternehmen, die der Autor angesprochen hat, ist ihre Reaktionszeit sehr gut. Spezielle Erfolgsfaktoren ließen sich jedoch nicht identifizieren.

\section{Process}

Die Kundenbeziehung bei Früchtebox kann als unkompliziert bezeichnet werden. Sobald eine Bestellung eingegangen ist, nimmt ein Mitarbeiter so rasch wie möglich Kontakt mit den Konsumenten auf. Dies kann per Mail oder per Telefon geschehen. Die Firma geht so vor, weil die eingereichte Online-Bestellung nicht vollständig ist. Lieferorte oder Früchtevorlieben kann der Verbraucher nicht online eingegeben. Früchtebox nutzt diese Lücke bewusst, um die Ansprüche ihrer Kunden besser kennenzulernen. Einerseits hat sich dieses Vorgehen bewährt. Andererseits stellt sich die Frage, ob diese unvollständigen Informationen den potenziellen Kunden nicht verunsichern und vom Kauf abhalten. Für die Personen, die nur Obst im Allgemeinen wünschen, ist dies kein Problem. Wie Oliver Stahl es sagt, handelt es sich aber um ein emotionales Produkt. Die Vorlieben spielen bei der Kaufentscheidung daher eine wesentliche Rolle. Flexibilität bei der Auswahl ist im ersten Schritt jedoch nicht vorhanden. Erst wenn Früchtebox den Kunden zurückruft, nimmt der Kunde die ganze Personalisierung wahr. Die in den Gesprächen gesammelten Informationen haben nur den Zweck, den Service zu personalisieren, und dadurch die Kundenbindung zu stärken. Aus Sicht der hohen Kundenbindung bei Früchtebox kann davon ausgegangen werden, dass eine einfache Prozessgestaltung äußerst wichtig ist (Stahl 2018, Z180-183).

Bei Kukimi könnte der Prozess klarer strukturiert werden, vor allem was die Zahlungsbedingungen und Anpassungsmöglichkeiten angeht. Der Verkaufsprozess stützt sich auf das „Beauty“-Profil. Es fällt den Kunden leicht, die Informationen zu geben. Wenn alle Daten und Vorlieben eingegeben wurden, soll der Kunde jedoch bezahlen, ohne die Zusammenstellung zu sehen. Dieser letzte Schritt scheint nicht überzeugend. Es fehlt an Klarheit und sobald Fragen auftauchen, geht in der Regel das Convenience-Gefühl verloren.

Daten werden in Zukunft immer wichtiger. All drei Firmen sehen dies als Chance, um die Kundenbedürfnisse noch besser zu erfüllen. In der aktuellen Situation gilt dieses neue Marketinginstrument jedoch noch nicht als Erfolgsfaktor. Bei kuratierten Online-Aboangeboten sollte entlang des Prozesses immer Convenience das Leitmotiv sein. Darum spielt der Prozess eine entscheidende Rolle. Daraus ergibt sich als Erfolgsfaktor, dass auf die Gestaltung einfacher Kaufprozesse wert gelegt wird.

\section{Physical Facilities}

Die Früchtebox-Webseite ist zwar nicht die modernste und benötigt laut dem Gründer eine Optimierung. Während der Untersuchungen ist im Vergleich mit anderen OnlineAbo-Anbietern jedoch ein Element aufgefallen. Abgesehen von der Frage, welche 
Früchte der Kunde in seiner Box haben wird, ist die Webseite einfach verständlich. Innerhalb von wenigen Minuten ist der Verbraucher in der Lage zu verstehen, worum es geht und was er bestellen kann. Ungewissheit bezüglich der Auswahl ist durch die Aussage „Abo jederzeit künd- und veränderbar“ unmöglich.

Das Design der Webseiten und der Boxen stimmt überein. Alle Online-Abo-Anbieter bieten Boxen, die bequem zu nutzen sind und nach dem Gebrauch einfach entsorgt werden können. Das Convenience-Konzept ist hier bis zum Ende durchgedacht worden. Physical Facilities sind zwar für das Konsumerlebnis wichtig, können aber nicht als Erfolgsfaktoren bezeichnet werden. Sie gehören vielmehr zum Standard.

\subsection{Zusammenfassung der gewonnenen Erkenntnisse}

Wird der Erfolg von den untersuchten Unternehmen genauer angeschaut, fällt auf, dass Bequemlichkeit und Flexibilität im Zentrum der Marketingmixgestaltung stehen. Das Leben der Kunden soll vereinfacht werden und jede Marketingdimension wird genutzt, um dieses Convenience-Versprechen einzuhalten. Die Cross-Case-Analyse ermöglicht es dabei, folgende Schlüsselfaktoren zu benennen:

- Convenience des Produkts und Flexibilität in der Zusammenstellung

- Begrenzte personalisierte Produktauswahl

- Persönlicher Kundenkontakt

- Verzicht auf den Begriff Abo

- Werbung über Google Adwords (nur mit Unterstützung von Google)

- Klare Kommunikation von Convenience und Flexibilität

- Aufbau einer effizienten Logistik

- Gestaltung einfacher Kaufprozesse

Anhand der Marketingsituationsanalyse können die wichtigsten Marketinginstrumente in Abb. 14.6 verortet werden.

\subsection{Reflexion und Ausblick}

Die Wissenschaftler erkennen die Online-Abo-Vermarktung als spannendes neues Forschungsfeld an. Bis heute sind kaum Informationen über branchenspezifische Marktstudien vorhanden. Die Aufbereitung der theoretischen Grundlagen zeigt auf, dass ein Unternehmen durch den Marketingmix einen wirtschaftlichen Erfolg erreichen kann (Kotler et al. 2016, S. 39).

Der theoretischen Analyse kann entnommen werden, dass die strategische Gestaltung eines Geschäftsmodells und hauptsächlich die Wahl der Produktkategorie entscheidend für eine erfolgreiche Online-Abo-Vermarktung sind. Dabei sollten Unternehmen zuerst 


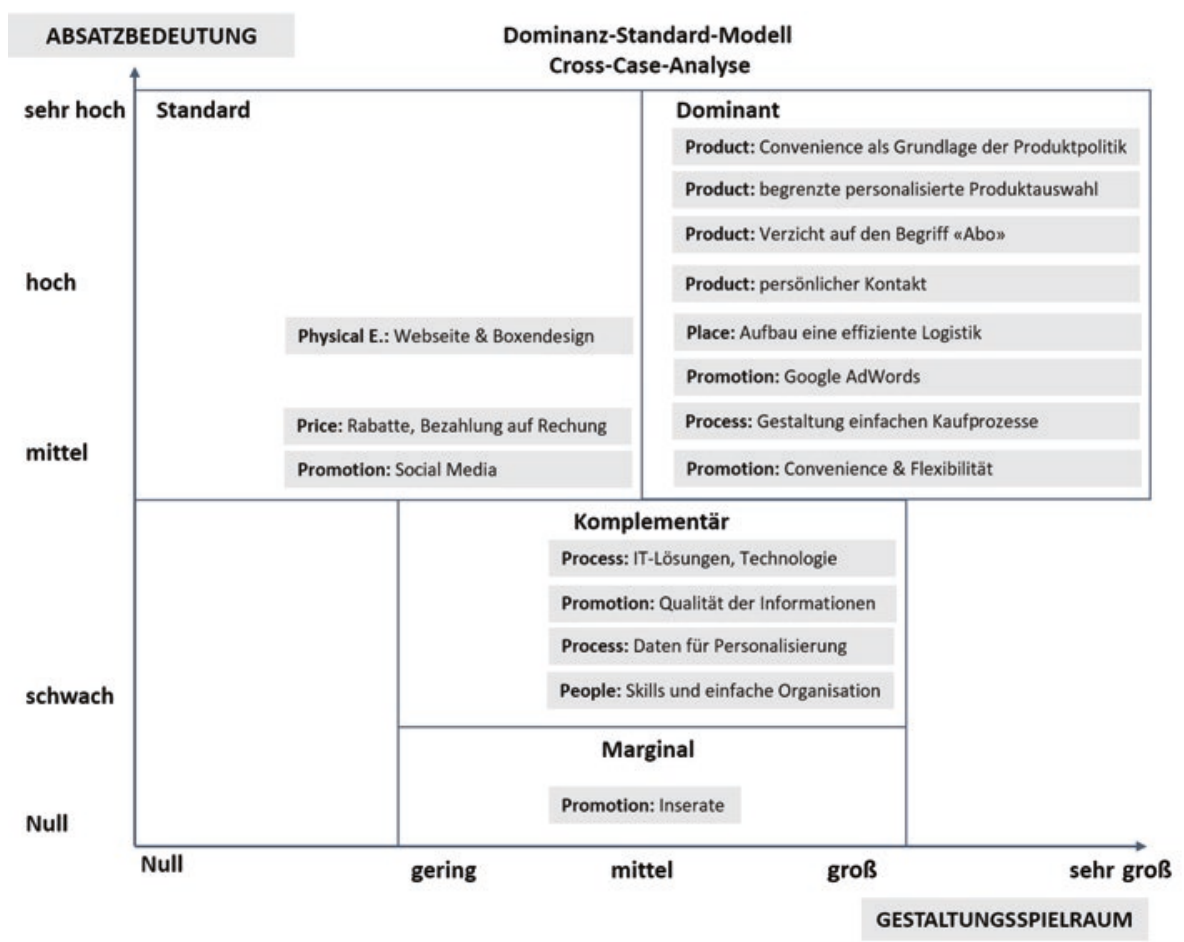

Abb. 14.6 Dominanz-Standard-Modell Cross-Case-Analyse. (Eigene Darstellung in Anlehnung an Kühn 2007, S. 48)

an die Kundenbedürfnisse denken, bevor sie die 7P's ausgestalten (Kotler et al. 2016, S. 126). Die empirische Untersuchung hat gezeigt, dass alle befragten Firmen viel Wert auf die Kundenorientierung legen. Ferner wurde aus der theoretischen Analyse abgeleitet, dass Convenience, reduzierte Komplexität, Inspiration, Teil einer Community zu sein und Geldeinsparungen die Gründe des Erfolgs von Online-Abo-Geschäftsmodellen sind (vgl. Abschn. 15.2). Die empirische Untersuchung bestätigt die drei erstgenannten Faktoren, aber zeigt auf, dass „Teil einer Community“ und „Geldeinsparungen“ nicht mit dem Unternehmenserfolg zusammenhängen. In der Literatur werden Faktoren wie „etwas Neues zu probieren“, die Produktzusammenstellung und personalisierte Abos als wichtige Beweggründe genannt, um Abos abzuschließen (Chen et al. 2018). Die Empire bestätigt diese Sichtweise. Die Literaturanalyse legt ferner nahe, dass finanzielle Anreize in Bezug auf vordefinierte und kuratierte Aboangebote als Kaufmotivation gelten (Chen et al. 2018, S. 5 f.; ING 2018, S. 33). Dies konnte durch die Untersuchung nicht bestätigt werden.

Eine harmonische Marketingmix-Gestaltung ist sowohl in der Theorie als auch in der Empirie als Erfolgsvoraussetzung bestätigt worden. Laut Butler hat ferner die Dis- 
tribution einen ausschlaggebenden Einfluss auf den langfristigen Erfolg einer Firma. Anhand der empirischen Erkenntnisse kann diese Ansicht belegt werden.

Die intensive Literaturanalyse hat ermöglicht, drei Stoßrichtungen für die Marketingmix-Gestaltung eines Online-Abo-Angebots zu eruieren: Kundenorientierung, Erlebnisse und Engagement. Die intensive Interaktion mit den Kunden spielt dabei eine besonders wichtige Rolle. Die empirische Analyse kann dies teilweise bestätigen. Die Interaktion mit dem Kunden durch persönliche Kontakte ist in der Praxis äußerst wichtig. Aber eine intensive Interaktion ist im Rahmen einer kuratierten Online-Abo-Vermarktung nicht notwendig, da der Kunde hauptsächlich nach Convenience sucht (vgl. Abschn. 14.5).

Eine weitere Erkenntnis der empirischen Analyse ist, dass der Begriff „Abo“ von den vier untersuchten Firmen nicht gern verwendet wird. Neue Bezeichnungen wurden entwickelt, um das mit „Abo“ bei vielen Kunden verbundene Zwang-Gefühl zu vermeiden.

Die Ergebnisse aus der theoretischen Analyse und der Fallstudienuntersuchung wurden von Experten als in sich plausibel bestätigt. Aufgrund der geringen Anzahl an Unternehmen kann die Studie allerdings nicht als repräsentativ betrachtet werden. Die gewonnenen Ergebnisse werden jedoch als aussagekräftige Referenz für Handlungsempfehlungen eingestuft. Anhand der eruierten Schlüsselfaktoren konnte aufgezeigt werden, wie der Marketingmix gestaltet werden sollte, um eine erfolgreiche Online-AboVermarktung für Konsumgüter ${ }^{1}$ zu ermöglichen.

Die rasante Entwicklung des Online-Abo-Geschäftsmodelles erschwert es, einen gesamten Überblick zu der Thematik zu erlangen. Das Thema Online-Abo-Vermarktung ist bis dato ein kaum erforschtes Themengebiet. Aus diesem Grund gibt es keine einheitlichen wissenschaftlichen Definitionen von „Abo-Modellen“ und identifizierten marktspezifischen Erfolgsfaktoren. Die durchgeführte qualitative Analyse soll einen Beitrag zur Klärung grundlegender Sachverhalte im Themenkontext leisten.

Insgesamt wurden im Rahmen der empirischen Untersuchung 26 Unternehmen angefragt, jedoch antworteten nur 8 und lediglich 4 waren zu einem Interview bereit. Die verspätete Zusage haben die Organisation und die Durchführung des empirischen Teils erschwert. Die untersuchten Firmen weisen markante Unterschiede auf, wie die Marktabdeckung, die finanziellen Kräfte und die Angebotsstruktur. Die systematische Herangehensweise der Untersuchung basiert auf dem 7P-Ansatz und der Formulierung von KPIs.

Bei der Analyse stellte sich heraus, dass sich die strategischen Überlegungen für die Marketingmix-Gestaltung zwischen Online-Abo-Unternehmen und klassischen OnlineAkteuren nicht deutlich unterscheiden. Um erfolgreich auf dem kuratierten OnlineAbo-Markt für Konsumgüter zu sein, sollten Unternehmen folgende Schlüsselfaktoren berücksichtigen:

- Convenience \& Flexibilität

\footnotetext{
${ }^{1}$ Die Erkenntnisse beziehen sich auf kuratierte Abomodelle.
} 
- Verzicht auf den Begriff „Abo“

- Die Bedeutung der persönlichen Kontakte mit den Konsumenten

Basierend auf diesen Schlüsselfaktoren können weitere Untersuchungen in Bezug auf den kuratierten Abomarkt für Konsumgüter und das hiermit verbundene Konsumverhalten einen Folgebeitrag leisten. Daraus könnte eruiert werden, ob sich die vorgeschlagenen Handlungsempfehlungen bestätigen lassen. Es ist davon auszugehen, dass die Online-Abo-Vermarktung in Zukunft noch weiter an Bedeutung gewinnen wird.

\section{Literatur}

Baxter, R. K. (2015). The membership economy: Find your super users, master the forever transaction, and build recurring revenue. New York: McGraw-Hill.

Bortz, J., \& Döring, N. (2006). Forschungsmethoden und Evaluation für Human- und Sozialwissenschaftler (4. überar. Aufl.). Berlin: Springer (H. S. M. Verlag, Ed.).

Bruhn, M. (2016). Marketing. https://doi.org/10.1007/978-3-658-09803-2.

CB Insight. (2016). Subscription e-Commerce market map: 57 startups In one infographic. Retrieved December 23, 2017. https://www.cbinsights.com/research/subscription-e-commerce-market-map-company-list/.

Chen, T., Fenyo, K., \& Yang, S. (2018). Thinking inside the subscription box: New research on e-commerce consumers. McKinsey \& Company, 1-9.

Clerc, I. (2017). Leitfadengestützte Interviews (Vol. WAFT 2).

Connexity. (2016). The rise of subscription box shopping.

CrateJoy. (2018). Sample subscription boxes: The end of a business model? I Cratejoy Blog. Retrieved June 4, 2018. https://www.cratejoy.com/sell/blog/sample-subscription-boxes-the-endof-a-business-model/.

DesMarais. (2016). Here's data showing the crazy growth of subscription box services (Infographic) I Inc.com. Retrieved May 20, 2018. https://www.inc.com/christina-desmarais/heres-data-showing-the-crazy-growth-of-subscription-box-services-infographic.html.

Elaguizy, A. (2014). Why subscription business models will transform every industry. Retrieved December 23, 2017. https://venturebeat.com/2014/12/17/why-subscription-business-models-will-transform-every-industry/.

Fetto, J. (2016). The rise of subscription box shopping.

Flick, U. (2007). Qualitative Sozialforschung: Eine Einführung. Reinbek bei Hamburg: Rowohlt.

Fruechtebox. (2018). Früchtebox Express: Früchte bestellen, Früchte Lieferservice. Retrieved June 9, 2018. https://www.fruechtebox-express.ch/.

Fueglistaller, U., Müller, C., Müller, S., \& Volery, T. (2016). Entrepreneurship. Entrepreneurship: Modelle - Umsetzung - Perspektiven Mit Fallbeispielen aus Deutschland, Österreich und der Schweiz. https://doi.org/10.1007/978-3-8349-4770-3_7.

Gurtner, A., \& Clerc, I. (2016). Gütekriterien im Forschungsverlauf.

Hofer, C. W., \& Schendel, D. (1978). Strategy formulation: Analytical concepts (M. W. P. St. Paul, Ed.). Berlin: Springer.

Homburg, C. (2017). Marketingmanagement: Strategie - Instrumente - Umsetzung - Unternehmensführung. Lehrbuch. https://doi.org/10.1007/978-3-8349-3628-8.

ING. (2018). Now that we subscribe to music, are tools and toiletries next? (April). https://www. ing.nl/media/ING_EBZ_opportunities-and-challenges-for-tangible-goods-subscriptions_ tcm162-143372.pdf. 
Janzer, A. H. (2015). Subscription marketing: Strategies for nutriting customer in a world of churn. Eiji

Kotler, P., Armstrong, G., Harris, L. C., \& Piercy, N. (2016). Grundlagen des Marketing. London: Pearson.

Kühn, R. (2007). Marketing. Analyse und Strategie. Zurich: Werd.

Kuss, A., \& Eisend, M. (2010). Marktforschung: Grundlagen der Datenerhebung und Datenanalyse. https://download.skopos.de/methoden/desk-research/SKOPOS_Desk_Research.pdf.

Maier, F. (2015). Studie zur Subscription Economy - Trend-Geschäftsmodell Abonnement. Retrieved December 28, 2017. https://www.computerwoche.de/a/trend-geschaeftsmodell-abonnement,3098415.

Mayring, P. (2016). Einführung in die qualitative Sozialforschung. Weinheim: Beltz.

McCarthy, E. J. (1964). Basic Marketing. IL: Richard D. Irwin.

Meffert, H., Christoph, B., \& Manfred, K. (2015). Marketing-Grundlagen marktorientierter Unternehmensführung. https://doi.org/10.1007/978-3-658-02344-7.

Noppeney, C. (2016). Gütekriterien methodischer Vorgehensweisen.

Patel, N. (2015, February). Four things you need to know before your start recurring billing. https://blog.kissmetrics.com/before-starting-recurring-billing/.

Rudolph, T., Bischof, S. F., Böttger, T. M., \& Weiler, N. (2017). Disruption at the door: A taxonomy on subscription models in retailing. Marketing Review St.Gallen (under Review), 34(5), $18-25$.

Saffron, A. (2017). Meat, fish, coffee and more: the best food subscription boxes delivered to your door. Retrieved December 23, 2017. http://www.telegraph.co.uk/food-and-drink/features/the15-best-subscription-boxes-delivered-to-your-door/.

Savage, B., \& Hanser, M. S. (2016). Amazon Prime: The world's leading subscription business.

Schifrin, M. (2016). Has the subscription box boom turned into a bubble? Retrieved May 20, 2018. https://www.forbes.com/sites/schifrin/2016/11/03/has-the-subscription-box-boom-turned-intoa-bubble/\#34226d5b49a9.

Woo, H., \& Ramkumar, B. (2018). Who seeks a surprise box? Predictors of consumers' use of fashion and beauty subscription-based online services (SOS). Journal of Retailing and Consumer Services, 41(November 2017), 121-130. https://doi.org/10.1016/j.jretconser.2017.11.011.

Yin, R. K. (2014). Case study research design and methods. Thousand Oaks: Sage.

Zuora. (2017). Subscription economy - Zuora. Retrieved December 28, 2017. https://www.zuora. com/vision/subscription-economy/.

Romain Descloux (M.Sc. BA/romaindescloux@hotmail.com) Konsekutives Masterstudium in Betriebsökonomie mit Vertiefung Geschäfts- und Unternehmensentwicklung an der Berner Fachhochschule. Vertiefte Auseinandersetzung mit der Thematik der Online Abo-Vermarktung. Hauptberuflich arbeitet er derzeit in der Unternehmensentwicklung (Projektleitung) bei Micarna SA (M-Industrie).

Rumo, Etienne J. (Dr./etienne.rumo@ hefr.ch) ist assoziierter Professor für Marketing und Sales Management an der Hochschule für Wirtschaft in Freiburg und Dozent am konsekutiven Masterstudiengang der Berner Fachhochschule mit Studien (MBA) an der University of Edinburgh (UK) und $(\mathrm{PhD})$ der Université de Lorraine (France). Seine Forschungsaktivitäten konzentrieren sich auf Sales Management und International Entrepreneurship. Seit über 10 Jahren begleitet er als Berater KMU's und Grossbetriebe in den Bereichen Verkauf, Vertrieb und International Business Development. 
Open Access Dieses Kapitel wird unter der Creative Commons Namensnennung 4.0 International Lizenz (http://creativecommons.org/licenses/by/4.0/deed.de) veröffentlicht, welche die Nutzung, Vervielfältigung, Bearbeitung, Verbreitung und Wiedergabe in jeglichem Medium und Format erlaubt, sofern Sie den/die ursprünglichen Autor(en) und die Quelle ordnungsgemäß nennen, einen Link zur Creative Commons Lizenz beifügen und angeben, ob Änderungen vorgenommen wurden.

Die in diesem Kapitel enthaltenen Bilder und sonstiges Drittmaterial unterliegen ebenfalls der genannten Creative Commons Lizenz, sofern sich aus der Abbildungslegende nichts anderes ergibt. Sofern das betreffende Material nicht unter der genannten Creative Commons Lizenz steht und die betreffende Handlung nicht nach gesetzlichen Vorschriften erlaubt ist, ist für die oben aufgeführten Weiterverwendungen des Materials die Einwilligung des jeweiligen Rechteinhabers einzuholen.

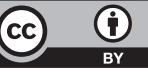




\title{
Social Influencer
}

\section{Eine Analyse ausgewählter visueller und auditiver Stile erfolgreicher Social Influencer auf YouTube}

\author{
Jonas von Rotz und Kim Oliver Tokarski
}

\begin{abstract}
Zusammenfassung
Visuelle Kommunikation gewinnt im Zusammenhang mit der Digitalisierung immer mehr an Bedeutung. So erfolgt die Kommunikation auf Social-Media-Plattformen wie Facebook, Instagram und YouTube fast nur noch mittels Bild- und Videosprache. Social Influencer nutzen diese Kommunikationsweise, um sich auf den genannten Kanälen eine große Community aufzubauen. Dabei entsteht ein Beziehungsverhältnis zwischen Social Influencer und Empfängern. Der vorliegende Beitrag untersucht, welche Faktoren dazu führen, dass Social Influencer „Communities“ von mehreren Tausend bis Millionen Menschen aufbauen können, von diesen als Vorbilder und Meinungsmacher angesehen werden und dass dabei ein Beziehungsverhältnis entsteht.

Die Ergebnisse zeigen, dass Social Influencer mittels aktivierender Wirkung des Gesagten die „Interaktion“ fördern. Durch eine selbstoffenbarende und wertschätzende Wirkung wird ein Storytelling realisiert, die Authentizität und Glaubwürdigkeit erhöht und die Entstehung einer Beziehung gefördert. Die Gestik, erhöhte Sprechlautstärke und das schnelle Sprechtempo sorgen dabei beim Empfänger für eine kompetent und glaubwürdig wirkende Erscheinung des Social Influencers, während die Mimik „Freude“ Glücksgefühle beim Adressaten auslöst. Die Ergebnisse liefern somit qualitativ-explorative Erkenntnisse über Stile von Social Influencern und deren Umsetzung auf dem Social-Media-Kanal YouTube. Sie bieten Ansätze zur Erklärung des Beziehungsverhältnisses zwischen YouTuber und Community.
\end{abstract}

J. von Rotz

Bern, Schweiz

E-Mail: jonas.vonrotz@gmx.ch

K. O. Tokarski ( ()

Departement Wirtschaft, Berner Fachhochschule, Bern, Schweiz

E-Mail: kim.tokarski@bfh.ch 


\subsection{Einleitung, Zielsetzung und Vorgehensweise}

YouTube, Facebook und Instagram stellen zum aktuellen Zeitpunkt die relevantesten Social-Media-Plattformen dar (Statista - The Statistics Portal 2018). Auf allen drei Plattformen ist die visuelle Kommunikation eine wichtige bzw. zentrale Grundlage für den Erfolg des Netzwerkes. Texte stehen im heutigen Internet (zumeist) nur noch an 2. Stelle (Kuch 2013). Durch die immer besseren und günstiger werdenden Kameras und die immer besser werdenden Auflösungen der Bildschirme wird diese Entwicklung der Bedeutung visueller Kommunikation noch verstärkt sowie beschleunigt.

Generell hat die Internetnutzung stark zugenommen. Gemäß der JAMES-Studie verbringen Schweizer Jugendliche unter der Woche täglich durchschnittlich 2 h und $30 \mathrm{~min}$ im Internet (2014: 2 Std.), am Wochenende 3 h und 40 min (2014: 3 Std.). Die täglichen Internetnutzungszeiten sind also im Vergleich zu 2014 jeweils um rund $25 \%$ gestiegen (zhaw/Swisscom, 2016). Dieser Trend stellt gleichzeitig ein großes Potenzial für neue Geschäftsmodelle dar. Zum Beispiel für die Social Influencer. Social Influencer sind Personen mit Vorbildfunktion für eine Große Social-Media-Community, auf Plattformen wie beispielsweise Instagram, Facebook oder YouTube. Mittels Videos, Bildern und Texten werden bspw. Meinungen und Empfehlungen zu Produkten, Dienstleistungen und Ferienzielen vermittelt. Es handelt sich um Blogger, Videomacher, Models, Schauspieler, aber auch um Journalisten oder Sportler (Noah Zygmont 2017). Diese konnten allein im Jahr 2017 ein hohes Wachstum verzeichnen. So nahmen bspw. im Jahr 2017 die Suchanfragen zu Influencer Marketing um $325 \%$ zu (Firsching 2017).

Vor diesem Hintergrund stellen sich unterschiedliche Fragen zum Phänomen ,Social Influencer": Wie ist es möglich, dass die Zuschauerzahlen von Social Influencern, wie beispielsweise beim YouTube-Video von Julien Bam „Everyday Saturday (Parodie)“ die 24 Mio. Marke übersteigen? Dies entspricht ungefähr eines 7-Fachen der Show „The Voice of Germany“ des Fernsehsenders ProSieben (Statista - Das Statistik Portal 2015). Für das Marketing ergeben sich in diesem Kontext neue Möglichkeiten spezifische Zielgruppen zu erreichen. So zeigen sich unterschiedliche Vorteile in diesem Kontext: Erstens wird mit Influencer Marketing meistens ein viel größeres Zielpublikum erreicht, als durch viele „klassische“ Marketingmaßnahmen. So hat beispielsweise die Influencerin Caro Daur 1,3 Mio. Follower auf Instagram und das Modemagazin Vogue Deutschland nur gerade mal ca. 220.000. Zweitens ist die Werbung aus Sicht des Zielpublikums glaubwürdiger, da sie durch bekannte Person vermittelt wird. Social Influencer sind die „,neuen Stars“ (im Internet).

Celie O’Neil-Hart (Contributor, Video Marketing bei Google) und Howard Blumenstein (Produkt Marketing Manager, Strategie und Erkenntnisse bei YouTube B2B) konnten zeigen, dass $70 \%$ der amerikanischen Jugendlichen YouTube-Stars mehr Vertrauen schenken als den gewohnten Berühmtheiten (Celie O'Neil-Hart 2016). Es ist also nicht verwunderlich, dass einige Unternehmungen, wie bspw. das Traditionshotel Waldhaus in Flims (Schweiz), bereits fast gänzlich auf Influencer Marketing setzen (Donati 2017). 
Doch was genau sind die Voraussetzungen, dass jemand allein durch Social Media so schnell so berühmt werden kann und einen so großen Einfluss erhält?

Die vorliegende Ausarbeitung liefert Hinweise zur Beantwortung der zuvor gestellten Frage in Form einer visuellen sowie auditiven Analyse von ausgewählten Videos der erfolgreichsten deutschen Social Influencer auf YouTube. Im Ziel analysiert und dargestellt werden, welche Faktoren dazu führen, dass Social Influencer „Communities von mehreren Tausend bis Millionen Menschen“ aufbauen, von diesen als Vorbilder und Meinungsmacher angesehen werden und dabei ein Beziehungsverhältnis entsteht. Die im empirischen Teil durchgeführte YouTube-Videoanalyse basiert auf definierten visuellen und auditiven Analysekriterien. Diese Analyse soll Muster, Gemeinsamkeiten und Unterschiede ersichtlich machen und zusammen auf Basis einer kurzen theoretischen Grundlage die gestellten Fragen beantworten.

\title{
15.2 Theoretische Grundlagen
}

\subsubsection{Social-Media-Plattformen und Social Influencer}

Das Ziel einer Social-Media-Plattform ist es, Menschen digital miteinander zu vernetzen. Es steht jedem Menschen frei, sich mit einem internetfähigen Gerät auf einer Social-Media-Plattform anzumelden und Informationen $\mathrm{zu}$ veröffentlichen. Das Veröffentlichen der Informationen funktioniert mittels Neuigkeiten in Textform, über Fotos, Videos von allgemeinen bis hin zu Special-Interest-Themen. Sie dienen bspw. dem Austausch der User, dem Diskutieren von Themen, dem Teilen von Tipps und der gegenseitigen Hilfe (Für-Gründer.de, kein Datum). Die Abb. 15.1 zeigt eine kurze Übersicht über die meistgenutzten Social-Media-Plattformen. Dabei ist allerdings anzumerken,

Monatlich aktive User: 2.13 Milliarden (31.12.2017) (investor.fb, 2018)
Jahreszuwachs an User: $14 \%$ (investor.fb, 2018)
Monatlich aktive User: 800 Millionen (30.09.2017) (Statista - The
Statistic Portal, 2017)
Jahreszuwachs an User: ca. 45\% (Statista - The Statistic Portal, 2017)

- YouTube Monatlich aktive User: 1.57 Milliarden (24.01.2018)

(www.omnicoreagency.com, 2018)

Jahreszuwachs an User: 9.2\% (Statista - The Statistic Portal, 2018)

\begin{abstract}
Abb. 15.1 Meistbenutzte Social-Media-Plattformen in der Übersicht. (Quelle: eigene Darstellung auf Basis von www.investor.fb 2018, Statista - The Statistic Portal 2017, www.omnicoreagency. com 2018, Statista - The Statistic Portal 2018, Rothe 2011, Glück 2010, Anja Linnenbürger 2018, Rhetores - Akademie der Sprecher)
\end{abstract}


dass die Entwicklungen und die Veränderungsgeschwindigkeit in diesem Kontext so hoch sind, dass die Darstellung vermutlich bei Publikation des Beitrags veraltet ist.

Obwohl Influencer Marketing, Social Influencing und Social Influencer aktuelle Begriffe im Zusammenhang mit Digital Marketing darstellen, lassen sie sich grundlegend (in anderer operativer Form) bis ins 18. Jahrhundert verfolgen. Bereits im Jahre 1760 nutzte Josiah Wedgwood, Gründer einer Töpferei, Influencer Marketing, indem er das englische Königshaus für seine Waren begeistern konnte, welches im Anschluss als Fürsprecher fungierte. Später wurden von Marken Charaktere erfunden, welche als „Influencer“ dienen sollten. So bspw. der bekannte weiß-rote „Santa Claus“ von CocaCola 1923 oder „,Tony the Tiger“ von Frosted Flakes 1952 (noGRE 2017). Der Begriff „Social Influencer“ hat mit dem Aufkommen der Social-Media-Plattformen allerdings in den letzten Jahren stark an Bedeutung gewonnen.

Die Definition des Begriffs „Social Influencer“ ist dabei nicht eindeutig und hat viele Einflussfaktoren. Dies zeigt auch die Studie von Olapic (2018) bzw. der Erörterungen von Connolly (2018) deutlich. Bei der Befragung von 4000 Teilnehmenden im Alter zwischen 16 und 61 Jahren in den Ländern Amerika, Großbritannien, Frankreich und Deutschland konnte Folgendes festgestellt werden: $53 \%$ der Befragten waren der Meinung, dass jemand mit mehr als 10.000 Followern auf einem Social-Media-Kanal als Social Influencer bezeichnet werden darf (wobei in Deutschland $34 \%$ der Meinung sind, dass dies erst ab 50.000 Followers gilt). Gemäß Connolly (2018) umfasst ein Influencer folgende Kriterien:

- Mindestens 10.000 Follower auf einem Social-Media-Kanal

- Vorhandene Partnerschaft mit einem Unternehmen

- Inhalte von Produkten oder Dienstleistungen werden geteilt

- Die betreffende Person kennt sich in ihrem Gebiet aus

Bei der Frage, was genau einen Social Influencer ausmacht, sagten $63 \%$ der Befragten, dass die Anzahl der Follower das maßgebende Kriterium sei, $42 \%$ waren der Meinung, dass der Informationsgehalt der Posts ausschlaggebend sei. Nur $39 \%$ meinten, dass die Posts bei einem Social Influencer von höherer Qualität seien. Weiter wurde die Erkenntnis erlangt, dass in Europa Social Influencer oftmals damit beschrieben werden, dass sie eine bezahlte Partnerschaft mit einer Firma haben, wobei in Amerika eher die Beliebtheit der Person maßgebend ist (Connolly 2018). Gemäß Hugi (2018), ist jedoch bereits ein Stammkunde in einer Kneipe, welcher vom Kneipenbesitzer gefragt wird, ob er einen guten Kommentar auf Instagram oder Tripadvisor hinterlassen kann, ein Influencer. Entscheidend ist laut Hugi folglich nicht die Reichweite des Influencers, sondern dessen Tätigkeit als Meinungsmacher (Hugi 2018). 


\subsubsection{Unterschiede zwischen Berühmtheiten und Social Influencern}

Obwohl beide, Social Influencer und Berühmtheiten, eine große Reichweite besitzen und von ihren Fans als Vorbilder angesehen werden, unterschieden sie sich. Fans von Berühmtheiten lieben es zu wissen, wo diese einkaufen, wo diese ihre Ferien verbringen, und welches ihre Designer sind. Dies bildet Markenbekanntheit, aber keinen direkten Umsatz. Fans von Social Influencern hingegen bilden sich langsam und organisch. Sie folgen ihnen auf ihren Social-Media-Kanälen oftmals aufgrund gleicher Interessen und Denkweisen und können sich somit mit ihnen identifizieren (Haines 2014). Influencer sind „Selfmade“-Stars auf Social-Media-Kanälen, während Berühmtheiten durch traditionelle Kanäle zum Star gemacht wurden (Hitz 2018). Dieser Unterschied bringt für Influencer einige Vorteile mit sich. Einer davon ist die Erfahrung der Influencer auf ihrem jeweiligen Gebiet. Während Berühmtheiten nicht unbedingt Experten in den Gebieten sind, für welche sie Werbung machen (Beispielsweise Justin Bieber, der für Calvin Klein wirbt), kennen sich die Influencer meistens sehr gut mit den beworbenen Produkten aus (Barker 2017). In der Beziehung zwischen Influencer und Follower geht es um gemeinsame Interessen. Dabei begegnen die Influencer den Followern als Fachmann auf ihrem Gebiet, werden jedoch gleichzeitig auch als ,,reale Menschen“ mit Wünschen, Träumen, und Krisen wahrgenommen. Follower können sich so mit dem jeweiligen Influencer identifizieren (Faltl und Freese 2017). Ein weiterer Punkt ist der Unterschied zwischen der analogen Kommunikation, wie sie jene der herkömmlichen Werbung durch Berühmtheiten darstellt, zur interaktiven Kommunikation, wie sie bei den Influencern zu beobachten ist. Der Influencer nutzt die Möglichkeit mit seinen Followern $\mathrm{zu}$ interagieren und $\mathrm{zu}$ kommunizieren. Ein weiterer wichtiger Unterschied sind das „Storytelling“ und die „Content Creation“. Berühmtheiten stellen zwar oft das Gesicht hinter einer Kampagne dar, sind jedoch nicht die Erschaffer des Inhaltes. Dies ist bei einem Influencer anders: Da sie Experten auf ihren Gebieten sind, erschaffen sie so auch die passenden Inhalte und Geschichten hinter den Produkten und Marken (Barker 2017).

Es entsteht eine Beziehung, welche (vermeintlich) nicht auf kommerzieller Ebene stattfindet, wie dies bspw. bei einer Kampagne von Calvin Klein durch Justin Bieber der Fall ist, sondern auf gemeinsamen Interessen basiert (Faltl und Freese 2017). Beide Gruppen haben jedoch oftmals eine große Reichweite. Diese unterscheidet sich jedoch. Während bei einer Werbung durch eine Berühmtheit ein Massenpublikum erreicht wird, ist dies beim Influencer eine Nischen-Zielgruppe zum jeweiligen Themengebiet. In manchen Fällen kann der Unterschied etwas verblassen bzw. es ergeben sich Überschneidungen. Beispielsweise wenn ein Athlet, welcher als Berühmtheit gilt, für eine Sportbekleidungsmarke wirbt. Das könnte auch als Influencer Marketing betrachtet werden (Barker 2017). Auch der YouTuber „LeFloid“ sieht einen Unterschied zwischen Berühmtheit und Influencer. Für ihn ist ein Influencer auf „Augenhöhe“ mit seinen Fans, hingegen ist dies bei einer Berühmtheit ganz anders (LeFloid 2014). Dies führt zu der 
bereits eingangs dargestellten Thematik, dass Social Influencern mehr Vertrauen geschenkt wird, als den ,gewohnten“ Berühmtheiten. (Celie O’Neil-Hart 2016).

Die Definitionen zum Begriff des Social Influencers sind nicht ganz trennscharf. Gleichwohl können, unabhängig von den quantifizierbaren Kategoriegrößen einer möglichen Klassifizierung von Social Influencern in Gruppen, zugeschriebene Kernkompetenzen von Social Influencern dargestellt werden.

\subsubsection{Kernkompetenzen und USP von Social Influencern}

Die möglichen Kernkompetenzen von Social Influencern im gewählten Untersuchungskontext folgen im Folgenden den Bereichen ,Zielpublikum Jugendliche, persönliche Beziehungen, Glaubwürdigkeit, Expertenwissen, Vorbildfunktion, Reichweite, Storytelling, Authentizität, Fotografie und Bildbearbeitung bzw. Filmen und Videobearbeitung, Erscheinungsbild und Interaktion":

Zielpublikum Jugendliche Gemäß Andreas Hugi, ist es heutzutage sehr schwierig, Jugendliche mittels herkömmlicher Werbung wie Plakatwerbung, Fernsehwerbung, Printmedienwerbung usw. zu erreichen. Influencer agieren jedoch auf Social-MediaPlattformen und stellen somit für Unternehmungen und deren Marketing-Anstrengungen ein großes Potenzial dar (Hugi 2018).

Persönliche Beziehungen Abonnenten und Follower der Influencer identifizieren sich oftmals mit ihren Idolen auf den Social-Media-Kanälen. YouTuber wie beispielsweise Bianca Heinicke vom YouTube-Beauty Blog „BibisBeautyPalace“ werden von ihren Abonnenten wie ein Star behandelt und gelten als absolutes Idol. Durch die Interaktion zwischen Community und Influencer entstehen persönliche Beziehungen, sogenannte parasoziale Beziehungen (Visscher 1998).

Glaubwürdigkeit Laut Statistik vom Statistikportal Statista geben $57 \%$ der Befragten in Deutschland im Jahr 2016 Influencer Marketing als glaubwürdig an. Vergleichsweise schneidet die ,klassische“ Werbung mit $47 \%$ schlechter ab (Statista.de 2016).

Expertenwissen Laut der Studie von Olapic (2018) geben $45 \%$ der befragten Amerikaner an, dass die Posts von Influencern mehr Informationsgehalt als übrige Posts haben. Influencer würden sich täglich mit der Nische auseinandersetzen, in der sie sich befinden. Dies mache sie zu Experten auf diesem Gebiet (Marco Nirschl 2018).

Vorbildfunktion Kiener (2018) begründet die Vorbildfunktion der Influencer damit, dass es Jugendlichen oftmals an eigenen Erfahrungen und Erfolgserlebnissen mangelt. Influencer dienen somit als Verhaltensmodelle, an deren Empfehlung man sich orientieren kann und eine gewisse Sicherheit erhält (Kiener 2018). 
Reichweite Die Reichweite kann sehr stark variieren und geht von wenigen Tausend (jedoch sehr zielgruppenspezifischen) Abonnenten bis zu mehreren Millionen Abonnenten. Mit dieser Reichweite erreichen die Influencer fast ausschließlich Abonnenten im betreffenden Nischenmarkt (Marco Nirschl 2018).

Storytelling Beim Storytelling will das menschliche Gehirn das Gehörte mit einer eigenen Erfahrung in Verbindung bringen. Dabei wird der Inselcortex des menschlichen Gehirns aktiviert, welcher dem Menschen hilft, die gehörte Geschichte mittels eigens Erlebtem lebendiger zu gestalten (Widrich 2012). Das Storytelling der Influencer bildet die Verbindung zwischen der Marke oder dem Produkt und dem Konsumenten, was für das Unternehmen von Vorteil ist (Casellas 2017).

Authentizität Authentizität bedeutet „Echtheit“ und „als Original empfunden“, und ist somit eine Grundvoraussetzung für die Glaubwürdigkeit eines Influencers. Während Hollywoodstars von der Mehrheit der Menschen als unberührbar und abgehoben wahrgenommen werden, wirken Social Influencer auf Augenhöhe und werden bspw. eher als „das freundliche Mädchen von nebenan“ wahrgenommen (Kamal 2018). Dabei wird die Authentizität durch Bewusstsein, Ehrlichkeit, konsequentes Handeln und Aufrichtigkeit erzielt (Mai 2013).

Fotografie und Bildbearbeitung bzw. Filmen und Videobearbeitung Influencer fotografieren oder filmen selten nur mit dem Smartphone, sondern verwenden dazu Digital-, Spiegelreflex- oder Kompaktkameras. Für die Bildbearbeitung werden Desktop Bildbearbeitungssoftwares wie beispielsweise Adobe Lightroom und/oder Adobe Photoshop oder Apps wie Snapseed, Square App usw. verwendet (Perry 2017).

Erscheinungsbild Während einige Influencer mittlerweile als Schönheitsbild fungieren, verschaffen sich andere Influencer auf anderem Weg Wiedererkennungswert. Ein gutes Erscheinungsbild ist für einen Influencer sehr wichtig. Er braucht also einen gewissen Wiedererkennungswert, um ein eigenes Branding aufbauen zu können (Affiliate Deals 2016).

Interaktion Die Interaktion dient dem Influencer dazu, ein großes Zielgruppenwissen aufzubauen. Sie dient zur Einschätzung, welche Inhalte und Themen aktuell für die Community spannend sind und welche nicht. Florian Mundt, alias LeFloid erklärt in einem Interview, dass es genau diese Interaktion ist, welche YouTube, vergleichsweise zum Fernsehen, so interessant macht. Von der Fanseite her entsteht dabei eine sogenannte parasoziale Beziehung, eine empfundene Freundschaft mit dem Influencer (Koschig 2016). 


\subsubsection{Visuelle und auditive Aspekte von Kommunikation}

Um Beziehungen zu ihrer Zielgruppe bzw. ihren Zielgruppen aufbauen zu können, bedarf es einer Kommunikation der Social Influencer über ihre jeweiligen Social-Media-Kanäle. Videos umfassen dabei eine Vielzahl visueller und auditiver Aspekte wie:

Mimik \& Gestik Unter Mimik versteht man sichtbare Bewegungen des Gesichts, welche als Unterstützung zur Kommunikation eingesetzt werden. Die Grundfunktionen der Mimik sind hauptsächlich die Vermittlung von Emotionen, die Rückmeldung des Empfängers für eine Botschaft an den Sender, eine Stellungnahme zu anderen Ereignissen und Personen und die Reaktion auf verbale Aussagen (Glück 2010). Der Begriff Gestik wird gemäß Metzler Lexikon Sprache (2010, S. 240) folgendermaßen bezeichnet: „Repertoire und System kommunikativ verwendeter Körperbewegungen und Körperhaltungen, v. a. Hand- und Armbewegungen“. Die Gestik stellt also eine Gesamtheit von nonverbalen Signalen dar, welche als Ausdruck von Körperbewegungen kommunikativ relevant sind (Glück 2010).

Sprechtempo \& Sprechlautstärke Das Sprechtempo und die Sprechlautstärke gehören zur paraverbalen Kommunikation und sind dabei wichtige Aspekte für eine erfolgreiche Kommunikation. Gleichzeitig ist das Sprechtempo die Schnittstelle zwischen dem Artikulationsorgan, der Neurolinguistik, der Neurophonetik und der Sprache selbst. Je nachdem wie schnell eine Person spricht, beeinflusst dies die Botschaft, welche übermittelt wird (Pfitzinger, Phonetische Analyse der Sprechgeschwindigkeit). Auch die Sprechlautstärke beeinflusst die Botschaft, welche übermittelt wird. So kann beispielsweise eine Lautstärkenvariation eine aktivierende Wirkung erzielen (Linnenbürger et al. 2018). Der Psychologe Howard Giles bewies, dass schnell sprechende Menschen oder auch laut sprechende Menschen als kompetent, sympathisch, gutaussehend und interessant wahrgenommen werden (Giles 1994).

Kommunikative Wirkungsweisen Die kommunikative Wirkungsweise beschreibt, welche Wirkung eine Person beim Sprechen tendenziell auf ihr Gegenüber erzielt. Die aktivierende Wirkung wird durch die Verwendung aktivierender Wörter (Begriffe, die Umsetzung und Handlung implementieren) sowie Verwendung von prosodischen Elementen wie Stimmvariation, Lautstärkenvariation, gezielten Pausen und einem vielfältigen Sprechrhythmus erzielt. Es entsteht eine aktivierende und dynamische Wirkung beim Zuhörer. Die wertschätzende Wirkung wird durch eine positive Haltung gegenüber anderen erzeugt. Der Sprechende wirkt sozial und das Publikum fühlt sich wohl und aufgehoben. Eine autoritäre Wirkung kann dadurch erzeugt werden, dass persönliche Ansichten und Standpunkte in den Vordergrund gerückt und klar preisgegeben werden. Somit wird eine dominante Haltung eingenommen und Autorität ausgestrahlt. Eine ausgeglichene Wirkung hingegen wird durch eine ruhige Herangehensweise erzielt. Es 
entsteht eine entspannte und gelassene Atmosphäre, was hitzige Diskussionen auf eine sachliche Ebene bringen kann. Bei der distanzierten Wirkung werden voreilige emotionale Einbindungen vermieden und die Unabhängigkeit vom Geschehen und von Gesprächspartnern wird hervorgehoben. Um eine informative Wirkung zu erzielen, wird auf Emotionen verzichtet, Argumente und Sachinformationen stehen im Vordergrund. Eigene Ansichten werden dabei auf eine bedächtige und sachliche Weise vermittelt. Bei der zielorientierten Wirkung wird das Gespräch vom Protagonisten in die gewünschte Richtung gelenkt. So kann ein Gespräch an einem anderen Punkt angesetzt werden und anschließend in die gewünschte Richtung gelenkt werden. Um eine emotional-offene Wirkung zu erzielen, werden eigene Ansichten und Empfindungen emotional statt rational ausgedrückt. Dies führt zu einer emotional-offenen und begeisternden Wirkung. Bei der verantwortlichen Wirkung ist die Kommunikation durch eine persönliche Pflichtwahrnehmung für die eigenen Aussagen geprägt. Die unterstützende Wirkung setzt einen direkten Gesprächspartner voraus. Dessen Ansichten wollen verstanden werden und werden nicht beeinflusst. Er fühlt sich dadurch persönlich wahrgenommen und unterstützt. Die zuversichtliche Wirkung zeichnet sich dadurch aus, dass positive und auf ein konkretes Ziel bezogene Elemente in den Vordergrund gerückt werden. Für die grundlegende Überzeugung, dass eine Herausforderung gemeistert werden kann, werden optimistische Elemente verwendet. Um eine selbstoffenbarende Wirkung zu erzielen, werden viele persönliche Charaktereigenschaften preisgegeben; so spielen beispielsweise persönliche Erlebnisse, Hintergründe und private Ereignisse eine wichtige Rolle (Linnenbürger et al. 2018).

Grußformeln/Abschiedsformeln Begrüßungen und Verabschiedungen können echte Wertschätzung gegenüber dem Empfänger ausdrücken (careerloft 2016). Influencer, insbesondere YouTuber begrüßen ihre Abonnenten häufig mit einer Grußformel und Abschiedsformel wie beispielsweise „Hallo ihr Lieben“ und „Das war's von mir, wir sehen uns morgen wieder".

\subsubsection{Forschungsfragen und Hypothesen}

Auf Basis der beschriebenen theoretischen Hintergründe der Social Influencer sowie der diversen Aspekte visueller Kommunikation, ergeben sich drei Fragestellungen im Untersuchungskontext:

- F1: Welche Stile führen dazu, dass Social Influencer von ihren Followern als Vorbilder und Meinungsmacher angesehen werden?

- F2: Wie führen diese Stile dazu, dass die Social Influencer von ihren Followern als Vorbilder und Meinungsmacher angesehen werden?

- F3: Wie entsteht zwischen Social Influencer auf YouTube und Zuschauern ohne reale Begegnung eine parasoziale Beziehung? 
Obwohl es sich bei der vorliegenden Studie um eine qualitative Studie handelt, wurden Hypothesen für die empirische Studie und somit den Untersuchungskontext formuliert. Die erstellten drei Hypothesen dazu lauten wie folgt:

- H1: Die Dialoge der Influencer sind emotional, aktiv, provokativ, aktivierend, selbstoffenbarend und teilweise informativ. So soll durch die emotionale, aktive, provokative Ausdrucksform ein einzigartiges und auffälliges Erscheinungsbild erzeugt werden. Die selbstoffenbarende Wirkung soll eine Beziehung zu den Abonnenten unterstützen. Sie soll die Authentizität des Influencer steigern, vor allem wenn es als Storytelling fungiert. Die informative Wirkung soll den Influencer wie einen Experten wirken lassen und die aktivierende Wirkung soll die Interaktion zwischen Zuschauer und YouTuber fördern.

- H2: Die Influencer sprechen schnell und laut. Damit soll ein kompetenter, sympathischer und kluger Eindruck hinterlassen werden. Dabei wird gezielte Gestik angewendet, um das Gesagte entsprechend zu betonen. Dies soll für eine starke Glaubwürdigkeit sorgen und das Erscheinungsbild stärken.

- H3: Fröhliche Emotionen stehen im Vordergrund. Dies soll eine ausdrucksstarke Erscheinung garantieren und so die Authentizität erhöhen.

\subsection{Empirie}

Der empirische Teil dieser Arbeit befasst sich mit der Durchführung einer visuellen und auditiven Analyse von YouTube-Videos. Die Analyse besteht einerseits aus einem visuellen Teil: Der Fokus liegt dabei auf den visuellen Analysekriterien Mimik und Gestik. Zusätzlich besteht die Analyse aus einem auditiven Teil. Der Fokus liegt dabei auf den Analysekriterien Sprechtempo, Sprechlautstärke, kommunikative Wirkungsweise und Grußformeln/Abschiedsformeln. Es gilt herauszufinden, wie die geschilderten Dimensionen in den Videos vorkommen und welche Wirkung sie mit sich bringen.

\subsubsection{Vorgehen und Prozessbeschreibung der Methodik}

\subsubsection{Analysekriterien, -objekte, Referenzwerte und Methodik}

Bei den untersuchten Faktoren dieser empirischen (explorativen) Studie handelte es sich um Medien- und Kommunikationswissenschaften, bei welchen Befragungen, Inhaltsanalysen und Beobachtungen die gängigsten Erhebungsmethoden darstellen (Möhring und Schlütz 2010). So wären auch Interviews eine Möglichkeit an die benötigten Daten heranzukommen. Jedoch wäre es schwierig, mit den entsprechenden Influencern ein Interview zu vereinbaren. Weiter wäre unklar, ob die betreffenden Influencer ihre Stile oder sogar Erfolgsfaktoren in einem Interview preisgeben würden. Demzufolge wird die 
Beobachtung als die beste Variante eingestuft, wobei eine Analyse der YouTube-Videos am sinnvollsten erscheint.

\subsubsection{Videoanalyse}

Die Analyse der YouTube-Videos wurde in eine visuelle und in eine auditive Forschung unterteilt. Durch die Ergebnisse der visuellen und auditiven Untersuchung der YouTube-Videos sollten im Anschluss Rückschlüsse auf die Stile ermöglicht werden. Für die Analyse wurden die Analysekriterien gemäß Tab. 15.1 als Referenzwerte definiert. In Tab. 15.1 sind die für die empirische Studie definierten Kriterien der visuellen und auditiven Analyse dargestellt.

\subsubsection{Analyseobjekte}

Als Analyseobjekte wurden per Zufallsprinzip ausgewählte Videos der 5 erfolgreichsten deutschen YouTuber gemäß Socialblade.com definiert. Es handelt sich dabei um:

- BibisBeautyPalace

- Julien Bam

- Dagi Bee

- ApeCrime bzw. ApeCrimeTV

- Simon Desue

Aufgrund der Theorie, welche größtenteils auf deutschen Quellen basiert, wurden auch für den empirischen Teil der Forschungsarbeit deutsche Analyseobjekte gewählt. Zur Homogenisierung wurden ausschließlich YouTuber untersucht, welche sich selbst auditiv sowie visuell in Dialogform als Hauptmotiv im Video platzieren. Alle anderen Formen der Selbstdarstellung, wie beispielsweise Gaming-Videos, Musikvideos etc., wurden ausgeschlossen, um ein einheitliches Analysevorgehen zu ermöglichen.

\subsubsection{Analysevorgehen}

Für die Auswahl der YouTube-Videos der verschiedenen YouTuber wurden alle zum Zeitpunkt der durchgeführten Untersuchung online bestehenden YouTube-Videos der YouTuber in einer Excel-Liste aufgelistet und durchnummeriert. Anschließend wurden mittels eines Zufallsgenerators (Rechneronline) 10 verschiedene Zufallszahlen generiert. Der Stichprobenumfang wurde aufgrund der Tatsache, dass eine qualitative Analyse vorlag und aufgrund zeitlich begrenzter Ressourcen, auf 10 Videos pro YouTuber beschränkt. Im Anschluss wurden die per Zufallsprinzip gewählten Videos mithilfe von einem MP4-Konverter heruntergeladen und in die Analysesoftware MAXQDA übertragen (Onlinevideokonverter). MAXQDA ist eine Software für qualitative Datenanalysen wie beispielsweise das Transkribieren und Codieren von Audio- und Videodateien. Mithilfe von MAXQDA wurden die Videos nach den definierten Analysekriterien codiert. Anhand der Codetabellen und Visual Tools sowie der Analyse der einzelnen codierten Filmausschnitte wurden die Analysekriterien ausgewertet und interpretiert. 
Tab. 15.1 Kriterien der visuellen und auditiven Analyse

\begin{tabular}{|c|c|}
\hline \multicolumn{2}{|l|}{ Visuelle Analyse } \\
\hline Analysekriterium & Referenzwerte \\
\hline Mimik & $\begin{array}{l}\text { Freude (Falten entlang der Nase, Lippenränder hoch- und zurück- } \\
\text { gezogen) } \\
\text { Wut (Augenbrauen gesenkt und gerade Linie ergebend, Unterlieder } \\
\text { angespannt, Lippen zusammengepresst) } \\
\text { Angst (Falten in der Stirnmitte, Augenbrauen hoch- und zusammen- } \\
\text { gezogen, Augenlider angespannt, Mund zurückgezogen) } \\
\text { Ekel (Nasenfalten, Wangen erhoben, angezogene Oberlippen) } \\
\text { Trauer (hochgezogene innere Augenbrauen, verlorener Augenfokus, } \\
\text { leicht nach unten gezogene Lippenecken) } \\
\text { Überraschung (erhobene Augenbrauen, weit geöffnete Augen, offener } \\
\text { und entspannter Mund) } \\
\text { Verachtung (einseitig hochgezogene und angespannte Lippenecke) }\end{array}$ \\
\hline Gestik & $\begin{array}{l}\text { Demonstrativgestik (Visualisierung von Gesagtem) } \\
\text { Betonungsgestik (Verstärkung des Gesagten) } \\
\text { Zeigegestik (Physisches Zeigen auf Objekte, Personen oder in Richtun- } \\
\text { gen) } \\
\text { Berührungsgestik (Physisches Berühren von Objekten oder Personen) }\end{array}$ \\
\hline \multicolumn{2}{|l|}{ Auditive Analyse } \\
\hline Analysekriterium & Referenzwerte \\
\hline $\begin{array}{l}\text { Kommunikative } \\
\text { Wirkungsweisen }\end{array}$ & $\begin{array}{l}\text { Aktivierende Wirkung (Begriffe, die Umsetzung und Handlung imple- } \\
\text { mentieren und Stimmvariationen) } \\
\text { Wertschätzende Wirkung (positive und wertschätzende Haltung) } \\
\text { Autoritäre Wirkung (dominante Haltung und persönlich Ansichten und } \\
\text { Standpunkte werden in den Vordergrund gerückt) } \\
\text { Ausgeglichene Wirkung (ruhige Herangehensweise) } \\
\text { Distanzierte Wirkung (voreilige emotionale Einbindungen werden } \\
\text { vermieden) } \\
\text { Informative Wirkung (Argumente und Sachinformationen stehen im } \\
\text { Vordergrund) } \\
\text { Zielorientierte Wirkung (Gespräch wird in die gewünschte Richtung } \\
\text { gelenkt) } \\
\text { Emotional-offene Wirkung (eigene Ansichten und Empfindungen wer- } \\
\text { den durch emotionale statt rationale Argumente ausgedrückt) } \\
\text { Verantwortliche Wirkung (Kommunikation ist durch eine persönliche } \\
\text { Pflichtwahrnehmung für die eigenen Aussagen geprägt) } \\
\text { Unterstützende Wirkung (Ansichten der Gesprächspartner werden ver- } \\
\text { sucht, verstanden zu werden und nicht beeinflusst) } \\
\text { Zuversichtliche Wirkung (positive und auf ein konkretes Ziel bezogene } \\
\text { Elemente werden in den Vordergrund gerückt) } \\
\text { Selbstoffenbarende Wirkung (persönliche Charaktereigenschaften, } \\
\text { Erlebnisse und Hintergrunde zu privaten Ereignissen werden preis- } \\
\text { gegeben) }\end{array}$ \\
\hline
\end{tabular}


Tab. 15.1 (Fortsetzung)

\begin{tabular}{l|l}
\hline Sprechtempo & $\begin{array}{l}\text { Langsam (weniger als 90 Wörter/min) } \\
\text { Normal (90-120 Wörter/min) } \\
\text { Schnell (mehr als 120 Wörter/min) }\end{array}$ \\
\hline Sprechlautstärke & $\begin{array}{l}\text { Leise/unverständlich (minimale Ausschläge der Tonspur) } \\
\text { Normale Lautstärke (normale Ausschläge der Tonspur) } \\
\text { Erhöhte Lautstärke (große Ausschläge der Tonspur) } \\
\text { Lautes Sprechen (sehr große Ausschläge der Tonspur) } \\
\text { Schreien (Ausschlagspitzen sind nicht mehr innerhalb der angezeigten } \\
\text { Tonspur) }\end{array}$ \\
\hline Grußformeln & Vorhanden/nicht vorhanden \\
\hline
\end{tabular}

Dabei wurde die erhaltene Anzahl Codings pro Video durch die Länge des Videos geteilt, um vergleichbare Werte pro Minute zu erhalten und eine Verzerrung der Resultate auszuschließen. Damit Muster, Gemeinsamkeiten und Unterschiede festgestellt werden können, wurden sogenannte Codelines und andere Visual Tools von MAXQDA herausgezogen und analysiert. Für die Analyse des Sprechtempos wurden in jedem Video die gesprochenen Wörter in der ersten Minute des Videos (Unterbrüche werden nicht mitberechnet) gezählt.

\subsubsection{Ziel der Analyse}

Die visuelle und auditive Analyse sollte dazu dienen, Regelmäßigkeiten und Muster in visuellen sowie auditiven Dimensionen der 5 erfolgreichsten YouTuber Deutschlands festzustellen. Aus den analysierten Regelmäßigkeiten und Muster werden zusammen mit dem gesammelten theoretischen Wissen aus dem ersten Teil der Arbeit Stile abgeleitet und Fazits geschlossen.

\subsubsection{Auswertung der analysierten YouTube-Videos}

Die Auswertung bezieht sich auf die 18 analysierten Videos, welche nach dem Literaturverzeichnis aufgelistet sind.

Betonungsgestik Die am meiste benutzte Gestik in den analysierten Videos ist mit insgesamt 769-mal deutlich die Betonungsgestik. Diese wird von allen YouTubern häufig eingesetzt, um lange gesprochene Monologpassagen für den Zuschauer aktiver und spannender zu gestalten.

Zeigegestik Die Zeigegestik ist die am zweitmeiste angewendete Gestik der YouTuber. Sie ist in den 18 verschiedenen analysierten Videos insgesamt 231-mal deutlich sichtbar. Die Zeigegestik wird von den YouTuber jedoch nicht nur dazu verwendet, auf Produkte 
oder Dinge zu zeigen, sondern auch um die Zuschauer anzusprechen. Während der YouTuber mit dem Zeigefinger auf den Zuschauer deutet, fühlt sich dieser angesprochen und zu einer Handlung aufgefordert. Eine weitere Funktion der Zeigegestik, welche von den YouTuber genutzt wird, ist die Visualisierung von deiktischen Ausdrücken wie „dort“, „gestern“, „,vorhin“, ,morgen“, „gleich“ etc.

Berührungsgestik Die Berührungsgestik wird insgesamt 61-mal verwendet. Sie liegt häufig einer emotionalen Absicht zugrunde. Während beispielsweise Dagi Bee die Zuschauer dazu auffordert, dem Video einen Daumen nach oben zu geben, was eine Demonstrativgestik darstellt (dazu später mehr), legt sie ihren Arm um ihre kleine Schwester um Sympathie und Zuneigung zu symbolisieren. Sie wirkt somit beim Zuschauer automatisch sympathischer und menschlicher.

Demonstrativgestik Die Demonstrativgestik ist in den analysierten Videos insgesamt 68-mal vorzufinden und hat dabei verschiedene Funktionen: Am häufigsten wird die Demonstrativgestik dazu verwendet, eine aktivierende Haltung zu erzeugen. Dies erfolgt meistens bei der Aufforderung an die Zuschauer, das aufgeschaltete Video mit ,gefällt mir“ zu markieren. Dabei wird vom YouTuber mit der Hand oder mit den Händen ein Daumen nach oben gestikuliert. Ein weiterer Verwendungszweck der Demonstrativgestik ist das Visualisieren von Produktefunktionen oder Handhabungen. So nutzt Bianca Heinicke beispielsweise eine Demonstrativgestik, um einen vorgestellten Make-up-Artikel und seine Handhabung zu visualisieren und zu demonstrieren. Demonstrativgestik wird von den YouTubern analog zur Berührungsgestik für schauspielerische Zwecke eingesetzt.

Mimik „Freude“ Bei der Mimik fällt auf, dass die Emotion Freude mit 440-mal mit Abstand am meisten zu sehen ist. Freude wird mittels fröhlichen Gesichtsausdrucks, Lächeln oder Lachen vermittelt und löst beim Zuschauer ähnliche Gefühle aus. Während beim Zuschauen das Gefühlt entsteht, dass die Protagonistin oder der Protagonist glücklich ist, erhält man als Betrachter zudem das Gefühl, dass der YouTuber Freude bei dem hat, was er gerade tut. Ein Beispiel dafür ist die Mimik von Bianka Heinicke mit ihrem natürlich wirkenden Lächeln.

Ähnlich wie bei der Gestik, wird auch die Mimik von den YouTuber auf schauspielerische Weise genutzt. Ein Beispiel dafür sind verschiedene Szenen im Video von Julien Bam „Die ULTIMATIVE STORY 2“. Dabei fällt auf, dass beim schauspielerischen Ausdruck von Freude die Mimik oftmals übertrieben nachgeahmt wird.

Mimik ,Überraschung“ Mit 43-mal ist die Überraschung die am zweithäufigsten vorkommende Mimik. Sie kommt vorwiegend schauspielerisch zum Vorschein und wird dabei meistens übertrieben dargestellt. Eine Überraschungs-Mimik ist jedoch nicht nur schauspielerisch zu verstehen, sondern kommt auch auf natürliche Art und Weise vor. So beispielsweise bei Bianca Heinicke als ein Schrei im Hintergrund sie erschreckt. Gleich 
darauf wird dieser Moment der Überraschung und des Erschreckens nochmals in Zeitlupe gezeigt. Dabei zieht sich die YouTuberin selbst ins Lustige, was sie im Auge des Betrachters sympathisch wirken lässt.

Mimik ,Wut", ,Angst" und ,Trauer“" Während die Mimik zur Emotion Freude oftmals auf natürliche Weise in den Videos vorkommt, ist dies bei der Wut, Angst und Trauer weniger der Fall. Sie ist insgesamt nur wenige Male in den Videos aufzufinden. Dabei kommen sie fast ausschließlich auf schauspielerische Weise zum Vorschein. Auch hier wird analog zur schauspielerischen Weise der Freude die Mimik übertrieben und sorgt für eine eher belustigte und amüsierte Reaktion.

Mimik ,Verachtung“" und ,Ekel“" Eine verachtend oder ekelnd wirkende Mimik ist in den Videos nur sehr selten zu erkennen. Sie kommen entweder als schauspielerische Art und Weise vor oder treten unabsichtlich auf.

Kommunikative Wirkungsweisen In den 18 verschiedenen analysierten Videos wurde insgesamt 306-mal eine kommunikative Wirkungsweise codiert. Jedoch wurden keine unterstützende Wirkungsweise, keine emotional offene Wirkungsweise, keine distanzierte Wirkungsweise und keine ausgeglichene Wirkungsweise wahrgenommen. Dies wird damit begründet, dass die unterstützende Wirkung einen Dialog und nicht Monolog voraussetzt. Die YouTube-Videos sind häufig als Monolog zum Zuschauer aufgebaut, lassen also keine unterstützende Wirkungsweise zu. Es wurde festgestellt, dass verschiedene Emotionen, hauptsächlich Freude, fast durchgehend in den Videos vertreten sind. Die distanzierte Wirkung schließt voreilige emotionale Einbindungen aus, es wird angenommen, dass dies eine Art Widerspruch darstellen würde und somit nicht in den Videos vorkommt. Weiter wird vermutet, dass auch die ausgeglichene Wirkung, welche eine ruhige Herangehensweise voraussetzt, ein Widerspruch zum schnell gemessenen Sprechtempo und zur teilweise lauten Sprechlautstärke darstellen würde und deshalb nicht vorkommt. Auf die emotional offene Wirkungsweise wird später genauer eingegangen.

Selbstoffenbarende Wirkung Mit 117 Codierungen ist mehr als ein Drittel der Wirkungsweisen selbstoffenbarend. Die Videos mit der stärksten selbstoffenbarenden Wirkung sind jene, in denen die Influencer private Seiten von sich zeigen. In zwei dieser Videos stellen die YouTuber persönliche (Kindheits-)Fotos von sich vor und erzählen etwas dazu, was eine selbstoffenbarende Wirkung zur Folge hat. Eine weitere Form der vorkommenden selbstoffenbarenden Sequenzen ist die Frage-Antwort-Runde. Dabei wird unter dem Video eine gestellte Frage eines Abonnenten eingeblendet und beantwortet. Dadurch entsteht eine wertschätzende und anschließend selbstoffenbarende Wirkung. Die selbstoffenbarenden Wirkungen haben zur Folge, dass beim Zuschauer des Videos eine parasoziale Beziehung entsteht. Dadurch, dass der Protagonist persönliche und vergangene Erlebnisse teilt, sei dies alte Kindheitsfotos oder Geschichten über sein Privatleben, fühlt es sich an, als würde man eine Freundschaft mit dieser Person 
aufbauen, da das Austauschen solcher privaten Informationen normalerweise nur unter Freunden geschieht.

Aktivierende Wirkung Die aktivierende Wirkung spielt in den Videos eine sehr wichtige Rolle. Sie kommt insgesamt 89-mal vor und hat dabei fast immer eine Call-to-action-Funktion. Das heißt, der Zuschauer bzw. die Zuschauerin wird dazu aufgefordert das Video mit einem ,gefällt mir“ zu markieren, den Kanal zu abonnieren etc. Ein Beispiel dazu kommt im Video von ApeCrimeTV vor (siehe Zitat unten).

(V12, 8:34-8:42) „Ihr könnt das Ganze natürlich freischalten, eine neue magische Zahl: 44.444 Likes, dann bestellen wir das Ganze und machen ein Teil 2 würde ich sagen.“

Das Gesagte tritt dabei häufig im Zusammenhang mit Bild- oder Schrifteinblendungen und der Zeigegestik auf, was den aktivierenden Effekt zusätzlich verstärkt. Weiter fällt auf, dass viele der aktivierenden Wirkungen im Zusammenhang mit einer Aufforderung zur Interaktion vorkommen. So beispielsweise im Video von Bianca Heinicke:

(V3, 9:39-9:42) „Schreibt mir unbedingt in die Kommentare ...“

Dabei wird der Zuschauer dazu aufgefordert, einen Kommentar unter das Video zu schreiben, was wiederum die Interaktionsrate des Videos und somit auch des Kanals erhöht. Zudem wird eine wertschätzende Wirkung erzielt, da man als Zuschauer das Gefühlt vermittelt bekommt, dass die eigene Meinung und eine Anmerkung erwünscht und wertvoll sind.

Wertschätzende Wirkung Genau wie die aktivierende Wirkung, spielt auch die wertschätzende Wirkung unter den YouTubern eine wichtige Rolle. So ist sie in den analysierten Videos insgesamt 67-mal ersichtlich. Dabei hat sie verschiedene Erscheinungsformen: Eine davon ist das Angesprochenwerden der Zuschauenden durch den YouTuber. Ein gutes Beispiel dafür liefern Bianca Heinicke und Dagi Bee in ihren Videos. So tut dies Bianca Heinicke beispielsweise fast immer, wenn sie ihre Zuschauer auf folgende Weise begrüßt:

(V1, 0:00-0:02) „Halli-hallo meine Lieben und willkommen zu meinem neuen Video ...“

Wertschätzung beim Zuschauer entsteht einerseits durch die Begrüßung selbst und andererseits durch die Worte ,meine Lieben“ und ,willkommen“. Eine andere Form der wertschätzenden Wirkung ist das Einblenden von geschriebenen Kommentaren. Dies kommt beispielsweise im Video „Heyju - Longboard Tour - Tag 5“ von Julien Bam oder „SO habe Ich meinen Freund kennengelernt Dagi Bee vor. Dabei wird ein Kommentar im Video eingeblendet und anschließend direkt vom YouTuber beantwortet. Als Zuschauer erhält man dabei ein wertschätzendes Gefühl, da man sich wahrgenommen, ernstgenommen und wichtig fühlt. Eine weitere Form der Wertschätzung ist in einem 
Beispiel von Simon Desue im Video“ „Der CHILLI Prank! - Revanche an Shirin David“ zu finden, wobei er Folgendes sagt:

(V13, 3:58-4:02) „Die coolsten werden wir auf jeden Fall liken, wir werden das meiste von euch angucken, definitiv ..."

Er verspricht hierbei, die geteilten Beiträge auf Instagram und Twitter unter dem Hashtag „FANTA86“ anzuschauen und zu liken. Dabei entstehen einerseits eine aktivierende Wirkung und andererseits eine wertschätzende Wirkung beim Zuschauer.

Autoritäre Wirkung In den angeschauten Videos ist insgesamt nur 6-mal eine autoritäre Wirkungsweise zu erkennen. Diese scheint in diesen Videokreisen eher eine sekundäre Rolle zu spielen.

Informative Wirkung Die informative Wirkung kommt insgesamt 13-mal vor. Alle informativen Wirkungsweisen mit Ausnahme von 2-mal kommen im Video „10 WHATS APP FACTS!“ von ApeCrime vor. Wie der Name des Videos bereits verrät, besteht der Inhalt dieses Videos darin, dass 10 Fakten/Informationen über die App „WhatsApp“ vorgestellt werden.

Zuversichtliche Wirkung Ähnlich wie bei der informativen Wirkung kommt auch die zuversichtliche Wirkung ausschließlich in einem einzigen Video vor. Diese entsteht im Zusammenhang mit einer selbstoffenbarenden Wirkung, als Dagi Bee in ihrem Video „SO habe Ich meinen Freund kennengelernt“" von ihrem neuen Onlineshop erzählt, wie im folgenden Beispiel zu sehen ist.

(V7, 8:07-8:09) ,es wird auf jeden Fall mega cool ...“

Verantwortliche und zielorientierte Wirkung Diese beiden Wirkungsweisen kommen insgesamt nur 3-mal vor. Es wird vermutet, dass der Grund für das seltene Vorkommen der lockere und nicht ernste Inhalt der Videos ist. Die zielorientierte Wirkung setzt hingegen einen Dialog voraus. Da die Videos meistens in Monolog-Form abgehalten sind, ist dies vermutlich der Grund, dass diese Wirkungsweise nicht oft vorkommt.

Grußformeln Mit Ausnahme von 3 Videos kommt überall eine Begrüßung der Zuschauenden vor, wobei sie unter den verschiedenen YouTubern sehr unterschiedlich ausfällt. Es fällt auf, dass einige YouTuber ihre Zuschauer immer auf dieselbe Art und Weise begrüßen. So macht dies beispielsweise Bianca Heinicke in allen drei Videos auf folgende Art:

(V1, 0:00-0:02) „Halli-hallo meine Lieben und willkommen zu meinem neuen Video ...“ 
Die Begrüßung schein immer auf die Zielgruppe abgestimmt zu sein. So wirkt die Begrüßung von Julien Bam lockerer und trendiger:

(V5, 0:02-0:04) „Jo Leute! Was geht? Willkommen ...“

Grundsätzlich fällt im Zusammenhang mit der Begrüßung auf, dass viel Gestik verwendet und tendenziell schneller und lauter gesprochen wird. Dies ist auch bei der Verabschiedung nicht anders. Diese kommt ähnlich wie die Begrüßung in jedem bis auf einem Video vor. Dabei hat jeder seine eigene Art, das Video zu beenden. Auch hier ist wieder der Unterschied zwischen männlichem und weiblichem YouTuber zu sehen. Während Dagi Bee eine lange und wertschätzende Verabschiedung macht, ist diese bei Simon Desue eher kurz und wirkt trendiger. Allgemein fällt bei der Verabschiedung auf, dass bei 14 von 18 Videos während oder direkt anschließend an die Verabschiedung mittels Bild- und Schrifteinblendungen oder Erwähnung auf andere Videos, einen anderen Kanal, einen Shop etc. verwiesen wird, was in einer aktivierenden Wirkung resultiert.

Sprechlautstärke Insgesamt kommt in den analysierten Videos 304-mal eine nach oben abweichende Lautstärke und 2-mal eine nach unten abweichende Lautstärke zur normalen Sprechlautstärke vor. Dabei wird 58-mal geschrien, 148-mal laut gesprochen, 154 mit einer erhöhten Lautstärke gesprochen und 2-mal leise und unverständlich gesprochen.

Sprechtempo Beim Sprechtempo fällt auf, dass in fast allen Videos in Dialogszenen über 180 Wörter pro Minute gesprochen werden. Einige YouTuber sprechen sogar mehr als 200 Wörter pro Minute. Wird als Referenzwert der Wert von Rhetores genommen, welcher mehr als 120 Wörter pro Minute als schnelles Sprechen definiert, sprechen alle YouTuber überdurchschnittlich schnell (Rhetores - Akademie der Sprecher).

\subsubsection{Ersichtliche Muster}

Grundsätzlich kann gesagt werden, dass in den analysierten Videos immer ungefähr dieselben Wirkungsmechanismen zum Vorschein kommen. So kommt häufig eine selbstoffenbarende, wertschätzende und aktivierende Wirkung vor, jedoch fast nirgendwo eine zuversichtliche, unterstützende, verantwortliche, emotional offene, zielorientierte, distanzierte, ausgeglichene oder autoritäre Wirkung. Auch bei der Mimik ist ein solches Muster zu erkennen. Während Freude in jedem Video und Überraschung in der Mehrheit der Videos vorzufinden sind, kommen Wut, Ekel, Angst, Trauer und Verachtung nur sehr selten vor. Bei der Gestik kommt zwar die Betonungsgestik mit Abstand am meisten vor, jedoch erscheinen auch Demonstrativ-, Zeige- und Berührungsgesten fast überall. Weiter ist ersichtlich, dass die Betonungsgestik in den meisten Videos, das am häufigsten vorkommende Analysekriterium darstellt. Ein weiteres Muster ist dahin gehend erkennbar, dass in den Videos oftmals eine kommunikative Wirkungsweise verstärkt vorkommt. 
Abb. 15.2 Ausschnitt Codeline „Haul“. (Eigene Darstellung)

Freude

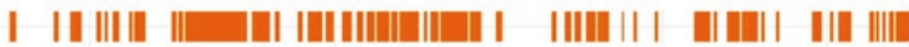

Abb. 15.3 Ausschnitt Codeline „Geschwister Battle“. (Eigene Darstellung)

So beispielsweise im Video „Meine PEINLICHSTEN TEENIE - FOTOS“ die selbstoffenbarende Wirkung, im Video „SO habe Ich meinen Freund kennengelernt“ die wertschätzende Wirkung und im Video „10 WHATS APP FACTS!“ die informative Wirkung. Schaut man die Codelines an, werden weitere Muster erkennbar: Beispielsweise, dass die aktivierende Wirkung vermehrt am Ende der Videos vorkommt, wie dies ein Beispiel von Bianca Heinicke in Abb. 15.2 visualisiert.

Weiter ist ersichtlich, dass die Mimik „Freude“ bei fast allen über das ganze Video verteilt vorkommt, gut erkennbar beim Video von Dagi Bee (siehe Abb. 15.3).

Dasselbe gilt für die Betonungsgestik, jedoch ist hier eine leichte Tendenz am Anfang und Ende der Videos feststellbar. Auch bei der Sprechlautstärke ist eine Tendenz Richtung Anfang und Ende der Videos zu erkennen; so tritt die erhöhte Lautstärke oder das laute Sprechen auch vermehrt mit der aktivierenden Wirkung auf. Eine weitere Beobachtung war, dass Zahlen sowie die Aufforderung ein Video mit „Gefällt mir“ zu markieren häufig im Zusammenhang mit Demonstrativgesten vorkommen.

\subsubsection{Gemeinsamkeiten}

In den analysierten Videos konnten mithilfe von MAXQDA verschiedene Gemeinsamkeiten festgestellt werden. Diese leiten sich direkt aus den vorgängig beschriebenen Mustern ab. Fast in jedem Video werden die Zuschauer und Abonnenten am Anfang herzlich willkommen geheißen und am Ende des Videos verabschiedet. Es ist auffallend, dass bei der Begrüßung häufig die Wörter ,ihr Lieben“ vorkommt. Beim Abschied ist auch gleich die zweite Gemeinsamkeit zu sehen: Dort fällt auf, dass der Abschied häufig mit einer aktivierenden Wirkung verbunden wird. So werden die Zuschauer aufgefordert, andere Videos, andere Kanäle anzuschauen oder das Video mit einem „Gefällt mir“ zu markieren. Die dritte Gemeinsamkeit besteht darin, dass in den Videos durchgehend sehr stark mit Betonungsgestik gearbeitet wird. Diese wird dabei häufig während des ganzen Videos intensiv verwendet. Als vierte Gemeinsamkeit zählt die Mimik „Freude“. Diese ist in fast ausnahmslos jedem Video sehr stark vertreten: Sei dies durch schauspielerische Tätigkeit oder durch natürliches Lächeln oder Lachen bei vlog-artigen Sequenzen. Auffällig ist, dass diese Mimik (ausgenommen die schauspielerische Mimik) immer sehr 
natürlich und authentisch wirkt. Ein weiteres gemeinsames Muster stellt die Tendenz der erhöhten Lautstärke oder des lauten Sprechens am Anfang und vor allem am Ende der Videos dar. Wie bereits angedeutet, haben die Videos die aktivierende Wirkung am Ende der Videos gemeinsam. Diese kommt häufig in Form von Grußformeln, jedoch aber auch in Form von Text- und Bildeinblendungen vor. Bei der kommunikativen Wirkungsweise ist eine weitere Gemeinsamkeit, dass von den YouTubern eine Wirkungsart fokussiert wird. So beispielsweise die informative Wirkung im Video „10 WHATS APP FACTS!“, die selbstoffenbarende Wirkung in den Videos mit den peinlichen Fotos und die wertschätzende Wirkung in den Videos mit den beantworteten Zuschauerfragen. Als weitere Gemeinsamkeit kann das schnelle Sprechtempo gezählt werden. In ausnahmslos allen analysierten Videos wird wesentlich schneller als 120 Wörter pro Minute gesprochen, was aufgrund vordefinierten Referenzwerten einem sehr schnellen Sprechtempo entspricht. Schaut man das Gesamtbild an, ist zu erkennen, dass immer dieselben Analysekriterien anzutreffen sind. So werden von den YouTubern die selbstoffenbarende, die wertschätzende und die aktivierende Wirkung immer wieder verwendet. Um diese Wirkungen zu erzielen, werden häufig dieselben Mittel verwendet: Beispielsweise peinliche Fotos oder Kindheitsfotos um eine selbstoffenbarende Wirkung zu erzielen, die Einblendung und Beantwortung von Zuschauerkommentaren um eine wertschätzende Wirkung zu erzielen und die Möglichkeit, mit einer gewissen Anzahl „Gefällt mir“ weitere Videos freizuschalten, um die aktivierende Wirkung zu erzielen. Weiter fällt auf, dass beispielsweise die Aufforderung das Video mit „Gefällt mir“ zu markieren häufig mit einem gestikulierten Daumen nach oben und Zahlen häufig mit der Anzahl aufgesteckter Finger visualisiert werden, was einer Demonstrativgestik entspricht.

Auch die erhöhte Sprechlautstärke und das laute Sprechen stellen immer wieder vorkommende Wirkungsmechanismen in Mittelteilen der Videos dar und können als Gemeinsamkeit gewertet werden. Alle übrigen Analysekriterien wurden in den analysierten Videos stark vernachlässigt, was gleichzeitig eine letzte Gemeinsamkeit darstellt.

\subsubsection{Unterschiede}

Unterschiede wurden bei der Begrüßung und Verabschiedung vor allem in der Art und Weise, wie diese umgesetzt werden, festgestellt, da einige YouTuber dies mittels persönlicher Ansprache und andere mittels Einblendungen machen. Auch bei der Gestik liegen die Unterschiede hauptsächlich darin, wie die Gestik aussieht und nicht ob sie gemacht wird. So sieht beispielsweise die Betonungsgestik bei jeder Person etwas anders aus. Weiter wurden Unterschiede in der Konstanz der verschiedenen YouTuber festgestellt. Bei einigen YouTubern, wie beispielsweise Bianca Heinicke, ist starke Konstanz darin erkenntlich, dass die Zuschauer immer persönlich begrüßt und verabschiedet werden. Dagegen wird dies bei ApeCrime sehr unterschiedlich gemacht. Einer der größten Unterschiede liegt darin, dass die Inhalte der Videos sehr verschieden sind, was jedoch nicht 
Teil der definierten Analysekriterien darstellt. So sind beispielsweise die Videos bei ApeCrime eher schauspielerisch aufgebaut, hingegen diejenigen bei BibisBeautyPalace und Dagi Bee eher vlog-mäßig.

\subsection{Fazit, Reflexion und Ausblick}

Das Ziel der Ausarbeitung war es, mittels Recherche und visueller und auditiver Analyse von YouTube-Videos der erfolgreichsten deutschen Social Influencer herauszufinden, welche Faktoren dazu führen, dass Social Influencer Communities von mehreren Tausend bis Millionen Menschen aufbauen, durch diese als Vorbilder und Meinungsmacher angesehen werden und dabei ein Beziehungsverhältnis entsteht. Dabei wurden die Videos anhand visueller und auditiver Analysekriterien nach deren Muster, Gemeinsamkeiten und Unterschieden untersucht. Anschließend wurden die Wirkungsmuster ausgewertet und die erkenntlichen Gemeinsamkeiten und Unterschiede festgehalten.

Es wurde herausgefunden, dass die kommunikativen Wirkungsweisen, die Gruß- und Abschiedsformel, die Mimik, die Gestik sowie die Sprechlautstärke und das Sprechtempo Stile der Social Influencer auf YouTube darstellen, mit denen sie eine parasoziale Beziehung mit den Zuschauern aufbauen. Es zeigte sich, dass die selbstoffenbarende, die wertschätzende und die aktivierende Wirkung bei den kommunikativen Wirkungsweisen am wichtigsten sind. Die selbstoffenbarende Wirkung ist ein wichtiger Bestandteil für die Entstehung einer parasozialen Beziehung des Zuschauers zum YouTuber, da man diesen dadurch besser kennenlernt, wie dies beispielsweise mittels Zeigen von Kindheitsfotos passiert. Es entsteht eine sogenannte, ,illusion of face-to-face relationship“. So fühlt sich der Zuschauer direkt vom YouTuber angesprochen, obwohl dieser eigentlich zu einer breiten Masse spricht (Diehl 2018).

Dies stützt zugleich auch die erste Hypothese. Die Gruß- und Abschiedsformeln verstärken dabei diesen Effekt. Auch die häufig vorkommende wertschätzende Wirkung hat darauf einen wichtigen Einfluss. Sie wird beispielsweise damit erzielt, dass in den Videos Kommentare der Zuschauer eingeblendet werden, diese dabei namentlich erwähnt werden, und ihre Fragen im Anschluss beantwortet werden. Eine Interaktion zwischen YouTuber und Zuschauer entsteht, wobei diese jedoch sehr asymmetrisch ist, da der YouTuber meistens nicht in der Lage ist, alle Reaktionen und Kommentare zu beobachten und zu beantworten. Das schmälert aber nicht den Effekt bei den Zuschauern (Diehl 2018). Durch die aktivierende Wirkung erhält der Zuschauer das Gefühl einflussreich zu sein. Diese Wirkung wird häufig mittels Aufforderung für ein „Gefällt mir“ des Videos, ein Abo des Kanals oder Partner-Kanals oder eines Kommentars erreicht. So können die Zuschauer dabei mit einer gewissen Anzahl an „Gefällt mir“ weitere Videos freischalten und haben somit einen Einfluss auf das Handeln der YouTuber. Dies stützt auch wieder die erste Hypothese.

Weiter wurde festgestellt, dass die Mimik „Freude“ einen oft vorkommenden Stil darstellt. Durch die Mimik „Freude“, also dem Lachen oder fröhlichen Gesichtsausdruck, entsteht auch beim Zuschauer ein Gefühl von Freude, denn: „Beobachten wir Mitmenschen 
beim Lachen, aktivieren sich im Gehirn die Regionen, die aktiv sind, wenn wir selbst lachen. Das bereitet uns darauf vor mitzulachen“ (Papousek 2016). Es entstehen also Glückgefühle beim Schauen der YouTube-Videos der Influencer. Dabei stützt dies indirekt die dritte Hypothese. Die Gestik verleiht dabei dem Gesprochenen Lebendigkeit und Überzeugungskraft und lässt den YouTuber kompetenter und glaubwürdiger erscheinen. Laut einer Studie vom Institut für Demoskopie kann nämlich die Körpersprache gesprochenen Text wesentlich beeinflussen und so beispielsweise auch weniger überzeugende Inhalte überzeugend wirken lassen (Institut für Demoskopie Allensbach 2006).

Bei der Analyse wurde herausgefunden, dass alle untersuchten Influencer gemäß Rhetores ein schnelles Sprechtempo aufweisen (Rhetores - Akademie der Sprecher). Das schnelle Sprechtempo trägt dazu bei, dass die YouTuber kompetenter, sympathischer und interessanter wahrgenommen werden (Giles 1994). Es hinterlässt einen positiv wertenden Eindruck beim Zuhörer (Sendlmeier 2012). Die zweite Hypothese wird also an diesem Punkt gestützt. Weiter wurde ersichtlich, dass lautes Sprechen ein oft vorkommender Stil ist. Wie das schnelle Sprechen, hat auch das laute Sprechen eine positive Wirkung auf die Zuhörer, denn der Psychologe Howard Giles bewies, dass Menschen, die schnell und laut sprechen, als kompetenter, sympathischer, klüger, besser aussehend und interessanter wahrgenommen werden (Giles 1994), was gerade am Anfang und Ende eines Videos für den ersten und letzten Eindruck wesentlich ist. Was abschließend die zweite aufgestellte Hypothese stützt.

In der Theorie wurde deutlich, dass die Interaktion mit der Community ein wichtiger Aspekt für das Entstehen einer parasozialen Beziehung darstellt. Dies wurde im empirischen Teil anhand von Beispielen gezeigt. Durch das Aufgreifen und Beantworten der Zuschauerkommentare wurde einerseits eine wertschätzende und andererseits eine selbstoffenbarende Wirkung erzielt. Beides trägt zum Entstehen einer solchen Beziehung bei. Weiter besagt die Theorie, dass das Storytelling der Influencer die Verbindung zwischen der Marke oder dem Produkt und dem Konsumenten schafft. Storytelling stellt also eine Verbindung her, welche allein durch das Unternehmen nicht machbar wäre. Vergleicht man diese Aussage mit den analysierten Dimensionen, kann man durchaus sagen, dass die selbstoffenbarende Wirkung als Storytelling gewertet werden kann. Diese Wirkungsweise wurde insgesamt 117-mal codiert, hat also durchaus seine Wichtigkeit in den 18 analysierten Videos. Im Zusammenhang mit dem Storytelling und der Identifikation mit dem Influencer wird zusätzlich die Authentizität in der Theorie stark gewichtet. Auch hier kann wieder die selbstoffenbarende Wirkungsweise als Beispiel betrachtet werden. Dadurch, dass die Influencer in den Videos mittels Kindheitsfotos, persönlicher Erfahrungen und Geschichten etliches von sich selbst preisgeben, entsteht gegenüber den Zuschauern eine Glaubwürdigkeit und Authentizität.

Für weitergehende Forschungsarbeiten könnten andere Analysedimensionen in die Forschung integriert werden, wie bspw. Kameraperspektive, Licht, Aussehen des Protagonisten und Inhalte der Videos und das Nachholen der Analyse der emotional-offenen Wirkung. Zudem wäre es interessant, genauer zu erforschen, warum die selten vorkommenden Analysekriterien von den Influencern nicht oder nur selten eingesetzt werden. 
Es wäre zudem spannend zu untersuchen, welche der analysierten Stile absichtlich eingesetzt werden und welche nicht. Zu einem späteren Zeitpunkt könnte ein Vergleich zwischen erfolgreichen und weniger erfolgreichen Influencern interessant sein.

Da das Konzept „Social Influencer“ aus zwei Parteien besteht und in dieser Arbeit nur jene der Social Influencer selbst untersucht wurde, wäre es interessant, die Seite der Fans, Abonnenten und Zuschauer besser zu untersuchen. Es stellt sich beispielsweise die Frage, ob die Zielgruppe der Influencer diese als Vorbild sehen, weil diese Charaktereigenschaften aufweisen, welche sie bei sich selbst unterdrücken oder gerne hätten. So bieten sich auch in Zukunft vielfältige Forschungsmöglichkeiten in diesem Gebiet. Dies gerade deshalb, weil vermutet wird, dass das Thema Social Influencer auch in Zukunft aktuell sein wird, da immer mehr Unternehmen darauf aufmerksam werden und sich diesen Marketingkanal zunutze machen wollen.

\section{Literatur}

Affiliate Deals. (2016). Mit diesen 50 Tipps zum YouTube-Experten - Wir zeigen Dir wie's geht. https://www.affiliate-deals.de/mit-diesen-50-tipps-zum-youtube-experten-wir-zeigen-dir-wiesgeht/. Zugegriffen: 26. März 2018.

Anja Linnenbürger, C. G. (2018). Psychologische Diagnostik durch Sprachanalyse. PRECIRE Technologies. Wiesbaden: Springer Fachmedien.

Barker, S. (2017). Why is influencer marketing better than celebrity endorsements? https://shanebarker.com/blog/influencer-marketing-celebrity-endorsements/. Zugegriffen: 29. März 2018.

careerloft. (30. November 2016). Grußformeln und ihre versteckte Botschaft. https://www.youtube.com/watch?v=DpP0FVJOsAI. Zugegriffen: 30. März 2018.

Casellas, S. (31. März 2017). Mit Der Marke Zum Erfolg - Storytelling Durch Influencer. (Tubesights, Hrsg.). https://tubesights.de/storytelling-influencer/. Zugegriffen: 30. März 2018.

Celie O'Neil-Hart, H. B. (2016). Why YouTube stars are more influential than traditional celebrities. (Google). https://www.thinkwithgoogle.com/consumer-insights/youtube-stars-influence/. Zugegriffen: 10. Apr. 2018.

Connolly, B. (2018). Why consumers follow, listen to, and trust influencers. http://www.olapic. com/resources/consumers-follow-listen-trust-influencers_article/. Zugegriffen: 20. März 2018.

Diehl, J. (Januar 2018). Parasoziale Beziehung: Das Verhältnis zur Community. (L. Shareman, Hrsg.). https://luckyshareman.com/blog/parasoziale-beziehung-das-verhaeltnis-zur-community/. Zugegriffen: 2. Apr. 2018.

Donati, M. K. (1. Mai 2017). Internetstars lösen Prominente ab. (S. Schweiz, Hrsg.). https://www. srf.ch/news/panorama/internetstars-loesen-prominente-ab. Zugegriffen: 8. Apr. 2018.

Faltl, \& Freese, M. (April 2017). Influencer Marketing - Evolution, Chancen und Herausforderungen der neuen Komponente im Kommunikationsmix. (Schweizerische Gesellschaft für Marketing). https://issuu.com/gfm-swiss-marketing/docs/a17-1202_forschungsbrosch_re_417. Zugegriffen: 15. März 2018.

Firsching, J. (16. Juni 2017). Der rasante Aufstieg von Influencer Marketing...geht immer weiter. (Futurebiz.de, Hrsg.). http://www.futurebiz.de/artikel/der-rasante-aufstieg-von-influencer-marketing-geht-immer-weiter-infografik/. Zugegriffen: 20. März 2018.

Giles, H. (1994). Human communication research. (Wiley-Blackwell, Hrsg.) Santa Barbara.

Glück, H. (2010). Metzler Lexikon Sprache. (G. Helmut, Hrsg.) Heidelberg: Metzler. 
Haines, R. (2014). Celebrity vs. social influencer. (H. Post, Hrsg.). https://www.huffpost.com/ entry/celebrity-vs-social-influ_b_6167776. Zugegriffen: 18. März 2018.

Hitz, L. (2018). How to work with influencers and celebrities on social media. (Sproutsocial, Hrsg.). https://sproutsocial.com/insights/how-to-work-with-influencers-on-social-media/. Zugegriffen: 29. März 2018.

Hugi, A. (März 2018). «nfluencer» Die umstrittenen Werbeträger der heutigen Jugend. (SRF, Hrsg.) Schweiz.

Institut für Demoskopie Allensbach. (2006). Welchen Anteil haben Text, Erscheinungsbild des Redners, Betonung und Gestik an der Gesamtwirkung eines Vortrags?. Mainz: VRdS.

Kamal, R. (2018). «Ifluencer» : Die umstrittenen Werbeträger der heutigen Jugend. (SRF, Hrsg.) Schweiz.

Kiener, U. (Januar 2018). Influencer: Idol oder Manipulator? (B. Lukesch, Interviewer, \& Sanitas, Hrsg.).

Koschig, T. (26. März 2016). Influencer Marketing - Definition und Anwendung. (Konsum-Marketing.de, Hrsg.). https://www.konsum-marketing.de/social-media/influencer-marketing/. Zugegriffen: 5. Apr. 2018.

Kuch, B. E. (19. Juni 2013). Bildkommunikation: Das Ende der Sprache im Social Web. Berlin: Springer.

Möhring, \& Schlütz, W. (2010). Die Befragung in der Medien- und Kommunikationswissenschaft (2. Aufl. Ausg.). Wiesbaden: VS Verlag.

Mai, J. (24. April 2013). Authentizität: Die Kunst authentisch zu sein. (K. Bibel, Hrsg.). https:// karrierebibel.de/authentizitat/. Zugegriffen: 29. März 2018.

Marco Nirschl, L. S. (2018). Einstieg in das Influencer Marketing. Wiesbaden: Springer Gabler.

Noah Zygmont, M. S. (25. April 2017). Influencer: Du empfiehlst, ich kaufe. (S. Schweiz, Hrsg.). https://www.srf.ch/news/wirtschaft/influencer-du-empfiehlst-ich-kaufe. Zugegriffen: 15. März 2018.

noGRE. (2017). Evolution of influencers. https://nogre.com/evolution-of-influencers/. Zugegriffen: 23. März 2018.

„,Wenn ich eine Frau wäre, wäre ich Beauty-Blogger"-LeFloid im Interview. (2014). https://www. vice.com/de_ch/article/5g438a/interview-mit-lefloid-wenn-ich-eine-frau-waere-waere-ichbeauty-blogger-058. Zugegriffen: 6. Apr. 2018.

Papousek, p. I. (2016). Warum ist Lachen ansteckend? (A. Grancy, Interviewer, \& D. Presse, Hrsg.)

Perry, J. (2017). Influencer Interview. (D. Brainstorms, Hrsg.). http://dailybrainstorms. com/2017/06/08/influencer-interview/. Zugegriffen: 15. März 2018.

Rothe, S. W. (2011). Die 7 Grundemotionen als Micro Expressions auf einen Blick. (P. Popkultur, Hrsg.). http://www.pantomime-popkultur.de/2011/09/die-7-grundemotionen-als-micro-expressions-auf-einen-blick/. Zugegriffen: 2. Apr. 2018.

Sendlmeier, P. D. (2012). Die psychologische Wirkung von Stimme und Sprechweise. (I. f. Kommunikationswissenschaft, Hrsg.) Berlin.

Statista - The Statistics Portal. (2018). https://www.statista.com/statistics/272014/global-socialnetworks-ranked-by-number-of-users/. Zugegriffen: 26. März 2018.

Statista - Das Statistik Portal. (2015). Statistiken zu ProSieben. https://de.statista.com/themen/2649/prosieben/. Zugegriffen: 24. März 2018.

Statista.de. (2016). Umfrage zur Glaubwürdigkeit von Marketing-Instrumenten in Deutschland 2016. https://de.statista.com/statistik/daten/studie/686056/umfrage/glaubwuerdigkeit-von-marketing-instrumenten-in-deutschland/. Zugegriffen: 27. März 2018. 
Visscher, A. (1998). Freunde in guten und schlechten Zeiten. Parasoziale Beziehungen von Vielsehern zu Charakteren einer Daily Soap. (V. V. Sozialwissenschaften, Hrsg.) Opladen: V. V. Sozialwissenschaften.

Widrich, L. (29. November 2012). The science of storytelling: What listening to a story does to our brains. (Buffer, Hrsg.). https://buffer.com/resources/science-of-storytelling-why-telling-a-storyis-the-most-powerful-way-to-activate-our-brains. Zugegriffen: 8. März 2018.

zhaw/Swisscom. (2016). www.zhaw.ch/psychologie/JAMES. (Z. Z. Psychologie, Hrsg.). https:// www.zhaw.ch/de/psychologie/forschung/medienpsychologie/mediennutzung/james/\#c77096. Zugegriffen: 13. März 2018.

\section{Weiterführende Literatur}

André Ruch, C. B. (16. Oktober 2017). Wie Influencer Schleichwerbung betreiben - noch. (SRF Schweiz). https://www.srf.ch/news/wirtschaft/bezahlte-produkt-platzierungen-wie-influencerschleichwerbung-betreiben-noch. Zugegriffen: 2. Apr. 2018.

Bam, J. (2018). (Instagram, Hrsg.).

Bauer, T. (28. Juli 2017). Die Follow-Unfollow-Strategie: Es gibt bessere Alternativen auf Instagram. (onlinemarketing.de, Hrsg.). https://onlinemarketing.de/news/follow-unfollow-mehr-follower-auf-instagram. Zugegriffen: 6. Apr. 2018.

Bea, F. X, \& Schweitzer, M. (2011). Allgemeine Betriebswirtschaftslehre (10. Aufl.). Stuttgart u. a.: UVK Lucius.

Bee, D. (2018). (Instagram, Hrsg.).

Boeing, N. (2017). Grossmacht des bewegten Bildes. Schweiz: NZZ.

Buchmann, P. (13. Oktober 2017). Magische Anziehungskräfte. (S. Schweiz, Hrsg.). https://www. srf.ch/sendungen/digital-plus/magische-anziehungskraefte. Zugegriffen: 10. Apr. 2018.

David Wagner, J.-M. S. (2016). Auf dem Weg zur vernetzten Organisation. Wiesbaden: Springer.

Dietrich, M. (2018). (Instagram, Hrsg.).

Für-Gründer.de. (kein Datum). Für-Gründer.de. https://www.fuer-gruender.de/wissen/unternehmen-fuehren/marketing/pr/social-media-plattformen/. Zugegriffen: 2. Apr. 2018.

Facebook. (2017). Facebook newsroom. https://newsroom.fb.com/company-info/. Zugegriffen: 10. März 2018.

Farooq, N. (2013). So wird man zum YouTube-Star. (D. Schurter, Interviewer, \& 2. Minuten, Hrsg.) Schweiz: 20 Minuten.

FOCUS Online/Wochit. (4. Januar 2017). Bibi, Caro, Pamela: So viel Geld verdienen die Social-Media-Stars. https://www.focus.de/finanzen/videos/mehr-als-die-meisten-im-monat-bekommen-bibisbeautypalace-und-caro-daur-so-viel-geld-verdienen-social-media-stars_id_6446022. html. Zugegriffen: 15. Apr. 2018.

Futurebiz. (2013). Futurebiz. http://www.futurebiz.de/artikel/warum-das-neue-facebook-logoauch-fur-marken-relevant-ist/. Zugegriffen: 28. März 2018.

Gabirano. (2. Februar 2017). Der Instagram-Star von nebenan. (M. Bürki, Interviewer, \& B. B. Zeitung, Hrsg.) Berner Zeitung.

Instagram. (2018). Instagram-Brand. https://en.instagram-brand.com/assets/glyph-icon. Zugegriffen: 27. März 2018.

investor.fb. (2018). Facebook Reports Fourth Quarter and Full Year 2017 Results. https://investor. fb.com/investor-news/press-release-details/2018/Facebook-Reports-Fourth-Quarter-and-FullYear-2017-Results/default.aspx. Zugegriffen: 4. Apr. 2018. 
Jean Burgess, J. G. (2013). YouTube: Online video and participatory culture (1. Aufl. Ausg.). (Polity, Hrsg.) Kansas: Polity.

Julian Kutzim, S. S. (12. Dezember 2017). Geschäftsmodel Influencer. (F. Online, Hrsg.). https:// www.focus.de/finanzen/news/neues-geschaeftsmodell-mode-macher-in-panik-so-mischen-instagram-girls-die-branche-auf_id_6520373.html. Zugegriffen: 10. März 2018.

Kanter, J. (2017). How to become famous on Instagram? https://gettinggrowth.com. Zugegriffen: 30. März 2018

Kreisblatt, H. (Hrsg.). (2018). Jugendzimmer werden zu Filmstudios: IT-Experte erklärt YouTube. https://www.haller-kreisblatt.de/lokal/steinhagen/22068893_Jugendzimmer-werden-zu-Filmstudios-IT-Experte-erklaert-YouTube.html. Zugegriffen: 5. Apr. 2018.

Kristy Sammis, C. L. (2015). Influencer marketing for dummies. (J. W. inc., Hrsg.) New Jersey.

Leahy, J. (2012). Social influence marketing and the brand-influencer-fan connection. (Engauge, Hrsg.). https://angelbc.files.wordpress.com/2013/08/engauge-enfluence.pdf. Zugegriffen: 20. März 2018.

Moreau, E. (2018). Lifewire. https://www.lifewire.com/what-is-instagram-3486316. Zugegriffen: 20. März 2018.

Oliver Kreuzer, S. L. (2014). Filmanalyse. (R. M. Oksana Bulgakowa, Hrsg.) Wiesbaden: VS Verlag.

Olsson, J. (2018). (Instagram, Hrsg.).

Onlinevideokonverter. (kein Datum). https://www.onlinevideoconverter.com/de. Zugegriffen: 5. Apr. 2018.

Paul, A. M. (2012). Your Brain on Fiction. (N. Y. Times, Hrsg.). https://www.nytimes. com/2012/03/18/opinion/sunday/the-neuroscience-of-your-brain-on-fiction.html. Zugegriffen: 10. Apr. 2018.

Pfitzinger, H. R. (März 2001). Phonetische Analyse der Sprechgeschwindigkeit. (I. f.-M.-U. München, Hrsg.) München.

Prof. Dr. Julian Kawohl, U. N. (2016). Strategien Digitaler Superstars- wie die erfolgreichsten Instagram-Blogs funktionieren. Berlin.

ProSieben. (2018). Bianca "Bibi" Heinicke. https://www.prosieben.ch/stars/star-datenbank/bianca-bibi-heinicke. Zugegriffen: 22. März 2018.

ProSieben. (2018). Dagi Bee. https://www.prosieben.ch/stars/star-datenbank/dagi-bee. Zugegriffen: 22. März 2018.

Rashid, B. (10. Juni 2017). 15 Top Instagram influencers you should follow. https://www.forbes.com/sites/brianrashid/2017/06/10/15-top-instagram-influencers-you-should-follow/. Zugegriffen: 10. März 2018.

Rechneronline. (kein Datum). Abgerufen am 30. März 2018 von https://rechneronline.de/zufallszahlen/. Zugegriffen: 30. März 2018.

Rhetores - Akademie der Sprecher. (29. März 2018). Sprechtempo. http://www.rhetores.de/footer/ glossar/sprechtempo/ abgerufen

Rusli, E. M. (9. April 2012). Facebook buys Instagram for \$1 Billion. NY Times.

Schärer, K. (2017). Diese Menschen waren Influencer - Lange vor Social Media. Schweiz.

Schärer, K. (13. Oktober 2017). Diese Menschen waren Influencer - lange vor Social Media. (S. Schweiz, Hrsg.). https://www.srf.ch/radio-srf-virus/aktuell/influencer-diese-menschen-waren-influencer-lange-vor-social-media. Zugegriffen: 2. Apr. 2018

Schröder, J. (29. September 2017). Sehdauer, Zuschauerzahlen, Marktanteile, Werbeumsätze: die großen Probleme der großen Privatsender. (meedia.de, Hrsg.). http://meedia.de/2017/09/29/ sehdauer-zuschauerzahlen-marktanteile-werbeumsaetze-die-grossen-probleme-der-grossen-privatsender/. Zugegriffen: 27. März 2018. 
Social Media Institute. (2016). Social Media Definitionen. http://socialmedia-institute.com/socialmedia-faq-begriffe-definitionen/. Zugegriffen: 5. Apr. 2018.

Social Media One. (kein Datum). Blogger, Influencer, Trendsetter - Wie wird man erfolgreicher Influencer? https://www.google.com/search?client=safari\&rls=en\&q=Blogger,+Influencer,+Trendsetter+-+Wie+wird+man+erfolgreicher+Influencer\%3F+social+media+one \&ie $=$ UTF-8\&oe $=U T F-8$. Zugegriffen: 14. März 2018.

SRF Schweiz. (4. August 2017). Bloggen und verdienen. https://www.srf.ch/news/panorama/ bloggen-und-verdienen. Zugegriffen: 19. März 2018.

Statista - The Statistic Portal. (2017). Number of monthly active Instagram users from January 2013 to September 2017 (in millions). https://www.statista.com/statistics/253577/number-of-monthly-active-instagram-users/. Zugegriffen: 24. März 2018.

Statista - The Statistic Portal. (2018). Annual growth of YouTube users worldwide from 2016 to 2021. https://www.statista.com/statistics/805671/youtube-viewer-number-growth-world/. Zugegriffen: 25. März 2018.

T3N. (2017). T3N. https://t3n.de/news/youtube-ueberrascht-852238/. Zugegriffen: 20. März 2018.

Trapp, F. T. (31. Januar 2018). Influencer Marketing - What drives Influencers? https://www.influencer-secrets.com. Zugegriffen: 28. März 2018.

Uwe Baltner, M. R. (2010). Social Media Lexikon - Von A wie Android bis Z wie Zattoo - 111 Begriffe kurz erklärt. (S. GmbH, Hrsg.) Ludwigsburg.

Weingarten, R. (9. März 2018). Instagram influencers are all starting to look the same. Here's why. (J. Brucculieri, Interviewer, \& Huffpost, Hrsg.).

www.omnicoreagency.com. (2018). YouTube by the numbers: Stats, demographics \& fun facts. https://www.omnicoreagency.com/youtube-statistics/. Zugegriffen: 15. März 2018.

Xeit GmbH. (2017). Xeit GmbH - Markeing \& Conversations. https://glossar.xeit.ch. Zugegriffen: 22. März 2018.

YouTube Wiki. (2016). Julien Bam. http://de.youtube.wikia.com/wiki/Julien_Bam. Zugegriffen: 26. März 2018.

YouTube Wiki. (2017). ApeCrime. http://de.youtube.wikia.com/wiki/ApeCrime. Zugegriffen: 29 2018.

YouTube Wiki. (2017). Simon Desue. http://de.youtube.wikia.com/wiki/Simon_Desue. Zugegriffen: 26. März 2018.

Von Rotz, Jonas (B.Sc. BA/jonas.vonrotz@gmx.ch) BSc Betriebsökonom 2018 (BFH (Bern), Unitec (Neuseeland), SeAMK (Finnland), Praktikum bei Bern Welcome im Bereich Kommunikation \& Content. Instagram Travel-Influencer unter dem Namen lets.capturetheworld. Während Studium 4 Jahre kaufmännische Erfahrung im Bereich Human Resources.

Tokarski, Kim Oliver (Prof. Dr./kim.tokarski@bfh.ch) Professor für Entrepreneurship und Unternehmensführung und Leiter der Abteilung Weiterbildung am Departement Wirtschaft der Berner Fachhochschule. Er ist Gastprofessor an der Wirtschaftsuniversität Bukarest (ASE Bukarest), Rumänien. Seine Forschungs- und Lehrtätigkeiten liegen in den Bereichen Entrepreneurship, Social Entrepreneurship, Innovation, Digitalisierung, Strategie sowie Leadership. Er ist Autor unterschiedlicher Publikationen in den genannten Themenkontexten. 
Open Access Dieses Kapitel wird unter der Creative Commons Namensnennung 4.0 International Lizenz (http://creativecommons.org/licenses/by/4.0/deed.de) veröffentlicht, welche die Nutzung, Vervielfältigung, Bearbeitung, Verbreitung und Wiedergabe in jeglichem Medium und Format erlaubt, sofern Sie den/die ursprünglichen Autor(en) und die Quelle ordnungsgemäß nennen, einen Link zur Creative Commons Lizenz beifügen und angeben, ob Änderungen vorgenommen wurden.

Die in diesem Kapitel enthaltenen Bilder und sonstiges Drittmaterial unterliegen ebenfalls der genannten Creative Commons Lizenz, sofern sich aus der Abbildungslegende nichts anderes ergibt. Sofern das betreffende Material nicht unter der genannten Creative Commons Lizenz steht und die betreffende Handlung nicht nach gesetzlichen Vorschriften erlaubt ist, ist für die oben aufgeführten Weiterverwendungen des Materials die Einwilligung des jeweiligen Rechteinhabers einzuholen.

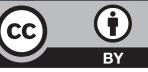

